\title{
Stream fish metacommunity and metapopulation ecology in a mined Appalachian watershed
}

\author{
Roy Worth Martin \\ West Virginia University
}

Follow this and additional works at: https://researchrepository.wvu.edu/etd

\section{Recommended Citation}

Martin, Roy Worth, "Stream fish metacommunity and metapopulation ecology in a mined Appalachian watershed" (2010). Graduate Theses, Dissertations, and Problem Reports. 3090.

https://researchrepository.wvu.edu/etd/3090

This Dissertation is protected by copyright and/or related rights. It has been brought to you by the The Research Repository @ WVU with permission from the rights-holder(s). You are free to use this Dissertation in any way that is permitted by the copyright and related rights legislation that applies to your use. For other uses you must obtain permission from the rights-holder(s) directly, unless additional rights are indicated by a Creative Commons license in the record and/ or on the work itself. This Dissertation has been accepted for inclusion in WVU Graduate Theses, Dissertations, and Problem Reports collection by an authorized administrator of The Research Repository @ WVU.

For more information, please contact researchrepository@mail.wvu.edu. 


\title{
STREAM FISH METACOMMUNITY AND METAPOPULATION ECOLOGY IN A MINED APPALACHIAN WATERSHED
}

\author{
Roy Worth Martin \\ Dissertation submitted to the \\ Davis College of Agriculture, Natural Resources and Design \\ At West Virginia University \\ in partial fulfillment of the requirements \\ for the degree of \\ Doctor of Philosophy \\ in
}

Forest Resources Management

Dr. J. Todd Petty, Committee Chair

Dr. E. James Harner

Dr. Michael P. Strager

Dr. Stuart A.Welsh

Dr. Paul F. Ziemkiewicz

Wildlife and Fisheries Resources Program

Morgantown, West Virginia

2010

Keywords: acid mine drainage (AMD), brook trout (Salvelinus fontinalis), capturemark-recapture, community ecology, habitat fragmentation, mass effect, metapopulation, metacommunity, mottled sculpin (Cottus bairdi), multistate models, neutral theory of biodiversity, patch dynamic, population dynamics, source-sink, species abundance distributions, species sorting, stream fish ecology, watershed management

Copyright 2010 Roy Worth Martin 


\title{
ABSTRACT \\ Stream Fish Metacommunity and Metapopulation Ecology in a Mined Appalachian Watershed
}

\author{
Roy Worth Martin
}

A central goal of the field of ecology is an improved understanding of the mechanisms responsible for determining patterns in species distribution, abundance and diversity. Understanding these mechanisms is crucial to the continued maintenance of valuable biological resources, such as recreational and commercial fisheries and aquatic biodiversity. The continued rapid loss of biodiversity on a global scale serves as a persistent reminder to scientists of the need for theoretical and empirical progress. Although threats are widespread across a diverse range of taxa, freshwater fishes are the most imperiled vertebrates worldwide and represent more than one quarter of the worldwide vertebrate fauna.

Arising from the need to predict the consequences of human-induced fragmentation of natural habitats, spatial or landscape ecology has emerged as a central paradigm in conservation ecology. However, empirical evaluation of theoretical landscape ecology lags far behind, particularly in studies of freshwater stream fishes. This is despite the obvious conservation benefits and the recognized tractability of stream systems to empirical testing given their well-defined topological structure and boundaries relative to terrestrial systems. 
There are few aquatic systems more conducive to research concerning the ecology of human-induced impairment than within the coal-mining region of Appalachia. Due to widespread bond forfeiture sites and abandoned mine lands, acid mine drainage (AMD) is a major source of pollution to surface waters in this region of West Virginia. AMD results from the extraction of high sulfur coal, which exposes an overburden layer of pyrite to oxygen and specialized bacteria. The result is a solution low in $\mathrm{pH}$ and high in sulfur and toxic metals draining from mines that can be detrimental to metal- and $\mathrm{pH}$-sensitive aquatic organisms. Local toxicity can be variable across the range of influx severity and dependent on local conditions such as alkalinity. While moderately impaired streams in this region can support a limited diversity of fishes and macroinvertebrates, severely impaired streams may be entirely devoid of either. In addition to direct local impairment, AMD can create chemical dispersal barriers, giving rise to fragmented networks, which may lead to local assemblage isolation.

The upper Monongahela River basin, specifically, is characterized by a highly variable range of local and regional conditions created by multi-scale variation in bedrock geology, coal geology, mining intensity and geography, acid precipitation, and stream thermal topology. This multi-scale variability results in a wide range of both local and watershed scale conditions that may be reflected in the fish community. For my dissertation, I used this system to address a series of broad research questions related to the theme of the spatial ecology of stream fishes. The dissertation is divided among three chapters. A common theme among the three chapters, however, is inquiry into the relative importance of local and regional processes driving stream fish population and 
assemblage dynamics in this system. The first two chapters are field tests of theory derived hypotheses, whereas the third chapter demonstrates the utility of a spatially and theoretically informed approach to managing stream fishes.

Chapter one is a direct empirical evaluation of metacommunity theory using stream fishes in a heavily mined and least impaired region of the basin. Metacommunity ecology, a new multi-scale paradigm, has provided important insights into mechanisms controlling regional patterns of assemblage structure. Metacommunity ecology offers four competing hypothetical models - neutral, patch dynamic, species sorting, and mass effect - each differing slightly in its underlying mechanistic framework. Modelspecific predictions were tested with a series of statistical analyses, which included: quantifying statistical relationships between $\alpha$ - and $\beta$-diversity and spatial and physicochemical gradients; fitting theoretical species abundance distribution curves to assemblage data; and testing for individual species response to local physicochemical conditions and spatial isolation.

Our results overwhelmingly supported the mass effect metacommunity model, which suggests that regional patterns of diversity are maintained by local habitat heterogeneity and dispersal among local assemblages. However, our findings also indicated that natural communities are structured simultaneously by a range of mechanisms, falling within a three dimensional continuum according to the relative importance of environmental heterogeneity, dispersal limitation, and competitive asymmetry. These findings emphasized that knowledge of habitat connectivity and spatial processes is crucial to the management of stream fish biodiversity in this system. To our knowledge, the study is the most complete direct evaluation of the competing 
metacommunity paradigms to date. Due to the pluralistic nature of the approach, the results will contribute significantly to the understanding of the mechanisms structuring local and regional diversity of stream fishes.

The second chapter details an experimental study in which we used field-based density perturbations to address questions concerning the importance of local and regional processes in determining local population dynamics. The relative importance of local controls (e.g., survival and recruitment) versus regional controls (e.g., dispersal) of stream fish population dynamics remains largely unknown. The overriding objective of this research was to quantify and characterize the extent to which the local population dynamics of two Appalachian, small stream fishes — brook trout (Salvelinus fontinalis) and mottled sculpin (Cottus bairdi) - were influenced by internal (i.e., population density, survival, and recruitment) versus external (i.e., immigration) processes as well as the extent to which those dynamics differed given the local (e.g. source or sink habitat) and regional context (i.e., proximity to a source versus sink habitat).

We used a 2 × 2 factorial design (density treatment $\mathrm{x}$ spatial location treatment), capture-mark-recapture techniques, and an information theoretic approach to quantify and characterize the effects of density perturbations on the population dynamics of the two study species. A key consideration in this study was whether population recovery differed depending on whether the focal stream was highly isolated in the headwaters or drained directly into a larger river mainstem because these areas represent important sources of colonizers for each species depending on the location.

Our results indicated that these small streams that are on the periphery of mottled sculpin distributions and within the core of the brook trout geographic distribution in this 
region may serve as functional sources for brook trout and functional sinks for mottled sculpin. We also demonstrated that, although the local context likely leads to predictable patterns in local processes, the regional context also modifies local dynamics. However, model inference provided little support for density dependence in estimates of apparent survival for either species. Nevertheless, there was some support for a density effect on the probability of transitioning among age classes both brook trout and mottled sculpin. Our study also resulted in some interesting, but unexpected, findings. For example, there was a nearly linear decreasing temporal trend in the immigration rate for both species among removal sites over the course of the study. This trend was coupled with a lagged increase in survival rates. Together, the findings suggested that the immediate recovery of the experimentally perturbed reaches was due an influx of predominantly mobile fish and that the subsequent return to a more locally driven dynamic over time was due to delayed accumulation of a more sedentary population segment.

Overall, our study suggested that stream fish population dynamics in these small streams are characterized by a strong source-sink dynamic coupled with relatively weak density dependence. The findings emphasize the importance of immigration and drainage network connectivity in the maintenance of Appalachian stream fish populations in small streams and were consistent with the view that knowledge of important source habitats and migration corridors is essential to the successful management of stream fishes.

The third chapter was a demonstration of the deflation of stream fish assemblages at the watershed scale as a result of accumulating local impacts. The specific objectives of the study were to: 1) develop models for predicting fish species occurrence and 
abundance as well as fish assemblage composition and integrity based on local (i.e., stream segment scale) and regional (i.e., drainage network scale) indicators of environmental quality; 2) quantify the relative "cost" of poor local and poor regional conditions on fish assemblages; and 3) identify thresholds at which the accumulation of impacts in the watershed affect biological conditions even in streams with good local conditions. Our results confirmed that there are measurable biological consequences to cumulative mining impacts within the regional stream network. We also observed substantial variability in species specific responses to local and regional conditions. Finally, we were able to generate explicit estimates of the relative biological costs associated with local and regional impairment on species abundance and likelihood of occurrence as well as assemblage composition and integrity. However, we did not observe threshold responses of fish metacommunities. Instead, watershed scale losses accumulated continuously across a range of watershed conditions. These findings indicated that effective management of stream fishes must consider the watershed context and should seek to affect local and regional conditions through the restoration and protection of interconnected drainage networks. 


\section{ACKNOWLEDGEMENTS}

I would first like to thank my close friends and family, especially my mother, Marilla Martin, father, Butch Martin, brother, Jack Martin, and partner, Jaci Gonzales, for supporting me unwaveringly throughout this lengthy and demanding endeavor. I would also like to especially thank my father and our long-time fishing partners and good friends, George MacMillan and Jack McHan, for impressing upon me at the age of approximately 9 years while standing on a bridge overlooking the Gallatin River near Big Sky, Montana that my future was in "stream ecology or aquatic entomology". At the time, I had little idea what those fields were, but it was the first notion that I had that a career related to streams was possible. In the end, had it not been for my father and his friends' willingness to let a young kid tag along on their many fishing adventures from the Blue Ridge Mountains of Georgia to the Rocky Mountains, I likely would not have developed a passion and interest in the outdoors, the natural sciences, and natural resources conservation and management.

I would also like to thank the graduate students, staff, and faculty of WVU, past and present, who provided field help, advice and friendship throughout my graduate career. Specifically, many thanks are in order for my friend, Dr. Jason Freund, with whom I worked with in collecting much of the data for this research. In fact, much of my research extends directly from Dr. Fruend's own innovative dissertation research. The years we worked together collecting data and collaborating will always be among my favorite memories of graduate school. Likewise, I would like to thank my friend and colleague Dr. George Merovich for his help in the field during the early phases of data collection for this project as well for his always helpful scientific and statistical advice. I would also like to thank Zena Hense, who finished her MS at WVU using data that we collected jointly for our research projects. Zina provided invaluable assistance in the field and also served as an important field crew leader supervising the collection of some of data related to the first and third chapters of this dissertation. Similarly, I would like to thank Ira Poplar-Jeffers, who also finished his MS at WVU, for his leadership and hard work in the collection of data used in this research. I would also like to thank Jen Fulton for her friendship, advice, and for keeping us all in order during her stint as the research coordinator in our lab. Jen helped in the collection and management of much of the water chemistry data used in the first and third chapters of this dissertation and also trained me in the fish marking techniques that were used in the mark-recapture study (chapter 2). I would also like to thank the many other staff members, undergraduates, and graduate students who helped in the field for their friendship, hard work, laughs and good times while camping and traveling around the state collecting important data, including Patrick Amick, Patrick Blosser, Luke Bolyard, Ryan Brahm, Jason Clingerman, Adam Cotchen, Seth Davis, Eric Gladwell, Jared Gregory, Tony Grubb, Zachary Golightly, Lee Hagerty, Tom Hardman, Chris Harvey, Mathias Hickman, Brandon Keplinger, Seth Lemley, Zach Liller, Sarah McClurg, Holy Morris, Chris Nelson, Cassie Nulph, Joe Osborne, Misty Phillips, Brock Reggi, Dan Ryan, John Safstrom, Alan Shipley, Dustin Smith, Dave Thomas, David Thorne, Josh Vance, and Logan Wamsley. I 
was extremely fortunate to get to know such an exceptional group of hard working and friendly people.

In addition, I would especially like to thank Dan Cincotta, Gina Graziani, Otto High, Susanna Scott, Aaron Yeager, and the West Virginia Division of Natural Resources for providing assistance with the collection of some of the data related to the markrecapture study. Without these hard working and kind folks, I likely would not have had enough hands to complete a crucial round of fish sampling in the fall of 2006. I am truly indebted to these people for offering to help me out when others at WVU were unavailable due to classes.

I also owe a great deal of gratitude to Dr. Jim Hines of the United States Geological Survey's Patuxent Wildlife Research Center for his technical assistance with the statistical analysis of the mark-recapture data. Dr. Hines provided me with a custom program for analyzing the data that very likely saved me months of time.

Finally, I would like to extend my sincere thanks to the members of my graduate committee - Dr. Jim Harner, Dr. Todd Petty, Dr. Mike Strager, Dr. Stuart Welsh, and Dr. Paul Ziemkiewicz - for their sound advice, guidance, enthusiasm and encouragement throughout the duration of my dissertation research. I thank Dr. Jim Harner for sparking my interest in applied statistical analysis and also for introducing me to the program R, which continues to be a passion of mine both as a hobby and in my work. I thank Dr. Strager not only for his friendship and eagerness to provide professional and technical advice, but also for his willingness to remain an enthusiastic member of my committee throughout both my MS and PhD projects. I also thank Dr. Stuart Welsh for remaining on my committee throughout my MS and $\mathrm{PhD}$ projects and for his willingness to lend both advice and many helpful textbooks that I used in my research. I also thank him for introducing me to the information theoretic approach, which served as a vital part of this dissertation. I thank Dr. Paul Ziemkiewicz for his input concerning this research and also for providing me with some of the most helpful and practical advice I received in graduate school during my comprehensive exams. Finally, words could not appropriately express the level of gratitude owed to my graduate advisor, Dr. Todd Petty, for his continued professional and personal support throughout both my MS and PhD careers. Specifically, his characteristically unrelenting enthusiasm for all things science and his ability to always inspire and encourage a creative, innovative, and, yet, practical approach to science and natural resource management are professional traits that I will continue to aspire to. A graduate student absolutely could not have a better mentor and, just as well, a person could not ask for a more admirable friend. I look forward to our continued friendship and professional collaboration.

Funding for this project was provided, in part, by the Electric Power and Research Institute, Allegheny Energy, the National Research Center for Coal and Energy, and the United States Environmental Protection Agency's Science to Achieve Results program. 


\section{TABLE OF CONTENTS}

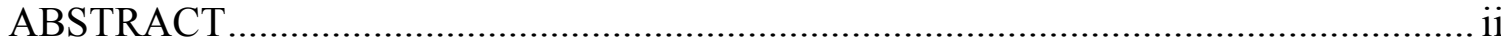

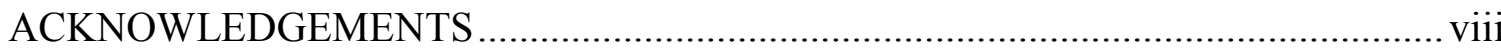

CHAPTER 1: An Empirical Evaluation of Metacommunity Theory: Stream Fish

Metacommunity Patterns in a Fragmented Appalachian Watershed ............................. 1

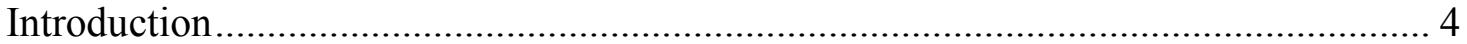

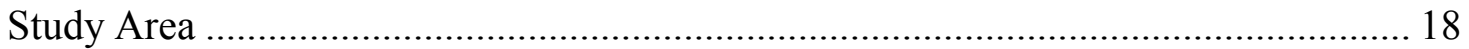

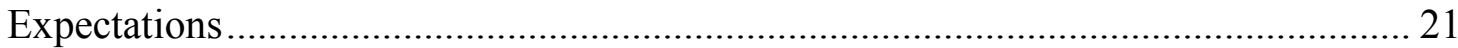

Patterns in diversity related to environmental and spatial gradients..................... 22

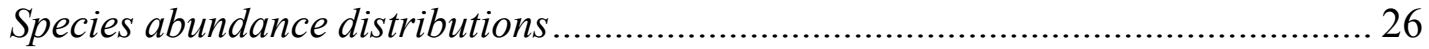

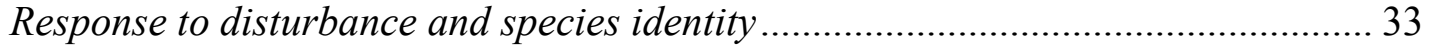

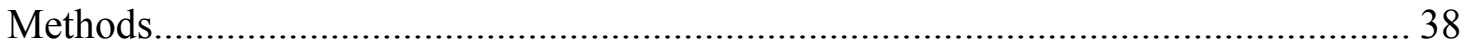

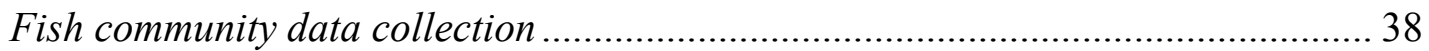

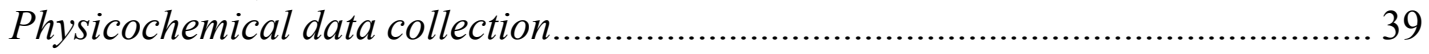

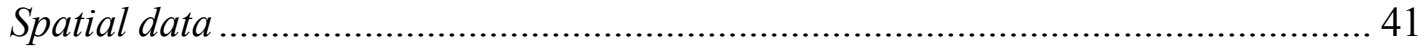

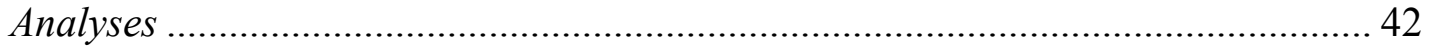

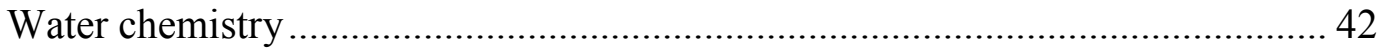

Response of diversity to physicochemical conditions and space........................ 43

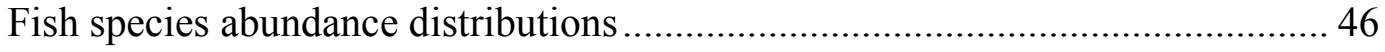

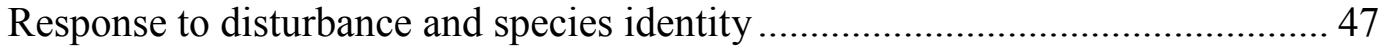

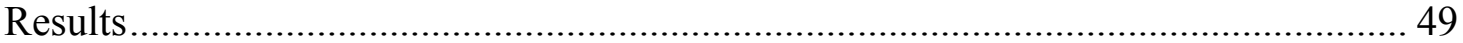

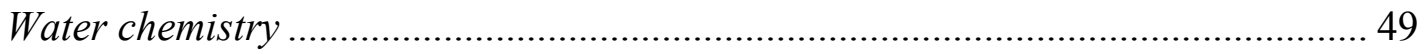

Correlating diversity to physicochemical and spatial gradients ............................ 50

Local species richness in the intact versus fragmented system .............................. 53

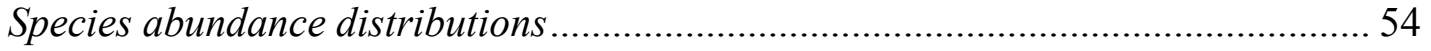

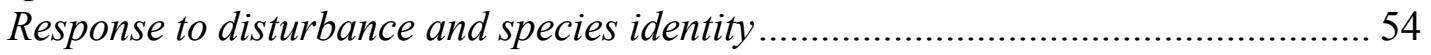

Summary of results pursuant of a priori expectations ........................................... 58

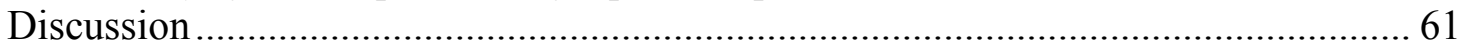

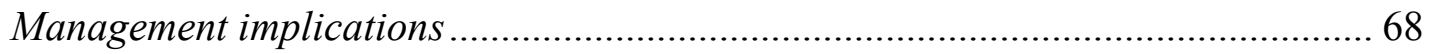

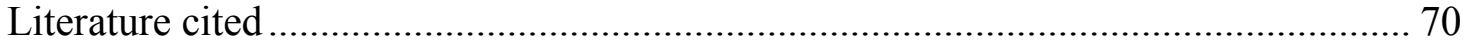

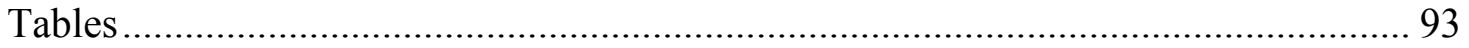

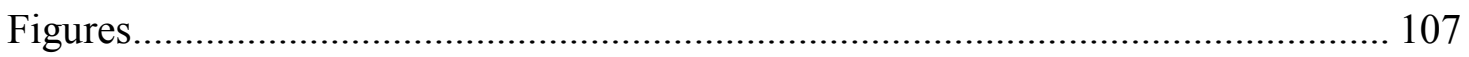

CHAPTER 2: Density Reduction Experiments Reveal Complex Metapopulation

Dynamics of Headwater Stream Fish Populations.................................................... 116

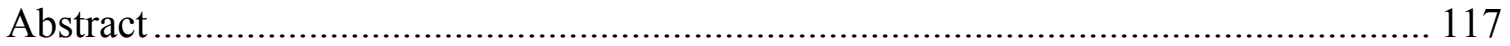

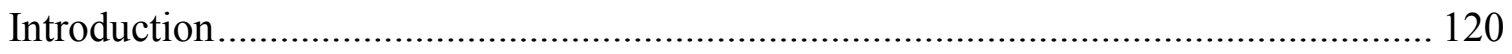

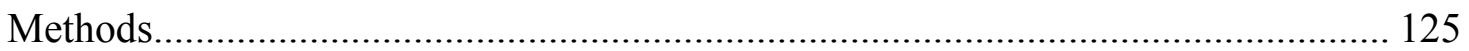

Brook trout, mottled sculpin, and small streams as a test system ........................ 125

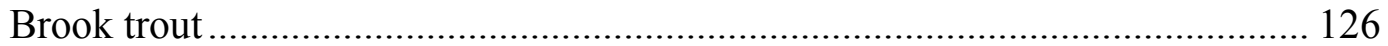

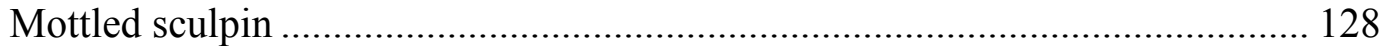

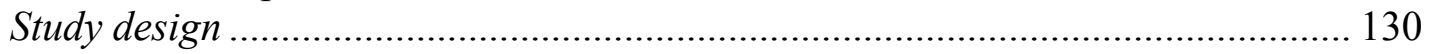

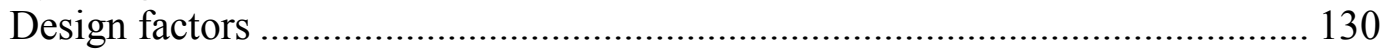

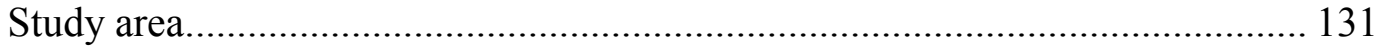

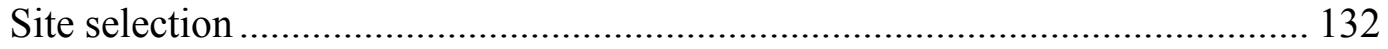

Fish removal and seasonal fish sampling and tagging..................................... 134 


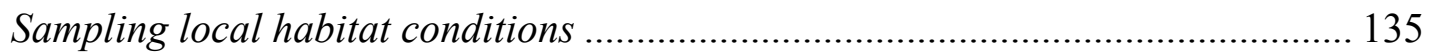

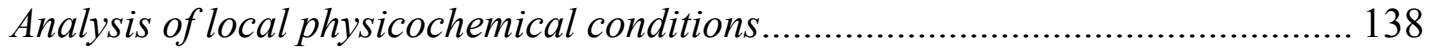

Model selection and inference methodology....................................................... 139

Estimating capture probability and abundance ................................................... 142

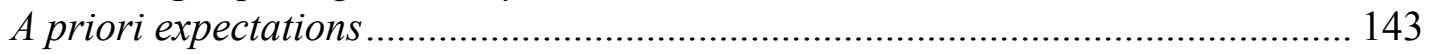

Population dynamics in the core versus periphery ………….......................... 143

Population dynamics and the regional context ............................................. 144

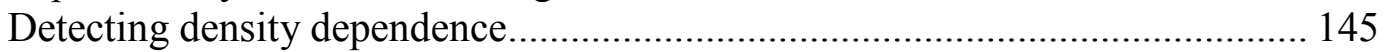

Density dependence in the core versus periphery ............................................. 145

Modeling the population response variables ...................................................... 146

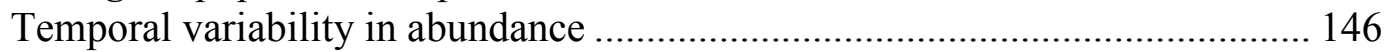

Population recovery following removal...................................................... 148

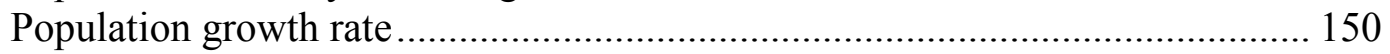

Apparent survival and probability of age class transition.................................... 151

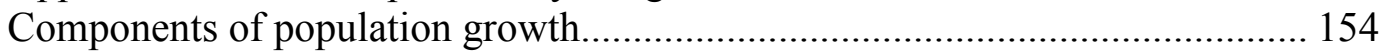

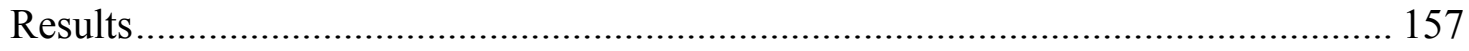

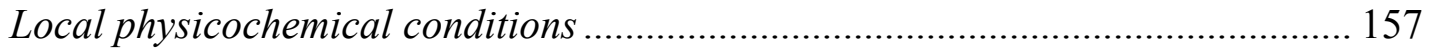

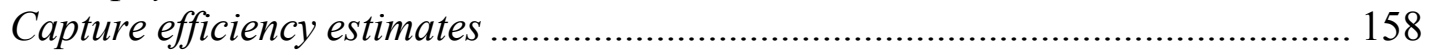

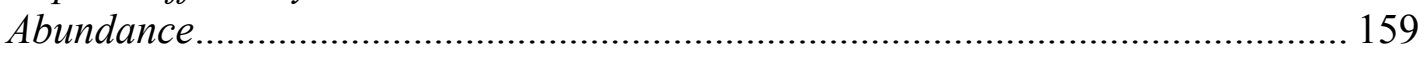

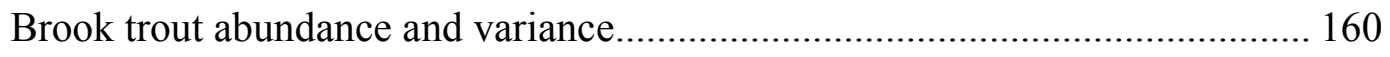

Mottled sculpin abundance and variance........................................................... 161

Comparison of abundance variability between species ....................................... 161

Population recovery following removal treatment ............................................... 162

Pre-and post-removal abundance estimates ........................................................ 162

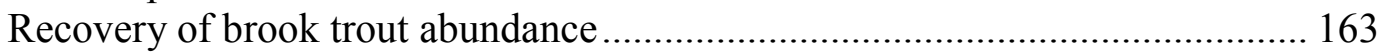

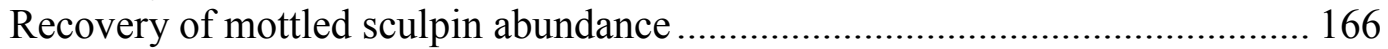

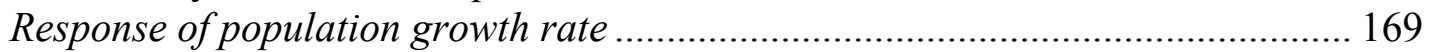

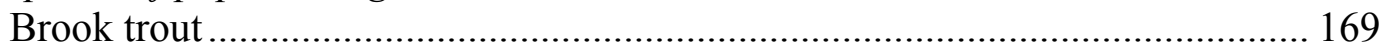

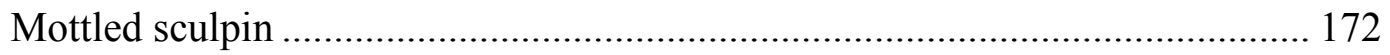

Response of apparent survival and age-class transition probability..................... 176

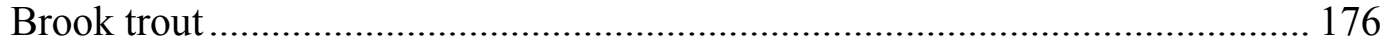

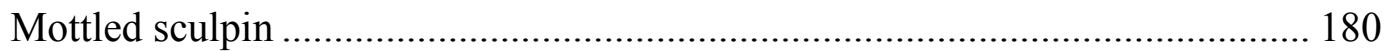

Response of the components of population growth................................................ 184

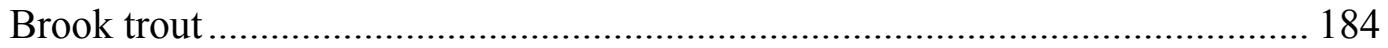

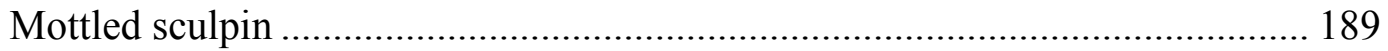

Instantaneous survival, local recruitment, and immigration rates ......................... 192

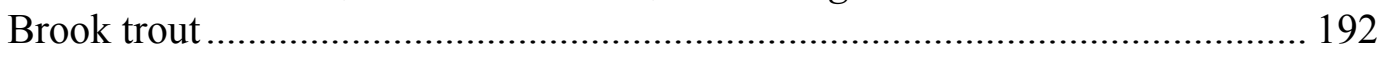

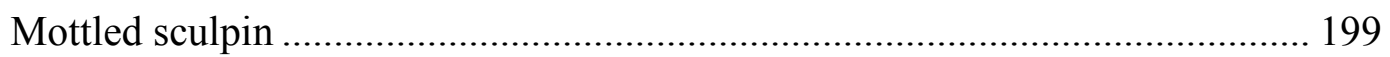

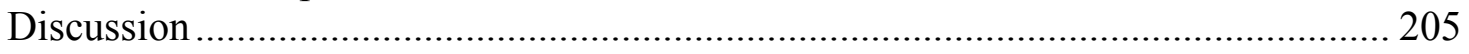

Local dynamics within the core versus the periphery ......................................... 205

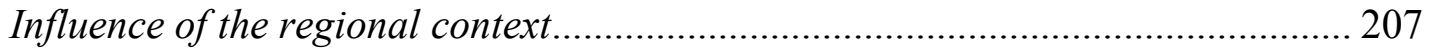

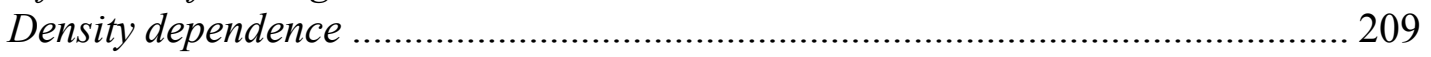

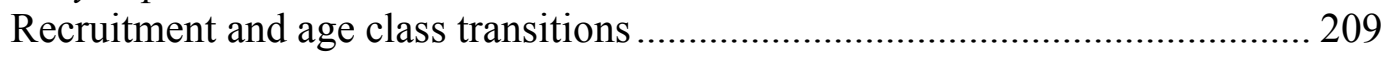

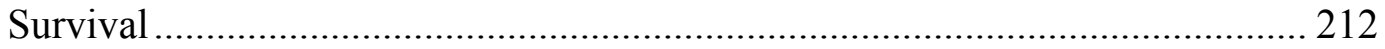

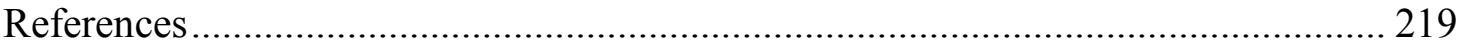

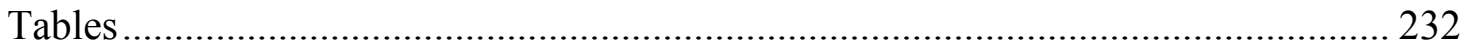




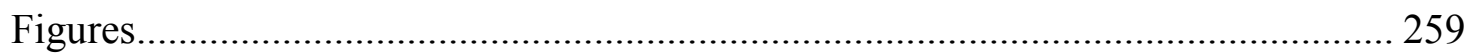

CHAPTER 3: Watershed Scale Deflation of Stream Fish Metacommunities in an

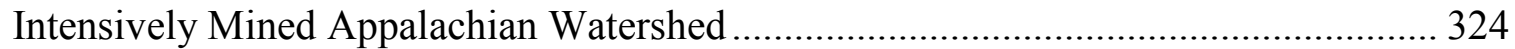

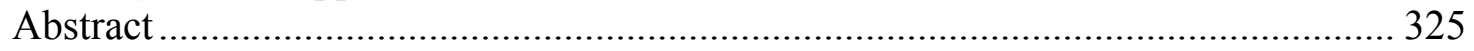

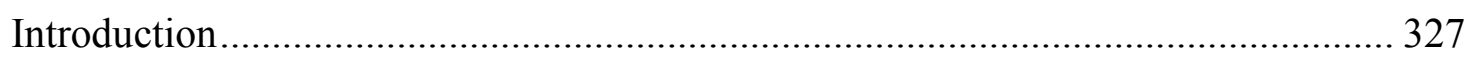

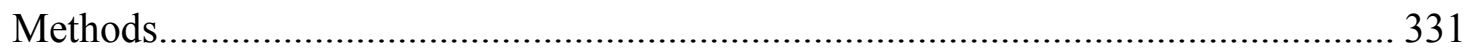

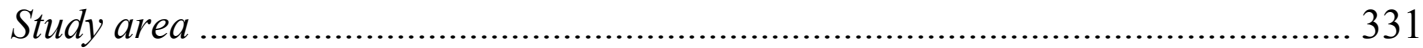

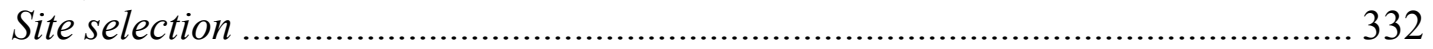

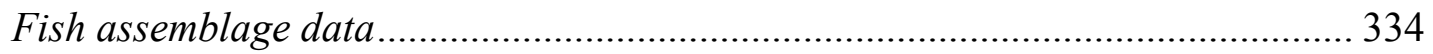

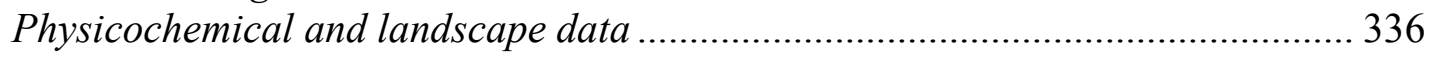

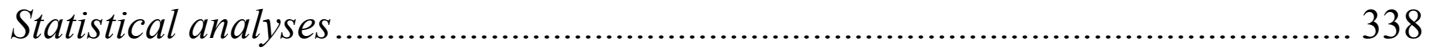

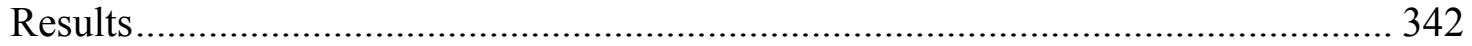

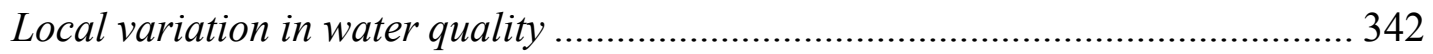

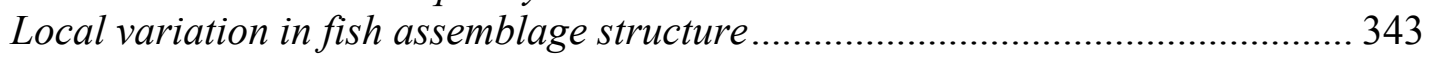

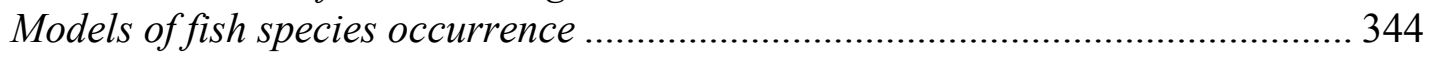

Models of species' abundance .............................................................................. 346

Summarizing the effects of local and regional condition on specie' distributions. 349

Fish community response to local and regional conditions ................................ 351

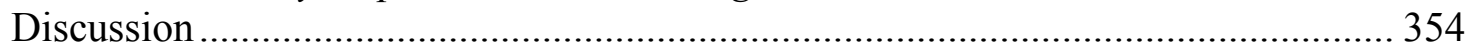

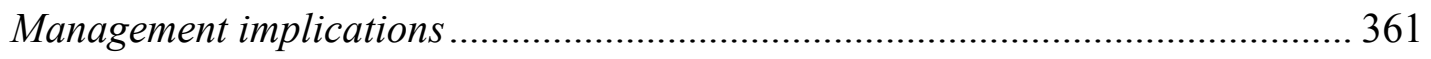

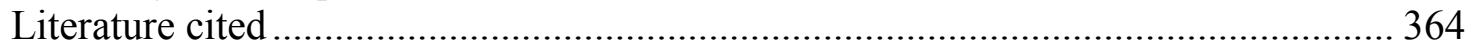

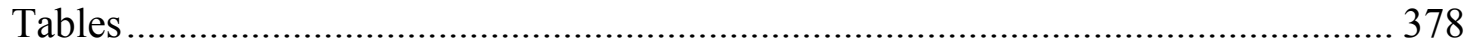

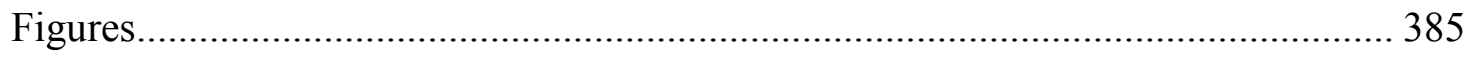

Appendix A: Summary of model-averaged capture efficiency model coefficients and

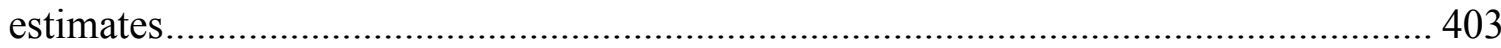

Appendix B: Length-frequency distributions of brook trout and mottled sculpin......... 429 Appendix C: Model-averaged coefficients for estimating log-transformed proportional recovery of abundance ....................................................................................... 431 Appendix D: Model-averaged coefficients for estimating population per capita growth

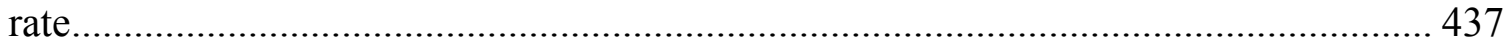

Appendix E: Survival and age class transition probability coefficients and estimates... 443 Appendix F: Model coefficients and parameter estimates for estimating components of instantaneous population growth rate 467 
CHAPTER 1: An Empirical Evaluation of Metacommunity Theory: Stream Fish Metacommunity Patterns in a Fragmented Appalachian Watershed

Roy W. Martin* and J. Todd Petty

Division of Forestry \& Natural Resources, West Virginia University, Morgantown, WV 26506

*Corresponding author: royworth@gmail.com 


\section{Abstract}

Metacommunity ecology, a new multi-scale paradigm, has provided important insights into mechanisms controlling local and regional patterns of assemblage structure. Metacommunity theory is comprised of four competing models — neutral, patch dynamic, species sorting, and mass effect — each differing slightly in its underlying mechanistic framework. Although theoretical development of these models has proceeded rapidly, empirical evaluation has lagged. Consequently, we conducted a direct test of competing metacommunity models using assemblages of stream fishes from a region heavily impacted by acid mine drainage (AMD). Model-specific predictions were tested with a series of statistical analyses, which included: quantifying statistical relationships between $\alpha$ - and $\beta$-diversity and spatial and physicochemical gradients; fitting theoretical species abundance distribution curves to assemblage data; and testing for individual species response to local physicochemical conditions and spatial isolation. In order to examine the extent to which dispersal limitation and environmental sorting due to AMD influenced diversity patterns, analyses were divided between a highly fragmented region of the study area and a least impaired, intact region.

Our analyses indicated that species sorting along environmental gradients and dispersal limitation interact to determine local and regional patterns of fish assemblage structure in this system. Our analyses also indicated that the zero sum multinomial (ZSM) species abundance distribution (a prediction of the neutral model) was consistently the best fitting model across both the fragmented and intact systems. However, the fit was likely unrelated to the assumptions of neutrality. An analysis of 
changes in species relative abundances and occupancy revealed significant, non-random inflation and deflation of some species in the fragmented relative to the intact system. Subsequent analyses of individual species' responses to isolation and local physicochemical variability suggested that many species' occurrence or abundance was related to both local physicochemical conditions and isolation. Also, the results indicated that some species may form deterministic metapopulations, meaning that their tendency to form metapopulations was presumably related to interactions with abiotic conditions or other species.

Our results overwhelmingly supported the mass effect metacommunity model, which suggests that regional patterns of diversity are maintained by local habitat heterogeneity and dispersal among local assemblages. However, our findings also indicated that natural communities are structured simultaneously by a range of mechanisms falling within a three dimensional continuum according to the relative importance of environmental heterogeneity, dispersal limitation, and competitive asymmetry. Our findings clearly emphasized that knowledge of habitat connectivity and spatial processes is crucial to the management of stream fish biodiversity. To our knowledge, our study is the most complete direct evaluation of the competing metacommunity paradigms to date. Due to the pluralistic nature of our approach, our results contribute significantly to our understanding of the mechanisms structuring local and regional diversity of stream fishes. 


\section{Introduction}

Two long standing goals for the discipline of ecology have been to develop sound theoretical and applied frameworks for (1) understanding the mechanisms giving rise to empirical patterns in diversity and (2) predicting patterns in diversity. There are two general reasons as to why ecologists continue to place so much emphasis on these problems. First, but not necessarily foremost, patterns in the kinds and nature of organisms and their genetic, phenotypic, or functional diversity are simply awe-inspiring and questions surrounding those patterns foster both scientific curiosity and a conservation ethic. Second, answers to questions surrounding the nature of diversity and its maintenance are crucial to the ongoing quest to understand the role of biodiversity in ecosystem function and the maintenance of ecosystem services (e.g., clean water, healthy fisheries, Naeem et al. 1997, Loreau et al. 2001, Naeem et al. 2002).

Scientists are increasingly realizing that the goal of predicting and understanding patterns in biodiversity looms more heavily with the passing time, as rapid, worldwide population declines and extinctions continue to mount with the ongoing loss of natural habitats (Koh et al. 2004). In fact, some have estimated that up to $50 \%$ of worldwide floral and faunal species will be lost in the next 50 years (Pimm and Raven 2000, Thomas et al. 2004). Perhaps the popular ecologist E. O. Wilson described the situation most succinctly in his book, The Future of Life (2001), when describing the discipline of conservation biology as "a discipline with a deadline".

Although threats to biodiversity are widespread across plant and animal taxa, it has been suggested that the threat to biodiversity loss in freshwater habitats may be 
grave enough to receive additional attention (Ricciardi and Rasmussen 1999, Abell 2002). For example, freshwater fishes are the most imperiled vertebrate worldwide (Allen and Flecker 1993, Leidy and Moyle 1998) and represent more than one quarter of the worldwide vertebrate fauna. North America, in particular, harbors the greatest diversity of temperature fishes worldwide, yet many have experienced an alarming decline for at least the last 50 years (Fausch et al. 2002).

Obviously, the goal of understanding and predicting patterns in diversity is of the utmost importance to its conservation. However, a unified theoretical framework for dealing with questions concerning biodiversity continues to elude ecologists, although substantial progress has been made. Though the theoretical foundation continues to evolve, the general patterns of diversity ecologists aim to explain have been long established due to several decades of important theoretical and empirical work. They include measures of diversity locally ( $\alpha$-diversity), among locations ( $\beta$-diversity), and regionally $(\gamma$-diversity $=$ sum of $\alpha$-diversity across all localities in the region, Whittaker 1960, Magurran 1988), their relationship with one another, and the extent to which they vary in conjunction with environmental, biological, temporal, and spatial factors or gradients (Holyoak et al. 2005a).

Initial theoretical and empirical research aimed at explaining diversity patterns, which still hold much influence today, were focused largely on explanations of localized patterns in diversity. Building heavily upon Hutchinson's (1957) foundational theory of the ecological niche, a number of scientists offered a theory of diversity that employed a list of "assembly rules", which were based on the ecological niches or functional roles of individual species in the community (e.g., MacArthur 1970, Levin 1970, Diamond 
1975). This view suggested that species coexist in an interactive equilibrium with other species in the community and that stability of the community and its resistance to disturbance were derived from the adaptive equilibrium of member species, each of which evolved to be the best competitor in its own ecological niche. As a result, nicheassembled communities were described as limited-membership assemblages within which interspecific competition for limited resources and other biotic interactions determine the occurrence and abundances of species the community. Nonetheless, while many of the details of niche theory continue to be debated in the current literature, it has been increasingly recognized that an important shortcoming of the early theoretical work is that it advanced largely under the assumption that localized and non-spatial processes, as opposed to spatial heterogeneity and/or migration, were the primary drivers of diversity (Holyoak et al. 2005a).

Since at least the 1960s and publishing of MacArthur and Wilson's $(1963,1967)$ paradigmatic theory of island biogeography, ecologists have questioned the extent to which spatial processes drive patterns in diversity. This is due, at least partly, to the realization that the failure to incorporate space has often lead to spurious predictions and conclusions about diversity (Holyoak et al. 2005a). For example, MacArthur and Wilson's $(1963,1967)$ theory of island biogeography explained what would have been, under the theory of the time period, an unexpected pattern in the diversity of birds on islands off the coast of New Guinea. At that time, the species area relationship, or the well known tendency for there to be a positive relationship between the number of species and habitat size, was a well documented empirical pattern in diversity and most of the theoretical attempts at explaining that pattern were based on local processes 
related to niche theory and competitive equilibrium (e.g., Preston 1962). However, MacArthur and Wilson $(1963,1967)$ demonstrated that, although island size was an important determinant of the diversity of birds off the coast of New Guinea, distance from the mainland, or the source of colonizers, helped explain why diversity often differed even among islands of the same size.

In the years since MacArthur and Wilson $(1963,1967)$ published their theory, the roles of space and dispersal, in particular, in biodiversity and population theory have grown substantially (e.g., Hanski 1997, Chesson 2000, Holt 2002, see Hoopes et al. 2005 for a thorough historical review). This is due, not only to the continued recognition that conclusions from the traditional, non-spatial, niche perspective often prove misleading, but also to the increasing awareness and concerns associated with human alteration and fragmentation of habitats at larger spatial scales (Holyoak et al. 2005a). One of the earliest of such models was Levins' (1970) classic metapopulation model. Although the model only accounts for a single species and is implicitly spatial, Levins' (1970) model, in addition to MacArthur and Wilson's (1963, 1967) model, continues to serve as the intellectual foundation upon which most current metapopulation and metacommunity models have been built.

The onset of metapopulation theory following Levins' (1970) classic model is generally recognized as a paradigm shift from the previously prevailing island biogeography theory (Hanksi 1989). Levins' (1970) model is now referred to in the greater context of the metapopulation biology field as a simple, "patch-occupancy" model, because the regional population is modeled as a collection of discrete populations, or patches, such that each has a finite probability of extinction (Levins 
1970, Hoopes et al. 2005). Each patch only has two states — occupied or empty — and, therefore, local dynamics are ignored and assumed to happen on a slower time scale than regional dynamics (Levins 1970). Again, space was modeled implicitly, meaning that it was assumed that each population was equally accessible from every other population in the region or metapopulation (Levins 1970). Additionally, each individual population, or patch, was assumed to be independent, but identical, meaning that patch colonization and extinction probabilities are fixed (Levins 1970).

Current models are distinguishable from Levins (1970) early formulation, and from one another, primarily by the extent to which the assumptions of (1) homogeneity/heterogeneity in patches (e.g., patch quality), (2) implicit/explicit local dynamics, and (3) implicit/explicit space are modified. For example, Hanski (1994) recognized that incorporating heterogeneity in patch quality could increase regional persistence of the metapopulation. Others also modeled local dynamics explicitly and found that heterogeneity in patch quality promotes source-sink dynamics (Pulliam 1988) and rescue effects (e.g., Holt 1985, Amarasekare 1998). Sources are areas within which species experience positive population growth and sinks are areas in which they experience negative population growth (Holt 1985, Pulliam 1988). Populations in sinks are maintained only by dispersal from sources (Holt 1985, Pulliam 1988). A rescue effect refers to the case where emigrants from patches of high density prevent low density patches from declining to extinction (Holt 1985). Finally, Bolker and Pacala (1997) demonstrated that making dispersal explicit enforces a dispersal limiting process, such that immigrants must come from a finite distance. They also demonstrated that incorporation of explicit space into single species models can lead to local and regional 
pattern formation (Bolker and Pacala 1997). In a similar manner to adding heterogeneity to patches, modeling space explicitly can also modify persistence of the metapopulation (Bolker and Pacala 1997).

Models with multiple species are concerned with not only the mechanisms that promote the persistence of individual species, but also the mechanisms that promote coexistence among two or more species. While early work examining coexistence was focused primarily at the local scale and typically concerned explanations following from traditional and non-spatial niche theory and the competitive exclusion principle (Hutchinson 1957, e.g., “Assembly rules”, Levin 1970, MacArthur 1970, Diamond 1975), more recent theory recognizes the importance of spatial patchiness and heterogeneity (Mouquet et al. 2005). This important realization that spatial structure leads to differentiation among local and regional processes recognition has since led to more comprehensive and realistic theories of coexistence (Mouquet et al. 2005).

Early formulations were multi-species extensions of Levin's (1970) original model, such that each species colonizes a single, homogeneous, patch in the regional community and coexistence is maintained by competition-colonization tradeoffs (Levins and Culver 1971, Hastings 1980, Tilman 1994). In other words, inferior competitors are able persist in the region by making quicker use of empty patches after local extinctions than superior competitors. One drawback to these earlier models, however, is that local dynamics are assumed to occur on a much faster time scale than regional dynamics because colonization and competitive exclusion is assumed instantaneous. Later models incorporated heterogeneity within patches by allowing co-occurrence of competing species within some patches, which allowed inferior competitors to colonize patches 
while increasing the extinction rate of superior competitors, increasing the likelihood of coexistence (Slatkin 1974). These models ultimately led to the realization that incorporation of spatial heterogeneity in species' abundances promotes "regional similarity" and, thus, coexistence (Mouquet and Loreau 2002). In other words, it was realized that, although species must differ in their abilities to compete within a patch, they must have compensating differences in those abilities that makes them similar at the regional scale in order to coexist (Mouquet and Loreau 2002).

These models and concepts then served as important precursors to source-sink models of coexistence (e.g., Levin 1974, Sale 1977, Schmida and Ellner 1984, Wilson 1992, Mouquet and Loreau 2002, 2003; Mouquet et al. 2005). As opposed to variation solely in species competitive ability, the source-sink formulation focuses on largely on spatial heterogeneity in the environment to give rise to spatial heterogeneity in abundances (Mouquet et al. 2005). These models are different from the more classic formulations in that there is (1) heterogeneity among patches, (2) local and regional dynamics operate on the same time scale and (3) migration is high and can change the outcome of local competition through mass effects (Schmida and Whittaker 1981, Mouquet and Loreau 2002, 2003; Mouquet et al. 2005).

Ultimately, the recent proliferation of spatial models of community coexistence led to a new paradigm in community ecology and the birth of the new sub-discipline of metacommunity ecology (Wilson 1992, Leibold et al. 2004, Holyoak et al. 2005b). The metacommunity ecology field is primarily concerned with the unification and development of these and other concepts for use in the advancement of a more complete theory of biodiversity (Leibold et al. 2004, Holyoak et al. 2005b). Leibold et al. (2004) 
define the metacommunity as "a set of local communities that are linked by dispersal of multiple potentially interacting species". The "metacommunity concept" describes the general framework within which scientists explore a suite of potential mechanisms that give rise to variability in local and regional patterns of species abundance and diversity (Leibold et al. 2004, Holyoak et al. 2005a). In a recent review, Leibold et al. (2004) characterized four metacommunity models that currently are driving the metacommunity paradigm forward: neutral, patch dynamics, species sorting and mass effect models. Each metacommunity model details the relative roles of local and regional processes in structuring ecological communities. The four models differ primarily in the extent to which they emphasize demographic or niche differences among species (e.g., competition-colonization vs. source-sink processes), stochastic processes, and dispersal limitation (e.g., local versus global dispersal, Leibold et al. 2004, Figure 1).

Species sorting models align closely with traditional niche-based explanations of community patterns, such that fitness and demographic rates are assumed closely tied to local abiotic environmental characteristics through which species "sort" themselves (i.e., akin to niche partitioning along environmental axes) so each is favored under a particular set of local conditions (Leibold 1998, Chase and Leibold 2003, Chase et al. 2005). Thus, the species sorting model explicitly assumes that abiotic heterogeneity is the primary driver of coexistence in the metacommunity and species traits, by contrast, in terms of colonizing or competitive ability (i.e., heterogeneity in competitive interactions), are largely ignored. Dispersal among the local communities is implied in these models, because it does not change the predictions (Chase et al. 2005). Specifically, dispersal is global, such that each species is expected to be able to reach 
any locality in the metacommunity within which it is favored by the local conditions even if the localities are far apart (Chase et al. 2005). Dispersal is sufficiently low and never to the extent at which a mass effect is produced, which enables persistence of inferior competitors in sink habitats (Schmida and Ellner 1984, see mass effect model framework discussed below). Pond plankton are thought to provide a good example of this type of metacommunity (Leibold et al. 2004) because (1) they are generally highly resistant to invasion (Shurin 2000, 2001), even under conditions with unusually high immigration rates (Cottenie et al. 2003), and (2) there is typically strong correlation between local composition and local abiotic conditions (Leibold 1999, Cottenie et al. 2003).

Neutral theory provides a stark contrast to niche-based theories of coexistence. There are several versions of neutral metacommunity models in ecology (Bell 2000, 2001, Hubbell 2001), but Hubbell's (2001) recent work on his "unified neutral theory of biodiversity" has received the most attention. Neutral theory, as described by Hubbell (2001), provides an alternative to models deriving coexistence from niche- (e.g., physicochemical habitat preference) or trait-based (e.g., fecundity, survival, dispersal, competitive rank) differences among species and, thus, and was developed, at least partly, as a null model for comparison. Hubbell's (2001) model, thus, works under an assumption of "ecological equivalence" such that trophically similar individuals are assumed to be demographically similar on a per capita basis. Hubbell's (2001) model was developed on the intellectual foundation originally described by MacArthur and Wilson's $(1963,1967)$ in their island biogeography model and, like the island biogeography model, is a class of "mainland-island" model. From the neutral 
perspective, the drivers of species coexistence and local and regional community composition are simply dispersal limitation and stochastic variation in demographic and evolutionary processes. It is, thus, assumed that environmental variation among localities does not lead to variation in species' birth or death rates. Essentially, each species in a local community is on a random walk to extinction, while immigration and speciation occur fast enough at the metacommunity scale to balance the rates of extinction and promote coexistence (Hubbell 2001). The regional metacommunity (i.e., "mainland"), thus, serves as a source of immigrants and new species to local communities (i.e., "islands"). Hubbell's (2001) model remains hotly debated in the ecological literature, due to its remarkable ability to predict diversity patterns (but see McGill 2003, McGill et al. 2006) while focusing solely on stochastic demography and localized dispersal and ignoring individual species' traits. Hubbell's (2001) model, therefore, strongly advocates for an important role of localized dispersal in metacommunity models. Early empirical support for neutral theory came primarily from Hubbell's (2001) own extensive inventory of tree diversity on Barro Colorado Island, Panama (Volkov et al. 2003). However, the extent to which those data fit the neutral assumptions remains debated (McGill 2003, McGill et al. 2006). Nonetheless, the model has been shown capable of accurately predicting diversity for a wide range of plant and animal taxa (Hubbell 2001).

Patch dynamic models are similar to the neutral approach in that they typically assume a network of identical patches (i.e., no effect of environmental variability) such that each species has a finite rate of extinction in a patch. Local diversity is then limited by either dispersal or species interactions (Chase et al. 2005). The emphasis of patch 
dynamic models is typically on tradeoffs in species' abilities to colonize, reproduce and disperse, which promotes coexistence through the formation of spatio-temporal niches (i.e., competition-colonization tradeoffs, Levins and Culver 1971, Hastings 1980, Tilman 1994, Moquet et al. 2002, Shurin et al. 2004; Chase et al. 2005). As described earlier, in a competition-colonization tradeoff model, a metacommunity comprised of both good and poor colonizers (e.g., based on dispersal or reproductive ecology) is able to sustain coexistence only when poorer competitors are the better colonizers. This leads to a situation where good colonizers are only able to make use of sites unoccupied by superior competitors, and coexistence is maintained by extinction dynamics. Like the species sorting model, dispersal is typically global in the patch dynamic model, such that colonizers are assumed able to reach all localities in the metacommunity. Hanski's (1998) work on butterfly metapopulations provides some of the more classic empirical examples of patch dynamic systems. Nouhys and Hanski (2005) also provide an interesting case study demonstrating interactions between butterflies, their host plants, and their parasitoids. This system provided a clear example of strong interspecific interactions within a metacommunity of 10 species of butterflies in the forms of tradeoffs in competitive ability and dispersal rate among species (Nouhys and Hanksi 2005).

Finally, the mass effect framework (Levin 1974, Sale 1977, Amarasekare and Nisbet 2001, Mouquet and Loreau 2002, 2003, Mouquet et al. 2006), like the species sorting framework, assumes that abiotic environmental heterogeneity results in some species being favored in specific localities over others. The mass-effect model, however, also allows species to persist as sink populations (sensu Pulliam 1988) in local 
habitats where they are not favored so long as they are maintained by immigration from those where they are favored (Mouquet and Loreau 2003, Leibold et al. 2004, Holyoak et al. 2005a, Mouquet et al. 2006). Like the species sorting and patch dynamic frameworks, dispersal has been modeled as global in current models (e.g., Mouquet et al. 2002, 2003). Some of the best examples of communities dominated by mass effects come from experimental fragmentation studies (Leibold et al. 2004). For example, Gonzalez et al. (1998) demonstrated that providing habitat corridors within a fragmented community of microarthropods mitigated losses of diversity. Similarly, Holyoak (2000) demonstrated that the rate of species loss in a protist community was reduced by adding corridors.

Early empirical testing of metacommunity theory suggests that each of the competing metacommunity models may be relevant to a range of natural ecosystems with specific applicability generally being system and/or scale dependent (Leibold et al. 2004, Chase et al. 2005, Cottenie 2005). A recent meta-analysis performed by Cottenie et al. (2005), demonstrated that a wide range of aquatic and terrestrial plant and animal systems were best described by species sorting processes, although a large number were also best fit by a combination of sorting and dispersal processes (i.e., mass effects). Interestingly, the importance of dispersal processes was generally linked to spatial scale, organism size, habitat type (e.g., aquatic versus terrestrial), and mode of dispersal (e.g., passive versus active, Cottenie et al. 2005). Likewise, although neutral processes were the dominant drivers of diversity patterns in only a small number of datasets, neutral effects generally became more important with increasing spatial scale, indicative of the increasing importance of dispersal limitation with increasing scale (Cottenie et al. 2005). 
Regarding direct tests of metacommunity theory for freshwater stream fishes, the most comprehensive research of Cottenie et al. (2005) discussed above. For the 11 stream fish datasets analyzed for that paper, four classified as species sorting and mass effect metacommunities, one as neutral or patch dynamics, and three were undetermined (Cottenie et al. 2005). Overall, the results emphasized the importance of both habitat heterogeneity and dispersal processes in the assembly of stream fish communities. More recently, Falke and Fausch (In Press) also found that a variability in local habitat variability and dispersal limitation were important mechanisms structuring an assemblage of fishes in a mid-western watershed that experienced a high degree of temporal variability in network connectedness due to periodic drought. These early findings are not surprising, however, given that stream fish ecologists have understood the combined importance of local environmental variability (Gorman and Karr 1978, Grossman et al. 1982, Poff and Allen 1995, Peres-Nato 2004) and regional dispersal processes (Osborne and Wiley 1992, Schlosser 1995, Lonzarich et al. 1998, Fagan et al. 2002, Grenouillet et al. 2004, Hitt and Angermeier 2008, Martin and Petty 2009) in stream fish community assembly for some time. For example, in the same year that Wilson (1992) coined the metacommunity term, Osborne and Wiley (1992) documented how small streams located nearer mainstem rivers tended to have more species than small streams positioned in the headwaters of the watershed. The effect on local diversity is presumably due to the proximity of the small stream to a species pool (i.e., large mainstem) and it is expected to be purely spatial in nature and independent of local habitat conditions, indicating what is likely a commonly reported mass effect in studies 
of stream fishes (Fausch et al. 1984, Osborne and Wiley 1992, Grenouillet et al. 2004, Hitt and Angermeier 2008).

Nevertheless, there has been some reluctance to formally incorporate a metacommunity perspective (but see Muneepeerakul et al. 2008, Falke and Fausch, In Press), despite the fact that a more fundamental understanding of the mechanisms driving local and regional stream fish biodiversity would provide invaluable insight to fisheries scientists and managers interested in predicting the consequences of local and regional impacts on stream fish biodiversity as a result of human alteration of stream habitats and watersheds. For example, a better understanding of the local and regional consequences of disturbance would help managers assess not only how poor local conditions can affect local assemblages but also how dispersal barriers and accumulated localized impacts can affect stream fish metacommunities at a regional scale. By the same token, it is important to understand how strategic, localized restoration efforts may result in regional benefits through the restoration of metacommunity linkages. Finally, direct empirical testing of metacommunity theory using stream fishes would not only provide additional insights into to the fundamental mechanisms structuring biodiversity across all taxa in a field where comprehensive and direct tests are currently lacking (Chase et al. 2005); it would also energize an important alignment between a fast moving and current ecological paradigm and the stream community ecology discipline.

Thus, in this paper, we used a "pluralistic" approach, suggestive of the recommendations of Chase et al. (2005), in a direct test of metacommunity theory using stream fish assemblages from a region heavily impaired and fragmented by acid mine drainage (AMD; Freund and Petty 2007, Merovich et al. 2007). Specifically, we used 
this community to test a series of a priori predictions related to (1) the response of diversity to environmental and spatial gradients, (2) theoretical relative abundance distributions, and (3) the response of individual species to isolation and physicochemical conditions. The overriding goal of this research was to determine which metacommunity model best described patterns in stream fish diversity in this system. The study area provided a unique setting for examining the roles of disturbance, network fragmentation, dispersal, and environmental and spatial gradients in the dynamics of freshwater stream fishes in the context of the metacommunity ecology and at a spatial scale relevant to fish conservation and management.

\section{Study Area}

The fish community data was collected from 79 reaches located in wadeable streams within the upper Monongahela River basin. The study encompassed two 8-digit hydrologic unit classification (HUCs, Seaber et al. 1987) watersheds that drain approximately $4,000 \mathrm{~km}^{2}$ of the north-central portion of West Virginia (Figure 1). The basin is characterized by a highly variable range of local (e.g., stream reach) and regional (e.g., stream network) conditions created by multi-scale variation in bedrock geology, coal geology, mining intensity and geography (Strager et al. 2009), AMD (Petty and Barker 2004, Freund and Petty 2007, Merovich et al. 2007), acid precipitation (Clayton et al. 1998, McClurg et al. 2007), and stream thermal topology (Martin 2004, Martin and Petty 2009). This multi-scale variability results in a wide range of both local 
and watershed scale conditions that may be reflected in the fish community (Freund and Petty 2007, McClurg et al. 2007, Martin and Petty 2009).

Due to a legacy of coal mining throughout this region, AMD is a major source of chemical pollution (Petty and Barker 2004, Freund and Petty 2007, Merovich et al. 2007, Merovich and Petty 2007). AMD results from the extraction of high sulfur coal, which exposes an overburden layer of pyrite to oxygen and specialized bacteria. The result is a solution low in $\mathrm{pH}$ and high in sulfur and toxic metals draining from mines that can be detrimental to metal- and $\mathrm{pH}$-sensitive aquatic organisms (Herlihy et al. 1990, Freund and Petty 2007, Merovich and Petty 2007). Local toxicity can be variable across the range of influx severity and dependent on local conditions such as alkalinity (Anderson et al. 2000, Petty and Barker 2004, Freund and Petty 2007, Merovich and Petty 2007). While moderately impaired streams in this region can support a limited number of fish species and macroinvertebrates, severely impaired streams may be entirely devoid of either (Herlihy et al. 1990, Anderson et al. 2000, Freund and Petty 2007, Merovich and Petty 2007). In addition to direct local impairment, AMD in this region can create chemical dispersal barriers, giving rise to fragmented networks, which may lead to local fish assemblage isolation (Freund 2004, Freund and Petty 2007).

Study sites were selected based on stream size, position within the drainage network, and expected local and regional impairment due to mining (Strager et al. 2009). Potential sites were first selected from an evaluation of the available pool of United States Geological Survey (USGS) National Hydrography Dataset (NHD) 1:24K stream segments (USGS 2000) based on the aforementioned properties. Selection of sampling reaches within these segments, however, was performed on-site with the intention of 
maximally representing conditions within each segment in terms of available habitat types (e.g., pools, riffles, runs; Barbour et al. 1999). Figure 1 details the distribution of the study reaches across the basin as well as the expected patterns of stream segment and watershed conditions based on an index of expected condition developed for the region and integrated at multiple scales by combining a multi-scale index of expected mining intensity (Strager et al. 2009), an index of expected conditions for local water chemistry (derived from Merovich et al. 2007), and an index of expected macroinvertebrate assemblage integrity (expected WV Stream Condition Index Score [WVSCI], Petty et al., unpublished data).

The condition index was integrated at the stream segment and 12- and 10-digit HUC scales, ranges from 0 to 100 , and was designed to be interpretable based on the common index of biotic integrity (IBI) scoring system (i.e., $<56=$ poor condition, $56-$ $72=$ fair, $72-85=$ good, $>85=$ excellent, Karr 1981). In order to contrast community dynamics among the fragmented versus intact portions of the basin, the study area was divided among two primary regions. In order to draw boundaries between the fragmented and intact regions for this study, watersheds were assessed for impairment based on the aforementioned HUC12 watershed condition index (WCI, Petty et al., unpublished data). Sites from contiguous 12-digit HUCs of good condition (WCI $\geq 72$ ) were assigned to the "intact" dataset $(\mathrm{n}=35)$. All other sites were assigned to the "fragmented" dataset $(n=44)$. Contiguity was assessed based on whether 12-digit HUCs were flowing into our out of one another. A 12-digit HUC draining another HUC with a WCI value lower than 72 was considered part of the fragmented region. The 
datasets derived from those two regions are hereafter referred to as the "intact" fish assemblage and the "fragmented" fish assemblage datasets.

Figure 3 illustrates the expected variability in local and regional conditions for each site based on the stream segment and HUC12 condition indices. This figure emphasizes the ideal nature of this system for examining questions related to metacommunity theory. Impairment in the system due to AMD provides a strong assemblage sorting mechanism across the range of local impairment and also serves as a strong regional isolating mechanism at elevated concentrations (Freund and Petty 2007).

\section{Expectations}

Because it is unlikely that community patterns in any given system are perfectly described by only one metacommunity framework (Leibold et al. 2004, Driscoll 2008, Falke and Fausch, in press) and because predictions across the four frameworks are often either similar or not well-defined for all models, we used a pluralistic approach to evaluating the applicability of these four models to our system (Chase et al. 2005, McGill et al. 2006). Following largely from the recommendations of Chase et al. (2005), we constructed a list of key model expectations for patterns in species diversity and abundances following from theoretical first principals as well from some speculation, given basic model assumptions, concerning empirical patterns not yet evaluated in a theoretical context (Table 1).

From these key assumptions, we then constructed a list of a priori expectations for empirical patterns that we were able to evaluate using our dataset (Table 2). These 
expectations were structured within three broad categories and were derived primarily from previous work and recommendations concerning empirical evaluation of metacommunity theory (Chase et al. 2005, McGill et al. 2006, Driscoll 2008, Driscoll and Lindenmayer 2009). Thus, we examined (1) empirical patterns in diversity along both environmental and spatial gradients (Chase et al. 2005), (2) patterns in the distribution of relative species abundances (Chase et al. 2005, McGill et al. 2006), and (3) patterns in species' abundances and occurrence in response to environmental gradients, disturbance, and isolation (Chase et al. 2005, MacNally 2007, Driscoll 2008, Driscoll and Lindenmayer 2009; Table 2). Within each of these categories, we conducted a series of specific quantitative and qualitative evaluations of our dataset in order to determine the extent to which each of the four metacommunity frameworks applied to this system (Table 2).

Patterns in diversity related to environmental and spatial gradients

The first avenue of investigation involved a comparison of the response of fish $\alpha$ and $\beta$-diversity to environmental and spatial gradients in the intact and fragmented regions. Again, $\alpha$-diversity is a local measure of diversity and refers simply to the number of locally occurring species (i.e., richness, Whittaker 1972), while $\beta$-diversity is a measure of site to site variability in diversity and refers to the number of species unique to each system being compared (Whittaker 1960, 1972).

Each of the four frameworks makes specific predictions regarding the relationship between these measures of diversity and increasing abiotic environmental 
heterogeneity (Table 1). Because both the neutral and patch dynamic model formulations assume local and regional homogeneity in patch resources (e.g., abiotic conditions, Figure 1), for example, each of these models predict both $\alpha$ - and $\beta$-diversity to be vary independent of heterogeneity in local abiotic conditions in nature (Table 1; Chase et al. 2005). In contrast, because coexistence in the mass-effect and species sorting model formulations is dependent on abiotic heterogeneity among local patches (Figure 1), each of these frameworks predicts $\alpha$ - and $\beta$-diversity to be significantly correlated with abiotic heterogeneity (Table 1; Chase et al. 2005). Therefore, we expected that, significant correlation between $\alpha$-diversity and local physicochemical conditions among sites in the intact and fragmented assemblages would indicate support for a species sorting or mass effect mechanism structuring diversity in this system (Table 2). Conversely, we expected that absence of a strong correlation would provide support for the neutral or patch dynamics models (Table 2).

Each of the four frameworks also offers specific predictions concerning the relationship between $\alpha$ - and $\beta$-diversity and a spatial isolation gradient (Table 1). For example, because dispersal is modeled as a local process in Hubbell's (2001) formulation (Figure 1), the neutral framework predicts $\beta$-diversity to be correlated with inter-site distance (Table 1). The neutral model, thus, predicts that, as sites become increasingly distant from one another, their local assemblages should become increasingly dissimilar due to a reduced exchange of species among them as a result of dispersal limitation. Similar to the neutral model, the mass effect model also predicts composition to correlate with inter-site distance (Table 1). This is because increased rates of dispersal or decreased dispersal limitation are expected to lead to increasing 
mass effects, whereas more localities are inhabited by species that may be poor local competitors, but are good dispersers or "regional competitors". These species maintain themselves in sink localities when dispersal is moderate or less limited so long as each also retains a source habitat in the region (Mouquet and Loreau 2003). These mass effects lead to spatial correlation of species abundances such that localities farther apart have less similar assemblages than those in closer proximity. The patch dynamic model, however, makes no specific predictions concerning the relationship between $\beta$-diversity and isolation because all local patches are assumed identical (Table 1). On the other hand, if dispersal were modeled locally, the prediction for the patch dynamic model would be identical to those of the neutral model (Table 1). Finally, because the species sorting model considers only global dispersal, $\beta$-diversity is predicted to be uncorrelated with increasing isolation (Table 1). However, $\beta$-diversity can appear spatially correlated in the species sorting model if underlying habitat heterogeneity is spatially autocorrelated. Based on these predictions, therefore, we expected that a strong correlation between inter-site distance and $\beta$-diversity, independent of the effects of local physicochemical conditions, would provide support for either neutral or mass effect mechanisms structuring diversity in this system (Table 2). Additionally, we expected that this correlation could be weaker in the fragmented assemblage relative to the intact assemblage due to increased dispersal limitation (neutral expectation) and reduced mass effects (mass effect expectation; Table 2).

Concerning the relationship between $\alpha$-diversity and isolation, while the species sorting model predicts no relationship between dispersal and local diversity, the other three models make similar predictions (Table 1). The species sorting model, again, 
assumes no relationship between dispersal and local diversity because dispersal is considered global and only sufficient to provide colonizers to recently vacated localities (i.e., due to extinction or disturbance; Figure 1). When dispersal is higher, however, mass effects contribute to local assembly and the species sorting model no longer applies (Figure 1). Therefore, the mass effect model predicts that reduced dispersal leads to lower local diversity (Table 1). This is due to a reduction in the number of regionally dominant competitors, or good dispersers, due to a regional reduction in their presence in sink habitats, which is dependent on less restricted dispersal. The neutral model predicts the same outcome as the mass effect due to reduced dispersal from the regional metacommunity (Table 1 ). The patch dynamic model, finally, also predicts $\alpha$ diversity to be lower with more restricted dispersal due to the increased regional displacement of good competitors with good dispersers (Table 1). Based on these predictions, we expected that local species richness would be similar, on average and given similar local physicochemical conditions, among localities in the fragmented region compared to local richness in the intact region should the species sorting model apply to this system (Table 2). Conversely, we expected that local species richness would be lower on average in the fragmented region should the neutral, mass effect, or patch dynamic models apply (Table 2). 


\section{Species abundance distributions}

Species abundance distribution analyses are one of the more comprehensive areas of direct empirical testing of metacommunity theory to date (Chave et al. 2002, McGill 2003, Mouquet and Loreau 2003, McGill et al. 2006). The resurgence stems largely from Hubbell's (2001) recent derivation of a new theoretical abundance distribution, the zero sum multinomial (ZSM), which he derived for describing the expected SAD for a neutral metacommunity of "ecologically equivalent" individuals undergoing what he terms "ecological drift" (Table 1). Abundances in Hubbell's (2001) neutral metacommunity diverge only through stochastic demography and dispersal limitation as opposed to asymmetrical partitioning along environmental or behavioral axes as was typically the case in historical niche-assembly models (e.g., Motomura 1932, Hutchinson 1957, Diamond 1975). Remarkably, Hubbell's (2001) ZSM has been shown to accurately fit the SAD for a range of taxa, all the while providing a vital link between biodiversity and evolutionary theory, yet ignoring the intricacies of individual species' traits. The onset of Hubbell's (2001) unified neutral theory of biodiversity represented an important divergence in biodiversity theory from a paradigm primarily focused on "niche assembly" to a paradigm that also recognized the implications of dispersal limitation.

The hypothesis that patterns in species relative abundances infer something of the underlying processes structuring natural communities has stimulated a long history of theoretical model construction and empirical evaluation. A wide range of species abundance distributions (SAD) have been proposed to describe rank-abundance patterns 
in nature (e.g., more than 20 SADs are presented in McGill 2003). A basic distinction is generally drawn between phenomenological and mechanistic interpretations of abundance patterns (Bell 2000). The phenomenological approach typically involves deriving a frequency distribution to fit an observed natural abundance pattern. For example, the Fisher et al. (1943) log-series model was developed to describe the abundance distribution of butterflies in Malaya, which emphasized a large number of rare species and a small number of common species. Preston (1948), in contrast, developed a model that emphasized a large number of species with intermediate abundances and fewer rare species.

Whittaker (1965) popularized the common modern method of presenting species relative abundances in what he termed the "dominance-diversity" curve, which plots the logarithm of abundances or relative abundances on the y-axis against the rank of abundance on the x-axis. With this plotting method, the Fisher's (1943) log-series model, for example, appears linear, whereas the log-normal model appears curvilinear and steepest over the low ranks of common species, shallower over the middle ranks, and steep again over the high ranks.

The mechanistic approach, on the other hand, attempts to identify an underlying ecological mechanism responsible for a particular abundance pattern and then derives a frequency distribution which might arise from that mechanism. For example, MacArthur (1957) developed what he termed a "broken-stick" SAD model derived from a niche partitioning mechanism such that species abundances are randomly divided among a finite resource axis. His model resulted in reasonable fits to a narrow range of bird communities (MacArthur 1960) and, more recently, to a few Texas stream fish 
guilds (Higgins and Strauss 2008). However, the distribution often proves too even to fit most species abundance data (Hubbell 2001, Higgins and Strauss 2008). Another popular niche-based model, the "niche-preemption model" developed originally by Motomura (1932) and made popular by Whittaker (1965), predicts a much steeper distribution of relative abundances. This model is conceptually similar to the broken stick approach except that the partitioning of resources is non-random such that the dominant species sequesters a fraction of a resource pool, $k$, while $1-k$ is left for the remaining species (May 1975). The second most abundant species then retains the same fraction, $k$, of the remaining resource and leaves fraction $(1-k)^{2}$ for the remaining species, and so on (May 1975). The dominance-preemption distribution has been shown to fit abundance patterns particularly well in primary succession (Whittaker 1972), disturbed (Whittaker 1965), and habitat-poor (Keely and Fotheringem 2003) plant communities. Additionally, abundances among fish guilds and species in some Texas streams also appear to approximate this model (Higgins and Strauss 2008).

Hubbell's (2001) ZSM is a mechanistic SAD model. The neutral model is essentially a mainland-island model, adapted from MacArthur and Wilson's island biogeography model $(1963,1967)$, which is also a "neutral" model; a point that is rarely emphasized in the current literature (Hubbell 2001). In the mainland-island framework, local communities may be considered as embedded within the much larger regional metacommunity (Figure 1) or as true islands. Nonetheless, the mainland-island nature of the model is important because the model actually predicts separate SADs for the mainland, or regional metacommunity, and the island or local community. The mainland formulation assumes that the number of individuals in the regional 
metacommunity $\left(J_{m}\right)$ is constant and that, at each time step, a single individual dies and is replaced by another single individual that is either a new species arising at the rate $v$ or a new offspring of a randomly selected individual already in the regional metacommunity with the probability $1-v$ (Hubbell 2001, McGill 2003). The SAD for the regional metacommunity is determined only by the parameter $\theta=J_{m} v$ termed the fundamental biodiversity number. Within the local community ( $J$; i.e., island), however, speciation does not occur and deaths are replaced by immigrants from the regional metacommunity with a probability $m$ or by births within the local community with the probability $1-m$ (Etienne 2005, Etienne 2007). The parameter $m$ is known as the migration parameter and represents the probability that a dead individual in the local community will be replaced by a disperser from the region. Thus, $m$ is ultimately a measure of dispersal limitation between the metacommunity and local community. The value ranges from 0 to 1 , where 1 represents unlimited dispersal. The ZSM is, therefore, conventionally defined by three parameters - $\theta, J$ and $m$.

The ZSM is similar in shape to a log-normal distribution, but generally predicts fewer common and fewer rarer species (Hubbell 2001, McGill 2003, 2006, Volkov et al 2003). Most empirical tests of neutral theory based on the ZSM have primarily utilized some version of the log-normal model as the phenomenological-based, null hypothesis for model comparison (McGill 2003, Volkov et al. 2003, McGill 2006). Nonetheless, the shape of the ZSM changes with the magnitude of the dispersal parameter, $m$, such that the curve becomes steeper and abundances less even when dispersal limitation is high and more curved with more even abundances when abundances are low. Generally, the ZSM has a flatter tail and more closely resembles the niche-preemption, 
or geometric SAD models relative to the lognormal when dispersal limitation is high, because ecological drift results in a loss of rare species and greater dominance by the numerically dominant species in the metacommunity. Conversely, a log-normal-like SAD is expected when dispersal limitation is low due to increased relative abundance of rare species and decreased relative abundance of more common species (Hubbell 2001).

Whether identifying the ZSM as the best fitting model in an empirical SAD provides strong evidence of underlying neutral processes remains at the forefront of debate. The controversy stems from three primary areas of dispute. The first is that it has been argued that statistically distinguishing among the subtleties of the ZSM and competing theoretical curves, such as the traditional, lognormal curve, may be impossible, especially when considering field sampling error and bias (Harte 2003, Volkov et al. 2005, McGill et al. 2006). However, the recent developments of exact analytical functions based on maximum likelihood estimation by Etienne $(2005,2007)$ enabled a more rigorous approach to discriminating among competing models using information theoretic approaches such as Akaike's information criterion (AIC; Akaike 1974). In addition to model comparisons using AIC, an "exact" test of neutrality was also recently developed by Etienne (2007; Table 1). The process is likened to a parametric bootstrap that allows comparing the likelihoods of artificially constructed neutral SADs to the empirical fit to obtain an estimate of the goodness-of-fit. This allows testing of the neutrality assumption without the need for comparisons with sampling formulas of alternative models (Etienne 2007).

A second criticism of ZSM curve fitting is that the $\theta$ parameter has been shown to systematically increase with scale (McGill 2006), which does not follow logically 
from the expected size of the metacommunity for a reasonable range of scales (Ricklefs 2006). The final area of controversy, however, stems from research indicating that similar SADs can be arrived at under competing mechanistic foundations (Chave et al. 2002, Mouquet and Loreau 2003, Chase et al. 2005, Volkov et al. 2006; Table 1). For example, Mouquet and Loreau (2003) demonstrated that, by altering dispersal rates in a source-sink (i.e., mass effect) metacommunity model, they could produce a range of SAD shapes, including some that are similar to the ZSM (Table 1). Mouquet and Loreau's (2003) model also predicts a flatter SAD at the regional scale, with increasing isolation, while approaching a log-series-like SAD at intermediate rates of dispersal (Table 1). Chave et al. (2002) produced very similar SAD curves to the ZSM for patch dynamic metacommunities by altering various model parameters (Table 1). Nonetheless, while a good fit of the ZSM may offer only weak support for Hubbell's (2001) model, its absence is thought to offer strong support for rejection of neutrality (McGill 2006). Therefore, the curve fitting approach still has a great deal of utility when comparing the neutral model to its competitors.

Regarding our a priori expectations for the SADs of the intact and fragmented assemblages, because the neutral framework explicitly predicts a ZSM SAD, we expected that, if neutral processes were important drivers of assemblage composition for stream fishes in this region, the ZSM would provide the best fit for the SAD data from both the intact and fragmented watersheds in comparison to the competing nichepreemption model (geometric; Motomura 1932) or phenomenological-based lognormal model (Preston 1948; Tables 2). Moreover, we expected that increased dispersal limitation in the fragmented assemblage relative to the intact assemblage would be 
reflected in the comparisons of the $m$ parameter, such that lower values would be estimated within the fragmented watershed (Table 2).

Similar to the neutral model, we expected that, if mass effect processes are important in this system, the geometric SAD model would provide a better fit to the fragmented assemblage, relative to the lognormal model, due to higher dispersal limitation resulting in a reduced number of transients (i.e., rare) and reduced assemblage evenness (Table 2). Conversely, we expected the lognormal model to better approximate the SAD of the intact assemblage (Table 2). The expectations following from the patch dynamic model was the same as the mass effect model (Table 2). For the patch dynamic model, we expected that an overall loss of poor colonizers in the fragmented region would result in a less even assemblage and a steeper SAD compared to the intact region (Table 2).

Although, the geometric and lognormal mechanistic models used for comparison in ultimately were developed to imply that niche-based mechanics give rise to such SADs, we expected that mass effect and patch dynamic metacommunities would approximate these shapes based on an examination of past theoretical work which produced qualitatively similar curves (Mouquet and Loreau 2002, Chave et al. 2002; Table 1). Thus, we did not intend to imply that niche-based mechanisms were responsible for fit. Essentially, we expected the geometric fit to represent a generally less even and steeper distribution and the lognormal fit to represent a more even distribution.

For the species sorting model, finally, there are no published specific predictions for the shape of the SAD, however, it is expected that the shape should vary with 
environmental conditions (Table 1). Therefore, we expected that, increased disturbance in the fragmented assemblage would lead to a regional homogenization of habitat, fewer available niches and, thus, a less even SAD (i.e., geometric). Conversely, we expected the SAD of the intact assemblage to be more even and include more rare species (i.e., lognormal; Table 2).

Regarding Etienne's (2007) exact test of neutrality, we expected that, should the neutral model not apply, the outcome of this test would indicate rejection of the null hypothesis of neutrality (Table 2). We, thus, expected that rejection of this test would strongly favor a non-neutral mechanism for the distribution of abundances in the intact and fragmented assemblages (Table 2).

Response to disturbance and species identity

Because shape comparisons of SAD can provide an incomplete representation of changes in species' relative abundances as a result of ignoring species-specific information (Fisher and Frank 2004, Wooten 2005, MacNally 2007), we used the approach of MacNally (2007) to compare species abundances between the intact and fragmented assemblages. Because the rank abundance approach orders species from most to least abundant regardless of identity, the most abundant species may not always be the same species in sequential plots, even though the shape of the distribution may remain the same (MacNally 2007). MacNally (2007), therefore, demonstrated that ordering and comparing abundances between samples where ranks are fixed is a useful method for comparing relative abundances while retaining species-specific information. 
MacNally (2007) described these simple plots as rank abundance "spectra" and demonstrated their utility in comparing abundances, for example, among assemblages pre- and post-disturbance (MacNally 2007). In a comparison of spectra, the most abundant species receives the top rank in the spectrum pre-impact and also retains that rank in the post-impact spectrum (MacNally 2007). This logic is followed for the second most abundant species and all of those that remain.

Comparison of relative abundances pre- and post-impact using spectra can provide valuable insight into the mechanisms driving diversity in a system (e.g., neutral versus niche-based, MacNally 2007) and is, therefore, a useful qualitative approach for evaluating predictions arising from metacommunity theory (Table 1). Prior to the assessment of spectra in this study, we developed models for predicting commonly sampled species' abundance and occurrence in both the intact and fragmented regions (see Methods section for analysis details). We then used those results to qualitatively inform the comparison of rank abundance spectra based on potential species' traits that were responsible for their inflation, deflation, or consistency in relative abundance or occupancy across the two assemblages (Table 2). The predictive models quantified the relationships between local physicochemical variability and dispersal limitation and individual species' occurrence and abundance across localities in the intact and fragmented regions. Specifically, abundance and occurrence was regressed with local measures of physicochemical variability and the extent of isolation from species' expected core habitat areas, or regional population pools (see Methods for detailed description). The models were developed to characterize individual species' tendencies to form metapopulations in the intact and fragmented regions (sensu Driscoll 2008) and 
their relationship to local abiotic variability. In combination, the rank abundance spectra and regression models provided a means of qualitatively assessing the response of species relative abundances and occupancy to disturbance and fragmentation in a metacommunity context.

For example, we expected that, should neutral dynamics be applicable in this system, species abundances in the fragmented system would reflect a random draw from the intact system, with the rarest species the most likely to be lost and the most common the least likely (Hubbell 2001, Chase et al. 2005, MacNally 2007; Table 2). In other words, we expected that, should the neutral model apply, change in species' abundances from the intact to the fragmented system, as a result of disturbance and isolation, would be independent of species' traits.

Conversely, the expectation for the remaining three models was that the organization of species' abundances in the fragmented assemblage with the intact assemblage would not reflect a random draw from the intact assemblage, but rather patterns consistent with species' traits. Should the patch dynamic or mass effect model apply, for example, we expected to observe a reduction in the relative abundances and the proportion of sites occupied by poorer dispersers in the fragmented system (Table 2). Specifically, we expected that, should the patch dynamic or mass effect model apply, we would observe an overall deflation of abundances and occupancy of species that tended to form metapopulations in the intact assemblages (i.e., tendency to demonstrate dispersal limitation at this scale) in the fragmented assemblage. However, because the patch dynamic paradigm emphasizes competitive trade-offs and stronger biotic interactions, we also expected a possible inflation of poorer competitors or potential prey species in the 
fragmented assemblage relative to the intact system should the patch dynamic model apply (Table 2). On the other hand, because the mass effect model emphasizes stronger sorting along abiotic (i.e., physicochemical) relative to biotic (i.e., interspecific competition, predation, etc.) gradients, we did not expect an inflation of abundances of inferior competitors or prey species in the fragmented region, but did expect a deflation of poor dispersers in addition to acid sensitive taxa (Table 2). Finally, because the species sorting framework emphasizes sorting along abiotic gradients, we expected species' relative abundances and occupancies in the fragmented region to reflect only a deflation in acid-sensitive taxa or a loss of species according to change in specific abiotic conditions from the intact region, should that framework apply in this system (Table 2).

While the predictive models were used to qualitatively assess the mechanisms through which species' relative abundances and occupancies differed in the intact versus fragmented watersheds, we also had specific expectations regarding the relationship between individual species' abundances or occurrence and local abiotic heterogeneity and isolation following from metacommunity theory (Table 1). Because neutral theory assumes that all individuals (i.e., not species) respond similarly, or in a zero-sum manner, to local abiotic heterogeneity, we expected that, should the neutral model apply in this system, the abundances or occurrences of all the commonly sampled species modeled would vary independent of local abiotic conditions or with a single dominant resource axis (Hubbell 2001, Muneepeerakul et al. 2008; Table 2). Likewise, because dispersal is local in the neutral model and because all individuals are equivalent in dispersal ability, we also expected that each species modeled would demonstrate a similar tendency to form metapopulations (sensu Driscoll 2008) in the intact and fragmented systems, 
meaning that they would show lower probabilities of occurrence or reduced abundances with increasing distance from their expected population cores (Table 2).

Similar to the neutral expectation, we expected that, should the patch dynamic model apply, species abundances and occurrence would vary independent of local abiotic conditions (Table 2). However, we expected that, should the patch dynamic model apply species would not demonstrate a similar tendency to form metapopulations because this tendency was expected to be influenced by tradeoffs in species traits related to interspecific interactions (Table 2). On the other hand, we expected that, should the mass effect model apply, abundances and occurrence would vary with both local abiotic conditions and isolation (Table 2). We expected that, should either the patch dynamic or mass effect frameworks apply, metapopulation formation could be deterministic (Driscoll 2008), meaning that species demonstrating isolation effects in one assemblage may not show them in another. For example, from the patch dynamic perspective, the tendency to form metapopulations could vary depending on which species are in the community, due presumably to interspecific interactions (Driscoll 2008). Loss of predatory species, for example, would be expected to lead to an inflation of prey species. Less restriction on prey distributions could then lead to a spatial structuring in their occurrence or abundance that was not possible in the presence of a key predator (DeRoos et al. 1998). Similarly, the formation of deterministic metapopulations could also result from mass effect processes. For example, we expected, should the mass effect model apply, that species demonstrating a tendency to form metapopulations in the intact assemblage could be less likely to do so in the fragmented assemblage due to alleviation of source-sink dynamics imposed by barriers to dispersal. Finally, we expected that, should the species sorting 
model apply, species would not form metapopulations and their abundances and occurrence would be best predicted only by measures local abiotic variability (Table 2).

\section{Methods}

Fish community data collection

Fish sampling protocols followed the EPA-EMAP guidelines set by McCormick et al. (2001), but with some modifications (Freund and Petty 2007, Hense et al., In Revision). Reach lengths were calculated as the estimated mean stream width (MSW) multiplied by 40 , with no reaches shorter than 150 meters or exceeding 300 meters in length (Lyons 1992, McCormick et al. 2001, Freund and Petty 2007). Greater sampling lengths do not typically result in higher species richness or IBI scores (Ohio EPA 1989). All fish community sampling was conducted over the period spanning July 10 through August 31 during the summers of 2001 to 2004 . This encompassed the time frame during which fish communities are thought to be stable and ideal for sampling (Barbour et al. 1999, Freund and Petty 2007). Concerning potential bias associated with annual variability in the assemblage data, using a subset of the sites from this study $(n=9)$, Freund and Petty (2007) found that annual variability in community structure, based on the Mid-Atlantic Highlands Index of Biotic Integrity (MAHIBI), was low ( $\mathrm{r}=0.95)$.

Sampling was conducted using one to two backpack electrofishing units, depending on mean stream width (Freund and Petty 2007, Hense et al., In Revision). 
Two units were used for streams with widths greater than 6 meters. No streams sampled were larger than 15 meters wide. A modified, single-pass technique was employed, such that sampling was conducted in an upstream direction moving away from a downstream blocking seine for a distance no longer than 10 meters. After moving upstream for 10 meters, the sampling crew kicked back downstream to the initial starting point and blocking seine. This continued for the length of the site, moving the seine in approximately 10 meter increments. The moveable blocking seine was employed primarily to increase the capture efficiency of smaller benthic fishes, which have a tendency to be underrepresented in electrofishing surveys due to cryptic coloration and relatively low buoyancy as a result of lacking a swim bladder. For each backpack electrofishing unit employed, another person assisted with a dip net for capturing the stunned fishes. Netted fishes were placed in ventilated buckets along the stream margin before being processed. All fishes collected were counted, weighed to the nearest $0.1 \mathrm{~g}$, and measured to the nearest $1 \mathrm{~mm}$ in standard length. Voucher specimens and unidentified specimens were preserved in $95 \%$ ethanol and returned to the lab for verification.

\section{Physicochemical data collection}

Water quality data were derived from samples expected to represent the most severe water quality condition among the most variable streams (i.e., moderately impaired, Petty and Barker 2004). The assumption was that the typical community structure in impaired streams is set by the worst periods of water quality impairment 
(Freund and Petty 2007). Late winter or early spring samples were, therefore, chosen as representative samples because water quality is thought to be poorest in moderately impaired streams during this season (Petty and Barker 2004, Freund and Petty 2007). Despite considerable seasonal variation, however, year to year variation in water chemistry has been shown to be remarkably low (Freund and Petty 2007). Freund and Petty (2007), for example, found that, among a subset of the sites used in this study, the correlation coefficient for specific conductance was approximately 0.97 across yearly samples and high correlations with other chemical constituents were observed as well.

For each water sampling occasion, temperature, dissolved oxygen, and total dissolved solids were measured on site using a YSI $650{ }^{\circledR}$ with a $600 \mathrm{xl}$ sonde (Yellow Springs, Ohio). Additionally, a $500 \mathrm{ml}$ sample was filtered using a Nalgene polysulfone filter holder and receiver using a mixed cellulose ester membrane $0.45 \mu \mathrm{m}$ pore size disk. Filtered samples were treated with $5 \mathrm{ml}$ of $1: 1$ nitric acid to force the $\mathrm{pH}$ below 2.0 to ensure that metals stay in solution. The filtered samples were used for laboratory analysis of aluminum, iron, manganese, nickel, cadmium, chromium, calcium and total hardness. A $1 \mathrm{~L}$ grab sample was also collected for laboratory analysis of alkalinity, acidity and sulfates. Unfiltered samples were kept on ice after collection and stored in the laboratory at $4{ }^{\circ} \mathrm{C}$ prior to analyses.

In addition to water chemistry data, we also included onsite evaluations of physical habitat based on the United States Environmental Protection Agency's (USEPA) protocol for rapid visual habitat assessment (RVHA, Barbour et al. 1999). Two observers trained in RVHA evaluated and collaborated on estimates in order to maximize 
repeatability (Roper and Scharnecchia 1995, Freund and Petty 2007). RVHA total score, 1 to 200 , was used for all sites and analyses.

\section{Spatial data}

All landscape variables were extracted from geographic databases using ArcGIS ${ }^{\circledR}$ Desktop (version 9.3, Environmental Systems Research Institute 2006). These variables included an index of expected summer thermal conditions or weekly mean July temperature (WMJT, Wehrly et al. 2003, Martin and Petty 2009), cumulative drainage area (AREA), reach gradient (GRAD), maximum reach elevation (MELE), cumulative percent land cover and geology type (Strager et al. 2009), and minimum distance to a potential population pool (MIN_POOL), which is based on previously documented information on the frequency distribution of abundances along a continuum of drainage area (Freund 2004, Hense 2007). Population cores were defined by the range of drainage areas encompassing the $25^{\text {th }}$ to $75^{\text {th }}$ percentile of abundances for a particular species (Freund 2004, Hense 2007). The population core concept was developed in analogy to the concept of the species pool and it was similarly expected that species abundances would deteriorate with increasing distance from the expected pool.

Minimum distances to the expected species' cores (i.e., segments of a particular drainage area) from each site were calculated using ESRI's ArcMap ${ }^{\circledR}$ (version 9.3) and the Network Analyst Extension ${ }^{\circledR}($ ESRI 2005). The distances were calculated while also accounting for expected barriers to dispersal due to severe AMD impairment. The evaluations of passability were based on both the previously developed index of expected 
mining intensity (Strager et al. 2009) and extensive empirical and field-based knowledge of AMD impairment and its effects on the biota in this region (Freund 2004, Merovich 2007, Freund and Petty 2007, Merovich and Petty 2007, Merovich et al. 2007, Martin and Petty 2009). If the stream path to a particular species' nearest core-sized stream from the focal site was evaluated to be impassible due to isolation by AMD impairment downstream, the estimate of minimum distance to the core for such a site was forced to the maximum value of the distance estimates where access was unimpeded.

Analyses

All physicochemical and spatial variables were screened for redundancy and colinearity and culled prior to further statistical analyses. We used a matrix of Pearson correlations to assess the strength of correlations among all of the independent variables (i.e., non-fish data) and identified and removed potentially redundant variables based on a correlation coefficient of 0.6 .

Water chemistry

We used principal components analysis (PCA) on the correlation matrix of the multivariate water chemistry dataset in order to identify and characterize important gradients in water chemistry variability (Merovich et al. 2007). Prior to the PCA, we assessed the individual chemistry variables for normality and applied transformations 
where applicable. The specific principal components discussed in the results were chosen based on the overall amount of variation in the data accounted for in those components as well as the interpretability of the component. Finally, we interpreted component loadings when loading values were greater than or equal to $|0.4|$. All water chemistry analyses were performed using the "stats" package of the R statistical programming language software, version 2.9.1.

Response of diversity to physicochemical conditions and space

We used partial Mantel tests (Mantel 1967) to determine the statistical significance of matrix correlations among environmental and spatial heterogeneity and fish community diversity. The Mantel correlation test statistic results from a distancebased matrix correlation procedure that uses permutations to estimate the probability that the correlations are random. The correlation coefficient is scaled from -1 to 1 , where a value of 1 indicates perfect positive correlation and a value of -1 indicates perfect negative correlation. Partial Mantel tests estimate the strength of correlation between two distance matrices after the effect of one or more matrices has been eliminated. Thus, these tests allowed us to isolate the effects of spatial and environmental heterogeneity on $\alpha$ - and $\beta$-diversity (Legendre and Legendre 1998, King et al. 2005, Hitt and Angermeier 2008).

Using the Network Analyst ${ }^{\circledR}$ extension provided in $\operatorname{ArcMap}^{\circledR}$ (version 9.3), we constructed a matrix of inter-site fluvial distances based on the topology of the aforementioned 1:24000 NHD network dataset for both the intact and fragmented 
datasets. To characterize diversity, we then constructed Bray-Curtis dissimilarity matrices for the intact and fragmented datasets as a measure of $\beta$-diversity in each region. We also calculated $\alpha$-diversity (i.e., species richness) for individual sites in the intact and fragmented datasets and converted the respective vectors to Euclidean distance matrices.

We then constructed 5 environmental distance matrices. Each of these matrices was created by converting the original vector data to a Euclidean distance matrix. The 5 matrices were constructed from (1) the water chemistry principal components, (2) a vector of predicted weekly mean July temperature values, (3) a vector of estimated drainage areas $\left(\mathrm{km}^{2}\right),(4)$ a vector of RVHA scores, and finally (5) a matrix combining all of the physicochemical variables in 1 through 4 .

Each of these five environmental matrices was then tested for significant partial Mantel correlations with the diversity matrices while accounting for the effect of space. Likewise, space was also tested for significant partial Mantel correlations with diversity while accounting for the effect of all physicochemical variables. We considered correlations as statistically significant based on an a priori alpha-level of 0.05 .

To visualize the relationships between physicochemical variables and community dissimilarity, we used non-metric multidimensional scaling (NMDS) to place samples in k-dimensional space while minimizing the differences between rank dissimilarities in the Bray-Curtis distance matrix and rank (Clarke 1993). NMDS is commonly recognized as one of the more robust unconstrained ordination methods in community ecology (Minchin 1987). We conducted the NMDS analyses with the goal of finding the minimum number of dimensions for each ordination, while also 
minimizing stress (Clarke 1993). We set our target maximum stress level at 14. Following the NMDS analyses for each dataset, we fit vectors for all of the physicochemical variables that demonstrated a significant relationship with diversity in the previous analysis using partial Mantel correlations (Oksanen et al. 2009). This process fits the vectors to the ordination in a manner that maximizes the correlation between the physicochemical variable and the ordination space (Oksanen et al. 2009). A permutation procedure is then used to estimate the statistical significance of each vector's fit to the ordination space (Oksanen et al. 2009). We used 1000 permutations and based our conclusions on an a priori alpha-level of 0.05 . We used these vector correlations to interpret the individual NMDS axes. We only interpreted variables where their correlation with the NMDS axes was greater than or equal to $|0.4|$.

In order to test the predictions concerning the comparison of $\alpha$-diversity in the intact and fragmented data, we used an analysis of covariance (ANCOVA) to determine whether the mean value for local richness for streams of similar drainage area and water chemistry (i.e., drainage area and chemistry covariates) differed among the intact and fragmented watersheds. The statistical null hypothesis was that the intercepts for the linear relationship between species richness and drainage area and chemistry did not differ between the intact and fragmented regions. We constructed an additive linear regression model fitting log transformed drainage area, two water chemistry principal components, and a two-level factor variable coding for the dataset each site was derived from (intact or fragmented) to local species richness for all sites from the two datasets $(\mathrm{N}=79)$. We also tested the null hypothesis that the slopes of the two regressions did not differ between the intact and fragmented datasets, by testing for an interaction 
between $\mathrm{WC} 1$ and the dataset factor variable. We first compared the additive model to the reduced model excluding the dataset factor variable using an ANOVA. We then compared the additive model to the model with the interaction using the ANOVA approach. We based our conclusions on an a priori alpha-level of 0.05 .

Fish species abundance distributions

The purpose of this analysis was to determine which of three competing SAD models — lognormal (Preston 1948), geometric (Wilson 1991), and ZSM (Hubbell 2001) — best fit the empirical data from the intact and fragmented regions as well as the two similar-sized, sub-watersheds within each of these broader assemblages (i.e., Horseshoe Run and Muddy Creek). We used the method of maximum likelihood estimation (MLE) to derive the best fitting parameters and log-likelihood estimates for each of the two datasets. For the ZSM model, we used the approach and PARI/GP code provided in Etienne (2007) to estimate the necessary parameters and log-likelihood estimates. For the lognormal and geometric models, we used the "radfit" function provided in the "vegan" package of the R statistical programming environment, version 2.6.0, to estimate the shape parameters and log-likelihoods. We used an AIC approach to compare the fits (Chave et al. 2006, MacNally 2007, Higgins and Strauss 2008).

In addition to SAD model comparisons, we utilized an "exact" test of neutrality described in Etienne (2007). Etienne's (2007) test involves simulating artificial abundance distributions using the MLE parameters from each of the empirical datasets and then using the MLE technique, again, to estimate new log-likelihood values for 
fitting the neutral model parameters to the simulated datasets. Following a sufficient number of simulations, the number of times the log-likelihood values for the simulated datasets were lower than those obtained for the empirical data is recorded. This proportion is, effectively, the P-value for the test (Etienne 2007). A test resulting in a Pvalue less than an a priori $\alpha$-level of 0.05 for 100 simulated datasets, for example, would indicate that more than 95 of 100 of the simulated datasets had log-likehood estimates lower than the log-likelihood value obtained for the estimates using the empirical data. This would confirm that the dataset is unlikely to have come from a local community conforming to the neutral assumptions (Etienne 2007). The test is considered "exact" in that the type I error can be precisely specified, while the type II error is minimized by increasing the number of simulated datasets (Etienne 2007). Therefore, although failure to reject neutrality can not imply acceptance, rejection yields a confident conclusion that the data do not fit the neutral assumptions (Etienne 2007). For each dataset, we used the code provided in Etienne (2007) to generate 100 distributions using the parameter estimates recorded for each dataset — intact and fragmented — and based our conclusions on an a priori alpha-level of 0.05 .

Response to disturbance and species identity

In order to statistically quantify the relationship between the environmental and spatial variables and individual species' occurrence and abundance, we used a stepwise selection procedure, based on Akaike's information criterion (AIC; Akaike 1974), to select among a list of generalized linear models (GLM, McCullough and Nelder 1989) 
for estimating the presence-absence and abundance of some commonly sampled species in the intact and fragmented regions (Faraway 2006, Driscoll 2008, Driscoll and Lindenmayer 2009). The GLM is a flexible generalization of ordinary least squares regression (OLS), which relates the distribution function of a dependant variable to linear predictors through a function called the link function (McCullough and Nelder 1989). It was formulated as a way of unifying various statistical models, including logistic regression and Poisson regression, under a single framework, such that a general algorithm for maximum likelihood estimation worked for all distribution models, thereby relaxing the constraints imposed by the linear relation between independent and dependent variables in OLS regression (McCullough and Nelder 1989). We assessed the responses of 14 fish species. These particular species were selected because they are commonly sampled throughout the region and because their spatial distributions have been extensively characterized, which made possible the estimation of individual species core distributions (Freund 2004, Hense 2007).

Due to the complications with regression analysis of abundances due to sparse data, we divided analyses among species that were widespread and those that were less common (Driscoll 2008). For species present in less than $75 \%$ of the sites sampled ( $\mathrm{n}=10$ in the intact and $\mathrm{n}=12$ in the fragmented), we modeled their presence-absence using a GLM with a binomial error distribution and employing the logit link function. For widespread species (i.e., $>75 \%$ ), we modeled their abundances using a GLM with a negative binomial error distribution and the log link function. In order to assess the fit for each model, we calculated the percent deviance explained by the final models (Faraway 2006, Driscoll 2008). Additionally, we verified the fit of each model by 
assessing the squared Pearson correlation of the fitted model values to the actual measured values (i.e., R-square). All GLM analyses were conducted using the "stats" (binomial GLM) and "MASS" (negative binomial GLM) packages of the R statistical programming environment, version 2.9.1.

Finally, because the rank abundance approach can provide an incomplete representation of changes as a result of ignoring species-specific information (Fisher and Frank 2004, MacNally 2007), we used the approach of MacNally (2007) to compare species abundance spectra between the intact and fragmented assemblages. We used this method to qualitatively assess changes in the patterns of percent relative abundance between the fragmented and intact assemblages. Also, the ratio of percent relative abundances for individual species between the two datasets was calculated in order to quantify the magnitudes of change for individual species. For example, the fragmented to intact ratio of a species with a percent relative abundance of $50 \%$ in the fragmented and $25 \%$ in the intact would indicate a two-fold increase in percent relative abundance.

\section{Results}

Water chemistry

For the Principal Components Analysis (PCA) of water chemistry variability among sites in the intact watershed, principal component 1 (WC1) accounted for $31 \%$ of the total variance in the solution and was the only interpretable axis for the intact 
assemblage PCA (Table 3). This component represented a hardness and alkalinity gradient characterized by a gradation of high to low specific conductivity (factor loading value $(l)=-0.43)$, total alkalinity $(l=-0.44)$, and calcium $(l=-0.43$, Table 3$)$.

For the PCA of water chemistry data in the fragmented, WC1 and WC2 accounted for $58 \%$ of the total variance in the solution (35\% and $23 \%$, respectively) and were the only interpretable axes in the solution (Table 3). WC1 represented a gradient of conductivity, hardness, and sulfates, with values for $\mathrm{SpCond}, \mathrm{SO} 4, \mathrm{Mg}$, and $\mathrm{Ca}$ decreasing with increasing WC1 values (Table 3). Sites with extremely low values on this axis tend to be associated with mining activity, but may be hard due to treatment (Merovich 2007). Sites with higher values on this axis, however, tend to be sites that are minimally impaired or not impacted by AMD (Petty and Barker 2004, Merovich 2007). Principal component axis 2 represents a gradient of $\mathrm{pH}$ and alkalinity, with low values of WC2 corresponding to higher $\mathrm{pH}$ (Table $3 ; l=-0.44)$. Sites with low values on this axis are likely unimpaired streams, while sites with high values may be impaired by AMD. Thus, higher quality sites should score moderate to higher on WC1 and low on WC2, while poorer quality sites should score moderate to low on $\mathrm{WC} 1$ and higher on WC2.

\section{Correlating diversity to physicochemical and spatial gradients}

In the intact region $(\mathrm{n}=35)$, WMJT (partial Mantel's $\mathrm{r}=0.42, \mathrm{P}<0.001)$, drainage area (partial Mantel's $\mathrm{r}=0.30, \mathrm{P}<0.001$ ) and all physicochemical variables combined (partial Mantel's $\mathrm{r}=0.28, \mathrm{P}<0.001$ ) were significantly correlated with local species richness, a surrogate of $\alpha$-diversity, independent of spatial variability (i.e., inter- 
site distance; Table 4). WMJT (partial Mantel's $r=0.42, \mathrm{P}<0.001$ ), drainage area (partial Mantel's $\mathrm{r}=0.35, \mathrm{P}<0.001)$, and the combined environmental data matrix $(\mathrm{r}=$ $0.39, \mathrm{P}<0.001)$ were also strongly and significantly correlated with Bray-Curtis dissimilarity, a surrogate of $\beta$-diversity, independent of space in the intact assemblage (Table 4). Interestingly, space (partial Mantel's $r=0.32, \mathrm{P}<0.01$ ) was also strongly and significantly correlated with Bray-Curtis dissimilarity, independent of all environmental variables in the intact region (Table 4). Finally, inter-site distance, water chemistry, and RVHA were not significantly correlated with either local species richness or Bray-Curtis dissimilarity in the intact dataset (Table 4).

In the fragmented region $(\mathrm{n}=44)$, WMJT (partial Mantel's $r=0.33, \mathrm{P}<0.001)$, drainage area (partial Mantel's $\mathrm{r}=0.39, \mathrm{P}<0.001$ ), and all physicochemical variables combined (partial Mantel's $r=0.24, \mathrm{P}<0.01$ ) were significantly and strongly correlated with local species richness independent of spatial autocorrelation (Table 4). Interestingly, local species richness was not correlated with the water chemistry, RVHA, or inter-site distance data matrices (Table 4). However, Bray-Curtis dissimilarity in the fragmented region was strongly and significantly correlated with the water chemistry data matrix independent of space (partial Mantel's $\mathrm{r}=0.36, \mathrm{P}<0.001$; Table 4). Additionally, although the magnitude of the mantel statistic was substantially lower than that reported for the intact region, Bray-Curtis dissimilarity also was significantly correlated with drainage area in the fragmented region (partial Mantel's $r=0.16, \mathrm{P}<0.05$; Table 4). The combined physicochemical data matrix also was strongly and significantly correlated with Bray-Curtis dissimilarity in the fragmented dataset (partial Mantel's $r=0.36, \mathrm{P}<$ 0.001; Table 4). However, unlike in the intact region, WMJT was not significantly 
correlated with Bray-Curtis dissimilarity in the fragmented region (Table 4). Finally, and also in contrast to the intact region, inter-site swim distance (i.e., space) was not significantly correlated with Bray-Curtis dissimilarity in the fragmented region (Table 4). NMDS ordination stress was minimized in three dimensions for both the intact and fragmented assemblages $(\mathrm{k}=3$, stress $=9.01$ in intact and 10.66 in fragmented; Table 5). Each of the variables demonstrated to be important correlates with community dissimilarity in the partial Mantel analyses above also were also significantly correlated with the ordination space in both the intact and fragmented regions (Table 5, Figure 4). The physicochemical vector fitting processes revealed that the fish assemblage in the intact region was sorted strongly along gradients of drainage area and WMJT (NMDS1; Table 5, Figure 4). Thus, the first ordination axis represented a gradient of stream size and temperature (Table 5) and fish species typically associated with larger and warmer streams, such as the cyprinids L. chrysocephalus, $N$. photogenis, $N$. rubellis, and $P$. notatus and the centrarchids L. macrochirus, and M. dolomieu, ordinate closer to one another and farther from those typically associated with smaller and cooler streams, such as $S$. fontinalis, C. bairdi, and R. obtusus (Figure 4). However, the second and third dimensions of the ordination for the intact region were less interpretable (Table 5).

The ordination for the fragmented assemblage also indicated that the assemblage sorts primarily along a gradient of temperature and drainage area (i.e., NMDS1; Table 5, Figure 4), however, this assemblage also sorted strongly along a second axis associated with water chemistry impairment (Table 5, Figure 4). The NMDS findings visualize the results of the partial Mantel tests, which concluded that, although local water chemistry is unrelated to local diversity, it was strongly related to $\beta$-diversity in the fragmented 
region. The ordination illustrates that a different subset of species is expected to be found in small streams with poor water chemistry than in small streams with good water chemistry (Figure 4). More specifically, S. fontinalis, R. cataractae, and C. bairdi, which were found in small streams with good local water chemistry, were replaced by $R$. obtusus, S. atromaculatus, and C. commersoni in small streams with poorer chemistry (Figure 4). In larger, warmer streams with good local chemistry in the fragmented system, one may expect to find $N$. photogenis, $N$. rubellus, and N. micropogon, for example, and A. natalis and P. notatus in larger, warmer streams with poorer local chemistry (Figure 4). In other words, species replacements rather than species losses can explain why water quality was not correlated with local species diversity but highly correlated with $\beta$-diversity in the Mantel correlation analyses (Table 4).

Local species richness in the intact versus fragmented system

The null hypothesis that mean species richness was not different between the intact region and fragmented region for streams of similar size and water chemistry was rejected following the ANCOVA and comparison of the full and reduced models (Table 6). The linear model coefficients also confirmed that, on average, there were approximately three more species in any given site in the intact region as compared to the fragmented region after accounting for the co-varying effects of drainage area and water chemistry (Table 6, Figure 5). 


\section{Species abundance distributions}

Results for SAD model comparisons indicated that the ZSM was the substantially better fitting model for both the intact and fragmented assemblages based on AIC (Table 7). Comparing only the lognormal and niche-preemption SAD model fits, the lognormal model was the better fit for the intact region, whereas the niche-preemption model was the better fit for the fragmented region (Table 7). Interestingly, estimates for the fundamental biodiversity parameter, $\theta$, were comparable among the two datasets $(\theta=$ 4.57 in the intact vs. 4.56 in the fragmented; Table 7). However, values for the migration parameter, $m$, indicated that the intact assemblage $(m=0.02)$ is more isolated from the region than the fragmented assemblage $(m=0.21$; Table 7).

Applying Etienne's (2007) exact test for neutrality revealed that the null hypothesis for neutrality could not be rejected for the SAD of the intact $(\mathrm{C}=100, \mathrm{P}=$ $0.4)$ or fragmented $(\mathrm{C}=100, \mathrm{P}=0.4)$ assemblages. It is important to note, again, that the results of this test do not confirm neutrality, only that the hypothesis of neutrality could not be rejected (Etienne 2007).

Response to disturbance and species identity

While both the intact and fragmented datasets were best fit by the ZSM, there were notable differences in the ranks of abundance and occupancy and the ratio of percent relative abundances of individual species when comparing the two datasets (Table 8). Comparing ratios of relative abundance and occupancy across the two 
systems, some species demonstrated notably lower percent relative abundance in the fragmented region compared to the intact region (ratio $<0.5$ ), or comprised less than half the percent relative abundance in the fragmented assemblage compared to the intact. These species were $C$. funduloides $($ Ratio $=0.01), M$. margarita $($ Ratio $=0.06), C$. anomalum $($ Ratio $=0.09)$, hybrid individuals $($ Ratio $=0.11), R$ cataractae $($ Ratio $=0.34)$, N. photogenis $($ Ratio $=0.40)$, and E. blennioides $($ Ratio $=0.41$; Table 8$)$. Conversely, several species demonstrated a notably higher ratio of percent relative abundance in the fragmented region compared to the intact region (ratio $>2$ ). These species were $P$. notatus $($ Ratio $=324.63)$, L. chrysocephalus $($ Ratio $=10.96)$, L. macrochirus $($ Ratio $=$ 10.41), S. atromaculatus $($ Ratio $=7.56)$, L. cyanellus $($ Ratio $=6.06)$, S. trutta $($ Ratio $=$ 3.47), A. rupestris $($ Ratio $=2.84), C$. commersoni $($ Ratio $=2.30)$, and $R$. obtusus $($ Ratio $=$ 2.00; Table 8). Additionally, species that retained relatively consistent proportions of the total abundance between the fragmented and intact regions, but demonstrated a lower proportional abundance in the fragmented region compared to the intact region (i.e., $0.5>$ ratio $<1)$ were $S$. fontinalis $($ Ratio $=0.62), N$. rubellus $($ Ratio $=0.65)$, C. bairdi $($ Ratio $=$ 0.66), O. mykiss $($ Ratio $=0.67), N$ micropogon $($ Ratio $=0.82)$, and E. flabellare $($ Ratio $=$ 0.86; Table 8). Finally, species that retained relatively consistent proportions of the total abundance between the fragmented and intact regions, but demonstrated slightly higher proportional abundance in the fragmented region (i.e., $2>$ ratio $>1$ ) were E. nigrum $($ Ratio $=1.89)$, L. gibbosus $($ Ratio $=1.73)$, M. dolomieu $($ Ratio $=1.51)$ and H. nigricans $($ Ratio $=1.35 ;$ Table 8$)$.

The results from GLM analysis of the intact dataset indicated that WMJT was the most common factor affecting species presence and abundance in this assemblage (Table 
9). The AIC-based, stepwise selection processes selected this predictor in 10 of the 14 species modeled, which accounted for $7 \%$ to $41 \%$ of the deviance in the dependent variables (Table 9). Of these 10 species, only S. fontinalis demonstrated a negative relationship with WMJT, indicating a tendency to be present more often in colder streams. The remaining 9 species' rates of presence all demonstrated a positive trend with WMJT (Table 9). Only two species, $R$. cataractae and $S$. fontinalis, demonstrated a relationship with RVHA (Table 9). Higher RVHA scores resulted in a higher rate of presence for S. fontinalis and higher abundances for R. cataractae (Table 9). Water chemistry principal component axis 1 (WC1) was selected as an important variable for only two species in the intact dataset, $C$. commersoni and $C$. funduloides. Both of these species demonstrated negative trends with $\mathrm{WC1}$, indicating an increased rate of presence in more alkaline streams (Table 9). Interestingly, six species demonstrated negative trends with distance from their expected population cores (Table 9). Finally, for two species, the widely occurring $R$. obtusus and $S$. atromaculatus, the independent variables used in this analysis did not result in a model superior to the null model (Table 9).

In the GLM analysis of species from the fragmented assemblage, WC1 was the most common variable selected by AIC for modeling species' abundances or presence (Table 9). This variable was selected as an important predictor in 10 of 14 models and explained anywhere from $4 \%$ to $35 \%$ of the deviance in the dependent variables in this dataset (Table 9). Of those species for which WC1 was selected, most (8 of 10) demonstrated a positive trend with WC1, indicating an increased likelihood of presence or increased abundance in sites with lower specific conductivity, sulfate concentrations, and hardness (Table 9). On the other hand, two species, C. commersoni and L. cyanellus, 
demonstrated a negative trend with $\mathrm{WC1}$, indicating increased rates of presence in streams with a stronger chemical mining signature (Table 9). WMJT remained a commonly selected predictor for species in the fragmented assemblage, as it was an important predictor for 9 of 14 species (Table 9). WMJT accounted for $6 \%$ to $24 \%$ of the deviance in the dependent variables in this dataset (Table 9). RVHA score was an important determinant in 7 of the 14 species modeled in the fragmented dataset and explained $1 \%$ to $16 \%$ of the deviance in the dependent variable for those species (Table 9). Of those seven species, four exhibited a negative trend with RVHA, indicating an increased rate of presence in streams with poorer local habitat condition (Table 9). The remaining three species demonstrated a positive trend with RVHA (Table 9). Four species demonstrated negative trends with distance to an expected population core in the fragmented assemblage, while one species, S. atromaculatus, demonstrated a positive trend with this variable (Table 9). Interestingly, there was no model selected superior to the null for C. funduloides in the fragmented system (Table 9).

In a comparison of species' responses to isolation from their respective cores in the two assemblages, the data indicated that, of the 9 species demonstrating a tendency to form metapopulations in the intact and fragmented systems, only two species, $C$.

anomalum and C. bairdi, consistently demonstrated the tendency in the both systems (Table 9). Although C. funduloides, H. nigricans, L. cyanellus, and R. cataractae demonstrated this tendency in the intact region, none of these species appeared to form metapopulations in fragmented system at this scale (Table 9). In fact, C. funduloides presence was unrelated to all of the independent variables modeled in the fragmented system (Table 9). Likewise, three species, E. flabellare, M. dolomieu, and $S$. 
atromaculatus demonstrated a relationship with isolation from their cores in the fragmented system, but not in the intact system (Table 9).

Summary of results pursuant of a priori expectations

The analyses related to the metacommunity model predictions concerning the relationship between $\alpha$ - and $\beta$-diversity and abiotic heterogeneity were not supportive of either the neutral or patch dynamic frameworks, but were supportive of both the species sorting and mass effect expectations (Table 10). Although, we expected that $\alpha$-diversity would be more strongly correlated with abiotic heterogeneity in the fragmented assemblage relative to the intact should the mass effect model apply (Table 2, Table 10), the results were difficult to interpret pursuant to this expectation due to the fact that the relative importance of individual abiotic gradients was generally inconsistent across assemblages (Table 4, Table 5). For instance, while WMJT was a strong sorting mechanism for the intact assemblage, it was not an important gradient in the fragmented assemblage (Table 5). Likewise, water chemistry became a dominant gradient in the fragmented assemblage, but was not in the intact system (Table 5). Nevertheless, cumulative drainage area, as expected given mass effect predictions, was more strongly correlated with diversity in the fragmented system (Table 4). This finding may lend some additional support, albeit very little, to our a priori expectation concerning mass effect theory (Table 2, Table 10). In any case, our results can not distinguish between a decreased strength of correlation due possibly to a reduction in mean local heterogeneity between the two systems or reduced mass effects. Therefore, our results concerning 
abiotic heterogeneity and diversity were equally supportive of the species sorting and mass effect predictions (Table 10).

Our results concerning dispersal and $\beta$-diversity were primarily supportive of the mass effect model prediction, although our finding demonstrating the relationship between dispersal and $\alpha$-diversity also provided support for the neutral and patch dynamic perspectives (Table 10). However, the finding that $\beta$-diversity in the fragmented system was not correlated with inter-site distance but was correlated in the intact system supports only the mass effect expectation. The mass effect model predicts that, as dispersal becomes more limited, mass effects are reduced and diversity is driven primarily by heterogeneity in abiotic conditions, thus, being reduced to a species sorting system (Mouquet and Loreau 2003, Table 2). Although the neutral model also predicts $\beta$ diversity to be correlated with distance and for $\beta$-diversity to decrease with decreasing dispersal, it does not predict a threshold relationship for dispersal and $\beta$-diversity as does the mass effect model and, therefore, does not support the finding of a different relationship for each assemblage (Figure 10). Interestingly, if we had considered the results separately for the intact and fragmented assemblages, the mass effect and neutral models would have been equally supported in the intact system and the species sorting and patch dynamic models would have been the only possible fits for the fragmented system.

The results of the comparison of theoretical and empirical SADs suggested that the ZSM provided a substantially better fit to both assemblages (Table 7) and, thus, the neutral model could not be ruled out as a candidate model for this system based on these results (Table 10). Because each of the other models also supports SADs similar to those 
predicted by the neutral model, however, none of those frameworks could be ruled out on this basis either (Table 10). Nevertheless, the fact that the neutral model fit both distributions well may provide some, albeit very little, additional support for neutral mechanisms in structuring the SADs (Table 10).

The results of the exact test of neutrality led to similar conclusions as the SAD model fitting (Table 10). The test could not be rejected for either the intact or fragmented assemblages. However, this finding does not lead unequivocally to the conclusion that neutral mechanisms are responsible for the structuring of the abundances in these two assemblages because the type I error rate is not specified for the test (Etienne 2007). Nevertheless, the finding may provide some minor additional support for a role for neutral processes in this system (Table 10).

Conversely, our results for species abundance and occupancy spectra demonstrating the inflation and deflation of individual species relative abundances and occupancies in the fragmented relative to intact assemblage did not support the neutral prediction that loss of species, or deflation of their occupancy or abundance, following disturbance would reflect a random draw from the assemblage pre-impact (i.e., intact assemblage; Table 10). This finding is supported by the three alternative models, although due to different mechanisms (Table 10).

The results detailing the response of individual species' abundances and occurrence to local abiotic conditions and distance from their core distribution provided complete support for only the mass effect prediction (Table 10). The results provided only partial support for the neutral, patch dynamic, and species sorting predictions. The mass effect model accounts for the finding that species abundances and occurrence were 
strongly related to both local physicochemical variability and isolation from the expected population core. Although the neutral model provides support for an effect of localized dispersal and a tendency to form metapopulations (i.e., distance to core) it does not support variability in that tendency among species. Likewise, although the species sorting framework accounts for the strong influence of local abiotic variability, it does not account for the tendency of some species to form metapopulations at this scale. The patch dynamic model accounts well for the tendency of species to form metapopulations and the variability in that tendency among species at this scale, but it does not account for the importance of local abiotic variability. Interestingly, the patch dynamic model, however, may also account for the inflation of S. atromaculatus with increasing isolation in the fragmented assemblage via a competitive release (e.g., loss of predatory or competitive species in completely isolated patches).

\section{Discussion}

Although the mass effect model was the framework receiving the most support following our analyses, the system specific applicability of the other models suggested that the mass effect model likely represents an intermediate region within a multidimensional spectrum of important mechanisms along which diversity is maintained in this system (Figure 7). For example, our results indicated that although the neutral and mass effect models each account for the importance of dispersal limitation in the intact system, the species sorting framework may more adequately describe dynamics in the fragmented system where dispersal was highly limited and sorting via chemical 
impairment was strong. Likewise, the patch dynamic perspective offers a mechanism for describing the inflation and deflation of individual species in isolated versus connected reaches based on their traits (e.g., inflation of prey species in isolated sites), although the phenomenon could also be related to manipulation of the source-sink landscape (Mouquet and Loreau 2003, Driscoll 2008). In any case, Cottenie (2005) came to a similar conclusion when assessing the importance of spatial versus environmental gradients in structuring a wide range of plant and animal communities, finding that the relative importance of species sorting, mass effect and neutral processes was largely system and scale specific. Others have also recently noted that a quantitative synthesis of the competing frameworks is most likely to lead to better approximations of a broader range of empirical patterns (Amerasekare et al. 2004, Mouquet et al. 2005).

Although the patch dynamic model was not well supported overall, two lines of evidence from our results suggested some role for species interactions in this system. First, our GLM results demonstrated that, while some species appeared to form metapopulations in the intact system, they did not appear to do so in the fragmented system (e.g., H. nigricans and $R$. cataractae; Table 9). Likewise, there were also some species that demonstrated an isolation effect in the fragmented system, but not in the intact system (e.g., E. flabellare and S. atromaculatus; Table 9). In a similar endeavor, Driscoll (2008) described this phenomenon within a metacommunity of Tasmanian beetles as a tendency for some species to form deterministic metapopulations (Thomas 1994), whereas isolation was suspected to interact with patch condition (i.e., mass effect) or interspecific interactions. In fact, the deterministic metapopulation was the most common type among the 40 species examined in that study (Driscoll 2008). We suggest 
that species interactions may also play a role in the extent to which fishes in this study form metapopulations. For example, S. atromaculatus abundances appeared to exhibit a positive relationship with distance from expected source streams in the fragmented assemblage, but not in the intact system (Table 9). This finding seemed, at first, counterintuitive to the nature of the isolation variable. However, given the logic of how this variable was constructed or, more specifically, given that sites that were completely isolated by AMD were also considered, by convenience, as being equivalent to the maximum distance for any site from a core size stream, it is intuitive that species that thrive in isolation would correlate positively with this "gradient". Inflation of $S$. atromaculatus abundances in isolation could be indicative of an important spatial consumer-resource interaction (Briggs and Hoopes 2004) attributable to a release from predation (Horwitz 1978, Anderson 1985, Power et al. 1985, Schlosser 1987) in disturbed habitats due to a loss of likely predatory species (e.g., S. fontinalis and M. dolomieu). In fact, the relative abundances of several potential prey species, including $L$. chrysocephalus and P. notatus, demonstrated notably inflated relative abundances and/or occupancy in the fragmented system relative to the intact (Table 8 , Figure 7).

Our results also provided mixed support for neutral processes structuring stream fish diversity in this system. The most compelling findings were related to the overall importance of dispersal limitation, the consistent fit of the ZSM, and the inability to reject the exact test of neutrality (Table 10; Etienne 2007). Very recent research by Muneepeerakul et al. (2008) demonstrated that stream fish communities in the Mississippi-Missouri drainage basin demonstrate patterns reminiscent of Hubbell's (2001) neutral model, where accurate predictions of diversity patterns were modeled 
using only estimates of habitat capacity (i.e., an estimate of local carrying capacity) and a mean dispersal kernel. With that study, the authors set an important precedent in placing empirical stream fish community patterns in a metacommunity context (Muneepeerakul et al. 2008). However, while that research detailed the importance of dispersal and stochastic demography in shaping fish communities at a near continental scale, the historical stream fish community ecology literature provides little support for a zero sum view given the common findings of habitat specialization (Gorman and Karr 1978), resource partitioning (Ross 1986, Schlosser 1987, Nisikawa and Nokano 1998) and habitat-diversity relationships (Horwitz 1978, Schlosser 1982) at finer spatial scales. Not surprisingly, the assumption of ecological equivalence and the zero-sum assumption, although recently relaxed (Etienne 2007b), has been a strong point of contention for ecologists from a range of ecological disciplines (Holyoak and Loreau 2006). In any case, our results suggest that neutral theory may not translate as well as competing theories at this intermediate spatial scale. Moreover, our findings from the fragmented system suggest that the neutral model is likely less applicable in highly disturbed systems or where dispersal is highly limited; a caution Hubbell (2001) himself noted.

The ZSM has been shown to approximate the SADs of a wide range of communities in nature, including neo-tropical trees (Hubbell 2001, Etienne and Olff 2005), Australian birds (MacNally 2007), a rocky intertidal community of the Pacific northwest (Wooton 2005), and neo-tropical fishes (Etienne and Olff 2005). To date, however, we know of no studies that have attempted fitting the ZSM to a freshwater stream fish community, although Muneepeerakul et al. (2008) noted qualitative similarities in the rank-occupancy distribution observed for Mississippi-Missouri basin 
fishes. Nevertheless, while the larger body of evidence in this paper suggests that the assumption of ecological equivalence is incompatible with this system, the remarkable fit of the ZSM to both datasets in this study adds to a growing list of studies characterizing the practical utility of the ZSM formulation in predicting diversity and abundance across a wide range of scales, ecosystems and trophic levels (Hubbell 2001, Latimer et al. 2005, Muneepeerakul et al. 2008). Nevertheless, as others have cautioned (Wooton 2005, Harpole and Tilman 2006, MacNally 2007), although empirical SADs may fit the ZSM quite well, fluctuations in individual species' relative abundance are likely to be poorly described by neutral mechanisms. Harpole and Tilman (2006) found that, for example, although the abundance distribution of plant species in a North American grassland were consistent with the ZSM model, species abundance ranks correlated strongly with expected competitive ranks based on competitive ability for nitrogen $(\mathrm{N})$. Wootton (2005) came to a similar conclusion following experimental examination of a rocky intertidal community, emphasizing an essential role for variation in species characteristics in community assembly. In the end, our results suggested that a combination of mechanisms related to dispersal limitation, differential tolerances to local conditions, source-sink dynamics, or common competition-colonization/fecundity tradeoffs (Chase and Leibold 2003, Tilman 1982, Yu and Wilson 2001) likely provide a more feasible explanation for the changes in species relative abundance patterns in this region. In support of this conclusion, several researchers have also shown that similar SADs, including the ZSM, can be arrived at under alternate theoretical foundations (Mouquet and Loreau 2003, Volkov et al. 2005, McGill 2006, Allouche and Kadmon 2009). 
Nevertheless, the mass effect framework received the most consistent support in this study and likely provided the best generalization of the mechanisms driving local and regional diversity in this system. Moreover, the mass effect model was the only model for which all specific predictions were unable to be rejected given the data. Our results consistently demonstrated that both $\alpha$ - and $\beta$-diversity in this system are influenced largely by an interaction between local physicochemical sorting processes and dispersal limitation. Other studies have noted the importance of dispersal to local and regional diversity (Harrison 1997, 1999, Chase 2003, Kneitel and Miller 2003, Staddon et al. 2010). For example, in a comparison of plant diversity between patches of serpentine grasslands varying in degree of isolation, Harrison $(1997,1998)$ found that both $\beta$ - and regional diversity were lower in more connected communities. Similarly, Chase (2003) found decreases in $\beta$ - and regional diversity and increases in local diversity in more closely aligned wetland amphibian and macroinvertebrate metacommunities. In a recent experiment, Staddon et al. (2010) found that modification of dispersal corridors affected the diversity of microarthropods across a range of trophic levels in a detrital food web. Our findings are also consistent with another recent study demonstrating the combined importance of dispersal and local physicochemical conditions to freshwater fish diversity (Falke and Fausch, in press). Falke and Fausch (in press) demonstrated that a metacommunity of stream fishes in a Great Plains watershed was structured primarily by processes related to temporal variability in dispersal opportunities resulting from an interaction between flow variability and dispersal. Finally, our conclusions are also generally supported by a growing body of literature indicating the importance of regional 
dispersal processes to the assemblage structure of stream fishes (Osborne and Wiley 1992, Snodgrass and Meffe 1998, Grenouillet et al. 2004, Hitt and Angermeier 2008). Recent direct evaluations of neutral theory have focused primarily on single avenues of evaluation, including simple curve fitting (e.g., Hubbell 2001, Bell 2003, McGill 2003), relationships between diversity and spatial and environmental gradients (e.g., Cottenie et al. 2005, Falke and Fausch, in press), and the response of relative abundances or individual species to experimental or natural disturbance (e.g., Wooten 2005, Driscoll 2008, Driscoll and Lindenmayer 2009). We believe our study is novel as we know of no other studies to date that have combined these approaches in a pluralistic approach to evaluating the predictions of metacommunity theory. By combining these approaches, we were able to evaluate a wide range patterns and hypotheses surrounding theoretical predictions outlined in the primary literature, ranging from simple curve fitting and null hypothesis testing to multiple complex predictions and competing model comparisons, as suggested by McGill (2006). Therefore, we believe our findings necessarily progress the metacommunity research program related to both freshwater stream fishes and the general field of ecology (Platt 1964, McGill 2006). Like others, we believe that future progression of metacommunity theory will necessarily hinge on similar pluralistic approaches (Chase et al. 2005, McGill 2006).

Although we were able to more clearly establish the importance of dispersal limitation and local physicochemical variability in explaining local and regional diversity in this system, we were unable to explicitly evaluate the strength of species interactions on diversity. For example, although we observed strong inflation and deflation of particular species abundances and occupancy in the fragmented assemblage compared to 
the intact assemblage that was highly inconsistent with neutral theory, we offered only anecdotal explanations for mechanisms (e.g., consumer-resource interactions versus modification of the source-sink landscape). We recognize, however, that explicit derivation of the mechanisms of inflation and deflation is only possible through carefully designed experimental studies. As such, we suggest that such approaches represent a promising avenue of future research. Further clarification of the mechanisms of inflation and deflation of individual species in response to disturbance and isolation will play an important role in determining where real communities fall within the three dimensional range of possibilities outlined in Figure 7, as well as the extent to which each of these three mechanisms are synthesized in future theoretical models.

\section{Management implications}

Our results indicate that knowledge of habitat connectivity and spatial processes is crucial to managing stream fish biodiversity. Like those from similar studies of metacommunity dynamics, our results imply that managers must take a multi-scale, landscape- or watershed-based approach to the protection and restoration of biodiversity. Specifically, managers of freshwater stream fish diversity should seek to avoid regional diversity losses as a result of accumulated local impacts and manipulation of the sourcesink and/or consumer-resource landscape. Approaches to conserving and protecting stream fish biodiversity should, therefore, prioritize protection of important dispersal corridors and species pools or source habitats. Likewise, managers should seek to restore local and regional stream fish diversity through targeted restoration of important stream 
migration corridors, with particular attention to the source-sink and consumer-resource landscape. 


\section{Literature cited}

Abell, R. 2002. Conservation biology for the biodiversity crisis: a freshwater follow-up. The Journal of the Society for Conservation Biology 16:1435-1437.

Allouche, O., and R. Kadmon. 2009. Demographic analysis of Hubbell's neutral theory of biodiversity. Journal of Theoretical Biology 258:274 - 280 .

Amarasekare, P. 1998. Interactions between local dynamics and dispersal: insights from single species models. American Naturalist 158: 572-584.

Amarasekare, P., and R. Nisbet. 2001. Spatial heterogeneity, source-sink dynamics, and the local coexistence of competing species. The American Naturalist 158:572584.

Anderson, C. S. 1985. The structure of sculpin populations along a stream size gradient. Environmental Biology of Fishes 13:93-102.

Anderson, R. M., T. F. Beer, M. E. Buckwalter, S. D. Clark, I. Mc Auley, J. I. Sams, and D. R. Williams. 2000. Water quality in the Allegheny and Monongahela River Basins, Pennsylvania, West Virginia, New York, and Maryland. U.S. Geological Survey Circular 1202, U.S. Department of Interior, U.S. Geological Survey. 
Angermeier, P. L., and I. J. Schlosser. 1989. Species-area relationship for stream fishes. Ecology 70:1450-1462.

Baker, J. P., and C. L. Schofield. 1981. Aluminum toxicity to fish in acid waters. Earth and Environmental Science 18:289-309.

Baker, J. P., J. Van Sickle, C. J. Gagen, D. R. DeWalle, W. E. Sharpe, R. F. Carline, B. P. Baldigo, P. S. Murdoch, D. W. Bath, W. A. Krester, H. A. Simonin, and P. J. Wigington. 1996. Episodic Acidification of Small Streams in the Northeastern United States: Effects on Fish Populations. Ecological Applications 6:422-437.

Barbour, M. T., J. Gerritsen, B. D. Snyder, and J. B. Stribling. 1999. Rapid Bioassessment Protocols for Use in Streams and Wadeable Rivers: Periphyton, Benthic Macroinvertebrates and Fish, Second Edition. EPA 841-B-99-002. U.S. Environmental Protection Agency; Office of Water; Washington, D.C.

Bell, G. 2000. The distribution of abundance in neutral communities. The American Naturalist 155:606-617.

Bell, G. 2001. Neutral macroecology. Science 293: 2413-2418.

Bojsen, B. H., and R. Barriga. 2002. Effects of deforestation on fish community structure in Ecuadorian Amazon streams. Freshwater Biology 47:2246-2260. 
Bolker, B. M., and S. W. Pacala. 1997. Using moment equations to understand stochastically driven spatial pattern formation in ecological systems. Theoretical Population Biology 52: 179-197.

Bouvier, L. D., K. Cottenie, and S. E. Doka. 2009. Aquatic connectivity and fish metacommunities in wetlands of the lower Great Lakes. Canadian Journal of Fisheries and Aquatic Sciences 66:933-948.

Briggs, C. J., and M. F. Hoopes. 2004. Stabilizing effects in spatial parasitoid-host and predator-prey models: a review. Theoretical Population Biology 65: 299-315.

Chase, J. M., and M. A. Leibold. 2003. Ecological Niches. University of Chicago Press, Chicago, IL, USA.

Chase, J. M., P. Amarasekare, K. Cottenie, A. Gonzalez, R. D. Holt, M. Holyoak, M. F. Hoopes, M. A. Leibold, M. Loreau, N. Mouquet, J. B. Shurin, and D. Tilman. 2005. Competing theories for competitive metacommunities. Pages 335-355 in M. Holyoak, M. A. Leibold, and R. D. Holt, editors. Metacommunities: spatial dynamics and ecological communities. University of Chicago Press, Chicago, IL, USA. 
Chave, J., H. Muller - Landau, and S. Levin. 2002. Comparing classical community models: theoretical consequences for patterns of diversity. The American Naturalist 159:1-23.

Chave, J. 2004. Neutral theory and community ecology. Ecology Letters 7:241-253.

Chave, J., D. Alonso, and R. S. Etienne. 2006. Comparing models of species abundance. Nature 441:E1-E2.

Chesson, P. 2000. General theory of competitive coexistence in spatially-varying environments. Theoretical Population Biology 58: 211-237.

Clarke, K. R., and R. H. Green. 1988. Statistical design and analysis for a 'biological effects' study. Marine Ecology Progress Series 46:213-226.

Clarke, K. R. 1993. Non-parametric multivariate analysis of changes in community structure. Australian Journal of Ecology 18:117-143.

Clayton, J. L., E. S. Dannaway, R. Menendez, H. W. Rauch, J. J. Renton, S. M. Sherlock, and P. E. Zurbuch. 1998. Application of limestone to restore fish communities in acidified streams. North American Journal of Fisheries Management 18:347-360. 
Connell, J. H. 1978. Diversity in Tropical Rain Forests and Coral Reefs. Science 199:1302-1310.

Cottenie, K. 2005. Integrating environmental and spatial processes in ecological community dynamics. Ecology Letters 8:1175-1182.

DeRoos, A. M. 1998. Pattern formation and the spatial scale of interaction between predators and their prey. Theoretical Population Biology 53: 108-130.

Diamond, J. M. 1975. Assembly rules of species communities. Pages 342-444 in M. L. Cody and J. M. Diamond, editors. Ecology and Evolution of Communities. Harvard University Press, Cambridge, MA.

Driscoll, D. A. 2008. The frequency of metapopulations, metacommunities and nestedness in a fragmented landscape. Oikos 117:297-309.

Driscoll, D. A., and D. B. Lindenmayer. 2009. Empirical tests of metacommunity theory using an isolation gradient. Ecological Monographs 79:485-501.

Eaton, J. G., J. H. McCormick, B. E. Goodno, D. G. O’Brien, H. G. Stefany, M. Hondzo, and R. M. Scheller. 1995. A field information-based system for estimating fish temperature tolerances. Fisheries 20:10-18. 
ESRI. 2007. ArcGIS Desktop version 9.3. Environmental Systems Research Institute, Redlands, California.

Etienne, R. S. 2005. A new sampling formula for neutral biodiversity. Ecology Letters 8:253-260.

Etienne, R. S., and H. Olff. 2005. Confronting different models of community structure to species-abundance data: a Bayesian model comparison. Ecology Letters 8:493-504.

Etienne, R. S. 2007. A neutral sampling formula for multiple samples and an 'exact' test of neutrality. Ecology Letters 10:608-618.

Etienne, R. S., D. Alonso, and A. J. McKane. 2007b. The zero-sum assumption in neutral biodiversity theory. Journal of Theoretical Biology 248:522-536.

Fagan, W. F., P. J. Unmack, C. Burgess, and W. L. Minckley. 2002. Rarity, fragmentation, and extinction risk in desert fishes. Ecology 83:3250-3256.

Falke, J. A., and K. D. Fausch.From metapopulations to metacommunities: linking theory with empirical observations of the spatial population dynamics of stream fishes. American Fisheries Society Symposium. 
Faraway, J. J. 2006. Extending the Linear Model with R. Chapman \& Hall/CRC, Boca Raton, FL, USA.

Fausch, K. D., J. R. Karr, and P. R. Yant. 1984. Regional application of an index of biotic integrity based on stream fish communities. Transactions of the American Fisheries Society 113: 39-55.

Fausch, K. D., C. Torgersen, C. V. Baxter, and H. W. Li. 2002. Landscapes to riverscapes: bridging the gap between research and conservation of stream fishes. BioScience 52:483-498.

Fisher, R. A., A. S. Corbet, and C. B. Williams. 1943. The relation between the number of species and the number of individuals in a random sample of an animal population. Journal of Animal Ecology 12:42-58.

Freund, J. G. 2004. Local and regional impairment of fish assemblages in a mined Appalachian watershed. Doctoral Dissertation. West Virginia Univeristy, Morgantown, WV, USA.

Freund, J. G., and J. Petty. 2007. Response of Fish and Macroinvertebrate Bioassessment Indices to Water Chemistry in a Mined Appalachian Watershed. Environmental Management 39:707-720. 
Grenouillet, G., D. Pont, and C. Herisse. 2004. Within-basin fish assemblage structure: the relative influence of habitat versus stream spatial position on local species richness. Canadian Journal of Fisheries and Aquatic Sciences 61:93-102.

Grossman, G. D., P. B. Moyle, and J. O. Whitaker. 1982. Stochasticity in structural and functional characteristics of an Indiana stream fish assemblage: a test of community theory. The American Naturalist 120:423-454.

Hanski, I. A. 1989. Metapopulation dynamics: does it help to have more of the same? Trends in Ecology \& Evolution 4:113-114.

Hanski, I. 1997. Metapopulation dynamics: from concepts and observations to predictive models. Pages 69-72 In I. Hanski and M. E. Gilpin Eds. Metapopulation biology: Ecology, Genetics, and Evolution. Academic Press, San Diego, CA.

Harpole, W. S., and D. Tilman. 2006. Non-neutral patterns of species abundance in grassland communities. Ecology Letters 9:15-23.

Harte, J. 2003. Ecology: Tail of death and resurrection. Nature 424:1006-1007.

Hense, Z. 2007. Stream fish populations in a watershed scale context for fish community dynamics in central Appalachian watersheds. Master's Thesis. West Virginia University, Morgantown, WV, USA. 
Hense, Z., R. W. Martin, and J. T. Petty. In Press. Electrofishing capture efficiencies for common stream fish species to support watershed scale studies in the central Appalachians. North American Journal of Fisheries Management I:I-I.

Herlihy, A. T., P. R. Kaufmann, M. E. Mitch, and D. D. Brown. 1990. Regional estimates of acid mine drainage impact on streams in the mid-Atlantic and Southeaster United States. Water, Air, \& Soil Pollution 50:91-107.

Higgins, C. L., and R. E. Strauss. 2008. Modeling stream fish assemblages with niche apportionment models: patterns, processes, and scale dependence. Transactions of the American Fisheries Society 137:696-706.

Hitt, N. P., and P. L. Angermeier. 2008. Evidence for fish dispersal from spatial analysis of stream network topology. Journal of the North American Benthological Society 27:304-320.

Holt, R. D. 1985. Population dynamics in two-patch environments: some anomalous consequences of an optimal habitat distribution. Theoretical Population Biology 28: $181-208$.

Holt, R. D. 2002. Food webs in space: on the interplay of dynamic instability and spatial processes. Ecological Research 17: 261-273. 
Holyoak, M., M. A. Leibold, and R. D. Hold (Eds.). 2005b. Metacommunities: Spatial Dynamics and Ecological Communities. University of Chicago Press.

Holyoak, M., M. A. Leibold, N. Mouquet, R. D. Holt, and M. F. Hoopes. 2005 a. Metacommunities: a framework for large-scale community ecology. Pages 1-31 in M. Holyoak, M. A. Leibold, and R. D. Holts editors. Metacommunities: Spatial Dynamics and Ecological Communities. University of Chicago Press, Chicago, Illinois, USA.

Holyoak, M., and M. Loreau. 2006. Reconciling empirical ecology with neutral community models. Ecology 87:1370-1377.

Hoopes, M. F., R. D. Holt, and M. Holyoak. 2005. The effects of spatial processes on two species interactions. Pages 35-67 in M. Holyoak, M. A. Leibold, and R. D. Holts editors. Metacommunities: Spatial Dynamics and Ecological Communities. University of Chicago Press, Chicago, Illinois, USA.

Horwitz, R. J. 1978. Temporal variability patterns and the distributional patterns of stream fishes. Ecological Monographs 48:307-321.

Hubbell, S. P. 2001. The Unified Neutral Theory of Biodiversity and Biogeography. Princeton University Press, Princeton, NJ. 
Keeley, J.E. and C.J. Fotheringham. 2003. Species-area relationships in Mediterranean climate plant communities. Journal of Biogeography 30: 1629-1657.

King, R. S., M. E. Baker, D. F. Whigham, D. E. Weller, T. E. Jordan, P. F. Kazyak, and M. K. Hurd. 2005. Spatial considerations for linking watershed land cover to ecological indicators in streams. Ecological Applications 15:137-153.

Kneitel, J. M., and T. E. Miller. 2003. Dispersal rates affect species composition in metacommunities of Sarracenia purpurea inquilines. American Naturalist 162: $165-171$.

Latimer, A. M., J. A. Silander, and R. M. Cowling. 2005. Neutral ecological theory reveals isolation and rapid speciation in a biodiversity hot spot. Science 309:1722-1725.

Legendre, P., and L. Legendre. 1998. Numerical Ecology, $2^{\text {nd }}$ edition. Elsevier Science B.V., Amsterdam, The Netherlands.

Leibold, M. A. 1998. Similarity and local co-existence of species in regional biotas. Evolutionary Ecology 12: 95-110. 
Leibold, M. A., M. Holyoak, N. Mouquet, P. Amarasekare, J. M. Chase, M. F. Hoopes, R. D. Holt, J. B. Shurin, R. Law, D. Tilman, M. Loreau, and A. Gonzalez. 2004. The metacommunity concept: a framework for multi-scale community ecology. Ecology Letters 7:601-613.

Levin, S. A. 1970. Community equilibria and stability, and an extension of the competitive exclusion principle. The American Naturalist 104:413.

Levin, S. A. 1974. Dispersion and population interactions. American Naturalist 108: 207-228.

Levins, R., and D. Culver. 1971. Regional coexistence of species and competition between rare species. Proceedings of the National Academy of Sciences 68: $1246-1248$.

Loreau, M., S. Naeem, P. Inchausti, J. Bengtsson, J. P. Grime, A. Hector, D. U. Hooper, M. A. Huston, D. Raffaelli, B. Schmid, D. Tilman, and D. A. Wardle. 2001. Biodiversity and ecosystem functioning: current knowledge and future challenges. Science 294:804-808.

MacArthur, R. H. 1957. On the Relative Abundance of Bird Species. Proceedings of the National Academy of Sciences of the United States of America 43:293-295. 
MacArthur, R. 1960. On the Relative Abundance of Species. The American Naturalist 94:25.

MacArthur, R. H., and E. O. Wilson. 1967. The Theory of Island Biogeography. Princeton University Press, Princeton, NJ, USA.

MacArthur, R. H. 1970. Species packing and competitive equilibrium for many species. Theoretical Population Biology 1: 1-11.

Mac Nally, R. 2007. Use of the abundance spectrum and relative - abundance distributions to analyze assemblage change in massively altered landscapes. The American Naturalist 170:319-330.

Magnuson, J. J., J. P. Baker, E. J. Rahel, and J. R. Kramer. 1984. A critical assessment of effects of acidification on fisheries in North America [and discussion]. Philosophical Transactions of the Royal Society of London. Series B, Biological Sciences 305:501-516.

Mantel, N. 1967. The Detection of Disease Clustering and a Generalized Regression Approach. Cancer Res 27:209-220.

Martin, R. W., and J. T. Petty. 2009. Local stream temperature and drainage network topology interact to influence the distribution of smallmounth bass and brook 
trout in a central Appalachian watershed. Journal of Freshwater Ecology 24:497508.

May, R. M. 1975. Patterns of species abundance and diversity. Pages 81-120 in C. L. Martin and J. M. Diamond, editors. Ecology and Evolution of Communities. Harvard University Press, Cambridge, MA.

McGill, B. J. 2003. A test of the unified neutral theory of biodiversity. Nature 422:881885.

McGill, B. J., B. A. Maurer, and M. D. Weiser. 2006. Empirical evaluation of neutral theory. Ecology 87:1411-1423.

McClurg, S. E., J. T. Petty, P. M. Mazik, and J. L. Clayton. 2007. Stream ecosystem response to limestone treatment in acid impacted watersheds of the Allegheny Plateau. Ecological Applications 17:1087-1104.

McCormick, F. H., R. M. Hughes, P. R. Kaufmann, D. V. Peck, J. L. Stoddard, and A. T. Herlihy. 2001. Development of an index of biotic integrity for the midAtlantic Highlands region. Transactions of the American Fisheries Society 130:857-877. 
McCullagh, P., and J. A. Nelder. 1989. Generalized Linear Models. John Wiley \& Sons, New York, New York, USA.

Merovich, G. T., Jr., and J. T. Petty. 2007. Interactive effects of multiple stressors and restoration priorities in a mined Appalachian watershed. Hydrobiologia 575:1331.

Merovich, G. T., J. M. Stiles, J. T. Petty, P. F. Ziemkiewicz, and J. B. Fulton. 2007. Water chemistry-based classification of streams and implications for restoring mined Appalachian watersheds. Environmental Toxicology and Chemistry: 1361-1369.

Minchin, P. R. 1987. An evaluation of relative robustness of techniques for ecological ordinations. Vegetatio 71:145-156.

Motomura, I. 1932. A statistical treatment of associations. Zoological Magazine, Japan 44:379-383.

Mouquet, N. J., and M. Loreau. 2002. Coexistence in metacommunities: the regional similarity hypothesis. American Naturalist 149: 420-426. 
Mouquet, N., J. L. Moore, and M. Loreau. 2002. Plant species richness and community productivity: why the mechanism that promotes coexistence matters. Ecology Letters 5:56-65.

Mouquet, N., and M. Loreau. 2003. Community patterns in source-sink metacommunities. The American Naturalist 162:544-557.

Mouquet, N., Hoopes, M. F., and P. Amarasekare. 2005. The world is patchy and heterogeneous! Pages 237-262 in M. Holyoak, M. A. Leibold, and R. D. Holts editors. Metacommunities: Spatial Dynamics and Ecological Communities. University of Chicago Press, Chicago, Illinois, USA.

Mouquet, N., T. E. Miller, T. Daufresne, and J. M. Kneitel. 2006. Consequences of varying regional heterogeneity in source-sink metacommunities. Oikos 113:481488.

Muneepeerakul, R., E. Bertuzzo, H. J. Lynch, W. F. Fagan, A. Rinaldo, and I. Rodriguez-Iturbe. 2008. Neutral metacommunity models predict fish diversity patterns in Mississippi-Missouri basin. Nature 453:220-222.

Naeem, S., and S. Li. 1997. Biodiversity enhances ecosystem reliability. Nature 390:507-509. 
Naeem, S., M. Loreau, and P. Inchausti. 2002. Biodiversity and ecosystem functioning: the emergence of a synthetic ecological framework. Pages 3-11 in M. Loreau, S. Naeem, and P. Inchausti, editors. Biodiversity and Ecosystem Functioning: Synthesis and Perspective. Oxford University Press, New York, New York, USA.

Nouhuys, S., and I. Hanski. 2005. Metacommunities of butterflies, their host plants, and their parasitoids. Pages 99-121 in M. Holyoak, M. A. Leibold, and R. D. Holts editors. Metacommunities: Spatial Dynamics and Ecological Communities. University of Chicago Press, Chicago, Illinois, USA.

Ohio Environmental Protection Agency. 1989. Biological criteria for the protection of aquatic life. Vol. III. Users manual for biological field assessment of Ohio surface waters. Division of Water Quality Monitoring and Assessment, Surface Water Section, Columbus, $\mathrm{OH}$.

Oksanen, J., R. Kindt, P. Legendre, B. O'Hara, G. L. Simpson, P. Solymos, M. H. H. Stevens, and H. Wagner. 2009. vegan: Community ecology package. R package version 1.15-2. http://cran.r-project.org/, http://vegan.r-forge.r-project.org.

Osborne, L. L. and M.J. Wiley. 1992. Influence of tributary spatial position on the structure of warmwater fish communities. Canadian Journal of Fisheries and Aquatic Sciences 49:671-681. 
Petty, J. T., and J. Barker. 2004. Water quality variability in tributaries of the Cheat River, a mined Appalachian watershed. Proceedings of the American Society of Mining and Reclamation 21:1484-1504.

Platt, J. R. 1964. Strong inference: certain systematic methods of scientific thinking may produce much more rapid progress than others. Science 146:347-353.

Poff, N. L., and J. D. Allan. 1995. Functional organization of stream fish assemblages in relation to hydrological variability. Ecology 76:606-627.

Power, M. E., W. J. Matthews, and A. J. Stewart. 1985. Grazing minnows, piscivorous bass, and stream algae: dynamics of a strong interaction. Ecology 66:1448-1456.

Pinder, M. J., and R. Morgan II. 1995. Interactions of $\mathrm{pH}$ and habitat on cyprinid distributions in Appalachian streams of Maryland. Transactions of the American Fisheries Society 124:94-102.

Preston, F. W. 1948. The commonness, and rarity, of species. Ecology 29:254-283.

Pulliam, H. R. 1988. Sources, sinks, and population regulation. The American Naturalist 132:652. 
Ricciardi, A., and J. B. Rasmussen. 1999. Extinction rates of North American freshwater fauna. Conservation Biology 13: 1220-1222.

Rahel, F. J., and W. A. Hubert. 1991. Fish Assemblages and Habitat Gradients in a Rocky Mountain-Great Plains Stream: Biotic Zonation and Additive Patterns of Community Change. Transactions of the American Fisheries Society 120:319332.

Ricklefs, R. E. 2006. The unified neutral theory of biodiversity: do the numbers add up? Ecology 87:1424-1431.

Roper, B. B., and D. L. Scarnecchia. 1995. Observer Variability in Classifying Habitat Types in Stream Surveys. North American Journal of Fisheries Management 15:49-53.

Ross, S. T. 1986. Resource Partitioning in Fish Assemblages: A Review of Field Studies. Copeia 1986:352-388.

Roxburgh, S. H., K. Shea, and J. B. Wilson. 2004. The Intermediate Disturbance Hypothesis: Patch Dynamics and Mechanisms of Species Coexistence. Ecology 85:359-371. 
Sale, P. F. 1977. Maintenance of high diversity in coral reef fish communities. American Naturalist 111: 337-359.

Schlosser, I. J. 1982. Fish community structure and function along two habitat gradients in a headwater stream. Ecological Monographs 52:395-414.

Schlosser, I. J. 1987. The role of predation in age- and size-related habitat use by stream fishes. Ecology 68:651-659.

Schlosser, I. J. 1995. Dispersal, boundary processes, and trophic-level interactions in streams adjacent to beaver ponds. Ecology 76:908-925.

Schmida, A., and S. Ellner. 1984. Coexistence of plant species with similar niches. Vegetatio 58: 29-55.

Schmida, A., and R. H. Whittaker. 1981. Pattern and biological microsite effects in two shrub communities, southern California. Ecology 62: 234-251.

Seaber, P., Kapinos, F.P., Knapp, G., 1987. Hydrologic Unit Maps. In: Water Supply Paper 2294. United States Department of Interior, US Geological Survey, 63 pp. 
Shea, K., S. H. Roxburgh, and E. S. J. Rauschert. 2004. Moving from pattern to process: coexistence mechanisms under intermediate disturbance regimes. Ecology Letters 7:491-508.

Shurin, J. B., P. Amarasekare, J. M. Chase, R. D. Holt, M. F. Hoopes, and M. A. Leibold. 2004. Alternative stable states and regional community structure. Journal of Theoretical Biology 227:359-368.

Slatkin, M. 1974. Competition and regional coexistence. Ecology 55: 128-134.

Strager, M. P., J. T. Petty, J. M. Strager, and J. Barker-Fulton. 2009. A spatially explicit framework for quantifying downstream hydrologic conditions. Journal of Environmental Management 90:1854-1861.

Thomas, C. 1994. Extinction, colonization, and metapopulations: environmental tracking by rare species. Conservation Biology 8:373-378.

Tilman, D. 1982. Resource Competition and Community Structure. Princeton University Press, Princeton, NJ, USA.

Torgersen, C. E., D. M. Price, H. W. Li, and B. A. McIntosh. 1999. Multiscale thermal refugia and stream habitat associations of Chinook salmon in northeastern Oregon. Ecological Applications 9:301-319. 
USGS, 2000. The National Hydrography Dataset: Concepts and Content. U.S. Geological Survey. Available from: http://nhd.usgs.gov/chapter1/chp1_data_users_guide.pdf.

Volkov, I., J. R. Banavar, S. P. Hubbell, and A. Maritan. 2003. Neutral theory and relative species abundance in ecology. Nature 424:1035-1037.

Volkov, I., J. R. Banavar, F. He, S. P. Hubbell, and A. Maritan. 2005. Density dependence explains tree species abundance and diversity in tropical forests. Nature 438:658-661.

Wehrly, K. E., M. J. Wiley, and P. W. Seelbach. 2003. Classifying regional variation in thermal regime based on stream fish community patterns. Transactions of the American Fisheries Society 132:18-38.

Wilson, D. S. 1992. Complex Interactions in Metacommunities, with Implications for Biodiversity and Higher Levels of Selection. Ecology 73:1984-2000.

Whittaker, R. H. 1960. Vegetation of the Siskiyou mountains, Oregon and California. Ecological Monographs 30:279-338. 
Whittaker, R. H. 1965. Dominance and Diversity in Land Plant Communities: Numerical relations of species express the importance of competition in community function and evolution. Science 147:250-260.

Whittaker, R. H. 1972. Evolution and measurement of species diversity. Taxon 21:213251.

Wootton, J. T. 2005. Field parameterization and experimental test of the neutral theory of biodiversity. Nature 433:309-312.

Yu, D. W., and H. B. Wilson. 2001. The competition - colonization trade - off is dead; long live the competition - colonization trade - off. The American Naturalist 158:49-63.

Yu, D. W., H. B. Wilson, and N. E. Pierce. 2001. An empirical model of species coexistence in a spatially structured environment. Ecology 82:1761-1771. 
Tables

Table 1. Predicted patterns of diversity and abundance following from published theoretical work (see superscripts) and speculation based on model formulations, but not yet backed by specific theory.

\begin{tabular}{|c|c|c|c|c|}
\hline \multirow[b]{2}{*}{ Effect } & \multicolumn{4}{|c|}{ Model Prediction } \\
\hline & Neutral & Patch dynamics & Species sorting & Mass effects \\
\hline $\begin{array}{l}\text { Abiotic heterogeneity } \\
\text { and } \alpha \text {-diversity }\end{array}$ & No effect ${ }^{\mathrm{a}}$ & No effect & Positive relationship & $\begin{array}{l}\text { Hump-shaped relationship and } \\
\text { interacting with dispersal }{ }^{\text {b,c }}\end{array}$ \\
\hline $\begin{array}{l}\text { Abiotic heterogeneity } \\
\text { and } \beta \text {-diversity }\end{array}$ & No effect ${ }^{\mathrm{a}}$ & No effect & Positive relationship & $\begin{array}{l}\text { Hump-shaped relationship and } \\
\text { interacting with dispersal }\end{array}$ \\
\hline Dispersal and $\alpha$-diversity & Positive relationship & Hump-shaped ${ }^{\mathrm{d}}$ & No effect & $\begin{array}{l}\text { Hump-shaped relationship and } \\
\text { interacting with heterogeneity }{ }^{b, c}\end{array}$ \\
\hline Dispersal and $\beta$-diversity & $\begin{array}{l}\text { (Local) Negative } \\
\text { relationship }\end{array}$ & $\begin{array}{l}\text { Global: no effect } \\
\text { Local: Negative relationship }\end{array}$ & No effect & $\begin{array}{l}\text { Global: Negative relationship } \\
\text { Local: Negative relationship }\end{array}$ \\
\hline $\begin{array}{l}\text { Expected relative species } \\
\text { abundance distribution }\end{array}$ & $\begin{array}{l}\text { Zero sum multinomial } \\
(\mathrm{ZSM})^{\mathrm{a}}\end{array}$ & $\begin{array}{l}\text { Depends on level of } \\
\text { migration and interspecific } \\
\text { interactions }^{\mathrm{e}}\end{array}$ & $\begin{array}{l}\text { Depends on abiotic } \\
\text { conditions }\end{array}$ & Depends on level of migration ${ }^{\mathrm{b}}$ \\
\hline $\begin{array}{l}\text { Response to disturbance } \\
\text { and fragmentation and } \\
\text { species' traits }\end{array}$ & $\begin{array}{l}\text { Traits not important. } \\
\text { Rare species lost first }{ }^{\mathrm{a}}\end{array}$ & $\begin{array}{l}\text { Function of biotic interactions } \\
\text { and isolation }\end{array}$ & $\begin{array}{l}\text { Function of changes in } \\
\text { abiotic heterogeneity }\end{array}$ & $\begin{array}{l}\text { Function of interaction between } \\
\text { abiotic heterogeneity and } \\
\text { isolation }\end{array}$ \\
\hline
\end{tabular}

${ }^{\mathrm{a}}$ Hubbell 2001, ${ }^{\mathrm{b}}$ Mouquet and Loreau 2003, ${ }^{\mathrm{c}}$ Mouquet et al. 2006, ${ }^{\mathrm{d}}$ Mouqet et al. 2002, ${ }^{\mathrm{e}}$ Chave et al. 2002 
Table 2 (continued on next page). Empirical patterns examined in the intact and fragmented watersheds in this study, related metacommunity model predictions (Table 1), and expected results based on the model predictions.

\begin{tabular}{|c|c|c|c|c|c|}
\hline \multirow{2}{*}{$\begin{array}{c}\text { Related } \\
\text { Prediction } \\
\text { (Table 1) } \\
\end{array}$} & \multirow[b]{2}{*}{ Response evaluated } & \multicolumn{4}{|c|}{ Expectation } \\
\hline & & Neutral & Patch dynamics & Species sorting & Mass effects \\
\hline $\begin{array}{l}\text { Abiotic } \\
\text { heterogeneity and } \\
\alpha \text {-diversity }\end{array}$ & $\begin{array}{l}\text { Correlation between local diversity } \\
\text { and local physicochemical conditions } \\
\text { (independent of space) in intact and } \\
\text { fragmented systems }\end{array}$ & No effect & No effect & $\begin{array}{l}\text { Positive and consistent } \\
\text { across both } \\
\text { assemblages }\end{array}$ & $\begin{array}{l}\text { Positive in both. } \\
\text { Possibly stronger in } \\
\text { fragmented } \\
\text { assemblage }\end{array}$ \\
\hline $\begin{array}{l}\text { Abiotic } \\
\text { heterogeneity and } \\
\beta \text {-diversity }\end{array}$ & $\begin{array}{l}\text { Correlation between community } \\
\text { dissimilarity and local } \\
\text { physicochemical conditions } \\
\text { (independent of space) in intact and } \\
\text { fragmented systems }\end{array}$ & No effect & No effect & $\begin{array}{l}\text { Positive and consistent } \\
\text { across both } \\
\text { assemblages }\end{array}$ & $\begin{array}{l}\text { Positive and consistent } \\
\text { across both } \\
\text { assemblages }\end{array}$ \\
\hline $\begin{array}{l}\text { Dispersal and } \alpha- \\
\text { diversity }\end{array}$ & $\begin{array}{l}\text { Local richness in fragmented versus } \\
\text { intact assemblage }\end{array}$ & Lower in fragmented & Lower in fragmented & $\begin{array}{l}\text { Consistent across both } \\
\text { assemblages given } \\
\text { similar local } \\
\text { physicochemical } \\
\text { conditions }\end{array}$ & Lower in fragmented \\
\hline $\begin{array}{l}\text { Dispersal and } \beta \text { - } \\
\text { diversity }\end{array}$ & $\begin{array}{l}\text { Correlation between community } \\
\text { dissimilarity and space (independent of } \\
\text { physicochemical variability) in intact } \\
\text { and fragmented systems }\end{array}$ & $\begin{array}{l}\text { Positive in both and } \\
\text { weaker in fragmented } \\
\text { assemblage }\end{array}$ & $\begin{array}{l}\text { No effect (same as } \\
\text { neutral if considering } \\
\text { local dispersal) }\end{array}$ & No effect & $\begin{array}{l}\text { Positive in intact and } \\
\text { no effect or positive, } \\
\text { but weaker, in } \\
\text { fragmented } \\
\text { assemblage }\end{array}$ \\
\hline
\end{tabular}




\begin{tabular}{|c|c|c|c|c|c|}
\hline \multirow{2}{*}{$\begin{array}{c}\text { Related Prediction } \\
\text { (Table 1) }\end{array}$} & \multirow[b]{2}{*}{ Response evaluated } & \multicolumn{4}{|c|}{ Expectation } \\
\hline & & Neutral & Patch dynamics & Species sorting & Mass effects \\
\hline \multirow[t]{2}{*}{$\begin{array}{l}\text { Species relative } \\
\text { abundance } \\
\text { distributions }\end{array}$} & $\begin{array}{l}\text { Relative species } \\
\text { abundance distribution in } \\
\text { fragmented versus intact } \\
\text { assemblage }\end{array}$ & $\begin{array}{l}\text { Zero sum multinomial } \\
\text { (ZSM) in intact and } \\
\text { fragmented, but lower } \\
\text { migration in fragmented } \\
\text { resulting in flatter, } \\
\text { steeper, distribution }\end{array}$ & $\begin{array}{l}\text { Lognormal in intact to } \\
\text { geometric in fragmented }\end{array}$ & $\begin{array}{l}\text { Lognormal in intact } \\
\text { to geometric in } \\
\text { fragmented }\end{array}$ & $\begin{array}{l}\text { Lognormal in intact } \\
\text { to geometric in } \\
\text { fragmented }\end{array}$ \\
\hline & Exact test of neutrality ${ }^{\mathrm{a}}$ & $\begin{array}{l}\text { Cannot reject null } \\
\text { hypothesis of neutrality }\end{array}$ & $\begin{array}{l}\text { Reject null hypothesis of } \\
\text { neutrality }\end{array}$ & $\begin{array}{l}\text { Reject null } \\
\text { hypothesis of } \\
\text { neutrality }\end{array}$ & $\begin{array}{l}\text { Reject null hypothesis } \\
\text { of neutrality }\end{array}$ \\
\hline \multirow[t]{2}{*}{$\begin{array}{l}\text { Local physicochemical } \\
\text { conditions, isolation, } \\
\text { and species' identity }\end{array}$} & $\begin{array}{l}\text { Species rank abundance } \\
\text { and occupancy to isolation } \\
\text { and disturbance }\end{array}$ & $\begin{array}{l}\text { Species lost in order of } \\
\text { rarity }\end{array}$ & $\begin{array}{l}\text { Non-random deflation and } \\
\text { inflation of individual } \\
\text { species according to } \\
\text { individual species' traits }\end{array}$ & $\begin{array}{l}\text { Non-random } \\
\text { deflation and } \\
\text { inflation of individual } \\
\text { species according to } \\
\text { changes in local } \\
\text { abiotic heterogeneity }\end{array}$ & $\begin{array}{l}\text { Non-random } \\
\text { deflation and inflation } \\
\text { of individual species } \\
\text { according to changes } \\
\text { in local abiotic } \\
\text { heterogeneity and } \\
\text { dispersal limitation }\end{array}$ \\
\hline & $\begin{array}{l}\text { Individual species' local } \\
\text { abundance and occurrence } \\
\text { in response to local } \\
\text { physicochemical } \\
\text { conditions and isolation } \\
\text { from expected core } \\
\text { distribution }\end{array}$ & $\begin{array}{l}\text { Function of isolation from } \\
\text { population core (i.e., } \\
\text { tendency to form } \\
\text { metapopulations) and } \\
\text { independent of local } \\
\text { abiotic conditions. Little } \\
\text { variability in response } \\
\text { among species }\end{array}$ & $\begin{array}{l}\text { Function of isolation and } \\
\text { independent of local } \\
\text { abiotic conditions. } \\
\text { Variability in species' } \\
\text { responses related to } \\
\text { competitive traits. }\end{array}$ & $\begin{array}{l}\text { Function of local } \\
\text { physicochemical } \\
\text { conditions only }\end{array}$ & $\begin{array}{l}\text { Function of both local } \\
\text { physicochemical } \\
\text { conditions and } \\
\text { isolation }\end{array}$ \\
\hline
\end{tabular}

${ }^{\mathrm{a}}$ Etienne 2007 
Table 3. Summary of eigenvalues, proportion of variance explained, and variable loadings for individual principal components resulting from the principal component analysis of water chemistry data from the intact and fragmented assemblage datasets. Component loadings that were interpreted when loadings where: $1>=|0.4|$. SpCond $=$ specific conductivity, TDS $=$ total dissolved solids, Alk = alkalinity, Acid $=$ total acidity, $\mathrm{SO} 4=$ sulfates, $\mathrm{Al}=$ aluminum, $\mathrm{Cr}=$ chromium, $\mathrm{Fe}=$ iron, $\mathrm{Mn}=$ manganese, $\mathrm{Ni}=$ nickel, $\mathrm{Ca}=$ calcium, $\mathrm{Mg}=$ magnesium.

\begin{tabular}{cccc}
\hline & Intact & \multicolumn{2}{c}{ Fragmented } \\
Variable & PC1 & PC1 & PC2 \\
\hline Eigenvalue & 4.0 & 4.6 & 3.0 \\
Variance Explained & 30.7 & 35.3 & 22.6 \\
\hline pH & & & -0.439 \\
SpCond & -0.430 & -0.361 & \\
TDS & & -0.352 & \\
Alk & -0.440 & & -0.374 \\
Acid & & & -0.373 \\
SO4 & & -0.425 & \\
$\mathrm{Al}$ & & & \\
$\mathrm{Cr}$ & & & 0.451 \\
$\mathrm{Fe}$ & & & \\
$\mathrm{Mn}$ & & & \\
$\mathrm{Ni}$ & & & \\
$\mathrm{Ca}$ & -0.428 & -0.409 & \\
$\mathrm{Mg}$ & & -0.433 & \\
\hline
\end{tabular}


Table 4. Summary results of partial Mantel tests for determining the independent effects of physicochemical versus spatial structure on diversity. Water chemistry = Euclidean matrix of water chemistry principal components, WMJT = Euclidean matrix of predicted weekly mean July temperature, RVHA = Euclidean matrix of rapid visual habitat assessment scores, Space $=$ matrix inter-site fluvial distances $(\mathrm{km})$, Physicochemical $=$ Euclidean matrix of combined physicochemical variables.

\begin{tabular}{|c|c|c|c|c|c|c|c|c|}
\hline \multirow[b]{3}{*}{ Independent variable } & \multicolumn{4}{|c|}{ Intact } & \multicolumn{4}{|c|}{ Fragmented } \\
\hline & \multicolumn{2}{|c|}{$\alpha$-diversity } & \multicolumn{2}{|c|}{$\beta$-diversity } & \multicolumn{2}{|c|}{$\alpha$-diversity } & \multicolumn{2}{|c|}{$\beta$-diversity } \\
\hline & Mantel's r & $\operatorname{Pr}>\mathbf{P}$ & Mantel's r & $\operatorname{Pr}>\mathbf{P}$ & Mantel's r & $\operatorname{Pr}>\mathbf{P}$ & Mantel's r & $\operatorname{Pr}>\mathbf{P}$ \\
\hline Water chemistry | Space & -0.10 & 0.94 & -0.07 & 0.74 & -0.03 & 0.52 & 0.36 & $<0.001$ \\
\hline WMJT | Space & 0.42 & $<0.001$ & 0.42 & $<0.001$ & 0.33 & $<0.001$ & 0.10 & 0.06 \\
\hline Drainage area | Space & 0.30 & $<0.001$ & 0.35 & $<0.001$ & 0.39 & $<0.001$ & 0.16 & $<0.05$ \\
\hline RVHA | Space & -0.01 & 0.50 & 0.04 & 0.29 & -0.03 & 0.61 & 0.09 & 0.13 \\
\hline Space | Physicochemical & 0.12 & 0.10 & 0.32 & $<0.01$ & -0.02 & 0.61 & 0.05 & 0.20 \\
\hline Physicochemical | Space & 0.28 & $<0.001$ & 0.39 & $<0.001$ & 0.24 & $<0.01$ & 0.36 & $<0.001$ \\
\hline
\end{tabular}


Table 5. Results of fitting physicochemical vectors to the non-metric multidimensional scaling (NMDS) ordinations of fish community dissimilarity (Bray-Curtis) in the intact (top) and fragmented assembles. WMJT = predicted weekly mean July temperature, $\mathrm{WC} 1 / \mathrm{W} 2=$ water chemistry principal components 1 and 2 .

\begin{tabular}{clcccc}
\hline Dataset & Variable & NMDS1 & NMDS2 & NMDS3 & P-value \\
\hline Intact & Drainage area & -0.68 & & & $<0.001$ \\
& WMJT & -0.88 & & & $<0.001$ \\
\hline Fragmented & Drainage area & 0.96 & & & $<0.001$ \\
& WMJT & 0.89 & -0.4 & & $<0.001$ \\
& WC1 & & 0.99 & & $<0.001$ \\
& WC2 & & 0.98 & $<0.01$ \\
\hline
\end{tabular}


Table 6. Results for ANCOVA and model comparison for predicting local species richness ( $\alpha$ diversity) as a function of log-transformed drainage area, two local water chemistry principal components, and, in the case of the full model, a two-level factor variable coding for the assemblage dataset (intact or fragmented). P-values are indicated for the individual coefficients for each model $(\operatorname{Pr}>|t|)$, the individual models $\left(\mathrm{P}_{\text {model }}\right)$, and the model comparison ANOVA $\left(\mathrm{P}_{\text {test }}\right)$.

\begin{tabular}{llccccc}
\hline Model & \multicolumn{1}{c}{ Term } & Estimate & $\operatorname{Pr}(>|\mathbf{t}|)$ & $\mathbf{R}^{\mathbf{2}}$ & $\mathbf{P}_{\text {model }}$ & $\mathbf{P}_{\text {test }}$ \\
\hline Full & Intercept & -2.39 & $<0.001$ & 0.60 & $<0.001$ & $<0.001$ \\
& log(Drainage area) & 3.2 & $<0.001$ & & & \\
& Water chemistry PC1 & 0.12 & $\mathrm{~ns}$ & & & \\
& Water chemistry PC2 & -0.03 & $\mathrm{~ns}$ & & & \\
& Assemblage & 2.9 & $<0.001$ & & & \\
\hline Reduced & Intercept & -0.98 & $<0.001$ & 0.54 & $<0.001$ & \\
& log(Drainage area) & 3.2 & $<0.001$ & & & \\
& Water chemistry PC1 & 0.53 & $<0.05$ & & & \\
& Water chemistry PC2 & -0.11 & $\mathrm{~ns}$ & & & \\
& & & & & & \\
& & & & & & \\
& & &
\end{tabular}


Table 7. Summary of results for fitting empirical species abundance distributions to the theoretical models and model comparisons using Akiake's Information Criterion (AIC) for the intact, fragmented, Horseshoe Run, and Muddy Creek fish assemblages. The model parameters estimated are indicated right of the model description, while estimates for those parameters are listed under the dataset labels.

\begin{tabular}{cccccc}
\hline SAD & Model & \multicolumn{2}{c}{ Intact } & \multicolumn{2}{c}{ Fragmented } \\
Model & Parameters & Estimate & AIC & Estimate & AIC \\
\hline Lognormal & $\log (\mu)$ & 5.79 & 2276.7 & 4.95 & 4672.2 \\
& $\log (\sigma)$ & 1.88 & & 1.91 & \\
Geometric & $\mathrm{a}$ & 0.027 & 5782.28 & 0.025 & 3425.38 \\
ZSM & $\mathrm{J}$ & 40596 & 285.52 & 24124 & 268.74 \\
& $\theta$ & 4.57 & & 4.56 & \\
& $\mathrm{~m}$ & 0.02 & & 0.21 & \\
\hline
\end{tabular}


Table 8 (continued on following page). Summary of abundance, proportional abundance, percent occupancy and rank of abundance and occupancy by species in the intact and fragmented assemblages. Differences $(\Delta)$ in rank of abundance, occupancy between the two datasets are noted as well as the proportional abundance of species in the fragmented compared to the intact assemblage. NA $=$ ratio could not be calculated because the species was not present in one of the datasets.

\begin{tabular}{|c|c|c|c|c|c|c|c|c|c|c|c|c|c|c|}
\hline Species & $\begin{array}{c}\text { Species } \\
\text { Code }\end{array}$ & $\begin{array}{c}\text { UA } \\
\text { Abundance }\end{array}$ & $\begin{array}{l}\text { UA } \\
\text { Ab. } \\
\text { Rank }\end{array}$ & $\begin{array}{c}\text { UA } \\
\text { Propn. } \\
\text { Ab. } \\
\end{array}$ & $\begin{array}{c}\text { UA } \\
\begin{array}{c}\text { Occupancy } \\
(\%)\end{array} \\
\end{array}$ & $\begin{array}{l}\text { UA } \\
\text { Occ. } \\
\text { Rank } \\
\end{array}$ & $\begin{array}{c}\text { FA } \\
\text { Abundance } \\
\end{array}$ & $\begin{array}{c}\text { FA } \\
\text { Ab. } \\
\text { Rank }\end{array}$ & $\begin{array}{c}\text { FA } \\
\text { Propn. } \\
\text { Ab. } \\
\end{array}$ & $\begin{array}{c}\text { FA } \\
\begin{array}{c}\text { Occupancy } \\
(\%)\end{array} \\
\end{array}$ & $\begin{array}{l}\text { FA } \\
\text { Occ. } \\
\text { Rank } \\
\end{array}$ & $\begin{array}{c}\Delta \\
\text { Rank. } \\
\text { Ab. }\end{array}$ & $\begin{array}{c}\Delta \\
\text { Rank. } \\
\text { Occ. }\end{array}$ & $\begin{array}{c}\text { FAVUA } \\
\text { Ratio } \\
\text { Propn. } \\
\text { Ab. } \\
\end{array}$ \\
\hline Cottus bairdi & COBA & 16033 & 1 & 0.394 & 94.3 & 1 & 6327 & 1 & 0.262 & 70.5 & 3 & 0 & -2 & 0.67 \\
\hline Rhinichthys obtusus & $\mathrm{RHOB}$ & 4758 & 2 & 0.117 & 85.7 & 3 & 5770 & 2 & 0.239 & 81.8 & 2 & 0 & 1 & 2.04 \\
\hline Campostoma anomalum & CAAN & 4644 & 4 & 0.114 & 65.7 & 6 & 248 & 14 & 0.010 & 22.7 & 14 & -10 & -8 & 0.09 \\
\hline Rhinichthys catarctae & $\mathrm{RHCA}$ & 4419 & 3 & 0.109 & 88.6 & 2 & 956 & 6 & 0.040 & 34.1 & 8 & -3 & -6 & 0.36 \\
\hline Nocomis micropogon & NOMI & 2740 & 5 & 0.067 & 57.1 & 7 & 1300 & 4 & 0.054 & 27.3 & 11 & 1 & -4 & 0.80 \\
\hline Clinostomus funduloides & CLFU & 1579 & 6 & 0.039 & 48.6 & 9 & 11 & 26 & 0.000 & 2.3 & 27 & -20 & -18 & 0.01 \\
\hline Semotilus atromaculatus & SEAT & 1076 & 7 & 0.026 & 80 & 4 & 4688 & 3 & 0.194 & 93.2 & 1 & 4 & 3 & 7.34 \\
\hline Hypentelium nigricans & HYNI & 981 & 8 & 0.024 & 57.1 & 8 & 762 & 7 & 0.032 & 36.4 & 6 & 1 & 2 & 1.31 \\
\hline Salvelinus fontinalis & SAFO & 756 & 9 & 0.019 & 77.1 & 5 & 269 & 13 & 0.011 & 29.5 & 9 & -4 & -4 & 0.60 \\
\hline Notropis rubellus & NORU & 746 & 10 & 0.018 & 31.4 & 15 & 279 & 12 & 0.012 & 2.3 & 28 & -2 & -13 & 0.63 \\
\hline Notropis photogenis & $\mathrm{NOPH}$ & 533 & 11 & 0.013 & 17.1 & 20 & 123 & 17 & 0.005 & 6.8 & 21 & -6 & -1 & 0.39 \\
\hline Etheostoma blennioides & ETBL & 505 & 12 & 0.012 & 48.6 & 10 & 120 & 18 & 0.005 & 11.4 & 18 & -6 & -8 & 0.40 \\
\hline Etheostoma flabellare & ETFL & 404 & 13 & 0.010 & 22.9 & 17 & 201 & 15 & 0.008 & 29.5 & 10 & -2 & 7 & 0.84 \\
\hline Micropterus dolomieu & MIDO & 375 & 14 & 0.009 & 45.7 & 12 & 326 & 10 & 0.014 & 25 & 12 & 4 & 0 & 1.47 \\
\hline Catostomus commersoni & CACO & 275 & 15 & 0.007 & 48.6 & 11 & 364 & 9 & 0.015 & 54.5 & 4 & 6 & 7 & 2.23 \\
\hline Ambloplites rubestris & AMRU & 189 & 16 & 0.005 & 40 & 13 & 309 & 11 & 0.013 & 36.4 & 7 & 5 & 6 & 2.76 \\
\hline Oncorhyncus mykiss & ONMY & 153 & 17 & 0.004 & 20 & 19 & 59 & 21 & 0.002 & 20.5 & 15 & -4 & 4 & 0.65 \\
\hline Lepomis cyanellus & LECY & 120 & 18 & 0.003 & 34.3 & 14 & 419 & 8 & 0.017 & 38.6 & 5 & 10 & 9 & 5.88 \\
\hline Phoxinus oreus & PHOR & 104 & 19 & 0.003 & 2.9 & 30 & & & & & & & & \\
\hline Hybrid Individuals & HYBR & 92 & 20 & 0.002 & 28.6 & 16 & 6 & 29 & 0.000 & 2.3 & 29 & -9 & -13 & 0.11 \\
\hline Salmo trutta & SATR & 45 & 21 & 0.001 & 22.9 & 18 & 90 & 19 & 0.004 & 25 & 13 & 2 & 5 & 3.37 \\
\hline Etheostoma nigrum & ETNI & 34 & 22 & 0.001 & 8.6 & 23 & 37 & 23 & 0.002 & 9.1 & 19 & -1 & 4 & 1.83 \\
\hline Margariscus margarita & MAMA & 29 & 23 & 0.001 & 11.4 & 21 & 1 & 34 & 0.000 & 2.3 & 30 & -11 & -9 & 0.06 \\
\hline Luxilus chrysocephalus & $\mathrm{LUCH}$ & 25 & 24 & 0.001 & 5.7 & 24 & 158 & 16 & 0.007 & 6.8 & 23 & 8 & 1 & 10.65 \\
\hline Etheostoma zonale & ETZO & 16 & 25 & 0.000 & 5.7 & 26 & & & & & & & & \\
\hline
\end{tabular}




\begin{tabular}{|c|c|c|c|c|c|c|c|c|c|c|c|c|c|c|}
\hline Species & $\begin{array}{c}\text { Species } \\
\text { Code }\end{array}$ & $\begin{array}{c}\text { UA } \\
\text { Abundance }\end{array}$ & $\begin{array}{l}\text { UA } \\
\text { Ab. } \\
\text { Rank }\end{array}$ & $\begin{array}{l}\text { UA } \\
\text { Propn. } \\
\text { Ab. }\end{array}$ & $\begin{array}{c}\text { UA } \\
\text { Occupancy } \\
(\%)\end{array}$ & $\begin{array}{l}\text { UA } \\
\text { Occ. } \\
\text { Rank }\end{array}$ & $\begin{array}{c}\text { FA } \\
\text { Abundance }\end{array}$ & $\begin{array}{c}\text { FA } \\
\text { Ab. } \\
\text { Rank }\end{array}$ & $\begin{array}{c}\text { FA } \\
\text { Propn. } \\
\text { Ab. }\end{array}$ & $\begin{array}{c}\text { FA } \\
\text { Occupancy } \\
(\%)\end{array}$ & $\begin{array}{l}\text { FA } \\
\text { Occ. } \\
\text { Rank }\end{array}$ & $\begin{array}{c}\Delta \\
\text { Rank. } \\
\text { Ab. }\end{array}$ & $\begin{array}{c}\Delta \\
\text { Rank. } \\
\text { Occ. }\end{array}$ & $\begin{array}{c}\text { FA/UA } \\
\text { Ratio } \\
\text { Propn. } \\
\text { Ab. }\end{array}$ \\
\hline Lepomis macrochirus & LEMA & 10 & 26 & 0.000 & 5.7 & 25 & 60 & 20 & 0.002 & 20.5 & 16 & 6 & 9 & 10.11 \\
\hline Lepomis gibbosus & LEGI & 9 & 27 & 0.000 & 11.4 & 22 & 9 & 27 & 0.000 & 6.8 & 22 & 0 & 0 & 1.69 \\
\hline Pimephales notatus & PINO & 6 & 28 & 0.000 & 2.9 & 27 & 1123 & 5 & 0.047 & 20.5 & 17 & 23 & 10 & 315.45 \\
\hline Etheostoma variatum & ETVA & 1 & 29 & 0.000 & 2.9 & 31 & & & & & & & & \\
\hline Micropterus punctatus & MIPU & 1 & 30 & 0.000 & 2.9 & 28 & 28 & 24 & 0.001 & 4.5 & 26 & 6 & 2 & 47.19 \\
\hline Micropterus salmoides & MISA & 1 & 31 & 0.000 & 2.9 & 29 & 19 & 25 & 0.001 & 9.1 & 20 & 6 & 9 & 32.02 \\
\hline Notropis buccata & NOBU & & & & & & 43 & 22 & 0.002 & 4.5 & 25 & & & NA \\
\hline Ameirus natalis & AMNA & & & & & & 7 & 28 & 0.000 & 6.8 & 24 & & & NA \\
\hline Percina caprodes & PECA & & & & & & 4 & 30 & 0.000 & 2.3 & 31 & & & NA \\
\hline Percina maculata & PEMA & & & & & & 3 & 31 & 0.000 & 2.3 & 32 & & & NA \\
\hline Moxostoma duquesni & MODU & & & & & & 2 & 32 & 0.000 & 2.3 & 33 & & & NA \\
\hline Notropis atherinoides & NOAT & & & & & & 2 & 33 & 0.000 & 2.3 & 34 & & & NA \\
\hline Notrpis volucellus & NOVO & & & & & & 1 & 35 & 0.000 & 2.3 & 35 & & & NA \\
\hline
\end{tabular}


Table 9. Summary of GLM results for presence-absence of commonly sampled species among sites in the intact and fragmented (following page) assemblage datasets. WMJT=modeled weekly mean July temperature, RVHA=Rapid Visual Habitat Assessment, WC1/WC2 = water chemistry principal component axes 1 and 2, Dist. Source = minimum distance to core stream. A positive relationship between an independent variable and presence or abundance is indicated by $(+)$ and a negative relationship is indicated by (-). Values left of the sign reflect the individual variable's contribution to overall deviance explained in the model (\%). NA $=$ not applicable, $\mathrm{nc}=$ variable not chosen by AIC-based selection. GLM model families are indicated as B (binomial) and $\mathrm{N}$ (negative binomial).

\begin{tabular}{|c|c|c|c|c|c|c|c|c|c|c|}
\hline Dataset & Dependent Variable & $\mathbf{n}$ & WMJT & RVHA & WC1 & WC2 & $\begin{array}{c}\text { Dist } \\
\text { Source }\end{array}$ & $\begin{array}{l}\text { Deviance } \\
\text { Explained }\end{array}$ & Verification & Family \\
\hline Intact & Ambloplites rupestris & 14 & $20.4(+)$ & $\mathrm{nc}$ & $\mathrm{nc}$ & NA & $\mathrm{nc}$ & 20.4 & 29.9 & $\mathrm{~B}$ \\
\hline \multirow[t]{13}{*}{$N=35$} & Campostoma anomalum & 23 & $30.6(+)$ & $\mathrm{nc}$ & $\mathrm{nc}$ & NA & $17.6(-)$ & 48.2 & 50.2 & B \\
\hline & Catostomus commersoni & 17 & $\mathrm{nc}$ & $\mathrm{nc}$ & $12.9(-)$ & NA & $\mathrm{nc}$ & 12.9 & 15.7 & $\mathrm{~B}$ \\
\hline & Clinostomus funduloides & 17 & $\mathrm{nc}$ & $\mathrm{nc}$ & $11.8(-)$ & NA & $9.0(-)$ & 20.8 & 25.5 & $\mathrm{~B}$ \\
\hline & Cottus bairdi & 33 & $25.9(+)$ & $\mathrm{nc}$ & $\mathrm{nc}$ & NA & $4.5(-)$ & 30.3 & 31.5 & $\mathrm{~N}$ \\
\hline & Etheostoma flabellare & 8 & $16.6(+)$ & $\mathrm{nc}$ & $\mathrm{nc}$ & NA & $\mathrm{nc}$ & 16.6 & 18.8 & $\mathrm{~B}$ \\
\hline & Hypentelium nigricans & 20 & $22.2(+)$ & $\mathrm{nc}$ & $\mathrm{nc}$ & NA & $5.2(-)$ & 27.4 & 34.8 & $\mathrm{~B}$ \\
\hline & Lepomis cyanellus & 12 & $6.8(+)$ & $\mathrm{nc}$ & $\mathrm{nc}$ & NA & $7.4(-)$ & 14.2 & 15.7 & B \\
\hline & Micropterus dolomieu & 16 & $40.7(+)$ & $\mathrm{nc}$ & $\mathrm{nc}$ & NA & $\mathrm{nc}$ & 40.7 & 56.1 & $\mathrm{~B}$ \\
\hline & Nocomis micropogon & 20 & $25.6(+)$ & $\mathrm{nc}$ & $\mathrm{nc}$ & NA & $\mathrm{nc}$ & 25.6 & 30.5 & $\mathrm{~B}$ \\
\hline & Rhinichthys obtusus & 30 & $\mathrm{nc}$ & $\mathrm{nc}$ & $\mathrm{nc}$ & NA & $\mathrm{nc}$ & NA & NA & $\mathrm{N}$ \\
\hline & Rhinichthys cataractae & 31 & $13.2(+)$ & $3.4(+)$ & $\mathrm{nc}$ & NA & $5.2(-)$ & 21.8 & 9.4 & $\mathrm{~N}$ \\
\hline & Salvelinus fontinalis & 27 & $13.4(-)$ & $12.5(+)$ & $\mathrm{nc}$ & NA & $\mathrm{nc}$ & 25.9 & 19.1 & $\mathrm{~N}$ \\
\hline & Semotilus atromaculatus & 28 & $\mathrm{nc}$ & $\mathrm{nc}$ & $\mathrm{nc}$ & NA & $\mathrm{nc}$ & NA & NA & B \\
\hline
\end{tabular}




\begin{tabular}{|c|c|c|c|c|c|c|c|c|c|c|}
\hline Dataset & Dependent Variable & $\mathbf{n}$ & WMJT & RVHA & WC1 & WC2 & $\begin{array}{c}\text { Dist } \\
\text { Source }\end{array}$ & $\begin{array}{l}\text { Deviance } \\
\text { Explained }\end{array}$ & Verification & Family \\
\hline Fragmented & Ambloplites rupestris & 16 & $20.3(+)$ & $13.3(-)$ & $5.6(+)$ & $\mathrm{nc}$ & $\mathrm{nc}$ & 39.2 & 56.9 & B \\
\hline \multirow[t]{13}{*}{$N=44$} & Campostoma anomalum & 10 & $24.0(+)$ & $1.8(-)$ & $\mathrm{nc}$ & $\mathrm{nc}$ & $9.8(-)$ & 35.6 & 35.0 & B \\
\hline & Catostomus commersoni & 24 & $6.1(+)$ & $\mathrm{nc}$ & $3.7(-)$ & $\mathrm{nc}$ & $\mathrm{nc}$ & 9.8 & 11.9 & B \\
\hline & Clinostomus funduloides & 1 & NA & NA & NA & NA & NA & NA & NA & B \\
\hline & Cottus bairdi & 31 & $\mathrm{nc}$ & $\mathrm{nc}$ & $14.5(+)$ & $\mathrm{nc}$ & $4.0(-)$ & 18.4 & 23.4 & $\mathrm{~B}$ \\
\hline & Etheostoma flabellare & 13 & $7.0(+)$ & $0.9(-)$ & $34.9(+)$ & $\mathrm{nc}$ & $21.9(-)$ & 64.7 & 68.7 & B \\
\hline & Hypentelium nigricans & 16 & $33.1(+)$ & $\mathrm{nc}$ & $17.2(+)$ & $\mathrm{nc}$ & $\mathrm{nc}$ & 50.3 & 52.7 & $B$ \\
\hline & Lepomis cyanellus & 17 & $5.8(+)$ & $\mathrm{nc}$ & $3.9(-)$ & $\mathrm{nc}$ & $\mathrm{nc}$ & 8.1 & 12.4 & B \\
\hline & Micropterus dolomieu & 11 & $14.6(+)$ & $12.0(-)$ & $20.6(+)$ & $\mathrm{nc}$ & $8.3(-)$ & 55.5 & 58.7 & B \\
\hline & Nocomis micropogon & 12 & $21.1(+)$ & $\mathrm{nc}$ & $17.9(+)$ & $\mathrm{nc}$ & $\mathrm{nc}$ & 39.0 & 41.6 & B \\
\hline & Rhinichthys obtusus & 36 & $\mathrm{nc}$ & $4.7(+)$ & $\mathrm{nc}$ & $\mathrm{nc}$ & $\mathrm{nc}$ & 4.7 & 8.0 & $\mathrm{~N}$ \\
\hline & Rhinichthys cataractae & 15 & $\mathrm{nc}$ & $13.9(+)$ & $16.7(+)$ & $\mathrm{nc}$ & $\mathrm{nc}$ & 30.5 & 33.0 & B \\
\hline & Salvelinus fontinalis & 12 & $13.9(-)$ & $16.2(+)$ & $25.4(+)$ & $\mathrm{nc}$ & $\mathrm{nc}$ & 55.5 & 57.7 & B \\
\hline & Semotilus atromaculatus & 41 & $\mathrm{nc}$ & $\mathrm{nc}$ & $\mathrm{nc}$ & $\mathrm{nc}$ & $4.9(+)$ & 4.9 & 9.2 & $\mathrm{~N}$ \\
\hline
\end{tabular}


Table 10 (continued on following page). Summary of supported model predictions based on a priori expectations for individual analyses outlined in Table 2. Only the expectations supported by the data are listed. Expectations listed in bold received some additional qualitative support relative to predictions in normal type.

\begin{tabular}{|c|c|c|c|c|c|}
\hline \multirow{2}{*}{$\begin{array}{l}\text { Related } \\
\text { Prediction } \\
\text { (Table 1) }\end{array}$} & \multirow[b]{2}{*}{ Response evaluated } & \multicolumn{4}{|c|}{ Expectation } \\
\hline & & Neutral & Patch dynamics & Species sorting & Mass effects \\
\hline $\begin{array}{l}\text { Abiotic } \\
\text { heterogeneity } \\
\text { and } \alpha \text {-diversity }\end{array}$ & $\begin{array}{l}\text { Correlation between local } \\
\text { diversity and local } \\
\text { physicochemical conditions } \\
\text { (independent of space) in } \\
\text { intact and fragmented systems }\end{array}$ & & & $\begin{array}{l}\text { Positive and consistent } \\
\text { across both assemblages }\end{array}$ & $\begin{array}{l}\text { Positive in both. } \\
\text { Possibly stronger in } \\
\text { fragmented assemblage }\end{array}$ \\
\hline $\begin{array}{l}\text { Abiotic } \\
\text { heterogeneity } \\
\text { and } \beta \text {-diversity }\end{array}$ & $\begin{array}{l}\text { Correlation between } \\
\text { community dissimilarity and } \\
\text { local physicochemical } \\
\text { conditions (independent of } \\
\text { space) in intact and fragmented } \\
\text { systems }\end{array}$ & & & $\begin{array}{l}\text { Positive and consistent } \\
\text { across both assemblages }\end{array}$ & $\begin{array}{l}\text { Positive and consistent } \\
\text { across both assemblages }\end{array}$ \\
\hline $\begin{array}{l}\text { Dispersal and } \\
\alpha \text {-diversity }\end{array}$ & $\begin{array}{l}\text { Local richness in fragmented } \\
\text { versus intact assemblage }\end{array}$ & Lower in fragmented & Lower in fragmented & & Lower in fragmented \\
\hline $\begin{array}{l}\text { Dispersal and } \\
\beta \text {-diversity }\end{array}$ & $\begin{array}{l}\text { Correlation between } \\
\text { community dissimilarity and } \\
\text { space (independent of } \\
\text { physicochemical variability) in } \\
\text { intact and fragmented systems }\end{array}$ & & & & $\begin{array}{l}\text { Positive in intact and no } \\
\text { effect or positive, but } \\
\text { weaker, in fragmented } \\
\text { assemblage }\end{array}$ \\
\hline
\end{tabular}




\begin{tabular}{|c|c|c|c|c|c|}
\hline \multirow{2}{*}{$\begin{array}{c}\text { Related } \\
\text { Prediction } \\
\text { (Table 1) } \\
\end{array}$} & \multirow[b]{2}{*}{ Response evaluated } & \multicolumn{4}{|c|}{ Expectation } \\
\hline & & Neutral & Patch dynamics & Species sorting & Mass effects \\
\hline \multirow[t]{2}{*}{$\begin{array}{l}\text { Species relative } \\
\text { abundance } \\
\text { distributions }\end{array}$} & $\begin{array}{l}\text { Relative species } \\
\text { abundance distribution } \\
\text { in fragmented versus } \\
\text { intact assemblage }\end{array}$ & $\begin{array}{l}\text { Zero sum multinomial } \\
\text { (ZSM) in intact and } \\
\text { fragmented, but lower } \\
\text { migration in fragmented } \\
\text { resulting in flatter, } \\
\text { steeper, distribution }\end{array}$ & $\begin{array}{l}\text { Lognormal in intact to } \\
\text { geometric in fragmented }\end{array}$ & $\begin{array}{l}\text { Lognormal in intact to } \\
\text { geometric in fragmented }\end{array}$ & $\begin{array}{l}\text { Lognormal in intact to } \\
\text { geometric in fragmented }\end{array}$ \\
\hline & Exact test of neutrality ${ }^{\mathrm{a}}$ & $\begin{array}{l}\text { Cannot reject null } \\
\text { hypothesis of neutrality }\end{array}$ & $\begin{array}{l}\text { Reject null hypothesis of } \\
\text { neutrality }\end{array}$ & $\begin{array}{l}\text { Reject null hypothesis of } \\
\text { neutrality }\end{array}$ & $\begin{array}{l}\text { Reject null hypothesis of } \\
\text { neutrality }\end{array}$ \\
\hline \multirow[t]{2}{*}{$\begin{array}{l}\text { Local } \\
\text { physicochemical } \\
\text { conditions, } \\
\text { isolation, and } \\
\text { species' identity }\end{array}$} & $\begin{array}{l}\text { Species rank abundance } \\
\text { and occupancy to } \\
\text { isolation and } \\
\text { disturbance }\end{array}$ & & $\begin{array}{l}\text { Non-random deflation and } \\
\text { inflation of individual } \\
\text { species according to } \\
\text { individual species' traits and } \\
\text { dispersal limitation }\end{array}$ & $\begin{array}{l}\text { Non-random deflation } \\
\text { and inflation of } \\
\text { individual species } \\
\text { according to changes in } \\
\text { local abiotic } \\
\text { heterogeneity }\end{array}$ & $\begin{array}{l}\text { Non-random deflation } \\
\text { and inflation of } \\
\text { individual species } \\
\text { according to changes in } \\
\text { local abiotic } \\
\text { heterogeneity and } \\
\text { dispersal limitation }\end{array}$ \\
\hline & $\begin{array}{l}\text { Individual species' local } \\
\text { abundance and } \\
\text { occurrence in response } \\
\text { to local } \\
\text { physicochemical } \\
\text { conditions and isolation } \\
\text { from expected core } \\
\text { distribution }\end{array}$ & & & & $\begin{array}{l}\text { Function of both local } \\
\text { physicochemical } \\
\text { conditions and isolation }\end{array}$ \\
\hline
\end{tabular}




\section{Figures}

Figure 1. Schematic representation of the four metacommunity frameworks for two competing species - species A and species B - modified from Leibold et al. (2004). The arrows connect populations to potential patches available for colonization, which are shown as the largest squares or circles. The degree to which either species is the competitive dominant in the patch is indicated by the shape of symbol surrounding it, indicating its habitat niche type. If the symbol surrounding the species' letter identification matches the shape of the patch surrounding it, that species is competitively dominant in that patch. For the neutral schematic, a larger hexagon is used to denote the regional metacommunity and mainland-island nature of the model. Dashed arrows indicate lower rates of dispersal among patches while solid arrows indicate higher dispersal. Double-headed arrows denote bi-directional movement. The four frameworks illustrated are patch-dynamics (PD), species-sorting (SS), mass-effects (ME), and neutral (N). The patch-dynamic schematic illustrates a competition-colonization tradeoff with species A being the superior competitor and species B the superior colonizer. Either species could colonize the empty patch, but species B is more likely given its superior ability to colonize. Species B will be replaced in its current patch by species A when species A colonizes. The species-sorting schematic illustrates species separation into spatial niches where dispersal is not sufficient to alter their distributions. The mass-effect schematic illustrates how each species is dominant in a particular habitat and also how both species are maintained in the patches they are not dominant in by high rates of dispersal from those within which they are dominant. Smaller letters indicate sink habitats for that particular species and larger letters denote sources. In the neutral model schematic, both species are present in all patches and neither is dominant in any patch. High rates of dispersal among patches are evident and are important to the maintenance of diversity. If not for immigration from the regional metacommunity, both species would eventually be lost. Speciation in the regional metacommunity could also lead to immigration of new species. 

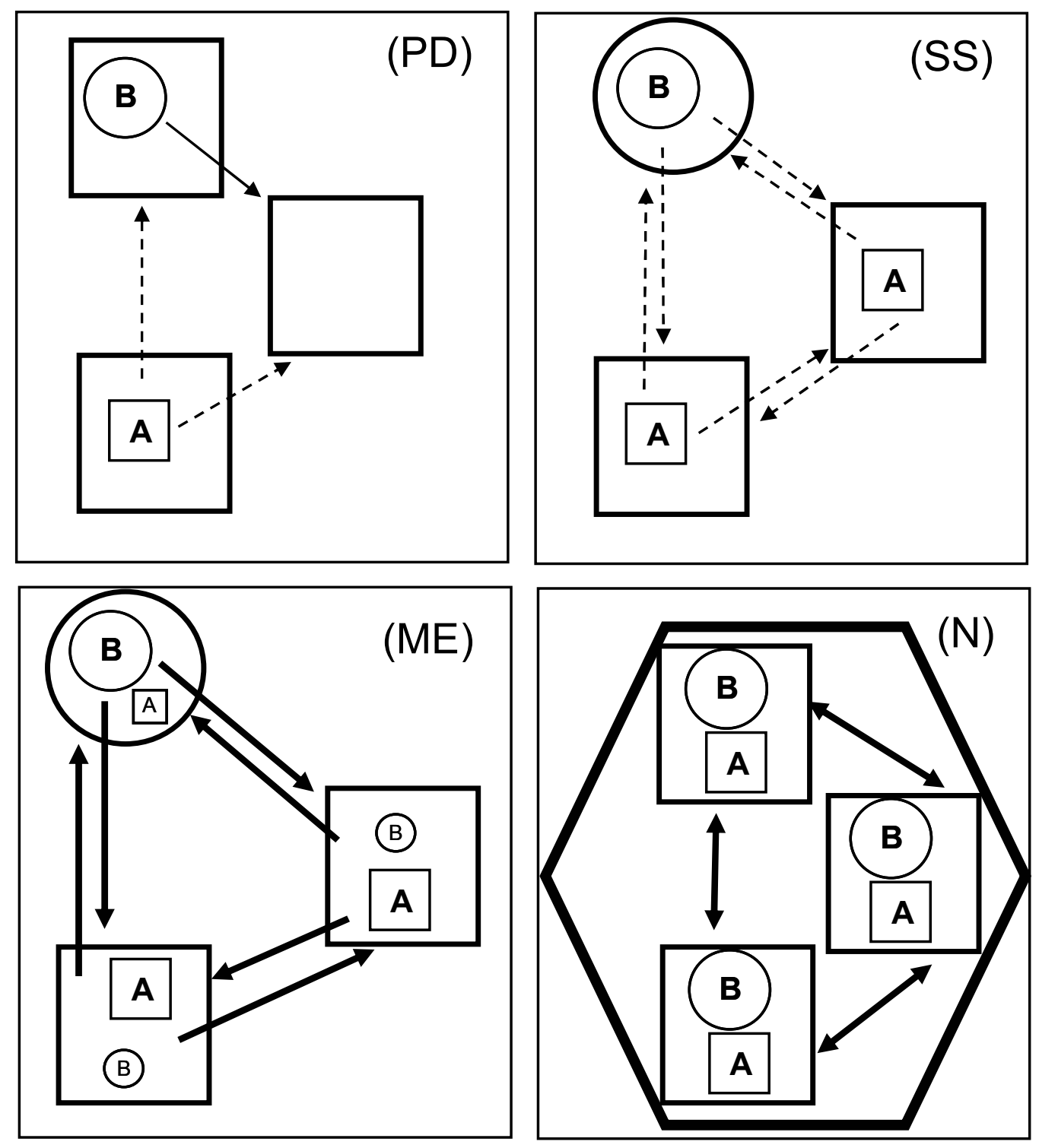
Figure 2. Map of the location of the study region within West Virginia and the distribution of study sites among the fragmented and intact regions of the upper Monongahela River basin.

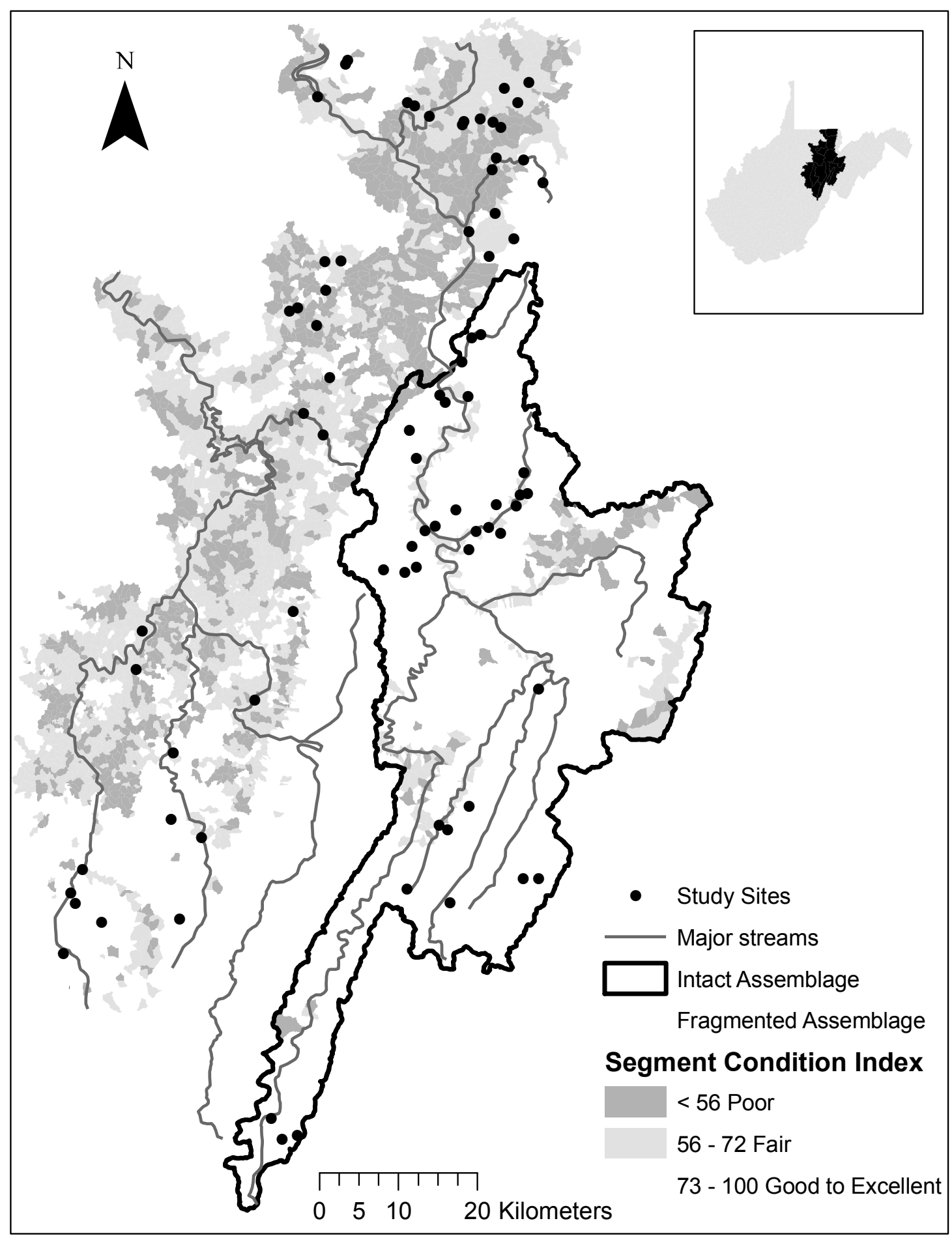


Figure 3. Plot of the variability in expected local and regional conditions based on the modeled, multimetric index values at the regional (HUC12 watershed) and local (stream segment) scales (Petty et al., unpublished data). Sites from the intact (filled circles) and fragmented (open circles) assemblage datasets are noted.

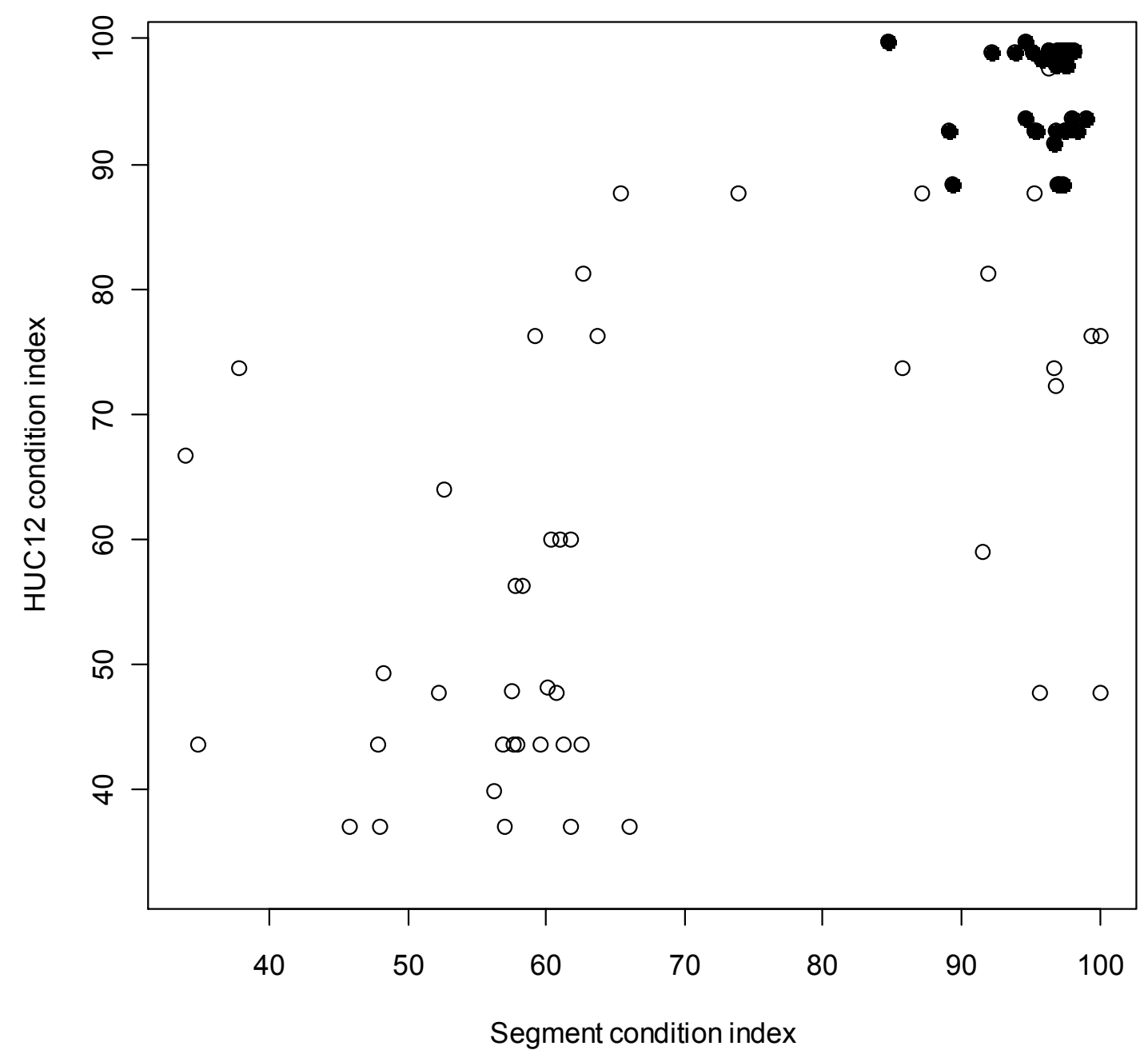


Figure 4. Non-metric multidimensional scaling ordination of sites (closed circles) and species (text) in the intact (top) and fragmented (bottom) assemblage datasets and fit of physicochemical variables to the ordination space. See table 5 for correlation strengths and significance of individual physicochemical variables. Area $=$ cumulative upstream drainage area $\left(\mathrm{km}^{2}\right), \mathrm{WMJT}=$ weekly mean July temperature $\left({ }^{\circ} \mathrm{C}\right), \mathrm{WC} 1=$ water chemistry principal component 1 (see Table 3 ).

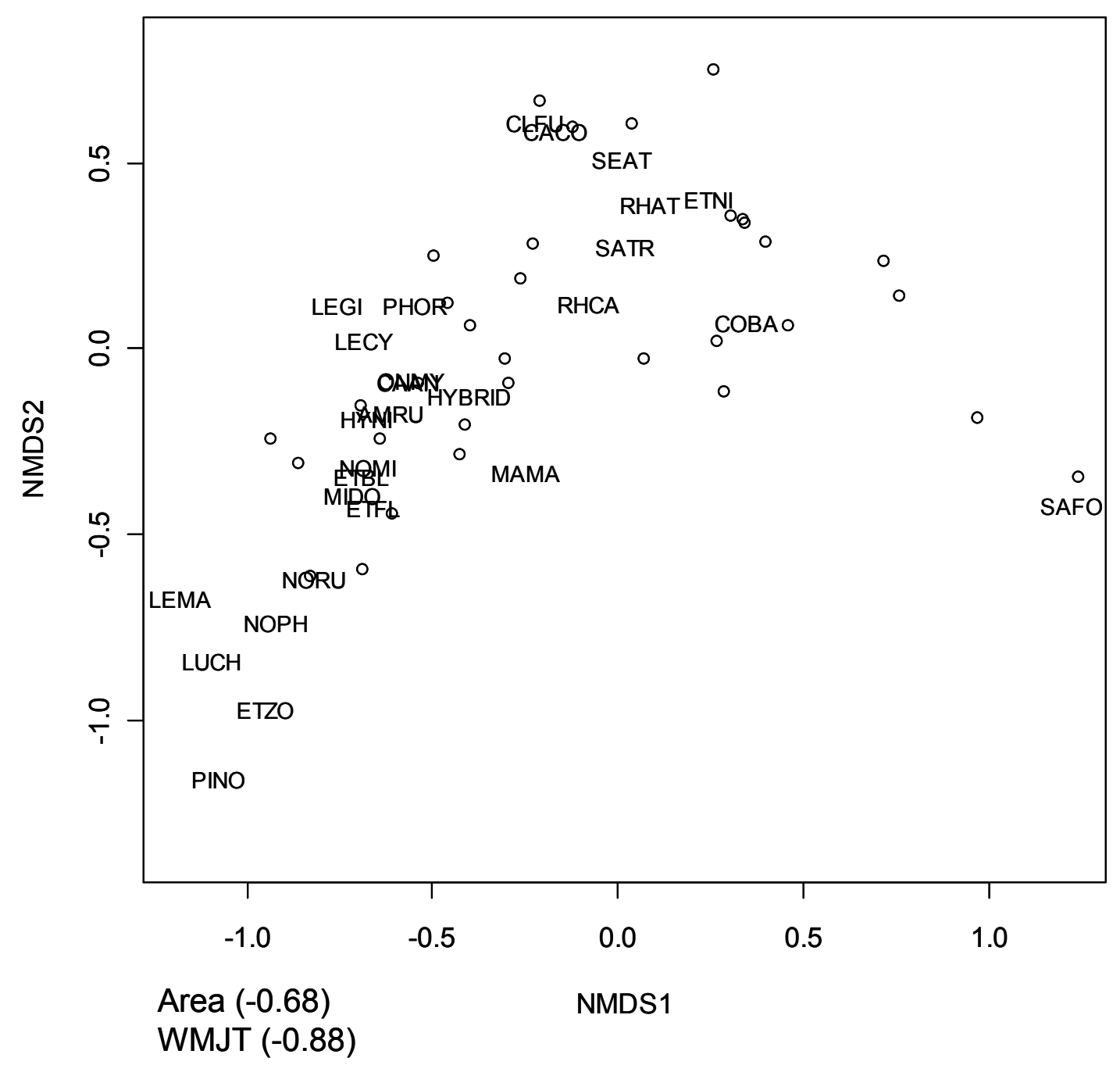




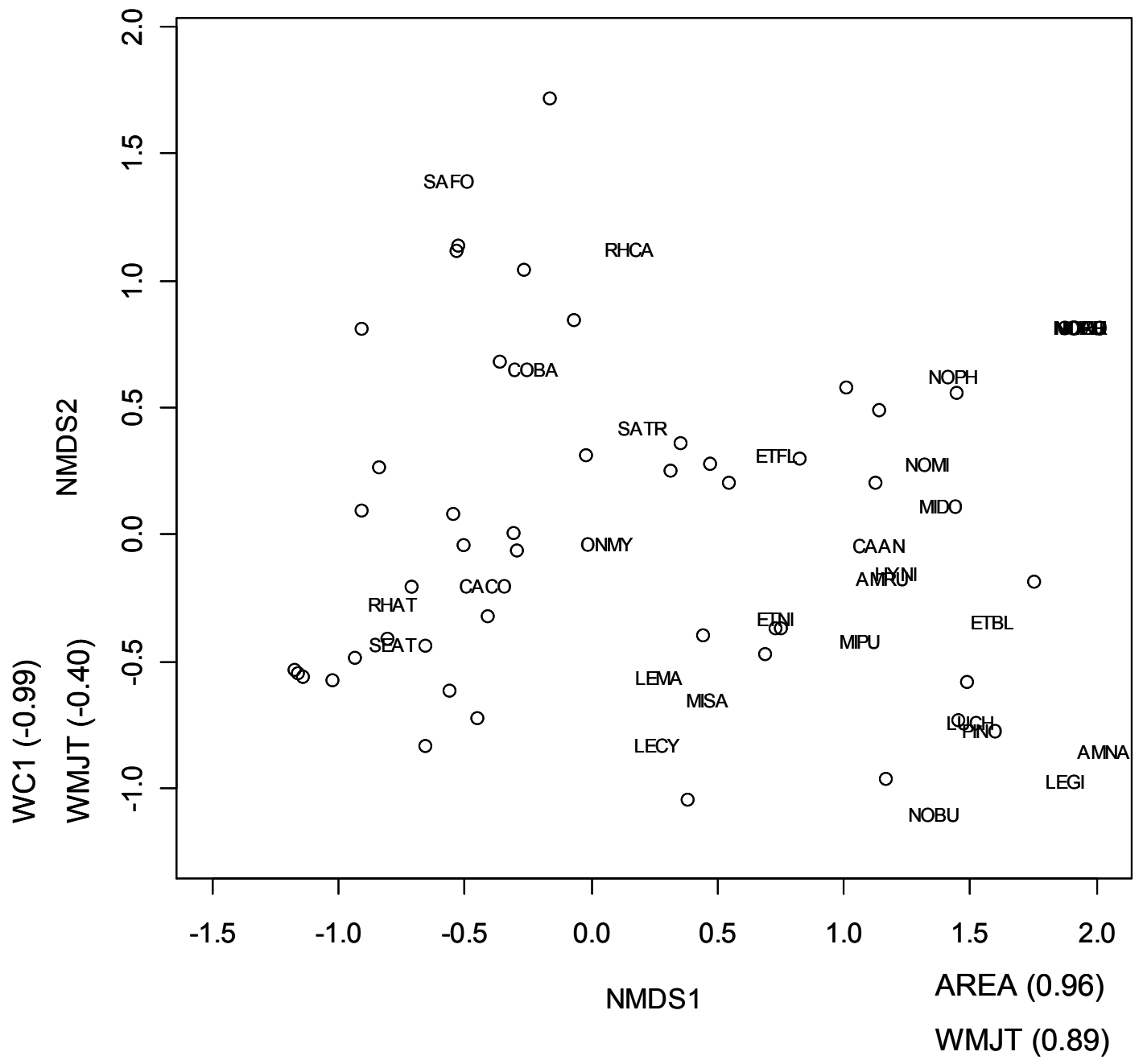


Figure 5. Plot of the relationship between richness and drainage area for the intact assemblage (closed circles) and fragmented assemblage (open circles) and trend lines fit to each group based on the ANCOVA linear model (Table 7).

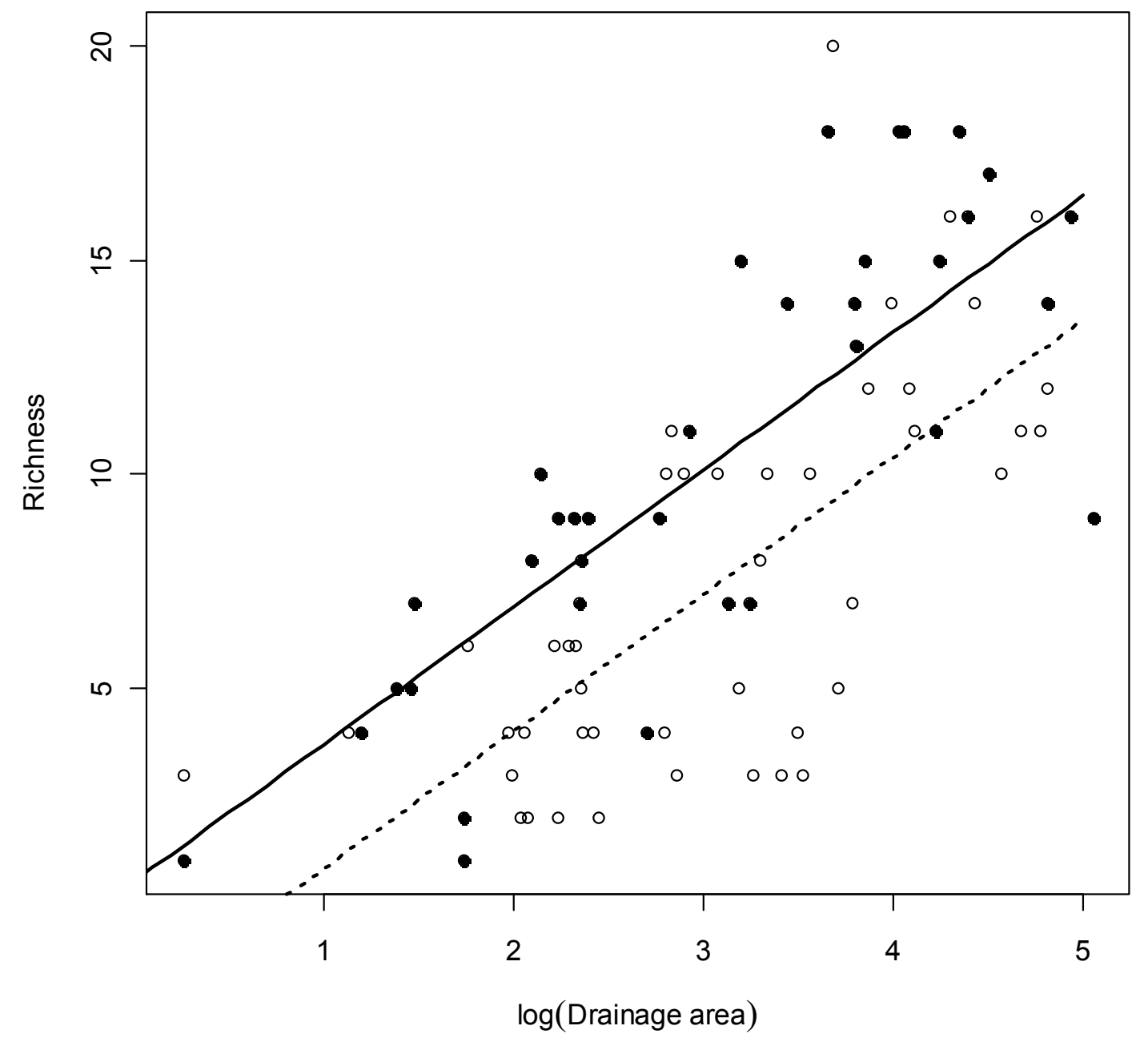


Figure 6. A comparison of species distributions in the intact and fragmented assemblage datasets via rank-abundance (top panel) and rank-occupancy (bottom panel) spectra (sensu MacNally 2007). Refer to Table 2 to identify individual species by code. Species are ordered based on rank of abundance/percent occupancy in the intact system. In the rank-abundance panel (top), because C. bairdi (COBA) were highly numerically dominant in the intact assemblage, their abundance was indicated by a text label to preserve scale.
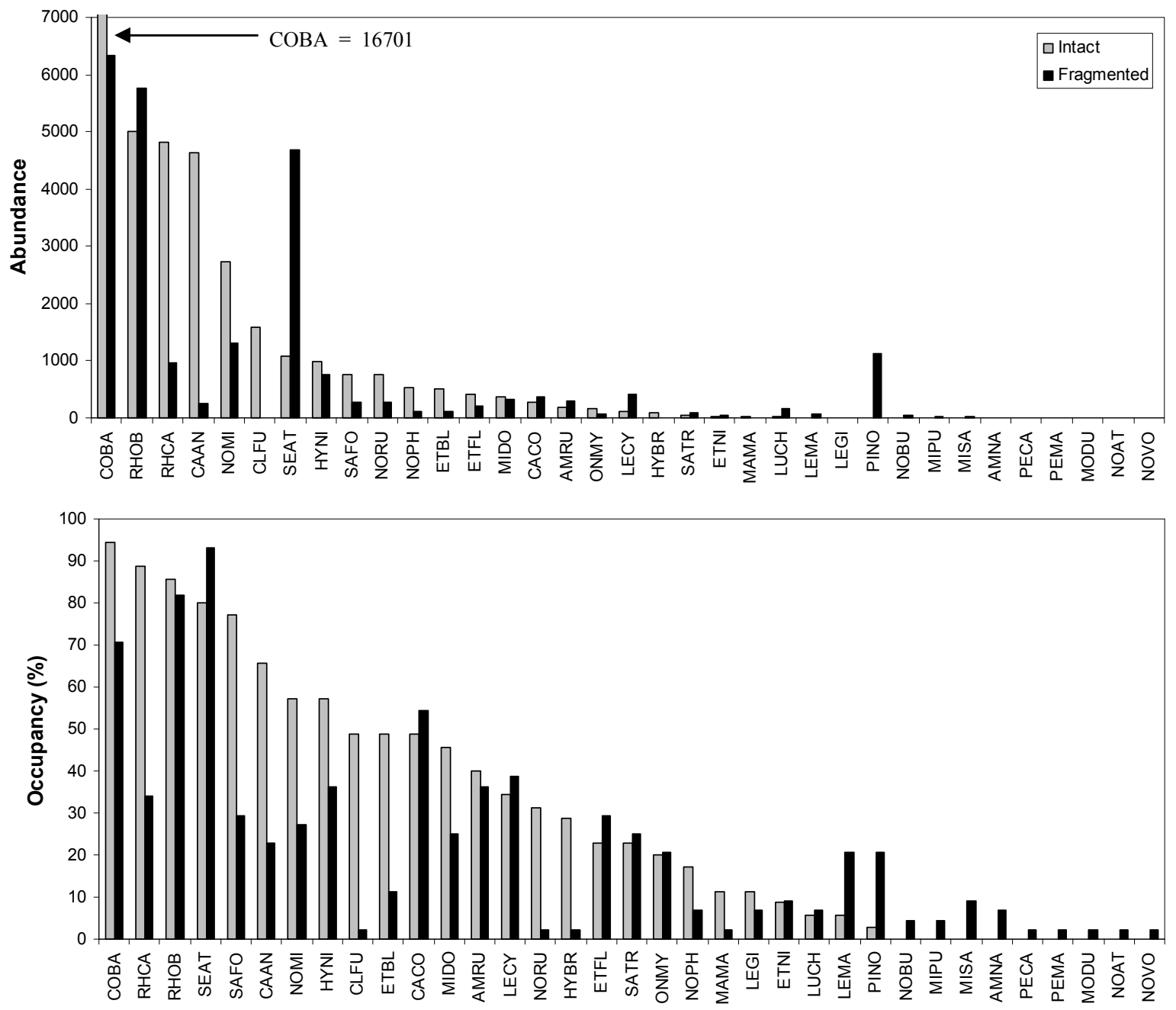
Figure 7. A three-dimensional schematic representation of the dominant mechanisms structuring stream fish metacommunities. The three axes represent gradients of increasing importance for indicated mechanisms of coexistence. Each of the four metacommunity frameworks focuses primarily on one or two dimensions within what is likely a multi-dimensional reality. The light gray, dotted " $x$ " represents the twodimensional region covered by patch dynamic models, while the dashed " $x$ " represents the two-dimensional region covered by mass effect models. The species sorting model covers only 1 dimension represented by the $\mathrm{x}$-axis. The neutral model also covers only one dimension represented by the $y$-axis. The results of this study suggested that real communities likely lie somewhere within the interior of the box (e.g., the location of the black dot).

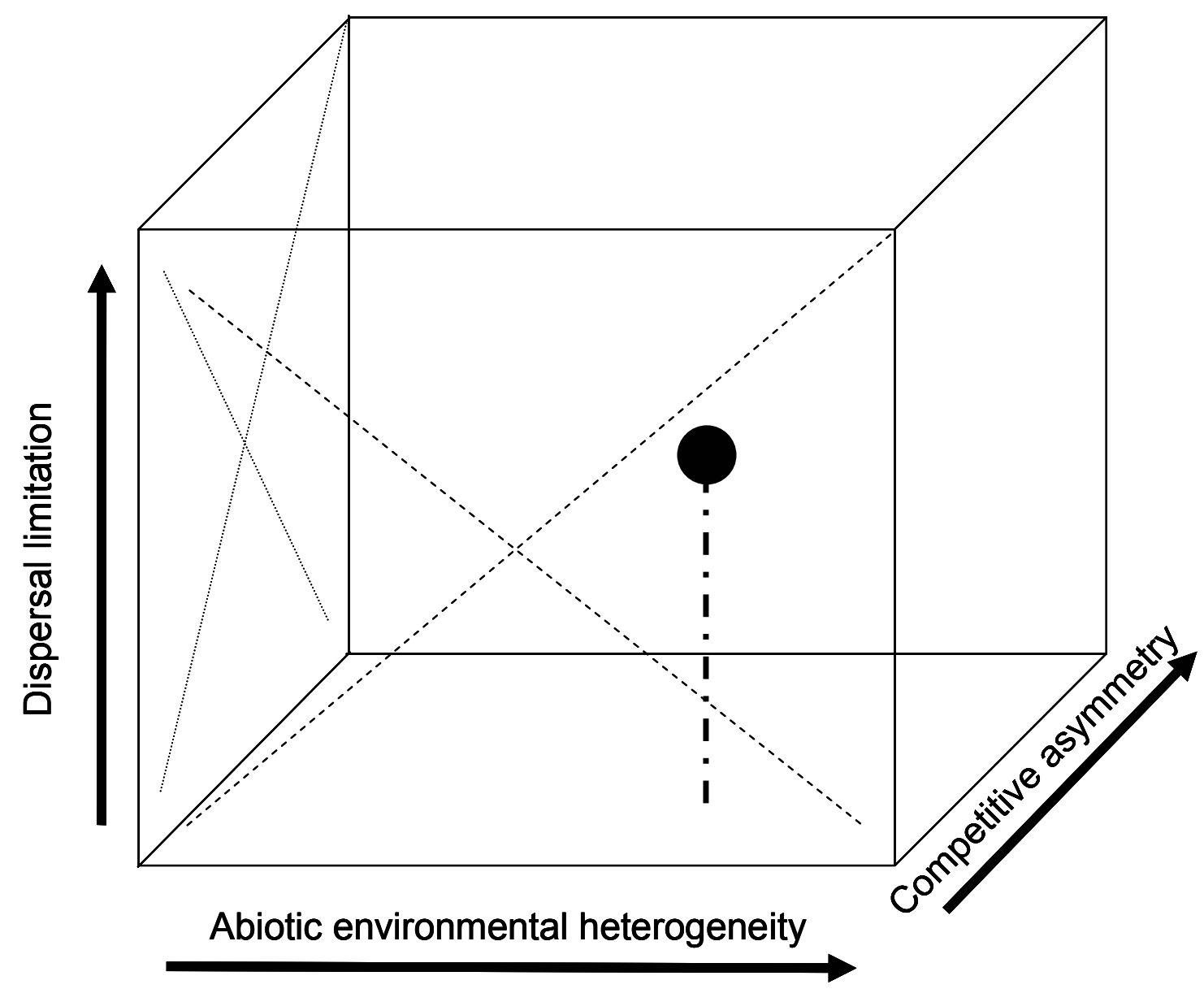


CHAPTER 2: Density Reduction Experiments Reveal Complex Metapopulation Dynamics of Headwater Stream Fish Populations

Roy W. Martin* and J. Todd Petty

Division of Forestry \& Natural Resources, West Virginia University, Morgantown, WV 26506

*Corresponding author: royworth@gmail.com 


\section{Abstract}

The relative importance of local controls (e.g., survival and recruitment) versus regional controls (e.g., dispersal) of stream fish population dynamics remains largely unknown. The overriding objective of this research was to quantify and characterize the extent to which the local population dynamics of two Appalachian, small stream fishes brook trout (Salvelinus fontinalis) and mottled sculpin (Cottus bairdi) - were influenced by internal (i.e., population density, survival, and recruitment) versus external (i.e., immigration) processes as well as the extent to which those dynamics differed given the local (e.g., core or periphery habitat) and regional context (i.e., proximity to a core versus periphery habitat). Specifically, we sought to quantify the extent to which 1) local population dynamics differed in the core (source habitat) versus the periphery (sink) of a species' distribution, 2) local population dynamics differ given the spatial context of the population (i.e., when the local population is in close proximity to its core versus its periphery), 3) density dependence is evident in local population dynamics, and 4) density dependence varies given the local context (i.e., in core versus periphery). A $2 \times 2$ factorial design (density treatment x spatial location treatment), capture-mark-recapture techniques, and an information theoretic approach were used to characterize the effects of density perturbations on the population dynamics of the two study species.

Our results indicated that small streams that are on the periphery of mottled sculpin distributions and within the core of brook trout distributions may serve as functional sources for brook trout and functional sinks for mottled sculpin. In a qualitative comparison, brook trout populations were less temporally variable than mottled sculpin and their dynamics were driven primarily by local processes such as 
survival and local recruitment. Conversely, mottled sculpin abundances were more temporally variable than brook trout, their dynamics were driven primarily by regional dispersal processes, and local recruitment was low. Additionally, apparent survival and the likelihood of transitioning among between age classes were qualitatively higher for brook trout than for mottled sculpin. We also demonstrated that recovery of abundances from the density perturbation for some age classes of each species was dependent on proximity to a presumed source of colonizers. Likewise, the data suggested that proximity to the presumed source of colonizers positively affected the proportion of local population growth due to immigration. These results suggest that regional dispersal processes play an important role in the local population dynamics of both species in these small streams.

Density dependence was not a substantial determinant of apparent survival for either species within either spatial context. However, we detected a significant effect of population density on the probability of transitioning among age classes for both brook trout and mottled sculpin. Interestingly, there was also some evidence that small adult sculpin transition rates were higher in headwater sites post removal compared to mainstem removal sites and control sites. This effect was presumably related to the fact that colonization of these sites farther from their core distribution and source of colonizers was delayed relative to mainstem sites.

The effect of the removal on apparent survival for both species was counterintuitive in that estimates were substantially lower in removal sites than in controls post-removal. This was presumably due to higher rates of population turnover as a result of migration within removal sites immediately post removal, which may have 
been related to an influx of a more mobile segment of fishes. Likewise, one of the most interesting trends in the data was a linearly decreasing temporal trend in the immigration rate for both species among removal sites over the course of the study. This trend was coupled with a lagged increase in survival rates. These findings suggested that the rapid initial recovery of the experimentally perturbed reaches was due to an influx of primarily mobile segment of the regional population and that the subsequent return to a more locally driven dynamic over time was due to delayed accumulation of a more sedentary population segment.

Overall, our study suggests that stream fish population dynamics in these small streams are characterized by a strong source-sink dynamic coupled with weak density dependence. Our findings emphasize the importance of immigration and drainage network connectivity in the maintenance of Appalachian stream fish populations in small streams. These results are consistent with the view that knowledge of important source habitats and migration corridors is essential to the successful management of stream fishes. 


\section{Introduction}

Despite a long held interest in uncovering the underlying rules governing the dynamics of natural populations, there is still a great deal that is unknown (Murdoch 1994, Hixon et al 2002, Turchin 2003, Grossman et al. 2006, Lobon-Cervia 2007). For example, two long standing theories concerning population regulation are still often at odds. On one side there is the endogenous regulation theory credited to Nicholson (1933, 1957) that focuses on population regulation through density-dependent mechanisms when organisms compete for limited resources. On the other side, there is the argument of Andrewartha and Birch (1954) that focuses largely on density-independent factors and environmental stochasticity. Although Nicholson $(1933,1957)$ argued for population self-regulation and an equilibrium state for population size, Andrewartha and Birch (1954) argued in favor of an abiotically determined non-equilibrium population.

Recent attempts to reconcile the density-dependent versus density-independent views of population control underscore the importance of interactions between the two mechanisms and suggest that each may be equally important (Bjornstad and Grenfell 2001). Others have argued that the importance of each may shift depending on the context of local conditions (Haldane 1956, Hixon et al. 2002). Specifically, Haldane (1956) argued that environmental factors should play a greater role in population dynamics when local conditions are less favorable and that density regulation should be most important when local conditions are more favorable. Building upon the hypothesis of Haldane (1956), Hixon et al. (2002) suggested that, although density dependence must certainly be acting somewhere in the regional population, it may be difficult to detect 
locally. Considering the potential for source-sink dynamics in metapopulations (Pulliam 1988), density-dependence may be especially difficult to detect in highly fluctuating sink populations (Hixon et al. 2002). However, as Hixon et al. (2002) suggested, even in the most extreme case in which one population is a source and all others are sinks, regulation need only occur in the source to sufficiently regulate the dynamics of the regional system.

There is little surprise that ecological systems at both the population and community scales are recognized increasingly as being more open (Roughgarden et al. 1994). Dispersal plays a prominent role in current population and community theory, including metapopulation (Levin 1976, Pulliam 1988, Hanski 1999) and metacommunity (Leibold et al. 2004, Holyoak et al. 2005) models. In the context of stream fish ecology, it is recognized that fish require access to a variety of resources that supplement and complement their life histories, which are distributed over a wide range of spatial and temporal scales (Schlosser 1991, Schlosser and Angermeier 1995). Consequently, stream fish ecologists increasingly describe stream ecosystems as dynamic "riverscapes" where spatial and temporal variability in complementary and supplementary resources interact with life-stage specific dispersal to ultimately determine fish population attributes at the local and regional scales (Fausch et al. 2002). Likewise, several empirical studies have demonstrated that specific areas in a drainage network can serve as important sources of individuals for other areas and, thus, ultimately influence population structure at the broader scale (Schlosser 1995, Snodgrass and Meffe 1998, Petty et al. 2005).

Stream fish population persistence at the scale of the region can be defined by the sum of local extinction and colonization events (e.g., Dunham et al. 1997, Dunham and Reiman 1999). However, local population persistence is quantified through four rate- 
dependent processes — mortality, natality, immigration and emigration — that are influenced by a wide range of environmental and biological variables (Shclosser and Angermeier 2005). A full understanding of the spatial and temporal variation in demographic factors within and between local populations and the environmental and biological processes driving that variation is crucial for effective management of stream fish populations (Labbe and Fausch 2000, Schlosser and Angermeier 1995, Fausch et al. 2002, Petty et al. 2005). However, the extents to which local (i.e., density dependence, survival, and recruitment) and regional (i.e., migration) dynamics are important to the local population dynamics of stream fishes are still largely unknown (Fausch et al. 2002). For example, although some studies have suggested a strong role for local processes such as density dependent feedbacks (Elliot 1994, Grossman et al. 2006, 2010, Utz and Hartman 2009), others have demonstrated a stronger role for regional processes such as dispersal (Labbe and Fausch 2000, Petty et al. 2005, Hitt and Angermeier 2008, Martin and Petty 2009).

Recent research on stream fishes in this region has suggested that local population persistence may be dependent on a combination of local density-dependent (Utz and Hartman 2009) and density-independent (Petty et al. 2005) factors in addition to regional dispersal patterns (Petty et al. 2005, Martin and Petty 2009, Martin, Chapter 1). For example, Petty et al. (2005) demonstrated that although local conditions related to reproductive success in headwater reaches were an important determinant of local persistence of juvenile and small adult brook trout (Salvelinus fontinalis), regional dispersal of large adults played an important role in distributions at the watershed scale. Specifically, Petty et al.'s (2005) findings suggest that the productivity of brook trout 
populations at the watershed scale is dependent on complementation of small headwater streams that serve as reproductive core habitats and larger streams that serve as foraging habitats for mobile adults. Martin and Petty (2009) also recently demonstrated that, although local thermal conditions play an important role in the watershed scale distribution of smallmouth bass (Micropterus dolomieu) and brook trout, regional dispersal often leads to an unexpected sympatric distribution patterns for these two species with distinctly different local thermal preferences. Finally, Martin (Chapter 1) demonstrated that stream fish metacommunity structure in this region is best described by a mass effect model, suggesting that local and regional diversity is driven primarily by an interaction between variability in local abiotic conditions and regional dispersal (Leibold et al. 2004, Mouqet and Loreau 2003).

Because local population dynamics are driven by a range of interacting processes, strategically designed experimental studies provide the only explicit means to tease apart the importance of these processes. Despite the obvious need, however, there have been relatively few studies focusing on the response of vital rates to experimental manipulation and most of those studies have focused on manipulation of only local factors (e.g., Grossman et al. 2006, Petty and Grossman 2009). Findings extending from natural and experimental perturbation studies, however, suggest an important role for regional processes in local dynamics. For instance, most of those studies have shown that recovery from disturbance is rapid ( $<1$ year), but that dispersal and mobility play an important role in local stream fish population and assemblage dynamics (Neimi 1990, Detenbeck et al. 1992, Lonzarich et al. 1998, Roghair et al. 2002 Albanese et al. 2009). What are needed to more fully understand the relative importance of both local and 
regional processes, however, are carefully designed experimental studies that simultaneously manipulate both local and regional factors.

We therefore conducted a field experiment designed to address the following central question: What are the relative contributions of local and regional processes in determining the dynamics of brook trout (Salvelinus fontinalis) and mottled sculpin (Cottus bairdi) populations inhabiting small, headwater streams. Specifically, we were interested in quantifying and characterizing the extent to which these species' local population dynamics were influenced by internal (i.e., population density, survival, and recruitment) versus external (i.e., immigration) processes as well as the extent to which those dynamics differed given the local (e.g., source or sink habitat) and regional context (i.e., proximity to a source versus sink habitat). We devised 4 specific goals to address our overriding objective. These goals were to quantify and characterize the extent to which:

1. local population dynamics differ in the core (e.g., source habitat) versus the periphery (e.g., sink) of a species' distribution,

2. local population dynamics differ given the regional context (i.e., when in close proximity to core versus the periphery),

3. density dependence is evident in local population dynamics, and

4. density dependence varies given the local context (i.e.,in core versus periphery).

To address these goals, we conducted field-based fish density reduction experiments combined with capture-mark-recapture techniques, a novel experimental 
design and a strong inference approach (Platt 1964) employing Akaike's Information Criterion (AIC, Akaike 1972, Burnham and Anderson 2002).

\section{Methods}

Brook trout, mottled sculpin, and small streams as a test system

We chose to examine brook trout and mottled sculpin populations within the context of our overriding research objective for several reasons. First, because mottled sculpin and brook trout are generally abundant and commonly occurring among many small, unimpaired, streams of this region (Freund 2004, Freund and Petty 2007, Martin Ch.1), they make excellent candidates for a mark-recapture study. More specifically, these two species are readily collected in small stream systems where an efficient balance is struck between being able to obtain an effective sample size of individual fish capture histories and having sufficient time to conduct a closed population survey and individually mark a large number of animals in a sufficiently sized stream reach. Additionally, small streams are perhaps more conducive to the study of population dynamics than larger systems due to reduced interspecific interactions (Schlosser 1982, Matthews et al. 1998).

Brook trout and mottled sculpin have been well studied in Appalachia and much is known about their respective ecologies relative to most small stream Appalachian fishes (e.g., Petty and Grossman 2004, Petty et al. 2005, Grossman et al. 2006, Utz and 
Hartman 2006, Utz and Hartman 2009, Grossman et al. 2010). In fact, our lab has collected an extensive amount of data throughout this region over recent years, which led to a number of research papers detailing the watershed scale patterns of abundance and occurrence for these two species (Freund 2004, Petty et al. 2005, Freund and Petty 2007, Hense 2007, Martin and Petty 2009, Martin Ch.1 and Ch.2). Likewise, colleagues in our lab have also collected data and published several studies detailing the spatial population dynamics of one of these species, brook trout, in this region (Lamothe 2002, Liller 2006, Petty et al. 2005). Consequently, we felt that we had a strong grasp of the individual ecologies and general population dynamics of these two species in this region. Thus, we believe that the a priori expectations we set out to address when designing this study were soundly informed by a reasonably extensive empirical background.

Brook trout

Throughout their central and southern Appalachian distribution brook trout have suffered substantial reductions in numbers due to a range of factors including physical and chemical habitat degradation and introduction of non-native species (Flebbe 1994, Marschall and Crowder 1996). In the central Appalachian region, acid precipitation is considered the most prominent stressor (Wigington et al. 1996). In the upper Monongahela watershed, however, both acid precipitation (Clayton et al. 1998, Petty et al. 2005, McClurg et al. 2007) and acid mine drainage (AMD, Freund and Petty 2007, Martin and Petty, Chapters 1 and 3) play prominent roles in their basin-wide geographic distribution. More specifically, regional loss of small stream habitats that provide 
supplementary adult spawning areas and YOY rearing habitats due to acidification (Petty et al. 2005, Petty and Thorne 2005) in addition impaired network connectivity (PoplarJeffers et al. 2010, Freund and Petty 2007, McClurg et al. 2007, Martin, Chapters 1 and 3) currently limit the geographic distribution of brook trout populations in this region.

In north-central West Virginia, the core distribution of brook trout has been estimated as limited to perennial streams draining less than approximately $15-20 \mathrm{~km}^{2}$ (Freund 2004, Hense 2007). However, presence in larger mainstems is not uncommon (Petty et al. 2005), and may be more likely when accessibility of small stream sources is high in the watershed (Martin and Petty 2009). These larger, downstream areas are thought to provide a greater scope for growth (Utz and Hartman 2006).

Three demographically relevant population segments are generally considered in studies on the population ecology of Appalachian brook trout. The young-of-the-year (YOY) segment is easily identified in the field and by using length frequency diagrams and represents a true age class of individuals produced in the previous fall (Petty et al. 2005, Utz and Hartman 2006, Utz and Hartman 2009). However, although YOY emerge in the spring and grow relatively rapidly to reproductively active adults, they seldom live beyond 2-3 years or grow longer than $200 \mathrm{~mm}$ in length (Whitworth and Strange 1983, Petty et al. 2005, Utz and Hartman 2009, Grossman et al. 2010). Thus, true age-class distinctions for age-1+ individuals are difficult based on length-frequency data (Petty et al. 2005, Utz and Hartman 2009). Nevertheless, in most populations, there is generally a readily distinguishable size structure within the age- $1+$ population. For many populations in this region, the distinction occurs around 110-120 mm standard length (SL; Petty et al. 2005). 
In this study, we used length-frequency data from spring fish population samples to distinguish the size structure for three demographic segments of brook trout used throughout the study (Appendix B1). Although the distinction among small and large adults may not represent a true age class, the break is thought to distinguish among demographically and ecologically relevant population segments. For example, larger adult brook trout are more often associated with habitats downstream in the drainage network and their advantage in mobility (Gowan and Fausch 1996) may play an important role in the spatial population ecology of the species (Petty et al. 2005).

The spatial population dynamics of brook trout in this region have been described as largely dependent on an interplay between the geographic distribution of suitable, small stream ( drainage area $<5 \mathrm{Km}^{2}$ ) spawning sites and post-reproductive dispersal of larger, age 1+ individuals throughout the network (Petty et al. 2005). At the scale of the typical stream reach $(\sim 0.1-1 \mathrm{Km})$, local brook trout populations have been shown to vary in their degree of demographic openness seasonally, by age class, and across their geographic distribution, with all demographic segments generally having higher apparent survival in upstream areas and immigration being generally high and variable across the network (Petty et al. 2005). Petty et al. (2005) also demonstrated that, in contrast to small adults, large adult distributions were not related to spawning intensity in the watershed and were more unstable over seasons and over the drainage continuum, reflecting a tendency toward higher mobility.

Mottled sculpin 
The mottled sculpin is a comparatively long-lived $(\sim 7+$ years, Grossman et al. 2002), territorial (Petty and Grossman 2004, Grossman et al. 2006, Petty and Grossman 2010), benthic invertivore (Stouder 1990) that is widely distributed and numerically dominant throughout this region (Hense 2007, Freund and Petty 2007, Martin Chapters 1 and 3). Sculpin are considered acid-sensitive (Magnuson et al. 1984, Van Sickle et al. 1996), and consequently are threatened in this region by acid precipitation and AMD (Freund and Petty 2007, Martin, Chapters 1 and 3). In fact, due to their sensitivity to acidification as well as their tendency to be restricted in movement (Petty et al. 2004), mottled sculpin presence is generally expected to provide a good indication of local water quality (Freund 2004).

Evidence from sculpin populations in $2^{\text {nd }}$ and $3^{\text {rd }}$ order southern Appalachian streams suggests that intraspecific interactions and local density-dependent population regulation play an important role in their population dynamics (Petty et al. 1996, Grossman et al. 2006, Petty et al. 2004, Petty et al. 2010). Petty and Grossman (2004, 2007, 2010), for example, demonstrated that large adults are highly sedentary and occupy the most profitable territories. They also demonstrated that when adults were experimentally removed from a subset of sites small conspecifics quickly shifted into territories previously occupied by adults (Grossman et al. 2006, Petty and Grossman 2010).

In an analysis of sculpin movement, Petty and Grossman (2004) found that, while sculpin generally exhibited highly restricted movements (mean daily movement $<5 \mathrm{~m}$ ), YOY and adults had higher rates of movement when large adult densities were lower. Intraspecific interactions generally result in higher survival and growth for dominant 
adults. As a result, populations in southern Appalachian streams are thought to be regulated largely through density dependent feedbacks resulting from intraspecific competitive interactions (Grossman et al. 2006, Petty and Grossman 2007, 2010).

Although mottled sculpin are ubiquitously distributed among the unimpaired watersheds of this region (Freund and Petty 2007), the core of their distribution is among streams ranging from approximately 25 to $90 \mathrm{~km}^{2}$ in drainage size (Hense 2007). Nevertheless, they are common among even $1^{\text {st }}$ order streams in this region and their presence in small streams is generally limited by either manmade barriers to dispersal such as road culverts (Poplar-Jeffers 2007) or natural barriers imposed by steep gradients and flow permanence (R. Martin, personal observation).

\section{Study design}

\section{Design factors}

The experiment was a $2 \times 2$ factorial design. One experimental factor was a fish removal treatment (removal vs. control) and the other factor was watershed position (headwater vs. mainstem). The study was conducted among 12 individual sites of which six received the density perturbation or fish removal treatment, hereafter referred to as "removal" sites. Of the six removal sites, three were mainstem tributaries and three were headwater tributaries. Likewise, of the remaining six control sites, three were headwater tributaries and three were mainstem tributaries. Thus, in total there were three mainstem 
control sites (MC), three mainstem removal treatment sites (MT), three headwater control sites (HC), and three headwater removal treatment sites (HT). Also, we divided the 12 sites equally over three sub-watersheds (HUC10) within the Cheat River watershed: Horseshoe Run, Gandy Creek, and Laurel Run.

The core study area on each study stream was a $200 \mathrm{~m}$ stream reach. During the first sampling occasion when fish were being captured and removed, we sampled a total length of $400 \mathrm{~m}$ to provide a $100 \mathrm{~m}$ upper and lower buffer zone (Peterson and Bayley 1993, Albanese et al. 2009). The lower extents of the sites were located 10 meters upstream from the approximate confluences with the downstream headwater or mainstem receiving streams. Therefore, due to the $100 \mathrm{~m}$ buffer zone, after the initial removal, the downstream extents of the six mainstem tributary sites, for example, were located $110 \mathrm{~m}$ upstream of the approximate confluence with the receiving mainstems.

Study area

Figure 1 portrays the geographic location of the Cheat River watershed in West Virginia and the distribution of the 12 study sites, their experimental grouping types, and spatial positions. The upper Cheat river watershed is located within the north central portion of West Virginia and is part of the upper Monongahela River basin (Figure 1). Although the lower Cheat River watershed is heavily impaired by AMD (Freund 2004, Freund and Petty 2007, Merovich et al. 2007, Martin, Chapters 1 and 3), the upper Cheat River watershed is characterized by relatively few impacts (Freund 2004, Martin and Petty 2009). In particular, the Gandy Creek, Glady Fork, Horseshoe Run subwatersheds 
boast some of the most productive streams in the region in terms of fish densities (Freund 2004, Hense 2007, Martin, Chapters 1 and 3) and are devoid of mining activity. Likewise, although acid precipitation is a concern in many poorly buffered watersheds throughout the Cheat basin (Clayton et al. 1998, McClurg et al. 2007), the watersheds from within which the sites used in this study were derived are comprised of sufficiently buffered high quality streams (Freund 2004, Hense 2007, Martin, Chapters 1 and 2).

Site selection

During site selection, we controlled for stream size and watershed position. All 12 of our sites had drainage areas ranging from 3 to $5 \mathrm{~km}^{2}$. This size encompasses the lower range of the core distribution of brook trout in this region (brook trout core range $=$ $2-23 \mathrm{~km}^{2}$, Hense 2007), but not the core distribution of sculpin (mottled sculpin core range $=11-50 \mathrm{~km}^{2}$, Hense 2007). We then selected study streams that differed with regard to position within the drainage network. Streams chosen for study could either be classified as headwater tributaries or mainstem tributaries (sensu Osborne and Wiley 1992, Grenuoillet et al. 2004, Hitt and Angermeier 2008). Mainstem tributaries were defined as streams that drained directly into a mainstem stream (defined as a stream reach with a drainage area exceeding $50 \mathrm{~km}^{2}$ ). Mainstems of this size are expected to encompass the core distribution of mottled sculpin in this region, yet fall on the periphery of the brook trout distribution (Hense 2007). Headwater tributaries were defined as streams that drained directly into another stream with a drainage area less than $10 \mathrm{~km}^{2}$ and were located at least $2 \mathrm{~km}$ swim distance from a mainstem river segment. 
Consequently, headwater tributaries were connected with streams that fall within the core distribution of brook trout but are highly isolated from the core distribution of mottled sculpin.

In summary, we studied small streams embedded within headwater drainage networks and small streams embedded within mainstem drainage networks. All studied stream segments represented "source" habitats for brook trout and "sink" habitats for mottled sculpin. This enabled us to examine brook trout population dynamics in potential source habitats while connected to other sources (headwater tributaries) and within sources situated adjacent to sink habitats (mainstem tributaries) (Pulliam 1988, Cornutt et al. 1996, Hixon et al. 2002). Similarly, with mottled sculpin, we examined their population dynamics in streams that are likely local sink habitats located adjacent to other likely sinks (headwater tributaries) versus local sink habitats located adjacent to source habitats (mainstem tributaries).

Finally, site selection was intended to minimize inter-site variability in local and regional environmental conditions. Stream fish assemblage and population dynamics are known to vary rather predictably along the downstream continuum (Schlosser 1982, Matthews 1998). Streams of similar size, however, particularly in the same region, are expected to have similar local physical and chemical properties (Vannote et al. 1980). Thus, our intent was to use the design to control as much as possible for site to site variability in environmental conditions such as habitat complexity, flow variability, and water temperature. 
Fish removal and seasonal fish sampling and tagging

Fishes were sampled at each of the 12 sites beginning late June 2006 and ending early October 2007. The initial sampling event was timed to be late enough to capture newly spawned brook trout and mottled sculpin. At the initial sampling occasion, we removed all fishes from $400 \mathrm{~m}$ segments within each of six sites assigned to the removal treatment. The first follow up sampling event occurred exactly 30 days later at each site during July-August 2006. A third sampling event occurred during October 2006. The following year (2007) we sampled each site three additional times, late spring (mid-May), mid-summer (mid-July), and mid-autumn (early October), giving at total of six sampling events. Follow up sampling was conducted within a central $200 \mathrm{~m}$ segment of each study area. The reach length sampled for control streams was also $400 \mathrm{~m}$ on the initial sampling occasion, although all fish were returned to the stream alive.

For each sampling event at each site, we blocked the study area with nets and used three-pass removal electrofishing to capture fishes (Petty et al. 2005). All captured fishes were immobilized with a dilute solution of clove oil and stream water and measured to the nearest millimeter standard length and weighed to the nearest 0.1 grams. Brook trout and mottled sculpin were tagged with a unique individual identifier tag, while other species were given a batch mark identifier specific to the sample date and site. All fishes were tagged using visual implant elastomer tags (VIE; Northwest Marine Technology, Shaw Island, Washington). Brook trout were tagged in the caudal fin (Petty et al. 2005, Utz et al. 2006), whereas sculpin were tagged on their abdomens (Petty and Grossman 2010). Fishes removed from the treatment sites on the initial sampling 
occasion were transported and released into reaches further than $10 \mathrm{~km}$ downstream. In one mainstem removal site — Lick Drain of the Horseshoe Run sub-watershed — all fishes removed were marked in order to roughly assess the likelihood of removed individuals repopulating the experimental sites at a later date. None of those marked fishes were subsequently recaptured in that site or in any of the other sites in the network.

\section{Sampling local habitat conditions}

Following each fish sampling occasion, we collected water chemistry data, including $\mathrm{pH}$, specific conductivity, total hardness, calcium hardness, and alkalinity. Both $\mathrm{pH}$ and specific conductivity were collected with a 600 XL Multi-Parameter Water Quality Monitor (YSI Incorporated, Yellow Springs, Ohio). Alkalinity was measured with a model AL-AP alkalinity test kit and total and calcium hardness were measured with a model HA-4P total and calcium hardness test kit (Hach Company, Loveland, CO). The kits are accurate to the nearest 1 grain/gallon $(17 \mathrm{mg} / \mathrm{L})$ for calcium and total hardness and to the nearest 5 grain/gallon $(86 \mathrm{mg} / \mathrm{L})$ for alkalinity.

To quantify variability in stream channel characteristics among sites, we conducted a thalweg habitat profile of each site following fish sampling in the spring of 2007 (Petty et al. 2001). The process involves measuring and recording a suite of instream habitat variables at evenly spaced points along the stream thalweg. Thalweg points were spaced every one mean stream width (MSW; Simonson et al. 1994). Individual habitat variables recorded along transects included thalweg water depth $(\mathrm{cm})$, average current velocity (velocity measured at $60 \%$ of the water depth, $\mathrm{m} / \mathrm{s}$ ), bottom 
current velocity (velocity measured on the streambed substrate, $\mathrm{m} / \mathrm{s}$ ), distance to fish cover (m), and channel unit (riffle, run, pool, or glide) (Petty et al. 2001). Current velocities were measured with a Flo-Mate water velocity meter (Marsh-McBirney Incorporated, Frederick, Maryland). Fish cover was defined as any cover feature large enough to conceal an approximately $200 \mathrm{~mm}$ fish (Simonson et al. 1994) and distance to cover was measured to the nearest 0.1 meters from the thalweg point. Prior to final statistical analyses, the thalweg profile data were summarized for the entire reach for each site by the mean estimates for each variable. We also calculated the coefficient of variation $(\mathrm{CV})$ of depth and of average current velocity and used these as measures of habitat complexity. Percent canopy cover was estimated using a spherical densiometer at 21 evenly spaced thalweg points within each reach (Barbour et al. 1999). Four measurements (facing upstream, downstream, stream left and stream right) at each thalweg location were taken and averaged to give a stream specific measure of canopy cover.

Pebble counts were conducted to characterize the streambed substrate. The procedure consisted of walking the length of the reach in a zigzag pattern and selecting pebbles at random (Bevenger and King 1995). A total of 100 pebbles were sampled over the entire reach and each pebble was tallied within one of six size classes based upon the diameter of the intermediate axis of the pebble: silt-clay $(<0.062 \mathrm{~mm})$, sand $(0.062-1.9$ $\mathrm{mm})$, gravel $(2-64 \mathrm{~mm})$, cobble $(65-256 \mathrm{~mm})$, boulder $(257-4000 \mathrm{~mm})$, and bedrock $(>$ $4000 \mathrm{~mm}$ ). Pebble count data was used to calculate median substrate size (D50) for each reach. 
Large woody debris within the active channel of each site was also quantified. All pieces of wood over $1.0 \mathrm{~m}$ in length and $10 \mathrm{~cm}$ in diameter were tallied. Each piece of debris was tallied within one of 12 size classes based upon diameter and length (length classes: 1-2 m, > 2-5 m, > 5-10 m, and > $10 \mathrm{~m}$; diameter classes: $10-20 \mathrm{~cm},>20-30 \mathrm{~cm}$, and $>30 \mathrm{~cm}$ ). From these data, we calculated the average volume of woody debris per 50 linear meters of stream for each site. To find this value we first calculated a wood volume to associate with each size class. This was calculated by squaring the mean radius for the diameter size range (e.g., $0.075 \mathrm{~m}$ for the $10-20 \mathrm{~cm}$ diameter size range) and multiplying by $\pi$, resulting in the mean cross-sectional area for the size class. We then multiplied this area by the mean of the length size range (e.g., $1.5 \mathrm{~m}$ for the 1-2 $\mathrm{m}$ size range), resulting in a volume $\left(\mathrm{m}^{3}\right)$. Within each size category we multiplied the associated volume by the number of pieces of wood tallied to arrive at a total volume within each category. Finally, we summed the total volume across all categories resulting in a total LWD volume for the site.

Water temperature regimes were measured at each site with $\mathrm{HOBO}^{\complement}$ Water Temp Pro stream temperature data loggers. Temperature loggers recorded hourly thermal data over the time period spanning June to September of 2007. For each site, we estimated the maximum weekly average summer temperature (MWAT) as a measure of average summertime thermal conditions at each site. This metric is calculated as the maximum value of the highest average of mean daily temperatures for any 7 day period and is considered a biologically meaningful index of summertime thermal conditions (Eaton et al. 1995, Welsh et al. 2001). 
Finally, for each site, we estimated the total site wetted area in both May 2007 and September 2007. To estimate the wetted area for each site, we first estimated the wetted width for a series of 21 equally spaced transects along the 200 meter length of the study reach. We then used the sequential estimates and the 10 meter intervals between them to estimate the two dimensional area between each measurement increment. To calculate the total wetted area for the site, we summed over all the increments. From these late spring (May) and early fall (September) estimates of wetted area we calculated the proportion of spring (May) wetted area in early fall as an indicator of stream flow variability among sites. Sites with a greater proportion of spring stream flow in the fall were expected to generally have more stable flows.

Analysis of local physicochemical conditions

We used principal components analysis (PCA) on the correlation matrix of the multivariate water chemistry dataset derived from all sites and sampling dates in order to identify and characterize important gradients in water chemistry variability among sites (Merovich et al. 2007). Water chemistry variables included in the analysis were $\mathrm{pH}$, specific conductivity, total hardness, calcium hardness and alkalinity. Prior to the PCA, we assessed the individual chemistry variables for normality and applied transformations where applicable. We interpreted component loadings when loading values were greater than or equal to 0.4 .

We also used PCA to quantify the multivariate variability in physical habitat conditions among sites. Variables included in the analysis were mean water depth, 
coefficient of variation in water depth, mean average current velocity, coefficient of variation in average current velocity, proportion of channel units as pool habitat, mean distance to fish cover, coefficient of variation in distance to fish cover, median substrate size, and large woody debris volume. All PCA analyses were performed using the "stats" package of the R statistical programming language software, version 2.9.1.

\section{Model selection and inference methodology}

We used AICc to evaluate biologically realistic candidate models (Burnham and Anderson 2002) useful for predicting capture efficiency, abundance, and demographic characteristics of each study species from combinations of measured biologically relevant covariates. For example, for models estimating the response of dependent variables related to the population dynamics of the two species, realistic combinations of the additive and interactive effects of the experimental design factors (removal treatment and headwater vs. mainstem tributary) and local physicochemical covariates were included as predictors.

For each set of models, a model including all of the experimental and covariate effects of interest and interactions was constructed and was the most highly parameterized model. It therefore served as the "global model" and baseline reference for subsequent, reduced (i.e., fewer parameters) models (Burnham and Anderson 2002). The global model was intended to maximally account for inter-site variability in the dependent variable based on all combinations of the experimental factors, covariates and interactions. The reduced models were constructed in order to attempt to account for 
variability in the dependent variable using fewer parameters than the global model. Employing AIC allowed comparison of the amount of information lost in each candidate model compared to the best fitting model.

The models were ranked and compared using $\triangle \mathrm{AICc}$ and AICc weights $\left(w_{i}\right.$, Burnham and Anderson 2002). While the $\triangle \mathrm{AICc}$ measures the magnitude of difference in AICc values between the highest ranked model and model of interest, the $w_{i}$ estimate the weight of evidence in favor of a particular model being the best in the set or, rather, the posterior probability that a given model is true (Burnham and Anderson 2002). The proportional value of $w_{i}$ for a higher ranked model to a lower ranked model is an estimate of the comparative likelihood between the two models (Burnham and Anderson 2002). For example, in a scenario where $w_{1}=0.4$ and $w_{2}=0.2, w_{1}$ is twice as likely to be the best model in the set compared to $w_{2}$ (i.e., $w_{1} / w_{2}=0.4 / 0.2=2$ ).

In order to account for model selection uncertainty, we averaged the model coefficients and fitted parameter estimates, as well as their standard errors and confidence limits, for the models comprising the 95\% confidence set (Burnham and Anderson 2002, Grossman et al. 2006, Grossman et al. 2010). The set is constructed by first ranking the candidate models from highest to lowest $w_{i}$ and cumulatively summing across the individual $w_{i}$. The $95 \%$ confidence set includes all models where $\Sigma w_{i} \leq 0.95$. Additionally, the relative importance of individual independent variables in the candidate set was estimated by summing their $w_{i}$ over all the interpretable models (i.e., the $95 \%$ confidence set, Burnham and Anderson 2002, Grossman et al. 2006, Grossman et al. 2010). 
For models used to estimate parameters associated with the population dynamics of the study species, in addition to the experimental factors, we included a consistent combination of the local physical and chemical covariates in an attempt to maximally account for site to site variability in local physicochemical conditions while assessing the effects of the experimental factors (Wiens et al. 2004, Parker and Weins 2005). This approach is synonymous with the common analysis of covariance (ANCOVA) approach to linear model construction and inference. Similarly, for some analyses, we incorporated "nuisance" covariates coding for age and temporal effects. These factors also included all two level interactions with the experimental factors when those experimental effects were in a model. The intention of the nuisance factors was to account for important variation in the data that was not necessarily of interest to the overriding research objective, but was considered biologically important. The effects of age class and season, for example, are generally well known and considered important for the dependent variables of interest in this study. Moreover, including these factors rather than experimental factors was essential to narrowing the field of candidate models to a reasonable number. Nevertheless, for some dependent variables, separate models were developed for each age class, while, for others, age was incorporated as a covariate factor. The specifics of the model development and selection process are described in more detail below in the sections specific to each dependent variable. 


\section{Estimating capture probability and abundance}

We analyzed electrofishing capture histories from three-pass depletion samples to estimate capture probability (p-hat) and population size (N-hat) for each fish species for all sites and sampling occasions and among species' size classes. Huggins' closed population estimator and maximum likelihood estimates for multinomial models in program MARK (White and Burnham 1999) were used. Because the Huggins' estimator is conditional only on the animals captured, it is regarded as having better statistical properties than other removal estimators when the number of animals captured is low (Huggins 1989, Peterson et al. 2004a, Hense et al., In Revision). In Huggins' model, Nhat is a derived parameter estimated from p-hat and the recapture probability (c-hat), both of which are calculated directly in the model likelihood (Huggins 1991). For all of our models, we fixed each c-hat at zero to account for the removal sampling design. All capture probability models employed the logit link function.

For the capture probability models of each species, we first used the design matrix in program MARK to parameterize a model that included the additive effects of "site", "age" and "date" (i.e., site + age + date, Lebreton et al. 1992, Peterson et al. 2004a, Hense et al., In Revision ). This model produced an estimate of p-hat and N-hat for each age class and site and was the most highly parameterized model. It therefore served as the "global model" and baseline reference for subsequent, reduced (i.e., fewer parameters) models (Burnham and Anderson 2002). The global model was intended to maximally account for inter-site variability in capture probability. The reduced models were constructed in an attempt to account for variability in p-hat using fewer parameters 
than the global model. The reduced models included all possible combinations of "site", "age", and "date" as well as a reduced age model, "age(2)", that treated age as a 2 level factor coding for YOY versus adults (i.e., as opposed to three levels with YOY, small and large adults). Additionally, a constant model, “.”, was also examined. The constant model was the simplest model (i.e., 1 parameter), which fit only a common intercept to all samples, thus, assuming capture probability to be constant across all age classes, sites, and sampling occasions. There were a total of 12 candidate models for each species.

\section{A priori expectations}

Population dynamics in the core versus periphery

This study was designed around a set of a priori expectations that we derived pursuant of the overriding research objective. These expectations were derived to address the four research goals. In order to address the first goal, which was to determine the extent to which local population dynamics differ for populations within the core of their distribution versus the periphery, we devised a set of expectations for the outcome of qualitative comparisons of brook trout and mottled sculpin population parameters (Table 1). Because we selected sites within the core of the brook trout distribution in this region, we expected temporal variability in their abundances to be lower over the duration of the experiment than those seen for mottled sculpin (Table 1). Also, because we expected brook trout population dynamics to be driven primarily by local processes, 
we expected them to exhibit higher survival than mottled sculpin (Table 1). Likewise, we expected survival and local recruitment to comprise a larger proportion of the total population growth rate compared mottled sculpin (Table 1). Finally, because all of our sites were located on the periphery of the mottled sculpin distribution in this region, we expected their populations to be driven largely by regional processes and, thus, we expected immigration to comprise a larger proportion of their overall local population growth rate compared to brook trout (Table 1).

Population dynamics and the regional context

In order to address the second goal of our study, which was to determine the extent to which local population dynamics differ given the regional context, we devised a set of expectations for the responses of both species to the experimental density manipulation (Table 2). We had expectations pertaining to how each species should respond to the removal given the whether they were near or far from core streams. Specifically, we expected that, if local conditions differ given the regional context, populations closer to their core would recover more completely and/ or more quickly from the density perturbation than populations further from the core (Table 2). In other words, for brook trout, we expected that they would recover more quickly in headwater removal sites compared to mainstem removal sites (Table 2). For mottled sculpin, since mainstems are within the core of their distribution, we expected the opposite (Table 2). Finally, we expected that the proportion of the population growth rate as immigration 
would be higher in removal sites closer to the species core than those farther away (Table 2).

Detecting density dependence

To address the third goal of the study, which was to examine the extent to which density dependence was evident in local population dynamics, we devised general expectations for how demographic rates for both species would respond to the removal (Table 3). Specifically, for both species, we expected survival and growth to be higher in the removal sites (Table 3). Additionally, we expected growth for both species to be higher in the removal sites relative to control sites (Table 3).

Density dependence in the core versus periphery

In order to address the fourth goal of our study, which was to evaluate the extent to which density dependence is detectable in the core versus periphery of a species range, we devised a list of a priori expectations regarding the qualitative differences in the response of brook trout versus mottled sculpin to the removal (Table 4). Specifically, we expected that, density dependence would be more likely to be detected or would be stronger in brook trout populations relative to mottled sculpin in this system because we expected their local dynamics to be driven primarily by local processes due to the fact that all of our sites were located within the core of their distribution (Table 4). Similarly, 
we expected that the effect of the removal on growth would be stronger for brook trout (Table 4).

\section{Modeling the population response variables}

Temporal variability in abundance

We analyzed temporal variation in the abundances of brook trout and mottled sculpin over the course of the study in relation to the experimental factors (treatment and network position). We used the coefficient of variation (C.V.) in abundance as a measure of temporal variability (Freeman et al. 1998, Grossman et al. 1990, Dauwalter et al. 2009). This metric scales population variation by the mean of abundance and is considered a superior metric for estimating population variability because it allows comparisons across populations with different abundances and because its values are less ambiguous compared to competing metrics (e.g., Kendall's W, Grossman et al. 1990). We subsequently constructed a full factorial analyses of variance (ANOVA) model to test for significant differences in the C.V. of abundance according to the experimental factors, species, and age classes. The model tested for significant differences in the C.V. of abundances estimates by species, age class, removal treatment and location.

The purpose of this analysis was to address the goal of determining the extent to which local population dynamics differ in the core versus the periphery (i.e., goal 1, Table 1). Our expectation was that, because our reaches were locally in the core of the brook trout distribution, variability in their abundances would be low compared to 
mottled sculpin estimates, which were from the periphery of their distribution. Again, this expectation follows from the reasoning that we expected regional processes to be more important in local sculpin population dynamics relative to brook trout, among all sites.

Although the ideal design for addressing this question would have included reaches representative of the core and periphery of both study species for comparison, we only had estimates from the core of brook trout to compare to the estimates from the periphery of mottled sculpin. Therefore, our expectation was based on the assumption that temporal variability in abundance would be of a similar magnitude for both species in their respective core or periphery habitats.

In addition, previous estimates of temporal variability in abundance from other systems suggested that variability in brook trout abundances (Dauwalter et al. 2009) may be higher, on average, than for mottled sculpin, which were exceptionally low compared to all sympatric species in a southern Appalachian system (Freeman et al. 1988, Grossman et al. 1990). Thus, we expected temporal variation in mottled sculpin abundances in this study to be higher than estimates reported for the larger southern Appalachian streams studied by Freeman et al. $\left(2^{\text {nd }}-3^{\text {rd }}\right.$ order, 1988), but possibly lower than estimates reported for brook trout in this study. On the other hand, we expected temporal variability in brook trout abundances to be similar to estimates for a range of systems reported by Dauwalter et al. (2009).

Finally, we included the age, removal, and location effects in these ANOVA tests as nuisance factors. 
Population recovery following removal

We modeled the recovery of abundances for individual age classes of each species over the course of the study as a function of the local habitat, age and sampling interval covariates and the experimental factors of interest. Specifically, we used the logtransformed proportion of total individuals at time $t$ versus the number pre-removal as the dependent variable. This measure was equivalent to one used in similar studies applying density perturbations (Peterson and Bayley 1993, Albanese et al. 2009); however, we normalized the response by applying a log-transformation. We refer to the dependent variable, henceforth, as proportional recovery or $\ln \left(\mathrm{R}_{\mathrm{p}}\right)$, which is calculated as the $\log$ transformed proportion of pre-removal abundance at time $t$.

Because we suspected that the site based estimates of $\ln \left(R_{p}\right)$ could be autocorrelated over the course of the study, we used a linear mixed effects modeling framework (LME) to model $\ln \left(\mathrm{R}_{\mathrm{p}}\right)$ in response to the independent variables of interest. LME models differ from traditional regression models in that they are able to account for potential sources of autocorrelation among samples within experimental units by including a random intercept for the experimental unit within which autocorrelation is suspected (Snijders and Bosker 1999). In the LME models for $\ln \left(\mathrm{R}_{\mathrm{p}}\right)$, the 12 study sites were our experimental units and, thus, "site" was modeled as a random factor while the independent variables, which included 7 local habitat covariates, a 5 level sampling interval factor, two experimental factors (i.e., removal treatment and watershed position) and select interactions, were all modeled as fixed factors. 
For each species and each age class, we constructed a candidate set of 13 biologically relevant models for predicting $\ln \left(\mathrm{R}_{\mathrm{p}}\right)$ (Table 1$)$. Each of the 13 models in the set contained all of the local habitat covariates and the sample interval factor (Table 1). Therefore, the model including only those effects was the most reduced model in the set. The local habitat covariates included were a single water chemistry principal component 1 (W1), three local physical habitat principal components (H1, H2, and H3), an index of local stream flow variability (PAREA), a measure of local canopy cover (CANP), and an index of local thermal conditions (MWAT, see Results section for details). The sampling interval factor included all 5 intervals following the removal. The global model for each set consisted of an additive removal effect, an additive watershed location effect, the interaction between removal and location, a removal-sampling interval interactive effect, a location-sampling interval effect, and a three way interaction among removal, location, and interval (Table 1). The global model was the most complex in the set and was intended to account for all of the possible experimental effects of interest and interactions. We included interactions of the experimental effects with sampling interval in order to detect temporal trends in the experimental effects as well as to maximize our ability to detect effects. For example, a short-lived experimental effect could be overwhelmed by temporal variance or the effect could alternate between positive and negative over the course of the study.

The purpose of this analysis was to inform the extent to which local population dynamics differ given the regional context (i.e., goal 2, Table 2). For brook trout, the expectation for adults (i.e., large and small) was that recovery of abundance would be more rapid and/or complete in headwater sites due to those sites being in closer proximity 
to other core streams and a source of colonizers. On the other hand, we did not expect any location effect on recovery of YOY brook trout as we expected their dynamics to be driven primarily by local recruitment (Petty et al. 2005).

For adult sculpin, we expected recovery of abundance to be more rapid and/or complete in mainstem sites due to those sites being in closer proximity to the core sculpin distribution. Similar to brook trout, we did not expect any location effect on the recovery of YOY sculpin since we expected their dynamics to be driven primarily by local recruitment.

Population growth rate

We modeled the response of the population growth for individual age classes of each species over the course of the study as a function of the local habitat, age and sampling interval covariates and the experimental factors of interest. Specifically, we used the per capita population growth rate (i.e., $r=\ln (\lambda)=\ln \left(\mathrm{N}_{\mathrm{t}+1} / \mathrm{N}_{\mathrm{t}}\right)$ ) as the dependent variable of interest (Grossman et al. 2006, Grossman et al. 2010). For each species and each age class, we again constructed a candidate set of 13 biologically relevant models for predicting $r$ (Table 1). As for the analysis for $\ln \left(\mathrm{R}_{\mathrm{p}}\right)$, each of the 13 models in the set contained all of the local habitat covariates and the sample interval factor (Table 5).

Therefore, the model including only those effects was the most reduced model in the set. The local habitat covariates included were a single water chemistry principal component 1 (W1), three local physical habitat principal components (H1, H2, and H3), an index of local stream flow variability (PAREA), a measure of local canopy cover (CANP), and an 
index of local thermal conditions (MWAT, see Results section for details). The sampling interval factor included all 5 intervals following the removal. For the first interval, $r$ was estimated for removal sites using abundance estimates for $\mathrm{N}_{1}$ that were immediately postremoval (i.e., $r=\ln (\lambda)=\ln \left(\mathrm{N}_{2} / \mathrm{N}_{1=\text { post-removal }}\right)$. The global model for each set consisted of an additive removal effect, an additive watershed location effect, the interaction between removal and location, a removal-sampling interval interactive effect, a location-sampling interval effect, and a three way interaction among removal, location, and interval (Table 1). The global model was the most complex in the set and was intended to account for all of the possible experimental effects of interest and interactions. We included interactions of the experimental effects with sampling interval in order to detect temporal trends in the experimental effects as well as to maximize our ability to detect effects.

The purpose for this analysis was to address the extent to which local population dynamics differ given the regional context (i.e., goal 2, Table 2). Our expectation for adult brook trout was that, following removal, $r$ would be higher in headwater removal sites than in mainstem removal sites, due to proximity to the core. Likewise, we expected no removal effect on YOY brook trout because we expected their dynamics to be driven largely by local recruitment as opposed to immigration.

Conversely, for mottled sculpin, our expectation was that, following removal, $r$ would be higher in mainstem removal sites than headwater removal sites. We also expected no removal effect on YOY sculpin.

Apparent survival and probability of age class transition 
We used the robust design approach (Pollack et al. 1978) to estimate survival and age class transition probabilities over the course of the study for brook trout and mottled sculpin. The approach assumes that populations are closed to gains (i.e., births and immigration) during each primary sampling occasion and open to gains between primary sampling occasions. Therefore, we used the Huggins closed population model estimates for age, species, and season-specific capture efficiencies to fix the appropriate capture probability parameters in open models used in estimating survival and transition (Petty et al. 2005).

We used the Amason Schwarz multistate model (Amason 1973, Schwarz et al. 1993) as implemented in Program MARK (White and Burnham 1999) to estimate apparent survival $(\varphi)$ and age-class transition probabilities $(\psi)$ for brook trout and mottled sculpin in each of the 12 sites. The Amason Schwarz multistate model is an extension of the Cormack Jolly Seber single-state models used to model survival and capture probabilities that permits estimation of stochastic transitions among states, which in our case was age class of mottled sculpin or brook trout (Williams et al. 2002, Schwarz et al. 2005). Like the single-state model, the multistate model uses maximum likelihood methods to estimate survival and recapture probabilities, but survival probabilities incorporate the probabilities of transitioning among states (i.e., age classes), with capture probabilities that are age-specific. Survival estimates are estimates of apparent survival and combine losses from mortality, permanent emigration, and complete tag loss. Survival and transition probabilities are separately estimated under the assumption that survival from $t$ to $t+1$ does not depend on age class at $t+1$. Estimates of $\psi$, therefore, 
quantify the probability of transitioning between age classes upon the condition of surviving the interval.

We parameterized 65 candidate models for estimating $\varphi$ and $\psi$ for brook trout and mottled sculpin. The linear model for estimating these parameters contains two intercept terms, one each for $\varphi$ and $\psi$. The portion of the global model for estimating S included all of the terms included in Table 1 in addition to an age effect, which coded for the three separate age classes for each species, and the interaction of the age term with the removal and location effects. The portion of the global model for estimating $\psi$, however, included only an additive age term indicating transition probability from the YOY or adult age class and the additive removal and location terms and their interaction (i.e., $\psi=$ age + removal + location + [removal $\mathrm{x}$ location $])$. We were unable to include all possible terms in the portion of the global model estimating $\psi$ because recapture data was sparse among some sites, preventing proper estimation of all parameters.

The purpose of this analysis was threefold. First, we conducted this analysis to qualitatively inform the goal to determine the extent to which local dynamics differ in the core and periphery (i.e., goal 1, Table 1). Because all sites were on the periphery of the sculpin distribution, we expected $\varphi$ to generally be lower for sculpin than for brook trout across the sites.

Second, we conducted the analysis to address the goal of determining the extent to which density dependent processes drove local population dynamics (i.e., goal 3, Tables 3 and 4). For brook trout, our expectation was that, due to density dependent feedbacks resulting from the experimental perturbation, $\varphi$ would be higher in removal streams relative to the control streams. Additionally, we expected that, as a result of density 
feedbacks on growth, both YOY and small adult brook trout would have a higher probability of transitioning to older age classes in the removal sites. For mottled sculpin, we also expected that $\varphi$ and $\psi$ would be higher in the removal sites compared to controls, as a result of density dependent feedbacks.

Third, we conducted this analysis to inform the goal of determining the extent to which the influence of density dependent processes varied given the local context (i.e., core or periphery; goal 4, Table 4). Although we did not directly test this hypothesis using AIC, we expected effect of the removal on brook trout $\varphi$ and $\psi$ to be larger than for mottled sculpin because all sites were on within the core of their distribution.

\section{Components of population growth}

We used the reverse-time capture-recapture methods (Pollack 1974, Nichols et al. 1986, Pradel 1996) and the methods outlined by Nichols et al. (2000) to directly estimate the contributions of survival $\left(\gamma_{\mathrm{ii}}\right)$, local recruitment $\left(\gamma_{\mathrm{ji}}\right)$, and immigration $\left(1-\gamma_{\mathrm{ji}}-\gamma_{\mathrm{ii}}\right)$ to the instantaneous population growth rates $(\lambda)$ of individual age classes. While survival rate estimation under capture-recapture models in forward time proceeds by conditioning on animal releases in earlier time periods and following their fate in later time periods, the reverse time approach proceeds under the realization that conditioning on animals caught later in time periods and observing their capture histories in earlier occasions lends inference to the recruitment process (Pollock et al. 1974). Specifically, Pollock et al. (1974) noted, "a backward process with recruitment and no mortality is statistically equivalent to a forward process with mortality and no recruitment". 
Therefore, while the forward-time approach results in the estimation of $\varphi$, the reverse-time analysis results in the estimation of $\gamma$, or the probability that an animal present in the population at time $t$ was also present in the population at $\mathrm{t}-1$. In the multistate reverse time model both $\gamma_{\mathrm{ii}}$ and $\gamma_{\mathrm{ji}}$ are estimated by analyzing capture histories in reverse time. The parameter $\gamma_{\mathrm{ii}}$ represents the probability that an animal in state $i$ and in the population at time $t$ was also in state $i$ and in the population at $t-1$ (Nichols et al. 2000). This is effectively the probability that, given that an animal survived the interval, it was in the population and in the same state as the previous interval. In our case, the state was age class.

The multistate reverse-time model also provides an estimate of $\gamma_{\mathrm{ji}}$, or the probability that, given that an animal survived the interval, it was present and in the population and in a younger age class in the previous interval. Additionally, each of these parameters represents a proportion of the instantaneous growth rate $(\lambda$, Nichols et al. 2000). Specifically, $\gamma_{\mathrm{ii}}$ is an estimate of the proportion of $\lambda$ attributable to survival from the current state. In our case, this was an estimate of the proportion of $\lambda$ for an age class attributable to survival within that age class from the previous interval. We produced estimates for $\gamma_{\mathrm{ii}}$ for three age classes for each species in our study. Likewise, $\gamma_{\mathrm{ji}}$ is an estimate of the proportion of $\lambda$ due to local recruitment or, rather, survivors from the lower age class that transitioned into the focal age class. Finally, $\left[1-\gamma_{\mathrm{ji}}-\gamma_{\mathrm{ii}}\right]$ is, by default, an estimate of the proportion of $\lambda$ due to immigration. We estimated contributions due to $\gamma_{\mathrm{ji}}$ for both the small and large adult segments of the trout and sculpin populations; however, we did not differentiate among local recruitment and immigration for YOY populations. We parameterized 65 candidate models for 
estimating $\gamma_{\mathrm{ii}}$ and $\gamma_{\mathrm{ji}}$ for both brook trout and mottled sculpin equivalent to those used in estimating $\varphi$ and $\psi$.

For brook trout, our a priori expectation was that, because we expected these streams to represent the core of their distribution and likely act as functional sources (Pulliam 1988), population growth in control streams would be driven largely by local survival and recruitment as opposed to being dependent on immigration for the region for sustainability (i.e., goal 1, Table 1). For mottled sculpin, we expected the opposite because we expected these streams to represent the periphery of their distribution and to act as functional sinks. As such, we expected mottled sculpin population maintenance to be largely dependent on immigration from the region.

We also expected the local dynamics of both mottled sculpin and brook trout to be modified by the regional context (i.e., goal 2, Table 2). Specifically, we expected that immigration rates would be higher for sculpin when adjacent to mainstems (i.e., sources) than when adjacent to headwater segments (i.e., sinks). Conversely, we expected that immigration rates would be higher for brook trout when adjacent to headwater segments (i.e., sources) as opposed to mainstem segments (i.e., sinks). We expected the removal to illuminate these dynamics, with immigration rates following the removal being stronger in removal streams adjacent to mainstems for mottled sculpin and in removal streams adjacent to headwater segments for brook trout.

Finally, we also expected to observe an increase in survival rate among removal sites for brook trout as a resulting from density dependent feedbacks. We also expected to see a density dependent effect on survival for sculpin, but to a lesser extent than for brook trout, given the local context of these small streams relative to their core. 


\section{Results}

\section{Local physicochemical conditions}

The results for the principal components analysis of local chemical conditions indicated that variation in local water chemistry among sites was generally low as is apparent both from the summary of individual variables measured at each site (Table 6) and by the finding that principal component 1 explained $74 \%$ of total variation in the dataset (Table 7). PC1 was, thus, the only component interpreted (Table 7). Specifically, the factor loadings for this component indicated a gradient of decreasing hardness and alkalinity with increasing PC1 values (Table 7). While average local chemical conditions varied little among sites, one site, Nan's Branch, a mainstem control site, was considerably more alkaline than the other sites (Table 7). This site had measurements for specific conductivity, total hardness, calcium hardness and alkalinity all several orders higher than the mean of other sites (Table 7). The disparity between the local conditions of this site and the others is also reflected in the principal components analysis of the water chemistry data as the mean PC1 score for the Nan's Branch was -6.102 with the next lowest score marginally less than zero (Lynn Run, -0.095).

Variability in local physical conditions as described by nine habitat measurements (Table 8) was summarized in three dimensions via a principal components analysis of the dataset (Table 9). The three components captured $88 \%$ of the total variation in the 
dataset. Principal component 1 (PC1) explained $58 \%$ of the variance in the dataset followed by PC2 (17\%) and PC3 (13\%). PC1 is best described as a gradient of channel complexity. Low scores on this gradient indicated greater channel complexity as characterized by increased variability in thalweg depth (CV_DEPTH) and distance to expected fish cover features (CV_DCOV; Table 9). PC2 is best described as a velocitydepth gradient, with sites scoring lower on this gradient having a greater proportion of pool area compared to riffle area and sites scoring higher having a greater average current velocity and more large woody debris (Table 9). PC3 can be described as a depth gradient with sites scoring higher on this principal component axis having both greater depth, on average, and a greater proportion of pool area (Table 9).

\section{Capture efficiency estimates}

Of the 12 candidate models for estimating capture efficiency (p-hat) for brook trout, three models comprised the $95 \%$ confidence set based on the Akaike weights (cumulative $w_{\mathrm{i}}>=0.95$, Table 11). These models included an age effect indicating that $\mathrm{p}$ hat differed among YOY and adult brook trout (i.e., effect term: "age(2)"), an age effect indicating that p-hat differed among YOY, small and large adults (i.e., "age"), a site effect indicating that p-hat differed among sites (i.e., "Site"), and a sample season effect indicating that $\mathrm{p}$-hat differed among sample dates (i.e., "date", Table 11). Summing the Akaike weights across the top models for individual model terms indicated that, of the top models, sample season had the most influence on p-hat $\left(\sum w_{\mathrm{i}}=1.0\right)$, followed closely by the site effect $\left(\sum w_{\mathrm{i}}=0.957\right.$, Table 11). Of the two age effects, the effect indicating 
that p-hat differed only among YOY and adults had the most support ( $\Sigma w_{\mathrm{i}}=0.447$ vs. $\Sigma w_{\mathrm{i}}$ $=0.27$, Table 11). Appendix A2 lists model-averaged estimates for $\mathrm{p}$-hat for all sites, age classes, and sample occasions for brook trout. The mean p-hat for brook trout was 0.688 and the maximum p-hat was 0.896 , while the minimum was 0.340 .

Of the 12 candidate models for estimating capture efficiency (p-hat) for mottled sculpin, 2 models comprised the $95 \%$ confidence set based on the Akaike weights (cumulative $w_{\mathrm{i}}>=0.95$, Table 12). Similar to the brook trout models, the top 2 models for sculpin included an age effect indicating that $\mathrm{p}$-hat differed among YOY and adult brook trout, an age effect indicating that $\mathrm{p}$-hat differed among YOY, small and large adults, a site effect indicating that $\mathrm{p}$-hat differed among sites, and a sample season effect indicating that $\mathrm{p}$-hat differed among sample dates (i.e., "date", Table 8). Summing the Akaike weights across the top models for individual model terms indicated that, again, of the top models, sample season had the most influence on p-hat $\left(\Sigma w_{\mathrm{i}}=1.0\right)$, which was tied by the site effect $\left(\Sigma w_{\mathrm{i}}=1.0\right.$, Table 12$)$. Of the two age effects, the effect indicating that $\mathrm{p}$-hat differed only among YOY and adults had the most support ( $\Sigma w_{\mathrm{i}}=0.673$ vs. $\Sigma w_{\mathrm{i}}$ $=0.303$, Table 12). Appendix A4 lists model-averaged estimates for $\mathrm{p}$-hat for all sites, age classes, and sample occasions for mottled sculpin. The mean p-hat for mottled sculpin was 0.525 and the maximum p-hat was 0.733 , while the minimum was 0.265 .

\section{Abundance}

The model-averaged estimates, standard errors and confidence intervals for the abundances derived from the Huggins' closed capture models are provided for all sites 
and sampling occasions and for both species can be found in the supplementary material for this chapter (Appendices A5 and A6, respectively).

Brook trout abundance and variance

Generally, brook trout abundance (N-hat) was variable within and among sites across all sampling occasions and age classes (Figure 2). Likewise, temporal variability in brook trout abundance within sites was relatively high for all sites and age classes as measured by the mean coefficient of variation (C.V.) in N-hat (mean $=0.662$, s.d. $=$ $0.235, \mathrm{n}=12$ ), which indicated that brook trout abundances across sites could be classified as "moderately fluctuating" according to the criteria of Freeman et al. (1988, $0.5<\mathrm{C} . \mathrm{V} .<=0.75)$. However, a detailed look at the range of C.V. among sites indicated some potentially important population characteristics. For instance, judging from the criteria of Freeman et al. (1988) for classifying variability in abundance based on C.V. there was some notable variation among age classes. First, none of the sites classified as "stable", according to these criteria, for any age class of brook trout (C.V. $<0.25$, Figure 3). Second, large adult brook trout demonstrated greater temporal stability in abundance with half of the sites classifying as having "moderately stable" large adult populations over the course of the study $(0.25<$ C.V. $<=0.5$, Figure 3$)$. Third, small adult populations appeared to be the least stable, with 8 of the 12 sites classifying as "fluctuating" and only one site classifying as "moderately stable" (Figure 3). Finally, temporal variability in YOY abundance appeared comparable to that of large adults, although there were slightly fewer sites classifying as "moderately stable" ( $n=4$ vs. n=6). 
Mottled sculpin abundance and variance

Mottled sculpin population size was also generally variable across sites and sample dates (Figure 4). Like brook trout, temporal variability in abundance within sites was relatively high as was indicated by the mean C.V. in mottled sculpin N-hat for all age classes $($ Figure 5, mean $=0.835$, s.d. $=0.421)$. Judging from the criteria of Freeman et al. (1988), mottled sculpin populations were generally classified as "fluctuating" among these sites for the duration of the study when averaged across all age classes (C.V.> $0.75)$.

A thorough look at the individual values for C.V. in N-hat among age classes of sculpin also highlighted differences in the degree of temporal stability in abundance (Figure 5). First, temporal variability in YOY abundance was classified as "fluctuating" among all sites (Freeman et al. 1988, Figure 5). Second, while small and large adult population stability appeared more similar, large adult abundance was generally more stable over time, with more sites classifying as "stable" $(n=1)$ to "moderately stable" $(n=4)$ than small adults $(n=2$, "moderately stable", Figure 5).

Comparison of abundance variability between species

Comparing temporal variability in the abundances of brook trout to mottled sculpin revealed a significant difference in temporal variability in N-hat between the two 
species (Table 13). As indicated by the full factorial ANOVA model coefficients, temporal variability in population size was lower on average for brook trout (Table 14). However, this difference was driven primarily by variability in the YOY age class as the model coefficients indicated that variability in brook trout N-hat was actually significantly higher for both small and large adult brook trout than for those age classes of sculpin (Table 14).

Population recovery following removal treatment

Pre-and post-removal abundance estimates

For the 6 sites receiving the removal treatment in July 2006, the number of individuals remaining following the removal was estimated based on the following formula:

[1] $\mathrm{N}_{\text {post-removal }}=\mathrm{N}$-hat - number of individuals collected.

For the sites where this value was less than 1, but greater than 0 given the standard error, we estimated $\mathrm{N}_{\text {post-removal }}$ to be 1 .

The number of individuals remaining after the initial removal treatment was near zero for both species and all age classes in most sites. For brook trout, only in Lick Drain was this estimate greater than $1(\mathrm{YOY}=1.165)$. For mottled sculpin, Otis Hollow (small 
adults $=1.013)$, Lick Drain $($ small adults $=2.733$, large adults $=3.315)$, swallow rock run $($ large adults $=1.08)$, Tanner Run $($ small adults $=1.496$, large adults $=1.451)$ all had abundances for some age classes greater than zero following the removal, although the numbers were still very low. The estimated abundances of brook trout and mottled sculpin across all sites pre- and immediately post-removal are shown in figures 6 and 7 , respectively.

Recovery of brook trout abundance

Generally, recovery of brook trout abundances to pre-removal estimates was highly variable among sites (Figures 8 and 9). The linear mixed ANCOVA models for predicting the log-transformed proportional recovery of YOY brook trout indicated that the fish removal treatment $\left(\Sigma w_{i}=0.511\right)$, spatial location $\left(\Sigma w_{i}=0.814\right)$, location-sample date interaction $\left(\Sigma w_{i}=0.296\right)$ and removal-location interaction $\left(\Sigma w_{i}=0.252\right)$ effects each had some support in the $95 \%$ confidence set of models (Table 15). The top ranking model included only the additive location effect in addition to the local habitat covariates and sampling date effects (Table 15). However, a closely competing model $\left(w_{1} / w_{i}<2.0\right)$ included the removal effect, the location effect, and their interaction (Table 13). This model fit the data well, as the proportion of variance explained in the measured values of $\ln \left(\mathrm{R}_{\mathrm{p}}\right)$ by the global model was $79 \%$ (Table 15$)$.

A plot of the model averaged estimates of $\ln \left(R_{p}\right)$ for YOY brook trout among the four experimental groups of sites over the course of the study illustrated the strength of the removal effect, as $\ln \left(R_{p}\right)$ for control groups was consistently higher than 0 for all 
sampling dates following the initial removal, while abundance among the removal groups did not recover to pre-removal levels (i.e., $\ln \left(R_{p}\right) \geq 0$ ) until the $3^{\text {rd }}$ sampling occasion following the removal, which was May of the following year (Figure 10). The location effect on $\ln \left(\mathrm{R}_{\mathrm{p}}\right)$ and its interaction with sampling date was also apparent. While $\ln \left(\mathrm{R}_{\mathrm{p}}\right)$ among headwater sites was higher, on average, than control sites at the first date postremoval, estimates were nearly identical in October 2006, but were higher among headwater sites at all sampling occasions the following year (May - October 2007, Figure 10). Interestingly, treatment sites remained lower, on average, over the entire year of 2007 (Figure 10). Finally, although the removal-location effect had some support in the set of models used for inference, the effect was not readily apparent in the plot of the model averaged estimates (Figure 10).

The comparison of models relating small adult brook trout $\ln \left(\mathrm{R}_{\mathrm{p}}\right)$ to the experimental factors revealed some support for the location $\left(\Sigma w_{i}=0.580\right)$, removal $\left(\sum w_{i}\right.$ $=0.312)$, and location-date $\left(\sum w_{i}=0.032\right)$ effects in the $95 \%$ confidence set of models (Table 13). As with YOY brook trout, the top ranking model in the set included only the additive location effect (Table 15). However, a model including only the additive removal effect was closely competing $\left(w_{l} / w_{i}<2.0\right.$, Table 15$)$. The model including the location-date interaction was relatively poorly supported $\left(w_{1} / w_{i} \approx 18.0\right.$, Table 15$)$. The model fit the data well, with the global model explaining $72 \%$ of the variance in the measured values of $\ln \left(R_{p}\right)$ (Table 15).

A plot of the model averaged estimates of $\ln \left(\mathrm{R}_{\mathrm{p}}\right)$ for small adult brook trout among the four experimental types of sites over the course of the study illustrated that recovery of pre-removal abundances occurred by the first sampling date following the 
removal (Figure 11), as $\ln \left(\mathrm{R}_{\mathrm{p}}\right)$ was greater than 0 , on average, for all experimental site types at that date (Figure 11). This plot also illustrates both the additive location effect and its interaction with sampling date on expected $\ln \left(\mathrm{R}_{\mathrm{p}}\right)$ as mainstem sites appeared to have substantially higher values for $\ln \left(R_{p}\right)$, on average, than headwater sites at all sampling occasions between August 2006 and October 2007 (Figure 11). The effect appeared strongest in October 2006 (Figure 11). The removal effect was also visible, although, $\ln \left(\mathrm{R}_{\mathrm{p}}\right)$ was actually higher among removal groups than among control groups (Figure 11), which is opposite the relationship observed for YOY (Figure 10).

The comparison of models relating large adult brook trout $\ln \left(\mathrm{R}_{\mathrm{p}}\right)$ to the experimental factors revealed some support for the removal $\left(\Sigma w_{i}=0.580\right)$ and location effects $\left(\Sigma w_{i}=0 . .798\right)$ as well as their interaction $\left(\sum w_{i}=0.500\right.$, Table 15). The top ranking model included all three experimental effects in combination (Table 15). However, a model including only the additive location effect was closely competing $\left(w_{1} / w_{i}<2.0\right.$, Table 15). Likewise, as in the model comparison results for YOY and small adults, there was also some support for a model including no experimental factors, but the top ranking model was nearly 4 times as likely $\left(w_{1} / w_{i} \approx 3.9\right.$, Table 15$)$. A model including only the additive effect of removal was also in the $95 \%$ confidence set of models, although it was weakly supported $\left(w_{1} / w_{i} \approx 6.7\right.$, Table 15$)$. This model also fit the data well, with the global model explaining $84 \%$ of the variance in the measured values of $\ln \left(R_{p}\right)($ Table 15).

A plot of the model averaged estimates of $\ln \left(R_{p}\right)$ for large adult brook trout among the four experimental types of sites over the course of the study suggested that complete recovery of pre-removal large adult abundances was never reached among 
either of the headwater group of sites nor the mainstem sites that received the removal treatment $\left(\ln \left(\mathrm{R}_{\mathrm{p}}\right)<0\right.$, Figure 12). Although the covariate effect of sampling date was not evaluated in the model comparison, the temporal trend resulting from the estimates suggested that abundances of large adults remained relatively consistent over the course of the study following the removal (Figure 12). The effect of the removal was also apparent in the estimates, as both headwater and mainstem removal sites had lower expected values of $\ln \left(R_{p}\right)$ than control sites (Figure 12). Interestingly, the removal-date interaction also appeared strong, as the effect size of removal on the mainstem site $\ln \left(R_{p}\right)$ appeared larger than for headwater sites (Figure 12). The removal-location interactive effect was also apparent in the model estimates as the removal effect on mainstem sites appeared to result in a greater difference $\ln \left(R_{p}\right)$ than was apparent from the removal effect among the headwater sites (Figure 12).

Recovery of mottled sculpin abundance

Concerning $\ln \left(\mathrm{R}_{\mathrm{p}}\right)$ of YOY mottled sculpin, there was some support for the removal $\left(\sum w_{i}=0.310\right)$, location $\left(\sum w_{i}=0.402\right)$, and removal-location interaction $\left(\Sigma w_{i}=\right.$ 0.074) effects (Table 16). However, the top model included no experimental effects and was more than 2 times as likely as the next best model $\left(w_{1} / w_{i} \approx 2.0\right.$, Table 16$)$, while, a model including only the additive removal effect and a model including only the additive location effect were closely competing with one another $\left(w_{i} / w_{j} \approx 1.0\right.$, Table 16$)$. This model fit the data well, as the global model explained $73 \%$ of the variation in the measured values of $\ln \left(R_{p}\right)$ (Table 16). 
A plot of the model averaged $\ln \left(\mathrm{R}_{\mathrm{p}}\right)$ for YOY mottled sculpin among the experimental groups illustrated a removal-location effect, such that $\ln \left(R_{P}\right)$ of YOY sculpin appeared to be lower in the headwater removal sites than headwater control sites among all post-removal dates, yet did not appear to differ between removal and control mainstem sites at any date post-removal (Figure 13$)$. In general, $\ln \left(\mathrm{R}_{\mathrm{p}}\right)$ fluctuated widely (Figure 13).

The top model for estimating small adult mottled sculpin $\ln \left(\mathrm{R}_{\mathrm{p}}\right)$ included a removal effect $\left(\Sigma w_{i}=0.608\right)$, a location effect $\left(\Sigma w_{i}=0.745\right)$, and their interaction (removal x location, $\Sigma w_{i}=0.528$, Table 16). This top model was 2.5 times more likely than the next best competing model based on the proportion of Akaike weights for those two models (Table 16). The next most likely model included only the additive location effect (Table 16). Also, the model including only the covariate effects and no experimental effects had some support in the $95 \%$ confidence set as did a model including only the additive removal effect (Table 16). Nonetheless, the top model was nearly 3 times more likely, given the data, than the model including no experimental effects $\left(w_{1} / w_{i} \approx 2.9\right.$, Table 16$)$. The model including only the additive removal effect received little support $\left(w_{1} / w_{i} \approx 7.0\right.$, Table 16$)$. The global model fit the data very well, explaining $85 \%$ of the variation in the measured values of $\ln \left(R_{p}\right)($ Table 16$)$.

Figure 14 illustrated the estimated mean value of $\ln \left(R_{p}\right)$ among the four experimental groups of sites and illustrates an interesting interaction between the removal treatment and site spatial location. Abundances of small adult mottled sculpin never recovered to pre-removal levels among the headwater sites receiving the removal treatment and also never approached the magnitude of $\ln \left(\mathrm{R}_{\mathrm{p}}\right)$ seen in the headwater 
control sites (Figure 16). Also, a result of the removal-location interaction, the effect size of removal on $\ln \left(R_{p}\right)$ was modeled as greater among the headwater removal sites than in mainstem removal sites (Figure 16). Interestingly, the removal effect on $\ln \left(\mathrm{R}_{\mathrm{p}}\right)$ was actually modeled as positive, on average, in mainstem removal sites, which was opposite the trend among headwater removal sites (Figure 10). Thus, the figure suggests that small adults in mainstem removal sites recovered their abundances both to a greater magnitude and more quickly than in headwater removal sites (Figure 14).

The top models for predicting large adult mottled sculpin $\ln \left(\mathrm{R}_{\mathrm{p}}\right)$ also indicated some support for removal $\left(\sum w_{i}=0.396\right)$, location $\left(\sum w_{i}=0.736\right)$, and removal-location interaction $\left(\Sigma w_{i}=0.325\right)$ experimental effects (Table 16). The highest ranking model included the additive location effect, but that model was very closely competing with the second ranked model that included all three of the supported experimental effects $\left(w_{l} / w_{i}\right.$ $\approx 1.3$, Table 16). The model with no experimental effects also was supported by the data in the $95 \%$ confidence set. The top ranked model was more than 2 times more likely to be the best model compared to the model with no experimental effects $\left(w_{1} / w_{i} \approx 2.1\right.$, Table 14). There was also some support for a model with only the additive removal effect $\left(w_{l} / w_{i} \approx 5.8\right.$, Table 16$)$. The global model, again, fit the data very well, explaining $85 \%$ of the variation in the measured values of $\ln \left(\mathrm{R}_{\mathrm{p}}\right)($ Table 16$)$.

Similar to the estimates modeled for small adult mottled sculpin, the figure illustrating modeled $\ln \left(\mathrm{R}_{\mathrm{p}}\right)$ for large adult mottled sculpin characterized the apparent influence of the removal treatment, spatial location, and their interactive experimental effect on the proportional recovery of abundances over the course of the study postremoval (Figure 15). As was the case with small adults, large adult sculpin abundance in 
headwater removal sites never fully recovered to pre-removal levels and $\ln \left(\mathrm{R}_{\mathrm{p}}\right)$ remained consistently lower in those sites, on average, than in the headwater control sites for all sampling dates post-removal (Figure 15). Also, headwater removal and control sites each had mean $\ln \left(R_{p}\right)$ values lower than those in the removal and control mainstem sites for all dates following removal (Figure 15), illustrating the strong additive effect of spatial location on $\ln \left(\mathrm{R}_{\mathrm{p}}\right)$ (Table 16). Interestingly, as was the case with small adult sculpin, the effect of removal in mainstem sites was the opposite as that in headwater sites, as $\ln \left(R_{p}\right)$ was higher among the removal sites than in the control sites for all dates post-removal (Figure 15).

Response of population growth rate

Brook trout

The $95 \%$ confidence set of models for estimating the per capita population growth rate $(r)$ for YOY brook trout suggested some support for the additive experimental effects of removal treatment $\left(\Sigma w_{i}=0.159\right)$ and spatial location $\left(\Sigma w_{i}=\right.$ 0.176) (Table 17). However, the basic local covariate model with a time interval effect was the highest ranking model and was more than 4 times as likely to be the best model than the next highest ranking model in the set, which included only the additive effect of location in addition to the basic local habitat and sample date covariates $\left(w_{1} / w_{i} \approx 4.4\right.$, Table 17). The second ranked model was closely competing with the next best model, 
which included only the additive removal effect $\left(w_{1} / w_{i} \approx 1.1\right.$, Table 17). Finally, the global model exhibited a fair fit to the data, as the proportion of the variance explained in the measured values of $r$ was $40 \%$ (Table 17).

A plot of expected $r$ for YOY brook trout among the experimental groups of sites over all sampling date intervals indicated that the additive effects of spatial location, while weakly supported in the model comparison, were not particularly interpretable in the plot (Figure 16). YOY $r$ appeared to alternate between periods of near doubling per capita growth (i.e., $r$ approaching 1.0) and periods of low to zero growth over the course of the study (Table 17, Figure 16).

The $95 \%$ confidence set of models for estimating $r$ for small adult brook trout indicated support for a removal effect $\left(\Sigma w_{i}=0.629\right)$, a location effect $\left(\Sigma w_{i}=0.155\right)$, and a removal-sampling interval interactive effect $\left(\sum w_{i}=0.541\right.$, Table 17). In fact, the top ranked model in the set included terms for all three of those experimental effects (Table 17). However, the standard covariate model was also closely competing with the top model $\left(w_{1} / w_{i}<2\right.$, Table 17). Although three other models were included in the $95 \%$ confidence set, including one with only the additive removal effect, one with the removal effect, location effect, and removal-interval interaction, and on with only the additive location effect, those models were relatively weakly supported by the data compared to the top two $\left(w_{i} / w_{j}>5\right.$, Table 17$)$. Finally, the global model provided a slightly better overall fit to the data compared to the global model for YOY brook trout, accounting for $54 \%$ of the variance in measured values of $r$ (Table 17 ).

A plot of the estimated values of $r$ among the experimental groups of sites indicated that the per capita growth rate was much higher and near doubling over the first 
interval following removal among the sites receiving the removal treatment (Figure 17). Over the second interval, however, $r$ was more similar among the removal and control sites (Figure 17). Over the last three intervals, $r$ was substantially lower and negative in the removal sites compared to the control sites, which also exhibited a negative $r$ over the two intervals spanning October 2006 to July 2007 (Figure 17). The rate of increase in control sites returned to a near doubling rate, however, over the final interval spanning July to October 2007 (Figure 17). Finally, there was a relatively small, but visible and additive effect of spatial location on $r$, with headwater streams having slightly higher rates of per capita increase compared to mainstem sites over all of the intervals (Figure 17).

Similar to small adult brook trout, the $95 \%$ confidence set of models for estimating large adult brook trout $r$ indicated some support for a removal $\left(\sum w_{i}=0.634\right)$, removal-interval interaction $\left(\sum w_{i}=0.526\right)$ and location $\left(\sum w_{i}=0.163\right)$ effect on $r$ (Table 15). The top ranked model included the additive removal effect and the removal-interval interactive effect (Table 17). The next best model, however, was the standard covariate model with no experimental effects and that model was closely competing with the top model $\left(w_{l} / w_{i}<2\right.$, Table 17). The third best model included only the additive removal term and the top model was more than 4 times as likely to be the best model compared to this model $\left(w_{1} / w_{i} \approx 4.3\right)$, while the next lowest and last model in the $95 \%$ confidence set was even less likely to be the best in the $\operatorname{set}\left(w_{l} / w_{i} \approx 5.8\right)$ and was the only model supporting the location effect (Table 17). Finally, the global model provided a moderate fit to the data, explaining approximately $63 \%$ of the variation in the measured values of $r$ among all sites and intervals (Table 17). 
A plot of the modeled $r$ across all intervals for large adult brook trout in the four experimental groups of sites indicated that, among the control sites, $r$ remained relatively consistent and positive over the first three intervals, but fell to negative over the fourth and then returned to a positive rate similar to the first three intervals over the fifth interval (Figure 18). Among the removal sites, however, $r$ was positive and much higher compared to the control groups of sites over the first interval post-removal, yet $r$ became substantially negative and much lower than in those sites over the second interval (Figure 18). Over the third interval spanning October 2006 to May 2007, $r$ among removal and control sites were indistinguishable (Figure 18). However, $r$ among the removal sites and control sites fell to a negative value again over the fourth interval (Figure 18). Over that interval spanning May to July 2007, $r$ for the mainstem removal group was discernibly lower than in the mainstem control group, but similar to $r$ in the headwater control group (Figure 18). Also, $r$ among the headwater removal group was discernibly lower than each of the three other experimental groups at that interval (Figure 18). The general differences among the four experimental group types remained similar over the final interval, however, $r$ appeared to increase a similar amount for all sites and returned to slightly to moderately positive among both control groups (Figure 18).

Mottled sculpin

The $95 \%$ confidence set of models for estimating $r$ for YOY mottled sculpin suggested a small amount of support for the additive effects of removal treatment $\left(\Sigma w_{i}=\right.$ $0.170)$ and spatial location $\left(\Sigma w_{i}=0.221\right.$, Table 18). However, the basic covariate model 
was the highest ranking model in the set and was more than 4 times as likely as the next best model (Table 18). A model with only the additive removal effect was ranked second, but was very closely competing with the third ranked model, which included only the additive location effect $\left(w_{i} / w_{j} \approx 1.1\right.$, Table 18$)$. The only other model in the $95 \%$ confidence set was a model including the additive location effect and the location-interval interaction, which comparably weakly supported $\left(w_{1} / w_{i} \approx 9.6\right.$, Table 18). The global model provided a fair fit to the data for YOY sculpin $r$ and explained approximately $40 \%$ of the variation in measured $r$ among all sites and sample intervals (Table 18).

A plot of the per capita growth rate for YOY sculpin indicated that $r$ was near equilibrium for all experimental groups over the first interval except the headwater removal group, which was substantially higher for that interval (Figure 19). From August to October 2006, however, all experimental groups had similar values of $r$ and growth was several orders of magnitude higher than over the first interval post-removal (Figure 19). Over the next interval spanning October 2006 to May 2007, however, the growth rate decreased, but was still nearly doubling, on average, for all sites (Figure 19).

The $95 \%$ confidence set of models for estimating $r$ for small adult mottled sculpin indicated support for a removal effect $\left(\sum w_{i}=0.497\right)$, weak support for a location effect $\left(\Sigma w_{i}=0.172\right)$, and some support for a removal-sampling interval interaction $\left(\Sigma w_{i}=\right.$ 0.417 , Table 18). The top ranked model included the additive removal effect and its interaction with sample interval (Table 18). This model was very closely competing with the standard covariate model with no experimental effects $\left(w_{1} / w_{i} \approx 1.3\right.$, Table 18). These two models, however, carried the great majority of the support compared to the remaining models and the next closest competing model to the top model, which included 
only the additive location effect, was more than 5 times less likely to be the best model by comparison $\left(w_{1} / w_{i} \approx 5.2\right.$, Table 18$)$. The final two models in the set were a model including the additive removal and location effects along with the removal-interval interactive effect and a model including only the additive removal effect (Table 18). Overall, the global model fit the data relatively poorly as it explained only $13 \%$ of the variation in the measured values of $r$ for small adult sculpin (Table 18).

A plot of the modeled $r$ across all intervals and at average values for local chemical and physical habitat covariates for the four experimental groups of sites indicated that, for all of the intervals, $r$ among the headwater and mainstem control groups of sites decreased very moderately from near 1 over the first interval to between 0 and 1 over the fifth and final interval (Figure 20). Over the fourth interval, $r$ was very slightly negative within the headwater control group of sites (Figure 20). Similar to the response of small and large adult brook trout among the removal site groups, $r$ was much higher over the first interval post removal in the removal sites compared to control sites for small adult sculpin (Figure 20). For the next two intervals spanning August 2006 to May $2007 r$ was relatively consistent and near 0 among the removal groups of sites and somewhat lower than the expected values of $r$ modeled for the control sites (Figure 20). At the fourth interval, $r$ was slightly higher, and positive, in the removal sites compared to control sites (Figure 20). Over the final interval, the pattern reversed, with $r$ being slightly lower among the removal sites, and negative, compared to the control groups (Figure 20). Finally, over all intervals, the per capita growth rate was slightly lower, on average, for headwater removal sites compared to mainstem removal sites as well as for 
headwater control sites compared to mainstem control sites, illustrating the contribution of the additive effect of spatial location on $r$ (Figure 20).

The $95 \%$ confidence set of models for estimating $r$ for large adult sculpin indicated support for an additive removal effect $\left(\sum w_{i}=0.465\right)$ and weaker support for the additive location $\left(\Sigma w_{i}=0.176\right)$ and removal-interval interaction $\left(\sum w_{i}=0.241\right)$ effects (Table 18). While the top ranked model was the standard covariate model without experimental effects, that model was closely competing with the next two models $\left(w_{1} / w_{i}\right.$ $<2$, Table 18), which included a model with only the additive removal effect and a model with the additive removal effect and the removal-interval interaction (Table 18). The fourth ranked model was one that included only the location effect and the top ranked model was more than 3 times as likely to be the best model by comparison ( $\psi$ Table 18 ). The final model in the $95 \%$ confidence set included additive terms for removal and location in addition to the interactive removal-interval effect (Table 18). The top model was more than 12 times as likely to be the best model by comparison $\left(w_{1} / w_{i} \approx 12.4\right.$, Table 18). Overall, the global model resulted in a fair fit to the measured values of large adult $r$, accounting for $31 \%$ of the variation in the data (Table 18$)$.

Subsequent plotting of the expected values for large adult sculpin $r$ across all intervals and at average values for local chemical and physical habitat covariates for the four classes of sites indicated that growth steadily declined in control sites, in general, from the first to fourth interval at which $r$ was negative for both mainstem and headwater control groups of sites (Figure 21). Over the final interval, $r$ became positive again for both groups of control sites (Figure 21). Again, the additive location effect was evident among both the control and removal groups of sites, such that $r$ was consistently lower 
among headwater sites in comparison to mainstem sites within the removal treatment groups (Figure 21). The removal interval effect was also pronounced in the modeled estimates, with $r$ in removal sites being somewhat higher compared to the control sites over the first interval post-removal, yet increasingly lower than control sites over the second and third intervals and remaining consistently lower over the final two intervals (Figure 21). In terms of magnitude, the per capita growth rate among the two experimental groups of removal sites was very high over the first interval, but nearer zero over the second interval post-removal (Figure 21). From that point, $r$ remained substantially negative among the removal sites for the remainder of the study (Figure 21).

\section{Response of apparent survival and age-class transition probability}

Brook trout

The set of forward-time multi-state models used for inference of brook trout apparent survival $(\varphi)$ and age-class transition $(\psi)$ included support for all of the experimental effects of interest, with the global model ranking in the $95 \%$ confidence set (Table 19). For the portion of the model estimating $\varphi$, there was unanimous support for a removal effect $\left(\Sigma w_{i}=1.000\right)$, a location effect $\left(\Sigma w_{i}=1.000\right)$, and a removal-interval interaction $\left(\Sigma w_{i}=1.000\right)$ as all models with any support (i.e., model likelihood $\left.>0.001\right)$ included those effects (Table 19). The location-interval $\left(\Sigma w_{i}=0.999\right)$ and removallocation $\left(\Sigma w_{i}=0.999\right)$ interaction effects were nearly as unanimously supported among 
the models in the likelihood (Table 19). Finally, the three way, removal-location-interval effect was also strongly supported $\left(\sum w_{i}=0.735\right)$. While the full model for $\varphi$ received the majority of the support from the data $\left(\Sigma w_{i}=0.669\right)$, a reduced model for $\varphi$ that did not include the removal-location-interval interaction made up the remainder of the support in the $95 \%$ confidence set $\left(\Sigma w_{i}=0.264\right.$, Table 19). Thus, only two models for $\varphi$ were included in the set of top models (Table 19).

For the portion of the model estimating $\psi$ for brook trout, there was support for a removal effect $\left(\Sigma w_{i}=0.380\right)$, a location effect $\left(\Sigma w_{i}=0.288\right)$, and very weak support for their interaction $\left(\Sigma w_{i}=0.035\right.$, Table 19). The model including no experimental effects was in the top ranked model and also received the majority of the support from the data $\left(\Sigma w_{i}=0.456\right)$, compared to a model including the additive effect of removal only $\left(\Sigma w_{i}=\right.$ $0.255)$, a model including the additive effect of location only $\left(\Sigma w_{i}=0.162\right)$, a model including the additive removal and location terms $\left(\Sigma w_{i}=0.066\right)$ and one including those additive terms as well as the interaction among the two experimental effects $\left(\Sigma w_{i}=\right.$ 0.043, Table 19).

Details concerning the precision of $\varphi$ and $\psi$ estimates for brook trout, in terms of standard errors and confidence limits, can be found in Appendix E2. A plot of the model averaged estimates of $\varphi$ for all age classes of brook trout and for all sites over all sampling intervals illustrated the variability in $\varphi$ among sites, with $\varphi$ generally being low over the first interval following the removal, increased over the second interval, highest over the third interval, lower again over the fourth and then higher over the fifth (Figure 22). Interestingly, when plotting estimates for $\varphi$ for all sites, the headwater treatment sites could be visually described as having consistently lower apparent survival over 
intervals one and two than any of the control sites or the mainstem treatment sites (Figure 22). This pattern was readily apparent for YOY, small adult, and large adult brook trout (Figure 22). Likewise, at interval three, $\varphi$ appeared to be higher among the mainstem removal treatment sites than among the others for all age classes (Figure 22).

A plot of the expected values for $\varphi$ among the experimental site types at mean values for the local habitat covariates illustrated patterns among these four types of sites and among the age classes more clearly (Figure 23). Among all intervals excepting the first one, $\varphi$ was consistently higher among mainstem control sites compared to headwater control sites for large adult brook trout, although the difference in modeled $\varphi$ between the two types of sites was relatively small to biologically negligible for the third fourth and fifth intervals (e.g., less than $\sim 10 \%$ difference in $\varphi$ ). The effect of location on $\varphi$ was less consistent among control sites, however, for YOY and small adult brook trout over the course of the study (Figure 23). For the first interval post-removal, $\varphi$ was zero among all removal sites and, over the second interval, $\varphi$ in the mainstem removal sites and headwater removal sites remained lower than in the mainstem control and headwater control sites, respectively (Figure 23).

Because the effect of location was generally inconsistent and small among mainstem and headwater control sites over the course of the study, except for large adult brook trout, it was difficult to logically discern whether differences among mainstem removal and mainstem control sites were biologically meaningful. Therefore, we did not interpret the effect of removal on mainstem sites as such. Interestingly, the effect size in headwater removal sites was estimated to be slightly smaller for small adults than for either YOY or large adult brook trout and $\varphi$ was somewhat higher, in general, over that 
interval for small adult trout compared to the other two age classes (Figure 23). Over the third interval, each of the four experimental groups of sites had similar estimates for $\varphi$, but the headwater removal sites remained slightly lower than the headwater control sites and both types of mainstem sites for large adults (Figure 23). Over the fourth interval, small adult $\varphi$ was more than $10 \%$ larger in headwater removal sites than for all other site types, yet the effect was very small to negligible for YOY and large adult trout (Figure 23).

Plotting the probability of age-class transition, given that an individual survived the interval, $(\psi)$ indicated that this probability, although variable among sites, was estimated to be lowest over the fourth and fifth intervals (modeled as constant May 07 Oct 07), highest over the third and low again over the first and second intervals (constant over July 06 - October 06, Figure 24). Over the interval spanning October 2006 - May 2007, estimates of $\psi$ ranged among sites from 0.463 to 0.922 (Appendix E2) and no clear patterns or differences were distinguishable when comparing estimates among the two age classes. Additionally, unlike the plot for $\varphi$ among sites and age classes (Figure 23), for $\psi$, there was no distinct pattern among sites given their experimental group types (Figure 24).

Nonetheless, aside from one headwater control site and one mainstem control site estimated relatively higher than other control sites and one headwater treatment site estimated relatively lower than other treatment sites, the remaining sites receiving the removal treatment did appear to have a higher probability of transition than the remaining control sites (Figure 24), which provides some reasoning as to why the set of top models used for inference included the removal effect (Table 19). There was no apparent pattern 
between the headwater and control sites to indicate any type of location effect, however (Figure 24). Finally, a plot of the expected mean values among the four experimental site types demonstrated more clearly the experimental effects of removal on $\psi$, which indicated that, although $\psi$ was estimated as slightly higher among removal sites compared to controls and among headwater sites compared to mainstem sites, the effect size was very slight $(<5 \%$ mean difference in expected $\psi$ for all experimental group comparisons, Figure 25).

Mottled sculpin

The set of forward-time multi-state models used for inference of mottled sculpin apparent survival $(\varphi)$ and age-class transition $(\psi)$ included support for all of the experimental effects of interest, with the global model ranking in the $95 \%$ confidence set and third among the 5 top models used for inference (Table 18). For the portion of the model estimating $\varphi$, there was unanimous support for a removal effect $\left(\sum w_{i}=1.000\right)$, a location effect $\left(\sum w_{i}=1.000\right)$ and a removal-interval interaction $\left(\sum w_{i}=1.000\right)$, as all models with any support (i.e., model likelihood $>0.001$ ) included those effects (Table 18). The location-interval $\left(\sum w_{i}=0.995\right)$, removal-location $\left(\sum w_{i}=0.989\right)$, and removallocation-interval $\left(\sum w_{i}=0.974\right)$ interaction effects were nearly as highly supported among the models in the likelihood (Table 20).

For the portion of the model used in estimating $\psi$ for mottled sculpin, there was support for a removal effect $\left(\sum w_{i}=0.540\right)$, a location effect $\left(\sum w_{i}=0.420\right)$, and a removallocation interaction $\left(\Sigma w_{i}=0.182\right.$, Table 20). For the portion of the model used in 
estimating $\varphi$, only the full model including all experimental effects and interactions of interest from the global model was included in the $95 \%$ confidence set (Table 20). However, for the portion of the multistate model constructed for estimating $\psi$, all possible combinations of experimental effects were included in the top set of models, but the model with no experimental effects was most likely (Table 20). Nonetheless, two other models for $\psi$ were also closely competing $\left(w_{1} / w_{i}<2\right)$, which included a model with only the additive effect of removal and a model with the additive effects of removal and treatment as well as their interaction (Table 20). Two other models for $\psi$ were also in the 95\% confidence set and those included an additive model with only the location effect and an additive model with both a removal and location effect (Table 20). Details concerning the precision of $\varphi$ and $\psi$ estimates for mottled sculpin, in terms of standard errors and confidence limits, can be found in Appendix E4.

A plot of $\varphi$ for mottled sculpin for all sites and over all sampling intervals demonstrated the variability in the model averaged estimates across all sites, sampling intervals, and age classes of mottled sculpin (Figure 26). Generally, small and large adult $\varphi$ was higher than YOY $\varphi$ for all sites across all sampling intervals (Figure 26). Among both experimental groups of control sites, $\varphi$ was moderate over the first interval postremoval, peaking over the second interval, and steadily declined over the final three intervals to values slightly lower than over the first interval (Figure 26). Interestingly, when plotting estimates for $\varphi$ for all sites, the mainstem removal sites could be described as having lower apparent survival over the intervals one to three, or from July 2006 to May 2007, than either of the control site types (Figure 20). One headwater removal site was also visibly distinguishable as having a much lower $\varphi$ than the control sites over the 
first two intervals, while the other 2 headwater removal sites were on the lower range of $\varphi$ for the control sites, but not visibly distinguishable from those sites (Figure 26). Over the third interval, the mainstem removal sites continued to have lower $\varphi$ than all other site types, while, interestingly, $\varphi$ among two of the headwater removal sites was highest among all site types, at least for adult sculpin, while the same headwater removal site that was lowest over the second interval, was similar in $\varphi$ to the control sites over the third interval (Figure 26).

A plot of $\varphi$ in terms of expected values among the four experimental group types at mean values for the local habitat covariates clarified the patterns among these four types of sites (Figure 27). Among the age classes, $\varphi$ was consistently higher for each of the experimental groups in large adults, although the difference in $\varphi$ between the large adult class and the others was greatest for the mainstem control sites (Figure 27). Apparent survival was, again, notably lower among both types of removal treatment sites over the first two intervals, but lower than control sites only in the mainstem removal sites over the third interval (Figure 21). Over those first two intervals, $\varphi$ was minimally divergent to negligible among the two removal treatment groups, but over the third interval, $\varphi$ in headwater removal sites returned to near the control site levels, while $\varphi$ in mainstem removal sites remained notably lower (i.e., minimum difference in expected $\varphi$ for mainstem removal sites versus next closest $>10 \%$, Figure 27).

Again, the effect of spatial location was notable in the plot of $\varphi$ for large adults, but less so for YOY or small adult sculpin (Figure 27). For large adults among the control sites, $\varphi$ was generally higher in the mainstem control sites than in headwater sites (Figure 27). This pattern was relatively consistent over the course of the study for large 
adults, however, the effect size was greatest over the first interval and smallest and nearly negligible over the last (Figure 27). The effect of location was less apparent among the removal experimental groups, although headwater and mainstem removal groups $\varphi$ diverged over the third interval for all age classes when the headwater removal group had values for $\varphi$ estimated as substantially higher than the mainstem removal group (Figure 27). This effect was greatest for small adult sculpin and smallest for YOY (Figure 27). For large adults, over the final interval, $\varphi$ was somewhat larger among the mainstem removal group than for the other groups (Figure 27).

A plot of the probability of age-class transition $(\psi)$ for YOY indicated that $\psi$ was generally very high across all sites, although highest and very nearly 1 for all sites over the third interval (Figure 28). There was less variability in $\psi$ among sites in the first two intervals than the fourth and fifth and $\psi$ covered a narrower range of probabilities all greater than 0.862 (Figure 28). Finally, there was no readily distinguishable pattern in $\psi$ in terms of experimental groupings in YOY $\psi$ (Figure 28). Probability of transition was more variable for the transition from small to large adults, with $\psi$ generally highest, again, over the third interval spanning October 2006 to May 2007 (Figure 28). Probability of transition was similar, within sites, among the first two intervals and again among the final two intervals (Figure 28). However, variability among sites was quite high among these intervals relative to the third interval (Figure 28).

Again, because variability among sites was generally high, there were no readily distinguishable patterns in $\psi$ among sites in terms of their experimental classification from this plot (Figure 28). However, plotting the expected values for $\psi$ among the four experimental groups of sites clarified the model selection results, which indicated some 
support for the removal, location, and removal-location interaction effects (Table 18, Figure 29). On average, while mainstem control sites tended to exhibit the highest probability of transition for YOY and small adults over the first two and last two intervals, the difference among experimental groups was minimal to negligible over the third interval. Over the first two intervals, $\psi$ for the small adult class was notably higher among the mainstem control sites than among the headwater removal sites, which, in turn, had expected values much higher than either the mainstem removal or headwater control sites. However, there were no distinguishable differences in the estimates for $\psi$ among the mainstem removal and headwater control groups (Figure 29). This general pattern was the same over the final two intervals, although the overall estimates for $\psi$ were lower for all experimental groups and the differences between their estimates were smaller (Figure 29). Again, differences in $\psi$ for the YOY age class among all experimental groups were largely negligible over the first two intervals and probability of transition was very nearly 1 for both of those intervals (Figure 29). Over the final two intervals, differences were somewhat more distinguishable and patterns were similar to those described for small adult $\psi$ (Figure 29).

Response of the components of population growth

Brook trout 
The final set of models selected for inference from the 65 candidate reverse-time multi-state models indicated that the data supported only three models for best estimating the proportional contribution of survival $\left(\gamma_{\mathrm{ii}}\right)$ and local recruitment $\left(\gamma_{\mathrm{ji}}\right)$ to the instantaneous population growth rate $(\lambda)$ of brook trout (Table 21). For the portion of the model estimating $\gamma_{\mathrm{ii}}$, three different models were supported by the data and were included in the $95 \%$ confidence set (Table 21). These models indicated unanimous support for a removal effect $\left(\Sigma w_{i}=1.000\right)$ and a removal-sampling interval interaction effect $\left(\sum w_{i}=\right.$ $1.000)$ along with reduced support for a spatial location effect $\left(\sum w_{i}=0.171\right)$ and very weak support for a removal-location interaction $\left(\sum w_{i}=0.086\right.$, Table 21$)$. The top model in the set included only the additive effect of removal and its interaction with sampling interval $($ Table 21$)$. That model received the vast majority of the support $(\mathrm{AICc}$ weight $=$ 0.710 ) and was more than 3 times as likely to be the best model given the data compared to the next best model in the set $\left(w_{1} / w_{2} \approx 3.5\right.$, Table 21$)$. The second ranked model included all of the terms as the top model in addition to the additive location term (Table 21). The third and lowest ranked model in the set included all of the terms in the top two models in addition to the removal-location interaction term, but this model was not likely compared to the top model $\left(w_{1} / w_{3} \approx 8.1\right.$, Table 21$)$. Finally, for the portion of the model constructed for estimating $\gamma_{\mathrm{ji}}$, only the model including no experimental effects was included in the $95 \%$ confidence set (Table 21). Details concerning the precision of $\gamma_{\mathrm{ii}}$ and $\gamma_{\mathrm{ji}}$ estimates for brook trout, in terms of standard errors and confidence limits, can be found in Appendix F2.

A plot of the estimated proportion of the instantaneous population growth rate $(\lambda)$ due to local survival $\left(\gamma_{\mathrm{ii}}\right)$ for YOY brook trout among all sites indicated that the 
contribution of survival was relatively low across all sites and intervals and was estimated as slightly, but distinctly lower among the removal treatment sites over the first two intervals (Figure 30). The strong support for the removal-interval interaction effect was also evidenced visually in this figure as $\gamma_{\mathrm{ii}}$ for removal sites was estimated to be higher over intervals four and five than over intervals one, two and three (Figure 30). Among control sites, $\gamma_{\mathrm{ii}}$ remained somewhat consistent over intervals one and two, but was lowest and least different from the removal sites at interval three and similar in magnitude to intervals one and two, again, over intervals four and five (Figure 30). In general, variability in the estimates of $\gamma_{\mathrm{ii}}$ for YOY brook trout appeared to be higher between the control sites than the removal sites, although, variability appeared to increase among the removal sites over intervals 4 and 5 compared to the first three intervals. Among control sites, there appeared to be a pattern in $\gamma_{\mathrm{ji}}$ indicating that headwater control sites may have slightly higher estimates of $\gamma_{\mathrm{ji}}$ than mainstem control sites, on average (Figure 30).

This effect of spatial location was clearer when comparing mainstem removal to headwater removal sites, as $\gamma_{\mathrm{ji}}$ was estimated to be higher among headwater removal sites than for mainstem removal site over all intervals (Figure 30). However, the distinction was least clear over the third interval (Figure 30). At intervals four and five, the separation among mainstem and headwater removal sites was much clearer than over intervals one and two (Figure 30). Again, because the contribution of local recruitment was not modeled for YOY, the plot of the contribution of immigration $\left(1-\gamma_{\text {ii }}\right)$ visualized correspondingly opposite patterns as those just described for $\gamma_{\mathrm{ii}}$ (Figure 30). 
A plot of the estimated proportion of the instantaneous population growth rate $(\lambda)$ due to survival $\left(\gamma_{\mathrm{ii}}\right)$ for small adult brook trout among all sites also helped visualize variability between sites in estimates (Figure 31 ). The strongly supported removalsampling interval effect was also apparent in the plot of $\gamma_{\mathrm{ji}}$ for small adults, as the effect of removal on $\gamma_{\mathrm{ii}}$ appeared to change over the course of the study (Figure 31). Again, $\gamma_{\mathrm{ii}}$ in control sites appeared lowest over the third interval, but similar over intervals one, two, four, and five (Figure 31). Also, variability in $\gamma_{\text {ii }}$ between all sites was lowest over the third interval (Figure 31). Interestingly, and as suggested by the strong support in the model comparisons for the removal term, the pattern in $\gamma_{\mathrm{ji}}$ over the course of the study suggested that $\gamma_{\mathrm{ji}}$ was estimated to be lower, on average, in all removal sites over intervals one, two, and higher over interval five, although the distinction was less clear among the latter two intervals (Figure 31). There was no clear distinction among the sites according to spatial location, although, among removal sites, there was very little variability between sites in $\gamma_{\mathrm{ji}}$ and estimates were generally slightly higher than for the mainstem removal sites over the course of the study with the exception of one mainstem removal site (Figure 31).

Estimates for the proportion of $\lambda$ due to immigration $\left(1-\gamma_{\mathrm{ji}}-\gamma_{\mathrm{ii}}\right)$ for small adult brook trout revealed an interesting pattern among the removal sites compared to the control sites, illustrating a strong and nearly linear decline in the proportion of $\lambda$ due to immigration over the course of the study (Figure 31). Additionally, there was no strongly discernable pattern among sites concerning the location effect (Figure 31). However, among the removal sites over the last 3 intervals estimates were somewhat higher for a couple of headwater removal sites compared to mainstem removal sites with the 
exception of one mainstem removal site that was estimated much higher than all other removal sites over those intervals (Figure 31). Among control sites, there was no visible pattern suggesting a location effect (Figure 31). Overall, variability between sites was higher for control sites compared to removal sites, although, much of that variability was driven by two headwater control sites that had much lower estimates, compared to the other control sites, over the final two intervals (Figure 31).

The plot of the proportional contribution of local recruitment $\left(\gamma_{\mathrm{ji}}\right)$ to $\lambda$ over time for small adult brook trout illustrated a great deal of variability in that parameter among sites (Figure 31). Interestingly, although no experimental effects were supported by the data, the plot suggests that the reason for that may be related to the disproportionately higher estimates for a couple of headwater control sites compared to the remaining sites (Figure 31). Other than those two sites, it appeared that most removal sites had estimates of $\gamma_{\mathrm{ji}}$ higher than those of the control sites (Figure 31).

Patterns in the contribution of $\gamma_{\mathrm{ii}}, \gamma_{\mathrm{ji}}$, and $1-\gamma_{\mathrm{ji}}-\gamma_{\mathrm{ii}}$ to $\lambda$ for large adult brook trout were very similar and largely indistinguishable from those for small adults (Figure 32). However, variability in $\gamma_{\mathrm{ii}}$ was generally greater among control sites for large adults than it was for small adults (Figures 31 and 32). Additionally, the location effect on both $\gamma_{\mathrm{ii}}$ and $1-\gamma_{\mathrm{ji}}-\gamma_{\mathrm{ii}}$ was slightly more discernable among experimental groups for large adults (Figure 31) compared to small adults (Figure 32). For the estimates of $\gamma_{\mathrm{ii}}$, there was greater separation among mainstem removal and headwater removal sites (Figure 32) compared to what was expected for small adults (Figure 31). Likewise, $\gamma_{\mathrm{ii}}$ was higher among the mainstem control sites than among the headwater control sites over all intervals (Figure 32). For $1-\gamma_{\mathrm{ji}}-\gamma_{\mathrm{ii}}$, there was also greater separation among the 
estimates for headwater removal compared to mainstem removal sites over the duration of the study for large adult trout (Figure 32) compared to small adults (Figure 31). Aside from one mainstem removal site, headwater removal sites had consistently higher estimates for $1-\gamma_{\mathrm{ji}}-\gamma_{\mathrm{ii}}$ compared to mainstem removal sites over all intervals (Figure $32)$.

Mottled sculpin

The $95 \%$ confidence set selected for inference from the 65 candidate reverse-time multi-state models indicated that the data supported only one model for estimating the proportional contribution of survival $\left(\gamma_{\mathrm{ii}}\right)$ and local recruitment $\left(\gamma_{\mathrm{ji}}\right)$ to the instantaneous population growth rate $(\lambda)$ of mottled sculpin (Table 22). Summing the Akaike weights for the experimental effects of interest across all models in the likelihood for the portion of the model used in estimating $\gamma_{\mathrm{ii}}$ indicated unanimous support for the additive removal effect $\left(\sum w_{i}=1.000\right)$, the removal-sampling interval interaction effect $\left(\sum w_{i}=1.000\right)$, and the additive location effect $\left(\Sigma w_{i}=1.000\right)$. For the portion of the model estimating $\gamma_{\mathrm{ji}}$, there was very little support for an additive location effect $\left(\sum w_{i}=0.022\right)$ and no other experimental effects were supported (Table 22). The top model in the set included the additive effect of removal and its interaction with sampling interval as well as the additive location effect (Table 22). The top model received the nearly all of the support (AICc weight $=0.978)$ and was nearly 50 times as likely to be the best model given the data compared to the next best model in the set $\left(w_{1} / w_{2} \approx 46.5\right)$. Details concerning the 
precision of $\gamma_{\mathrm{ii}}$ and $\gamma_{\mathrm{ji}}$ estimates for mottled sculpin, in terms of standard errors and confidence limits, can be found in Appendix F4.

A plot of the estimated proportion of the instantaneous population growth rate $(\lambda)$ due to local survival $\left(\gamma_{\mathrm{ii}}\right)$ for YOY mottled sculpin among all sites indicated that the proportional contribution of survival was very nearly zero across all dates and, thus, the contribution due to immigration was necessarily very nearly one across those same intervals and sites (Figure 36).

Plotting the estimated contribution of the various components of $\lambda$ for small adult sculpin revealed more interesting patterns than the uniformity seen in YOY estimates. Estimates for $\gamma_{\mathrm{ii}}$ among the control sites, for instance, were relatively consistent over time (Figure 37). Estimates were slightly higher, however, over the final two intervals compared to the first three (Figure 37). Among the control sites, there was no clear pattern in $\gamma_{\mathrm{ii}}$ according to spatial location (Figure 37). Removal sites, on the other hand, appeared to demonstrate a pattern according to location. Aside from one mainstem removal site, for instance, all headwater removal sites had values for $\gamma_{\mathrm{ii}}$ estimated higher than for mainstem removal sites (Figure 37). Concerning the removal effect, there was no clear separation between the removal and control sites aside from the first interval where $\gamma_{\mathrm{ii}}$ was 0 for all removal sites (Figure 37). However, aside from the one headwater removal site with much higher estimates over the second to fifth intervals compared to the other removal sites, the removal sites, as a group, appeared to have slightly lower estimates than those seen in control sites. Nonetheless, estimates for $\gamma_{\text {ii }}$ were generally low across all sites and intervals $(\max =0.478$, Figure 37$)$. 
Estimates for small adult $1-\gamma_{\mathrm{ji}}-\gamma_{\mathrm{ii}}$ indicated similar, but opposite, patterns compared to those seen for $\gamma_{\mathrm{ii}}$ (Figure 37). Again, the proportional contribution of immigration was generally high and largely consistent over the course of the study for the control and removal sites, although, decreasing somewhat over time (Figure 37).

Likewise, $1-\gamma_{\mathrm{ji}}-\gamma_{\mathrm{ii}}$ was necessarily estimated at 1 for all removal sites at the first interval post-removal (Figure 37). Again, estimates appeared somewhat higher overall, and as a group, across all intervals for the removal sites compared to the controls (Figure 37).

Concerning the proportional contribution of recruitment from the YOY class to small adult sculpin $\lambda$, estimates were generally quite low across all sites $(\max =0.175$, Figure 37). Also, there appeared to be no discernable pattern among either removal or control sites (Figure 37).

Estimates for $\gamma_{\mathrm{ii}}$ for large adult sculpin demonstrated much more variability among sites and covered a wider range of values (Figure 38). As a group, the estimates for large adults were generally higher than those for small adults (Figure 37 versus Figure 38). The removal effect was, of course, most pronounced over the first interval, where all removal sites had estimates for $\gamma_{\mathrm{ii}}$ at 0 (Figure 38). Beyond that interval, any removal effect on $\gamma_{\mathrm{ii}}$ was difficult to discern, although, the removal sites appeared to be estimated somewhat lower, on average, over the fourth interval compared to controls (Figure 38). Again, among the control sites, there was no discernable effect of location (Figure 38). On the other hand, aside from on mainstem removal sites having estimates similar to the headwater removal sites, headwater removal sites appeared, on average, to have somewhat higher estimates than mainstem removal sites (Figure 38). 
Patterns in the estimates of the proportional contribution of immigration to large adult $\lambda$ were similar, but opposite, to those for $\gamma_{\mathrm{ii}}$, with generally more variability between sites (Figure 38). Estimates for large adult mottled sculpin $\gamma_{\mathrm{ji}}$ were somewhat higher, on average, than estimates for small adults (Figure 38 versus Figure 37). Again, however, there was no discernable pattern in $\gamma_{\mathrm{ji}}$ related to the experimental effects (Figure 38).

Instantaneous survival, local recruitment, and immigration rates

Brook trout

While the proportional contributions to $\lambda$ indicated interesting patterns among the experimental groups and between sites and age classes, the estimates were more informative when converted to instantaneous rates of survival $(\Phi)$, recruitment $(\Omega)$, and immigration $(\Gamma)$ by multiplying the estimated proportions for the experimental groups at each interval and age class by the appropriate estimates of $\lambda$ for the same groups, age classes and intervals $(\lambda=\ln (r)$, see results for Response of the population growth rate). For YOY brook trout, estimates of $\Phi$ for the two control groups were generally highest over the first interval, lowest over the third, and next highest over the sixth (Figure 33). Also, $\Phi$ was somewhat higher among the headwater control group compared to mainstem control group over all intervals ( $\min$ difference $=0.1$ and $\max =0.8$, Figure 33). This was also generally the case in the headwater removal group compared to the mainstem removal group, excepting the first interval where $\Phi$ was zero for both groups (Figure 33). 
Comparing estimates of $\Phi$ for removal versus control groups, $\Phi$ was much lower among the removal groups compared to controls for the first interval $(\min$ difference $=0.4)$ and slightly lower to negligible over the third and fourth (max difference $<0.2$, Figure 33). Over the second interval, $\Phi$ was somewhat higher in the headwater control group compared to the headwater removal group, but the difference between the mainstem control and mainstem removal groups over that interval was negligible (difference $<0.1$, Figure 33). Interestingly, over the final interval, $\Phi$ was somewhat higher in the mainstem removal group compared to the mainstem control group (difference $=0.2)$ and higher in the headwater removal group compared to the headwater control group (difference $=0.4$, Figure 33).

Concerning YOY brook trout $\Gamma$ estimates, rates were generally similar at all intervals when comparing the headwater control group to the mainstem control group, excepting the third interval where $\Gamma$ was estimated higher for the headwater control group (difference $=0.4$, Figure 33). Among the removal groups, however, $\Gamma$ was consistently higher in the headwater removal group, compared to the mainstem removal group, over the first three intervals ( $\min$ difference $=0.2$ and $\max =0.7$, Figure 33 ). Over the fourth interval, on the other hand, estimates were similar between the two removal groups (Figure 33). Over the final interval, $\Gamma$ was slightly higher in the mainstem removal group (difference $=0.2$, Figure 33).

Because we did not differentiate between true immigration and local recruitment for YOY brook trout, we could not characterize the population dynamics of this age class in terms of the degree to which dynamics were internal or external. For the same reason, we also could not determine the extent to which the YOY population was self-sustaining. 
Estimates of $\Phi$ for small adult brook trout were consistently higher among the control experimental groups compared to the removal groups over all intervals, although the difference was minimal (expected difference $<1$ ) and not likely meaningful over the third or fourth intervals, where $\Phi$ was very low among all groups $(\Phi<0.2$, Figure 34). The difference was greatest over the first interval post-removal, where $\Phi$ was necessarily 0 among the removal experimental groups (Figure 34). The difference among removal and control groups was also notable over the fifth interval, particularly between the two headwater groups (expected difference $\approx 0.5$, Figure 34 ). Estimates for $\Phi$ were also generally higher among the headwater experimental groups compared to the mainstem groups over all intervals, excluding the first interval for the removal groups where $\Phi$ was 0 (Figure 34). Again the differences were minimal over the third and fourth intervals where $\Phi$ was generally very low among all groups (Figure 34). Finally, the results for small adult brook trout indicated that estimates of $\Phi$ were consistently lower than what would be required to maintain those populations over time. For instance, $\Phi$ estimates were consistently less than 1 for all experimental groups and intervals, excepting the final interval for headwater control sites, and were regularly as low as or lower than 0.5 for many intervals (Figure 34).

Plots for small adult brook trout indicated that estimates of $\Gamma$ over all sampling intervals for the mainstem control groups were very slightly $(0.1<$ expected differences $<$ 0.3), but consistently, higher than those estimated for the headwater control groups (Figure 34). Among the two removal experimental groups, $\Gamma$ was estimated as substantially higher (expected difference $\approx 0.5$ ) over the first interval post-removal in the headwater removal group compared to the mainstem removal group, yet slightly higher in 
mainstem removal group over the second $(0.2<$ expected difference $<0.3)$, fourth and fifth $(0<$ expected difference $<0.2)$ intervals (Figure 34). Among both removal groups, however, $\Gamma$ declined steadily over the course of the study following removal (Figure 34 ). In fact, over the final two intervals, $\Gamma$ was very nearly 0 among the headwater removal group and not much higher, over those intervals, for the mainstem removal group (Figure 34). Among the control groups, $\Gamma$ was highest over the second and fifth intervals, lowest over the fourth, slightly higher over the third and then very slightly higher over the first (Figure 34).

Estimates of $\Omega$ for small adult brook trout were generally low across all intervals, but were over the third and fifth intervals (Figure 34). Additionally, $\Omega$ was generally higher among control sites relative to removal sites, but differences were only meaningful over the third and fifth intervals $(0.2<$ expected differences $<0.3)$, whereas recruitment was, again, generally low to negligible over the other intervals (Figure 34). Differences according to spatial location were not discernable (Figure 34). Finally, estimates of $\Omega$ generally demonstrated that local recruitment contributed little to population maintenance across all groups, on average, and relative to survival and immigration, over the course of the study (Figure 34).

The population dynamics of small adult brook trout in mainstem control sites was characterized as slightly to moderately external over all intervals (Figure 34). Over the first four intervals populations were moderately self-sustaining; however, populations were completely self-sustaining over the final interval (Figure 34). The pattern in selfsustainability was similar among the headwater control group; however, dynamics were slightly internal across all intervals (Figure 34). Among the headwater removal group, 
growth was due completely to external dynamics over the fist interval post-removal and was not self-sustaining (Figure 34). Over the second interval, the dynamics were slightly external and moderately self-sustaining (Figure 34). Over the third interval, the dynamics were largely internal and the populations were weakly self-sustaining (Figure 34). Over the fifth interval, the dynamics were completely internal and moderately self-sustaining (Figure 34). Within the mainstem removal group, over the first interval, growth was again due to completely external dynamics (Figure 34). The dynamics over the second interval were largely external and the population was moderately self-sustaining (Figure 34). Over the third interval, the dynamics were slightly internal and moderately selfsustaining (Figure 34). Over the fourth interval, the dynamics were largely internal and weakly self-sustaining (Figure 34). Finally, over the fifth interval, the dynamics were largely internal and moderately self-sustaining (Figure 34). In general, among the removal groups, small adult population dynamics became increasingly internal over the course of the study (Figure 34).

For large adult brook trout, $\Gamma$ was relatively consistent over all intervals for the control groups, excepting the fourth interval, where it was several orders of magnitude lower compared to estimates for the other four intervals $(0.2<$ differences $<0.5$, Figure 35). Among the two control groups, $\Gamma$ was estimated slightly lower for all intervals among the headwater control group compared to the mainstem control group, but differences were generally not meaningful (max difference $<0.1)$, excepting estimates at the third interval (difference $\approx 0.3$, Figure 35 ). Comparing estimates among the control versus removal experimental groups indicated that, excepting the first and third intervals, $\Gamma$ was higher among the control groups (Figure 35). However, over the fourth interval 
that difference was slight to negligible (max difference $<0.1$, Figure 35 ). Over the second and fifth intervals, on the other hand, the differences were more substantial (max difference $\approx 0.5$, Figure 35 ). Interestingly, $\Gamma$ was many orders of magnitude higher in the removal groups compared to control groups over the first interval post-removal (Figure 35). Estimates were also higher for the removal groups compared to controls over the third interval, although, the differences were relatively slight (max expected difference $<$ 0.2 , Figure 35). Within the removal groups, $\Gamma$ was lower for the headwater groups compared to the mainstem groups for all intervals, although, differences were minimal over the second, fourth, and fifth intervals where $\lambda$ was very low overall (max difference $<0.2$, Figure 35$)$. The difference was substantially large over the first interval (difference $\approx 2.0$ ) and notable over the third (difference $\approx 0.6$, Figure 35 ).

Estimates of $\Phi$ for large adult brook trout among both control experimental groups were generally highest over the first two intervals, lowest over the third, low again over the fourth, and high again over the fifth (Figure 35). Among the removal groups, however, $\Phi$ generally increased over each interval and was highest over the fifth (Figure 35). Among the control groups, $\Phi$ estimates were generally higher across all intervals within the mainstem group compared to the headwater control group, although differences were somewhat small (max difference $\approx 0.3$ ), particularly over the fourth interval, where $\lambda$ was low overall for both groups (difference $<0.2$, Figure 35 ). This trend was also somewhat apparent among the removal groups over all intervals after the first one, although, the differences were very small to negligible (max difference $<0.2$ ). Estimates of $\Phi$ were also higher, for all intervals, in the control groups compared to the removal groups (Figure 35). The greatest magnitudes of difference in $\Phi$ estimates 
between the control and removal groups occurred over the first interval post-removal, where the estimates of $\Phi$ among the removal groups were necessarily 0 , but the control groups ranged from 0.7 in the headwater control group to 1.0 in the mainstem control group (Figure 35). Estimates of $\Phi$ were also notably higher among the control groups compared to the removal groups over the second interval post-removal, where $\Phi$ was 0.5 to 0.7 in the control groups and still less than 0.2 among the removal groups (Figure 35). After those first two intervals, however, differences among control and removal sites in the estimates of $\Phi$ were negligible (differences $<0.1$, Figure 35).

Estimates of $\Omega$ for large adult brook trout were highest over the third interval as local recruitment from the small adult class appears to have contributed strongly to the maintenance of the large adult population over that interval (Figure 35). The estimates were next highest over the final interval, although, the rate was relative small among all groups $(\Omega<0.2$, Figure 35). Estimates of $\Omega$ were also greater than 0 over the fourth interval for all groups, excepting the headwater removal group, over the fourth interval, although, the magnitude was negligible $(\Omega<0.1$, Figure 35). Estimates for $\Omega$ also differed somewhat between the mainstem and headwater groups, with estimates generally higher for the mainstem groups (Figure 35). While differences were notable over the third interval ( $\max$ differences $\approx 0.3$ ), they were negligible over the other intervals where $\Omega$ was greater than 0 (differences $<0.1$, Figure 35 ). On the other hand, there were no discernable differences in $\Omega$ among removal and control groups (Figure 35).

It was generally apparent that the population dynamics of large adult brook trout across most intervals for the mainstem control group was characterized as slightly internal to slightly external as well as largely to completely self-sustaining across most 
intervals, excepting the fourth interval, which classified as moderately self sustaining (Figure 35). Likewise, the headwater control group was characterized as slightly internal to slightly external and moderately self-sustaining across all intervals (Figure 35). In contrast, population dynamics in the mainstem removal group fluctuated temporally relative to the controls and were characterized as external to completely external and weakly to not self sustaining over the first two intervals post-removal, slightly external and moderately self-sustaining over the third, slightly internal, but weakly self-sustaining over the fourth, and largely internal and moderately self-sustaining over the fifth (Figure 35). Generally speaking, population dynamics among both removal groups became increasingly internal following the removal (Figure 35). Over the first two intervals for the mainstem removal group, and similar to the headwater removal group, dynamics were completely external to external again and weakly to not self-sustaining (Figure 35). Over the third interval, dynamics were, as in the headwater removal group, slightly external, but the population was completely self-sustaining over that interval for this group (Figure 35). Finally, dynamics were slightly internal, but weakly self-sustaining over the fourth interval and internal and largely self-sustaining over the fifth (Figure 35).

\section{Mottled sculpin}

Estimates for $\Phi$ for YOY mottled sculpin were generally negligible or 0 among all intervals and for all experimental groups (Figure 39). On the other hand, estimates for $\Gamma$ across all sites and dates demonstrated interesting variability. In general, $\Gamma$ was somewhat higher among control groups compared to removal groups over all intervals 
(Figure 39). Additionally, $\Gamma$ was somewhat higher among the mainstem groups compared to the headwater groups for all intervals (Figure 39). Differences were generally greatest where $\Gamma$ was highest and least where $\Gamma$ was lowest (Figure 39).

Among the experimental control groups for small adult sculpin, estimates for $\Phi$ were fairly similar for all intervals, although, estimates at most intervals were slightly higher in the mainstem control group compared to the headwater control group (max difference $<0.2$, Figure 40 ). This slight location effect on $\Phi$ was also apparent when comparing mainstem removal and headwater removal sites, where mainstem removal sites had consistently higher estimates for $\Phi($ max difference $<0.2$, Figure 40$)$. Comparing the estimates between the removal and control groups also indicated that, excepting the first interval post-removal, estimates of $\Phi$ were quite similar among the four groups of sites (Figure 40). Overall, estimates for $\Phi$ were lower among the removal sites than among the control sites, although, the differences were slight to negligible (max difference $<0.2$, Figure 40 ).

Comparing estimates of $\Gamma$ among the experimental groups for small adult sculpin revealed more striking patterns. Estimates for $\Gamma$ were consistently higher in the mainstem groups relative to the headwater groups (Figure 40). The differences were greater between the mainstem control and headwater control groups (max difference $=0.8$ and $\min =0.3$ ), but they were also substantial between the two removal groups (max difference $=7.9$ and $\min =0.2$, Figure 40 ). Interestingly, $\Gamma$ declined rather steadily over the course of the study among the control groups, excepting the slight increase over the fifth interval (Figure 40). On the other hand, $\Gamma$ was more variable over time in the removal groups (Figure 40). Nonetheless, aside from the first interval and the fourth 
interval, $\Gamma$ was consistently higher among the control groups than the removal groups (Figure 40). At the first interval, however, $\Gamma$ was extremely high for both removal groups compared to the controls and, interestingly, $\Gamma$ was considerably higher in the mainstem removal group compared to the headwater removal group over that interval (Figure 40).

In general, local recruitment contributed very little to growth among all experimental groups (Max $\Omega=0.14$ ). However, $\Omega$ was generally higher in the control groups compared to the removal groups, though actual differences in magnitude were negligible (max difference $<0.1$, Figure 40).

Concerning the overall dynamics of small adult sculpin among the experimental groups, the population dynamics among all intervals and groups, excepting the first interval among the removal groups, were classified as largely external (Figure 40). Over the first interval post-removal in the removal groups, the dynamics were necessarily completely external (Figure 40). Likewise, for all intervals among the two control groups, the dynamics were classified as moderately self-sustaining (Figure 40). Removal groups were more variable concerning sustainability, however. In both removal groups, growth over the first interval post-removal was classified as not self-sustaining (Figure 40). In the mainstem removal group, growth was weakly self-sustaining over the second interval post-removal, moderately self-sustaining over the next two intervals, and then weakly self-sustaining over the last (Figure 40). In the headwater removal group, growth was weakly self-sustaining over both the second and third intervals, moderately selfsustaining over the fourth, and weakly self-sustaining again over the final interval (Figure 40). 
Estimates for $\Phi$ for large adult mottled sculpin were generally more variable than for small adult or YOY (Figure 41). With the control groups, $\Phi$ generally was near maximum (i.e., $\Phi \approx 1$ ) over the first two intervals, substantially lower over the second two (min difference $=0.4)$, and then high again over the final interval (Figure 41). Within the mainstem control group, $\Phi$ was approximately maximum again over the final interval (Figure 41). Within both control groups, $\Phi$ generally decreased steadily over the first three intervals with $\lambda$, head steady between the third and fourth, and then rose again somewhat over the fifth (Figure 41). Within both removal groups, $\Phi$ was essentially 0 over the first interval post-removal (Figure 41). The estimate rose somewhat over the second interval, then plummeted back to near zero again over the third and fourth intervals before finally rising slightly again over the final interval (Figure 40). Likewise, $\Phi$ was comparably lower within the removal groups, at each interval, compared to the control groups (min difference $=0.2)$ and differences were more substantial over the earlier intervals compared to the later (Figure 41). Also, there was a noticeable location effect, as estimates for mainstem sites were consistently higher than those for headwater sites, although differences in some cases, particularly over the third and fourth intervals when $\lambda$ was small, were likely biologically negligible (i.e., differences $<0.1$, Figure 41 ).

Estimates for $\Gamma$ for large adult sculpin were also variable among the experimental groups of sites (Figure 41). Similar to the case with $\Phi$, estimates for $\Gamma$ in the control groups generally declined over the first four intervals, but increased slightly again over the final interval (Figure 41). Generally, $\Gamma$ was very high in both control groups over the first two intervals post-removal (Figure 41). However, estimates were considerably lower over the next three intervals compared to the first two (Figure 41). For both 
removal groups, $\Gamma$ was extremely high over the first interval post-removal, somewhat low over the second interval, and very low to nearly zero over the final three intervals (Figure 41). Comparing the removal groups to the control groups, $\Gamma$ in both removal groups was considerably higher than in either of the control groups over the first interval postremoval (Figure 41). On the other hand, over the remainder of the study, $\Gamma$ was higher in the control groups relative to the removal groups (Figure 41). Finally, the location effect was also apparent as estimates for $\Gamma$ in the mainstem groups were consistently higher than estimates in the headwater groups across all intervals and both treatment groups (Figure 41). However, the difference was small to negligible over the third and fourth intervals where $\Gamma$ was generally low among all groups (Figure 41). That also described the case fittingly for the removal sites over the final intervals (Figure 41).

Although $\Omega$ did contribute measurably to large adult $\lambda$, the overall contribution was generally very slight $(\Omega<0.2)$ (Figure 41$)$. Nonetheless, the contribution was greatest over the second and third intervals post-removal in the control groups and only discernable in the removal groups over the second interval (Figure 41).

Concerning the general characterization of the population dynamics of large adult mottled sculpin across the experimental groups and over the course of the study, the dynamics may be described as slightly to largely external, on average (Figure 41). Interestingly, the dynamics in the control groups could be described as moderately to completely self-sustaining, on average, and the internal dynamic was driven almost entirely by survival as local recruitment was generally extremely low to negligible (Figure 41). More specifically, the dynamics in the mainstem control group was largely external over the first interval and the dynamics in the headwater control group was 
slightly external (Figure 41). For both groups over that interval, however, the populations were completely self-sustaining, on average (Figure 41). Over the second interval, the dynamics in both control groups were classified as slightly external and completely self-sustaining (Figure 41). Over the final three intervals for both control groups, the dynamics classified as slightly internal (Figure 41). Over intervals two and three, both control groups were moderately self-sustaining (Figure 41). Over the final interval, the headwater control group was moderately self-sustaining, while the mainstem control group was completely self-sustaining (Figure 41).

The dynamics within the removal groups, on the other hand, were somewhat different than what was observed in the control groups. First, the dynamics were obviously completely external and not self-sustaining over the first interval post-removal (Figure 41). Over the second interval, the dynamics in the mainstem removal group were slightly external and moderately self-sustaining in comparison to the headwater removal group which was slightly internal and moderately self-sustaining (figure 41). In both removal groups, the dynamics were slightly internal and weakly self-sustaining over the third and fourth intervals (Figure 41). Over the fifth interval, the dynamics in the mainstem group were slightly internal and moderately self-sustaining, while dynamics in the removal group were slightly internal and weakly self-sustaining (Figure 41). 


\section{Discussion}

Local dynamics within the core versus the periphery

Although we expected that mottled sculpin population persistence in the control streams would be driven largely by regional immigration and less by local processes such as recruitment and survival, the patterns were largely age-class specific. For instance, temporal variability in abundance was generally greater across all sites for mottled sculpin relative to brook trout when combining all age classes. On the other hand, much of the temporal variability in sculpin abundances was attributable to the highly fluctuating YOY class. Yet, comparison of the estimates of the C.V. in abundance for mottled sculpin from this study with those from considerably larger, third and fourth order southern Appalachian streams (Freeman et al. 1988, Grossman et al. 1990), likely within the core distribution of sculpin in that region, revealed that overall variability was nearly twice as high, on average, among sites in our system (e.g., mean C.V. [N-hat] for all age classes in this study $\approx 0.84$ versus $\max =0.44$, Freeman et al. 1988). Nonetheless, differences in adult C.V. were not as extreme, although they remained higher (mean C.V. $[\mathrm{N}-$ hat $]=0.67$ for small adults and 0.52 for large adults in this study, Freeman et al. 1988).

Immigration was the dominant driver of sculpin population growth in these streams, however, the extent to which it was the primary driver of population maintenance (i.e., $\lambda \geq 1$ ) differed between small and large adult sculpin. Small adult dynamics followed our expectation for sculpin in periphery streams as they were driven 
largely by immigration and survival was less important. Large adult dynamics, on the other hand, were driven largely by survival, and immigration was much less important. Nonetheless, immigration rates of large adult sculpin were still very high throughout the study. The dynamics of YOY sculpin, finally, were driven almost entirely by immigration, although we did not distinguish among in situ versus true immigration for YOY.

Finally, YOY survival was very low following immigration/recruitment, and local transition of YOY to the small adult class was very low. Thus, although survival was generally high enough to maintain the persistence of large adult sculpin throughout this study, because these populations were not effectively self-recruiting from the YOY class, the long term persistence of sculpin in these streams is likely dependent on immigration from the regional drainage network. Thus, from a metapopulation perspective, sculpin populations in these streams would likely be classified as functional "sinks" (Pulliam 1988). Thus, the overall local population dynamics of sculpin, among control sites, supported our a priori expectation concerning the dynamics of a species on the periphery of its distribution.

By comparison, temporal variability in brook trout population size was also high. Although we had no reference for comparison from Appalachian streams, the estimates across sites in this study (mean C.V. $[\mathrm{N}-$ hat $] \approx 0.66$ ) were on the upper end of the range of estimates provided from a meta-analysis covering a range of Rocky Mountain and Midwestern brook trout populations in Dauwalter et al. $(0.17 \leq \mathrm{C} . \mathrm{V}$. $[\mathrm{N}-$ hat $] \leq 0.82$, 2009). In contrast to sculpin, brook trout population dynamics across the control sites were far more locally driven and, assuming much of the YOY immigration was in situ, 
also self-recruiting. For instance, estimates of apparent survival $(\varphi)$ and instantaneous rates of survival $(\Phi)$ were generally higher for brook trout across all age classes compared to sculpin and were high enough to maintain persistence over some intervals. Immigration was also an important contributor, although rates were generally lower and less variable than for sculpin. Finally, local recruitment was also an important contributor to adult brook trout population dynamics and was a large proportion of the growth rate contributing to persistence from fall to spring in both years. Therefore, because these brook trout populations were self-recruiting and persistence was attributed largely to local processes, they could be characterized as functional "sources" (Pulliam 1998). Our results therefore support our a priori expectation for the population dynamics of species in the core of its distribution.

\section{Influence of the regional context}

Overall, our results clearly indicated that the regional context modified local population dynamics. First, recovery of both brook trout and mottled sculpin abundances in the removal sites was often faster and/or more complete when the sites were closer to their respective cores. The candidate model for estimating proportional recovery with the removal and location interaction effect was often well supported by the data. For brook trout, the interaction was well-supported for YOY, but the effect size was quite small. For large adults, however, the interaction was strongly supported (i.e., top model in the set) and the model averaged result suggested that, although the pre-removal abundance was never fully recovered in either the headwater or mainstem removal sites, on average, 
the headwater sites recovered a greater proportion of their abundance relative to the headwater control group. Mainstem control sites, nevertheless, had much higher abundances than the pre-removal level throughout the study.

On the other hand, there was very little support for the interactive effect of location and removal on small adult brook trout. One reason the removal-location interaction was strongest for large adults could have been related to their lower overall abundances throughout the study. Although, some studies have shown that larger bodied individuals tend to recover more quickly following disturbance (Lonzarich et al. 1998, Albanese et al. 2009), other similar studies have also shown that the least abundant taxa are also often the slowest to repopulate following disturbance (Meffe and Sheldon 1990, Albanese et al. 2009). Although the interactive effect was relatively weak for YOY and small adult brook trout, it may have been more apparent given a larger scale perturbation or over narrower sampling intervals.

The removal also highlighted the importance of the regional context to mottled sculpin population dynamics. Although there was no support for the removal-location interaction for YOY sculpin, it was included in the top model for small adult sculpin and also had strong support in the model for large adult recovery. Interestingly, recovery in mainstem sites for both classes of adults exceeded pre-removal levels as well as the levels of the control sites, yet headwater sites never fully recovered, on average. The slow or incomplete recovery of small adults in headwater systems is likely due to both their low abundances in those sites, on average, and restricted mobility (Petty and Grossman 2004, Albanese et al. 2009). Although the shortest distance to the lower or upper end of the perturbed reaches was only $100 \mathrm{~m}$, the average daily movement recorded for sculpin in a 
southern Appalachian stream was less than 5 m (Petty and Grossman 2004). Again, rarity and restricted mobility are likely the least desirable population characteristics for fast recovery of abundance (Albanese et al. 2009). Our study is not the first to experimentally demonstrate that proximity to a source of colonizers is an important determinant of recovery (Lonzarich et al. 2009). However, to our knowledge, it is the first to explicitly demonstrate, through capture-mark-recapture techniques, the extent to which immigration is the mechanism.

\section{Density dependence}

Recruitment and age class transitions

Our models provided very little support for a density reduction effect on either population growth rate or recruitment for brook trout. The forward-time probability of transition models did provide some support for an advantage in growth in the removal sites in terms of a slightly higher likelihood of transitioning among age classes in those sites. Although higher probabilities of transitioning among age classes should translate into a greater contribution of recruitment to population growth, the reverse-time model comparisons for estimating recruitment did not support a removal effect. One explanation for the discrepancy in these two related estimates may be related to temporary emigration. For instance, like apparent survival, probability of transition in the forward-time models was not conditional on remaining in the population over adjacent time steps. However, in the reverse-time formulation, $\gamma_{\mathrm{ji}}$ is interpreted as the 
probability that, given that an animal survived to time $t$, it was in the population and of a lower age class at time $t-1$. This suggests that, perhaps, seasonal migrants gained an advantage in growth among the removal sites that was not realized in less mobile individuals.

Nonetheless, our overall findings demonstrate that the local population processes affecting vital rates of brook trout in these very small streams were operating largely independent of density feedbacks over the course of the study. Likewise, the degree of variability among sites in both transition probabilities and the contribution of recruitment indicated that site to site variability in local environmental and/or physical conditions likely played a more meaningful role in local persistence than density feedbacks. On the other hand, numerous studies have detected density dependent growth in brook trout (Imre and Grant 2005), including in populations from this region (Utz et al. 2009). Thus, although we were unable to detect any substantial effect on population vital rates, density regulation was likely present, even if faint, in the populations we studied.

Conversely, we did find a slightly more substantial effect of density on the transition rates for YOY and small adult sculpin following the removal. However, the advantage in growth was only detected in the headwater removal sites. This interesting, location-specific, phenomenon may have been attributable the fact that it generally took adults longer to repopulate the headwater reaches compared to the mainstem reaches. Intraspecific competition, particularly interference by dominant large adults, is known to be a strong factor influencing sculpin growth among southern Appalachian streams where populations are dense (Petty and Grossman 2004, 2007, 2010). For instance, Petty and Grossman (2004) suggested that, when adult densities are low, smaller individuals should 
enjoy both greater mobility and higher growth rates. Thus, we suggest that, because adults generally recovered more slowly and less completely in the headwater areas, the YOY and small adults that were quickest to colonize post-disturbance realized a slight advantage in growth in those areas, which translated into a higher probability of transition to the older age classes. Conversely, the advantage was unable to be realized in mainstem removal sites due to a more rapid and more complete recovery.

As for brook trout, the reverse-time estimate of the contribution to recruitment did not support a density effect. Again, this may indicate that more migrant individuals in the local population are more likely to gain an advantage in growth. Petty and Grossman (2004), for instance, demonstrated that mobile juvenile sculpin generally exhibited higher growth rates than their less mobile counterparts in a southern Appalachian system.

Overall, the contribution of recruitment to population maintenance of small and large adults was very small in these streams and, thus, the minor density dependent feedback we observed in the probability of transition ultimately provided an insubstantial contribution to the local dynamics of sculpin throughout the study. Therefore, like brook trout, we suggest that local growth and recruitment within sculpin populations in these systems is maintained primarily by factors largely independent of density.

However, the conclusion that, with mottled sculpin, the removal effect was only apparent in the headwater sites where recovery was most delayed suggests that, for both brook trout and sculpin, there is some chance that a larger scale or more chronic perturbation may have elucidated a greater or more consistent response from growth. For instance, in a study of brook trout populations before and after a catastrophic debris flow in a similar-sized Virgina stream, Roghair et al. (2002) found significant differences in 
mean length at age for brook trout in the affected area in the first year following the disturbance. The affected area in that study, however, was more than 4 times the size of the areas receiving the experimental perturbations in our study. Nevertheless, the discrepancy in findings between our study and theirs may be related to the scale of the disturbances and, ultimately, may indicate that the temporal and/or spatial scale at which density dependent feedbacks become important to the local persistence of fishes in small streams is relatively large.

Survival

Interestingly, patterns in apparent survival among the removal sites over the first two to three intervals post-removal suggested the opposite pattern than would be expected from negative density dependence. For both species, apparent survival was substantially lower in the removal streams over those intervals for most age classes and also lower in streams closer to the respective cores for the two species. However, because apparent survival does not distinguish between losses due to death and losses due to emigration, it is likely that this pattern seen in the removal sites was due to an initial flux of transients following the removal. We believe these unexpected patterns in survival immediately post removal reflect an interesting contrast in patterns of movement in streams next to the core versus those next to a periphery stream for both species. Specifically, transience appeared to be higher (i.e., lower apparent survival) when adjacent to the core for both species. Interestingly, one explanation is that these transients were part of a group of potential colonists involved in an initial 'scramble' for 
space that resulted in quick turnover of individuals. Another explanation may be that the initial colonists were part of a group of more innately mobile segment of the greater population that may always be present, but transient, in the system, but less detectible when assessed in combination with a dominant group of local residents, most of which whom were removed in this case.

On the other hand, the data did suggest that apparent survival was slightly higher in the removal sites relative to controls in the second year post-removal over the interval spanning May to July for both YOY and small adult brook trout. There also appeared to be a slight advantage in survival in the mainstem removal sites relative to the controls over the interval from October 2006 to May 2007, although that effect was considerably weak to negligible for large adult brook trout. However, the instantaneous rates of survival did fully corroborate the findings for apparent survival and generally indicated no removal effect other than a slight advantage for YOY trout in removal streams in the second year. The slight discrepancy between the trends in apparent survival and the instantaneous estimates may, again, be related to temporary emigration. Specifically, as the unexpected pattern of lower apparent survival estimates over the first two intervals indicated, if animals in the removal sites were consistently moving in and out of the study area, any advantage in survival would not be captured by the instantaneous rate for the intervals over which they were unavailable for capture. However apparent survival estimates would account for an increased rate of survival for a cohort over an interval for which some may have left the study area so long as those individuals are captured alive at another date. 
Large adult sculpin demonstrated a similar pattern in survival to brook trout and, in fact, large adults in headwater sites demonstrated a slight advantage in survival, relative to headwater control sites, for each of the final two intervals. Again, this pattern in headwater sites may be related to the fact that these sites demonstrated the slowest and least complete recovery and, thus, below average densities persisted for a longer duration compared to other sites. Large adult sculpin also demonstrated an advantage in apparent survival in mainstem removal sites over the final interval relative to controls.

Interestingly, neither YOY nor small adult sculpin demonstrated a removal effect in apparent survival. In addition to the evidence for density regulation of brook trout survival, these results for large adult brook trout suggests that density regulation may be most evident when local dynamics are the dominant drivers of persistence or when in the core. Therefore, our expectation that density dependence would be stronger in the core versus the periphery was somewhat supported by the data although, the effect of density dependence was quite small. However, we didn't expect large adult sculpin persistence in these small streams to be so locally driven. For the YOY and small adult sculpin population segments, however, where regional processes dominated local population dynamics, density dependent apparent survival was not evident, as expected. Nonetheless, as for the results for brook trout, instantaneous survival rates did not corroborate the positive removal effect.

\section{Conclusions}


The overriding objective of this paper was to examine the roles of population density and watershed spatial location in the population dynamics of two Appalachian headwater stream fishes with the specific intent of advancing our understanding of the extent to which the local population dynamics of fishes in these small streams are influenced by local (i.e., population density and local physicochemical conditions) versus regional (i.e., immigration) processes and the extent to which those dynamics differ given the local (e.g., core/periphery or source/sink habitat) and regional context (i.e., position in the watershed or proximity to a source versus sink habitat). Our results pursuant of this objective can be characterized by three important findings. First, the extent to which local (i.e., survival, recruitment) versus regional (i.e., migration) processes influence population persistence likely depends on the local context or where the population is located relative to the core versus periphery of its distribution. Studies of other organisms have shown similar patterns in population dynamics over the range of geographic distributions (Cornutt et al. 1996, Williams et al. 2003). Cornutt et al. (1996), for example, demonstrated that populations of song sparrows in the mid-west were most abundant and least variable at the core of their distribution and abundance and variance increased from the core to the periphery, which they attributed to a likely source-sink dynamic. We suggest a similar dynamic for stream fishes at the watershed scale. For instance, our results strongly suggest that these small streams act as population "sources" for brook trout at the scale of the watershed and play an important role in the maintenance of populations at the watershed scale. Likewise, these small streams are likely "sink" habitats for mottled sculpin, overall, but may provide an important 
complement or supplement to the population at the scale of the watershed (Schlosser and Angermeier 1995).

A second important conclusion was that, while local processes had varying degrees of importance in the population persistence of each species, the regional context was also an important determinant of the local dynamic for both species. Throughout the study, immigration made up a large component of the population growth rate for both species, however, the extent of that importance was modified by the regional context. For both species, sites adjacent to the core generally realized a stronger regional influence than those adjacent to the periphery. This conclusion also supports a practical "sourcesink" view of fish populations at the watershed scale in this region (Pulliam 1988). While the local context (core/periphery or source/sink) is an important determinant of the extent to which local population dynamics are driven by local (survival and recruitment) versus regional (immigration) processes, the regional context (core/periphery or source/sink) also modifies the local dynamic.

Third, although our a priori expectation was that density dependence would be stronger in brook trout populations relative to sculpin, because their local persistence was expected to be more locally driven, we found little evidence of meaningful density feedbacks on vital rates in response to the perturbation from either species. As a result, we concluded that both brook trout and sculpin populations in these small systems are driven primarily by processes independent of density feedbacks. Rather, the dominant pattern in the data for both species emphasized more the importance of dispersal processes and the influence of local and regional spatial position on overall population persistence in these small Appalachian streams. The strong component of migration for 
both species is consistent with a highly mobile community in these small streams that is thought characteristic of fish assemblages in headwater or frequently disturbed habitats (Schlosser 1987). Likewise, the relatively weak influence of density feedbacks is generally expected under the hypothesis of Haldane (1956) who, again, suggested that density dependence should be weakest in unfavorable or variable environments.

In addition to the conclusions following from the overall objective, our study also resulted in some interesting, unexpected, findings. One of the most interesting and predominant trends in the data was a nearly linearly decreasing temporal trend in the immigration rate for both species among removal sites over the course of the study. This trend was coupled with a lagged increase in survival rates. These findings suggested that the immediate recovery of the experimentally perturbed reaches was due an influx of predominantly mobile fish and that the subsequent return to a more locally driven dynamic over time was due to delayed accumulation of a more sedentary segment. This suggests that population level movements in these systems may strongly follow a leptokurtic pattern (Rodriguez 2002, Petty et al. 2004) as a result of the tendency of these populations to be comprised of a segment of mobile and sedentary animals (Fraser et al. 2001, Petty et al. 2004). This view is consistent with the broader importance of dispersal in structuring populations and assemblages in this region (Petty et al. 2005, Martin, Chapters 1 and 3).

Finally, most studies of fish population recovery following disturbance have noted relatively rapid recovery similar to the extent we observed in this study. However, to our knowledge, no other studies have tracked the response of population vital rates to perturbation. While our results indicated that recovery of abundance was rather rapid, 
they also emphasized explicitly the extent to which immigration was important to recovery as well as the extent to which the regional context modifies the magnitude of immigration. This finding emphasizes the importance of immigration and drainage network connectivity in the maintenance of headwater stream fish populations. Thus, managers should seek to affect fish assemblages in these small Appalachian streams, first, through the restoration and protection of interconnected drainage networks. Moreover, our findings suggest that additional research into the spatial variability in population vital rates for other members of the broader community represents an important future direction for research that will be necessary for making management decisions related to the restoration and protection of species-specific habitats and dispersal corridors at the watershed scale. 


\section{References}

Akaike, H. 1974. A new look at the statistical model identification. IEE Transactions on Automatic Control 19:716-723.

Albanese, B., P. L. Angermeier, and J. T. Peterson. 2009. Does mobility explain variation in colonisation and population recovery among stream fishes? Freshwater Biology 54:1444-1460.

Andrewartha, H. G., and L. C. Birch. 1954. The distribution and abundance of animals. University of Chicago Press, Chicago, Illinois, USA.

Arnason, A. N. 1973. The estimation of population size, migration rates, and survival in a stratified population. Researches on Population Ecology 15:1-8.

Barbour M. T., J. Gerritsen, B.D. Snyder, and J.B. Stribling. 1999. Rapid bioassessment protocols for use in streams and wadeable rivers: periphyton, benthic macroinvertebrates and fish, Second edition. EPA 841-B-99-002. U.S. EPA; Office of Water; Washington, D.C.

Bevenger, G. S., and R. M. King. 1995. A pebble count procedure for assessing watershed cumulative effects. USDA Forest Service Research Paper RM-319: 117. 
Burnham, K. P., and D. R. Anderson. 2002. Model selection and inference: a practical information-theoretic approach, 2nd edition. Springer-Verlag, New York, New York, USA.

Clayton, J. L., E. S. Dannaway, R. Menendez, H. W. Rauch, J. J. Renton, S. M. Sherlock, and P. E. Zurbuch. 1998. Application of limestone to restore fish communities in acidified streams. North American Journal of Fisheries Management 18:347-360.

Curnutt, J. L., S. L. Pimm, and B. A. Maurer. 1996. Population variability of song sparrows in space and time. Oikos 76:131-144.

Dauwalter, D. C., F. J. Rahel, and K. G. Gerow. 2009. Temporal variation in trout populations: implications for monitoring and trend detection. Transactions of the American Fisheries Society 138:38-51.

Detenbeck, N.E., DeVore, P.W., Niemi, G.J., and Lima, A. 1992. Recovery of temperate stream fish communities from disturbance: a review of case studies and synthesis of theory. Environ. Manage. 16: 33-53. Dunham, J. B., G. L. Vinyard, and B. E. Reiman. 1997. Habitat fragmentation and extinction risk of Lahontan cutthroat trout. North American Journal of Fisheries Management 17: 1126-1133. 
Dunham, J. B., M. M. Peacock, B. E. Reiman, and R. E. Schroeter. 1999. Local and geographic variability in the distribution of stream-living Lahontan cutthroat trout. Transactions of the American Fisheries Society 128: 875-889.

Eaton, J. G., J. H. McCormick, B. E. Goodno, D. G. O'Brien, H. G. Stefany, M. Hondzo, and R. M. Scheller. 1995. A field information-based system for estimating fish temperature tolerances. Fisheries 20:10-18.

Falke, J. A., and K. D. Fausch. From metapopulations to metacommunities: linking theory with empirical observations of the spatial population dynamics of stream fishes. American Fisheries Society Symposium.

Flebbe, P. A. 1994. A regional view of the margin: salmonid abundance and distribution in the southern Appalachian Mountains of North Carolina and Virginia. Transactions of the American Fisheries Society 123:657-667.

Freeman, M. C., M. K. Crawford, J. C. Barrett, D. E. Facey, M. G. Flood,]. Hill, D.J. Stouder, and G. D. Grossman. 1988. Fish assemblage stability in a Southern Appalachian stream. Canadian Journal of Fisheries Aquatic Sciences 45: 19491958.

Freund, J. G. 2004. Local and regional impairment of fish assemblages in a mined Appalachian watershed. Doctoral Dissertation. West Virginia Univeristy, Morgantown, WV, USA. 
Freund, J. G., and J. T. Petty. 2007. Response of Fish and Macroinvertebrate Bioassessment Indices to Water Chemistry in a Mined Appalachian Watershed. Environmental Management 39:707-720.

Gowan, C., and K. D. Fausch. 1996. Long-term demographic responses of trout populations to habitat manipulation in six Colorado streams. Ecological Applications 6: 931-946.

Grenouillet, G., D. Pont, and C. Herisse. 2004. Within-basin fish assemblage structure: the relative influence of habitat versus stream spatial position on local species richness. Canadian Journal of Fisheries and Aquatic Sciences 61:93-102.

Grossman, G. D., J. F. Dowd, and M. C. Crawford. 1990. Assemblage stability in stream fishes: a review. Environmental Management 14: 661-671.

Grossman, G. D., R. E. Ratajczak Jr., J. T. Petty, M. D. Hunter, J. T. Peterson, and G. Grenouillet. 2006. Population dynamics of mottled sculpin, Pisces, in a variable environment - information theoretic approaches. Ecological Monographs 76:217234. 
Grossman, G. D., R. E. Ratajczak, C. M. Wagner, and J. T. Petty. 2010. Dynamics and regulation of the southern brook trout (Salvelinus fontinalis) population in an Appalachian stream. Freshwater Biology 9999.

Haldane, J. B. S. 1953. Animal populations and their regulation. New Biology 15:9-24.

Hansbarger, J. L., Petty, J. T., and P. M. Mazik. 2010. Microhabitat use by brook trout inhabiting small tributaries and a large river mainstem: implications for stream habitat restoration in the central Appalachians.

Hense, Z. 2007. Stream fish populations in a watershed scale context for fish community dynamics in central Appalachian watersheds. Master's Thesis. West Virginia University, Morgantown, WV, USA.

Hitt, N. P., and P. L. Angermeier. 2008a. Evidence for fish dispersal from spatial analysis of stream network topology. Journal of the North American Benthological Society 27:304-320.

Hixon, M. A., S. W. Pacala, and S. A. Sandin. 2002. Population regulation: historical context and contemporary challenges of open vs. closed systems. Ecology 83:1490-1508. 
Huggins, R.M. 1991. Some practical aspects of a conditional likelihood approach to capture experiments. Biometrics. 47: 725-732.

Imre, I., J. W. A. Grant, and R. A. Cunjak. 2005. Density-dependent growth of young-ofthe-year Atlantic salmon, Salmo salar, in Catamaran Brook, New Brunswick. Journal of Animal Ecology 74:508-516.

Labbe, T. R., and K. D. Fausch. 2000. Dynamics of intermittent stream habitat regulate persistence of a threatened fish at multiple scales. Ecological Applications 10:1774-1791.

Lamothe, P. J. 2002. Spatial population dynamics of brook trout (Salvelinus fontinalis) in a central Appalachian watershed. Master's Thesis. West Virginia University, Morgantown, WV, USA.

Lebreton, J. D., K. P. Burnham, J. Clobert, and D. R. Anderson. 1992. Modeling survival and testing biological hypotheses using marked animals: a unified approach with case studies. Ecological Monographs. 62: 67-118.

Levin, S. A. 1976. Population dynamic models in heterogeneous environments. Annual Review of Ecology and Systematics 7:287-311. 
Liller, Z. W. 2006. Spatial variation in brook trout (Salvelinus fontinalis) population dynamics and juvenile recruitment potential in an Appalachian watershed. Master's Thesis. West Virginia University, Morgantown, WV, USA.

Lobón-Cerviá, J. 2007. Numerical changes in stream-resident brown trout (Salmo trutta): uncovering the roles of density-dependent and density-independent factors across space and time. Canadian Journal of Fisheries \& Aquatic Sciences 64:1429-1447.

Lonzarich, G. D., M. W. Warren, and M. R. E. Lonzarich. 1998. Effects of habitat isolation on the recovery of fish assemblages in experimentally defaunated stream pools in Arkansas. Canadian Journal of Fisheries and Aquatic Sciences 55: 21412149.

Martin, R. W., and J. T. Petty. 2009. Local stream temperature and drainage network topology interact to influence the distribution of smallmounth bass and brook trout in a central Appalachian watershed. Journal of Freshwater Ecology 24:497508.

Marschall, E. A. and L. B. Crowder. 1996. Assessing population responses to multiple anthropogenic effects: a case study with brook trout. Ecological Applications 6: $152-167$. 
McClurg, S. E., J. T. Petty, P. M. Mazik, and J. L. Clayton. 2007. Stream ecosystem response to limestone treatment in acid impacted watersheds of the Allegheny Plateau. Ecological Applications 17:1087-1104.

Meffe G.K. \& Sheldon A.L. (1990) Post-defaunation recovery of fish assemblages in southeastern blackwater streams. Ecology, 71, 657-667.

Murdoch, W. W. 1994. Population regulation in theory and practice. Ecology 75:271287.

Niemi, G.J., DeVore, P., Detenbeck, N., Taylor, D., Lima, A., Pastor, J., Yount, J.D., and Naiman, R.J. 1990. Overview of case studies on recovery of aquatic systems from disturbance. Environ. Manage. 14: 571-588.

Nichols, J. D., R. W. Morris, C. Brownie, and K. H. Pollock. 1986. Sources of variation in extinction rates, turnover and diversity of marine invertebrate families during the Paleozoic. Paleobiology 12:421-432.

Nichols, J. D., J. E. Hines, J. Lebreton, and R. Pradel. 2000. Estimation of contributions to population growth: a reverse-time capture-recapture approach. Ecology 81: $3362-3376$.

Nicholson, A. J. 1933. The balance of animal populations. Journal of Animal Ecology 2:132-178. 
Nicholson, A. J. 1957. The self-adjustment of populations to change. Cold Spring Harbor Symposium on Quantitative Biology 22:153-172.

Parker, K. R., and J. A. Weins. 2005. Assessing recovery following environmental accidents: environmental variation, ecological assumptions, and strategies. Ecological Applications 15: 2037-2051.

Peterson J.T. \& Bayley P.B. 1993. Colonization rates of fishes in experimentally defaunated warmwater streams. Transactions of the American Fisheries Society, 122, 199-207.

Peterson, D.P., K.D. Fausch, and G.C. White. 2004. Population ecology of an invasion: effects of brook trout on native cutthroat trout. Ecological Applications 14: 754772.

Petty, J. T., P. J. Lamothe, and P. M. Mazik. 2005. Spatial and seasonal dynamics of brook trout populations inhabiting a central Appalachian watershed. Transactions of the American Fisheries Society 134:572-587.

Petty, J. T., and D. Thorne. 2005. An ecologically based approach to identifying restoration priorities in an acid-impaired watershed. 
Petty, J. T., and G. D. Grossman. 2009. Giving-up densities and ideal pre-emptive patch use in a predatory benthic fish. Freshwater Biology 9999.

Platt, J. R. 1964. Strong Inference: Certain systematic methods of scientific thinking may produce much more rapid progress than others. Science 146:347-353.

Pollock, K. H., D. L. Solomon, and D. S. Robson. 1974. Tests for mortality and recruitment in a K-sample tag-recapture experiment. Biometrics 30:77-87.

Poplar-Jeffers, I. O. 2005. Culvert inventory and effects on fish assemblages in a central Appalachian watershed. Master's Thesis. West Virginia University, Morgantown, WV, USA.

Poplar-Jeffers, I. O., J. T. Petty, J. T. Anderson, S. J. Kite, M. P. Strager, and R. H. Fortney. 2008. Culvert Replacement and Stream Habitat Restoration: Implications from Brook Trout Management in an Appalachian Watershed, U.S.A. Restoration Ecology 17:404-413.

Pradel, R. 1996. Utilization of capture-mark-recapture for the study of recruitment and population growth rate. Biometrics 52:703-709.

Pulliam, H. R. 1988. Sources, sinks, and population regulation. American Naturalist 132:652-661. 
Roghair, C. N., C. A. Dolloff, and M. K. Underwood. 2002. Response of a brook trout population and instream habitat to a catastrophic flood and debris flow. Transactions of the American Fisheries Society 131:718-730.

Roughgarden, J., T. Pennington, and S. Alexander. 1994. Dynamics of the rocky intertidal zone with remarks on generalization in ecology. Philosophical Transactions of the Royal Society of London B 343:79-85.

Sagarin, R. D., S. D. Gaines, and B. Gaylord. 2006. Moving beyond assumptions to understand abundance distributions across the ranges of species. Trends in Ecology \& Evolution 21:524-530.

Schlosser, I. J. 1991. Stream fish ecology: a landscape perspective. BioScience 41:704712.

Schlosser, I. J. 1987. The role of predation in age- and size-related habitat use by stream fishes. Ecology 68:651-659.

Schlosser, I. J., and P. L. Angermeier. 1995. Spatial variation in demographic processes of lotic fishes: conceptual models, empirical evidence, and implications for conservation. Page 435 in J. L. Nielsen, editor. Evolution and the Aquatic 
Ecosystem: Defining Unique Units in Population Conservation. American Fisheries Society, Bethesda, Maryland, USA.

Schwarz, C. J., J. F. Schweigert, and A. N. Arnason. 1993. Estimating migration rates using tag-recovery data. Biometrics 49:177-193.

Schwarz, C. F. 2005. Multistate models. Pages $165-195$ in S. C. Amstrup, T. L. McDonald, and B. F. J. Manly, editors. Handbook of capture-recapture analysis. Princeton University Press, Princeton, New Jersey.

Snodgrass, J. W., and G. K. Meffe. 1998. Influence of beavers on stream fish assemblages: effects of pond age and watershed position. Ecology 79:928-942.

Utz, R. M., and K. J. Hartman. 2006. Temporal and spatial variation in the energy intake of a brook trout (Salvelinus fontinalis) population in an Appalachian watershed. Canadian Journal of Fisheries and Aquatic Sciences 63: 2675-2686.

Utz, R. M., and K. J. Hartman. 2009. Density-dependent individual growth and size dynamics of central Appalachian brook trout (Salvelinus fontinalis). Canadian Journal of Fisheries and Aquatic Sciences 66: 1072-1080.

Vannote, R. L., G. W. Minshall, K. W. Cummins, J. R. Sedell, and C. E. Cushing. 1980. The river continuum concept. Canadian Journal of Fisheries and Aquatic Sciences 37: 130-137. 
Wiens, J. A., R. H. Day, S. M. Murphy, and K. R. Parker. 2004. Changing habitat and habitat use by birds after the Exxon Valdez oil spill, 1989-2001. Ecological Applications 14:1806-1825.

White, G.C., and K.P. Burnham. 1999. Program MARK: survival estimation from populations of marked animals. Bird Study 46 Supplement: 120-138.

Whitworth, W.E., and Strange, R.J. 1983. Growth and production of sympatric brook and rainbow trout in an Appalachian stream. Trans. Am. Fish. Soc. 112(4): 469-475.

Wigington, P. J., Jr., J. P. Baker, D. R. DeWalle, W. A. Kretser, P. S. Murdoch, H. A. Simonin, J. Van Sickle, M. K. McDowell, D. V. Peck, and W. R. Barchet. 1996. Episodic acidification of small streams in the northeast United States: episodic response project. Ecological Applications 6: 374-388.

Williams, B. K., J. D. Nichols, and M. J. Conroy, editors. 2002. Analysis and management of animal populations. Academic Press, San Diego, California. 


\section{Tables}

Table 1. A priori expectations for the qualitative comparison of individual population parameters between brook trout and mottled sculpin under the assumption that local population dynamics differ in the core versus the periphery of a species' distribution. These expectations address the goal of the study concerning determining the extent to which population dynamics differ in the core (i.e., brook trout) versus the periphery (i.e., mottled sculpin) of a species' distribution (i.e., goal 1).

\begin{tabular}{cccccc} 
& $\begin{array}{c}\text { Temporal } \\
\text { variability in } \\
\text { abundance }\end{array}$ & Survival & \multicolumn{2}{c}{$\begin{array}{c}\text { Contribution of } \\
\text { survival to } \lambda\end{array}$} & $\begin{array}{c}\text { Contribution of } \\
\text { recruitment to } \lambda\end{array}$ immigration to $\lambda$ \\
\hline imecies & Contribution of \\
\hline Brook trout & Lower & Higher & Higher & Higher & Lower \\
Mottled sculpin & Higher & Lower & Lower & Lower & Higher \\
\hline
\end{tabular}


Table 2. A priori expectations for the expected response of brook trout (top) and mottled sculpin (bottom) to the experimental removal under the assumption that the regional context has an influence on local population dynamics. These expectations address the goal of the study concerning determining the extent to which local population dynamics differ given the regional context (i.e., goal 2).

\begin{tabular}{cccc}
\hline & \multicolumn{3}{c}{ Brook trout } \\
\hline Site type & $\begin{array}{c}\text { Recovery of } \\
\text { abundance }\end{array}$ & $\begin{array}{c}\text { Population } \\
\text { growth rate }\end{array}$ & $\begin{array}{c}\text { Contribution of } \\
\text { immigration to } \lambda\end{array}$ \\
\hline $\begin{array}{c}\text { Mainstem } \\
\text { Removal }\end{array}$ & $\begin{array}{c}\text { Slower/Less } \\
\text { complete }\end{array}$ & Lower & Lower \\
$\begin{array}{c}\text { Headwater } \\
\text { Removal }\end{array}$ & $\begin{array}{c}\text { Faster/More } \\
\text { complete }\end{array}$ & Higher & Higher \\
\hline
\end{tabular}

\begin{tabular}{cccc}
\hline & \multicolumn{2}{c}{ Mottled sculpin } \\
\hline Site type & $\begin{array}{c}\text { Recovery of } \\
\text { abundance }\end{array}$ & $\begin{array}{c}\text { Population } \\
\text { growth rate }\end{array}$ & $\begin{array}{c}\text { Contribution of } \\
\text { immigration to } \lambda\end{array}$ \\
\hline $\begin{array}{c}\text { Mainstem } \\
\text { Removal }\end{array}$ & $\begin{array}{c}\text { Faster/More } \\
\text { complete }\end{array}$ & Lower & Lower \\
$\begin{array}{c}\text { Headwater } \\
\text { Removal }\end{array}$ & $\begin{array}{c}\text { Slower/Less } \\
\text { complete }\end{array}$ & Higher & Higher \\
\hline
\end{tabular}


Table 3. A priori expectations for the expected response of brook trout (top) and mottled sculpin (bottom) to the experimental removal under the assumption that density dependent feedbacks are an important feature driving local population dynamics (i.e., goal 3).

\begin{tabular}{ccc}
\hline & \multicolumn{2}{c}{ Brook trout } \\
\hline Treatment & Survival & Growth \\
\hline Control & Lower & Lower \\
Removal & Higher & Higher \\
\hline & \\
\hline \multicolumn{3}{c}{ Mottled sculpin } \\
\hline Treatment & Survival & Growth \\
\hline Control & Lower & Lower \\
Removal & Higher & Higher \\
\hline
\end{tabular}


Table 4. A priori expectations for the expected difference in effect size of brook trout (top) and mottled sculpin (bottom) response to the experimental removal under the assumption that density dependent feedbacks are an important feature driving local populations in this system and the assumption that density dependent feedbacks are stronger in the core of a species' distribution than the periphery.

\begin{tabular}{ccc}
\hline Species & $\begin{array}{c}\text { Removal effect } \\
\text { on survival }\end{array}$ & $\begin{array}{c}\text { Removal effect } \\
\text { on transition }\end{array}$ \\
\hline Brook trout & Larger & Larger \\
Mottled sculpin & Smaller & Smaller \\
\hline
\end{tabular}


Table 5. List of candidate models used for predicting two dependent variables (DV) proportional recovery of abundance $\left(\ln \left(\mathrm{R}_{\mathrm{p}}\right)=\ln \left(\mathrm{N}_{\mathrm{t}} / \mathrm{N}_{1}\right)\right.$ and the per capita population growth rate $\left(\mathrm{r}=\ln (\lambda)=\ln \left(\mathrm{N}_{\mathrm{t}} / \mathrm{N}_{\mathrm{t}+1}\right)\right)$ - for three age classes of brook trout and mottled sculpin following the density perturbation. The global model is highlighted in bold.

\begin{tabular}{|c|c|}
\hline Model & Description \\
\hline Local $+\mathrm{t}$ & $\begin{array}{l}\text { DV is independent of either the removal or location } \\
\text { effects. }\end{array}$ \\
\hline Local + g + t & DV is an additive function of the removal factor. \\
\hline Local $+\mathrm{I}+\mathrm{t}$ & DV is an additive function of the location factor. \\
\hline Local $+g+t+g^{*} t$ & $\begin{array}{l}\mathrm{DV} \text { is a function of the removal factor and that effect } \\
\text { varies depending on the sampling interval. }\end{array}$ \\
\hline Local $+1+t+I^{*} t$ & $\begin{array}{l}\text { DV is a function of the location factor and that effect } \\
\text { varies depending on the sampling interval. }\end{array}$ \\
\hline Local $+g+I+t+I^{*} t$ & $\begin{array}{l}\text { DV is an additive function of the removal factor. DV is } \\
\text { also function of the location effect and the effect of } \\
\text { location varies with sampling interval and is } \\
\text { independent of the removal effect }\end{array}$ \\
\hline Local $+g+I+t+g^{*} t$ & $\begin{array}{l}\text { DV is a function of the removal factor and that effect } \\
\text { varies depending on the sampling interval. DV is also } \\
\text { an additive function of the location effect and is } \\
\text { independent of the removal effect. }\end{array}$ \\
\hline Local $+g+I+t+g^{*} \mid$ & $\begin{array}{l}\text { DV is a function of the removal factor and its effect is } \\
\text { dependent on the location factor (and vise versa). The } \\
\text { extent to which the removal effect is dependent on the } \\
\text { location effect is consistent throughout the study }\end{array}$ \\
\hline Local $+g+I+t+g^{*} \mid+I^{*} t$ & $\begin{array}{l}\text { DV is a function of the removal factor and the effect is } \\
\text { dependent on the location factor (and vise versa). The } \\
\text { extent to which the removal effect is dependent on the } \\
\text { location effect is consistent throughout the study. DV is } \\
\text { also a function of the location effect, but the effect of } \\
\text { location varies with sampling interval and the removal } \\
\text { effect. }\end{array}$ \\
\hline Local $+g+I+t+g^{*} t+I^{*} t$ & $\begin{array}{l}\text { DV is a function of the removal factor, but varies } \\
\text { depending on sampling interval. DV is also a function } \\
\text { of the location effect and the effect of location varies } \\
\text { with sampling interval and is independent of the } \\
\text { removal effect. }\end{array}$ \\
\hline Local $+g+I+t+g^{*} \mid+g^{*} t$ & $\begin{array}{l}\text { DV is a function of the removal factor and the removal } \\
\text { effect is dependent on the location factor (and vise } \\
\text { versa) and on the sampling interval. The extent to } \\
\text { which the removal effect is dependent on the location } \\
\text { effect is consistent throughout the study. DV is also a } \\
\text { function of the location factor and the effect of location } \\
\text { is consistent over time. }\end{array}$ \\
\hline
\end{tabular}


Local $+g+I+t+g^{*} I+g^{*} t+I^{*} t$

Local $+g+I+t+g^{*} I+g^{*} t+I^{*} t+g^{*} I^{\star} t$
DV is a function of the removal factor and the removal effect is dependent on the location effect (and vise versa) and the sampling interval. The extent to which the removal effect is dependent on the location effect is consistent throughout the study. DV is also a function of the location factor and the effect of location is dependent on the sampling interval and removal effect.

DV is a function of the removal factor and the removal effect is dependent on the location effect (and vise versa) and the sampling interval. The extent to which the removal effect is dependent on the location effect is dependent on the sampling interval. DV is also a function of the location factor and the effect of location is dependent on the sampling interval and the removal effect. 
Table 6. Summary of mean values and standard errors for all chemical variables measured at each site throughout the study. Type $=$ (MC) mainstem - control, (MT) mainstem - removal treatment, (HC) headwater - control, (HT) headwater - removal, SpCond = specific conductivity.

\begin{tabular}{lcccccc} 
Site & Type & pH & SpCond & Hardness & Ca Hardness & Alkalinity \\
\hline Lynn Run & MC & $7.2(0.17)$ & $46.4(5.1)$ & $40.0(7.1)$ & $24.0(4.5)$ & $20.0(5.9)$ \\
Zinn Hollow & MC & $6.9(0.10)$ & $26.7(3.4)$ & $30.0(6.8)$ & $20.0(0.0)$ & $10.8(1.5)$ \\
Nan's Branch & MC & $7.9(0.23)$ & $141.8(17.7)$ & $108.0(16.7)$ & $80.0(10.0)$ & $80.0(7.9)$ \\
Otis Hollow & MT & $7.0(0.13)$ & $24.3(3.2)$ & $40.0(11.6)$ & $20.0(0.0)$ & $8.8(1.0)$ \\
Lick Drain & MT & $7.1(0.34)$ & $41.5(5.9)$ & $40.0(8.2)$ & $25.0(5.0)$ & $16.3(2.4)$ \\
Laurel Lick Run & MT & $6.9(0.12)$ & $30.3(7.4)$ & $46.7(10.8)$ & $23.3(3.7)$ & $14.2(3.0)$ \\
Mudlick Run & HC & $7.0(0.20)$ & $37.0(5.0)$ & $36.7(9.8)$ & $23.3(3.7)$ & $14.2(2.5)$ \\
Big Run & HC & $7.0(0.17)$ & $24.5(3.6)$ & $35.0(7.8)$ & $20.0(0.0)$ & $11.3(1.0)$ \\
Glady Fork & HC & $6.9(0.15)$ & $25.7(3.6)$ & $30.0(4.9)$ & $20.0(0.0)$ & $11.7(2.3)$ \\
Swallow Rock Run & HT & $7.4(0.23)$ & $25.8(4.3)$ & $35.0(8.6)$ & $20.0(0.0)$ & $10.0(1.8)$ \\
Tanner Run & HT & $6.9(0.11)$ & $31.0(3.3)$ & $40.0(8.2)$ & $20.0(0.0)$ & $14.0(1.7)$ \\
Daniels Run & HT & $6.9(0.11)$ & $24.4(3.4)$ & $32.0(8)$ & $20.0(0.0)$ & $12.0(1.2)$
\end{tabular}


Table 7. Summary of mean principal component scores (mean across all dates) for individual sites (top) and factor loadings for individual principal components (bottom). Principal component 1 (PC1) explained $74 \%$ of the variance in the dataset and was the only component interpreted. Only loading factors with values $>|0.4|$ were used to interpret the component. Type $=(\mathrm{MC})$ mainstem control, (MT) mainstem treatment, (HC) headwater control, (HT) headwater treatment, SpCond = specific conductivity.

\begin{tabular}{lcc} 
Site & Type & PC1 \\
\hline Lynn Run & MC & $-0.095(0.374)$ \\
Zinn Hollow & MC & $0.866(0.094)$ \\
Nan's Branch & MC & $-6.102(0.389)$ \\
Otis Hollow & MT & $0.778(0.137)$ \\
Lick Drain & MT & $0.108(0.468)$ \\
Laurel Lick Run & MT & $0.453(0.290)$ \\
Mudlick Run & HC & $0.362(0.313)$ \\
Big Run & HC & $0.737(0.130)$ \\
Glady Fork & HC & $0.883(0.166)$ \\
Swallow Rock Run & HT & $0.455(0.212)$ \\
Tanner Run & HT & $0.572(0.060)$ \\
Daniels Run & HT & $0.886(0.057)$
\end{tabular}

\begin{tabular}{lc}
\multicolumn{1}{c}{ Variable } & PC1 loading \\
\hline $\mathrm{pH}$ & - \\
SpCond & -0.489 \\
Total Hardness & - \\
Ca Hardness & -0.493 \\
Alkalinity & -0.501 \\
\hline
\end{tabular}


Table 8. Values for physical habitat variables measured at each site and used in principal component analysis of local physical habitat conditions (Table 4). Type $=(\mathrm{MC})$ mainstem control, $(\mathrm{MT})$ mainstem treatment, $(\mathrm{HC})$ headwater control, $(\mathrm{HT})$ headwater treatment, SpCond = specific conductivity, M_DEP = mean thalweg depth, CV_DEP = coefficient of variation in thalweg depth, M_ACV = mean average current velocity, $\mathrm{CV}_{-}^{-} \mathrm{ACV}=$ coefficient of variation in average current velocity, PRP_POOL $=$ proportion of total area as pool channel units, M_DCOV = mean distance to cover feature, CV_DCOV = coefficient of variation in distance to cover feature, $\mathrm{D} 50=$ median substrate size, $\mathrm{LWD}=$ woody debris volume

\begin{tabular}{|c|c|c|c|c|c|c|c|c|c|c|}
\hline Site & Type & M_DEP & CV_DEP & M_ACV & CV_ACV & PRP_POOL & M_DCOV & CV_DCOV & D50 & LWD \\
\hline Nan's Branch & MC & 10.53 & 0.55 & 0.25 & 0.63 & 0.23 & 1.09 & 0.97 & 3.576 & 7.070 \\
\hline Lynn Run & MC & 7.65 & 0.46 & 0.19 & 0.69 & 0.17 & 1.33 & 0.76 & 277 & 27.716 \\
\hline Zinn Hollow & $\mathrm{MC}$ & 10.19 & 0.41 & 0.27 & 0.57 & 0.22 & 0.90 & 0.70 & 36.67 & 24.072 \\
\hline Lick Drain & MT & 10.89 & 1.11 & 0.17 & 0.91 & 0.21 & 2.65 & 1.15 & 205.2 & 62.327 \\
\hline Laurel Lick Run & MT & 11.58 & 0.69 & 0.16 & 0.74 & 0.25 & 0.50 & 0.95 & 185.6 & 29.809 \\
\hline Otis Hollow & MT & 10.61 & 0.40 & 0.46 & 0.49 & 0.07 & 0.63 & 0.74 & 25.08 & 27.460 \\
\hline Mudlick Run & $\mathrm{HC}$ & 10.83 & 0.66 & 0.27 & 0.59 & 0.20 & 1.24 & 0.93 & 98.94 & 28.408 \\
\hline Big Run & $\mathrm{HC}$ & 11.97 & 0.44 & 0.30 & 0.59 & 0.25 & 0.70 & 0.75 & 57.73 & 28.763 \\
\hline Glady Fork & $\mathrm{HC}$ & 13.84 & 0.29 & 0.58 & 0.48 & 0.19 & 0.89 & 0.66 & 48.02 & 29.551 \\
\hline Daniel's Run & $\mathrm{HT}$ & 10.59 & 0.71 & 0.16 & 0.77 & 0.33 & 1.23 & 0.85 & 15.6 & 28.119 \\
\hline Tanner Run & $\mathrm{HT}$ & 11.09 & 0.64 & 0.28 & 0.50 & 0.39 & 1.33 & 0.74 & 34.63 & 15.529 \\
\hline Swallow Rock Run & $\mathrm{HT}$ & 12.02 & 0.50 & 0.33 & 0.67 & 0.29 & 0.61 & 0.82 & 81.31 & 20.698 \\
\hline
\end{tabular}


Table 9. Summary of principal component scores by site for analysis of local physical habitat data (top) and summary of factor loadings for individual principal components (bottom). Principal component 1 (PC1) explained $58 \%$ of the variance in the dataset followed by PC2 (17\%) and PC3 (13\%). Only these three components were interpreted. Only loading factors with values $>|0.4|$ were used to interpret the components. M_DEP $=$ mean thalweg depth, CV_DEP = coefficient of variation in thalweg depth, $\mathrm{M} \_\mathrm{ACV}=$ mean average current velocity, CV_ACV $=$ coefficient of variation in average current velocity, PRP_POOL $=$ proportion of total area as pool channel units, $\mathrm{M} \_\mathrm{DCOV}=$ mean distance to cover feature, CV_DCOV = coefficient of variation in distance to cover feature, $\mathrm{D} 50=$ median substrate size, $\mathrm{LWD}=$ large woody debris volume.

\begin{tabular}{ccccc} 
Site & Type & PC1 & PC2 & PC3 \\
\hline Nan's Branch & MC & -0.815 & 0.021 & -0.157 \\
Lynn Run & MC & -0.040 & -0.806 & -2.739 \\
Zinn Hollow & MC & 1.159 & -0.006 & -0.908 \\
Lick Drain & MT & -6.202 & 1.691 & 0.416 \\
Laurel Lick Run & MT & -1.105 & -0.658 & 0.529 \\
Otis Hollow & MT & 2.153 & 1.943 & -1.259 \\
Mudlick Run & HC & -0.656 & 0.381 & -0.128 \\
Big Run & HC & 1.689 & -0.686 & 0.517 \\
Glady Fork & HC & 3.263 & 1.946 & 1.404 \\
Daniel's Run & HT & -0.896 & -2.068 & 0.329 \\
Tanner Run & HT & 0.670 & -1.311 & 1.014 \\
Swallow Rock Run & HT & 0.778 & -0.447 & 0.984
\end{tabular}

\begin{tabular}{cccc} 
Variable & PC1 & PC2 & PC3 \\
\hline M_DEP & - & - & 0.80 \\
CV_DEP & -0.42 & - & - \\
M_ACV & - & 0.50 & - \\
CV_ACV & - & - & - \\
PRP_POOL & - & -0.60 & 0.52 \\
M_DCOV & - & - & - \\
CV_DCOV & -0.41 & - & - \\
D50 & -0.42 & - & - \\
LWD & - & 0.49 & - \\
\hline
\end{tabular}


Table 10. Values for additional local habitat covariates measured for each site. PAREA $=$ proportion of spring wetted area (May 2007) in early fall (August 2007), CANP = mean percent canopy cover, MWAT = maximum weekly average temperature (MWAT). Sites are ordered from lowest to highest habitat PC1 score. Covariate values for MWAT listed in bold were estimates assigned for missing data based on the mean value of all other sites. Values in bold face type indicate sites where temperature logging devices were lost, resulting in those sites receiving the mean value for MWAT for all sites with data.

\begin{tabular}{ccccc} 
SITE & TYPE & PRP_AREA & CANP & MWAT \\
\hline Nan's Branch & MC & 0.88 & 88 & 15.82 \\
Lynn Run & MC & 1.06 & 83 & 18.32 \\
Zinn Hollow & MC & 0.25 & 95 & 16.46 \\
Lick Drain & MT & 1.05 & 79 & 17.74 \\
Laurel Lick Run & MT & 0.53 & 88 & 18.13 \\
Otis Hollow & MT & 0.85 & 94 & 16.02 \\
Mudlick Run & HC & 1.06 & 79 & $\mathbf{1 7 . 3 9}$ \\
Big Run & HC & 0.64 & 96 & $\mathbf{1 7 . 3 9}$ \\
Glady Fork & HC & 0.25 & 92 & 17.86 \\
Daniel's Run & HT & 0.45 & 78 & 18.3 \\
Tanner Run & HT & 0.89 & 85 & 17.53 \\
Swallow Rock Run & HT & 0.45 & 94 & 17.7
\end{tabular}


Table 11. Summary of model selection results for estimating capture probability of brook trout. age = YOY, small adult or large adult. age $(2)=$ YOY or adult (large + small). $()=$. constant model. Models in boldface accounted for at least $95 \%$ of the support based on the cumulative Akaike weights and were used for model averaging of model coefficients and parameter estimates.

\begin{tabular}{cccccccc} 
Model & AICc & Delta AICc & AICc Weights & Model Likelihood & Num. Par & Deviance & Cum. Weight \\
\hline \{age(2) + site + date\} & $\mathbf{3 0 4 0 . 4 8 3}$ & $\mathbf{0}$ & $\mathbf{0 . 4 3 5}$ & $\mathbf{1}$ & $\mathbf{1 8}$ & $\mathbf{8 6 0 0 . 7 9 3}$ & $\mathbf{0 . 4 3 5}$ \\
\{site + date\} & $\mathbf{3 0 4 1 . 4 4 0}$ & $\mathbf{0 . 9 5 7}$ & $\mathbf{0 . 2 7 0}$ & $\mathbf{0 . 6 2 0}$ & $\mathbf{1 7}$ & $\mathbf{8 6 0 3 . 7 6 1}$ & $\mathbf{0 . 7 0 4}$ \\
\{age + site + date\} & $\mathbf{3 0 4 1 . 5 6 7}$ & $\mathbf{1 . 0 8 4}$ & $\mathbf{0 . 2 5 3}$ & $\mathbf{0 . 5 8 2}$ & $\mathbf{1 9}$ & $\mathbf{8 5 9 9 . 8 6 4}$ & $\mathbf{0 . 9 5 7}$ \\
age + date\} & 3046.925 & 6.442 & 0.017 & 0.040 & 8 & 8627.322 & 0.975 \\
\{date\} & 3047.450 & 6.967 & 0.013 & 0.031 & 6 & 8631.857 & 0.988 \\
\{age(2) + date\} & 3047.665 & 7.182 & 0.012 & 0.028 & 7 & 8630.067 & 1.000 \\
$\{\}$. & 3090.897 & 50.414 & 0 & 0 & 1 & 8685.317 & 1.000 \\
$\{$ site\} & 3091.497 & 51.014 & 0 & 0 & 12 & 8663.867 & 1.000 \\
\{age(2)\} & 3092.316 & 51.832 & 0 & 0 & 2 & 8684.734 & 1.000 \\
age(2) + site\} & 3092.370 & 51.887 & 0 & 0 & 13 & 8662.732 & 1.000 \\
age & 3093.014 & 52.531 & 0 & 0 & 3 & 8683.431 & 1.000 \\
\{age + site\} & 3093.875 & 53.391 & 0 & 0 & 14 & 8662.227 & 1.000
\end{tabular}


Table 12. Summary of model selection results for estimating capture probability of mottled sculpin. age $=$ YOY, small adult or large adult. age (2) $=$ YOY or adult (large + small). (.) = constant model. Models in boldface accounted for at least $95 \%$ of the support based on the cumulative Akaike weights and were used for model averaging of model coefficients and parameter estimates.

\begin{tabular}{|c|c|c|c|c|c|c|c|}
\hline Model & $\mathrm{AlCc}$ & Delta AICc & AICc Weights & Model Likelihood & Num. Par & Deviance & Cum. Weight \\
\hline$\{$ age $(2)+$ site + date $\}$ & 11675.413 & 0.000 & 0.673 & 1.000 & 18 & 39081.788 & 0.673 \\
\hline \{age + site + date $\}$ & 11677.007 & 1.594 & 0.303 & 0.451 & 19 & 39081.378 & 0.977 \\
\hline \{site + date $\}$ & 11682.143 & 6.730 & 0.023 & 0.035 & 17 & 39090.522 & 1.000 \\
\hline$\{\operatorname{age}(2)+$ site $\}$ & 11731.267 & 55.854 & 0 & 0 & 13 & 39147.659 & 1.000 \\
\hline$\{$ age + site $\}$ & 11733.258 & 57.845 & 0 & 0 & 14 & 39147.647 & 1.000 \\
\hline$\{\operatorname{age}(2)+$ date $\}$ & 11734.740 & 59.327 & 0 & 0 & 7 & 39163.145 & 1.000 \\
\hline$\{$ age + date $\}$ & 11736.676 & 61.263 & 0 & 0 & 8 & 39163.079 & 1.000 \\
\hline$\{$ date\} & 11740.873 & 65.461 & 0 & 0 & 6 & 39171.280 & 1.000 \\
\hline$\{$ site $\}$ & 11753.677 & 78.265 & 0 & 0 & 12 & 39172.072 & 1.000 \\
\hline$\{\operatorname{age}(2)\}$ & 11805.091 & 129.678 & 0 & 0 & 2 & 39243.501 & 1.000 \\
\hline \{age\} & 11806.822 & 131.410 & 0 & 0 & 3 & 39243.231 & 1.000 \\
\hline$\{\}$, & 11828.260 & 152.848 & 0 & 0 & 1 & 39268.671 & 1.000 \\
\hline
\end{tabular}


Table 13. Results for the full factorial analysis of variance (ANOVA) testing the null hypothesis of no significant difference in coefficient of variation in site abundance by species, age class, removal treatment or spatial location. $\mathrm{a}=$ age effect, $\mathrm{g}=$ removal treatment, $1=$ spatial location, $\mathrm{p}=$ species, $\mathrm{a}^{*} \mathrm{~g}=$ age $\mathrm{x}$ removal treatment interaction, $\mathrm{a}^{* 1}$ $=$ age $\mathrm{x}$ location, $\mathrm{g}^{*} \mathrm{l}=$ removal $\mathrm{x}$ location, $\mathrm{a}^{*} \mathrm{p}=$ age $\mathrm{x}$ species, $\mathrm{g}^{*} \mathrm{p}=$ removal $\mathrm{x}$ species, $1^{*} \mathrm{p}=$ location $\mathrm{x}$ species, $\mathrm{a}^{*} \mathrm{~g}^{*} \mathrm{l}=$ age $\mathrm{x}$ removal $\mathrm{x}$ location, $\mathrm{a}^{*} \mathrm{~g}^{*} \mathrm{p}=$ age $\mathrm{x}$ removal $\mathrm{x}$ species, $\mathrm{a}^{*} \mathrm{l}^{*} \mathrm{p}=$ age $\mathrm{x}$ location $\mathrm{x}$ species, $\mathrm{g}^{*} \mathrm{l}^{*} \mathrm{p}=$ removal $\mathrm{x}$ location $\mathrm{x}$ species, $\mathrm{a}^{*} \mathrm{~g} \mathrm{l}^{*} \mathrm{p}=$ age $\mathrm{x}$ removal $\mathrm{x}$ location $\mathrm{x}$ species.

\begin{tabular}{cccccc} 
Term & Df & Sum Sq & Mean Sq & F value & $\operatorname{Pr}(>F)$ \\
\hline $\mathrm{a}$ & 2 & 3.219 & 1.610 & 25.137 & $<0.001$ \\
$\mathrm{~g}$ & 1 & 0.015 & 0.015 & 0.239 & 0.627 \\
$\mathrm{I}$ & 1 & 0.067 & 0.067 & 1.048 & 0.311 \\
$\mathrm{p}$ & 1 & 0.539 & 0.539 & 8.414 & $<0.01$ \\
$\mathrm{a}^{*} \mathrm{~g}$ & 2 & 0.164 & 0.082 & 1.281 & 0.287 \\
$\mathrm{a}^{*} \mathrm{l}$ & 2 & 0.022 & 0.011 & 0.168 & 0.846 \\
$\mathrm{~g}^{*} \mid$ & 1 & 0.004 & 0.004 & 0.059 & 0.810 \\
$\mathrm{a}^{*} \mathrm{p}$ & 2 & 1.317 & 0.658 & 10.281 & $<0.001$ \\
$\mathrm{~g}^{*} \mathrm{p}$ & 1 & 0.008 & 0.008 & 0.130 & 0.720 \\
$\mathrm{I}^{*} \mathrm{p}$ & 1 & 0.037 & 0.037 & 0.578 & 0.451 \\
$\mathrm{a}^{*} \mathrm{~g}^{*} \mid$ & 2 & 0.049 & 0.025 & 0.385 & 0.682 \\
$\mathrm{a}^{*} \mathrm{~g}^{*} \mathrm{p}$ & 2 & 0.118 & 0.059 & 0.923 & 0.404 \\
$\mathrm{a}^{*} \mathrm{I}^{*} \mathrm{p}$ & 2 & 0.011 & 0.006 & 0.089 & 0.915 \\
$\mathrm{~g}^{*} \mathrm{I}^{*} \mathrm{p}$ & 1 & 0.004 & 0.004 & 0.054 & 0.817 \\
$\mathrm{a}^{*} \mathrm{~g}^{*} \mathrm{I}^{*} \mathrm{p}$ & 2 & 0.014 & 0.007 & 0.108 & 0.897
\end{tabular}


Table 14. Model coefficients, standard errors, and p-values for the full factorial analysis of variance (ANOVA) model illustrating a significant difference in temporal variability of abundance between mottled sculpin and brook trout and among age classes of the two species. $\mathrm{a} 2=$ small adults effect, $\mathrm{a} 3=$ large adults, $\mathrm{g}=$ removal treatment, $\mathrm{l}=$ spatial location, $\mathrm{p}=$ species, $\mathrm{a} 2 * \mathrm{~g}=$ small adults $\mathrm{x}$ removal interaction, $\mathrm{a} 3 * \mathrm{~g}=$ large adults $\mathrm{x}$ removal, $\mathrm{a} 2 * 1=$ small adults $\mathrm{x}$ location, $\mathrm{a} 3 * 1=$ large adults $\mathrm{x}$ location, $\mathrm{g} * \mathrm{l}=$ removal $\mathrm{x}$ location, $\mathrm{a} 2 * \mathrm{p}=$ small adults $\mathrm{x}$ species, $\mathrm{a} 3 * \mathrm{p}=$ large adults $\mathrm{x}$ species, $\mathrm{g} * \mathrm{p}=$ removal $\mathrm{x}$ species, $1 * \mathrm{p}=$ location $\mathrm{x}$ species, a $2 * \mathrm{~g} * 1=$ small adults $\mathrm{x}$ removal $\mathrm{x}$ location, $\mathrm{a} 3 * \mathrm{~g} * 1=$ large adults $x$ removal $x$ location, $a 2 * g * p=$ small adults $x$ removal $x$ species, $a 3 * g * p=$ large adults $\mathrm{x}$ removal $\mathrm{x}$ location, $\mathrm{a} 2 * 1 * \mathrm{p}=$ small adults $\mathrm{x}$ location $\mathrm{x}$ species, $\mathrm{a} 3 * 1 * \mathrm{p}=$ large adults $\mathrm{x}$ removal $\mathrm{x}$ species, $\mathrm{g} * 1 * \mathrm{p}=$ removal $\mathrm{x}$ location $\mathrm{x}$ species, $\mathrm{a} 2 * \mathrm{~g} * 1 * \mathrm{p}=$ small adults $\mathrm{x}$ removal $\mathrm{x}$ location $\mathrm{x}$ species, $\mathrm{a} 3 * \mathrm{~g} * 1 * \mathrm{p}=$ large adults $\mathrm{x}$ removal $\mathrm{x}$ location $\mathrm{x}$ species.

\begin{tabular}{|c|c|c|c|c|}
\hline Coefficient & Estimate & Std. Error & $t$ value & $\operatorname{Pr}(>|t|)$ \\
\hline Intercept & 1.515 & 0.146 & 10.369 & $<0.001$ \\
\hline a2 & -0.912 & 0.207 & -4.415 & $<0.001$ \\
\hline a3 & -1.027 & 0.207 & -4.969 & $<0.001$ \\
\hline g (removal) & -0.341 & 0.207 & -1.652 & 0.105 \\
\hline I (mainstem) & -0.101 & 0.207 & -0.491 & 0.626 \\
\hline $\mathrm{p}$ (brook trout) & -0.653 & 0.207 & -3.159 & $<0.01$ \\
\hline$a 2^{*} g$ & 0.535 & 0.292 & 1.829 & 0.074 \\
\hline$a 3^{*} g$ & 0.336 & 0.292 & 1.148 & 0.256 \\
\hline$a 2 * \mid$ & 0.158 & 0.292 & 0.539 & 0.592 \\
\hline a3*I & 0.098 & 0.292 & 0.335 & 0.739 \\
\hline$g^{*} \mid$ & 0.086 & 0.292 & 0.293 & 0.771 \\
\hline$a 2^{*} p$ & 0.678 & 0.292 & 2.322 & $<0.05$ \\
\hline$a 3^{*} p$ & 0.874 & 0.292 & 2.992 & $<0.01$ \\
\hline$g^{*} p$ & 0.270 & 0.292 & 0.925 & 0.360 \\
\hline$I^{*} p$ & -0.041 & 0.292 & -0.141 & 0.888 \\
\hline$a 2^{*} g^{*} \mid$ & -0.309 & 0.413 & -0.747 & 0.458 \\
\hline$a 3^{*} g^{*} \mid$ & 0.055 & 0.413 & 0.133 & 0.894 \\
\hline$a 2^{*} g^{*} p$ & -0.430 & 0.413 & -1.040 & 0.304 \\
\hline$a 3^{*} g^{*} p$ & -0.335 & 0.413 & -0.812 & 0.421 \\
\hline$\left.a 2^{*}\right|^{*} p$ & -0.137 & 0.413 & -0.330 & 0.743 \\
\hline$\left.a 3^{*}\right|^{*} p$ & -0.095 & 0.413 & -0.230 & 0.819 \\
\hline$\left.g^{*}\right|^{*} p$ & 0.002 & 0.413 & 0.005 & 0.996 \\
\hline$\left.a 2^{*} g^{*}\right|^{*} p$ & 0.208 & 0.584 & 0.356 & 0.723 \\
\hline$\left.a 3^{*} g^{*}\right|^{*} p$ & -0.048 & 0.584 & -0.082 & 0.935 \\
\hline
\end{tabular}


Table 15. Summary of model selection results for comparing among linear mixed models estimating the relationship between logtransformed proportional recovery (i.e., abundance at time $t$ divided by pre-removal abundance) of brook trout and fixed effects of spatial location, defaunation treatment, and their interactions while modeling site as a random factor and incorporating fixed local physical and chemical habitat effects. Selection results are listed for young-of-the-year (YOY, top), small adult (middle), and large adult (bottom) brook trout. Only the 95\% confidence model sets and the global models are listed in the tables. The global model, as well as its fit to the data $\left(\mathrm{R}^{2}\right)$, is highlighted in boldface type. $\mathrm{g}=$ treatment, $\mathrm{l}=$ spatial location, $\mathrm{t} 3-\mathrm{t} 6=$ sampling date, $\mathrm{g} * \mathrm{t}=$ removal $\mathrm{x}$ date interaction, $1 * \mathrm{t}=$ location $\mathrm{x}$ date, $\mathrm{g} * \mathrm{l}=$ removal $\mathrm{x}$ location, $\mathrm{g} \mathrm{l}^{*} \mathrm{t}=$ removal $\mathrm{x}$ location $\mathrm{x}$ date, Local $=$ all local physical and chemical habitat covariates, including $\mathrm{W} 1=$ water chemistry principal component $1, \mathrm{H} 1-\mathrm{H} 3=$ physical habitat principal components 1 to 3, PAREA = proportion of spring wetted channel area in early fall, CANP = mean percent canopy cover, MWAT = maximum weekly average temperature $\left({ }^{\circ} \mathrm{C}\right)$.

\begin{tabular}{|c|c|c|c|c|c|c|c|}
\hline Model & $\mathrm{AlCc}$ & $\triangle \mathrm{AlCc}$ & $\begin{array}{c}\text { AlCc } \\
\text { Weight }\end{array}$ & $\begin{array}{c}\text { Model } \\
\text { Likelihood }\end{array}$ & $\begin{array}{l}\text { Num. } \\
\text { Par }\end{array}$ & $\begin{array}{c}\text { Cum. } \\
\text { Weight }\end{array}$ & R-square \\
\hline Local + I + t & 218.272 & 0 & 0.306 & 1 & 15 & 0.306 & \\
\hline Local $+g+I+t+g^{*}$ & 219.090 & 0.817 & 0.204 & 0.665 & 17 & 0.510 & \\
\hline Local $+g+I+t+I^{*} t$ & 220.007 & 1.734 & 0.129 & 0.420 & 20 & 0.639 & \\
\hline Local + g + t & 220.128 & 1.856 & 0.121 & 0.395 & 15 & 0.760 & \\
\hline Local $+1+t+I^{*} t$ & 220.141 & 1.868 & 0.120 & 0.393 & 19 & 0.880 & \\
\hline Local + t & 221.464 & 3.192 & 0.062 & 0.203 & 14 & 0.942 & \\
\hline Local + g + I+t + g*I + I*t & 222.061 & 3.788 & 0.046 & 0.150 & 21 & 0.988 & \\
\hline Local $+g+\left|+t+g^{*}\right|+g^{*} t+\left.\right|^{*} t+\left.g^{*}\right|^{*} t$ & 237.184 & 18.912 & 0 & 0 & 29 & 1 & 0.79 \\
\hline Local $+\mathrm{I}+\mathrm{t}$ & 181.888 & 0 & 0.521 & 1 & 15 & 0.521 & \\
\hline Local $+\mathrm{g}+\mathrm{t}$ & 183.137 & 1.249 & 0.279 & 0.536 & 15 & 0.800 & \\
\hline Local + t & 179.228 & 2.660 & 0.138 & 0.264 & 14 & 0.938 & \\
\hline Local $+I+t+I^{*} t$ & 187.685 & 5.796 & 0.029 & 0.055 & 19 & 0.967 & \\
\hline Local + g + I + t + g*I + $g^{*} t+\left.\right|^{*} t+\left.g^{*}\right|^{*} t$ & 219.287 & 37.399 & 0 & 0 & 29 & 1 & 0.72 \\
\hline Local + g + I+t + g*I & 176.447 & 0 & 0.496 & 1 & 17 & 0.496 & \\
\hline Local + I+ t & 177.520 & 1.073 & 0.290 & 0.585 & 15 & 0.786 & \\
\hline Local + t & 179.155 & 2.709 & 0.128 & 0.258 & 14 & 0.914 & \\
\hline Local $+\mathrm{g}+\mathrm{t}$ & 180.265 & 3.819 & 0.074 & 0.148 & 15 & 0.988 & \\
\hline Local $+g+I+t+g^{*} I+g^{*} t+I^{*} t+g^{*} I^{*} t$ & 204.891 & 28.444 & 0 & 0 & 29 & 1 & 0.84 \\
\hline
\end{tabular}


Table 16. Summary of model selection results for comparing among linear mixed models estimating the relationship between logtransformed proportional recovery (i.e., abundance at time $t$ divided by pre-removal abundance) and fixed effects of spatial location, defaunation treatment, and their interactions while modeling site as a random factor and incorporating fixed local physical and chemical habitat effects. Selection results are indicated for young-of-the-year (YOY, top), small adult (middle), and large adult (bottom) mottled sculpin. Only the 95\% confidence model sets and the global models are listed in the tables. The global model, as well as its fit to the data $\left(\mathrm{R}^{2}\right)$, is highlighted in boldface type. $\mathrm{g}=$ treatment, $\mathrm{l}=$ spatial location, $\mathrm{t} 3-\mathrm{t} 6=$ sampling date, $\mathrm{g} * \mathrm{t}=$ removal $\mathrm{x}$ date interaction, $1 * \mathrm{t}=$ location $\mathrm{x}$ date, $\mathrm{g} * \mathrm{l}=$ removal $\mathrm{x}$ location, $\mathrm{g} * 1 * \mathrm{t}=$ removal $\mathrm{x}$ location $\mathrm{x}$ date, Local $=$ all local physical and chemical habitat covariates, including $\mathrm{W} 1=$ water chemistry principal component $1, \mathrm{H} 1-\mathrm{H} 3=$ physical habitat principal components 1 to 3, PAREA = proportion of spring wetted channel area in early fall, CANP = mean percent canopy cover, MWAT = maximum weekly average temperature $\left({ }^{\circ} \mathrm{C}\right)$.

\begin{tabular}{|c|c|c|c|c|c|c|c|}
\hline Model & AlCc & $\triangle \mathrm{AICc}$ & $\begin{array}{c}\text { AlCc } \\
\text { Weight }\end{array}$ & $\begin{array}{c}\text { Model } \\
\text { Likelihood }\end{array}$ & $\begin{array}{c}\text { Num. } \\
\text { Par }\end{array}$ & $\begin{array}{c}\text { Cum. } \\
\text { Weight }\end{array}$ & R-square \\
\hline Local $+\mathrm{t}$ & 217.247 & 0 & 0.394 & 1 & 14 & 0.394 & \\
\hline Local $+\mathrm{g}+\mathrm{t}$ & 218.652 & 1.405 & 0.195 & 0.495 & 15 & 0.590 & \\
\hline Local + I + t & 218.691 & 1.444 & 0.192 & 0.486 & 15 & 0.781 & \\
\hline Local $+I+t+I^{*} t$ & 219.917 & 2.670 & 0.104 & 0.263 & 19 & 0.885 & \\
\hline Local $+g+\left|+t+g^{*}\right|$ & 221.177 & 3.930 & 0.055 & 0.140 & 17 & 0.940 & \\
\hline Local $+g+I+t+I^{*} t$ & 222.369 & 5.122 & 0.030 & 0.077 & 20 & 0.971 & \\
\hline Local + g + I+ t + $g^{*} I+g^{*} t+\left.\right|^{*} t+g^{*} I^{*} t$ & 242.565 & 25.318 & 0 & 0 & 29 & 1 & 0.73 \\
\hline Local $+g+\left|+t+g^{*}\right|$ & 189.461 & 0 & 0.521 & 1 & 17 & 0.521 & \\
\hline Local $+\mathrm{I}+\mathrm{t}$ & 191.269 & 1.809 & 0.211 & 0.405 & 15 & 0.731 & \\
\hline Local + t & 191.592 & 2.131 & 0.179 & 0.345 & 14 & 0.911 & \\
\hline Local $+\mathrm{g}+\mathrm{t}$ & 193.339 & 3.878 & 0.075 & 0.144 & 15 & 0.985 & \\
\hline Local + g + I+ t + $g^{*} I+g^{*} t+\left.\right|^{*} t+\left.g^{*}\right|^{*} t$ & 221.233 & 31.773 & 0 & 0 & 29 & 1 & 0.85 \\
\hline Local $+\mathrm{I}+\mathrm{t}$ & 173.164 & 0 & 0.407 & 1 & 15 & 0.407 & \\
\hline Local $+g+I+t+g^{*} \mid$ & 173.629 & 0.466 & 0.323 & 0.792 & 17 & 0.730 & \\
\hline Local $+\mathrm{t}$ & 174.647 & 1.484 & 0.194 & 0.476 & 14 & 0.924 & \\
\hline Local $+\mathrm{g}+\mathrm{t}$ & 176.685 & 3.521 & 0.070 & 0.172 & 15 & 0.994 & \\
\hline Local + g + I+t + $g^{*} I+g^{*} t+I^{*} t+g^{*} I^{*} t$ & 212.904 & 39.741 & 0 & 0 & 29 & 1 & 0.85 \\
\hline
\end{tabular}


Table 17. Summary of model selection results comparing regression models for estimating the relationship between young-of-theyear (YOY, top), small adult (middle), and large adult (bottom) brook trout per capita growth rate $\left(\mathrm{r}=\ln \left(\mathrm{N}_{t+1} / \mathrm{N}_{\mathrm{t}}\right)\right.$ ) and fixed effects of site spatial location, defaunation treatment, and their interactions, while accounting for the effects local physical and chemical habitat covariates and sampling date. Only the $95 \%$ confidence model sets and the global models are listed. The global model, as well as its fit to the data $\left(\mathrm{R}^{2}\right)$, is highlighted in boldface type. $\mathrm{g}=$ treatment, $1=$ spatial location, $\mathrm{t} 3-\mathrm{t} 6=$ sampling date, $1 * \mathrm{t} 3-1^{*} \mathrm{t} 6=$ location $\mathrm{x}$ sampling date interaction, $\mathrm{g} * \mathrm{l}=$ removal $\mathrm{x}$ location interaction, $\mathrm{g} * \mathrm{l}^{*} \mathrm{t}=$ removal $\mathrm{x}$ location $\mathrm{x}$ sampling date, Local $=$ all local physical and chemical habitat covariates: $\mathrm{W} 1=$ water chemistry principal component $1, \mathrm{H} 1-\mathrm{H} 3=$ physical habitat principal components 1 to 3, PAREA $=$ proportion of spring wetted channel area in early fall, CANP = mean percent canopy cover, MWAT $=$ maximum weekly average temperature $\left({ }^{\circ} \mathrm{C}\right)$.

\begin{tabular}{|c|c|c|c|c|c|c|c|}
\hline Model & $\mathrm{AlCc}$ & $\triangle \mathrm{AICc}$ & $\begin{array}{c}\text { AlCc } \\
\text { Weight }\end{array}$ & $\begin{array}{c}\text { Model } \\
\text { Likelihood }\end{array}$ & $\begin{array}{l}\text { Num. } \\
\text { Par }\end{array}$ & $\begin{array}{c}\text { Cum. } \\
\text { Weight }\end{array}$ & R-square \\
\hline Local + t & 222.635 & 0 & 0.674 & 1 & 13 & 0.674 & \\
\hline Local + I+t & 225.588 & 2.953 & 0.154 & 0.228 & 14 & 0.828 & \\
\hline Local + g + t & 225.698 & 3.063 & 0.146 & 0.216 & 14 & 0.974 & \\
\hline Local + $g+I+t+g^{*} I+g^{*} t+I^{*} t+\left.g^{*}\right|^{*} t$ & 262.758 & 40.123 & 0 & 0 & 28 & 1 & 0.4 \\
\hline Local $+g+t+g^{*} t$ & 192.025 & 0 & 0.455 & 1 & 18 & 0.455 & \\
\hline Local + t & 192.851 & 0.826 & 0.301 & 0.662 & 13 & 0.757 & \\
\hline Local + g + t & 195.318 & 3.293 & 0.088 & 0.193 & 14 & 0.844 & \\
\hline Local $+g+I+t+g^{*} t$ & 195.673 & 3.648 & 0.073 & 0.161 & 19 & 0.918 & \\
\hline Local $+\mathrm{I}+\mathrm{t}$ & 195.923 & 3.899 & 0.065 & 0.142 & 14 & 0.983 & \\
\hline Local + $g+\left|+t+g^{*}\right|+g^{*} t+\left.\right|^{*} t+\left.g^{*}\right|^{*} t$ & 226.841 & 34.816 & 0 & 0 & 28 & 1 & 0.54 \\
\hline Local $+g+t+g^{*} t$ & 193.163 & 0 & 0.443 & 1 & 18 & 0.443 & \\
\hline Local $+\mathrm{t}$ & 194.012 & 0.849 & 0.290 & 0.654 & 13 & 0.733 & \\
\hline Local + g+t & 196.072 & 2.910 & 0.103 & 0.233 & 14 & 0.837 & \\
\hline Local + I+t & 196.689 & 3.526 & 0.076 & 0.171 & 14 & 0.913 & \\
\hline Local + $g+\left|+t+g^{*}\right|+g^{*} t+\left.\right|^{*} t+\left.g^{*}\right|^{*} t$ & 208.938 & 15.776 & 0 & 0 & 28 & 1 & 0.63 \\
\hline
\end{tabular}


Table 18. Summary of model selection results comparing regression models for estimating the relationship between young-of-theyear (YOY, top), small adult (middle), and large adult (bottom) mottled sculpin per capita growth rate $\left(\mathrm{r}=\ln \left(\mathrm{N}_{\mathrm{t}+1} / \mathrm{N}_{\mathrm{t}}\right)\right)$ and fixed effects of site spatial location, defaunation treatment, and their interactions, while accounting for the effects local physical and chemical habitat covariates and sampling date. Only the $95 \%$ confidence model sets and the global models are listed. The global model, as well as its fit to the data $\left(\mathrm{R}^{2}\right)$, is highlighted in boldface type. $\mathrm{g}=$ treatment, $1=$ spatial location, $\mathrm{t} 3-\mathrm{t} 6=$ sampling date, $1^{*} \mathrm{t} 3$ $-1 * \mathrm{t} 6=$ location $\mathrm{x}$ sampling date interaction, $\mathrm{g} * \mathrm{l}=$ removal $\mathrm{x}$ location interaction, $\mathrm{g} * 1 * \mathrm{t}=$ removal $\mathrm{x}$ location $\mathrm{x}$ sampling date, Local $=$ all local physical and chemical habitat covariates: $\mathrm{W} 1=$ water chemistry principal component $1, \mathrm{H} 1-\mathrm{H} 3=$ physical habitat principal components 1 to 3, PAREA = proportion of spring wetted channel area in early fall, CANP = mean percent canopy cover, MWAT $=$ maximum weekly average temperature $\left({ }^{\circ} \mathrm{C}\right)$.

\begin{tabular}{|c|c|c|c|c|c|c|c|}
\hline Model & AICc & $\triangle \mathrm{AICC}$ & $\begin{array}{c}\text { AlCc } \\
\text { Weight }\end{array}$ & $\begin{array}{c}\text { Model } \\
\text { Likelihood }\end{array}$ & $\begin{array}{l}\text { Num. } \\
\text { Par }\end{array}$ & $\begin{array}{c}\text { Cum. } \\
\text { Weight }\end{array}$ & R-square \\
\hline Local $+\mathrm{t}$ & 235.142 & 0 & 0.630 & 1 & 13 & 0.630 & \\
\hline Local $+g+t$ & 238.020 & 2.878 & 0.149 & 0.237 & 14 & 0.779 & \\
\hline Local $+\mathrm{I}+\mathrm{t}$ & 238.234 & 3.092 & 0.134 & 0.213 & 14 & 0.913 & \\
\hline Local $+I+t+I^{*} t$ & 239.641 & 4.499 & 0.066 & 0.105 & 18 & 0.979 & \\
\hline Local $+g+I+t+g^{*} I+g^{*} t+I^{*} t+g^{*} I^{*} t$ & 279.969 & 44.827 & $\mathbf{0}$ & 0 & 28 & 1 & 0.4 \\
\hline Local $+g+t+g^{*} t$ & 213.416 & 0 & 0.421 & 1 & 18 & 0.421 & \\
\hline Local $+\mathrm{t}$ & 213.898 & 0.482 & 0.331 & 0.786 & 13 & 0.752 & \\
\hline Local $+\mathrm{I}+\mathrm{t}$ & 216.709 & 3.293 & 0.081 & 0.193 & 14 & 0.833 & \\
\hline Local $+g+1+t+g^{*} t$ & 216.836 & 3.420 & 0.076 & 0.181 & 19 & 0.910 & \\
\hline Local $+\mathrm{g}+\mathrm{t}$ & 216.926 & 3.510 & 0.073 & 0.173 & 14 & 0.982 & \\
\hline Local + $g+I+t+g^{*}\left|+g^{*} t+\right|^{*} t+\left.g^{*}\right|^{*} t$ & 245.964 & 32.548 & 0 & 0 & 28 & 1 & 0.13 \\
\hline Local $+\mathrm{t}$ & 203.988 & 0 & 0.409 & 1 & 13 & 0.409 & \\
\hline Local + g + t & 205.287 & 1.299 & 0.214 & 0.522 & 14 & 0.622 & \\
\hline Local $+g+t+g^{*} t$ & 205.398 & 1.410 & 0.202 & 0.494 & 18 & 0.824 & \\
\hline Local $+\mathrm{I}+\mathrm{t}$ & 206.396 & 2.409 & 0.123 & 0.300 & 14 & 0.947 & \\
\hline Local $+g+I+t+g^{*} t$ & 209.002 & 5.014 & 0.033 & 0.082 & 19 & 0.980 & \\
\hline Local $+g+I+t+g^{*} I+g^{*} t+\left.\right|^{*} t+\left.g^{*}\right|^{*} t$ & 238.329 & 34.341 & 0 & 0 & 28 & 1 & 0.31 \\
\hline
\end{tabular}


Table 19. Summary of model comparison results for multi-state models estimating apparent survival $(\varphi)$ and age-class transition probability $(\psi)$ of brook trout. Only the $95 \%$ confidence model set and the global models are listed. The global model is highlighted in boldface type. $\mathrm{a}=3$ age classes (YOY, small and large adult), $\mathrm{g}=$ removal treatment, $1=$ spatial location (mainstem or headwater tributary site), $\mathrm{t}=$ sample intervals ( 5 intervals) $\mathrm{t}(3)=3$ sample intervals for transition probability, $\mathrm{a}^{*} \mathrm{~g}=$ age $\mathrm{x}$ removal interaction, $\mathrm{a}^{*} \mathrm{l}=$ age $\mathrm{x}$ location, $\mathrm{g} * \mathrm{t}=$ removal $\mathrm{x}$ date, $1^{*} \mathrm{t}=$ location $\mathrm{x}$ date, $\mathrm{g} * 1=$ removal $\mathrm{x}$ location, $\mathrm{g} * 1^{*} \mathrm{t}=$ removal $\mathrm{x}$ location $\mathrm{x}$ date, Local $=$ all local physical and chemical habitat covariates: $\mathrm{W} 1=$ water chemistry principal component $1, \mathrm{H} 1-\mathrm{H} 3=$ physical habitat principal components 1 to 3, PAREA = proportion of spring wetted channel area in early fall, CANP = mean percent canopy cover, MWAT $=$ maximum weekly average temperature $\left({ }^{\circ} \mathrm{C}\right)$.

\begin{tabular}{|c|c|c|c|c|c|c|c|}
\hline$\varphi$ & $\psi$ & $\mathrm{AlCc}$ & $\begin{array}{l}\text { Delta } \\
\text { AICc }\end{array}$ & $\begin{array}{c}\text { AlCc } \\
\text { Weights }\end{array}$ & $\begin{array}{c}\text { Model } \\
\text { Likelihood }\end{array}$ & $\begin{array}{c}\text { Num. } \\
\text { Par }\end{array}$ & $\begin{array}{l}\text { Cum. } \\
\text { Weight }\end{array}$ \\
\hline Local $+a+g+\left|+t+a^{*} g+a^{*}\right|+g^{*}\left|+g^{*} t+\right|^{*} t+\left.g^{*}\right|^{*} t$ & Local $+a+g+t(3)$ & 2327.140 & 1.174 & 0.187 & 0.556 & 43 & 0.524 \\
\hline Local $+a+g+\left|+t+a^{*} g+a^{*}\right|+g^{*}\left|+g^{*} t+\right|^{*} t+\left.g^{*}\right|^{*} t$ & Local+a+l+t(3) & 2328.035 & 2.069 & 0.120 & 0.355 & 43 & 0.763 \\
\hline Local $+a+g+\left|+t+a^{*} g+a^{*}\right|+g^{*}\left|+g^{*} t+\right|^{*} t$ & Local+a+g+t(3) & 2329.168 & 3.201 & 0.068 & 0.202 & 40 & 0.831 \\
\hline Local $+a+g+\left|+t+a^{*} g+a^{*}\right|+g^{*}\left|+g^{*} t+\right|^{*} t+\left.g^{*}\right|^{*} t$ & Local $+a+g+l+t(3)$ & 2329.229 & 3.263 & 0.066 & 0.196 & 44 & 0.897 \\
\hline Local $+a+g+\left|+t+a^{*} g^{2} a^{*}\right|+g^{*}\left|+g^{*} t+\right|^{*} t$ & Local $+a+1+t(3)$ & 2330.095 & 4.128 & 0.043 & 0.127 & 40 & 0.940 \\
\hline
\end{tabular}


Table 20. Summary of model comparison results for multi-state models estimating apparent survival (S) and age-class transition probability $(\psi)$ of mottled sculpin. . Only the $95 \%$ confidence model set and the global models are listed. The global model is highlighted in boldface type. $\mathrm{a}=3$ age classes (YOY, small and large adult), $\mathrm{g}=$ removal treatment, $1=$ spatial location (mainstem or headwater tributary site), $\mathrm{t}=$ sample intervals ( 5 intervals), $\mathrm{t}(3)=3$ sample intervals for transition probability, $\mathrm{a}^{*} \mathrm{~g}=\mathrm{age} \mathrm{x}$ removal interaction, $\mathrm{a}^{*} \mathrm{l}=$ age $\mathrm{x}$ location, $\mathrm{g}^{*} \mathrm{t}=$ removal $\mathrm{x}$ date, $\mathrm{l}^{*} \mathrm{t}=$ location $\mathrm{x}$ date, $\mathrm{g} * 1=$ removal $\mathrm{x}$ location, $\mathrm{g}^{*} 1^{*} \mathrm{t}=$ removal $\mathrm{x}$ location $\mathrm{x}$ date, Local $=$ all local physical and chemical habitat covariates: $\mathrm{W} 1=$ water chemistry principal component $1, \mathrm{H} 1-\mathrm{H} 3=$ physical habitat principal components 1 to 3, PAREA = proportion of spring wetted channel area in early fall, CANP $=$ mean percent canopy cover, MWAT $=$ maximum weekly average temperature $\left({ }^{\circ} \mathrm{C}\right)$.

\begin{tabular}{|c|c|c|c|c|c|c|c|}
\hline$\varphi$ & $\psi$ & $\mathrm{AICc}$ & $\begin{array}{l}\text { Delta } \\
\text { AlCc }\end{array}$ & $\begin{array}{c}\text { AlCc } \\
\text { Weights }\end{array}$ & $\begin{array}{c}\text { Model } \\
\text { Likelihood }\end{array}$ & $\begin{array}{c}\text { Num. } \\
\text { Par }\end{array}$ & $\begin{array}{c}\text { Cum. } \\
\text { Weight }\end{array}$ \\
\hline Local+a+g+|+t+a* $g^{*} a^{*}\left|+g^{*}\right|+g^{*} t+\left.\right|^{*} t+\left.g^{*}\right|^{*} t$ & Local+a+t & 7903.275 & 0 & 0.312 & 1 & 45 & 0.31163 \\
\hline Local+a+g+1+t+a* $g^{*}+a^{*}\left|+g^{*}\right|+g^{*} t+\left.\right|^{*} t+\left.g^{*}\right|^{*} t$ & Local $+a+g+t$ & 7903.707 & 0.433 & 0.251 & 0.806 & 46 & 0.56266 \\
\hline Local $+a+g+\left|+t+a^{*} g+a^{*}\right|+g^{*}\left|+g^{*} t+\right|^{*} t+\left.g^{*}\right|^{*} t$ & Local+a+g+l+t+g*I & 7904.369 & 1.094 & 0.180 & 0.579 & 47 & 0.74299 \\
\hline Local+a+g+|+t+a* $g^{*} a^{*}\left|+g^{*}\right|+g^{*} t+\left.\right|^{*} t+\left.g^{*}\right|^{*} t$ & Local+a+l+t & 7904.948 & 1.673 & 0.135 & 0.433 & 46 & 0.87801 \\
\hline Local+a+g+|+t+a* $g+a^{*}\left|+g^{*}\right|+g^{*} t+\left.\right|^{*} t+\left.g^{*}\right|^{*} t$ & Local $+a+g+1+t$ & 7905.638 & 2.363 & 0.096 & 0.307 & 47 & 0.97363 \\
\hline
\end{tabular}


Table 21. Summary of model comparison results for multi-state models estimating the proportion of the instantaneous population growth rate $(\lambda)$ due to local survival $\left(\gamma_{\mathrm{ii}}\right)$ and local recruitment $\left(\gamma_{\mathrm{ji}}\right)$ of brook trout. Only the $95 \%$ confidence model set and the global models are listed. The global model is highlighted in boldface type. $\mathrm{a}=3$ age classes (YOY, small and large adult), $\mathrm{g}=$ removal treatment, $1=$ spatial location (mainstem or headwater tributary site), $\mathrm{t}=$ sample intervals $(5$ intervals), $\mathrm{t}(3)=3$ sample intervals for transition probability, $\mathrm{a}^{*} \mathrm{~g}=$ age $\mathrm{x}$ removal interaction, $\mathrm{a} * \mathrm{l}=$ age $\mathrm{x}$ location, $\mathrm{g} * \mathrm{t}=$ removal $\mathrm{x}$ date, $1 * \mathrm{t}=$ location $\mathrm{x}$ date, $\mathrm{g} * 1=$ removal $\mathrm{x}$ location, $\mathrm{g}{ }^{*}{ }^{*} \mathrm{t}=$ removal $\mathrm{x}$ location $\mathrm{x}$ date, Local $=$ all local physical and chemical habitat covariates: $\mathrm{W} 1=\mathrm{water}$ chemistry principal component $1, \mathrm{H} 1-\mathrm{H} 3$ = physical habitat principal components 1 to 3 , PAREA = proportion of spring wetted channel area in early fall, $\mathrm{CANP}=$ mean percent canopy cover, MWAT $=$ maximum weekly average temperature $\left({ }^{\circ} \mathrm{C}\right)$.

\begin{tabular}{|c|c|c|c|c|c|c|c|}
\hline$\gamma_{i i}$ & $\gamma_{j i}$ & $\mathrm{AlCc}$ & $\triangle \mathrm{AICC}$ & $\begin{array}{c}\text { AlCc } \\
\text { Weight }\end{array}$ & $\begin{array}{c}\text { Model } \\
\text { Likelihood }\end{array}$ & $\begin{array}{l}\text { Num. } \\
\text { Par }\end{array}$ & $\begin{array}{l}\text { Cum. } \\
\text { Weight }\end{array}$ \\
\hline Local $+a+g+t+a^{*} g+g^{*} t$ & Local+a+t(3) & 1162.895 & 0 & 0.710 & 1 & 32 & 0.710 \\
\hline Local $+a+g+\left|+t+a^{*} g+a^{*}\right|+g^{*} t$ & Local+a+t(3) & 1165.401 & 2.506 & 0.203 & 0.286 & 35 & 0.912 \\
\hline Local $+a+g+\left|+t+a^{*} g+a^{*}\right|+g^{*} \mid+g^{*} t$ & Local+a+t(3) & 1167.080 & 4.185 & 0.088 & 0.123 & 36 & 1 \\
\hline Local+a+g+l+t+a* $a^{*}+a^{*}\left|+g^{*}\right|+g^{*} t+\left.\right|^{*} t+\left.g^{*}\right|^{*} t$ & Local+a+g+l+t(3)+g*I & 1285.707 & 122.812 & 0 & 0 & 47 & 1 \\
\hline
\end{tabular}


Table 22. Summary of model comparison results for multi-state models estimating the proportion of the instantaneous population growth rate $(\lambda)$ due to local survival $\left(\gamma_{\mathrm{ii}}\right)$ and local recruitment $\left(\gamma_{\mathrm{ji}}\right)$ of mottled sculpin. Only the $95 \%$ confidence model set and the global models are listed. The global model is highlighted in boldface type. $\mathrm{a}=3$ age classes (YOY, small and large adult), $\mathrm{g}=$ removal treatment, $1=$ spatial location (mainstem or headwater tributary site), $\mathrm{t}=$ sample intervals $(5$ intervals), $\mathrm{t}(3)=3$ intervals for transition probability, $\mathrm{a}^{*} \mathrm{~g}=$ age $\mathrm{x}$ removal interaction, $\mathrm{a}^{*} \mathrm{l}=$ age $\mathrm{x}$ location, $\mathrm{g} * \mathrm{t}=$ removal $\mathrm{x}$ date, $1^{*} \mathrm{t}=$ location $\mathrm{x}$ date, $\mathrm{g} * 1=$ removal $\mathrm{x}$ location, $\mathrm{g}{ }^{*}{ }^{*} \mathrm{t}=$ removal $\mathrm{x}$ location $\mathrm{x}$ date, Local $=$ all local physical and chemical habitat covariates: $\mathrm{W} 1=\mathrm{water}$ chemistry principal component $1, \mathrm{H} 1-\mathrm{H} 3$ = physical habitat principal components 1 to 3 , PAREA = proportion of spring wetted channel area in early fall, $\mathrm{CANP}=$ mean percent canopy cover, MWAT $=$ maximum weekly average temperature $\left({ }^{\circ} \mathrm{C}\right)$.

\begin{tabular}{|c|c|c|c|c|c|c|c|}
\hline$\gamma_{\text {ii }}$ & $\gamma_{\mathbf{j i}}$ & AIC & $\triangle \mathrm{AlCc}$ & $\begin{array}{c}\text { AlCc } \\
\text { Weight }\end{array}$ & $\begin{array}{c}\text { Model } \\
\text { Likelihood }\end{array}$ & $\begin{array}{c}\text { Num. } \\
\text { Par }\end{array}$ & $\begin{array}{c}\text { Cum. } \\
\text { Weight }\end{array}$ \\
\hline Local $+a+g+\left|+t+a^{*} g+a^{*}\right|+g^{*} t$ & Local+a+t & 2584.439 & 0 & 0.978 & 1 & 35 & 0.978 \\
\hline Local $+a+g+\left|+t+a^{*} g+a^{*}\right|+g^{*}\left|+g^{*} t+l^{*} t+g^{*}\right|^{*} t$ & Local+a+g+l+t+g*I & 2526.771 & 95.059 & 0 & 0 & 49 & 1 \\
\hline
\end{tabular}


Table 23. Summary of results pursuant of a priori expectations for the qualitative comparison of individual population parameters between brook trout and mottled sculpin under the assumption that local population dynamics differ in the core versus the periphery of a species' distribution. These expectations address the goal of the study concerning determining the extent to which population dynamics differ in the core (i.e., brook trout) versus the periphery (i.e., mottled sculpin) of a species' distribution (i.e., goal 1).

Expectations supported by the data are italicized.

\begin{tabular}{ccccccc}
\hline & $\begin{array}{c}\text { Temporal } \\
\text { variability in } \\
\text { abundance }\end{array}$ & Survival & $\begin{array}{c}\text { Age } \\
\text { Transition }\end{array}$ & $\begin{array}{c}\text { Contribution of } \\
\text { survival to } \lambda\end{array}$ & $\begin{array}{c}\text { Contribution of } \\
\text { recruitment to } \lambda\end{array}$ & $\begin{array}{c}\text { Contribution of } \\
\text { immigration to } \lambda\end{array}$ \\
\hline Brook trout & Lower & Higher & Higher & Higher & Higher & Lower \\
Mottled sculpin & Higher & Lower & Lower & Lower & Lower & Higher \\
\hline
\end{tabular}


Table 24. Summary of results pursuant of a priori expectations for the expected response of brook trout (top) and mottled sculpin (bottom) to the experimental removal under the assumption that the regional context has an influence on local population dynamics. These expectations address the goal of the study concerning determining the extent to which local population dynamics differ given the regional context (i.e., goal 2). Expectations supported by the data are italicized. Expectations supported by a top model are also highlighted in bold.

\begin{tabular}{cccc}
\hline & \multicolumn{3}{c}{ Brook trout } \\
\hline Site type & $\begin{array}{c}\text { Recovery of } \\
\text { abundance }\end{array}$ & $\begin{array}{c}\text { Population } \\
\text { growth rate }\end{array}$ & $\begin{array}{c}\text { Contribution of } \\
\text { immigration to } \lambda\end{array}$ \\
\hline $\begin{array}{c}\text { Mainstem } \\
\text { Removal }\end{array}$ & $\begin{array}{c}\text { Slower/Less } \\
\text { complete }\end{array}$ & Lower & Lower \\
$\begin{array}{c}\text { Headwater } \\
\text { Removal }\end{array}$ & $\begin{array}{c}\text { Faster/More } \\
\text { complete }\end{array}$ & Higher & Higher \\
\hline
\end{tabular}

\begin{tabular}{cccc}
\hline & \multicolumn{3}{c}{ Mottled sculpin } \\
\hline Site type & $\begin{array}{c}\text { Recovery of } \\
\text { abundance }\end{array}$ & $\begin{array}{c}\text { Population } \\
\text { growth rate }\end{array}$ & $\begin{array}{c}\text { Contribution of } \\
\text { immigration to } \lambda\end{array}$ \\
\hline $\begin{array}{c}\text { Mainstem } \\
\text { Removal }\end{array}$ & $\begin{array}{c}\text { Faster/More } \\
\text { complete }\end{array}$ & Lower & Lower \\
$\begin{array}{c}\text { Headwater } \\
\text { Removal }\end{array}$ & $\begin{array}{c}\text { Slower/Less } \\
\text { complete }\end{array}$ & Higher & Higher \\
\hline
\end{tabular}


Table 24. Summary of results pursuant of a priori expectations for the expected response of brook trout (top) and mottled sculpin (bottom) to the experimental removal under the assumption that density dependent feedbacks are an important feature driving local population dynamics (i.e., goal 3). Expectations supported by the data are italicized. Although the removal effect was included in the top ranked model for survival, the trend was the opposite expected as is indicated by the (-) symbol.

\begin{tabular}{clc}
\hline & \multicolumn{2}{c}{ Brook trout } \\
\hline Treatment & Survival & Growth \\
\hline Control & Lower (-) & Lower \\
Removal & Higher (-) & Higher \\
& & \\
& & \\
\hline \multicolumn{3}{c}{ Mottled sculpin } \\
\hline Treatment & Survival & Growth \\
\hline Control & Lower (-) & Lower \\
Removal & Higher (-) & Higher
\end{tabular}


Table 25. Summary of results pursuant of a priori expectations for the expected relative effect size of the removal term on brook trout (top) and mottled sculpin (bottom) under the assumption that density dependent feedbacks are an important feature driving local populations in this system and the assumption that density dependent feedbacks are stronger in the core of a species' distribution than the periphery (i.e., goal 4).

\begin{tabular}{ccc}
\hline Species & $\begin{array}{c}\text { Removal effect on } \\
\text { survival }\end{array}$ & $\begin{array}{c}\text { Removal effect on } \\
\text { transition }\end{array}$ \\
\hline Brook trout & Larger $\left(^{*}\right)$ & Larger $(-)$ \\
Mottled sculpin & Smaller $\left(^{*}\right)$ & Smaller $(-)$ \\
\hline
\end{tabular}




\section{Figures}

Figure 1. Location of the 12 study sites in the Cheat River watershed, location of the Cheat River watershed in West Virginia, and the experimental groupings and spatial location of sites within the Gandy Creek sub-watershed.

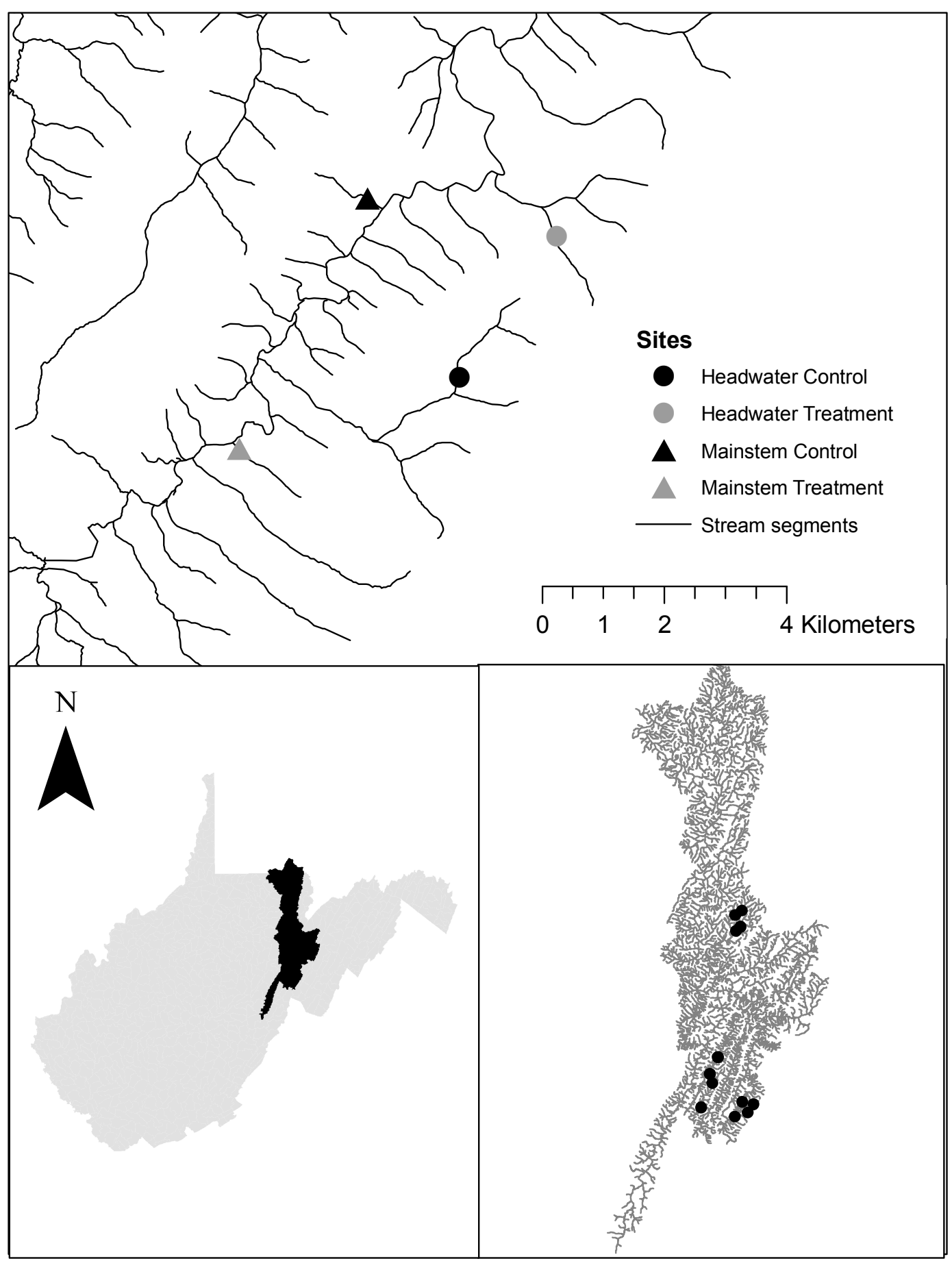


Figure 2. Abundance of young-of-the-year (YOY), small, and large adult brook trout among all sites and sampling occasions. Refer to Appendix $A 3$ for information regarding precision of estimates. Standard errors in abundance estimates were generally low (mean $=$ 0.55 , $\max =3.6$ for N-hat of 25). Categories of treatment and spatial location for sites are indicated above each site in panel 1 (July 2006). $\mathrm{MC}=$ mainstem tributary control site, $\mathrm{MT}=$ mainstem tributary treatment site, $\mathrm{HC}=$ headwater tributary control site, $\mathrm{HT}=$ headwater tributary treatment site. Site abbreviations are NB = Nan's Branch, LR = Lynn Run, ZH = Zinn Hollow, OH = Otis Hollow, LD = Lick Drain, LL = Laurel Lick, BR = Big Run, MR = Mudlick Run, GF = Glady Fork, SR = Swallow Rock Run, TR = Tanner Run, DR = Daniels Run. 


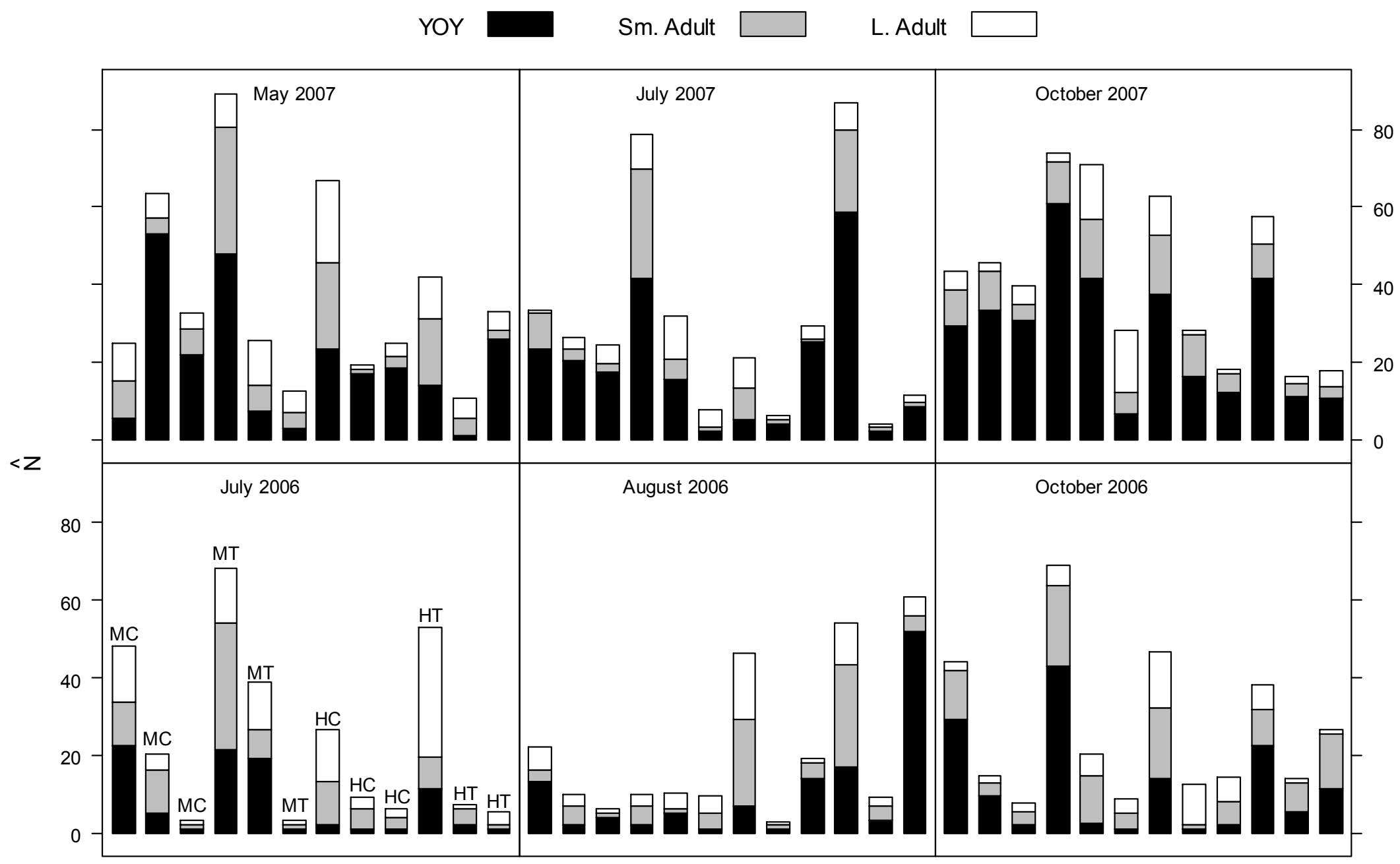

NB LR ZH OH LD LL BR MR GF SR TR DR NB LR ZH OH LD LL BR MR GF SR TR DR NB LR ZH OH LD LL BR MR GF SR TR DR 
Figure 3. Coefficient of variation in abundance of young-of-the-year (YOY), small, and large adult brook trout among all sites estimated across all sampling occasions. Site abbreviations are NB $=$ Nan's Branch, LR $=$ Lynn Run, ZH $=\mathrm{Zinn} \mathrm{Hollow}, \mathrm{OH}=\mathrm{Otis}$ Hollow, LD = Lick Drain, LL = Laurel Lick, BR = Big Run, MR = Mudlick Run, GF = Glady Fork, SR = Swallow Rock Run, TR = Tanner Run, DR = Daniels Run. Horizontal dashed lines indicate arbitrary cutoffs for "stable" (C.V. $<=0.25)$, "moderately stable" $(0.25<\mathrm{C} . \mathrm{V} .<=0.5)$, "moderately fluctuating" $(0.5<\mathrm{C} . \mathrm{V} .<=0.75)$, and "fluctuating" $(\mathrm{C} . \mathrm{V} .>0.75)$ abundance according to Freeman et al. (1988).

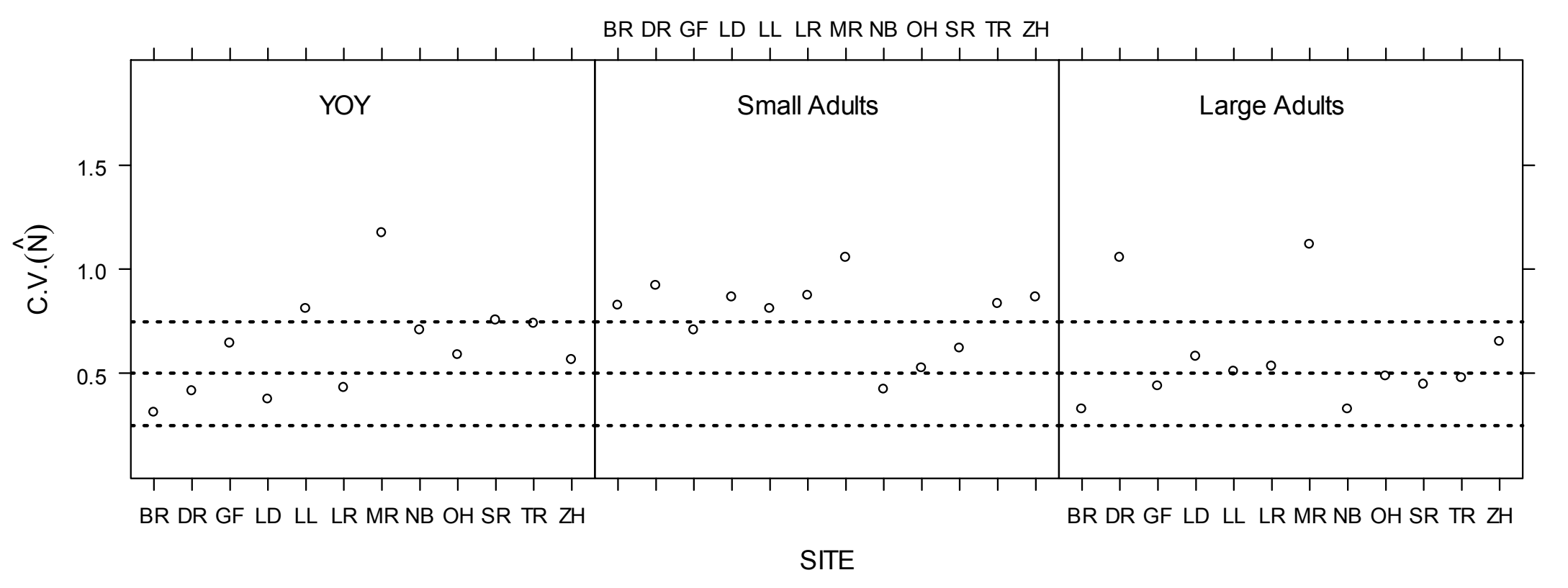


Figure 4. Abundance of young-of-the-year (YOY), small, and large mottled sculpin among all sites and sampling occasions. Refer to Appendix $A 3$ for information regarding precision of estimates. Standard errors in abundance estimates were generally low (mean $=$ 0.55 , $\max =3.6$ for $\mathrm{N}$-hat of 25). Categories of treatment and spatial location for sites are indicated above each site in panel 1 (July 2006). $\mathrm{MC}=$ mainstem tributary control site, $\mathrm{MT}=$ mainstem tributary treatment site, $\mathrm{HC}=$ headwater tributary control site, $\mathrm{HT}=$ headwater tributary treatment site. Site abbreviations are NB = Nan's Branch, LR = Lynn Run, ZH = Zinn Hollow, OH = Otis Hollow, LD = Lick Drain, LL = Laurel Lick, BR = Big Run, MR = Mudlick Run, GF = Glady Fork, SR = Swallow Rock Run, TR = Tanner Run, DR = Daniels Run. 


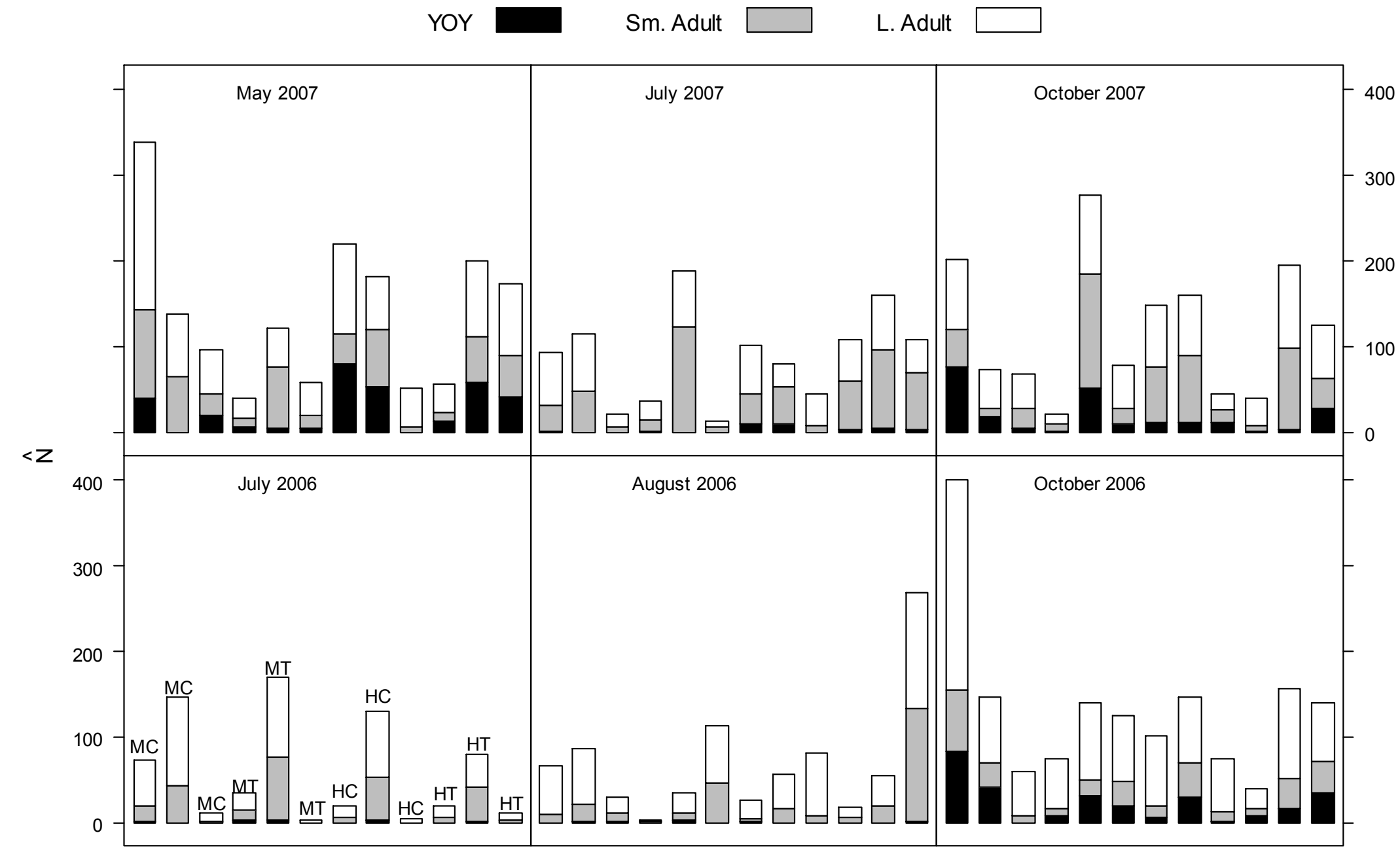

NB LR ZH OH LD LL BR MR GF SR TR DR NB LR ZH OH LD LL BR MR GF SR TR DR NB LR ZH OH LD LL BR MR GF SR TR DR 
Figure 5. Coefficient of variation in abundance of young-of-the-year (YOY), small, and large adult mottled sculpin among all sites estimated across all sampling occasions. Site abbreviations are NB $=$ Nan's Branch, LR $=$ Lynn Run, ZH $=$ Zinn Hollow, OH $=$ Otis Hollow, LD = Lick Drain, LL = Laurel Lick, BR = Big Run, MR = Mudlick Run, GF = Glady Fork, SR = Swallow Rock Run, TR = Tanner Run, DR = Daniels Run. Horizontal dashed lines indicate arbitrary cutoffs for "stable" (C.V. $<=0.25)$, "moderately stable" $(0.25<$ C.V. $<=0.5)$, "moderately fluctuating" $(0.5<$ C.V. $<=0.75)$, and "fluctuating" $(\mathrm{C} . \mathrm{V} .>0.75)$ abundance according to Freeman et al. (1988).

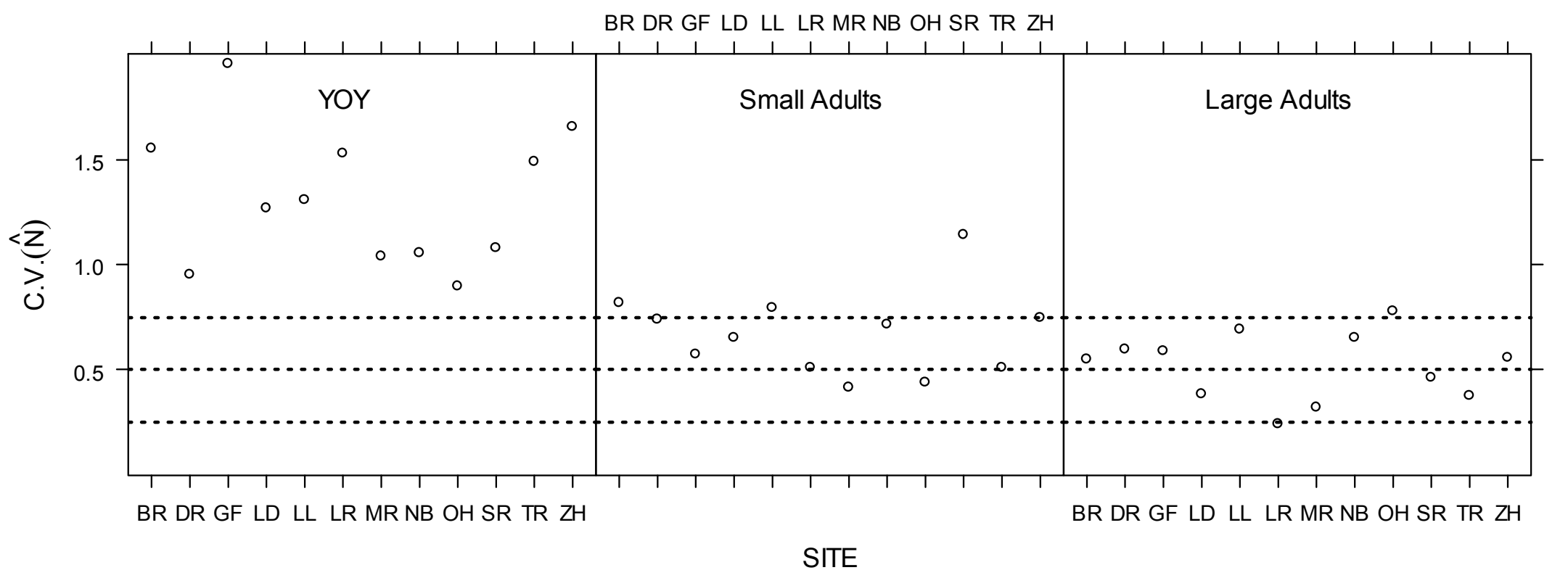


Figure 6. Estimated abundance of young-of-the-year (YOY), small, and large adult brook trout in all sites on the first sampling occasion in July 2006 both pre-removal (i.e., N-hat) and post-removal (i.e., N-hat - total number caught (n)). Where N-hat - n was less than 1 (i.e., given standard error in N-hat), N-hat $-\mathrm{n}$ was estimated to be 1 . Site abbreviations are indicated under the panel for "Pre-removal" (left), while categories of treatment and spatial location for the sites are indicated in each "Post-removal" panel (right). Refer to Appendix A3 for information regarding precision of abundance estimates. Site abbreviations are NB = Nan's Branch, LR = Lynn Run, ZH = Zinn Hollow, OH = Otis Hollow, LD = Lick Drain, LL = Laurel Lick, BR = Big Run, MR = Mudlick Run, GF = Glady Fork, SR $=$ Swallow Rock Run, TR $=$ Tanner Run, DR = Daniels Run. Standard errors in abundance estimates were generally low $($ mean $=0.55, \max =3.6$ for $\mathrm{N}$-hat of 25$) . \mathrm{MC}=$ mainstem tributary control site, $\mathrm{MT}=$ mainstem tributary treatment site, $\mathrm{HC}=$ headwater tributary control site, $\mathrm{HT}=$ headwater tributary treatment site. 
Pre-removal - YOY

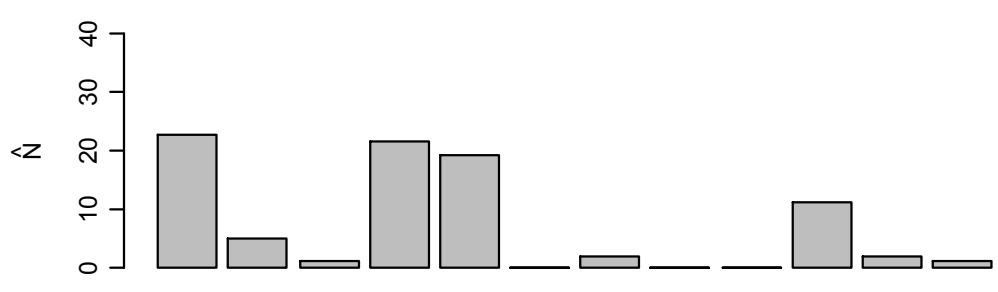

$\begin{array}{llllllllllll}N B & L R & Z H & O H & L D & L L & B R & M R & G F & S R & T R & D R\end{array}$ Site

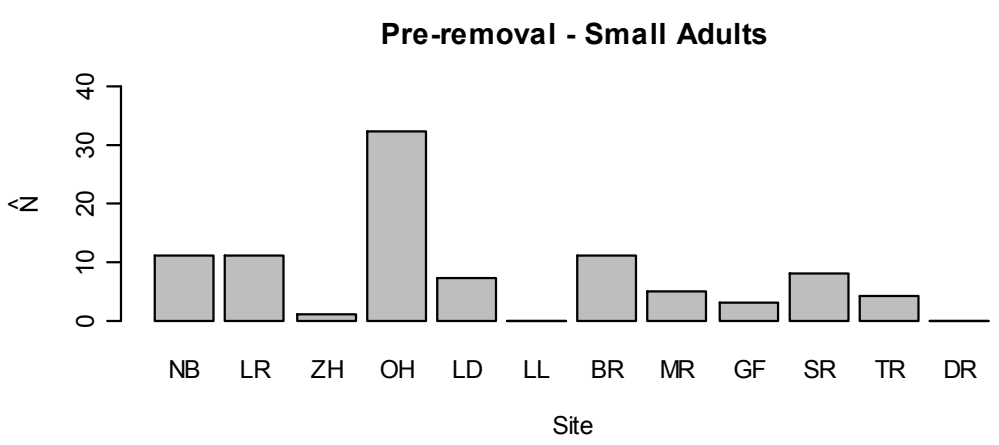

Pre-removal - Large Adults

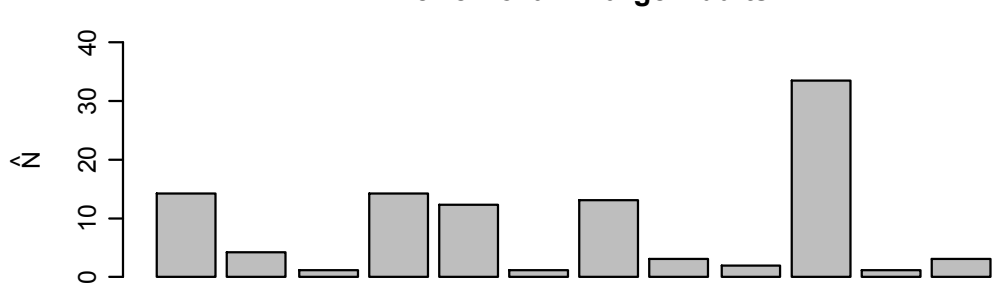

$\begin{array}{llllllllllll}\mathrm{NB} & \mathrm{LR} & \mathrm{ZH} & \mathrm{OH} & \mathrm{LD} & \mathrm{LL} & \mathrm{BR} & \mathrm{MR} & \mathrm{GF} & \mathrm{SR} & \mathrm{TR} & \mathrm{DR}\end{array}$ Site
Post-removal - YOY

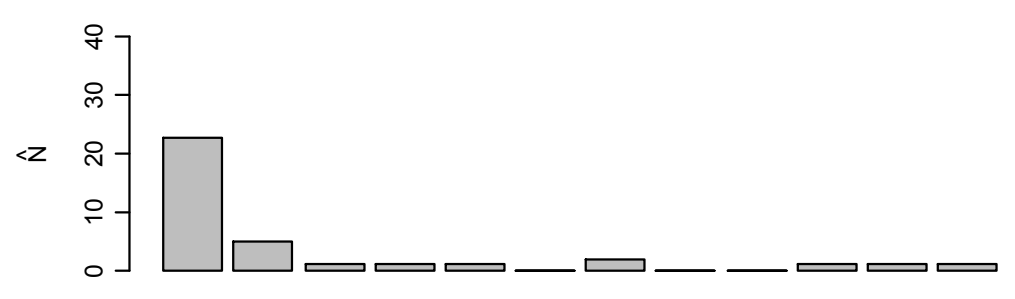

MC MC MC $\mathrm{MT}$ MT $\mathrm{MT}$ HC $\mathrm{HC}$ HC $\mathrm{HT}_{\mathrm{H}}$ HT

Site

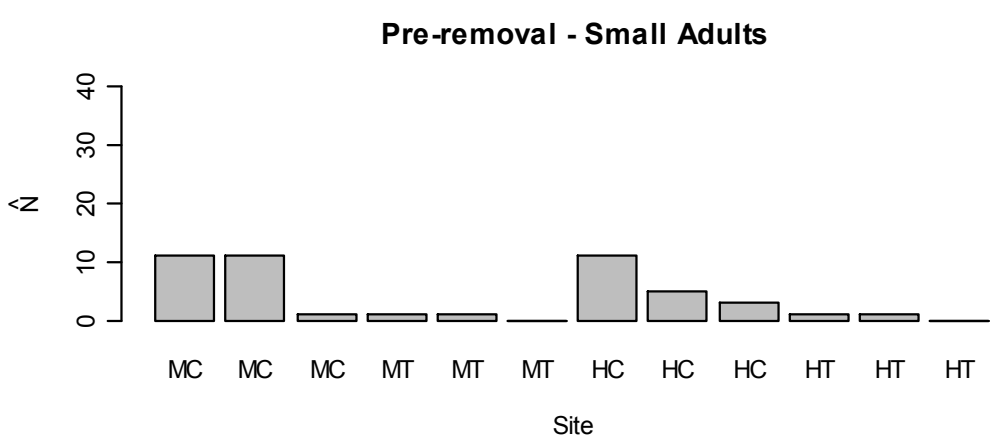

Post-removal - Large Adults

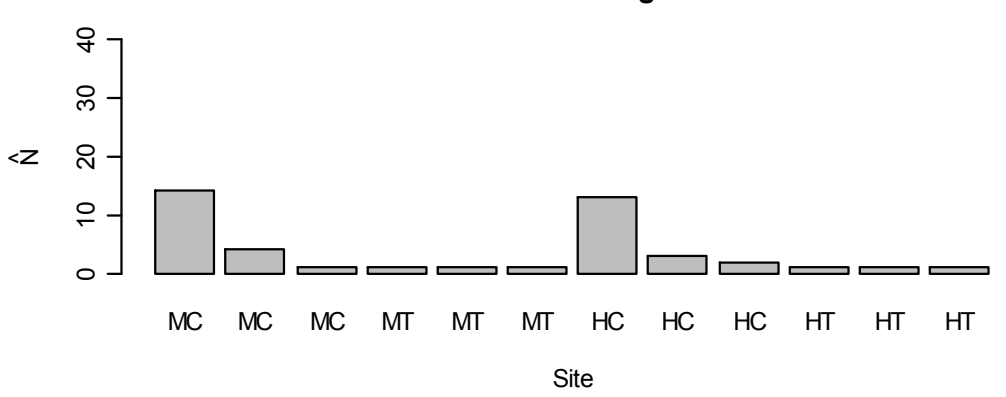


Figure 7. Estimated abundance of young-of-the-year (YOY), small, and large adult mottled sculpin in all sites on the first sampling occasion in July 2006 both pre-removal (i.e., N-hat) and post-removal (i.e., N-hat - total number caught (n)). Where N-hat - n was less than 1 (i.e., given standard error in N-hat), N-hat $-\mathrm{n}$ was forced to 1 . Site abbreviations are indicated under the panel for "Preremoval" (left), while categories of treatment and spatial location for the sites are indicated in each "Post-removal" panel (right).

Refer to Appendix A3 for information regarding precision of abundance estimates. Site abbreviations are NB = Nan's Branch, LR = Lynn Run, ZH = Zinn Hollow, OH = Otis Hollow, LD = Lick Drain, LL = Laurel Lick, BR = Big Run, MR = Mudlick Run, GF = Glady Fork, SR $=$ Swallow Rock Run, TR $=$ Tanner Run, DR = Daniels Run. Standard errors in abundance estimates were generally low $($ mean $=0.55, \max =3.6$ for $\mathrm{N}$-hat of 25$) . \mathrm{MC}=$ mainstem tributary control site, $\mathrm{MT}=$ mainstem tributary treatment site, $\mathrm{HC}=$ headwater tributary control site, $\mathrm{HT}=$ headwater tributary treatment site. 
Pre-removal - YOY

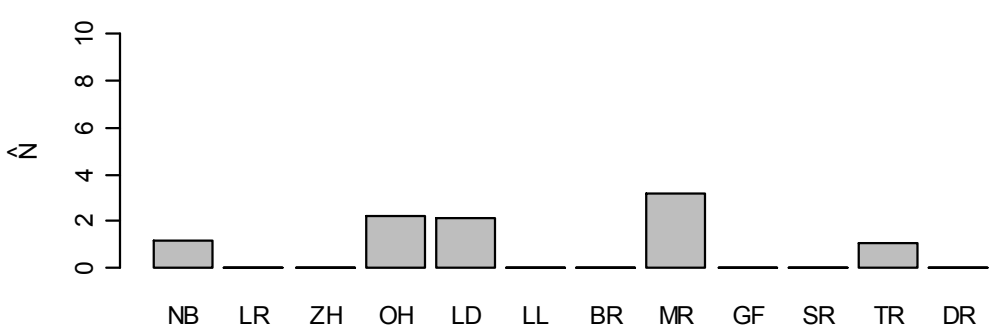

Site

Pre-removal - Small Adults

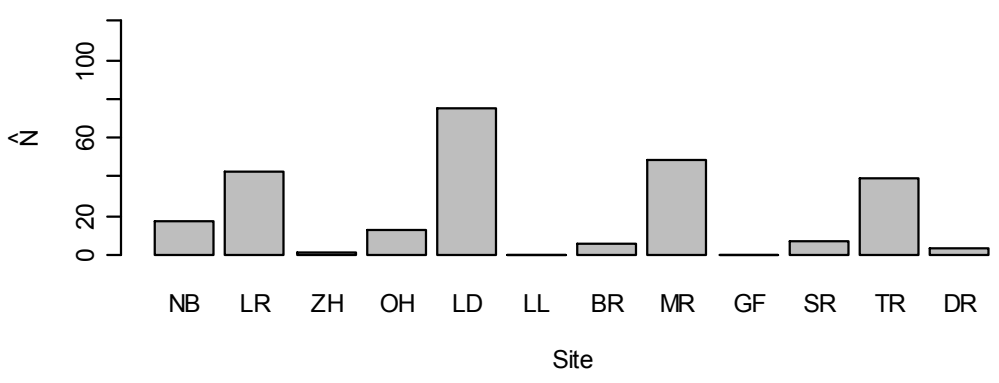

Pre-removal - Large Adults

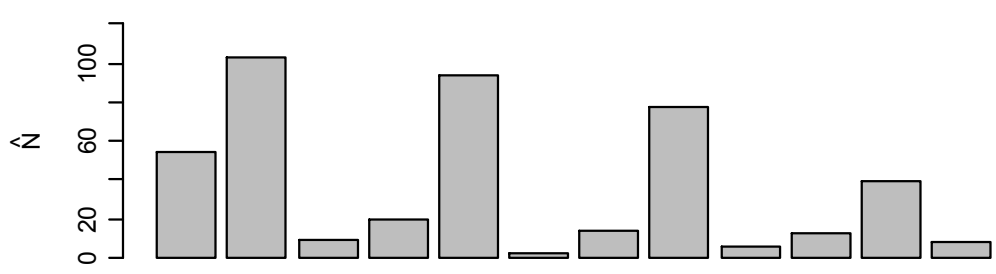

$\begin{array}{lllllllllllll}N B & L R & \mathrm{ZH} & \mathrm{OH} & \mathrm{LD} & \mathrm{LL} & \mathrm{BR} & \mathrm{MR} & \mathrm{GF} & \mathrm{SR} & \mathrm{TR} & \mathrm{DR}\end{array}$ Site
Post-removal - YOY

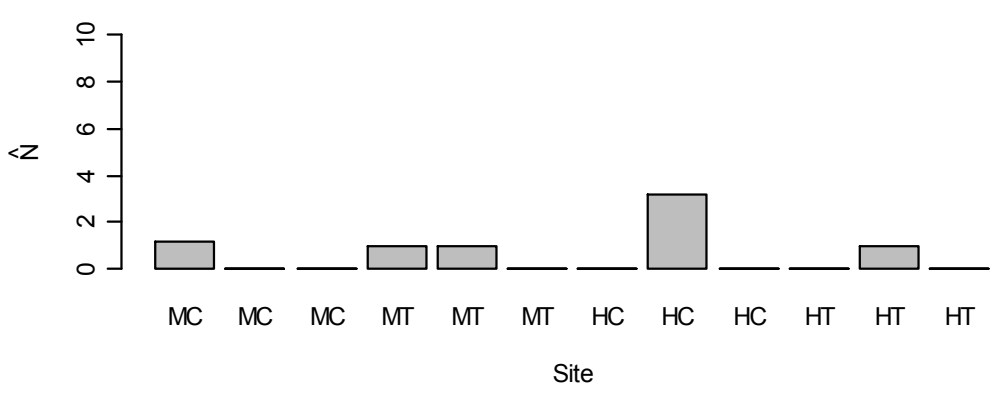

Post-removal - Small Adults

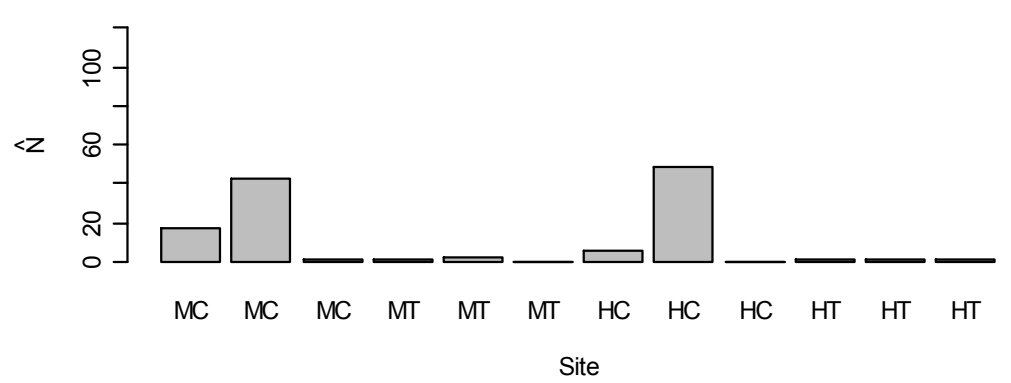

Post-removal - Large Adults

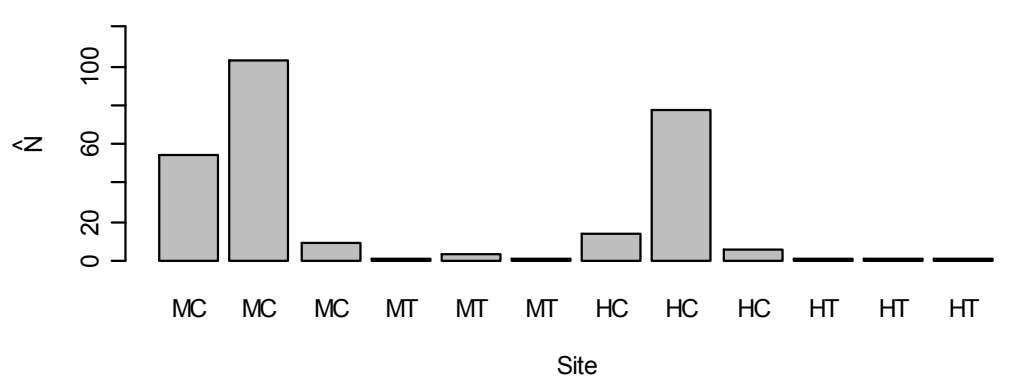


Figure 8. Log-transformed proportional recovery among all sites for all age classes of brook trout. YOY = young-of-the year age class, $\mathrm{SAD}=$ small adults, $\mathrm{LAD}=$ large adults. Site abbreviations are NB $=$ Nan's Branch, LR $=\mathrm{Lynn} \mathrm{Run}, \mathrm{ZH}=\mathrm{Zinn} \mathrm{Hollow}, \mathrm{OH}=$ Otis Hollow, LD = Lick Drain, LL = Laurel Lick, BR = Big Run, MR = Mudlick Run, GF = Glady Fork, SR $=$ Swallow Rock Run, $\mathrm{TR}=$ Tanner Run, DR = Daniels Run. Standard errors in abundance estimates were generally low ( $\operatorname{mean}=0.55$, max $=3.6$ for N-hat of 25). $\mathrm{MC}=$ mainstem tributary control site, $\mathrm{MT}=$ mainstem tributary treatment site, $\mathrm{HC}=$ headwater tributary control site, $\mathrm{HT}=$ headwater tributary treatment site. 


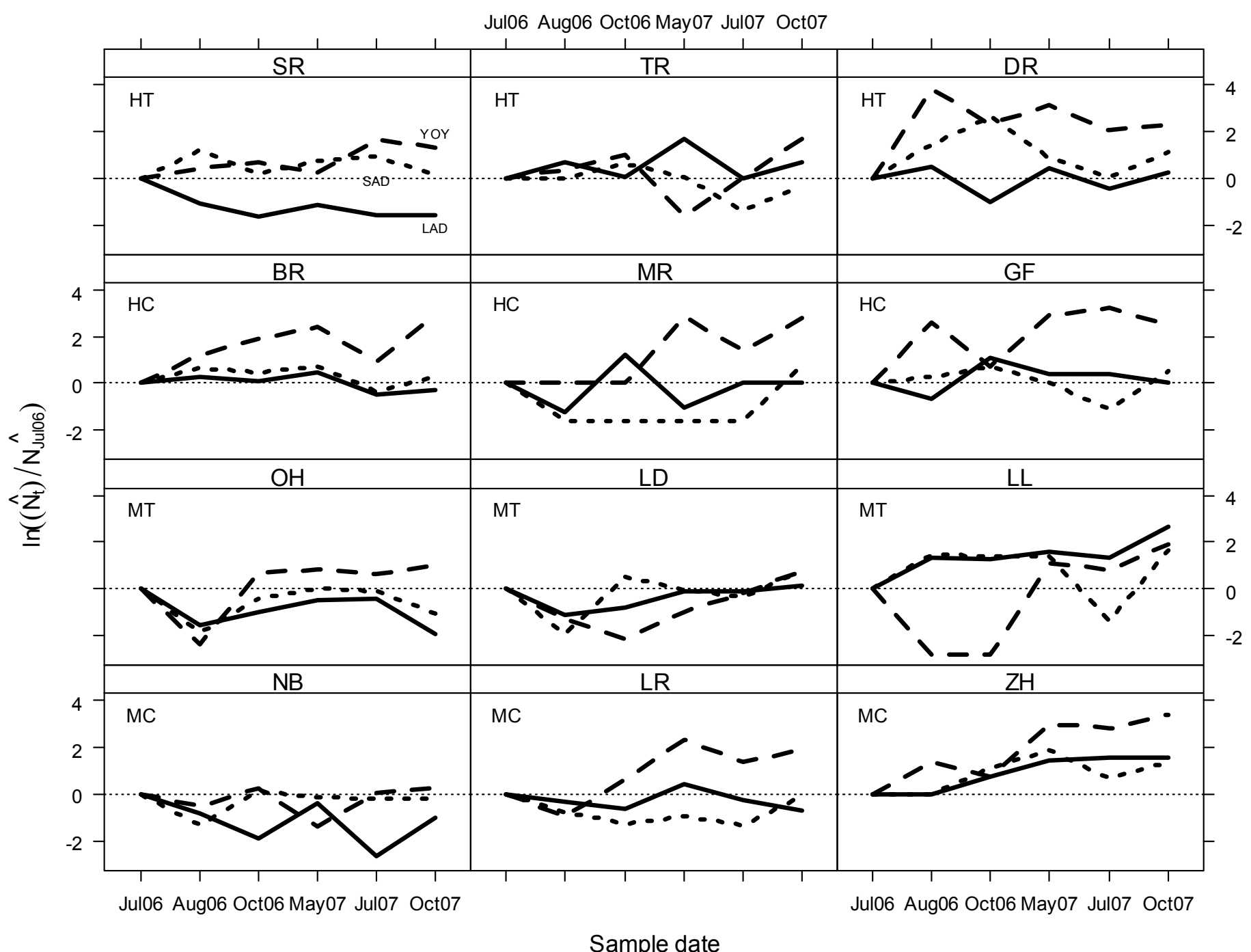


Figure 9. Log-transformed proportional recovery among all sites for all age classes of brook trout. YOY = young-of-the year age class, $\mathrm{SAD}=$ small adults, $\mathrm{LAD}=$ large adults. Site abbreviations are NB $=$ Nan's Branch, LR $=$ Lynn Run, ZH $=$ Zinn Hollow, OH $=$ Otis Hollow, LD = Lick Drain, LL = Laurel Lick, BR = Big Run, MR = Mudlick Run, GF = Glady Fork, SR $=$ Swallow Rock Run, $\mathrm{TR}=$ Tanner Run, DR = Daniels Run. Standard errors in abundance estimates were generally low ( $\operatorname{mean}=0.55$, max $=3.6$ for N-hat of 25). $\mathrm{MC}=$ mainstem tributary control site, $\mathrm{MT}=$ mainstem tributary treatment site, $\mathrm{HC}=$ headwater tributary control site, $\mathrm{HT}=$ headwater tributary treatment site. 


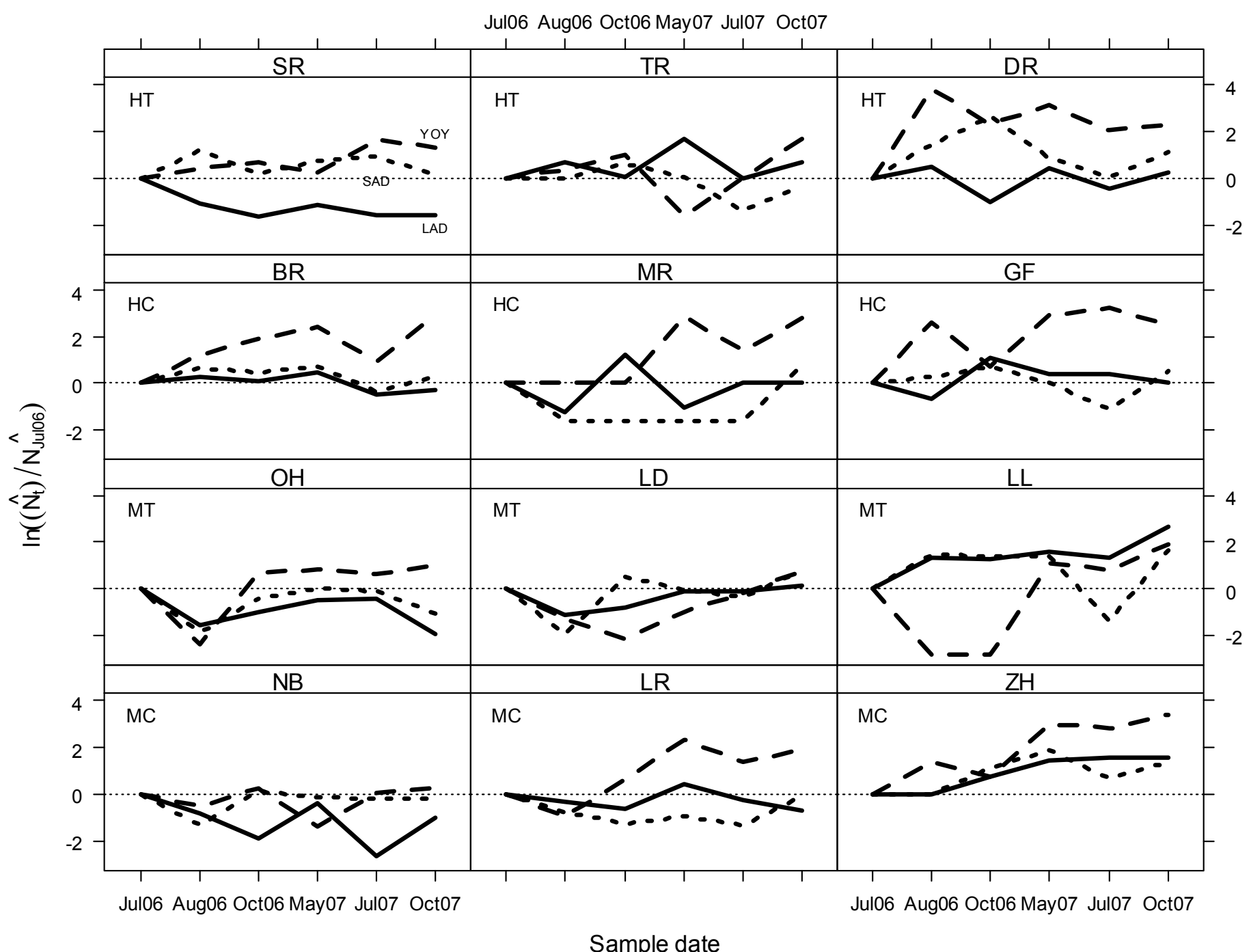


Figure 10. Predicted proportional recovery (i.e., abundance at time $t$ or $\mathrm{N}_{\mathrm{t}}$ divided by preremoval abundance or $\mathrm{N}_{\text {rem }}$ ) of young-of-the-year brook trout among mainstem tributary control $(\mathrm{MC}, \mathrm{n}=3)$ sites, mainstem removal treatment $(\mathrm{MT}, \mathrm{n}=3)$ sites, headwater tributary control sites $(\mathrm{HC}, \mathrm{n}=3)$ and headwater treatment $(\mathrm{HT}, \mathrm{n}=3)$ for all sampling dates following the initial removal at average values for local physical and chemical habitat covariates based on the $95 \%$ confidence set of models used for inference (Table 13) and their model-averaged coefficients (Appendix D). The horizontal dashed line at 0 indicates where $\mathrm{N}_{\mathrm{t}}=\mathrm{N}_{\mathrm{rem}}$ or $\mathrm{N}_{\mathrm{t}} / \mathrm{N}_{\mathrm{rem}}=1$.

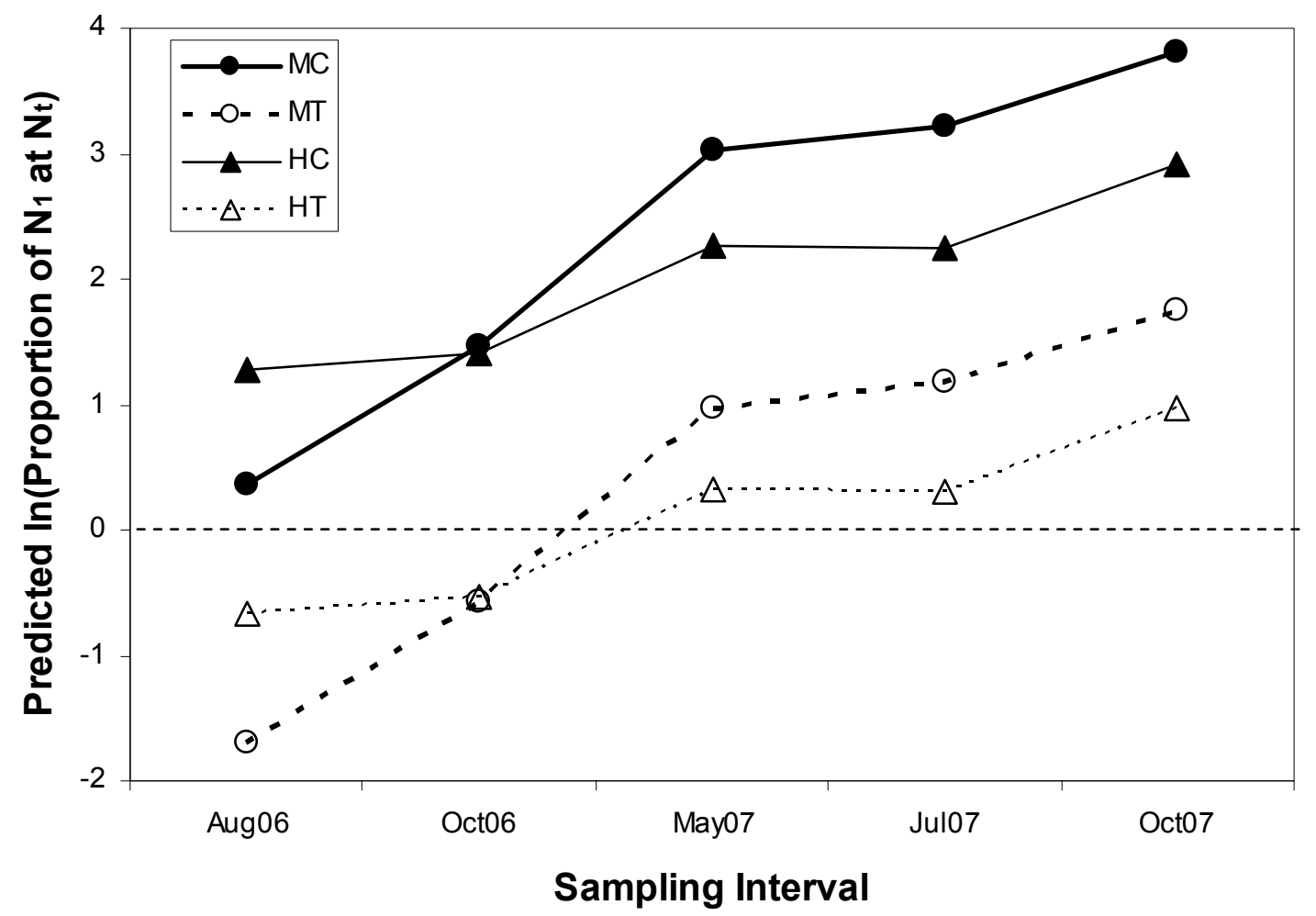


Figure 11. Predicted proportional recovery (i.e., abundance at time $t$ divided by preremoval abundance) of small adult brook trout among mainstem tributary control (MC, $\mathrm{n}=3$ ) sites, mainstem removal treatment $(\mathrm{MT}, \mathrm{n}=3)$ sites, headwater tributary control sites $(\mathrm{HC}, \mathrm{n}=3)$ and headwater treatment $(\mathrm{HT}, \mathrm{n}=3)$ for all sampling dates following the initial removal at average values for local physical and chemical habitat covariates based on the $95 \%$ confidence set of models used for inference (Table 13) and their model-averaged coefficients (Appendix D). The horizontal dashed line at 0 indicates where $\mathrm{N}_{t}=\mathrm{N}_{\text {rem }}$ or $\mathrm{N}_{\mathrm{t}} / \mathrm{N}_{\mathrm{rem}}=1$.

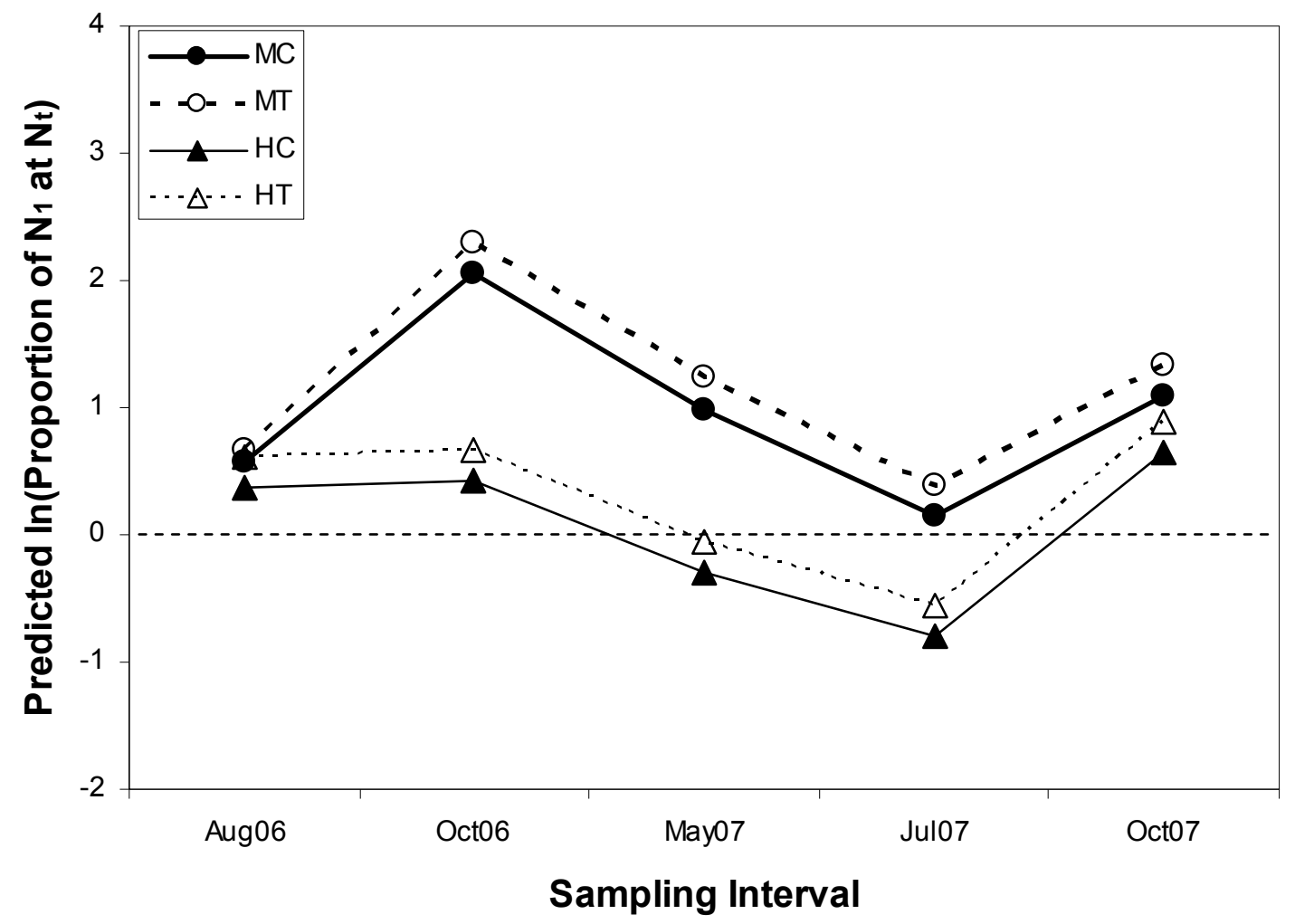


Figure 12. Predicted proportional recovery (i.e., abundance at time $t$ divided by preremoval abundance) of large adult brook trout among mainstem tributary control (MC, $\mathrm{n}=3$ ) sites, mainstem removal treatment $(\mathrm{MT}, \mathrm{n}=3)$ sites, headwater tributary control sites $(\mathrm{HC}, \mathrm{n}=3)$ and headwater treatment $(\mathrm{HT}, \mathrm{n}=3)$ for all sampling dates following the initial removal at average values for local physical and chemical habitat covariates based on the $95 \%$ confidence set of models used for inference (Table 13) and their model-averaged coefficients (Appendix D). The horizontal dashed line at 0 indicates where $\mathrm{N}_{\mathrm{t}}=\mathrm{N}_{\mathrm{rem}}$ or $\mathrm{N}_{\mathrm{t}} / \mathrm{N}_{\mathrm{rem}}=1$.

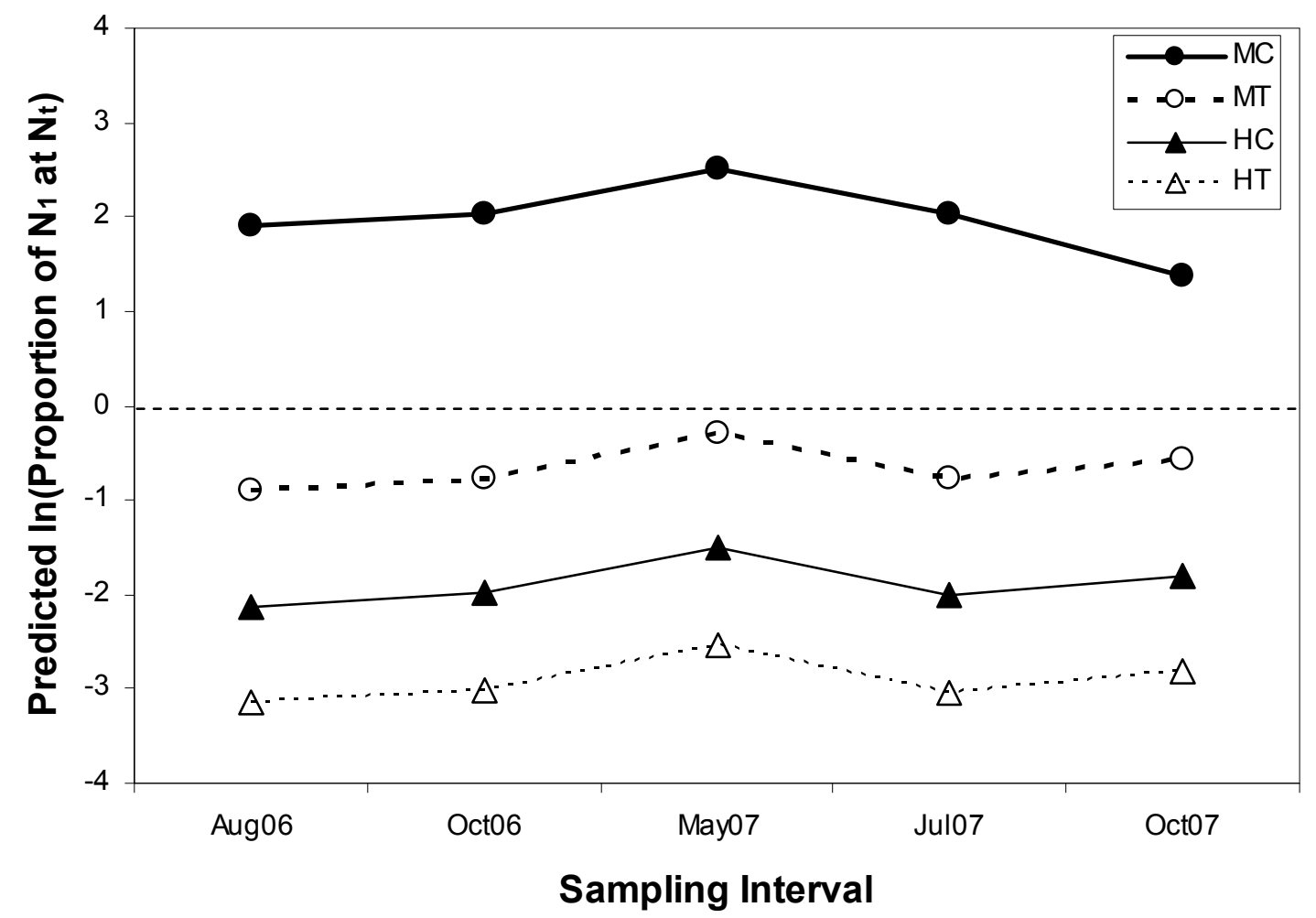


Figure 13. Predicted proportional recovery (i.e., abundance at time $t$ or $\mathrm{N}_{\mathrm{t}}$ divided by preremoval abundance or $\mathrm{N}_{\text {rem }}$ ) of young-of-the-year mottled sculpin among mainstem tributary control $(\mathrm{MC}, \mathrm{n}=3$ ) sites, mainstem removal treatment $(\mathrm{MT}, \mathrm{n}=3)$ sites, headwater tributary control sites $(\mathrm{HC}, \mathrm{n}=3)$ and headwater treatment $(\mathrm{HT}, \mathrm{n}=3)$ for all sampling dates following the initial removal at average values for local physical and chemical habitat covariates based on the $95 \%$ confidence set of models used for inference (Table 14) and their model-averaged coefficients (Appendix D). The horizontal dashed line at 0 indicates where $\mathrm{N}_{\mathrm{t}}=\mathrm{N}_{\text {rem }}$ or $\mathrm{N}_{\mathrm{t}} / \mathrm{N}_{\text {rem }}=1$.

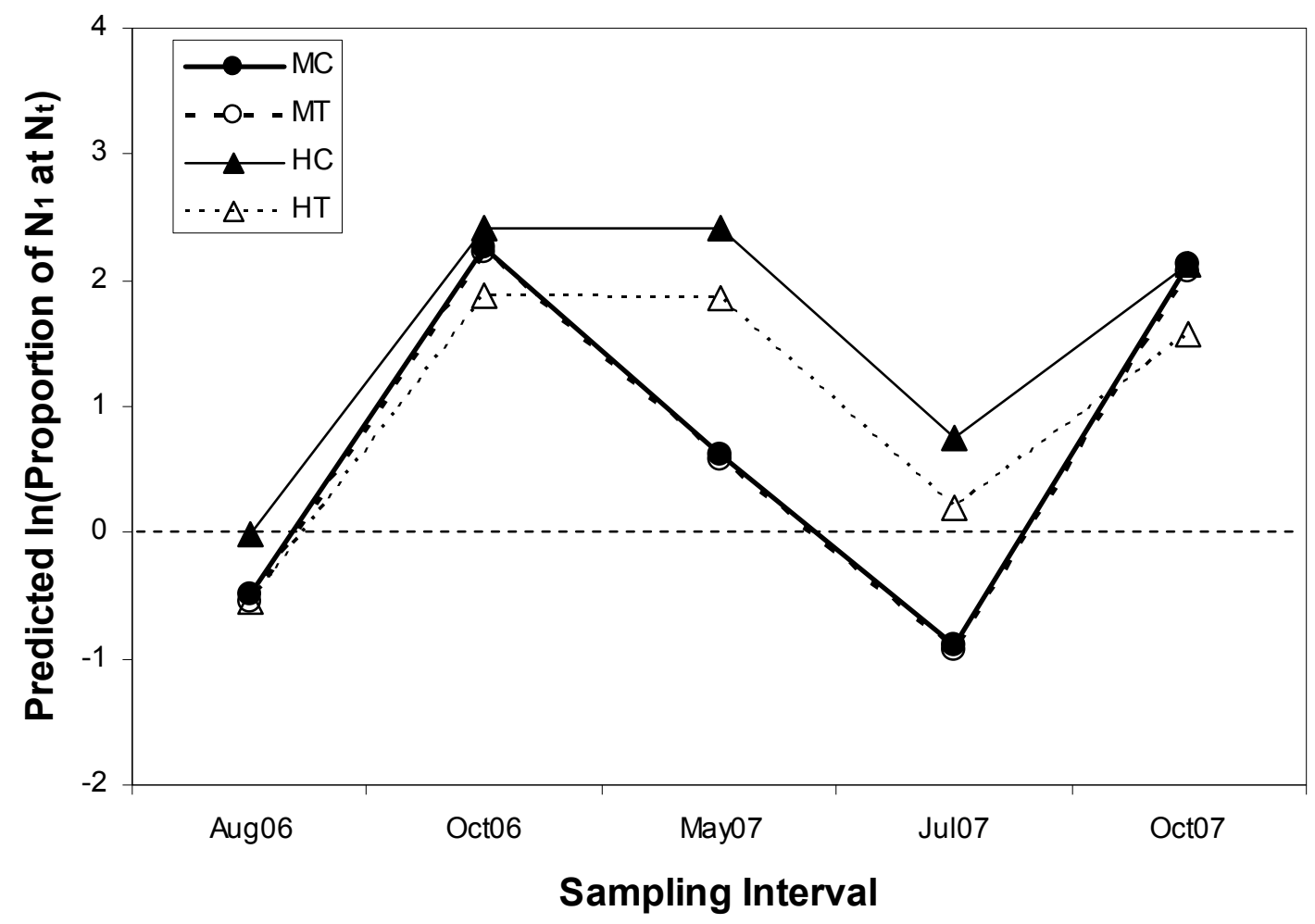


Figure 14. Predicted proportional recovery (i.e., abundance at time $t$ or $\mathrm{N}_{\mathrm{t}}$ divided by preremoval abundance or $\mathrm{N}_{\text {rem }}$ ) of small adult mottled sculpin among mainstem tributary control (MC, $n=3)$ sites, mainstem removal treatment $(M T, n=3)$ sites, headwater tributary control sites $(\mathrm{HC}, \mathrm{n}=3)$ and headwater treatment $(\mathrm{HT}, \mathrm{n}=3)$ for all sampling dates following the initial removal at average values for local physical and chemical habitat covariates based on the $95 \%$ confidence set of models used for inference (Table 14) and their model-averaged coefficients (Appendix D). The horizontal dashed line at 0 indicates where $\mathrm{N}_{\mathrm{t}}=\mathrm{N}_{\mathrm{rem}}$ or $\mathrm{N}_{\mathrm{t}} / \mathrm{N}_{\mathrm{rem}}=1$.

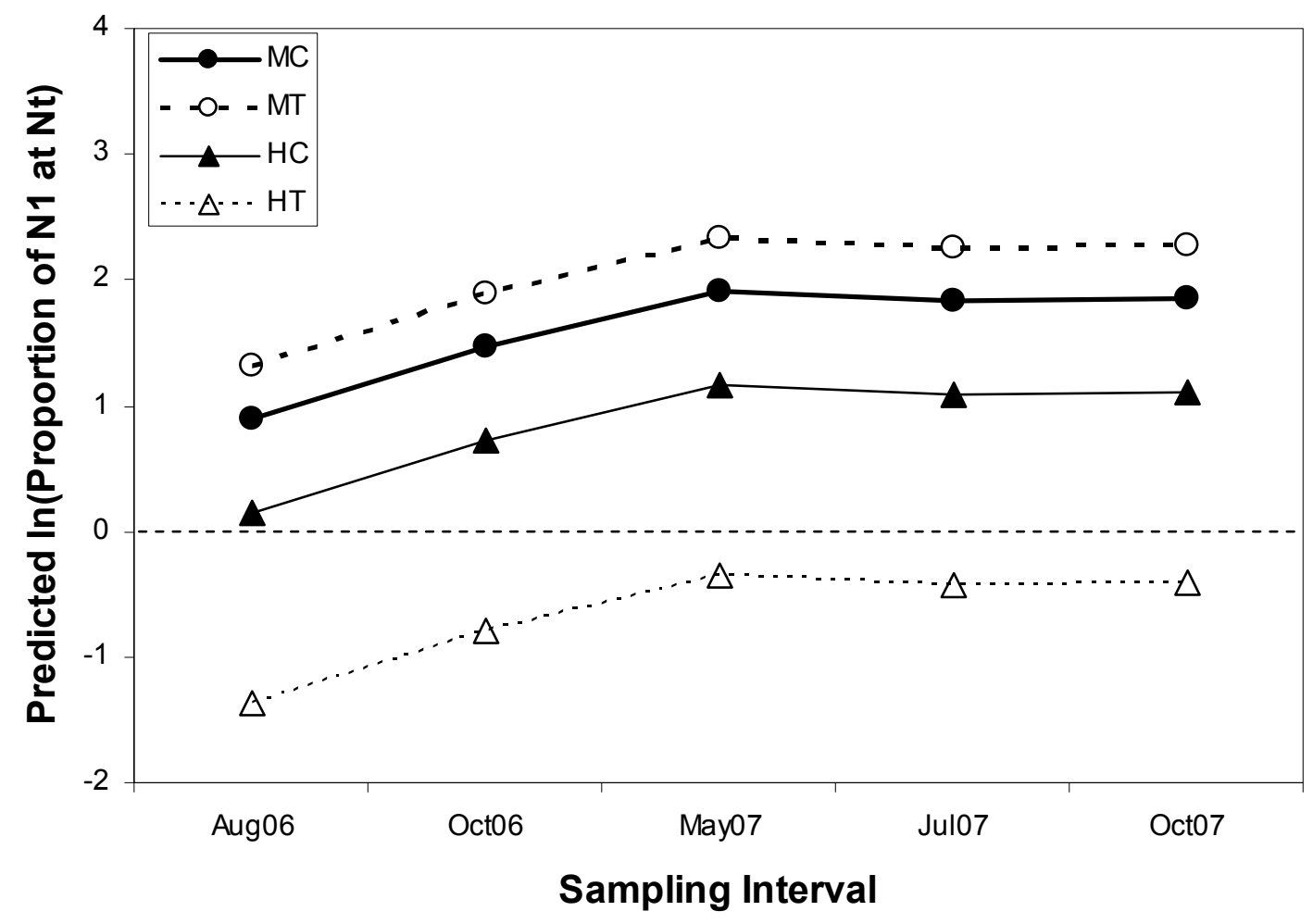


Figure 15. Predicted proportional recovery (i.e., abundance at time $t$ or $\mathrm{N}_{\mathrm{t}}$ divided by preremoval abundance or $\mathrm{N}_{\text {rem }}$ ) of large adult mottled sculpin among mainstem tributary control $(M C, n=3)$ sites, mainstem removal treatment $(M T, n=3)$ sites, headwater tributary control sites $(\mathrm{HC}, \mathrm{n}=3)$ and headwater treatment sites $(\mathrm{HT}, \mathrm{n}=3)$ for all sampling dates following the initial removal at average values for local physical and chemical habitat covariates based on the $95 \%$ confidence set of models used for inference (Table 14) and their model-averaged coefficients (Appendix D). The horizontal dashed line at 0 indicates where $\mathrm{N}_{\mathrm{t}}=\mathrm{N}_{\mathrm{rem}}$ or $\mathrm{N}_{\mathrm{t}} / \mathrm{N}_{\mathrm{rem}}=1$.

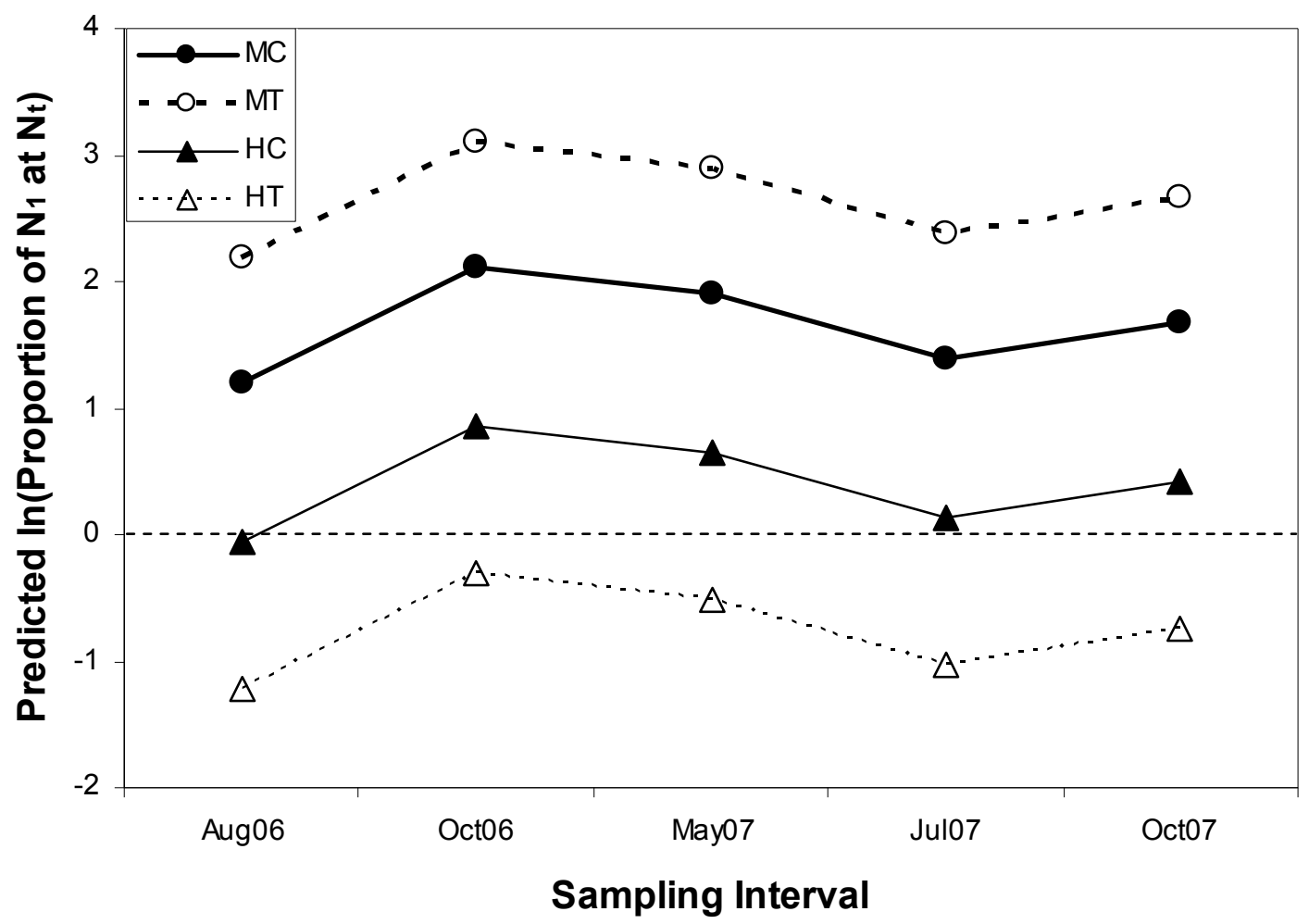


Figure 16. Predicted per capita growth rate $\left(r=\ln \left(\mathrm{N}_{\mathrm{t}+1} / \mathrm{N}_{\mathrm{t}}\right)\right)$ of young-of-the-year brook trout among mainstem tributary control $(\mathrm{MC}, \mathrm{n}=3)$, mainstem removal treatment (MT, $\mathrm{n}=3)$, headwater tributary control, $(\mathrm{HC}, \mathrm{n}=3)$ and headwater treatment $(\mathrm{HT}, \mathrm{n}=3)$ sites for all sampling intervals at fixed mean values for local physical and chemical habitat covariates based on the $95 \%$ confidence set of models used for inference (Table 15) and their model-averaged coefficients (Appendix E). The horizontal line is at $r=0$ indicates unchanging population size (i.e., $\mathrm{N}_{\mathrm{t}+1}=\mathrm{N}_{\mathrm{t}}$ ).

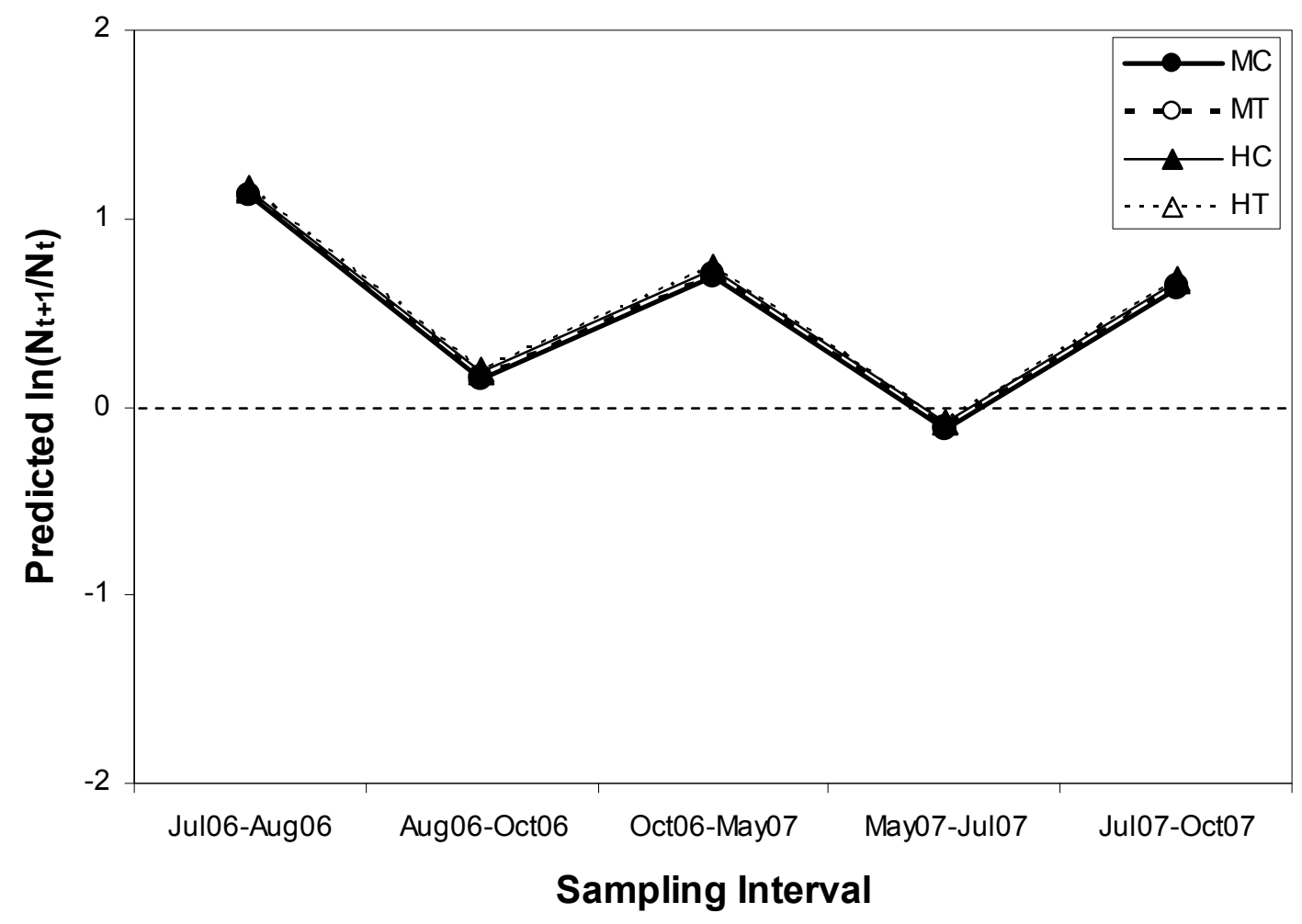


Figure 17. Predicted per capita growth rate $\left(r=\ln \left(\mathrm{N}_{\mathrm{t}+1} / \mathrm{N}_{\mathrm{t}}\right)\right)$ of small adult brook trout among mainstem tributary control $(\mathrm{MC}, \mathrm{n}=3)$, mainstem removal treatment $(\mathrm{MT}, \mathrm{n}=3)$, headwater tributary control, $(\mathrm{HC}, \mathrm{n}=3)$ and headwater treatment $(\mathrm{HT}, \mathrm{n}=3)$ sites for all sampling intervals at fixed mean values for local physical and chemical habitat covariates based on the $95 \%$ confidence set of models used for inference (Table 15) and their model-averaged coefficients (Appendix E). The horizontal line is at $r=0$ indicates unchanging population size (i.e., $\mathrm{N}_{\mathrm{t}+1}=\mathrm{N}_{\mathrm{t}}$ ).

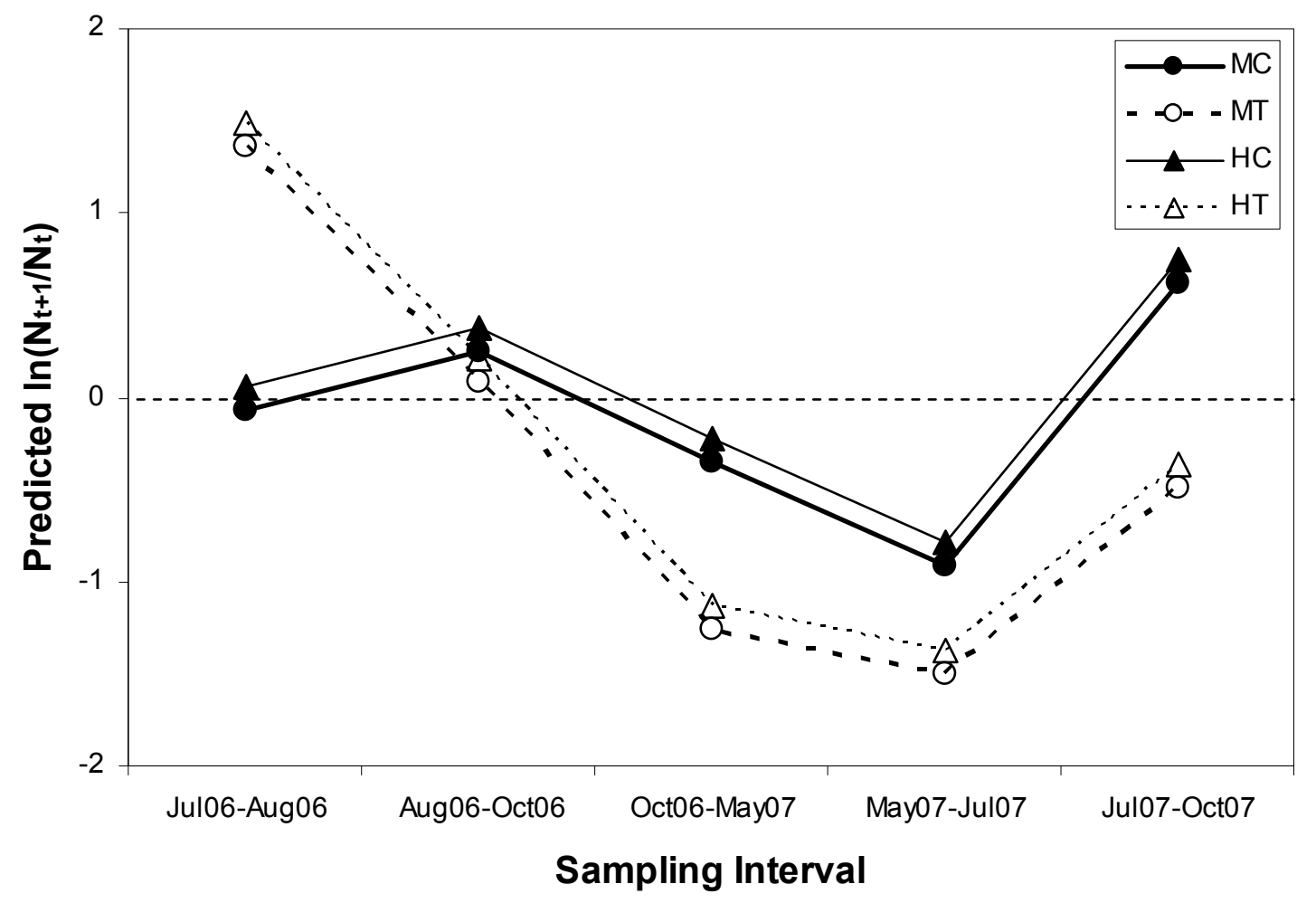


Figure 18. Predicted per capita growth rate $\left(r=\ln \left(\mathrm{N}_{\mathrm{t}+1} / \mathrm{N}_{\mathrm{t}}\right)\right)$ of large adult brook trout among mainstem tributary control $(\mathrm{MC}, \mathrm{n}=3)$, mainstem removal treatment $(\mathrm{MT}, \mathrm{n}=3)$, headwater tributary control, $(\mathrm{HC}, \mathrm{n}=3)$ and headwater treatment $(\mathrm{HT}, \mathrm{n}=3)$ sites for all sampling intervals at fixed mean values for local physical and chemical habitat covariates based on the $95 \%$ confidence set of models used for inference (Table 15) and their model-averaged coefficients (Appendix E). The horizontal line is at $r=0$ indicates unchanging population size (i.e., $\mathrm{N}_{\mathrm{t}+1}=\mathrm{N}_{\mathrm{t}}$ ).

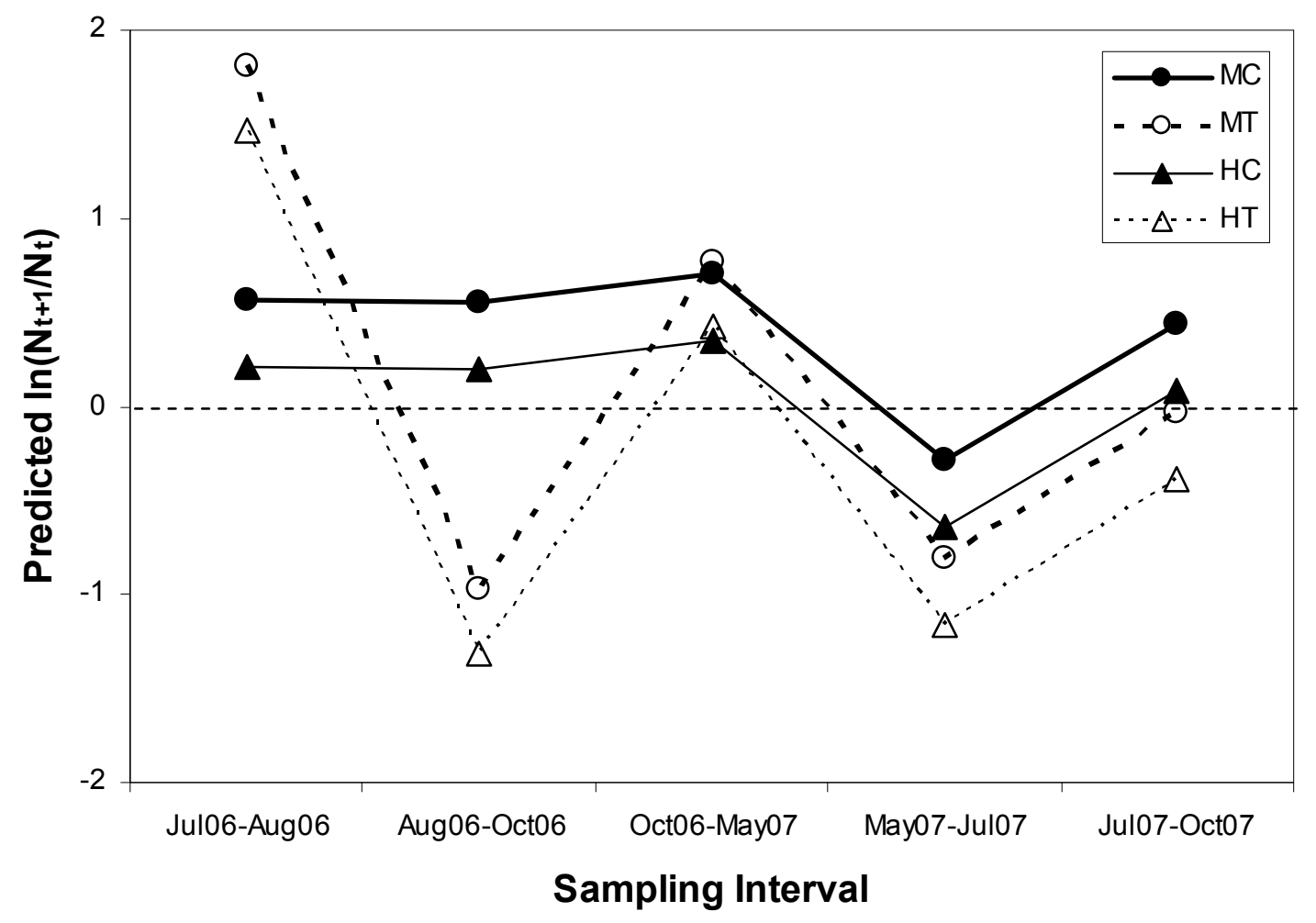


Figure 19. Predicted per capita growth rate $\left(r=\ln \left(\mathrm{N}_{\mathrm{t}+1} / \mathrm{N}_{\mathrm{t}}\right)\right)$ of young-of-the-year mottled sculpin among mainstem tributary control $(\mathrm{MC}, \mathrm{n}=3)$, mainstem removal treatment (MT, $n=3)$, headwater tributary control, $(\mathrm{HC}, \mathrm{n}=3)$ and headwater treatment $(\mathrm{HT}, \mathrm{n}=3)$ sites for all sampling intervals at fixed mean values for local physical and chemical habitat covariates based on the $95 \%$ confidence set of models used for inference (Table 16) and their model-averaged coefficients (Appendix E). The horizontal line is at $r=0$ indicates unchanging population size (i.e., $\mathrm{N}_{\mathrm{t}+1}=\mathrm{N}_{\mathrm{t}}$ ).

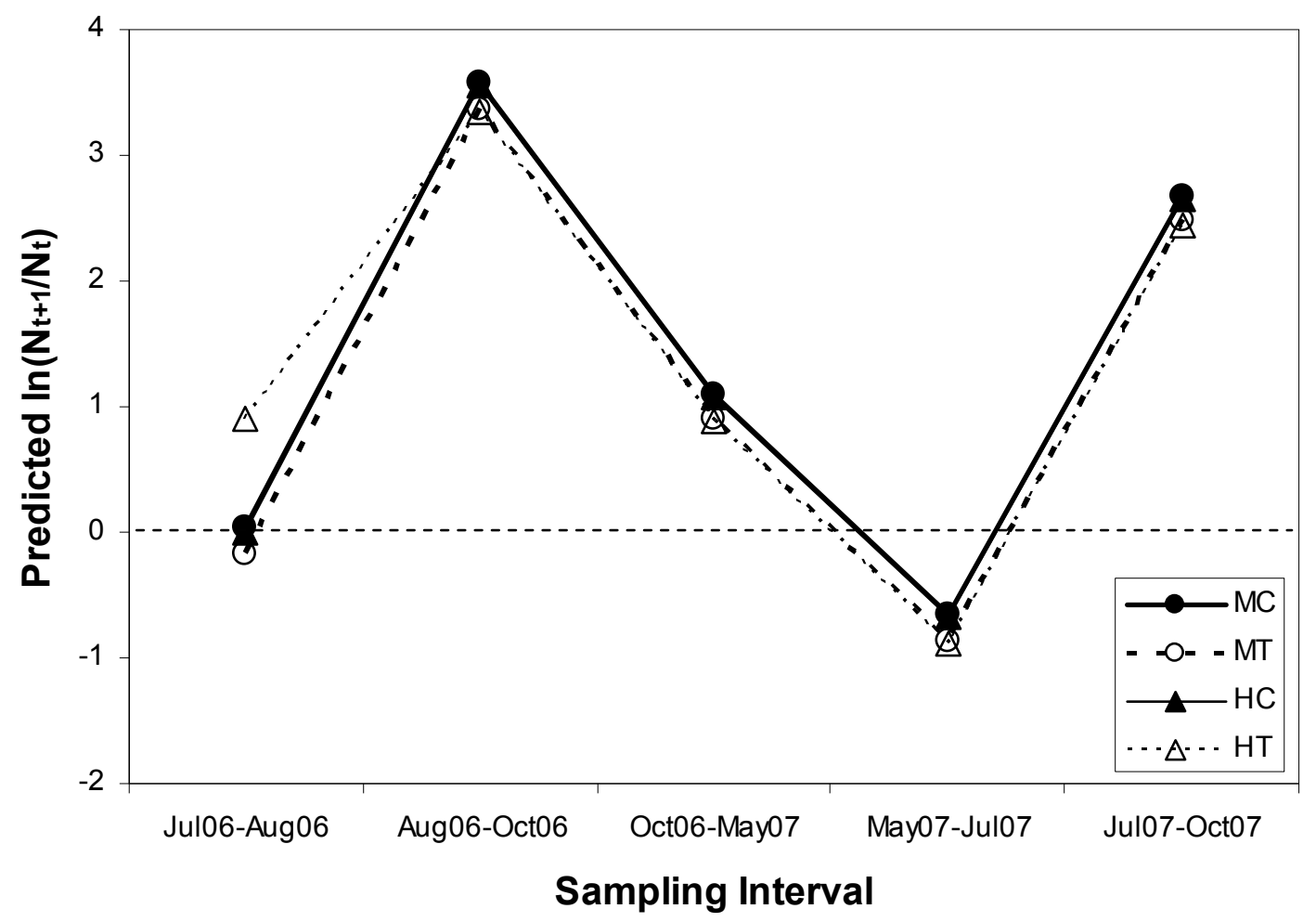


Figure 20. Predicted per capita growth rate $\left(r=\ln \left(\mathrm{N}_{\mathrm{t}+1} / \mathrm{N}_{\mathrm{t}}\right)\right)$ of small adult mottled sculpin among mainstem tributary control $(\mathrm{MC}, \mathrm{n}=3$ ), mainstem removal treatment (MT, $\mathrm{n}=3$ ), headwater tributary control, $(\mathrm{HC}, \mathrm{n}=3)$ and headwater treatment $(\mathrm{HT}, \mathrm{n}=3)$ sites for all sampling intervals at fixed mean values for local physical and chemical habitat covariates based on the $95 \%$ confidence set of models used for inference (Table 16) and their model-averaged coefficients (Appendix E). The horizontal line is at $r=0$ indicates unchanging population size (i.e., $\mathrm{N}_{\mathrm{t}+1}=\mathrm{N}_{\mathrm{t}}$ ).

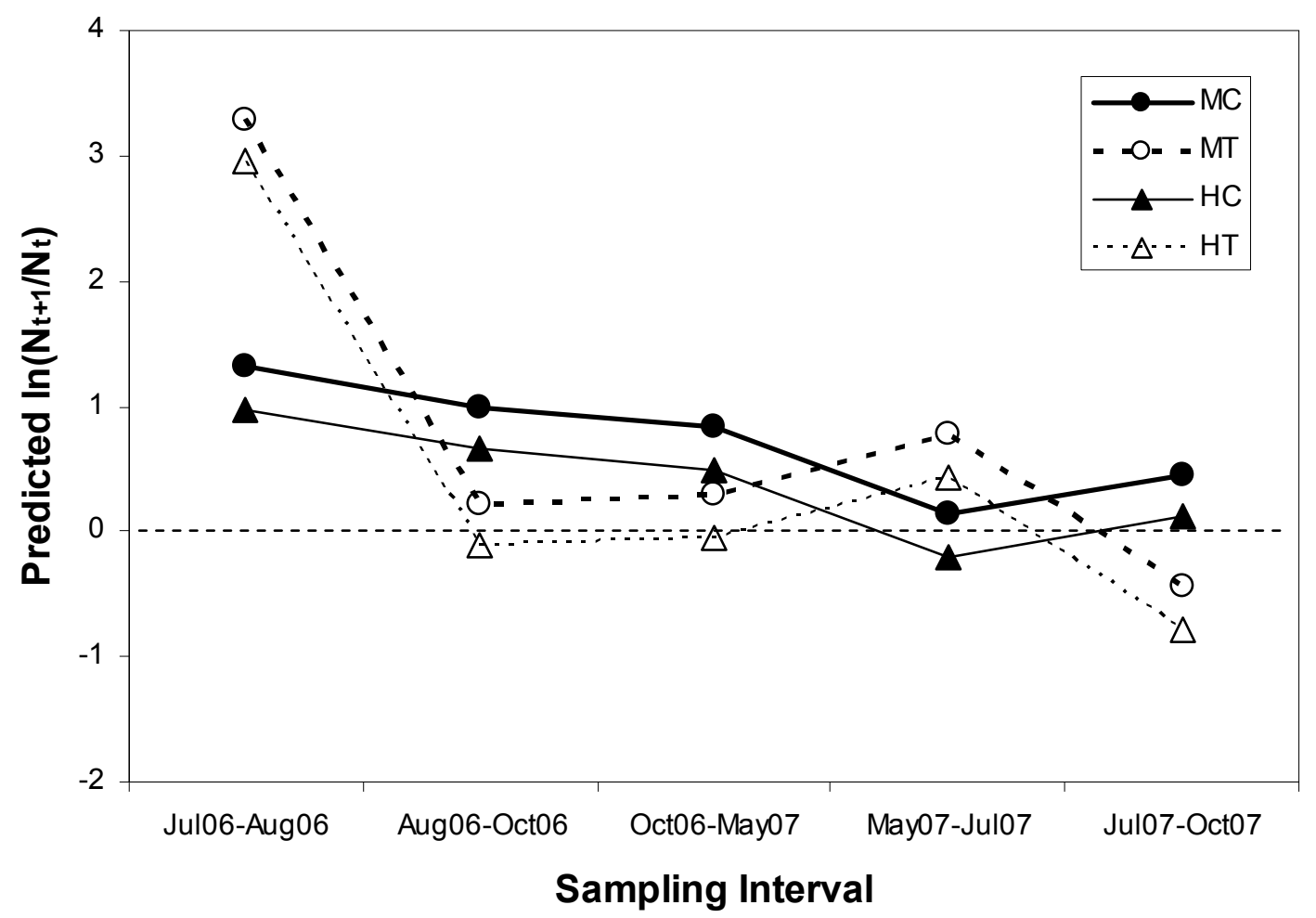


Figure 21. Predicted per capita growth rate $\left(r=\ln \left(\mathrm{N}_{\mathrm{t}+1} / \mathrm{N}_{\mathrm{t}}\right)\right)$ of large adult mottled sculpin among mainstem tributary control $(\mathrm{MC}, \mathrm{n}=3)$, mainstem removal treatment (MT, $n=3)$, headwater tributary control, $(H C, n=3)$ and headwater treatment $(H T, n=3)$ sites for all sampling intervals and at fixed mean values for local physical and chemical habitat covariates based on the $95 \%$ confidence set of models used for inference (Table 16) and their model-averaged coefficients (Appendix E). The horizontal line is at $r=0$ indicates unchanging population size (i.e., $\mathrm{N}_{\mathrm{t}+1}=\mathrm{N}_{\mathrm{t}}$ ).

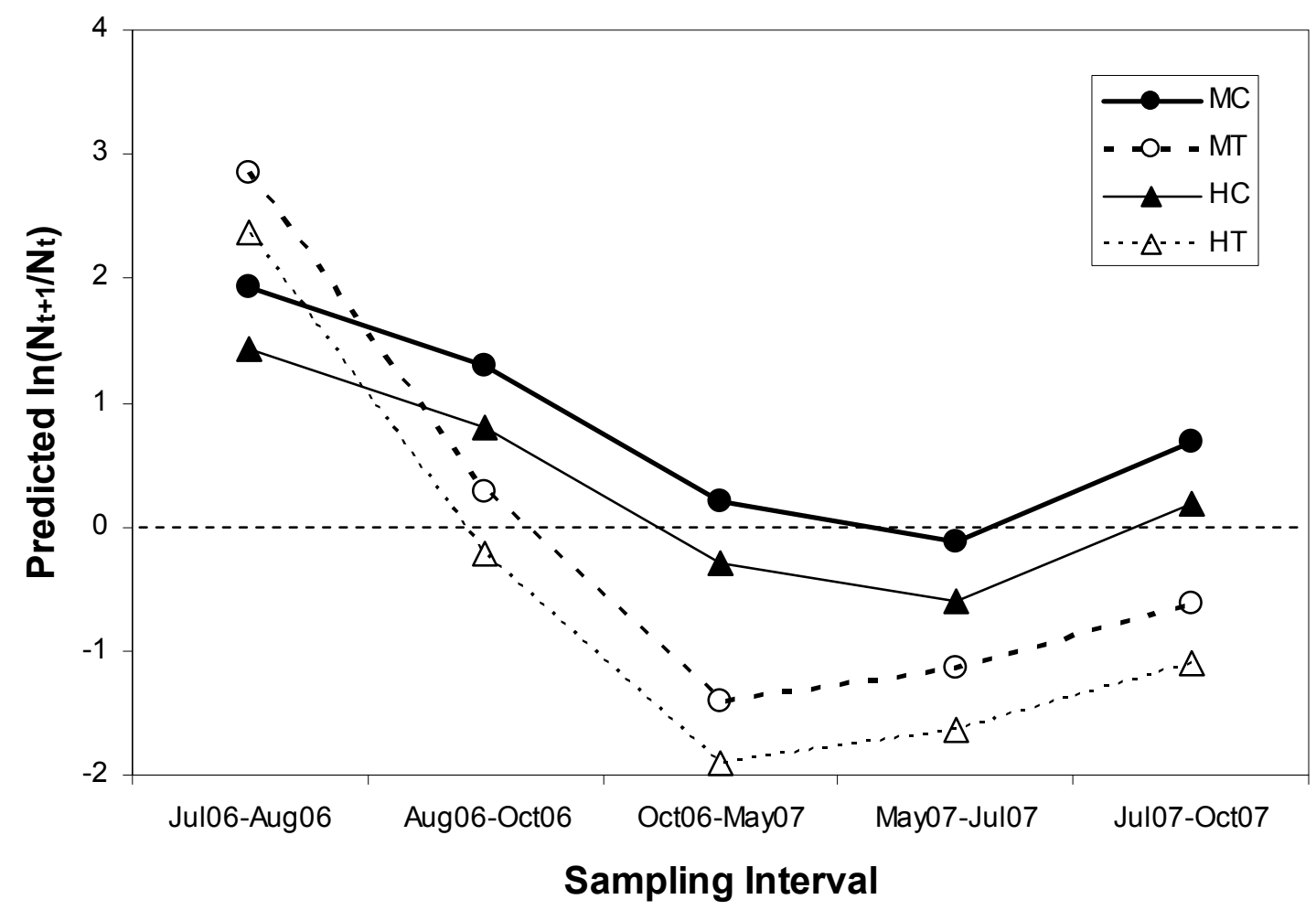


Figure 22. Estimated apparent survival (S) of young-of-the-year (YOY, top left), small adult (top right), and large adult (bottom left) brook trout among all sites for all sampling intervals based on the 95\% confidence set of models used for inference (Table 17) and their model-averaged coefficients (Appendix E). $\mathrm{MC}=$ mainstem tributary control site, $\mathrm{MT}$ = mainstem tributary treatment site, $\mathrm{HC}=$ headwater tributary control site, $\mathrm{HT}=$ headwater tributary treatment site. 

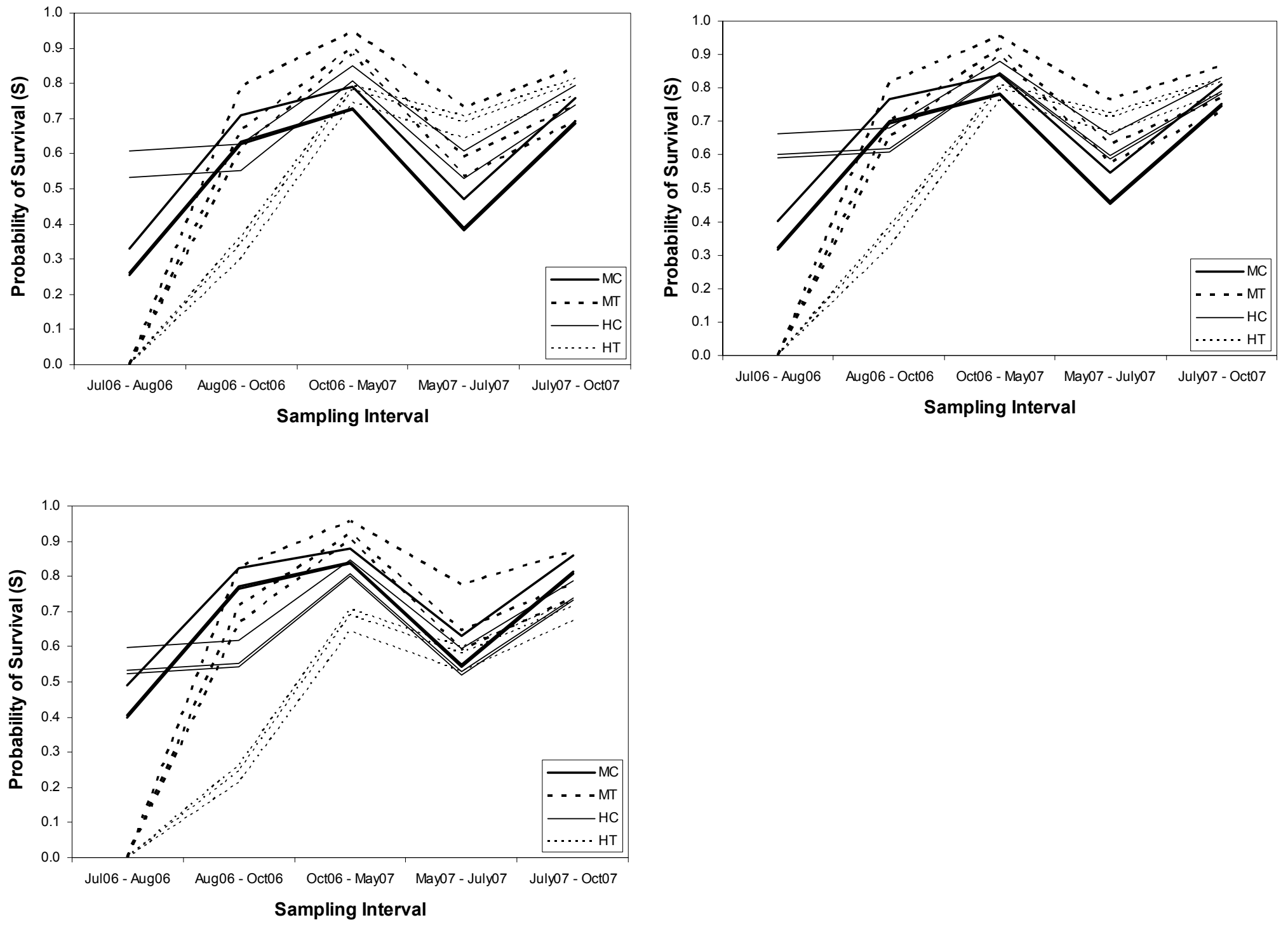
Figure 23. Estimated apparent survival (S) of young-of-the-year (YOY, top left), small adult (top right), and large adult (bottom left) brook trout among mainstem tributary control ( $\mathrm{MC}, \mathrm{n}=3)$, mainstem removal treatment (MT, $\mathrm{n}=3$ ), headwater tributary control, (HC, $\mathrm{n}=3$ ) and headwater treatment (HT, $\mathrm{n}=3$ ) sites for all sampling intervals and at fixed mean values for local physical and chemical habitat covariates and based on the 95\% confidence set of models used for inference (Table 17) and their model-averaged coefficients (Appendix E). 

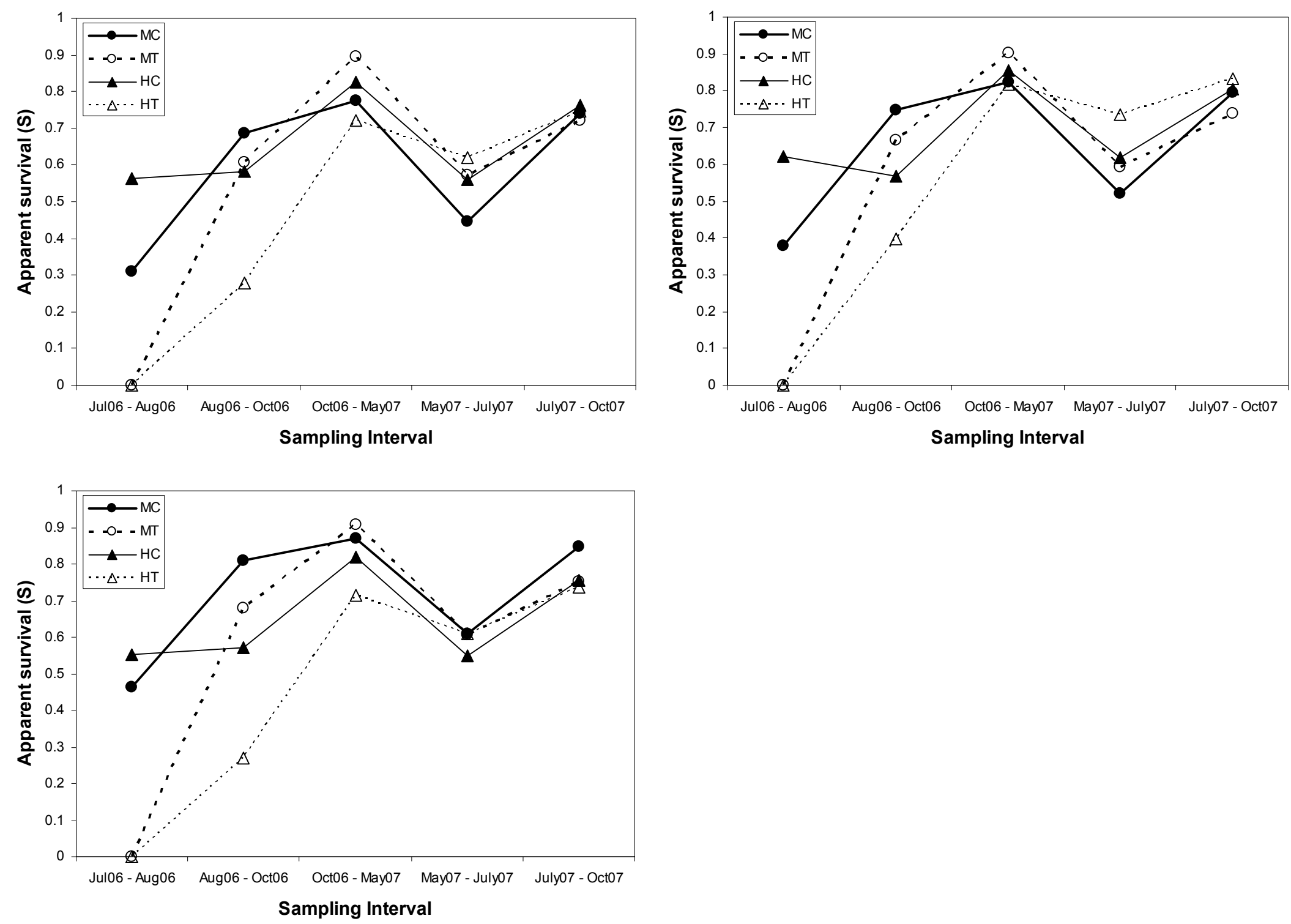
Figure 24. Estimated probability of transitioning from the young-of-the-year (YOY, top) or small adult (bottom) age classes into the small adult or large adult age classes, respectively, among all sites and sampling intervals based on the $95 \%$ confidence set of models used for inference (Table 17) and their model averaged coefficients (Appendix $\mathrm{E}) . \mathrm{MC}=$ mainstem tributary control site, $\mathrm{MT}=$ mainstem tributary treatment site, $\mathrm{HC}=$ headwater tributary control site, HT = headwater tributary treatment site. 

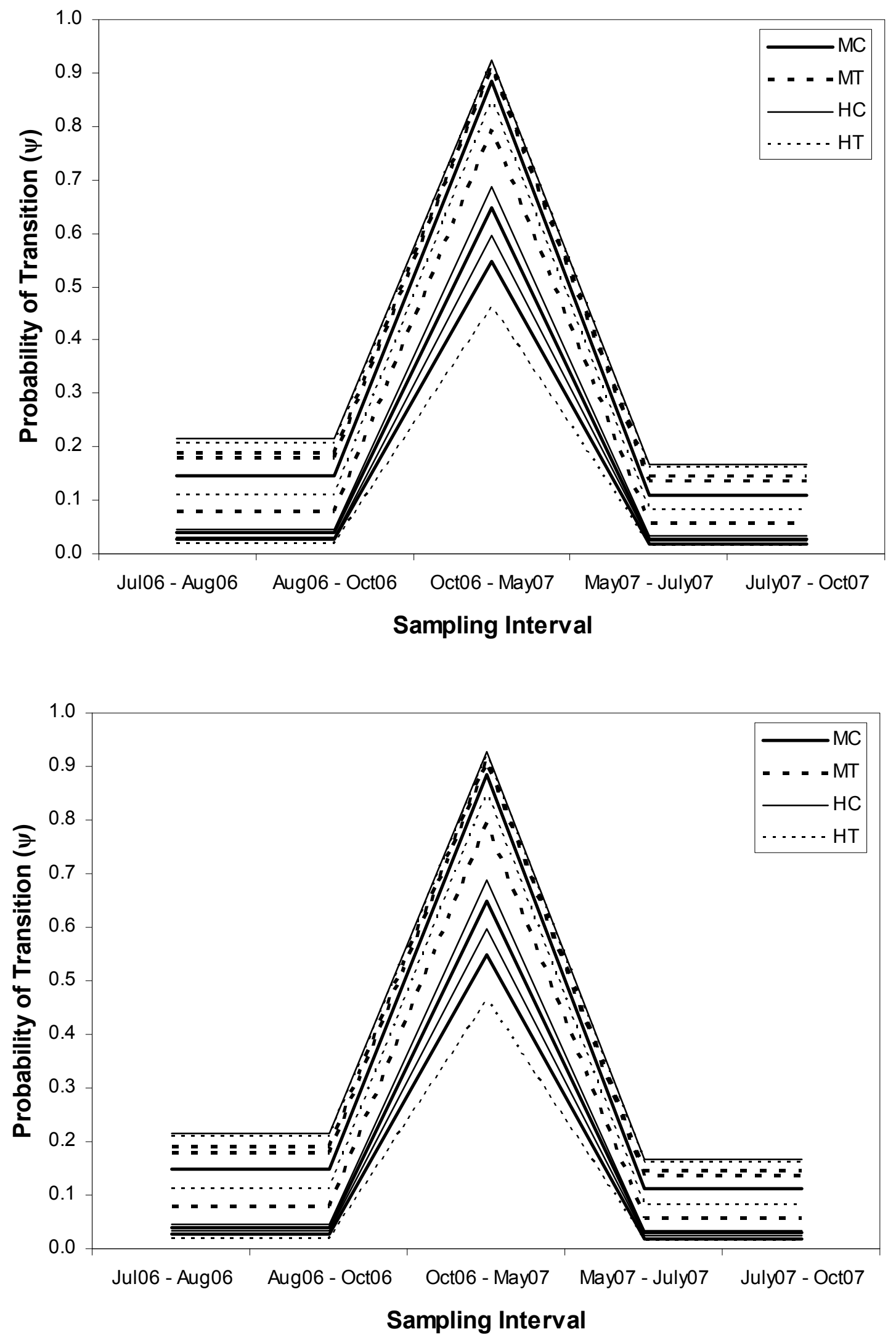
Figure 25. probability of transitioning from the young-of-the-year (YOY, top) or small adult (bottom) age classes into the small adult or large adult age classes, respectively, among mainstem tributary control $(\mathrm{MC}, \mathrm{n}=3)$, mainstem removal treatment $(\mathrm{MT}, \mathrm{n}=3)$, headwater tributary control, $(\mathrm{HC}, \mathrm{n}=3)$ and headwater treatment $(\mathrm{HT}, \mathrm{n}=3)$ sites for all sampling intervals and at fixed mean values for local physical and chemical habitat covariates and based on the $95 \%$ confidence set of models used for inference (Table 17) and their model-averaged coefficients (Appendix E). 

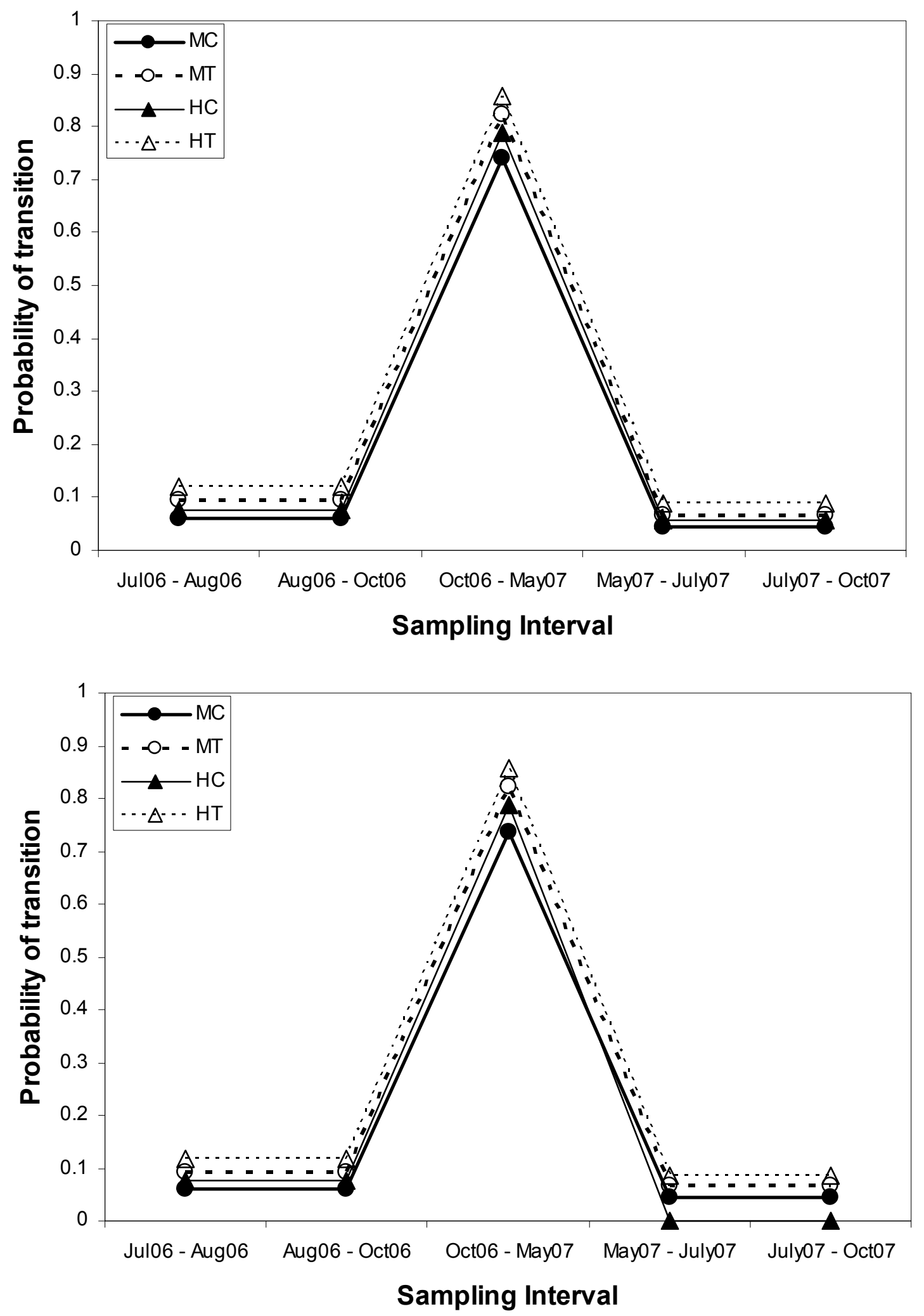
Figure 26. Estimated apparent survival (S) of young-of-the-year (YOY, left) small adult (top right), and large adult (bottom left) mottled sculpin among all sites for all sampling intervals based on the $95 \%$ confidence set of models used for inference (Table 18) and their model-averaged coefficients (Appendix $\mathrm{E}$ ). $\mathrm{MC}=$ mainstem tributary control site, $\mathrm{MT}=$ mainstem tributary treatment site, $\mathrm{HC}=$ headwater tributary control site, $\mathrm{HT}=$ headwater tributary treatment site. 


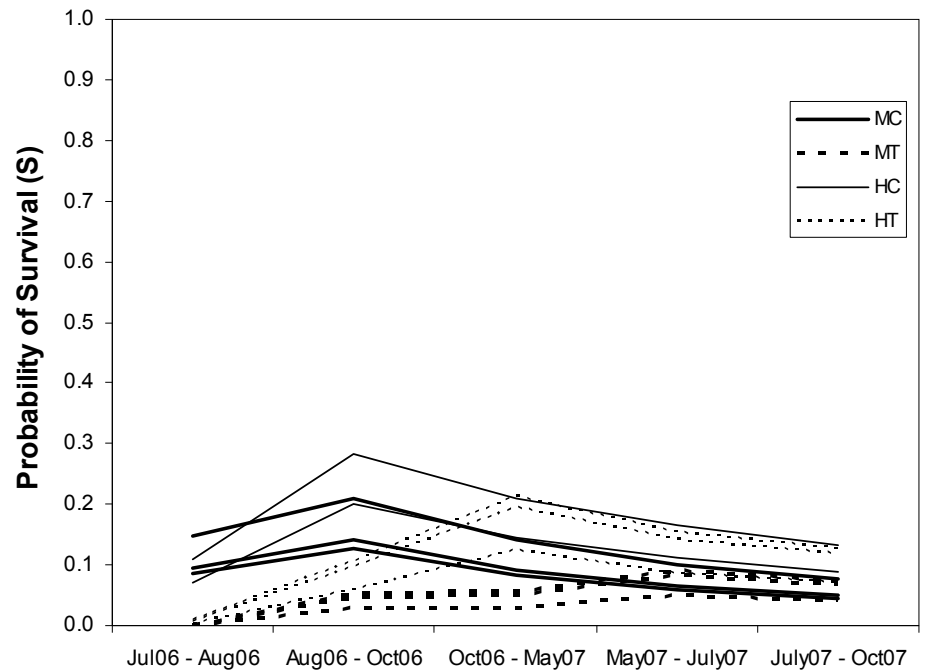

Sampling Interval

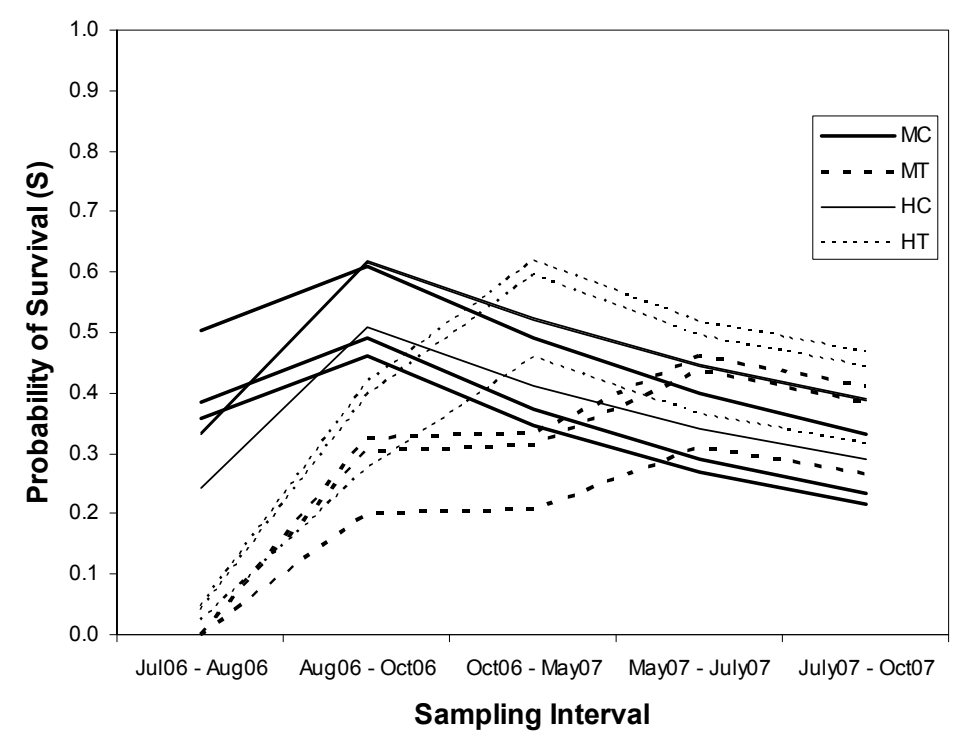

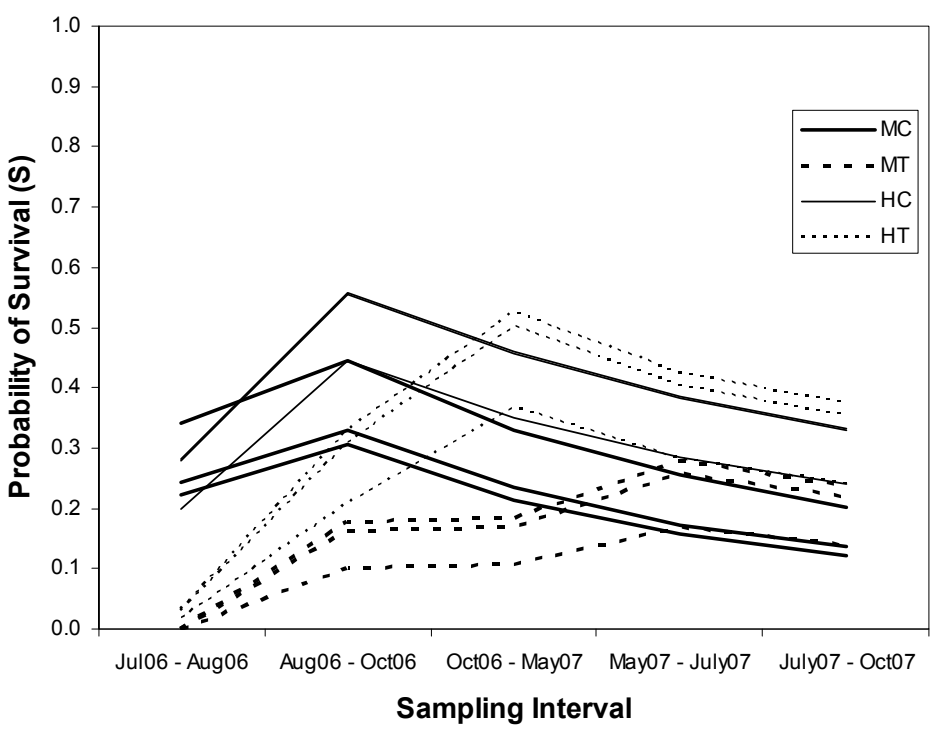

Sampling Interva 
Figure 27. Estimated apparent survival (S) of adult mottled sculpin among mainstem tributary control $(\mathrm{MC}, \mathrm{n}=3$ ), mainstem removal treatment $(\mathrm{MT}, \mathrm{n}=3)$, headwater tributary control, $(\mathrm{HC}, \mathrm{n}=3)$ and headwater removal treatment $(\mathrm{HT}, \mathrm{n}=3)$ sites for all sampling intervals at fixed mean values for local physical and chemical habitat covariates and based on the $95 \%$ confidence set of models used for inference (Table 18) and their model-averaged coefficients (Appendix E). 

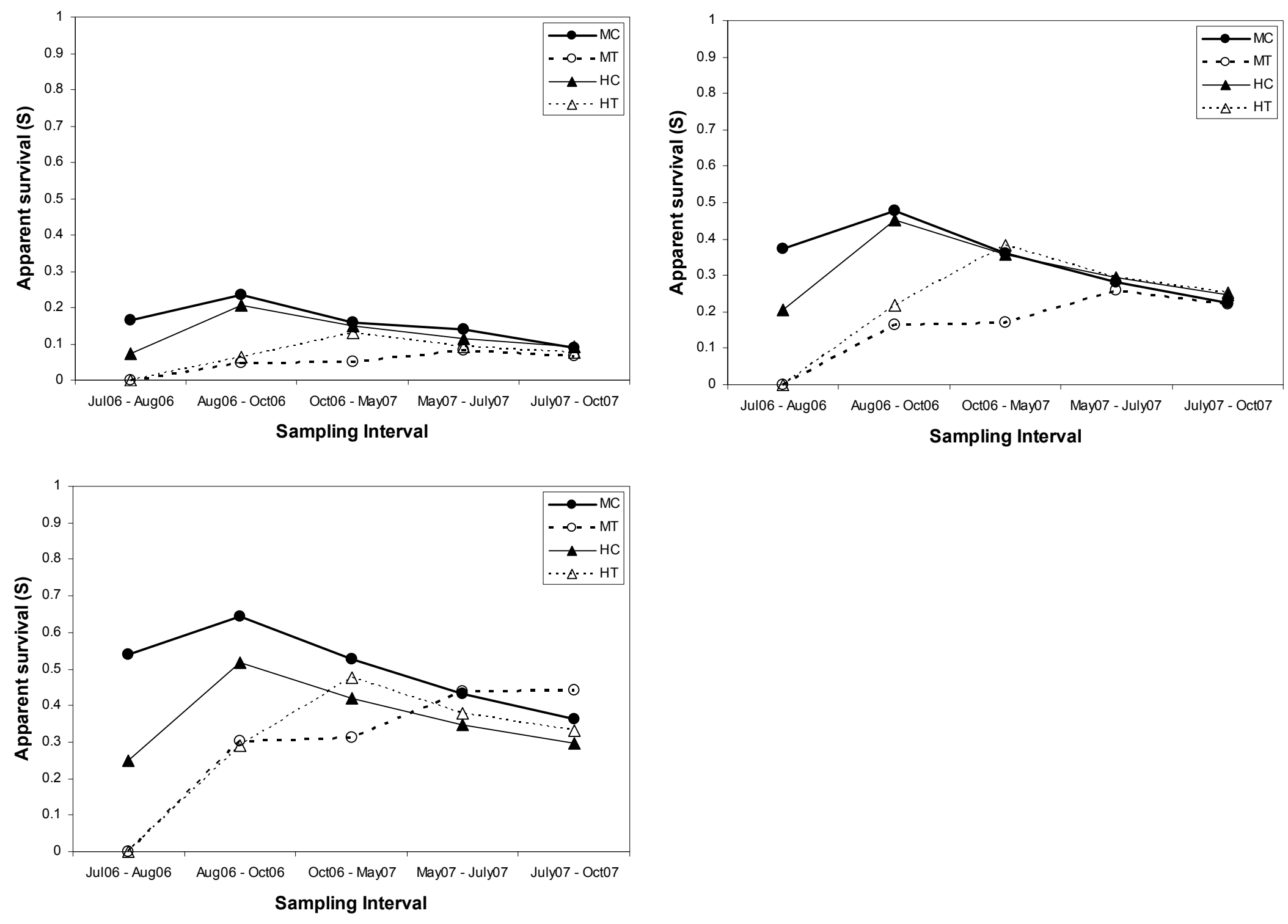
Figure 28. Estimated probability of transitioning from the young-of-the-year (YOY) age classes into the small adult age class among all sites and sampling intervals for mottled sculpin based on the $95 \%$ confidence set of models used for inference (Table 18) and their model averaged coefficients (Appendix E). MC = mainstem tributary control site, $\mathrm{MT}=$ mainstem tributary treatment site, $\mathrm{HC}=$ headwater tributary control site, $\mathrm{HT}=$ headwater tributary treatment site. 

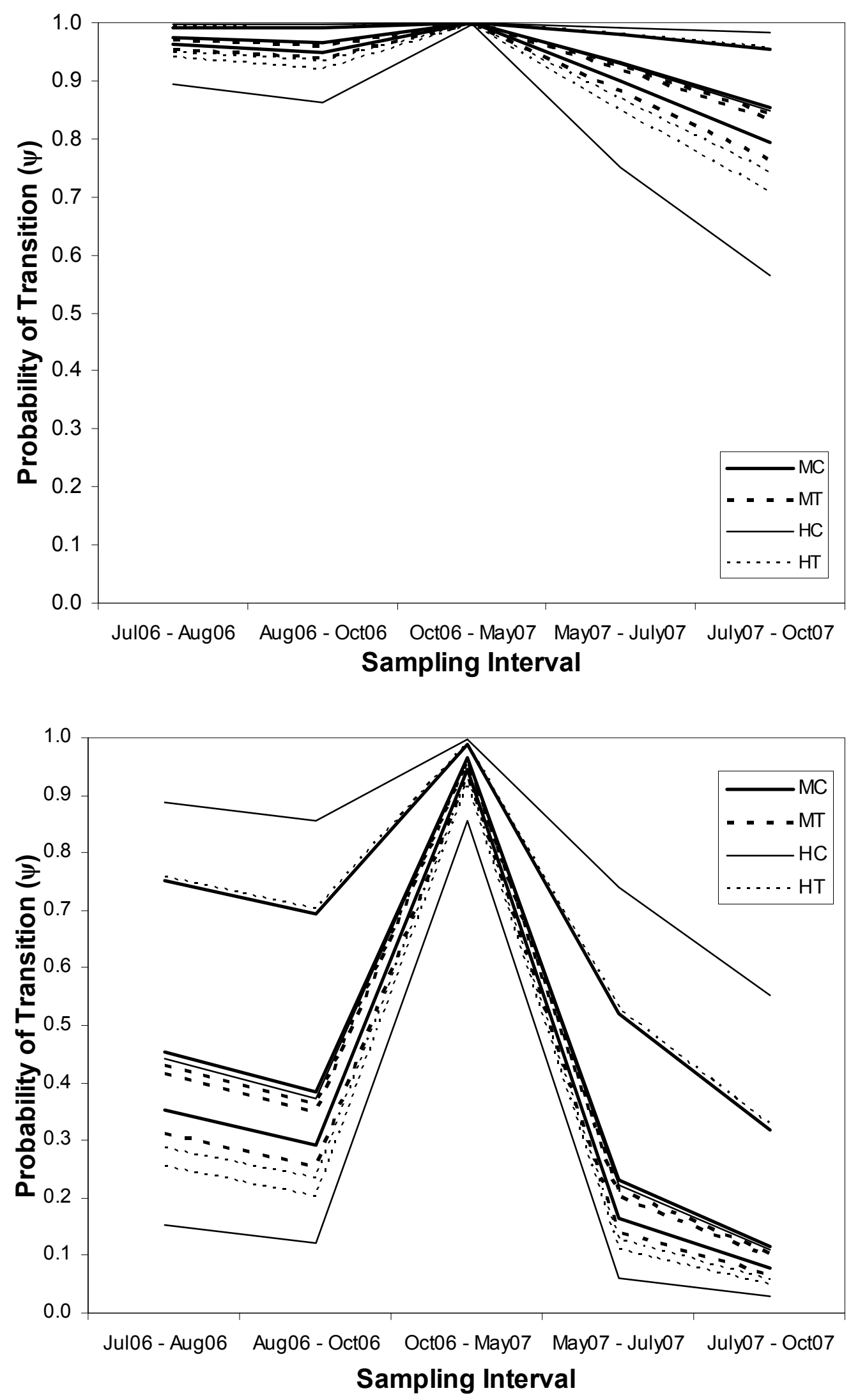
Figure 29. Estimated probability of transitioning from the young-of-the-year (YOY) age class into the small adult classes for mottled sculpin among mainstem tributary control $(\mathrm{MC}, \mathrm{n}=3)$, mainstem removal treatment $(\mathrm{MT}, \mathrm{n}=3)$, headwater tributary control, $(\mathrm{HC}$, $\mathrm{n}=3)$ and headwater treatment $(\mathrm{HT}, \mathrm{n}=3)$ sites for all sampling intervals at fixed mean values for local physical and chemical habitat covariates and based on the $95 \%$ confidence set of models used for inference (Table 18) and their model-averaged coefficients (Appendix E). 

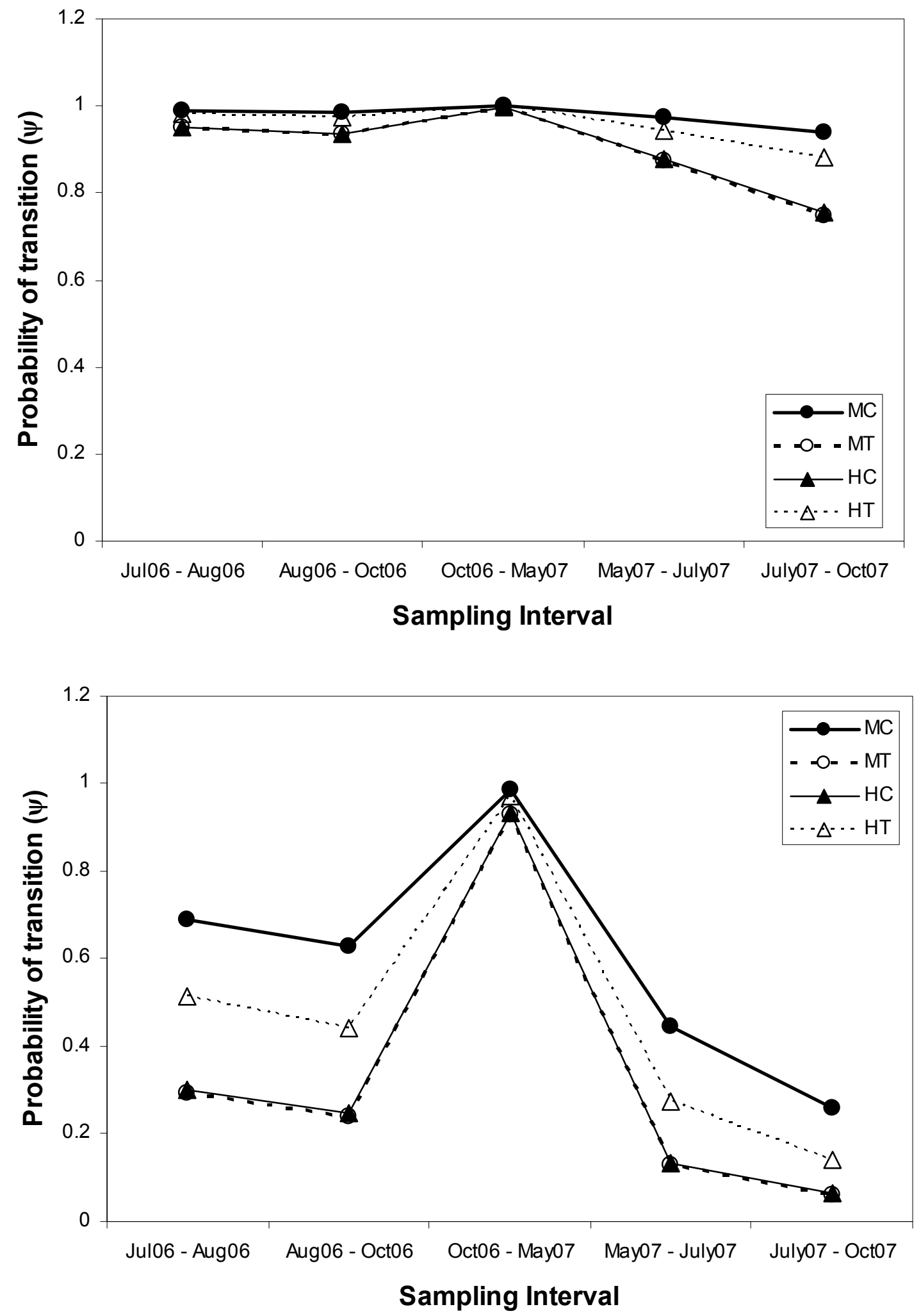
Figure 30. Estimated proportion of the instantaneous population growth rate $(\lambda)$ due to survival ( $\gamma_{\mathrm{ii}}$, top left) and immigration $(1-\gamma \mathrm{ii}-$ $\gamma \mathrm{ji}$, top right) for young-of-the-year (YOY) brook trout among all sites based on the $95 \%$ confidence set of models used for inference (Table 19) and their model-averaged coefficients (Appendix F).
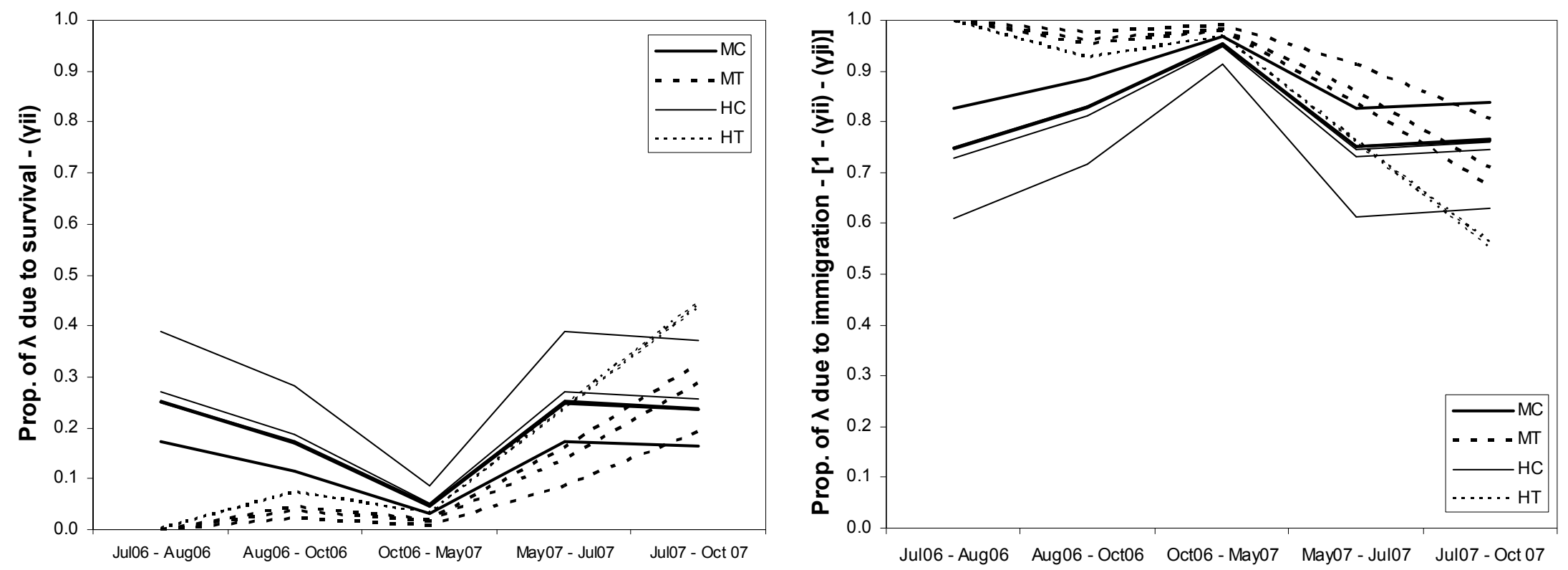
Figure 31. Estimated proportion of the instantaneous population growth rate $(\lambda)$ due to survival $\left(\gamma_{\mathrm{ii}}\right.$, top left), local recruitment $\left(\gamma_{\mathrm{ji}}\right.$, bottom left), and immigration ( 1 - $\gamma \mathrm{ii}-\gamma \mathrm{ji}$, top right) for small adult brook trout among all sites based on the $95 \%$ confidence set of models used for inference (Table 19) and their model-averaged coefficients (Appendix F). 

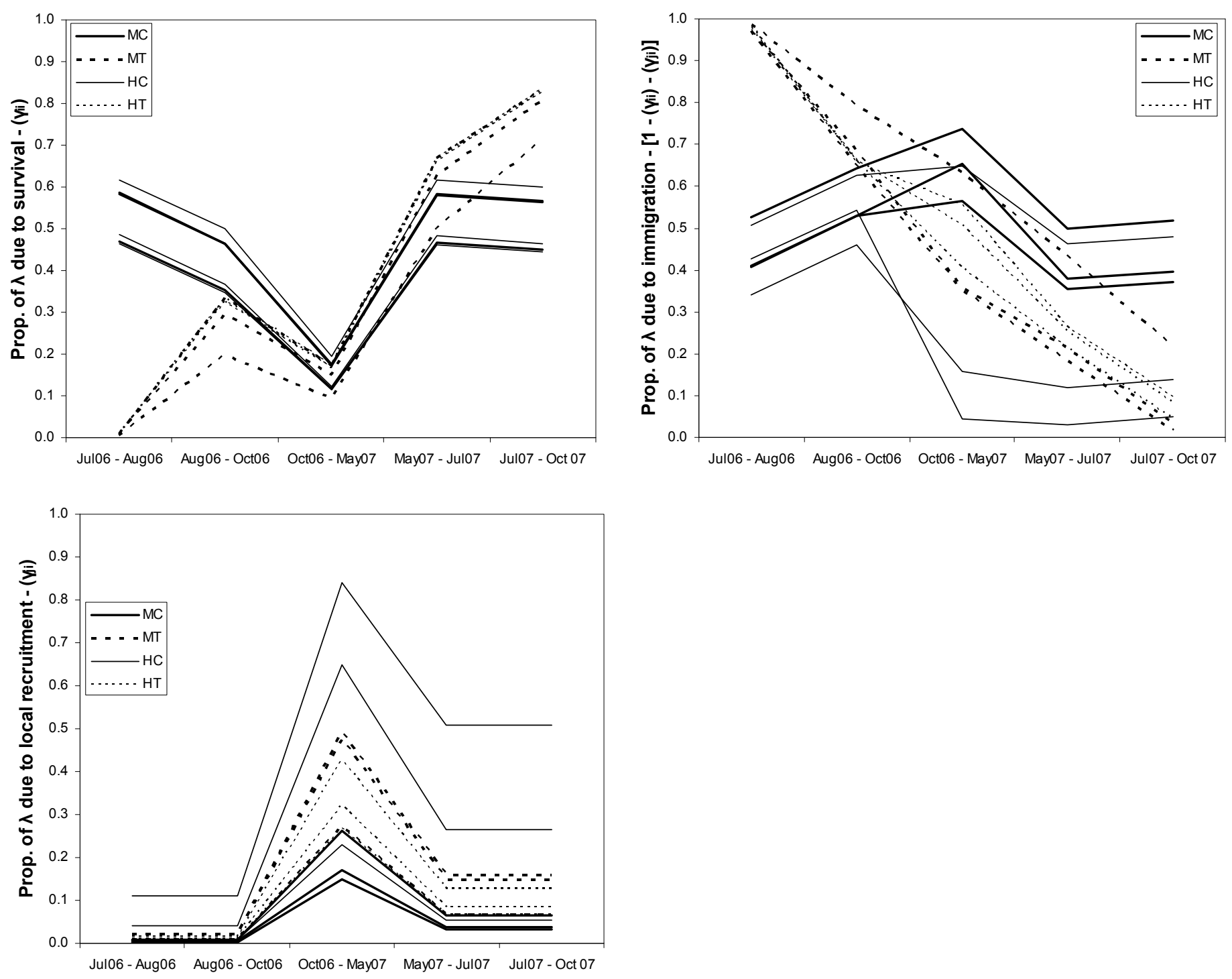
Figure 32. Estimated proportion of the instantaneous population growth rate $(\lambda)$ due to survival $\left(\gamma_{\mathrm{ii}}\right.$, top left), local recruitment $\left(\gamma_{\mathrm{ji}}\right.$, bottom left), and immigration ( $1-\gamma_{\mathrm{ii}}-\gamma_{\mathrm{ji}}$, top right) for large adult brook trout among all sites based on the $95 \%$ confidence set of models used for inference (Table 19) and their model-averaged coefficients (Appendix F). 

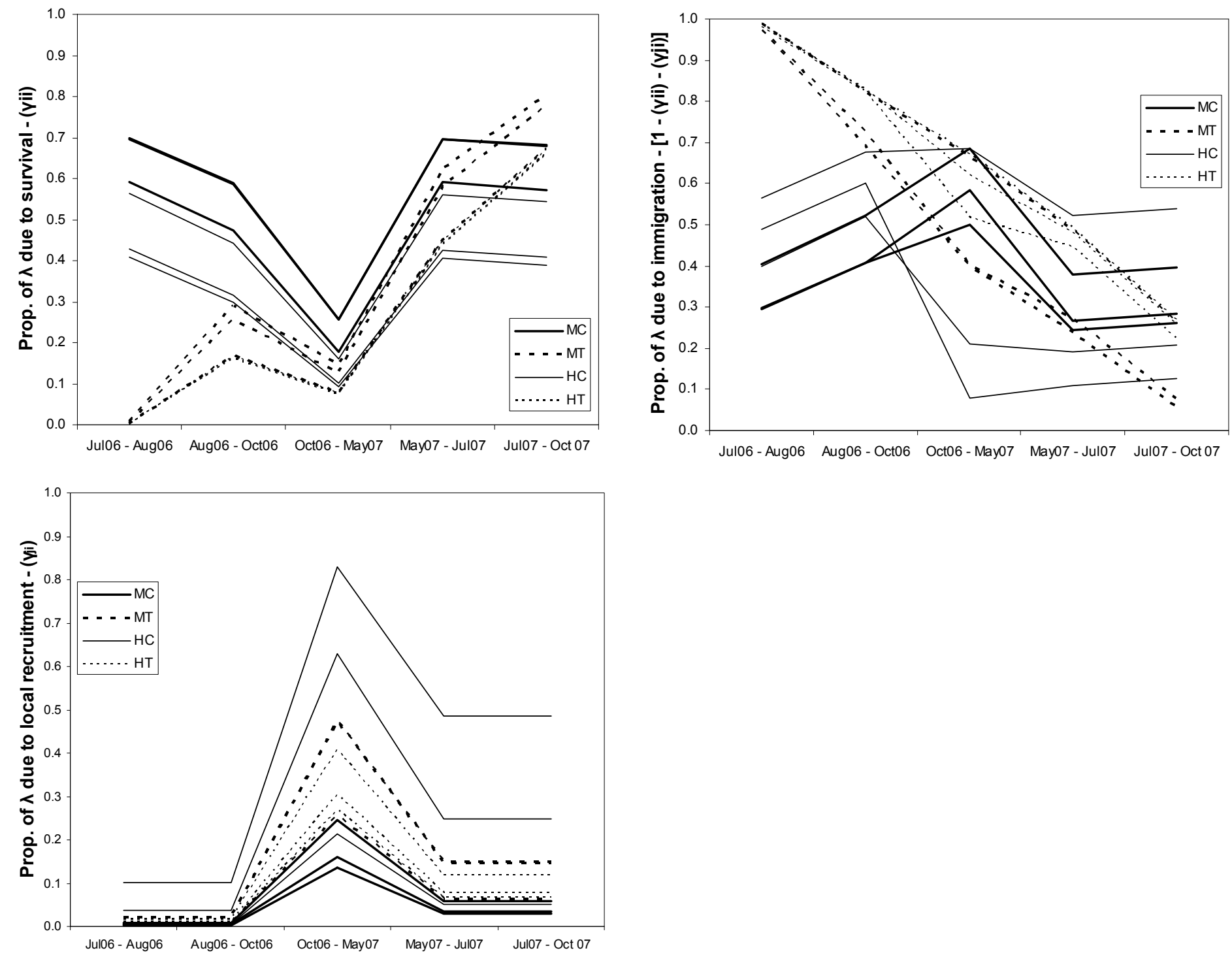
Figure 33. Estimated instantaneous survival $\left(\Phi=\lambda \times \gamma_{\mathrm{ii}}\right)$ and immigration rates $\left(\Gamma=\lambda \times\left[1-\gamma_{\mathrm{ii}}\right]\right)$ of young-of-the-year book trout among mainstem tributary control $(\mathrm{MC}, \mathrm{n}=3)$, mainstem removal treatment $(\mathrm{MT}, \mathrm{n}=3)$, headwater tributary control, $(\mathrm{HC}, \mathrm{n}=3)$ and headwater treatment $(\mathrm{HT}, \mathrm{n}=3)$ sites for all sampling intervals and at fixed mean values for local physical and chemical habitat covariates. The estimates for the instantaneous rates of survival and immigration were derived from the product of the logtransformed estimates of the population growth rates among MC, MT, HC, and HT sites (i.e., $\ln (\mathrm{r})=\lambda=\mathrm{N}_{\mathrm{t}+1} / \mathrm{N}_{\mathrm{t}}$, Figure 16 ) and the model averaged estimates for the proportion of $\lambda$ due to survival $\left(\gamma_{\mathrm{ii}}\right)$ and immigration $\left(1-\gamma_{\mathrm{ji}}-\gamma_{\mathrm{ii}}\right)$ among MC, MT, HC, and HT sites (Figure 30). The horizontal dashed line at $\lambda=0$ indicates unchanging population size (i.e., $\mathrm{N}_{\mathrm{t}+1}=\mathrm{N}_{\mathrm{t}}$ ). 

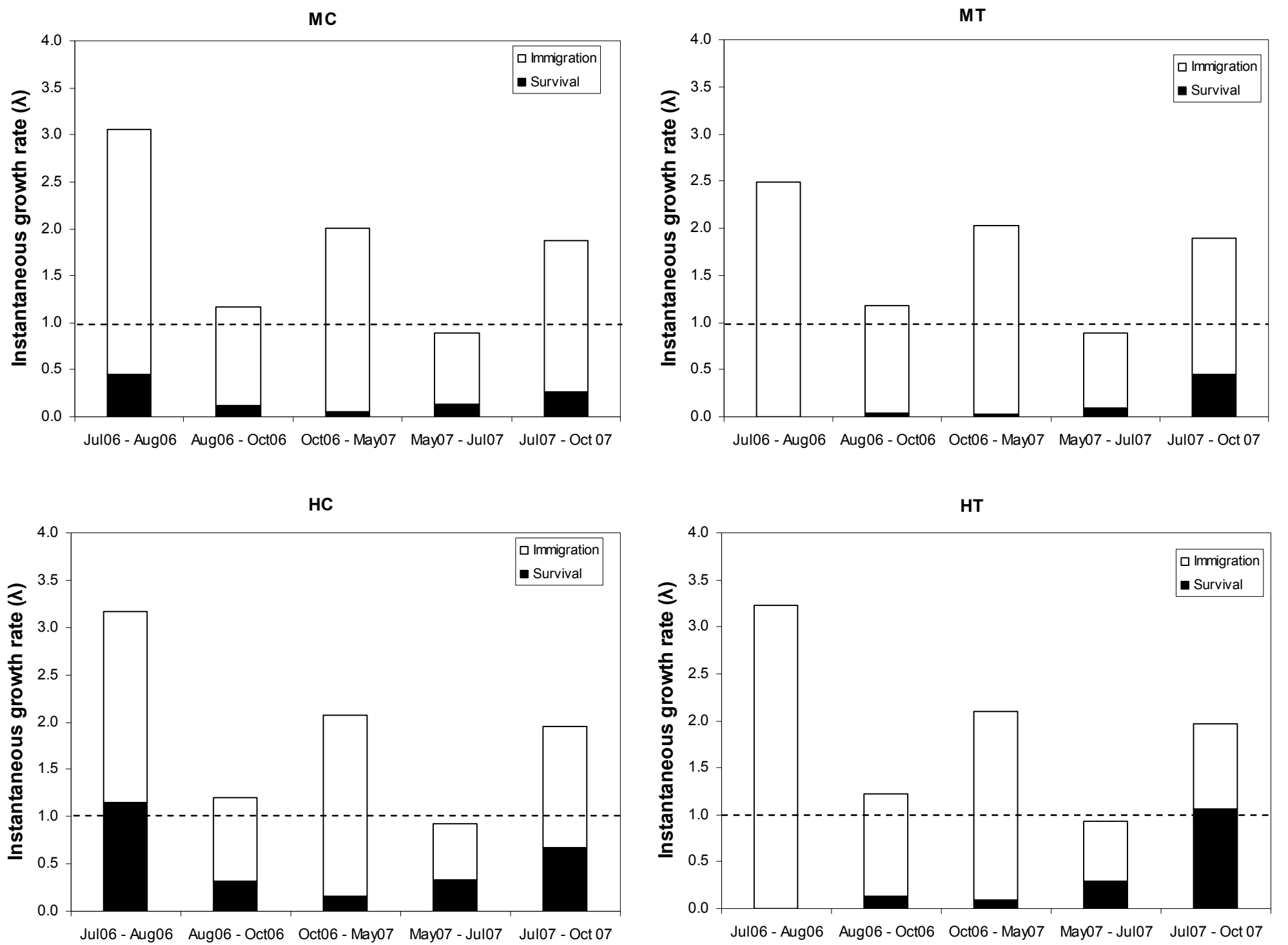
Figure 34. Estimated instantaneous survival $\left(\Phi=\lambda \times \gamma_{\mathrm{ii}}\right)$, local recruitment $\left(\Omega=\lambda \times \gamma_{\mathrm{ji}}\right)$, and immigration rates $\left(\Gamma=\lambda \times\left[1-\gamma_{\mathrm{ji}}-\gamma_{\mathrm{ii}}\right]\right)$ of small adult brook trout among mainstem tributary control $(\mathrm{MC}, \mathrm{n}=3)$, mainstem removal treatment $(\mathrm{MT}, \mathrm{n}=3)$, headwater tributary control, $(\mathrm{HC}, \mathrm{n}=3)$ and headwater treatment $(\mathrm{HT}, \mathrm{n}=3)$ sites for all sampling intervals and at fixed mean values for local physical and chemical habitat covariates. The estimates for the instantaneous rates of survival, recruitment and immigration were derived from the product of the log-transformed estimates for population growth rates among MC, MT, HC, and HT sites (i.e., $\ln (\mathrm{r})=\lambda=\mathrm{N}_{\mathrm{t}+1} / \mathrm{N}_{\mathrm{t}}$, Figure 17) and the model averaged estimates for the proportion of $\lambda$ due to survival $\left(\gamma_{\mathrm{ii}}\right)$, local recruitment $\left(\gamma_{\mathrm{ji}}\right)$, and immigration $(1-$ $\left.\gamma_{\mathrm{ji}}-\gamma_{\mathrm{ii}}\right)$ among MC, MT, HC, and HT sites (Figure 31). The horizontal dashed line at $\lambda=0$ indicates unchanging population size (i.e., $\mathrm{N}_{\mathrm{t}+1}=\mathrm{N}_{\mathrm{t}}$ ). 
MC

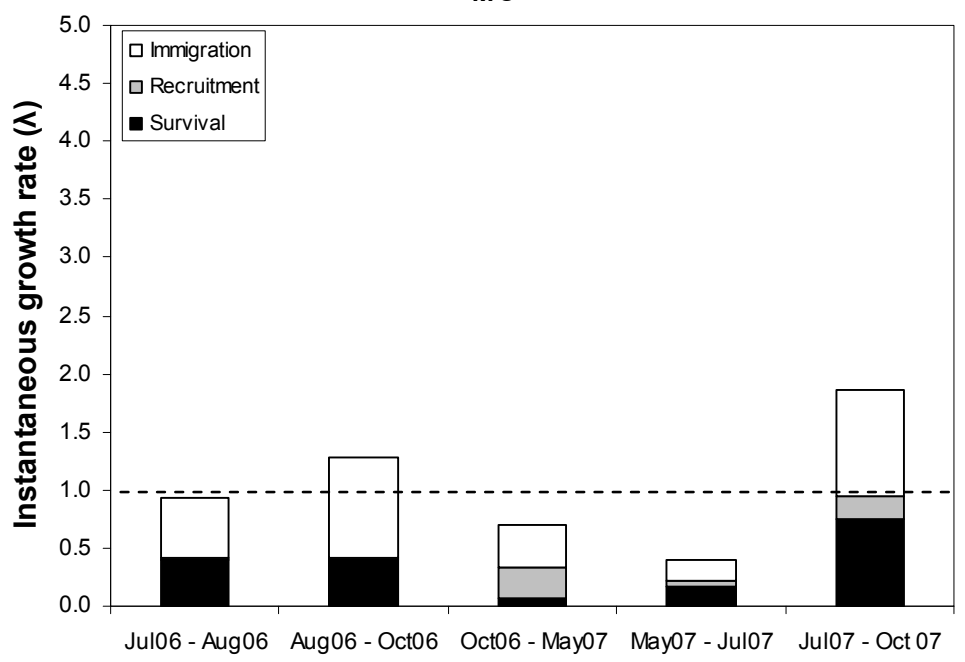

HC

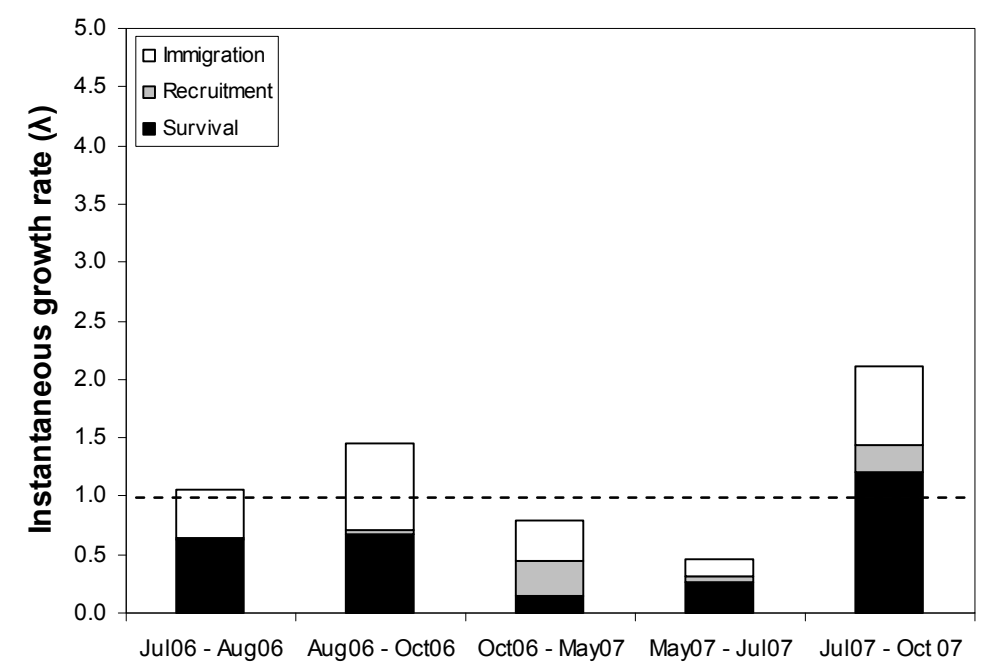

MT

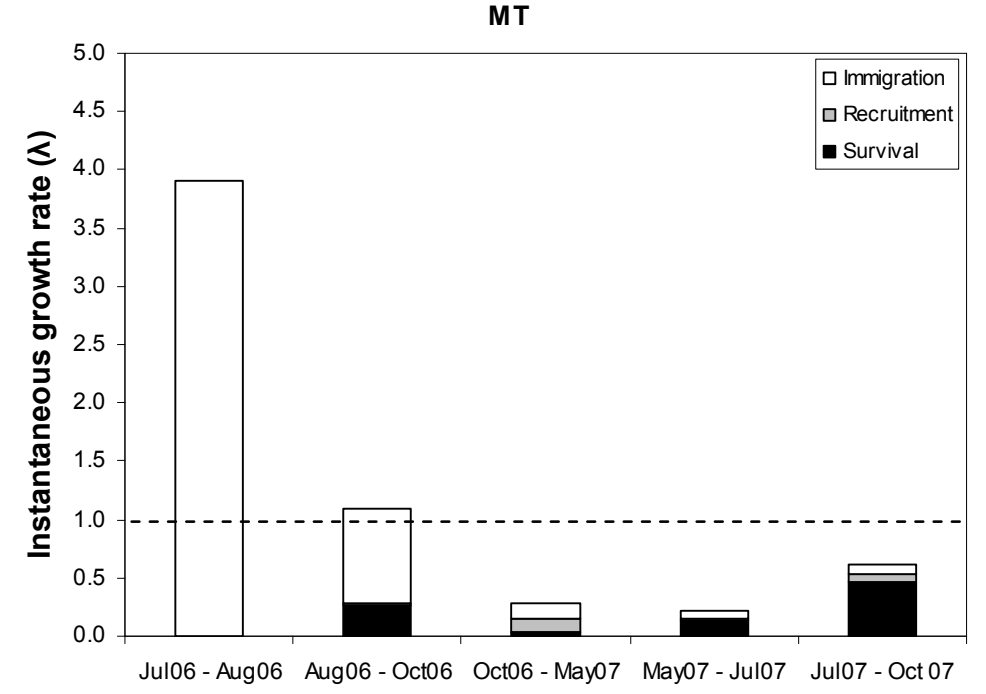

HT

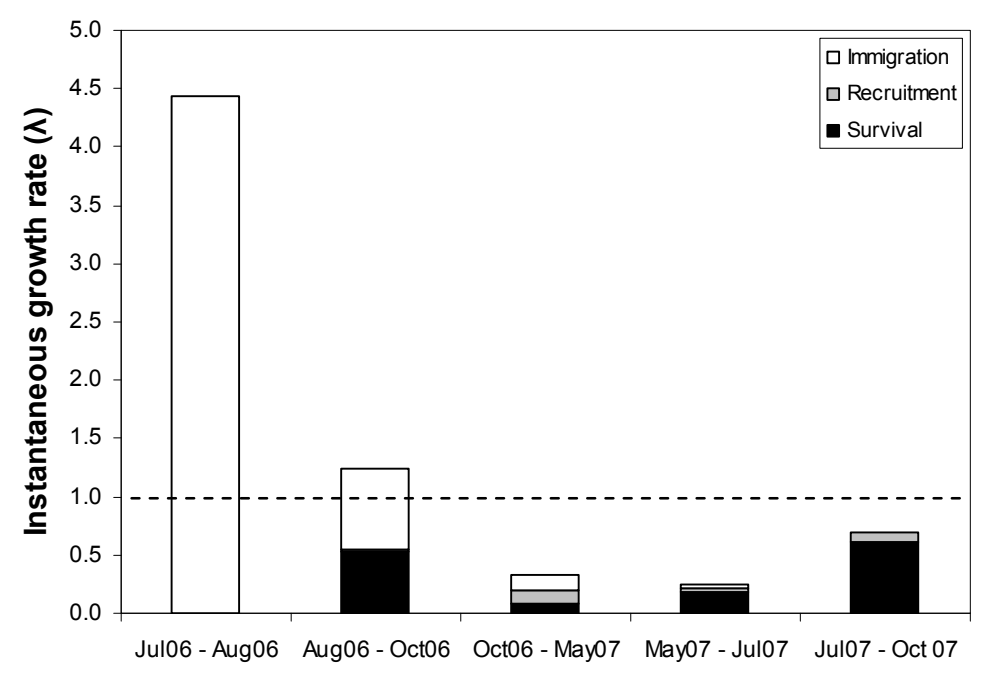


Figure 35. Estimated instantaneous survival $\left(\Phi=\lambda \times \gamma_{\mathrm{ii}}\right)$, local recruitment $\left(\Omega=\lambda \times \gamma_{\mathrm{ji}}\right)$, and immigration rates $\left(\Gamma=\lambda \times\left[1-\gamma_{\mathrm{ji}}-\gamma_{\mathrm{ii}}\right]\right)$ of large adult brook trout among mainstem tributary control (MC, $n=3)$, mainstem removal treatment $(\mathrm{MT}, \mathrm{n}=3)$, headwater tributary control, $(\mathrm{HC}, \mathrm{n}=3)$ and headwater treatment $(\mathrm{HT}, \mathrm{n}=3)$ sites for all sampling intervals and at fixed mean values for local physical and chemical habitat covariates. The estimates for the instantaneous rates of survival, recruitment and immigration were derived from the product of the log-transformed estimates for population growth rates among MC, MT, HC, and HT sites (i.e., $\ln (\mathrm{r})=\lambda=\mathrm{N}_{\mathrm{t}+1} / \mathrm{N}_{\mathrm{t}}$, Figure 18) and the model averaged estimates for the proportion of $\lambda$ due to survival $\left(\gamma_{\mathrm{ii}}\right)$, local recruitment $\left(\gamma_{\mathrm{ji}}\right)$, and immigration $(1-$ $\left.\gamma_{\mathrm{ji}}-\gamma_{\mathrm{ii}}\right)$ among MC, MT, HC, and HT sites (Figure 32). The horizontal dashed line at $\lambda=0$ indicates unchanging population size (i.e., $\mathrm{N}_{\mathrm{t}+1}=\mathrm{N}_{\mathrm{t}}$ ). 
MC

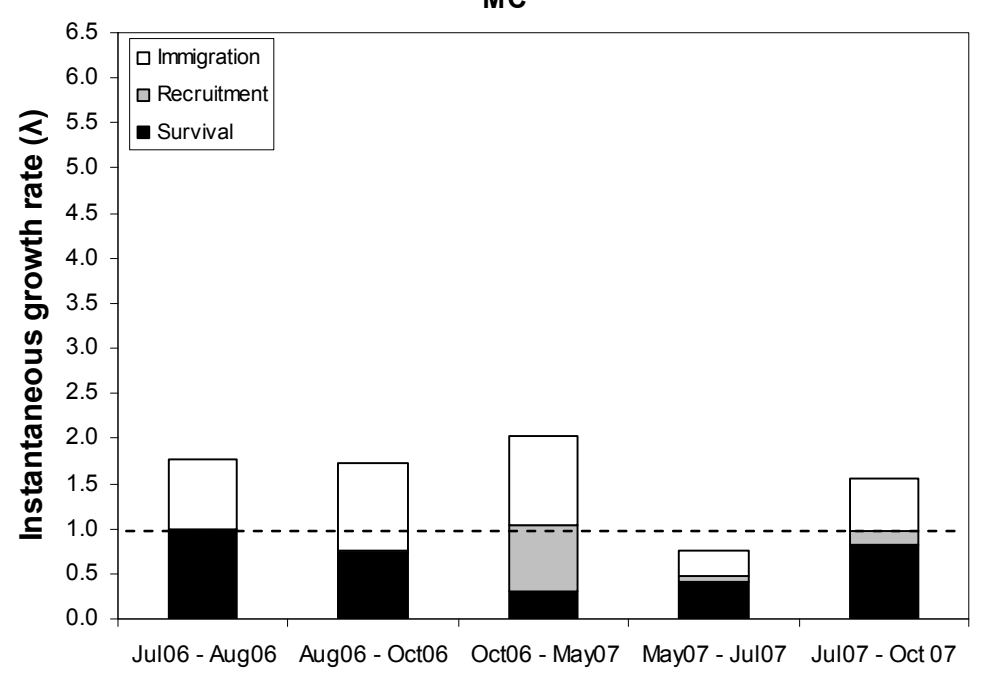

HC

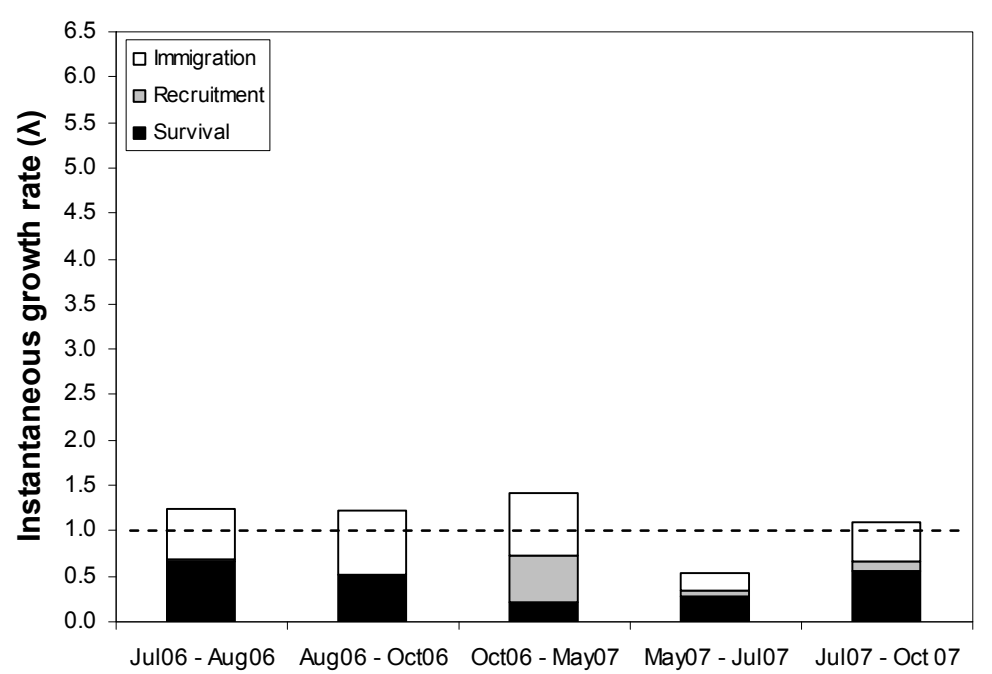

MT

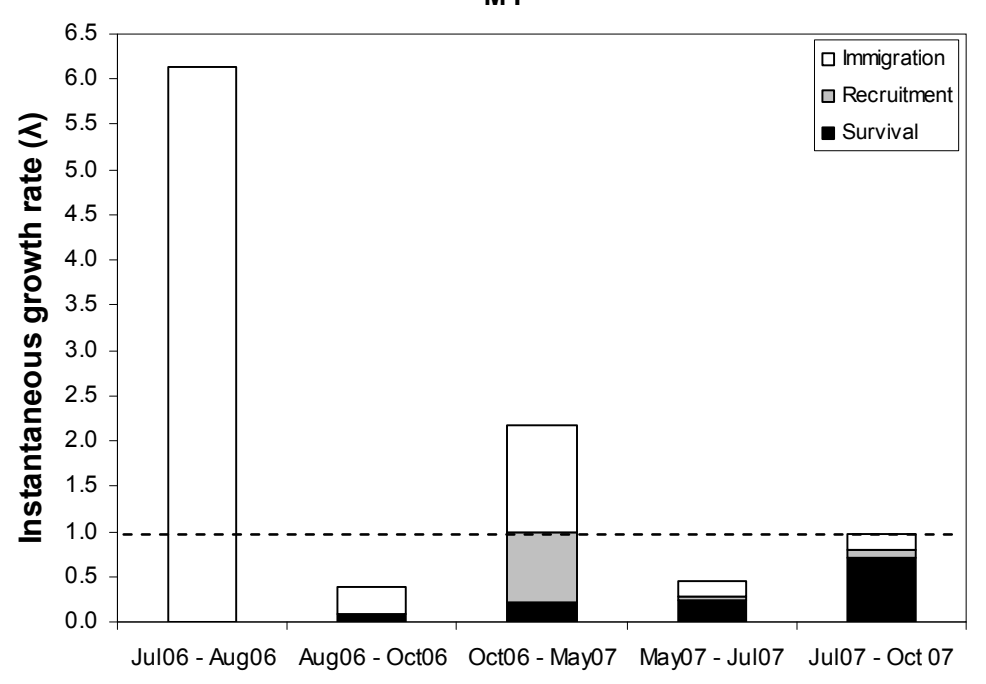

HT

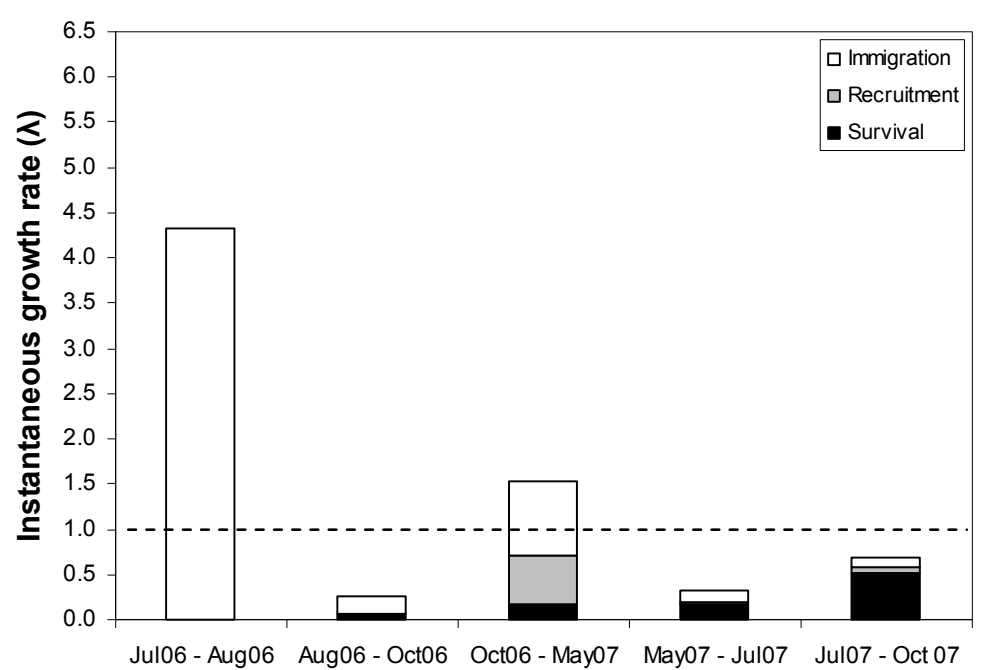


Figure 36. Estimated proportion of the instantaneous population growth rate $(\lambda)$ due to survival ( $\gamma_{\mathrm{ii}}$, top left) and immigration $(1-\gamma \mathrm{ii}-$ $\gamma \mathrm{ji}$, top right) for young-of-the-year (YOY) mottled sculpin among all sites based on the 95\% confidence set of models used for inference (Table 20) and their model-averaged coefficients (Appendix F).
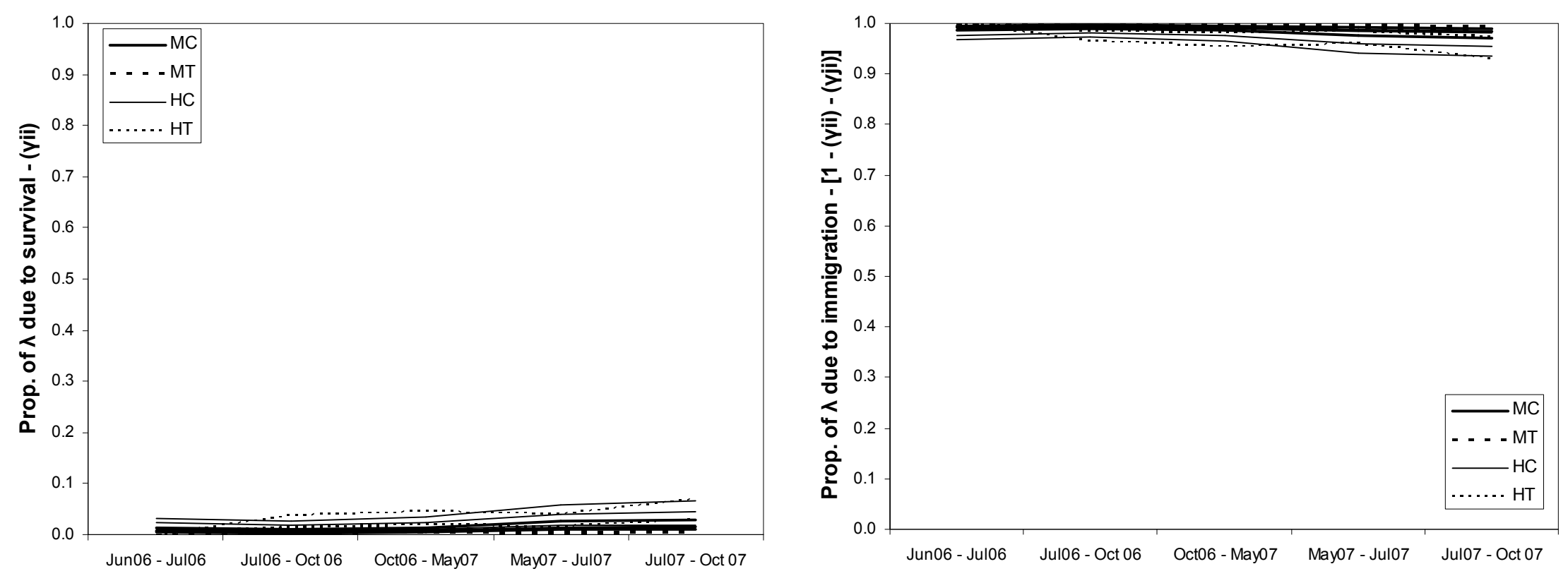
Figure 37. Estimated proportion of the instantaneous population growth rate $(\lambda)$ due to survival $\left(\gamma_{\mathrm{ii}}\right.$, top left), local recruitment $\left(\gamma_{\mathrm{ji}}\right.$, bottom left), and immigration ( 1 - $\gamma \mathrm{ii}-\gamma \mathrm{ji}$, top right) for small adult mottled sculpin among all sites based on the $95 \%$ confidence set of models used for inference (Table 20) and their model-averaged coefficients (Appendix F). 

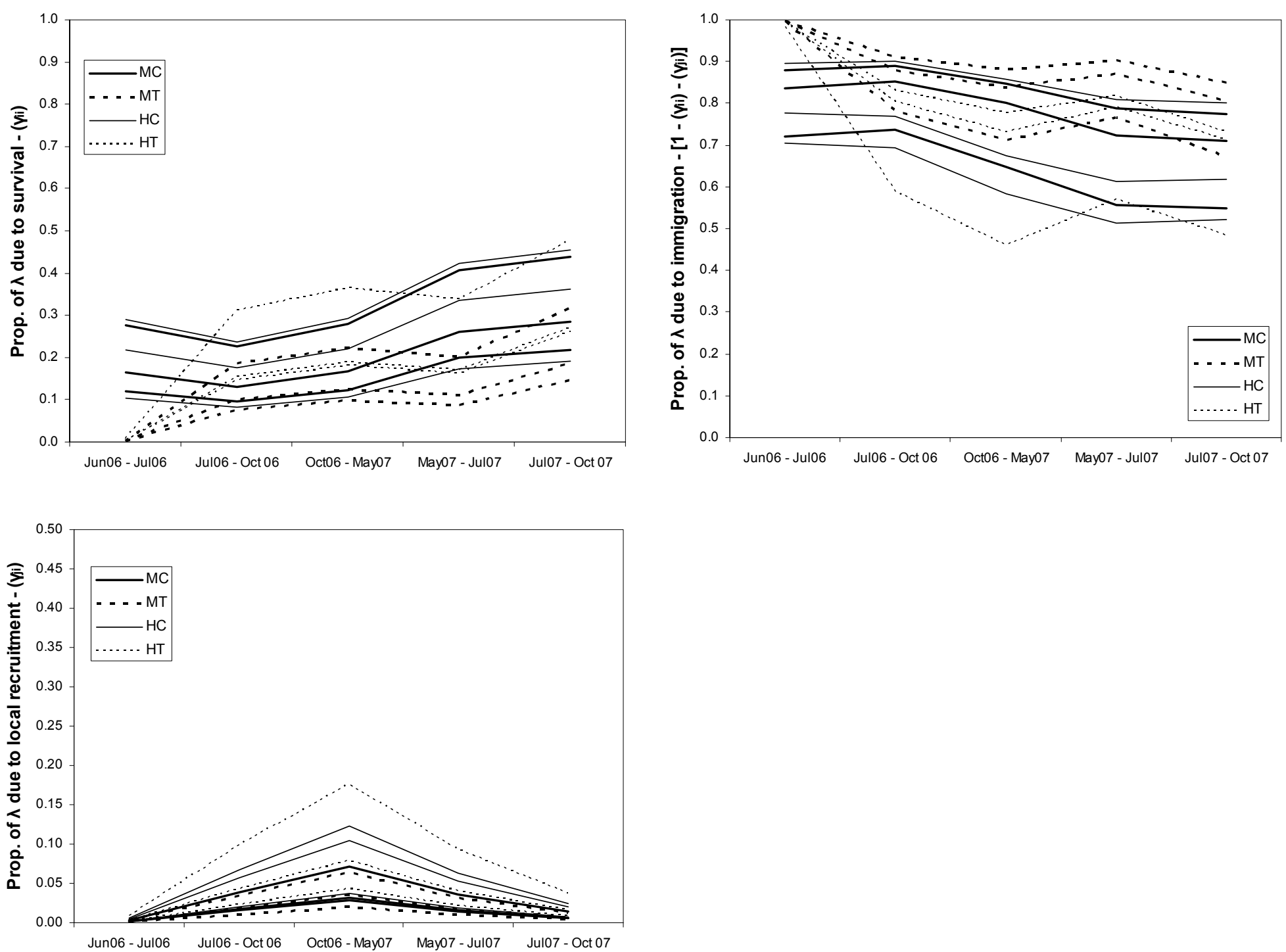
Figure 38. Estimated proportion of the instantaneous population growth rate $(\lambda)$ due to local survival $\left(\gamma_{\mathrm{ii}}\right.$, top left), local recruitment $\left(\gamma_{\mathrm{ji}}\right.$, bottom left), and immigration ( $1-\gamma_{\mathrm{ii}}-\gamma_{\mathrm{ji}}$, top right) for large adult mottled sculpin among all sites based on the $95 \%$ confidence set of models used for inference (Table 20) and their model-averaged coefficients (Appendix F). 

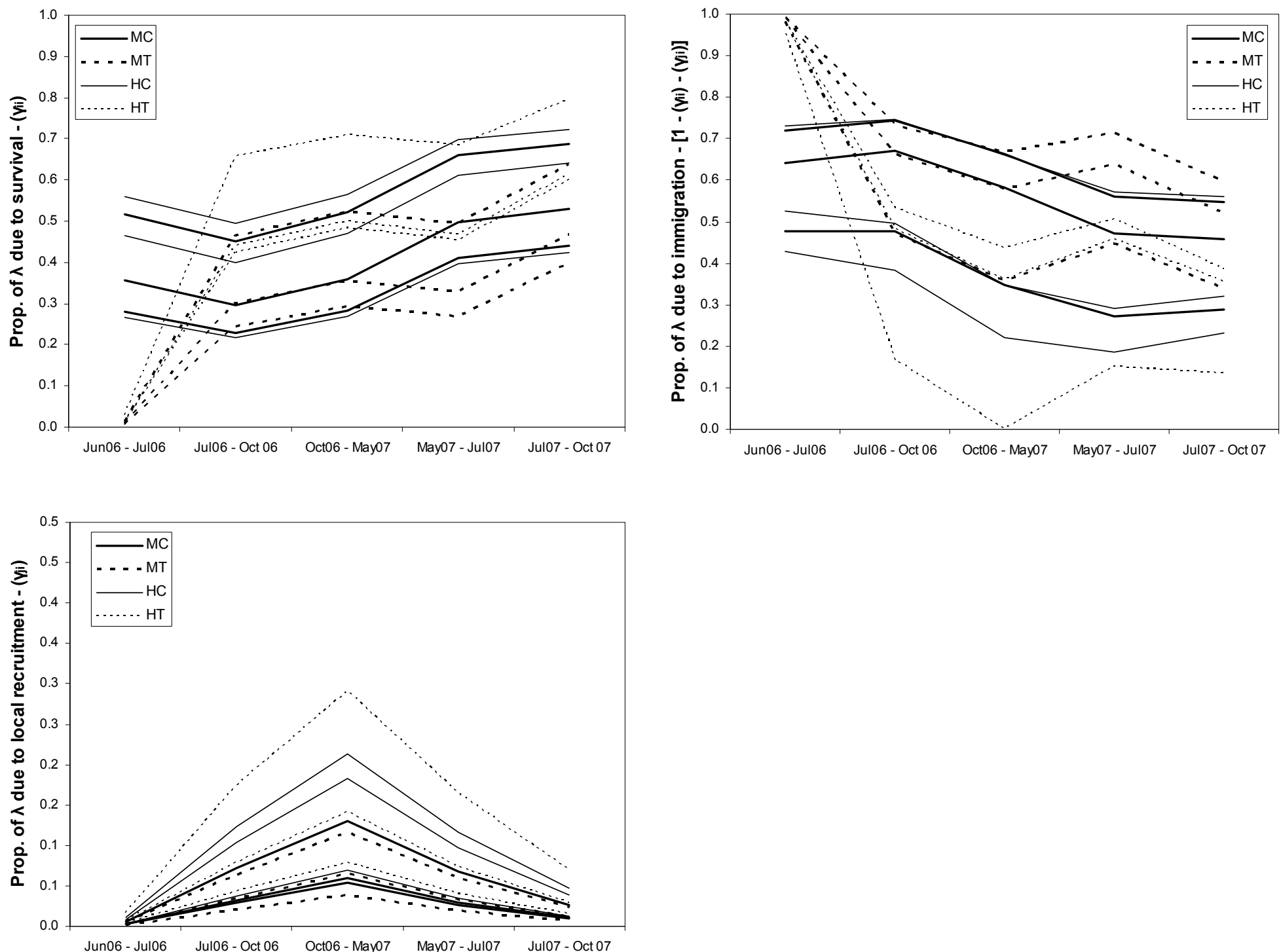
Figure 39. Estimated instantaneous survival $\left(\Phi=\lambda \times \gamma_{\mathrm{ii}}\right)$ and immigration rates $\left(\Gamma=\lambda \times\left[1-\gamma_{\mathrm{ii}}\right]\right)$ of young-of-the-year sculpin among mainstem tributary control (MC, $n=3)$, mainstem removal treatment (MT, n=3), headwater tributary control, $(\mathrm{HC}, \mathrm{n}=3)$ and headwater treatment $(\mathrm{HT}, \mathrm{n}=3)$ sites for all sampling intervals and at fixed mean values for local physical and chemical habitat covariates. The estimates for the instantaneous rates of survival and immigration were derived from the product of the log-transformed estimates of the population growth rates among MC, MT, HC, and HT sites (i.e., $\ln (\mathrm{r})=\lambda=\mathrm{N}_{\mathrm{t}+1} / \mathrm{N}_{\mathrm{t}}$, Figure 19) and the model averaged estimates for the proportion of $\lambda$ due to survival $\left(\gamma_{\mathrm{ii}}\right)$ and immigration $\left(1-\gamma_{\mathrm{ji}}-\gamma_{\mathrm{ii}}\right)$ among MC, MT, HC, and HT sites (Figure 36). The horizontal dashed line at $\lambda=0$ indicates unchanging population size (i.e., $\mathrm{N}_{\mathrm{t}+1}=\mathrm{N}_{\mathrm{t}}$ ). 
MC

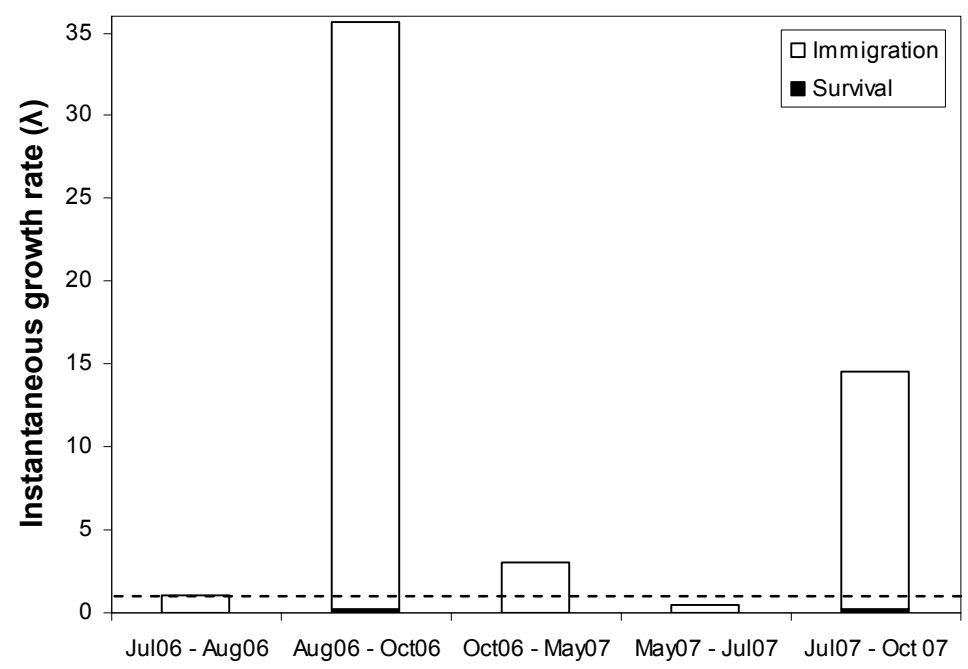

HC

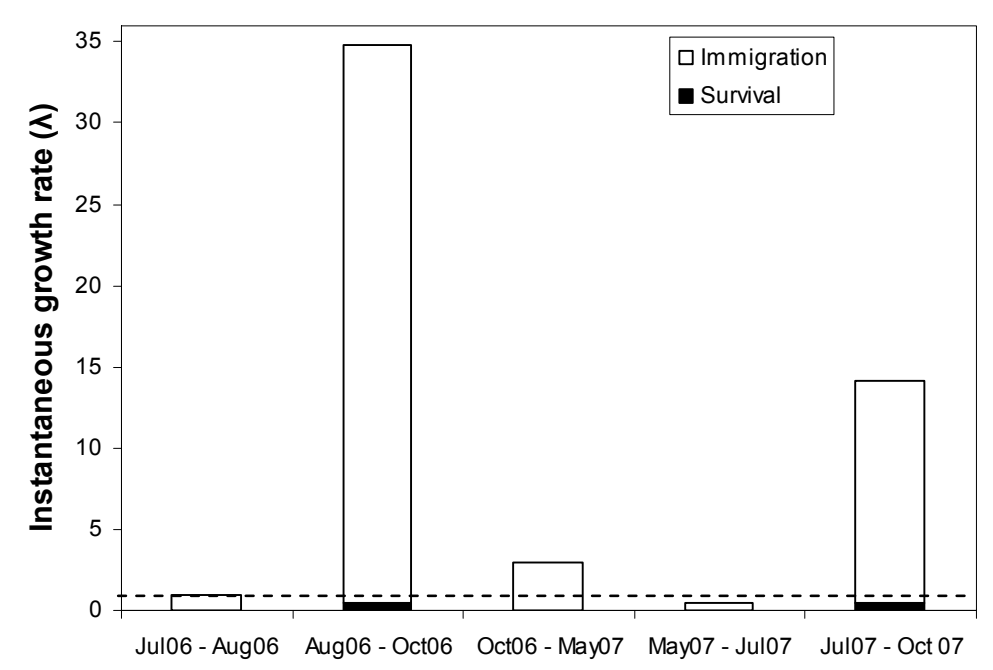

MT

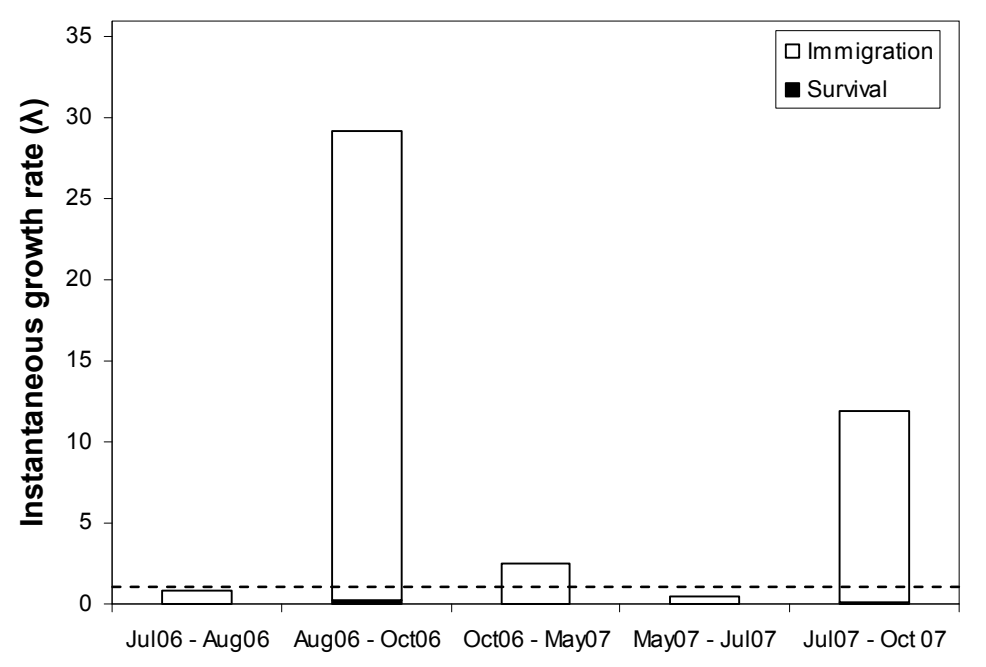

HT

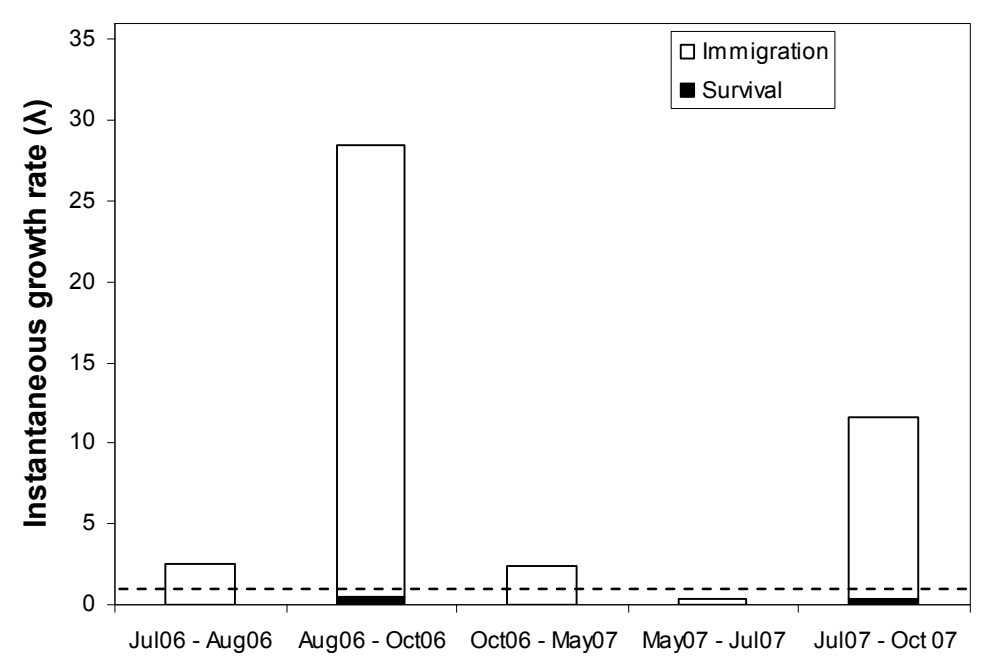


Figure 40. Estimated instantaneous survival $\left(\Phi=\lambda \times \gamma_{\mathrm{ii}}\right)$, local recruitment $\left(\Omega=\lambda \times \gamma_{\mathrm{ji}}\right)$, and immigration rates $\left(\Gamma=\lambda \times\left[1-\gamma_{\mathrm{ji}}-\gamma_{\mathrm{ii}}\right]\right)$ of small adult mottled sculpin among mainstem tributary control $(\mathrm{MC}, \mathrm{n}=3)$, mainstem removal treatment $(\mathrm{MT}, \mathrm{n}=3)$, headwater tributary control, $(\mathrm{HC}, \mathrm{n}=3)$ and headwater treatment $(\mathrm{HT}, \mathrm{n}=3)$ sites for all sampling intervals and at fixed mean values for local physical and chemical habitat covariates. The estimates for the instantaneous rates of survival, recruitment and immigration were derived from the product of the log-transformed estimates for population growth rates among MC, MT, HC, and HT sites (i.e., $\ln (r)=$ $\lambda=\mathrm{N}_{\mathrm{t}+1} / \mathrm{N}_{\mathrm{t}}$, Figure 20) and the model averaged estimates for the proportion of $\lambda$ due to survival $\left(\gamma_{\mathrm{ii}}\right)$, local recruitment $\left(\gamma_{\mathrm{ji}}\right)$, and immigration $\left(1-\gamma_{\mathrm{ji}}-\gamma_{\mathrm{ii}}\right)$ among MC, MT, HC, and HT sites (Figure 37). The horizontal dashed line at $\lambda=0$ indicates unchanging population size (i.e., $\mathrm{N}_{\mathrm{t}+1}=\mathrm{N}_{\mathrm{t}}$ ). Because $\lambda$ was disproportionately higher over the first interval for the removal sites the scale of the yaxis was set lower than the maximum value over that interval in order to preserve scale among the remaining estimates. As such, the actual values for $\lambda$ for the removal sites are noted on the bars that exceed the range of the axis. 

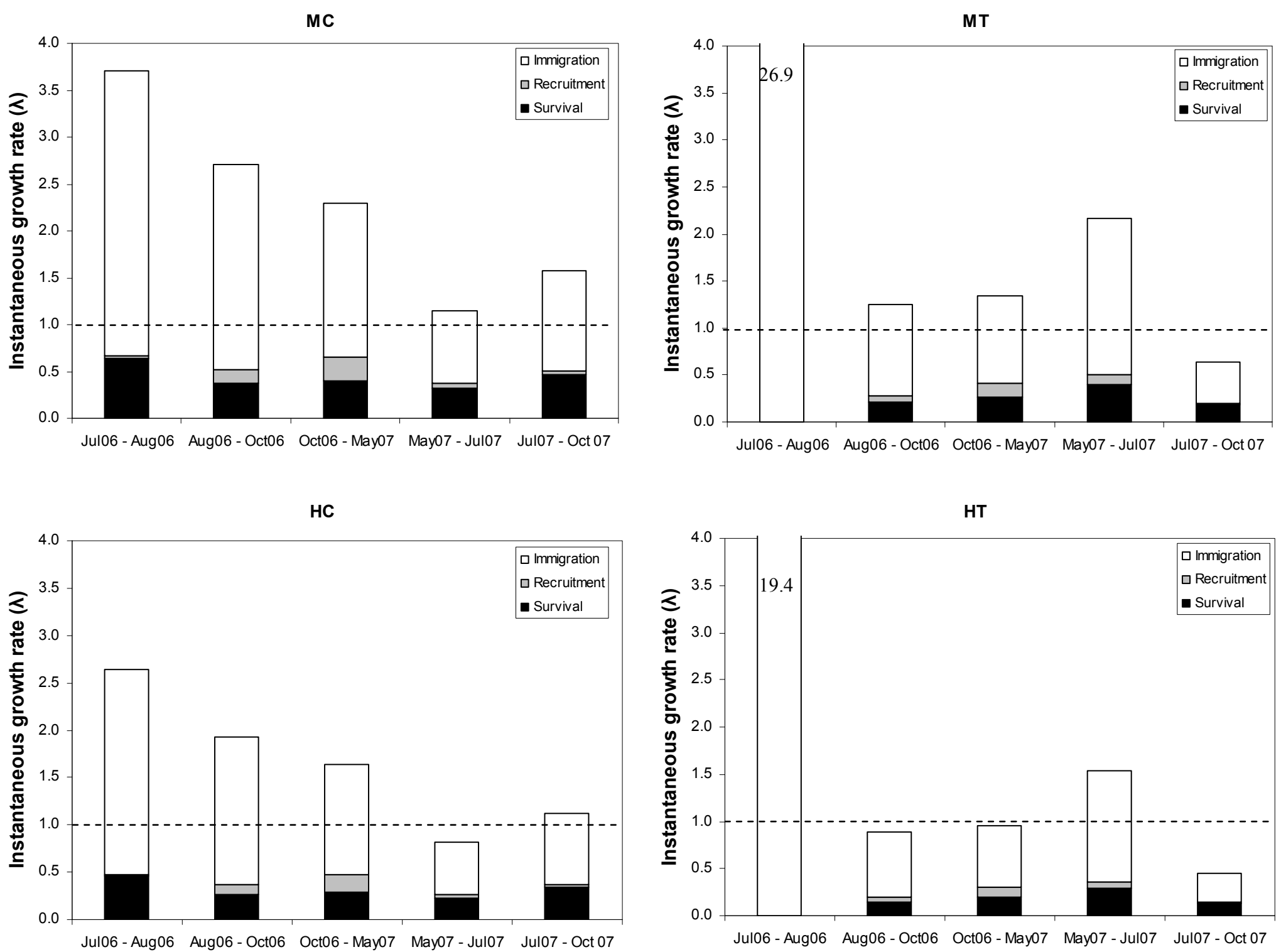
Figure 41. Estimated instantaneous survival $\left(\Phi=\lambda \times \gamma_{\mathrm{ii}}\right)$, local recruitment $\left(\Omega=\lambda \times \gamma_{\mathrm{ji}}\right)$, and immigration rates $\left(\Gamma=\lambda \times\left[1-\gamma_{\mathrm{ji}}-\gamma_{\mathrm{ii}}\right]\right)$ of large adult mottled sculpin among mainstem tributary control (MC, n=3), mainstem removal treatment (MT, $n=3)$, headwater tributary control, $(\mathrm{HC}, \mathrm{n}=3)$ and headwater treatment $(\mathrm{HT}, \mathrm{n}=3)$ sites for all sampling intervals and at fixed mean values for local physical and chemical habitat covariates. The estimates for the instantaneous rates of survival, recruitment and immigration were derived from the product of the log-transformed estimates for population growth rates among MC, MT, HC, and HT sites (i.e., ln(r) = $\lambda=\mathrm{N}_{\mathrm{t}+1} / \mathrm{N}_{\mathrm{t}}$, Figure 21) and the model averaged estimates for the proportion of $\lambda$ due to survival $\left(\gamma_{\mathrm{ii}}\right)$, local recruitment $\left(\gamma_{\mathrm{ji}}\right)$, and immigration $\left(1-\gamma_{\mathrm{ji}}-\gamma_{\mathrm{ii}}\right)$ among MC, MT, HC, and HT sites (Figure 38). The horizontal dashed line at $\lambda=0$ indicates unchanging population size (i.e., $\mathrm{N}_{\mathrm{t}+1}=\mathrm{N}_{\mathrm{t}}$ ). Because $\lambda$ was disproportionately higher over the first interval for the removal sites the scale of the yaxis was set lower than the maximum value over that interval in order to preserve scale among the remaining estimates. As such, the actual values for $\lambda$ for the removal sites are noted on the bars that exceed the range of the axis. 
MC

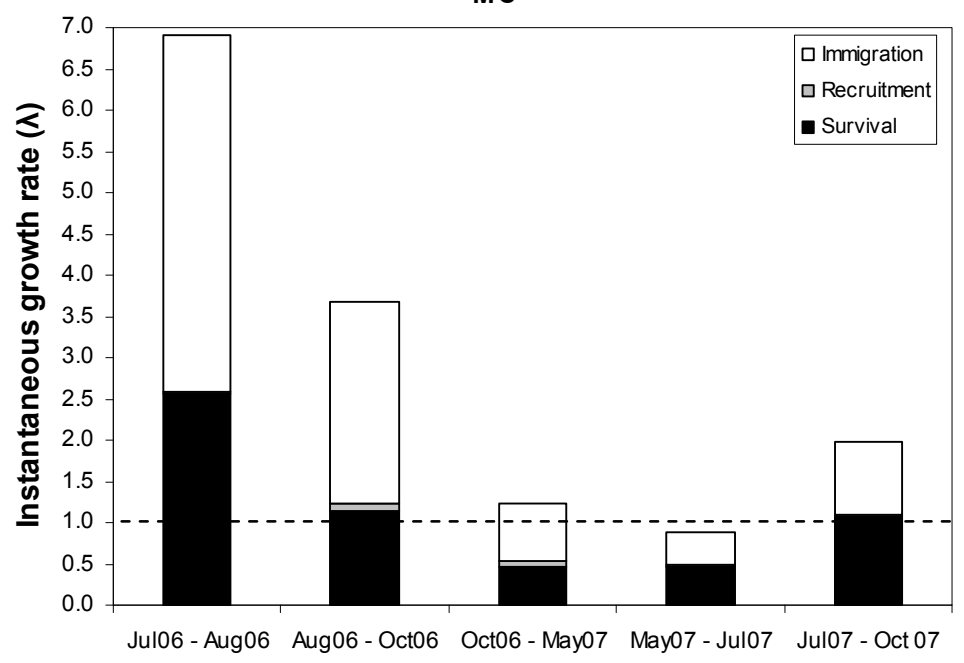

HC

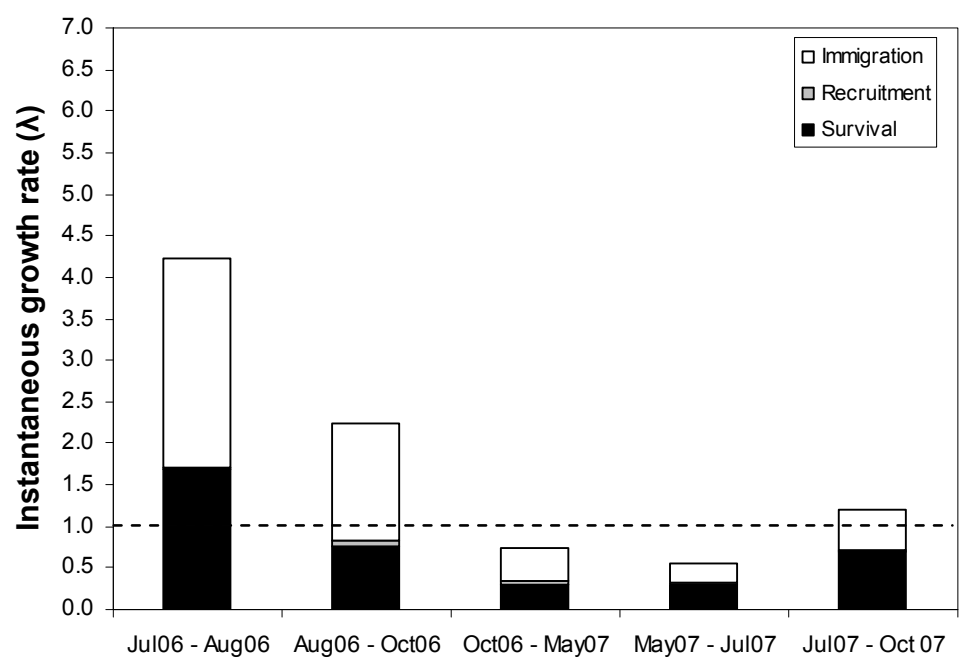

MT

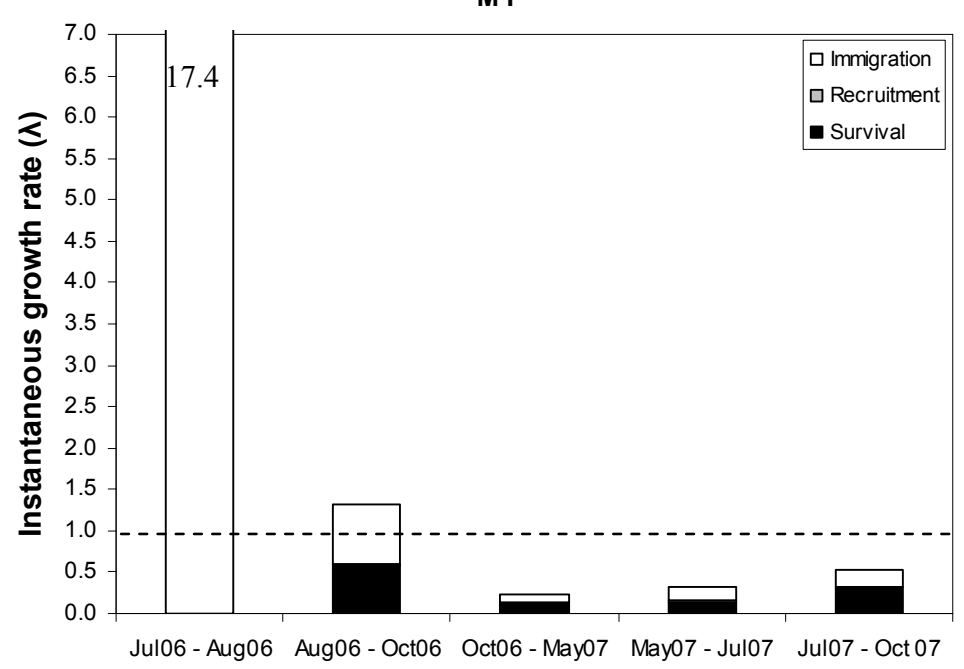

HT

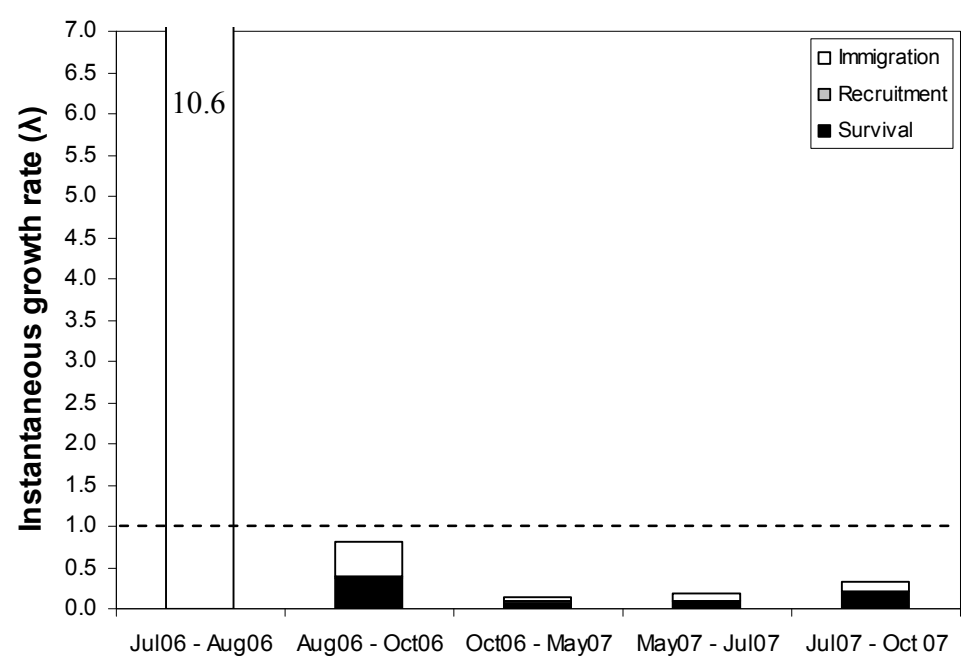


CHAPTER 3: Watershed Scale Deflation of Stream Fish Metacommunities in an Intensively Mined Appalachian Watershed

Roy W. Martin* and J. Todd Petty

Division of Forestry \& Natural Resources, West Virginia University, Morgantown, WV 26506

*Corresponding author: royworth@gmail.com

To be submitted to Ecological Applications 


\section{Abstract}

Theoretically, localized disturbances can produce watershed scale deflation of stream fish metacommunities and metapopulations through disruption of dispersal and source-sink dynamics. However, few empirical studies have sought to explicitly quantify watershed scale losses of aquatic diversity resulting from an accumulation of localized or stream scale impacts. Consequently, the specific objectives of this study were to: 1) develop models for predicting fish species occurrence and abundance as well as fish assemblage composition and integrity based on local (i.e., stream segment scale) and regional (i.e., drainage network scale) indicators of environmental quality; 2) quantify the relative "cost" of poor local and poor regional conditions on fish assemblages; and 3) identify thresholds at which the accumulation of impacts in the watershed to affect biological conditions even in streams with good local conditions.

Our results confirmed that there are measurable biological consequences to cumulative mining impacts within the regional stream network. We also observed substantial variability in species specific responses to local and regional conditions. Finally, we were able to generate explicit estimates of the relative biological costs associated with local and regional impairment on species abundance and likelihood of occurrence as well as assemblage composition and integrity. However, we did not observe threshold responses of fish metacommunities. Instead, watershed scale losses accumulated continuously across a range of watershed conditions. These findings indicate that effective management of stream fishes must consider the watershed context 
and should seek to affect local and regional conditions through the restoration and protection of interconnected drainage networks. 


\section{Introduction}

Recent research has suggested an important role for both local and regional processes in structuring stream fish assemblages (Osborne and Wiley 1992, Schlosser 1995, Snodgrass and Meffe 1998, Fausch et al. 2002, Freund and Petty 2007, Martin and Petty 2009, Falke and Fausch, in press, Martin, chapters 1 and 2). For example, local species richness is often a function of both a stream's size and its position within the drainage network (Gorman 1986, Fausch et al. 1984, Osborne and Wiley 1992, Matthews and Robison 1998, Grenouillet et al. 2004, Hitt and Angermeier 2008a). Similarly, others have demonstrated the importance of the regional availability of specific habitats to local fish assemblage composition (Snodgrass and Meffe 1998, Schlosser 1995, Martin and Petty 2009). Both Schlosser (1995) and Snodgrass and Meffe (1998), for example, demonstrated how the position of a focal reach relative to the position of productive beaver ponds can be an important determinant of fish assemblage composition in that reach. Beaver ponds acted as important sources of immigrants for peripheral stream reaches (Schlosser 1995). Likewise, the peripheral stream reaches often functioned as sink habitats (sensu Pulliam 1988) for the fishes emigrating from beaver ponds (Schlosser 1995). Similarly, Martin and Petty (2009) recently demonstrated how interconnectivity of coldwater and warmwater stream segments strongly influenced the distribution of brook trout and smallmouth bass in a West Virginia watershed. High rates of sympatry of these two species occurred at the juxtaposition of small coldwater tributaries and larger warmwater mainstems due to an interaction between local thermal conditions and dispersal (Martin and Petty 2009). 
Advances in our understanding of the ecology of stream fishes at broader spatial scales have benefited greatly from theoretical and empirical advances within the fields of metapopulation (Pulliam 1988, Hanski and Simberloff 1997, Schlosser and Angermeier 1995, Reiman and McIntyre 1995) and metacommunity ecology (Leibold et al. 2004, Muneepeerakul et al. 2008, Falke and Fausch, In Press, Martin, Chapter 1). It is now well understood, for example, that because individuals require access to spatially and temporally variable resources, dispersal among geographically separated important habitats is vital to population productivity and persistence (Pulliam 1988, Dunning et al. 1992, Schlosser and Angermeier 1995, Hanski and Simberloff 1997). Moreover, due to the linear and dendritic topological structure of stream systems (Fagan 2002), temporary and permanent barriers to movement, both natural and manmade, may constrain the accessibility of important habitats through time, which may have consequences for the persistence of fishes throughout the riverscape (Schlosser 1995, Taylor 1997, Poff and Allan 1995, Reiman and Dunham 2000, Fagan et al. 2002, Fausch et al. 2002). In fact, stream fish populations (Schlosser and Angermeier 1995, Ganio et al. 2005, Petty et al. 2005) and assemblages (Muneepeerakul et al. 2008, Bouvier et al. 2009, Falke and Fausch, In Press, Martin, chapter 1) are often spatially structured on the riverscape largely as a result of these processes. Consequently, a more complete understanding of fish community and population dynamics necessarily requires a multi-scale, watershedbased perspective that emphasizes the interactions between local and regional environmental conditions and fish dispersal (Fausch et al. 2002).

Both metapopulation and metacommunity theory suggest that localized disturbances (e.g., disturbances at the scale of a stream segment $<1 \mathrm{~km}$ in length) can 
have both local and regional consequences (e.g., consequences at the scale of drainage networks $>10-100 \mathrm{~km}$ ) for stream fish populations and assemblages. For example, source-sink metapopulation theory (Pulliam 1988) suggests that direct impacts to important source habitats and/or an increased frequency of isolation of source localities will result in regional deflation of the metapopulation. Similarly, metacommunity theory (Hubbell 2001, Mouquet and Loreau 2003, Leibold et al. 2004, Holyoak et al. 2005) predicts that habitat fragmentation, which results in increased dispersal limitation and reduced habitat heterogeneity, can result in landscape scale deflation of assemblage diversity. For example, Martin (chapter 1) demonstrated that excessive stream network fragmentation may inhibit regional dispersal, which can disrupt the influence of mass effects (i.e., regional migration between source and sink habitats) on local and regional stream fish diversity patterns.

Recent studies assessing stream restoration effectiveness provide some empirical evidence supportive of these theoretical expectations (Freund and Petty 2007, McClurg et al. 2007, Poplar-Jeffers et al. 2009). McClurg et al. (2007), for example, emphasized that the effectiveness of acid stream restoration would be maximized if efforts were focused at the scale of the network. Fish and macroinvertebrate communities in acidified streams never fully recovered to reference conditions following application of limestone fines to neutralize acidity, which the authors suggest was due at least partly to the fact that these acidified streams remain in acidified networks and lack sufficient connectivity to potential colonizers. Similarly, Freund and Petty (2007) found that streams with good local chemistry in fragmented networks often had fewer species than those in intact 
watersheds, which they attributed to reduced network connectivity and a degraded regional species pool.

We know of only one study that has addressed the possibility of regional community deflation as a result of accumulated localized impacts for freshwater fishes (Whittier 1997); however, we know of no studies that have explicitly addressed the issue for freshwater stream fishes. A sound understanding of this issue is extremely important for managing aquatic ecosystems for two reasons. First, the Clean Water Act (CWA) generally allows some level of localized impact to occur as a result of development activities, such as mining. However, the CWA makes it clear that localized impacts cannot be allowed to accumulate to the point that watershed scale processes become degraded. Unfortunately, our current understanding of watershed scale deflation is so poor that we have no effective means to implement this CWA mandate. Second, the number of stream restoration projects being implemented and the amount of money being spent on restoration has increased dramatically (Bernhardt et al. 2007). Most restoration projects are designed to improve aquatic communities and overall biological conditions. Project implementation, unfortunately, tends to occur on an opportunistic, ad hoc, stream-to-stream basis rather than on an integrated, watershed scale basis. If fish assemblages are controlled largely by watershed scale processes, however, then we would expect to observe minimal benefits resulting from spatially uninformed, localized restoration activities. Instead, we need to design integrated restoration programs that seek to "inflate" local assemblages through re-establishment of functioning networks (Petty and Thorne 2005, McClurg et al. 2007, Poplar-Jeffers et al. 2009). 
To address these current shortcomings, we quantified the response of stream fish assemblages inhabiting a central Appalachian riverscape heavily fragmented by intensive mining (Freund and Petty 2007, Merovich and Petty 2007, Merovich et al. 2007, Strager et al. 2009, Petty et al, in press) to both local environmental variability and an index of watershed scale condition (Strager et al. 2009). The specific objectives of this study were to: 1) develop models for predicting fish species occurrence and abundance as well as fish assemblage composition and integrity based on local (i.e., stream segment scale) and regional (i.e., drainage network scale) indicators of environmental quality; 2) quantify the relative "cost" of poor local and poor regional conditions on fish assemblages; and 3) identify watershed scale thresholds at which the accumulation of regional impacts affects biological conditions in this system.

\section{Methods}

Study area

Data for this study was collected from 90 wadeable streams distributed throughout the upper Monongahela River watershed in north central West Virginia. The study area was comprised of two 8-digit hydrologic unit classification watersheds (HUCs) that drain approximately $4000 \mathrm{~km}^{2}$ : Cheat River and Tygart Valley River. Due to a legacy of coal mining throughout this region, acid mine drainage (AMD) is a major source of chemical pollution in both watersheds (Merovich and Petty 2007). AMD results from the extraction of high sulfur coal, which exposes an overburden layer of 
pyrite to oxygen, water, and bacteria. The resulting water chemistry is a solution low in $\mathrm{pH}$ and high in sulfates and toxic metals that can be detrimental to metal- and $\mathrm{pH}-$ sensitive aquatic organisms (Herlihy et al. 1990, Freund and Petty 2007). However, toxicity can be variable across the range of influx severity and dependent on local conditions such as alkalinity (Williams 1999, Anderson et al. 2000). While moderately impaired streams in this region can support a limited number of fish species and macroinvertebrates, severely impaired streams may be entirely devoid of either (Herlihy et al. 1990, Anderson et al. 2000, Freund and Petty 2007, Merovich and Petty 2007). In addition to direct local impairment, AMD can create chemical dispersal barriers, giving rise to fragmented networks, which may lead to local community isolation (Freund and Petty 2007, Martin, chapter 1).

Site selection

Study sites were selected based on stream size, position within the drainage network, and expected local and regional impairment due to mining (Strager et al. 2009). Potential sites were first selected from an evaluation of the available pool of United States Geological Survey (USGS) National Hydrography Dataset (NHD) 1:24K stream segments (USGS 2000) based on the aforementioned properties. On-site selection of sampling reaches within these segments, however, was performed with the intention of maximally representing conditions within each segment in terms of available habitat types (e.g., pools, riffles, runs; Barbour et al. 1999). 
Figure 1 details the distribution of the 90 study reaches across the basin as well as the expected patterns of stream segment and watershed scale mining impairment based on an index of mining intensity developed for the region and integrated at multiple scales (Strager et al. 2009). Petty et al. (In Press) demonstrated that the mining index is strongly correlated to local water quality and benthic macroinvertebrate species richness in the study system. At the local (i.e., stream segment) scale, the index characterizes the total upstream area of mining related features, including coal seam outcrops, mine permit boundaries, abandoned mine land locations, and bond forfeiture sites (Strager et al. 2009). The calculation results in a value for each segment in the study area that varies between zero and 100 and can be interpreted as a percentage of the highest possible mining intensity in the study area (Strager et al. 2009). The index was also applied at the regional (i.e., watershed) scale by summing the index for all stream segments within the watershed and dividing by the total length of streams in the watershed (Strager et al. 2009). Coal seam outcrops were mapped from a series of county-based geologic maps from the West Virginia Geological and Economic Survey (Sisler and Reger,1931) and only included the mapped acidic coal seams that contribute to acid mine drainage related issues, including the Bakerstown, Lower Kittanning, Pittsburgh, Sewell, Upper Freeport, and Upper Kittanning seams (Strager et al. 2009). The mine permit boundaries, abandoned mine locations and bond forfeiture sites were obtained from web-based spatial data clearinghouses maintained by the West Virginia Department of Environmental Protection (WVDEP, 2004). Figure 2 illustrates the degree of variability in local and regional conditions due to mining intensity across the study area. The figure emphasizes 
the ideal nature of the study area for examining questions related to regional population and assemblage deflation as a result of accumulated local impacts.

\section{Fish assemblage data}

Fish sampling protocols followed the EPA-EMAP guidelines set by McCormick et al. (2001), but with some modifications (Freund and Petty 2007, Hense et al., In Revision). Reach lengths were calculated as the estimated mean stream width (MSW) multiplied by 40 , with no reaches shorter than 150 meters or exceeding 300 meters in length (Lyons 1992, McCormick et al. 2001, Freund and Petty 2007). Greater sampling lengths do not typically result in higher species richness or IBI scores (Ohio EPA 1989). All fish community sampling was conducted over the period spanning July 10 through August 31 during the summers of 2001 to 2004 . This encompassed the time frame during which fish communities are thought to be stable and ideal for sampling (Barbour et al. 1999, Freund and Petty 2007).

Sampling was conducted using one to two backpack electrofishing units, depending on MSW. Two units were used for streams with MSW greater than 6 meters. No streams sampled were larger than 15 meters wide. A modified, single-pass technique was employed, such that sampling was conducted in an upstream direction moving away from a downstream blocking seine for a distance no longer than 10 meters. After moving upstream for 10 meters, the sampling crew kicked back downstream to the initial starting point and blocking seine. This continued for the length of the site, moving the seine in approximately 10 meter increments. The moveable blocking seine was employed 
primarily to increase the capture efficiency of smaller benthic fishes, which have a tendency to be underrepresented in electrofishing surveys due to cryptic coloration and relatively low buoyancy as a result of lacking a swim bladder. For each backpack electrofishing unit employed, another person assisted with a dip net for capturing the stunned fishes. Netted fishes were placed in ventilated buckets along the stream margin before being processed. All fishes collected were counted, weighed to the nearest $0.1 \mathrm{~g}$, and measured to the nearest $1 \mathrm{~mm}$ in standard length. Voucher specimens and unidentified specimens were preserved in $95 \%$ ethanol and returned to the lab for verification.

For each site sampled, we also calculated a Mid-Atlantic Highlands Index of Biotic Integrity (MAHIBI) based on the methods and metrics described by McCormick et al. (2001). Metric scoring equations were based on those provided by the United States Environmental Protection Agency (USEPA) and the MAHIBI (McCormick et al. 2001, Freund and Petty 2007). Important metrics in the MAHIBI include: number of native cyprinid species, number of native benthic species, proportion of individuals in the family Cottidae, sensitive species richness, proportion of tolerant individuals, proportion of nonindigenous individuals, proportion of invertivore-piscivore individuals, proportion of macro-omnivores, and proportion of (clean) gravel spawning species (McCormick et al. 2001). Freund and Petty (2007) demonstrated that IBI score was consistent in subsequent sampling seasons in a subset of sites $(r=0.95, n=9)$ and, therefore, year-to-year variability in IBI score was expected to have little influence on our results relative to site to site variability. 


\section{Physicochemical and landscape data}

Water quality data were derived from late winter to early spring water samples taken over the span from 2002 to 2004 (Petty and Barker 2004, Merovich et al. 2007). Although late summer samples tend to capture the worst conditions among severely impaired AMD streams (Petty and Barker 2004), there was less concern over variability in these streams, which are likely devoid of fishes. Late winter samples were chosen as representative samples, because water quality has been shown to be poorest in moderately impaired streams during this season (Petty and Barker 2004). Thus, the water quality samples chosen to represent each site in this study were expected to reflect the most severe water quality condition among the most variable streams (Merovich et al. 2007).

At the time of the sample, temperature, dissolved oxygen, and total dissolved solids were measured on site using a YSI $650^{\circledR}$ with a $600 \mathrm{xl}$ sonde (Yellow Springs, Ohio). Additionally, a $500 \mathrm{ml}$ sample was filtered using a Nalgene polysulfone filter holder and receiver using a mixed cellulose ester membrane $0.45 \mu \mathrm{m}$ pore size disk. Filtered samples were treated with $5 \mathrm{ml}$ of $1: 1$ nitric acid to force the $\mathrm{pH}$ below 2.0 to ensure that metals remained in solution. The filtered samples were used for laboratory analysis of aluminum, iron, manganese, nickel, cadmium, chromium, calcium and total hardness. A 1 L grab sample was also collected for laboratory analysis of alkalinity, acidity and sulfates. Unfiltered samples were kept on ice after collection and stored in the laboratory at $4{ }^{\circ} \mathrm{C}$ prior to analyses. 
On-site evaluations of physical habitat conditions were also conducted using the United States Environmental Protection Agency's (USEPA) protocol for rapid visual habitat assessment (RVHA, Barbour et al. 1999). The RVHA variable used to visual characterize the local physical habitat condition in this study is a composite of 10 metrics characterizing epifaunal substrate/available cover, embeddedness, velocity and depth regime, sediment deposition, channel flow status, channel alteration, frequency of riffles (or bends), bank stability, vegetative protection, and riparian vegetative zone width (Barbour et al. 1999). Two observers trained in RVHA evaluated and collaborated on estimates for all sites in order to maximize repeatability (Roper and Scharnecchia 1995, Freund and Petty 2007). RVHA total score, 1 to 200, was used for all sites and analyses.

All landscape variables used in our analyses were extracted from geographic databases with ArcGIS ${ }^{\circledR}$ Desktop (version 9.3, Environmental Systems Research Institute 2006). These variables included an index of expected summer thermal conditions or weekly mean July temperature (WMJT, Wehrly et al. 2003, Martin and Petty 2009), cumulative drainage area (AREA), reach gradient (GRAD), maximum reach elevation (MELE), cumulative percent land cover and geology type (WVGAP 1996, Strager et al. 2009), and an index of mining intensity (MI, Strager et al. 2009, see Site selection).

All physicochemical and spatial variables were screened and culled to minimize variable redundancy and colinearity in further statistical analyses. We used a matrix of Pearson correlations to assess the strength of correlations among all variables and identified and removed potentially redundant variables based on a correlation value of 0.6 . 


\section{Statistical analyses}

We used principal components analysis (PCA) on the correlation matrix of the multivariate water chemistry dataset to characterize gradients in water chemistry variability. Prior to the PCA, we assessed the individual chemical variables for normality and applied transformations where applicable to best approximate normality for the multivariate dataset. The specific principal components discussed in the results were chosen based on the overall amount of variation in the data accounted for in those components as well as the interpretability of the component. Finally, we interpreted component loadings where loading values were greater than or equal to 0.4 . All water chemistry analyses were performed using the "stats" package of the R statistical programming language software, version 2.9.1.

We conducted non-metric multidimensional scaling (NMDS) analysis on the Bray-Curtis dissimilarity matrix of species abundances to characterize gradients in the fish community in relation to physicochemical variables. NMDS is a non-linear technique that ordinates multivariate observations onto multi-dimensional space based on the rank distances of the community dissimilarity matrix (Clark 1993). The final dimensionality of the ordination solution is based on a stress evaluation (Clark 1993). We minimized the number of dimensions of the solution while ensuring that the stress level was less than an a priori level set at 14\%. Additionally, we used a NMDS technique employing multiple random starts to maximize the probability of finding the globally minimum stress value (Clark 1993). Following the NMDS ordination, we fit vectors for each of the physicochemical covariates. This process fits vectors to the 
ordination in a manner that maximizes the correlation between the physicochemical variable and the entire ordination space. A permutation procedure was used to estimate the statistical significance of each vector's fit to the ordination space. We used 1000 permutations and based our conclusions on an a priori alpha-level of 0.05 . Only statistically significant vectors were interpreted.

An information theoretic framework was used to derive models for characterizing the effects of local and regional environmental condition on IBI scores and individual species distributions and abundances. We used stepwise variable selection based on Akiake's Information Criterion (AIC) to produce generalized linear models (GLM, McCullough and Nelder 1989) for predicting each dependent variable (Faraway 2006, Driscoll 2008, Driscoll and Lindenmayer 2009). The GLM is a flexible generalization of ordinary least squares regression (OLS), which relates the distribution function of a dependent variable to linear predictors through a function called the link function (McCullough and Nelder 1989). It was formulated as a way of unifying various statistical models, including logistic regression and Poisson regression, under a single framework, such that a general algorithm for maximum likelihood estimation was applicable for all distribution models, thereby relaxing the constraints imposed by the linear relation between independent and dependent variables in OLS regression (McCullough and Nelder 1989). The stepwise selection process used employed a "forward" and "backward" selection procedure that sequentially added and removed independent predictor variables on the basis of the overall model AIC value (Faraway 2006). The AIC value is based on the model log-likelihood and a penalization term based on the number of parameters in the model (Akaike 1974, Burnham and Anderson 2002). 
The process is iterated until all combinations of the available parameters are exhausted. The procedure, thus, results in a final, most parsimonious model where the log-likelihood is minimized using fewest possible number of predictors.

For predicting IBI scores among sites, we used a GLM with a Gaussian link function. We modeled species presence-absence using a GLM with a binomial error distribution employing the logit link function. For sites where species were present, we modeled abundances using a GLM with a negative binomial error distribution and the log link function. We predicted presence and abundance for 17 commonly sampled species (i.e., species present in at least $15 \%$ of all sample sites). In order to assess the fit for each final GLM, we calculated the percent deviance explained as well as the relative contribution of each variable selected to the overall proportion of deviance explained (Dobson 1999, Faraway 2006, Driscoll 2008). All GLM analyses were conducted using the "stats" (binomial GLM) and "MASS" (negative binomial GLM) packages of the R statistical programming environment, version 2.9.1.

In order to visualize the effect of HUC10 mining intensity (i.e., regional environmental condition) on local biological conditions, we constructed several plots of local physicochemical variables (e.g., water chemistry principal component scores) versus the dependent variables of interest: IBI score, species abundance, and species probability of presence. For each plot, we fit three regression lines based on model structure and coefficients of the final AIC-selected GLMs mentioned above. These three regression lines illustrated the modeled effect of the local covariate of interest (x-axis) given average conditions of other covariates in the GLM given the (1) minimum, (2) maximum, and (3) average values of HUC10 mining intensity in the dataset. Finally, in 
order to further illustrate and quantify the effects of watershed mining condition on the IBI score given local environmental conditions, we also conducted and analysis of covariance (ANCOVA) using median HUC10 mining index as a cutoff for value for a factor variable indicating high or low HUC10 mining index and modeled this factor with the other covariates indicated as important in the previous GLM analysis for predicting IBI score. The null hypothesis was that, given average values for these other locally important covariates, mean IBI score did not differ among sites with HUC10 mining index above or below the median value. We based our conclusions on an a priori alpha level of 0.05 . For visual purposes, we also constructed a plot of the relationship between local water chemistry and IBI score and fit trend lines for sites with HUC10 mining index above and below the median value. We interpreted the coefficient values of the regression equation to quantify the relative effect of being in a poor (i.e., HUC10 mining index below median) versus less impaired (i.e., HUC10 mining index above median) watershed in IBI units.

Finally, we used a threshold analysis technique to identify nonlinear trends in the relationship between increasing regional impairment (i.e., HUC10 mining index) and IBI score in sites with better versus poorer local chemistry (i.e., water chemistry principal component score). First, the sites were divided into two groups representing better and poorer local chemistry according to the natural break in the frequency distribution of water chemistry PC1 scores. Next, we utilized the significant zero crossings (SiZer) approach, a derivative-based technique, to approximate response functions of IBI score to the HUC10 mining index gradient and to examine how those functions change across that gradient (Sonderegger et al. 2009). This method produces a map of both the first and 
second derivatives of these functions, which aid in calibrating smoothing parameters in non-parametric regression fits to the response gradient (Sonderegger et al. 2009). The technique results in an estimate of a non-parametric response function that is data driven and requires only weak assumptions (Sonderegger et al. 2009).

\section{Results}

Local variation in water quality

The results of the PCA of water chemistry data indicated that principal component 1 (WC1) and 2 (WC2) accounted for $47 \%$ of the total variance in the solution $(29 \%$ and $18 \%$, respectively, Table 1) and were the only interpretable axes in the solution. WC1 represented a gradient of increasing specific conductivity, sulfates, total calcium, and total magnesium (Table 1). Sites with extremely low values on this axis tend are likely associated with mining activity, but may be hard due to treatment signatures (Merovich et al. 2007; Figure 3). Sites with higher values on this axis, however, are likely sites that are minimally or unimpaired by AMD (Merovich 2007 et al. 2007; Figure 3). WC2 represented a gradient of low to high $\mathrm{pH}$ and alkalinity (Table 1). Sites with low values on this axis are likely unimpaired streams (Figure 3). Sites with intermediate values may be slightly or moderately impaired by AMD or may be naturally soft streams (Merovich et al. 2007; Figure 3). Sites with high values are likely severely impaired by AMD (Merovich et al. 2007; Figure 3). Thus, highest quality sites scored moderate to high on 
the WC1 axis and moderate to low on the WC2 axis, whereas poorer quality sites scored moderate to low on WC1 and high on WC2.

\section{Local variation in fish assemblage structure}

For the NMDS ordination, stress was minimized in the three dimensional solution $(\mathrm{k}=3$, stress $=11.79)$. The vector fitting processes revealed that NMDS axis 1 represents a gradient of stream size, WMJT, and total species richness, whereas NMDS axis 2 represents a gradient of impairment and was most strongly correlated with HUC10 mining intensity, WC1, and site IBI score (Table 2, Figure 4). Thus, this ordination characterizes a fish community that is sorted along the continuum of stream size and expected temperature and also along a second continuum related to mining activity and impairment (Figure 4). Along NMDS axis 1, richness was lower among smaller, colder, streams and higher among larger, warmer streams (Table 2, Figure 4). Along axis 2 there were fewer and different species in streams with poor water chemistry or in watersheds impaired by mining (Figure 4). Considering both dimensions, the upper right hand corner of the plot is characterized by larger, warmer, less impaired streams with higher richness and IBI and the lower right hand corner is characterized by larger, warmer, impaired streams with lower richness and IBI (Figure 4). The upper left hand corner is characterized by smaller, colder, less impaired sites with low richness and higher IBI and the lower left hand corner is characterized by smaller, colder, impaired streams with lower richness and lower IBI (Figure 4). More specifically, based on species scores on these axes, one may expect to find $S$. fontinalis, $R$. cataractae, and $C$. bairdi in small 
streams in un-mined watersheds and $R$. obtusus, S. atromaculatus, and C. commersoni in smaller streams in mined regions (Figure 4). In larger streams in un-mined regions one may expect to find $N$. photogenis, $N$. rubellus, and $N$. micropogon, and A. natalis and $P$. notatus in larger streams in disturbed regions (Figure 4). It should be noted, however, that, while this analysis is essential in demonstrating the dominant gradients along which the community sorts in this system, it can not unequivocally distinguish among the local and regional effects of disturbance.

\section{Models of fish species occurrence}

For GLM results predicting presence-absence of 17 common species (Table 3) predicted weekly mean July temperature (WMJT) was an important variable and was selected by AIC as a parsimonious predictor for 15 of 17 species. All species but brook trout (Salvelinus fontinalis) demonstrated a positive relationship with WMJT, indicating that the probability of species' presence was higher in streams with higher local WMJT. Local habitat quality (RVHA) also was an important predictor for seven species (Table 3). While the probability of presence of longnose dace (Rhinichthys cataractae) and brook trout was positively related to RVHA score, rock bass, (Ambloplites rupestris), fantail darters (Etheostoma flabellare), smallmouth bass (Micropterus dolomieu), bluntnose minnows (Pimephales notatus), and creek chubs were all less likely to be present with increasing RVHA score (Table 3). Water chemistry principal component 1 (WC1) was an important determinant of presence in seven species as well, including mottled sculpin (Cottus bairdi), fantail darter, northern hogsucker (Hypentelium nigricans), smallmouth bass, river chub (Nocomis micropogon), longnose dace, and 
brook trout (Table 3). Each of these species were less likely to be present in streams with higher WC1 scores (i.e., streams with a stronger AMD chemical signature, refer to Table 1) than those with lower scores. Water chemistry principal component 2 (WC2) was an important predictor for four species, including central stoneroller (Campostoma anomalum), white sucker, mottled sculpin, and bluegill sunfish (Lepomis macrochirus; Table 3). While central stonerollers and mottled sculpin presence increased in streams with higher lower WC2 scores, white sucker and bluegill sunfish were more likely to be present in streams with higher WC2 scores (Table 3).

The HUC10 mining index, which is a measure of regional or watershed scale condition, was an important predictor for 11 of the 17 species modeled (Table 3). Only two species - bluntnose minnow (Pimephales notatus) and bluegill sunfish demonstrated a positive trend with this index, indicating an increasing likelihood of presence in sites situated in HUC10 sub-watersheds that are more intensively mined (Table 3). The remaining 10 species — rock bass, central stoneroller, rosyside dace (Clinostomus funduloides), mottled sculpin, greenside darter (Etheostoma blennioides), northern hogsucker, green sunfish, longnose dace, and brook trout - demonstrated a negative relationship with this index (Table 3).

Figure 5 illustrates the effect of poor, average, and good watershed condition (i.e., HUC10 mining index) on the probability of presence for two commonly sampled species that demonstrated a negative relationship between presence and regional impairment. When local water chemistry is very good (i.e., low $\mathrm{WC} 1$ score), the probability of presence for both longnose dace and brook trout ranged from less than $40 \%$ to nearly $100 \%$, depending on the HUC10 condition score (Figure 5). Figure 6 illustrates a similar 
phenomenon, but for two commonly sampled species where the HUC10 mining index facilitates their presence. Bluntnose minnows and bluegill sunfish were more likely to be present in streams embedded within highly impaired HUC10 watersheds (Figure 6). In other words, these results indicate that poor conditions at the HUC10 scale resulted in a deflation of longnose dace and brook trout distributions, whereas poor HUC10 conditions resulted in and inflation in the distribution of bluntnose minnows and bluegill sunfish (Figures 5 and 6).

\section{Models of species' abundance}

For the GLM results predicting the abundance of 17 common species in sites where they were present (Table 4), weekly mean July temperature (WMJT) was, again, the most common variable selected by AIC and was an important predictor of abundance for 10 of 17 species. While two species, white sucker and brook trout, were more abundant in sites with lower WMJT values, the remaining 8 species, rock bass, central stoneroller, mottled sculpin, northern hogsucker, bluegill sunfish, smallmouth bass, bluntnose minnow, and longnose dace, had higher abundances in streams with higher WMJT values (Table 4). RVHA score was an important determinant of abundance for eight species, with rosyside dace, mottled sculpin, bluegill sunfish, blacknose dace (Rhinichthys obtusus), longnose dace and brook trout all demonstrating higher abundances in streams with higher quality habitat (Table 4). Interestingly, the only two species whose presence was determined by RVHA score (refer to Table 3) also had abundances dependent on this variable — longnose dace and brook trout (Table 4). The 
abundance of two species, green sunfish and bluntnose minnow, was negatively related to local habitat quality (Table 4). Six species abundances were negatively related to WC1 (Table 4) indicating higher abundances in those streams with higher total calcium and magnesium and lower specific conductivity and sulfates (refer to Table 1). WC2 was an important predictor of abundance for six species as well (Table 4). Interestingly, the abundance of bluntnose minnows was associated with lower local pH (i.e., higher WC2 scores).

HUC10 mining index was an important predictor of eight species' abundances among sites where they were present (Table 4). While the abundances of rosyside dace and longnose dace were negatively associated with this index, as were their presences (refer to Table 3), the abundances of green sunfish, bluegill sunfish and bluntnose minnows were positively associated with increasing HUC10 mining index (Table 4). Interestingly, although the probability of presence for green sunfish was negatively associated with this index, their abundance among those sites where they were present was negatively associated with the HUC10 index (Table 4). The remaining three species whose abundances were predicted by HUC10 mining index were white suckers, river chubs, and creek chubs (Table 4). All three species' abundances increased with HUC10 mining index in those streams where they were present (Figure 4).

Figure 7 illustrates the effect of poor, average, and good watershed condition (i.e., HUC10 mining index $=0,4.2$, and 11.8 , respectively) on the abundance of two commonly sampled species that demonstrated a negative relationship between abundance and regional impairment (i.e., decreased abundance with increasing HUC10 mining index values). When local water chemistry was very good (i.e., low WC1 score), the 
abundance of longnose dace ranged from just under 200 to nearly zero individuals, depending on the relative watershed condition (i.e., minimum to maximum HUC10 mining index; Figure 7). Similarly, even when local habitat quality is maximized, the abundance of rosyside dace is expected to vary from nearly zero to just under 400 individuals depending on watershed condition with respect to mining (Figure 7). Although both species responded significantly and positively to the watershed mining index, the expected effect size of the HUC10 mining index covariate was slightly larger for rosyside dace than for longnose dace (Table 4, Figure 7). That is, abundances of rosyside dace varied somewhat more widely over the range of HUC10 mining index values and under similar local chemical conditions (i.e., WC2 scores) than did the abundances of longnose dace (Figure 7).

Figure 8 illustrates the effect of watershed condition on species abundance for two species whose abundances increased with increasing HUC10 mining index. While the abundance of white suckers is expected to be highest in sites with better water quality (i.e., lower WC2 scores), white sucker abundance varied widely depending on the condition of the watershed with respect to the mining index (Figure 8). Green sunfish, on the other hand, were predicted to have higher abundances in streams with poorer water quality (i.e., higher WC2 scores), yet abundance fluctuated widely depending on HUC10 mining index (Figure 8).

Finally, figures 5 through 8 aid in the visualization of the relative importance of each of the local and regional predictors to the occurrence and abundance, respectively, of the individual species modeled in the GLM-based analyses. 
Summarizing the effects of local and regional condition on specie' distributions

Table 5 summarizes the effects of the local water chemistry covariates (i.e., $\mathrm{WC} 1$ and WC2) and the regional, HUC10 mining index covariate on species occurrence and abundance among all sites. This table simply collapsed WC1 and WC2 into a single local response category intended to represent local conditions resulting from mining activity. Likewise, the HUC10 mining index represents the regional condition resulting from the cumulative effects of mining activity in the watershed. This table summarizes the results across all species in an attempt to group species that respond similarly to local and regional conditions due to mining activity. Table 5 demonstrates that some species occurrences, such as mottled sculpin, central stoneroller, brook trout, longnose dace, and northern hogsucker, were affected negatively by deteriorating local and regional conditions. On the other hand, some species' occurrences, such as river chub, smallmouth bass, and fantail darter, were negatively affected primarily by deteriorating local conditions and the regional condition appears to play no role (Table 5).

Likewise, several species' occurrences, including greenside darter, rosyside dace, green sunfish, and rock bass, were negatively affected primarily by conditions at the scale of the watershed (Table 5). Interestingly, white suckers and bluegill sunfish demonstrated a positive response of occurrence to deteriorating local conditions (Table 5). Bluegill sunfish and bluntnose minnows also demonstrated a positive response to deteriorating regional conditions (Table 5). Finally, the presence of some species, such as the blacknose dace and creek chub, was independent of either local or regional condition (Table 5). 
While some species' presences were dependent on both local and regional conditions, their abundances were dependent on the local or regional condition or neither (Table 5). For example, while mottled sculpin, central stoneroller, and brook trout occurrence was dependent on both the local and regional condition, all three of these species abundances were negatively affected by only the local condition (Table 5). Similarly, while longnose dace presence was related to both local and regional conditions, their abundance in those sites where they were present was, interestingly, only affected by the regional condition (Table 5). Northern hogsucker abundances, on the other hand, were not affected by either the local or regional condition in those sites where they were present (Table 5).

Interestingly, of the three species whose presences were affected only by the local condition, only river chub abundances were related to these metrics (Table 5). River chub abundance was negatively related to local conditions, but positively related to HUC10 mining intensity. Smallmouth bass and fantail darter abundances, however, were not affected by the local or regional condition in those sites where they were present (Table 5).

Abundances of species whose presence was related only to the regional condition demonstrated a variety of responses to the local and regional condition in those sites where they were present (Table 5). For example, greenside darter abundances were lower in streams with poorer local conditions and did not respond to HUC10 mining index. Rosyside dace abundances, conversely, decreased with deteriorating regional conditions, but were not affected by the local condition (Table 5). 
While white sucker and bluntnose dace rate of occurrence was higher in streams located in HUC10 watersheds of poorer condition, their abundances in those sites were negatively related to the poorer local conditions, yet were higher, again, when the regional condition was poorer (Table 5). In other words, white sucker and bluntnose dace populations thrived in high quality streams located within poor quality watersheds. In contrast, bluegill sunfish populations tended to thrive in poorer quality streams located within poorer quality watersheds (Table 5). Finally, creek chub, whose occurrence was unrelated to either local or regional condition, had higher abundances in sites located in more heavily mined watersheds.

Fish community response to local and regional conditions

NMDS 1 score was best predicted by WMJT, RVHA, WC1, and HUC10 mining index score and the resulting model explained approximately $48 \%$ of the total deviance in NMDS 1 (Table 6). NMDS 2 was best modeled by WMJT, RVHA, WC1, WC2, and HUC10 mining index score and this model explained approximately $51 \%$ of the total deviance in NMDS 2 (Table 6). WMJT explained most of the deviance in NMDS 1 (approx. 33\%), whereas WC1 explained most of the deviance in NMDS 2 (approx. 25\%) (Table 6).

HUC10 mining index explained around 6\% of the deviance in NMDS 1 and $8 \%$ of the deviance in NMDS 2. The coefficient for the HUC10 mining index covariate in the model for NMDS 1 indicated that a one unit change in this index results in an approximately 0.05 unit decrease in NMDS 1 score (Table 6). Combined with the results 
of the fit of the environmental vectors to the NMDS axes (Table 2, Figure 4), this result would suggest that increasing HUC10 index values result in a progression from a community that may resemble a larger, more diverse stream, to a community that resembles a smaller, less diverse one.

The coefficient for the HUC10 mining index covariate in the model for NMDS 2 was substantially larger than the one for the model predicting axis 1 score and indicated that a one unit change in HUC10 mining index results in an approximately 0.04 unit decrease in NMDS 2 score (Table 6). Combined with the results in Table 2 and Figure 4, this coefficient would indicate that increasing HUC10 index values lead from a community characterized sensitive taxa, such as brook trout, sculpin, and darters, to a community dominated by tolerant taxa, such as blacknose dace and creek chubs. In larger streams, with decreasing HUC10 condition there is likely a shift towards communities dominated by bluntnose minnows, yellow bullheads, and striped shiners.

The results of the Gaussian GLM for predicting IBI among sites using the local and regional environmental covariates demonstrated that IBI score is best modeled using WMJT, WC1, WC2, and the HUC10 mining index (Table 6). This final model accounted for over $40 \%$ of the total deviance in IBI score. While WMJT accounted for a minor amount of the overall deviance in IBI score $(<0.1 \%)$, the local water chemistry covariates, $\mathrm{WC} 1$ and $\mathrm{WC} 2$, accounted for most of the variance in the model $(>32 \%$, collectively). Interestingly, the regional mining index also accounted for more than $7 \%$ of the overall deviance (Table 6). Judging from the model coefficients, holding the other covariates in the model constant, one would expect a one unit increase in WC1 score to result in a 3.3 unit reduction in local IBI score (Table 6). Likewise, holding other 
covariates constant, a one unit increase in WC2 score is expected to result in a 2.6 unit reduction in IBI score (Table 6). Finally, holding the covariates for local conditions constant, a one unit increase in HUC10 mining intensity is expected to result in a 1.2 unit reduction in IBI score (Table 6).

Figure 11 demonstrates the effect of watershed condition on site IBI score. While IBI score is expected to be highest in sites with the good water quality (i.e., sites with a low WC1 score), IBI scores in sites with the lowest WC1 scores still varied considerably depending on the HUC10 mining index. For example, for the lowest WC1 values, IBI score varied from less than 70 to more 80 , depending on whether the HUC10 mining index was at its minimum value for the dataset (0) or its maximum (11.8).

The results of the ANCOVA analysis indicated that, while accounting for other local environmental covariates, such as WMJT, RVHA, WC1, WC2, IBI scores differed significantly among sites with HUC10 mining index values greater than the median value compared to those sites with an index value less than the median (Table 7). The coefficient value for the HUC10 mining index grouping factor indicated that sites with an index value greater than the median had an IBI score approximately 8.5 units lower than those sites below the median. The additive model including this factor variable explained $39 \%$ of the variation in IBI score and was a statistically significant improvement over the reduced model not including the grouping factor $(\mathrm{P}<0.01$; Table 7$)$. The additive model explained $6 \%$ more variance in IBI score than the reduced model, indicating that the grouping factor accounted for $6 \%$ of the variation in IBI score. The test for the interaction between IBI score and WC1, however, was not statistically significant, indicating that the slope of the relationship between IBI and WC1 for sites with HUC10 
mining index above and below the median were statistically indistinguishable (Table 7). Figure 12 illustrates the variability in IBISCORE along the gradient of degrading local water quality (i.e., WC1) for sites above and below the median HUC10 index value.

The result of the SiZer analysis indicated that the relationship between HUC10 mining index value and IBI score was both linear and negative (Figure 13). The panel illustrating the change in the first derivative indicated that there was no detectable threshold value at which HUC10 mining index value produced a reduction in IBI score (Figure 13). Likewise, the panel for the first derivative indicated that IBI score was reduced with every HUC10 mining index value greater than zero and declined linearly

thereafter (Figure 13). In other words, there was no evidence of a threshold effect of HUC10 condition on IBI. Instead, IBI scores declined linearly with increasing levels of HUC10 scale degradation.

\section{Discussion}

Extensive impacts to aquatic resources due to mining in this region have been well documented both in terms of water chemistry degradation (Petty and Barker 2004, Merovich et al. 2007, Strager et al. 2009) and reduced integrity of fish and macroinvertebrate assemblages (Freund and Petty 2007, Merovich and Petty 2007). Studies in other regions have shown similar consequences of mining-related chemical impairment on aquatic resources (Maret and McCoy 2002, Maret et al. 2003, Bruns 2005). As expected, our results clearly indicated that water chemistry impairment due to mining is a dominant determinant of both population and assemblage structure in this 
system. However, our study is novel in that we have demonstrated that local fish assemblages were affected simultaneously and independently by local environmental conditions (e.g., water chemistry, physical habitat quality, water temperature) and regional impairment as defined by the HUC10 scale mining index. Although numerous studies have demonstrated the consequences of human activities at the landscape scale on aquatic resources (Lammert and Allen 1995, Schlosser 1995, McCormick et al. 2001, King et al. 2005), most of these studies have focused primarily on linking upstream landscape disturbance to instream physical and chemical properties that affect biological conditions. We know of no other studies that have demonstrated how impacts to the region, which may not directly degrade local physicochemical properties, can affect local biological conditions.

Potential mechanisms driving the effect of regional impairment on local biological condition can be found in the metapopulation (Hanski and Gilpin 1997, Schlosser and Angermeier 1995) and metacommunity (Holyoak et al. 2005, Falke and Fausch, in press) literature. For example, among the severely impaired streams of the region, $\mathrm{pH}$ rarely exceeds 5 and concentrations of metals often remain well above toxic levels throughout much of the year (Petty and Barker 2004, Merovich et al. 2007, Freund and Petty 2007). As a result, those streams likely serve as temporary to permanent barriers to dispersal (Freund and Petty 2007, Martin and Petty, chapter 1), leading to the decreased persistence and/or abundance of species whose population persistence at this scale is dependent on regional dispersal (Schlosser and Angermeier 1995, Reiman and Dunham 2000, Hanksi and Gilpin 1997, Driscoll 2008, Martin, chapter 1). Likewise, cumulative localized impacts throughout the watershed network can incrementally reduce 
the quality and/or spatiotemporal availability of important complementary and supplementary habitats for some species (Dunning et al. 1992, Shlosser and Angermeier 1995), which may lead to a disruption of source-sink dynamics at this scale (Pulliam 1988, Schlosser 1995, Schlosser and Angermeier 1995).

In addition to the mechanisms offered in the metapopulation literature, metacommunity theory suggests that regional habitat loss and fragmentation is expected to result in reduced local and regional biodiversity, independent of local processes (Hubbell 2001, Mouquet and Loreau 2002, 2006, Freund and Petty 2007, Martin, chapter 1). The results of this study, along with a previous study of metacommunity dynamics in this system (Martin, chapter 1), in fact, suggest that the most probable mechanism for regional deflation is a disruption of the source-sink dynamics of sensitive taxa (e.g., brook trout, rosyside dace) at the scale of the watershed that leads to their extirpation or reduced abundance in high quality streams. Disruption of the source-sink dynamic and subsequent loss of sensitive taxa may also lead to the disruption of important spatial consumer-resource dynamics (DeRoos et al. 1998) and interspecific interactions, resulting in inflation of tolerant or generalist taxa (e.g., blacknose dace, creek chub) in regionally isolated, but locally favorable reaches (Martin, chapter 1).

Our findings demonstrating the wide range of variability in species' responses to local and regional impairment are presumably a result of species level differences in tolerance to local pollution, local habitat preferences, competitive rank, and dispersal tendencies. Consequently, variability in responses to impairment demonstrated among species in this study suggests that the occurrence or abundance of specific taxa may provide indication of the presence and magnitude of local and regional impacts. For 
example, ranking taxa in order of the proportion of variance explained in their probability of presence by the HUC10 mining index (e.g., Figures 9 and 10) may provide a means to assigning sensitivity levels to regional impairment to individual taxa. In this region, rosyside dace, central stoneroller, and longnose dace may serve as early indicators of impairment at the watershed scale based on this logic. Additionally, individual regression coefficients representing effect sizes (e.g., Tables 3, 4, and 6) can inform assignment of sensitivity. For example, because the effect size of watershed condition on longnose dace is much greater than for brook trout they likely provide a better indicator of degrading watershed condition.

Finally, taxa that are most likely to be present in the absence of degraded local or regional conditions, but least likely to be present when conditions are degraded will likely prove to be the most practical indicators. For example, although rosyside dace abundance is clearly affected strongly by conditions at the watershed scale, they are also generally more patchily distributed than some other potential indicator taxa and their abundance and occurrence is presumably dependent on a number of other factors not measured in this study (i.e., relatively low overall proportion of variance explained). Longnose dace and central stoneroller, on the other hand, are generally widespread in this system and have an overall higher likelihood of presence in any given wadeable stream. The effect size of degrading watershed condition is also high for these two species and they are very unlikely to be present in degraded watersheds, particularly when watershed condition is very poor. Absence of these two fishes within any given wadeable stream in a watershed, consequently, may serve as a practical indication of biological impairment at the watershed scale in this system. 
Several studies aimed at developing stream fish IBIs have indirectly described local inflation and deflation, respectively, of "tolerant" and "intolerant" species as a result of increased disturbance due to human activity (McCormick et al. 2001, Bosjen and Barriga 2002, Whittier et al. 2007). Although these studies often incorporate landscape predictors into metric development (McCormick et al. 2001, Bosjen and Barriga 2002, Whittier et al. 2007) distinction among individual taxa tolerance to local versus watershed scale degradation has not been considered in metric development. For example, few fishes are likely to withstand even dilute levels of toxic metals associated with AMD impairment (Freund and Petty 2007). In fact, most cyprinids, such as blacknose dace and creek chub, which are typically classified as tolerant species in these metrics (e.g., McCormick et al. 2001), are known to be acid-intolerant (Pinder and Morgan 1995, Magnuson et al. 1984). Although current metric formulations would suggest that presence of these species is likely a result of local degradation, our results suggest, rather, that their local proliferation is a better indicator of watershed scale impairment. In a related paper, Hitt and Angermeier (2008b) recently demonstrated that fish dispersal is likely an important determinant of metric performance. Our findings, thus, corroborate the assertion of Hitt and Angermeier (2008b) that factors limiting dispersal can affect bioassessment performance. Other authors have come to similar conclusions (Fausch et al. 1984, Osborne et al. 1992). Our results, however, extend this concept by demonstrating consequences to assemblage integrity, and individual taxa, due to impairment of stream network connectivity and regional habitat degradation resulting from the accumulation of localized impacts at the watershed scale. Thus, our findings 
provide further support for the conclusion that a more spatially explicit approach to bioassessment is necessary.

In addition to quantifying and characterizing the independent effects of local and regional impairment on biological conditions in this system, our modeling framework also facilitates explicit quantification of the "cost" of the accumulation of impacts at the watershed scale in biological units, including species' probability of occurrence, species' expected abundance, assemblage structure (i.e., NMDS score), and assemblage integrity (i.e., IBI score). Moreover, using a recently developed threshold analysis technique (Sonderegger et al. 2009), we also concluded that regional impairment due to mining is linearly related to biological integrity and the effect is likely immediate, in regard to the amount of mining activity in the watershed needed to produce a local effect. This combination of findings suggests that minimal mining activity at the scale of the HUC10 watershed will produce predictable and measurable consequences for local fish assemblages and that integrity will decline at a constant rate with accumulating regional impacts. Thus, the cost of localized mining activities to both local and watershed biological condition is explicitly estimable in biological units using a simple, linear formula.

Concerning the biological response to local impairment due to mining, although the fish assemblage sorted broadly along two gradients of local chemical condition (i.e., a conductivity/sulfate concentration gradient or $\mathrm{WC} 1$ and a $\mathrm{pH}$ gradient or $\mathrm{WC2}$ ) our analyses indicated that individual species differed in their response to these two gradients. Of the 7 species whose occurrences or abundances were significantly affected by WC1 (Table 2), for example, all of those species' occurrences were negatively related 
to the $\mathrm{WC} 1$ gradient, suggesting they are particularly sensitive to degraded local chemical conditions associated with increased specific conductivities and sulfate levels. Freund and Petty (2007) found that such chemical signatures, particularly elevated sulfate levels, serve as robust indicators of local biological degradation associated with temporally variable, mining-related toxins (e.g., aluminum, iron, and nickel). Additionally, because $\mathrm{WC1}$ is orthogonal to and independent of the second principal component describing a gradient of $\mathrm{pH}$ (i.e., WC2), these species may be absent even where $\mathrm{pH}$ is locally suitable, such as in treated or episodically acidified streams (Petty and Barker 2004, Merovich et al. 2007). For example, brook trout are known to be quite sensitive to aluminum toxicity associated with acid deposition (Baker and Schofield 1981, Gagen et al. 1993, Van Sickle et al. 1996, Baldigo et al. 2007), but are relatively tolerant of low pH (Magnuson et al. 1984, Gagen et al. 1993, Van Sickle et al. 1996) compared to some commonly sympatric species, such as blacknose dace (Baker and Schofield 1981, Magnuson et al. 1984, Van Sickle et al. 1996, Pinder and Morgan 1995).

Interestingly, although several species' occurrences were unrelated to WC1, many of those species, nevertheless, demonstrated reduced abundance in those streams scoring higher on WC1. Indeed, few fishes can tolerate even dilute sources of dissolved metals (Pinder and Morgan 1995, Maret and McCoy 2002). Because toxin levels may vary considerably over time from slightly to moderately impaired streams (Petty and Barker 2004, Merovich et al. 2007), however, some of these species may be able to persist marginally in these streams, particularly if they are able to frequently migrate to and from nearby, less impaired, refuge areas during spates of increased local toxicity (Freund and Petty 2007). 
Our results indicated that reduced $\mathrm{pH}$ (i.e., higher WC2 scores) was less important in determining species' occurrence compared to WC1 in this study. Only central stoneroller and mottled sculpin exhibited a negative relationship with this gradient. Most cyprinids, such as the central stoneroller, are commonly thought to be intolerant of low pH conditions (Pinder and Morgan 1995). Conversely, some adult cottids are thought to be relatively tolerant of low pH (Gagen et al. 1993, Van Sickle et al. 1996). However, Kaeser and Sharpe (2001) found that, even though adult slimy sculpin were often able to persist through episodically acidic spring conditions in a Pennsylvania stream, loss of juvenile year classes as a result of recruitment failure eventually led to their local extirpation.

Interestingly, the occurrences of two species — bluegill sunfish and white sucker - were associated with higher scores on WC2. Not surprisingly, both white sucker (Baker and Schofield 1981, Magnuson et al. 1984) and bluegill sunfish (Magnuson et al. 1984) are known to be relatively tolerant of low $\mathrm{pH}$. Their increased rate of occupancy among more acidic sites is also presumably related to release from local or regional competition or predation from less tolerant species. Nevertheless, the finding that relatively few species' responded to this gradient may be due to the fact that only 2 sites of the 90 sampled had $\mathrm{pH}$ values lower than 5.5 at the time of water sampling, which occurred at early spring base flow (see Methods). In general, most species are able to persist in streams near this $\mathrm{pH}$ level (Magnuson et al. 1984).

\section{Management implications}


The results of this study indicate that the effect of watershed impairments on local biological conditions is largely independent of local conditions. This finding clearly emphasizes that a watershed scale approach to the management of stream fishes is crucial. Specifically, managers should seek to affect fish populations and assemblages at the watershed scale through the restoration and protection of regionally important habitats and interconnected drainage networks (Schlosser and Angermeier 1995, Fausch et al. 2002, Martin, chapters 1 and 2). We have shown that local impairment of chemical habitat and the loss of important dispersal corridors leads to assemblage deflation at the watershed scale. However, by the same token, targeted restoration of crucial corridors and restoration of regionally important habitats at the watershed scale is expected to lead to regional inflation of fish populations and assemblages. We believe that our results detailing the responses of individual species and assemblage properties to local and regional degradation will provide an important foundation for discerning which corridors and habitats should be prioritized for restoration and protection in mined Appalachian watersheds.

Our results should also provide an important framework and currency for use in prioritizing restoration and protection activities in this region. A real estate analogy applies. Plans to improve the overall value of houses (e.g., stream reaches) in a neighborhood (i.e., watershed), for example, should explicitly consider of the condition of the other houses in the neighborhood if the goal is to maximize the biological benefits gained from restoration efforts given the cost or if the goal is to minimize the ecological costs expected to result from accumulating impairments (Strager et al. 2009). In real estate operations repairing poor houses in poor neighborhoods results in a lower financial 
return than equal investment into poor houses in good neighborhoods. Similarly, because the effectiveness of localized stream restoration efforts are dependent on conditions at the watershed scale, restoring poor streams in poor watersheds, for example, is expected to result in a lower return of biological units than restoration of poor streams in good watersheds where a more healthy population of colonists may facilitate more complete recovery (McClurg et al. 2007). Therefore, prioritization of restoration and protection plans for stream fish assemblages should proceed on a neighborhood (i.e., watershed) to house (i.e., stream reach or segment) basis.

Therefore, our findings suggest an important role for stream fishes in bioassessment programs. While macroinvertebrates are superior indicators of local conditions, we believe that a detailed analysis of the stream fish community will often reveal important conclusions concerning human activities at the scale of the watershed (Freund and Petty 2007). Thus, we believe that a stream fish community sampling will prove to be a cost-effective approach to bioassessment, given that they provide important information about processes occurring at a scale at which management decisions are increasingly directed (Wiens 2001, Fausch et al. 2002). As others have noted (Fausch et al. 1984, Osborne and Wiley 1992, Hitt and Angermeier 2008), in the future, a more spatially informed approach to bioassessment for stream fishes will prove vital for improving managers' ability to make decisions across the relevant range of spatial scales at which stream fish assemblages integrate the effects of stressors. 


\section{Literature cited}

Akaike, H. 1974. A new look at the statistical model identification. IEE Transactions on Automatic Control 19:716-723.

Anderson, R. M., T. F. Beer, M. E. Buckwalter, S. D. Clark, I. Mc Auley, J. I. Sams, and D. R. Williams. 2000. Water quality in the Allegheny and Monongahela River Basins, Pennsylvania, West Virginia, New York, and Maryland. U.S. Geological Survey Circular 1202, U.S. Department of Interior, U.S. Geological Survey.

Angermeier, P. L. 1987. Spatiotemporal variation in habitat selection by fishes in small Illinois streams. Pages 52-60 in W. J. Matthews and D. C. Heins, editors. Community and evolutionary ecology of North American stream fishes. University of Oklahoma Press, Norman, Oklahoma, USA.

Baker, J. P., and C. L. Schofield. 1981. Aluminum toxicity to fish in acid waters. Earth and Environmental Science 18:289-309.

Baldigo, B. P., and G. B. Lawrence. 2001. Effects of stream acidification and habitat on fish populations of a North American river. Aquatic Sciences - Research Across Boundaries 63:196-222. 
Barbour, M. T., J. Gerritsen, B. D. Snyder, and J. B. Stribling. 1999. Rapid Bioassessment Protocols for Use in Streams and Wadeable Rivers: Periphyton, Benthic Macroinvertebrates and Fish, Second Edition. EPA 841-B-99-002. U.S. Environmental Protection Agency; Office of Water; Washington, D.C.

Bernhardt, E. S., E. B. Sudduth, M. A. Palmer, J. D. Allan, J. L. Meyer, G. Alexander, J. Follastad-Shah, B. Hassett, R. Jenkinson, R. Lave, J. Rumps, and L. Pagano. 2007. Restoring rivers one reach at a time: results from a survey of U.S. river restoration practitioners. Restoration Ecology 15:482-493.

Bouvier, L. D., K. Cottenie, and S. E. Doka. 2009. Aquatic connectivity and fish metacommunities in wetlands of the lower Great Lakes. Canadian Journal of Fisheries and Aquatic Sciences 66:933-948.

Bruns, D. 2005. Macroinvertebrate response to land cover, habitat, and water chemistry in a mining-impacted river ecosystem: A GIS watershed analysis. Aquatic Sciences - Research Across Boundaries 67:403-423.

Burnham, K. P., and D. R. Anderson. 2002. Model selection and inference: a practical information-theoretic approach, 2nd edition. Springer-Verlag, New York, New York, USA. 
Clarke, K. R. 1993. Non-parametric multivariate analysis of changes in community structure. Australian Journal of Ecology 18:117-143.

Danehy, R. J., N. H. Ringler, S. V. Stehman, and J. M. Hassett. 1998. Variability of fish densities in a small catchment. Ecology of Freshwater Fish 7:36-48.

DeRoos, A. M. 1998. Pattern formation and the spatial scale of interaction between predators and their prey. Theoretical Population Biology 53: 108-130.

Driscoll, D. A. 2008. The frequency of metapopulations, metacommunities and nestedness in a fragmented landscape. Oikos 117:297-309.

Driscoll, D. A., and D. B. Lindenmayer. 2009. Empirical tests of metacommunity theory using an isolation gradient. Ecological Monographs 79:485-501.

ESRI. 2007. ArcGIS Desktop version 9.3. Environmental Systems Research Institute, Redlands, California.

Fagan, W. F., P. J. Unmack, C. Burgess, and W. L. Minckley. 2002. Rarity, fragmentation, and extinction risk in desert fishes. Ecology 83:3250-3256. 
Falke, J. A., and K. D. Fausch. In Press. From metapopulations to metacommunities: linking theory with empirical observations of the spatial population dynamics of stream fishes. American Fisheries Society Symposium.

Faraway, J. J. 2006. Extending the Linear Model with R. Chapman \& Hall/CRC, Boca Raton, FL, USA.

Fausch, K. D., J. R. Karr, and P. R. Yant. 1984. Regional Application of an Index of Biotic Integrity Based on Stream Fish Communities. Transactions of the American Fisheries Society 113:39-55.

Fausch, K. D., C. Torgersen, C. V. Baxter, and H. W. Li. 2002. Landscapes to riverscapes: bridging the gap between research and conservation of stream fishes. BioScience 52:483-498.

Freund, J. G. 2004. Local and regional impairment of fish assemblages in a mined Appalachian watershed. Doctoral Dissertation. West Virginia Univeristy, Morgantown, WV, USA.

Freund, J. G., and J. T. Petty. 2007. Response of Fish and Macroinvertebrate Bioassessment Indices to Water Chemistry in a Mined Appalachian Watershed. Environmental Management 39:707-720. 
Gagen, C. J., W. E. Sharpe, and R. F. Carline. 1993. Mortality of brook trout, mottled sculpins, and slimy sculpins during acidic episodes. Transactions of the American Fisheries Society 122:616-628.

Ganio, L. M., C. E. Torgersen, and R. E. Gresswell. 2005. A geostatistical approach for describing spatial pattern in stream networks. Frontiers in Ecology and the Environment 3:138-144.

Gorman, O. T. 1986. Assemblage organization of stream fishes: the effect of rivers on adventitious streams. The American Naturalist 128:611-616.

Grenouillet, G., D. Pont, and C. Herisse. 2004. Within-basin fish assemblage structure: the relative influence of habitat versus stream spatial position on local species richness. Canadian Journal of Fisheries and Aquatic Sciences 61:93-102.

Hanski, I. A., and D. Simberloff. 1997. The metapopulation approach, its history, conceptual domain, and application to conservation. Pages 5-26 in I. A. Hanski and M. E. Gilpin, editors. Metapopulation Biology: Ecology, Genetics, and Evolution. Academic Press, San Diego, California, USA.

Hense, Z., R. W. Martin, and J. T. Petty. Electrofishing capture efficiencies for common stream fish species to support watershed scale studies in the central Appalachians. North American Journal of Fisheries Management I:I-I. 
Herlihy, A. T., P. R. Kaufmann, M. E. Mitch, and D. D. Brown. 1990. Regional estimates of acid mine drainage impact on streams in the mid-Atlantic and Southeaster United States. Water, Air, \& Soil Pollution 50:91-107.

Hitt, N. P., and P. L. Angermeier. 2008a. Evidence for fish dispersal from spatial analysis of stream network topology. Journal of the North American Benthological Society $27: 304-320$.

Hitt, N. P., and P. L. Angermeier. 2008b. River-Stream Connectivity Affects Fish Bioassessment Performance. Environmental Management 42:132-150.

Holyoak, M., M. A. Leibold, and R. D. Hold (Eds.). 2005b. Metacommunities: Spatial Dynamics and Ecological Communities. University of Chicago Press.

Hubbell, S. P. 2001. The Unified Neutral Theory of Biodiversity and Biogeography. Princeton University Press, Princeton, NJ, USA.

Karr, J. R. 1981. Assessment of biotic integrity using fish communities. Fisheries 6:2127. 
Kaeser, A. J., and W. E. Sharpe. 2001. The influence of acidic runoff episodes on slimy sculpin reproduction in Stone Run. Transactions of the American Fisheries Society 130:1106-1115.

Lammert, M., and J. D. Allan. 1999. Assessing Biotic Integrity of Streams: Effects of Scale in Measuring the Influence of Land Use/Cover and Habitat Structure on Fish and Macroinvertebrates. Environmental Management 23:257-270.

Lyons, J., L. Wang, and T. Simonsin. 1996. Development and validation of an index of biotic integrity for coldwater streams in Wisconsin. North American Journal of Fisheries Management 16:241-256.

Magnuson, J. J., J. P. Baker, E. J. Rahel, and J. R. Kramer. 1984. A critical assessment of effects of acidification on fisheries in North America [and discussion]. Philosophical Transactions of the Royal Society of London. Series B, Biological Sciences 305:501-516.

Maret, T. R., and D. E. MacCoy. 2002. Fish assemblages and environmental variables associated with hard-rock mining in the Coeur d'Alene River Basin, Idaho. Transactions of the American Fisheries Society 131:865-884.

Maret, T. R., D. J. Cain, D. E. MacCoy, and T. M. Short. 2003. Response of benthic invertebrate assemblages to metal exposure and bioaccumulation associated with 
hard-rock mining in northwestern streams, USA. Journal of the North American Benthological Society 22:598-620.

Martin, R. W., and J. T. Petty. 2009. Local stream temperature and drainage network topology interact to influence the distribution of smallmounth bass and brook trout in a central Appalachian watershed. Journal of Freshwater Ecology 24:497508.

Martin, R. W. and J. T. Petty. In Review. An empirical evaluation of metacommunity theory using an assemblage of stream fishes in a central Appalachian riverscape fragmented by mining activities: advancing a metacommunity approach to stream fish ecology. Ecological Monographs.

Matthews, W. J., and H. W. Robison. 1998. Influence of drainage connectivity, drainage area and regional species richness on fishes of the Interior Highlands in Arkansas. American Midland Naturalist 139:1-19.

McCormick, F. H., R. M. Hughes, P. R. Kaufmann, D. V. Peck, J. L. Stoddard, and A. T. Herlihy. 2001. Development of an index of biotic integrity for the mid-Atlantic Highlands region. Transactions of the American Fisheries Society 130:857-877. 
McClurg, S. E., J. T. Petty, P. M. Mazik, and J. L. Clayton. 2007. Stream ecosystem response to limestone treatment in acid impacted watersheds of the Allegheny Plateau. Ecological Applications 17:1087-1104.

McCullagh, P., and J. A. Nelder. 1989. Generalized Linear Models. John Wiley \& Sons, New York, New York, USA.

Meador, M. R., and D. M. Carlisle. 2007. Quantifying tolerance indicator values for common stream fish species of the United States. Ecological Indicators 7:329338.

Merovich, G. T., J. M. Stiles, J. T. Petty, P. F. Ziemkiewicz, and J. B. Fulton. 2007. Water chemistry-based classification of streams and implications for restoring mined Appalachian watersheds. Environmental Toxicology and Chemistry:13611369.

Mouquet, N., and M. Loreau. 2003. Community patterns in source - sink metacommunities. The American Naturalist 162:544-557.

Mouquet, N., T. E. Miller, T. Daufresne, and J. M. Kneitel. 2006. Consequences of varying regional heterogeneity in source-sink metacommunities. Oikos 113:481488. 
Muneepeerakul, R., E. Bertuzzo, H. J. Lynch, W. F. Fagan, A. Rinaldo, and I. RodriguezIturbe. 2008. Neutral metacommunity models predict fish diversity patterns in Mississippi-Missouri basin. Nature 453:220-222.

Ohio Environmental Protection Agency. 1989. Biological criteria for the protection of aquatic life. Vol. III. Users manual for biological field assessment of Ohio surface waters. Division of Water Quality Monitoring and Assessment, Surface Water Section, Columbus, $\mathrm{OH}$.

Osborne, L. L., and M. J. Wiley. 1992. Influence of tributary spatial position on the structure of warmwater fish communities. Canadian Journal of Fisheries and Aquatic Sciences 49:671-681.

Osborne, L. L., Kohler, S. L., Bayley, P. B., Day, D. M., Bertrand, W. A., and Wiley, M. J. 1992. Influence of stream location in a drainage network on the index of biotic integrity. Transactions of the American Fisheries Society 12: 635-643.

Petty, J. T., and J. Barker. 2004. Water quality variability in tributaries of the Cheat River, a mined Appalachian watershed. Proceedings of the American Society of Mining and Reclamation 21:1484-1504. 
Petty, J. T., P. J. Lamothe, and P. M. Mazik. 2005. Spatial and seasonal dynamics of brook trout populations inhabiting a central Appalachian watershed. Transactions of the American Fisheries Society 134:572-587.

Pinder, M. J., and R. Morgan II. 1995. Interactions of $\mathrm{pH}$ and habitat on cyprinid distributions in Appalachian streams of Maryland. Transactions of the American Fisheries Society 124:94-102.

Poff, N. L., and J. D. Allan. 1995. Functional organization of stream fish assemblages in relation to hydrological variability. Ecology 76:606-627.

Poplar-Jeffers, I. O., J. T. Petty, J. T. Anderson, S. J. Kite, M. P. Strager, and R. H. Fortney. 2008. Culvert Replacement and Stream Habitat Restoration: Implications from Brook Trout Management in an Appalachian Watershed, U.S.A. Restoration Ecology 17:404-413.

Pulliam, H. R. 1988. Sources, sinks, and population regulation. The American Naturalist 132:652.

Rieman, B. E., and J. D. McIntyre. 1995. Occurrence of bull trout in naturally fragmented habitat patches of varied size. Transactions of the American Fisheries Society 124:285-296. 
Rieman, B. E., and J. B. Dunham. 2000. Metapopulations and salmonids: a synthesis of life history patterns and empirical observations. Ecology of Freshwater Fish 9:5164.

Roper, B. B., and D. L. Scarnecchia. 1995. Observer Variability in Classifying Habitat Types in Stream Surveys. North American Journal of Fisheries Management 15:49-53.

Schlosser, I. J. 1995. Dispersal, boundary processes, and trophic-level interactions in streams adjacent to beaver ponds. Ecology 76:908-925.

Schlosser, I. J., and P. L. Angermeier. 1995. Spatial variation in demographic processes of lotic fishes: conceptual models, empirical evidence, and implications for conservation. Page 435 in J. L. Nielsen, editor. Evolution and the Aquatic Ecosystem: Defining Unique Units in Population Conservation. American Fisheries Society, Bethesda, Maryland, USA.

Sisler, J.D., Reger, D.B., 1931. Maps of General and Economic Geology. West Virginia Economic and Geological Survey, Morgantown, WV.

Snodgrass, J. W., and G. K. Meffe. 1998. Influence of beavers on stream fish assemblages: effects of pond age and watershed position. Ecology 79:928-942. 
Sonderegger, D. L., H. Wang, W. H. Clements, and B. R. Noon. 2009. Using SiZer to detect thresholds in ecological data. Frontiers in Ecology and the Environment 7:190-195.

Strager, M. P., J. T. Petty, J. M. Strager, and J. Barker-Fulton. 2009. A spatially explicit framework for quantifying downstream hydrologic conditions. Journal of Environmental Management 90:1854-1861.

Taylor, C. M. 1997. Fish species richness and incidence patterns in isolated and connected stream pools: effects of pool volume and spatial position. Oecologia 110:560-566.

Van Sickle, J., J. P. Baker, H. A. Simonin, B. P. Baldigo, W. A. Kretser, and W. E. Sharpe. 1996. Episodic acidification of small streams in the northeastern United States: fish mortality in field bioassays. Ecological Applications 6:408-421.

Wehrly, K. E., M. J. Wiley, and P. W. Seelbach. 2003. Classifying regional variation in thermal regime based on stream fish community patterns. Transactions of the American Fisheries Society 132:18-38.

Wiens, J. A. 2002. Riverine landscapes: taking landscape ecology into the water. Freshwater Biology 47:501-515. 
Whittier, T. R., D. B. Halliwell, and S. G. Paulsen. 1997. Cyprinid distributions in northeast USA lakes: evidence of regional-scale minnow biodiversity losses. Canadian Journal of Fisheries and Aquatic Sciences 54:1593-1607.

Whittier, T. R., R. M. Hughes, G. A. Lomnicky, and D. V. Peck. 2007. Fish and amphibian tolerance values and an assemblage tolerance index for streams and rivers in the western USA. Transactions of the American Fisheries Society $136: 254-271$.

WVDEP, 2004. Mining Permit Data. Division of Mining and Reclamation, West Virginia Department of Environmental Protection, Charleston, WV. Available from: $<$ http://gis.wvdep.org/data/omr.html > (accessed 09.01.08) [ESRI shapefile]. 


\section{Tables}

Table 1. Summary of loadings for the principal component analysis of water chemistry data. Component loadings that were interpreted $(1>=|0.4|)$ in the results section are highlighted in bold. SpCond $=$ specific conductivity, TDS = total dissolved solids, Alk = alkalinity, $\mathrm{Acid}=$ total acidity, $\mathrm{SO} 4=$ sulfates, $\mathrm{Al}=$ aluminum, $\mathrm{Cr}=$ chromium, $\mathrm{Fe}=$ iron, $\mathrm{Mn}=$ manganese, $\mathrm{Ni}=$ nickel, $\mathrm{Ca}=$ calcium, $\mathrm{Mg}=$ magnesium.

\begin{tabular}{ccc}
\hline Variable & WC1 & WC2 \\
\hline Eigenvalue & 4.1 & 2.5 \\
Variance Explained & 29.2 & 17.7 \\
\hline pH & & -0.46 \\
SpCond & 0.395 & \\
TDS & & \\
Alk & & -0.42 \\
Acid & & \\
SO4 & 0.454 & \\
$\mathrm{Al}$ & & \\
$\mathrm{Cd}$ & & \\
$\mathrm{Cr}$ & & 0.403 \\
$\mathrm{Fe}$ & & \\
$\mathrm{Mn}$ & & \\
$\mathrm{Ni}$ & & \\
$\mathrm{Ca}$ & 0.425 & \\
$\mathrm{Mg}$ & 0.464 & \\
\hline
\end{tabular}


Table 2. Summary of results for fitting environmental and biological variable vectors to the non-metric multidimensional scaling (NMDS) ordinations of the fish community. WMJT_PRED = predicted weekly mean July temperature, AREA_KM = upstream drainage area $(\mathrm{Km} 2), \mathrm{RVHA}=$ rapid visual habitat assessment score, $\mathrm{WC} 1 / \mathrm{WC} 2=$ water chemistry principal component scores for components 1 and 2, MI_HUC10 = HUC10 mining index score, IBISCORE = Mid-Atlantic Highlands index of biotic integrity score, RICHNESS $=$ local species richness. Values highlighted in bold are those that were interpreted in the results $(r>0.6, \mathrm{P}<0.05)$.

\begin{tabular}{lccccc}
\hline \multicolumn{1}{c}{ Variable } & NMDS1 & NMDS2 & NMDS 3 & $\mathbf{R}^{2}$ & $\operatorname{Pr}(>\mathbf{r})$ \\
\hline WMJT_PRED & 0.743 & & & 0.4782 & $<0.001$ \\
AREA_KM & 0.953 & & & 0.5293 & $<0.001$ \\
RVHA & & & & 0.0784 & 0.071 \\
WC1 & & -0.952 & & 0.3722 & $<0.001$ \\
WC2 & & - & -0.931 & 0.0983 & 0.032 \\
MI_HUC10 & & -0.951 & & 0.2941 & $<0.001$ \\
RICHNESS & 0.971 & & & 0.7815 & $<0.001$ \\
IBISCORE & & 0.802 & & 0.5175 & $<0.001$ \\
\hline
\end{tabular}


Table 3. Summary of binomial GLM results for predicting presence-absence of commonly sampled species. WMJT=modeled weekly mean July temperature, RVHA=Rapid Visual Habitat Assessment, $\mathrm{WC} 1 / \mathrm{WC} 2$ = water chemistry principal component axes 1 and 2, MI_HUC10 = watershed mining index. Percentages reflect the individual variable's contribution to overall deviance explained in the model. NA = not applicable, $(-)=$ variable not chosen by AIC-based selection.

\begin{tabular}{|c|c|c|c|c|c|c|c|c|}
\hline Species & $\mathbf{n}$ & Intercept & WMJT & RVHA & WC1 & WC2 & MI_HUC10 & $\begin{array}{l}\text { Deviance } \\
\text { Explained }\end{array}$ \\
\hline Ambloplites rupestris & 38 & -15.48 & $1.021(21.2 \%)$ & $-0.024(3.2 \%)$ & - & - & $-0.158(3.9 \%)$ & $28.2 \%$ \\
\hline Campostoma anomalum & 42 & -12.99 & $0.767(7.7 \%)$ & - & - & $-0.277(2.2 \%)$ & $-0.387(20.5 \%)$ & $30.5 \%$ \\
\hline Catostomus commersoni & 54 & -7.20 & $0.407(6.5 \%)$ & - & - & $0.269(2.8 \%)$ & - & $9.3 \%$ \\
\hline Clinostomus funduloides & 26 & 0.08 & - & - & - & - & $-0.292(14.0 \%)$ & $14.0 \%$ \\
\hline Cottus bairdi & 74 & -3.28 & $0.355(<0.1 \%)$ & - & $-0.487(20.2 \%)$ & $-0.350(2.1 \%)$ & $-0.269(8.5 \%)$ & $30.8 \%$ \\
\hline Etheostoma blennioides & 27 & -14.08 & $0.734(13.1 \%)$ & - & - & - & $-0.199(6.3 \%)$ & $19.4 \%$ \\
\hline Etheostoma flabellare & 31 & -11.06 & $0.789(10.6 \%)$ & $-0.032(2.7 \%)$ & $-0.655(12.5 \%)$ & - & - & $25.7 \%$ \\
\hline Hypentelium nigricans & 44 & -18.33 & $0.998(15.4 \%)$ & - & $-0.555(13.0 \%)$ & - & $-0.143(2.6 \%)$ & $30.9 \%$ \\
\hline Lepomis cyanellus & 44 & -13.49 & $0.747(14.7 \%)$ & - & - & - & $-0.150(4.0 \%)$ & $18.7 \%$ \\
\hline Lepomis macrochirus & 15 & -29.01 & $1.346(30.4 \%)$ & - & - & $0.422(5.4 \%)$ & $0.161(2.5 \%)$ & $38.3 \%$ \\
\hline Micropterus dolomieu & 34 & -17.70 & $1.244(19.7 \%)$ & $-0.044(4.4 \%)$ & $-0.718(13.6 \%)$ & - & - & $37.6 \%$ \\
\hline Nocomis micropogon & 36 & -15.20 & $0.769(10.1 \%)$ & - & $-0.768(13.7 \%)$ & - & - & $23.9 \%$ \\
\hline Pimephales notatus & 15 & -19.65 & $1.301(27.9 \%)$ & $-0.057(11.1 \%)$ & - & - & $0.185(3.9 \%)$ & $42.9 \%$ \\
\hline Rhinichthys obtusus & 78 & - & - & - & - & - & - & NA \\
\hline Rhinichthys cataractae & 55 & -15.01 & $0.595(<0.1 \%)$ & $0.040(3.0 \%)$ & $-0.971(2.7 \%)$ & - & $-0.387(14.3 \%)$ & $44.5 \%$ \\
\hline Salvelinus fontinalis & 49 & 2.26 & $-0.386(8.2 \%)$ & $0.039(4.6 \%)$ & $-0.654(15.6 \%)$ & - & $-0.181(4.4 \%)$ & $32.1 \%$ \\
\hline Semotilus atromaculatus & 81 & 0.92 & $0.361(6.3 \%)$ & $-0.034(5.2 \%)$ & - & - & - & $11.5 \%$ \\
\hline
\end{tabular}


Table 4. Summary of negative binomial GLM results for predicting abundance of commonly sampled species among sites where they were present. WMJT=modeled weekly mean July temperature, RVHA=Rapid Visual Habitat Assessment, WC1/WC2 = water chemistry principal component axes 1 and 2, MI_HUC10 = watershed mining index. Percentages reflect the individual variable's contribution to overall deviance explained in the model. NA $=$ not applicable, $(-)=$ variable not chosen by AIC-based selection.

\begin{tabular}{|c|c|c|c|c|c|c|c|c|}
\hline Species & $\mathbf{n}$ & Intercept & WMJT & RVHA & WC1 & WC2 & MI_HUC10 & $\begin{array}{l}\text { Deviance } \\
\text { Explained }\end{array}$ \\
\hline Ambloplites rupestris & 38 & -0.583 & $0.164(8.3 \%)$ & - & - & - & - & $8.30 \%$ \\
\hline Campostoma anomalum & 42 & 3.505 & $0.064(9.2 \%)$ & - & $-0.322(<0.1 \%)$ & - & - & $9.20 \%$ \\
\hline Catostomus commersoni & 54 & 9.031 & $-0.363(8.9 \%)$ & - & - & $-0.164(0.5 \%)$ & $0.116(6.8 \%)$ & $16.20 \%$ \\
\hline Clinostomus funduloides & 26 & -1.253 & - & $0.038(11.9 \%)$ & - & - & $-0.305(19.8 \%)$ & $31.70 \%$ \\
\hline Cottus bairdi & 74 & 0.255 & $0.204(4.4 \%)$ & $0.009(0.5 \%)$ & $-0.487(19.2 \%)$ & - & - & $24.00 \%$ \\
\hline Etheostoma blennioides & 27 & 3.028 & - & - & - & $-0.324(9.4 \%)$ & - & $9.40 \%$ \\
\hline Etheostoma flabellare & 31 & - & - & - & - & - & - & NA \\
\hline Hypentelium nigricans & 44 & -0.052 & $0.196(7.5 \%)$ & - & - & - & - & $7.50 \%$ \\
\hline Lepomis cyanellus & 44 & 3.446 & - & $-0.011(0.6 \%)$ & - & $0.339(12.5 \%)$ & $0.149(17.3 \%)$ & $30.50 \%$ \\
\hline Lepomis macrochirus & 15 & -14.469 & $0.612(14.6 \%)$ & $0.015(5.8 \%)$ & $-0.410(12.9 \%)$ & $0.281(28.1 \%)$ & $0.144(12.9 \%)$ & $74.20 \%$ \\
\hline Micropterus dolomieu & 34 & -3.435 & $0.334(13.6 \%)$ & - & - & - & - & $13.60 \%$ \\
\hline Nocomis micropogon & 36 & 3.443 & - & - & $-0.691(<0.1 \%)$ & $-0.425(7.0 \%)$ & $0.147(8.1 \%)$ & $15.10 \%$ \\
\hline Pimephales notatus & 15 & -12.100 & $0.876(10.5 \%)$ & $-0.024(23.5 \%)$ & - & $-0.273(<0.1 \%)$ & $0.216(18.5 \%)$ & $52.50 \%$ \\
\hline Rhinichthys obtusus & 78 & 2.786 & - & $0.015(5.8 \%)$ & - & - & - & $5.80 \%$ \\
\hline Rhinichthys cataractae & 55 & -3.112 & $0.324(9.3 \%)$ & $0.012(1.6 \%)$ & - & - & $-0.085(3.3 \%)$ & $13.10 \%$ \\
\hline Salvelinus fontinalis & 49 & 6.542 & $-0.450(23.6 \%)$ & $0.024(10.3 \%)$ & $-0.776(14.8 \%)$ & - & - & $48.70 \%$ \\
\hline Semotilus atromaculatus & 81 & 3.446 & - & - & - & - & $0.158(15.6 \%)$ & $15.60 \%$ \\
\hline
\end{tabular}


Table 5. Summary of individual species responses to local (i.e., water chemistry principal components 1 or 2, WC1 and WC2, respectively) and regional (i.e., HUC10 mining index) mining-induced impairment from GLM models for predicting species occurrence and abundance (refer to Tables 2 and 3). A (+) symbol denotes that a species demonstrated a positive, statistically significant relationship with condition and a (-) symbol indicates a negative, statistically significant relationship. The local relationship was summarized to characterize the relationship with WC1, WC2, or both. Since, for both WC1 and WC2, negative values were associated with better local chemical condition and positive values indicated poorer local chemical conditions, these two variables were interchangeable for this characterization. A positive relationship for either the local or regional indices imply better conditions with respect to mining activity, while a negative relationship implies deteriorating conditions.

\begin{tabular}{ccccc}
\hline & \multicolumn{2}{c}{ Presence-Absence } & \multicolumn{2}{c}{ Abundance } \\
Species & Local & Watershed & Local & Watershed \\
\hline Cottus bairdi & $(-)$ & $(-)$ & $(-)$ & $\mathrm{ns}$ \\
Campostoma anomalum & $(-)$ & $(-)$ & $(-)$ & $\mathrm{ns}$ \\
Salvelinus fontinalis & $(-)$ & $(-)$ & $(-)$ & $\mathrm{ns}$ \\
Rhinichthys cataractae & $(-)$ & $(-)$ & $\mathrm{ns}$ & $(-)$ \\
Hypentelium nigricans & $(-)$ & $(-)$ & $\mathrm{ns}$ & $\mathrm{ns}$ \\
Nocomis micropogon & $(-)$ & $\mathrm{ns}$ & $(-)$ & $(+)$ \\
Micropterus dolomieu & $(-)$ & $\mathrm{ns}$ & $\mathrm{ns}$ & $\mathrm{ns}$ \\
Etheostoma flabellare & $(-)$ & $\mathrm{ns}$ & $\mathrm{ns}$ & $\mathrm{ns}$ \\
Etheostoma blennioides & $\mathrm{ns}$ & $(-)$ & $(-)$ & $\mathrm{ns}$ \\
Clinostomus funduloides & $\mathrm{ns}$ & $(-)$ & $\mathrm{ns}$ & $(-)$ \\
Lepomis cyanellus & $\mathrm{ns}$ & $(-)$ & $(+)$ & $(+)$ \\
Ambloplites rupestris & $\mathrm{ns}$ & $(-)$ & $\mathrm{ns}$ & $\mathrm{ns}$ \\
Catostomus commersoni & $(+)$ & $\mathrm{ns}$ & $(-)$ & $(+)$ \\
Lepomis macrochirus & $(+)$ & $(+)$ & $(+)$ & $(+)$ \\
Pimephales notatus & $\mathrm{ns}$ & $(+)$ & $(-)$ & $(+)$ \\
Rhinichthys obtusus & $\mathrm{ns}$ & $\mathrm{ns}$ & $\mathrm{ns}$ & $\mathrm{ns}$ \\
Semotilus atromaculatus & $\mathrm{ns}$ & $\mathrm{ns}$ & $\mathrm{ns}$ & $(+)$ \\
\hline
\end{tabular}


Table 6. Summary of Gaussian GLM results for predicting non-metric multidimensional scaling axis scores (NMDS 1 and 2) and index of biotic integrity (IBI) scores among all sites sampled $(\mathrm{n}=90)$. WMJT=modeled weekly mean July temperature,

RVHA=Rapid Visual Habitat Assessment, WC1/WC2 = water chemistry principal component axes 1 and 2, MI_HUC10= watershed mining index. Percentages in parentheses reflect the individual variable's contribution to the overall deviance explained in the model. $(-)=$ variable not chosen by AIC-based selection.

\begin{tabular}{cccccccc}
\hline $\begin{array}{c}\text { Dependent } \\
\text { Variable }\end{array}$ & Intercept & WMJT & RVHA & WC1 & WC2 & MI_HUC10 & $\begin{array}{c}\text { Deviance } \\
\text { Explained }\end{array}$ \\
\hline NMDS 1 & -3.505 & $0.238(33.1 \%)$ & $-0.005(2.1 \%)$ & $-0.053(6.8 \%)$ & - & $-0.045(6.4 \%)$ & $48.41 \%$ \\
NMDS 2 & 0.775 & $-0.054(11.4 \%)$ & $.003(3.5 \%)$ & $-0.101(25.2 \%)$ & $-0.055(3.0 \%)$ & $-0.043(8.4 \%)$ & $51.40 \%$ \\
IBI Score & 51.319 & $1.343(<0.1 \%)$ & - & $-3.329(26.7 \%)$ & $-2.591(6.8 \%)$ & $-1.230(7.1 \%)$ & $40.50 \%$ \\
\hline
\end{tabular}


Table 7. Results for ANCOVA and model comparison for linear models predicting local IBI score as a function of weekly mean July temperature (WMJT), two local water chemistry principal components (WC1 and WC2), a factor variable coding for HUC10 mining intensity values above and below the median value for the dataset (MI_Group), and an interaction term for a group by WC1 effect. Pvalues are indicated for the individual variables in each model $(\operatorname{Pr}>|t|)$, the individual models $\left(\mathrm{P}_{\text {model }}\right)$, and the model comparison ANOVA $\left(\mathrm{P}_{\text {test }}\right)$.

\begin{tabular}{|c|c|c|c|c|c|c|}
\hline Model & Variable & Estimate & $\operatorname{Pr}(>|t|)$ & $\mathbf{R}^{2}$ & $\mathbf{P}_{\text {model }}$ & $\mathbf{P}_{\text {test }}$ \\
\hline \multirow[t]{7}{*}{ Interaction } & Intercept & 36.714 & $<0.001$ & 0.39 & $<0.001$ & ns \\
\hline & WMJT & 1.555 & ns & & & \\
\hline & RVHA & -0.008 & ns & & & \\
\hline & WC1 & -3.317 & $<0.001$ & & & \\
\hline & WC2 & -2.401 & $<0.01$ & & & \\
\hline & MI_Group & 8.481 & $<0.01$ & & & \\
\hline & MI_Group*WC1 & -0.076 & ns & & & \\
\hline \multirow[t]{6}{*}{ Additive } & Intercept & 36.804 & $<0.001$ & 0.39 & $<0.001$ & $<0.01$ \\
\hline & WMJT & 1.553 & ns & & & \\
\hline & RVHA & -0.008 & ns & & & \\
\hline & WC1 & -3.33 & $<0.001$ & & & \\
\hline & WC2 & -2.408 & $<0.01$ & & & \\
\hline & MI_Group & 8.518 & $<0.01$ & & & \\
\hline \multirow[t]{5}{*}{ Reduced } & Intercept & 56.667 & $<0.001$ & 0.33 & $<0.001$ & \\
\hline & WMJT & 0.906 & ns & & & \\
\hline & RVHA & -0.015 & ns & & & \\
\hline & WC1 & -4.115 & $<0.001$ & & & \\
\hline & WC2 & -2.599 & $<0.01$ & & & \\
\hline
\end{tabular}




\section{Figures}

Figure 1. Map of the 90 sites distributed throughout the upper Monongahela River basin in north-central West Virginia, location of the study area within the state of West Virginia (upper left inset), predicted watershed mining index values among the 10-digit subwatersheds (HUCs) in the region and among individual stream segments (bottom left

inset; Strager et al. 2009). For display purposes, classes of mining intensity were defined by natural breaks in the data. 


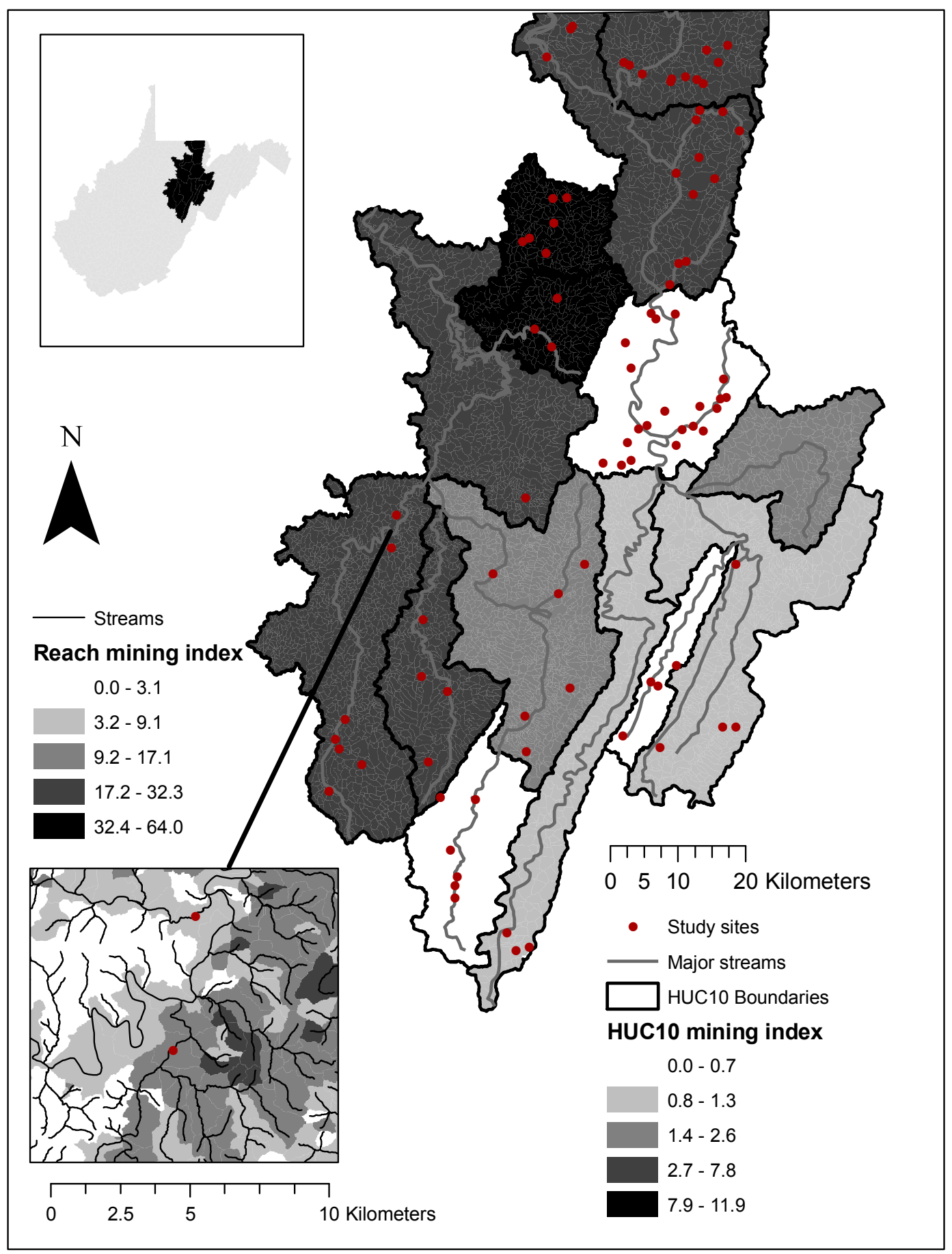


Figure 2. Plot of variability in expected mining intensity at the regional (HUC12 watershed) and local (stream segment) scales (Strager et al. 2009) among the 90 study sites.

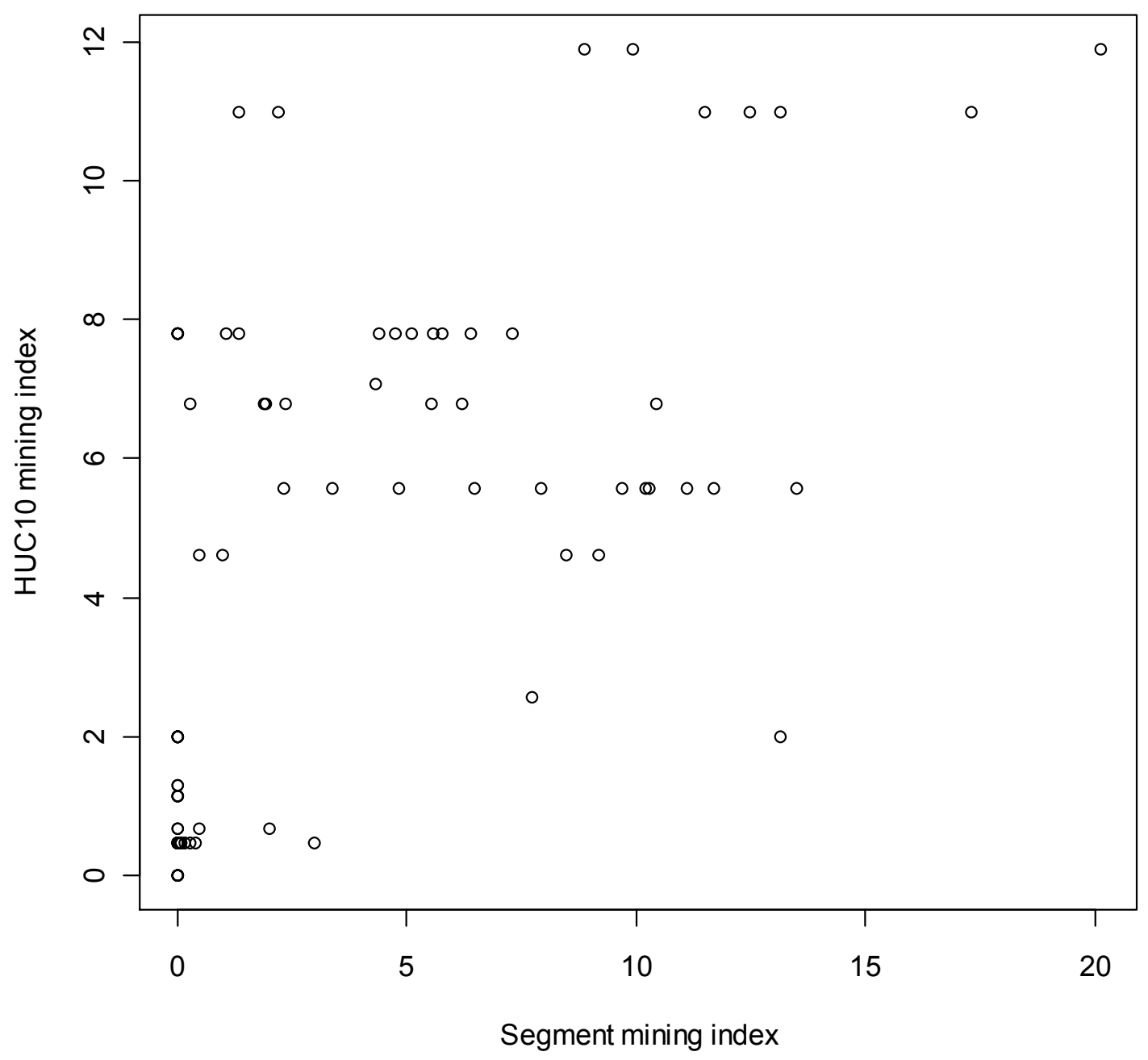


Figure 3. Plot of water chemistry principal components 1 and 2 scores for all 90 sites. SpCond $=$ specific conductivity, $\mathrm{SO} 4=$ sulfates. Chemical variables with high factor loadings $(\geq 0.4)$ are shown on the corresponding axes.

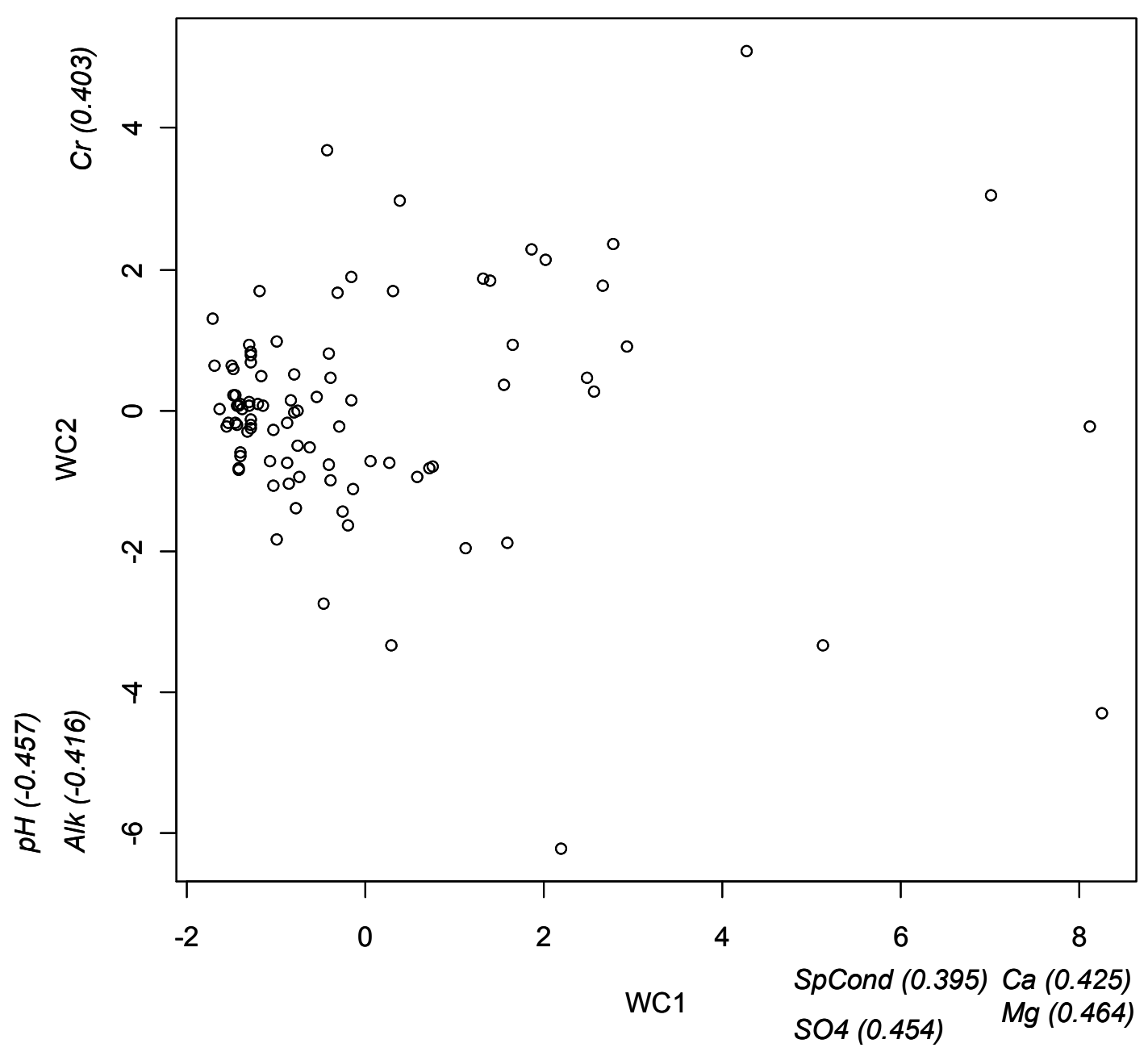


Figure 4. Non-metric multidimensional scaling ordination of species and sites. Strongly correlated vectors $(\mathrm{r}>0.6, \mathrm{P}<0.05)$ of environmental and biological variables are noted along both NMDS axes based on the direction of correlation. See table 2 for correlation strengths and significance of individual variables. Small black dots represent the ordination of the 90 sites sampled. Characters are acronyms for individual species and represent the direction and magnitude of strongest correlation of individual abundances in the ordination space. WMJT = predicted weekly mean July temperature, AREA = upstream drainage area $\left(\mathrm{Km}^{2}\right), \mathrm{WC1}=$ water quality principal component 1 score, MI_HUC10 = HUC10 mining index score, RICHNESS = local species richness, IBISCORE $=$ index of biotic integrity score, AMNA = Ameirus natalis, AMRU = Ambloplites rupestris, CAAN = Campostoma anomalum, COBA = Cottus bairdi, ETBL $=$ Etheostoma blennioides, $\mathrm{ETFL}=$ Etheostoma flabellare, $\mathrm{ETNI}=$ Etheostoma nigrum, $\mathrm{HYNI}=$ Hypentelium nigricans, LECY = Lepomis cyanellus, LEGI = Lepomis gibbosus, $\mathrm{LUCH}=$ Luxilus chrysocephalus, $\mathrm{MIDO}=$ Micropterus dolomieu, MISA = Micropterus salmoides, $\mathrm{NOMI}=$ Nocomis micropogon, $\mathrm{NOPH}=$ Notropis photogenis, $\mathrm{NORU}=$ Notropis rubellus, PINO = Pimephales notatus, RHAT = Rhinichthys obtusus, RHCA = Rhinichthys cataractae, SAFO = Salvelinus fontinalis, SEAT $=$ Semotilus atromaculatus.

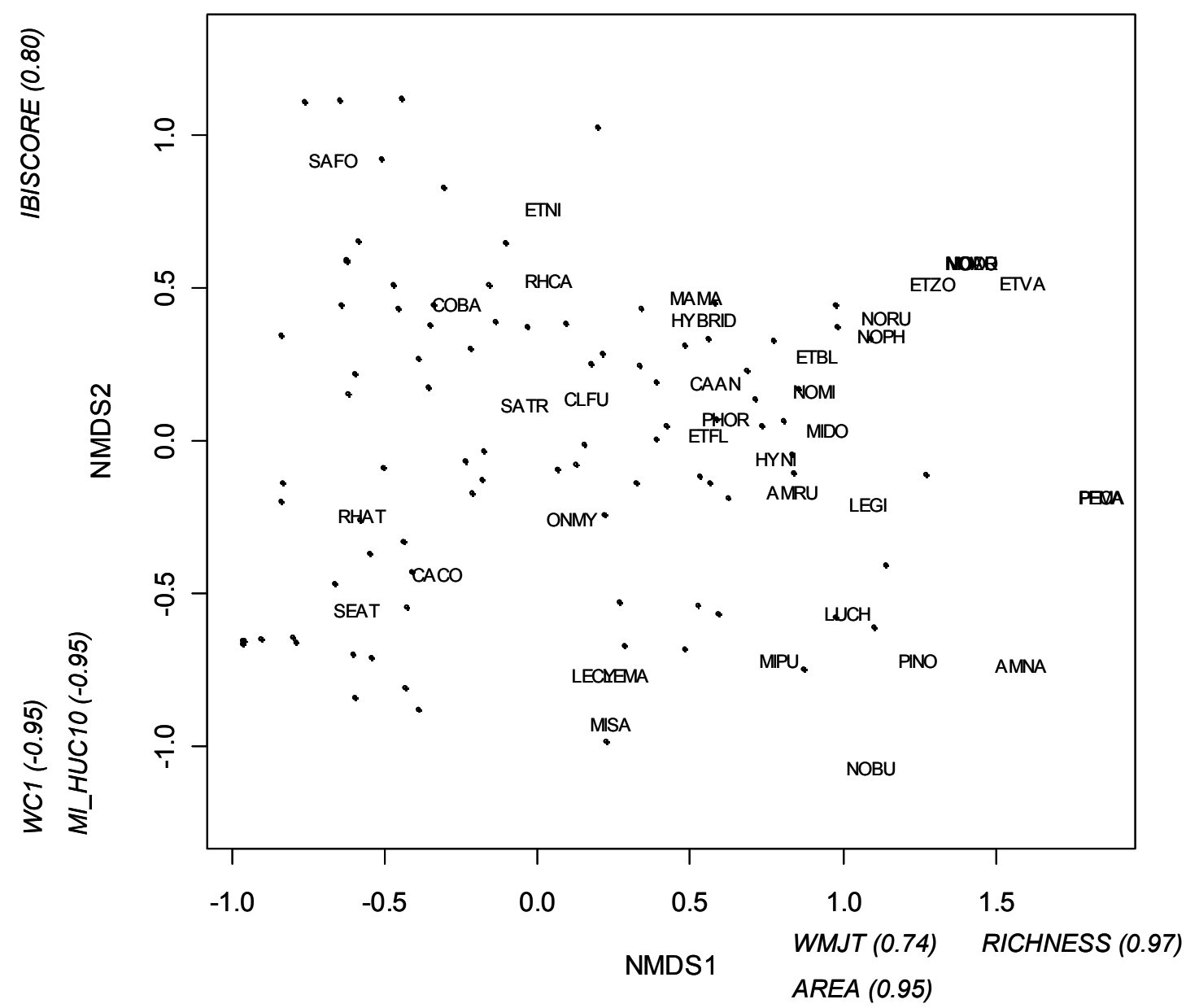


Figure 5. Plot of local water chemistry principal component 1 (WC1) versus probability of presence for two commonly sampled species - longnose dace (Rhinichthys cataractae, top panel) and brook trout (Salvelinus fontinalis, bottom panel) demonstrating a negative relationship between probability of presence and HUC10 mining index based on the binomial GLM results presented in Table 2. The trend lines represent the relationship between probability of presence and $\mathrm{WC} 1$ at mean conditions for other the variables in the final models (see Table 2), while setting HUC10 mining index (HUC10 MI) to the minimum (0), maximum (11.9), and mean (4.2) values for all sites in the dataset.

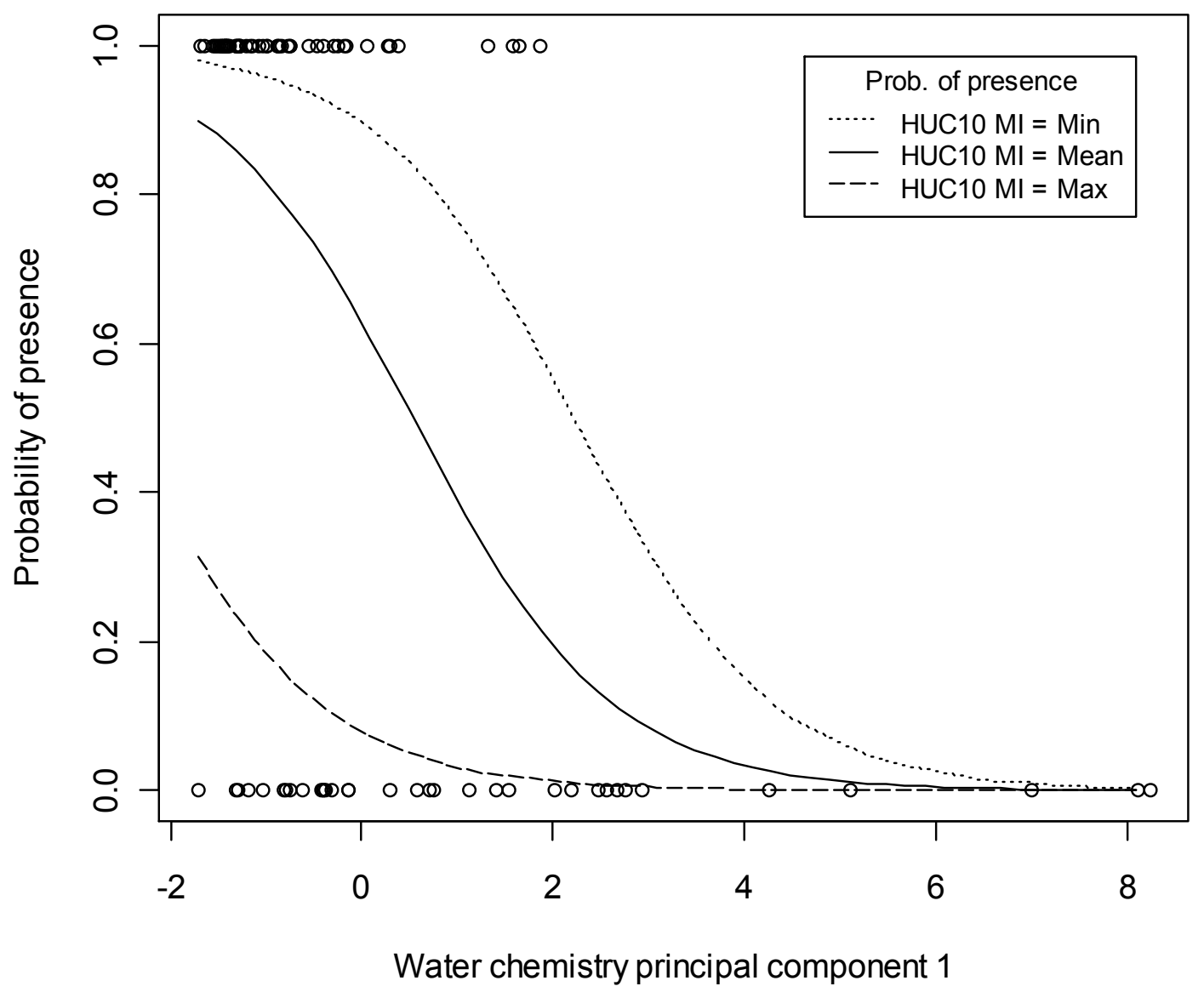




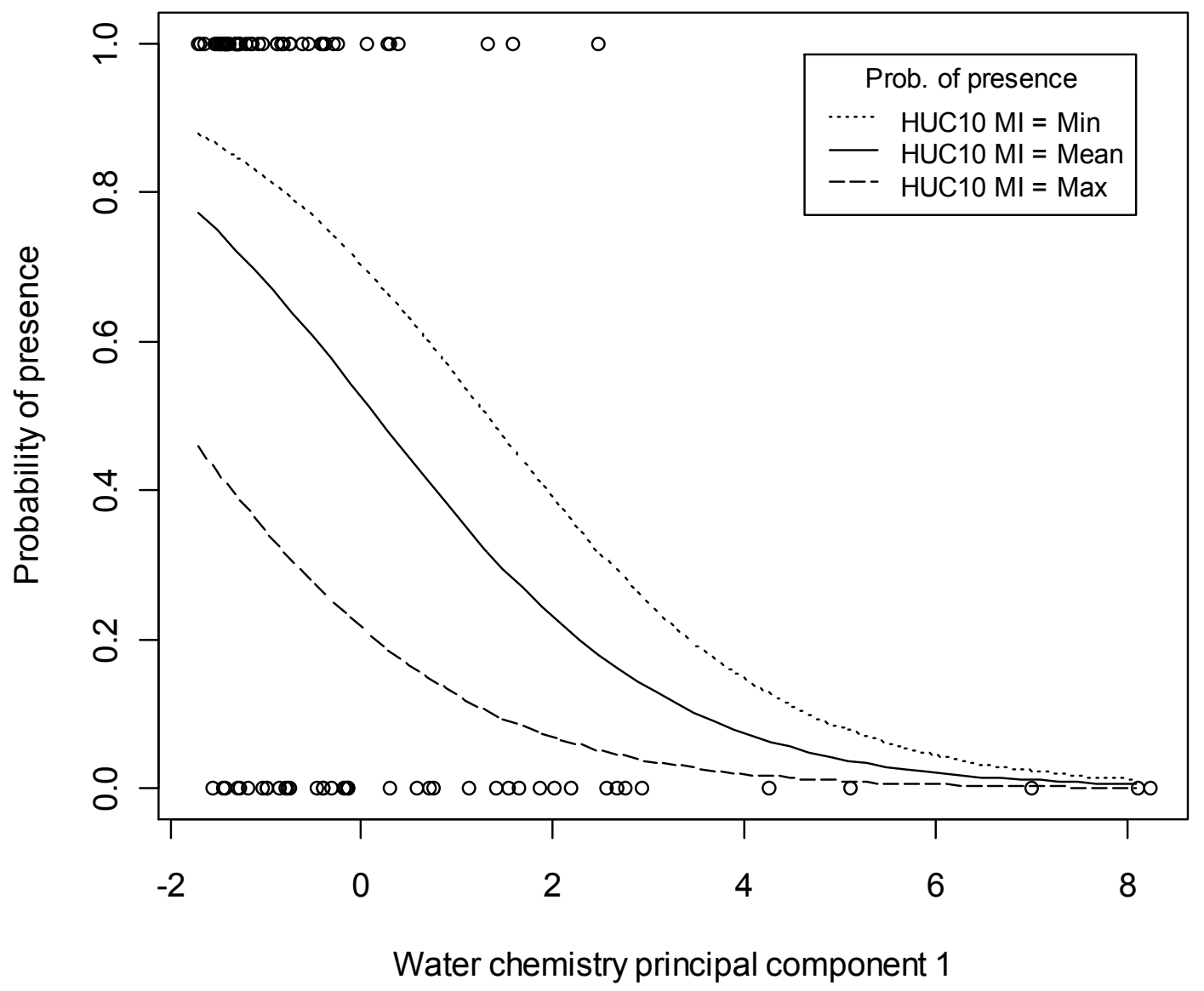


Figure 6. Plot of local rapid visual habitat assessment (RVHA) score (top panel) and local water chemistry principal component 2 score (WC2, bottom panel) versus probability of presence for two commonly sampled species - bluntnose minnow (Pimephales notatus, top) and bluegill sunfish (Lepomis macrochirus, bottom) - that demonstrated a positive relationship between probability of presence and HUC10 mining index in the binomial GLM results presented in Table 2. The trend lines represent the relationship between probability of presence and RVHA score at mean conditions for other the variables in the final model for this species (see Table 2), while setting HUC10 mining index (HUC10 MI) to the minimum (0), maximum (11.9), and mean (4.2) values for all sites in the dataset.

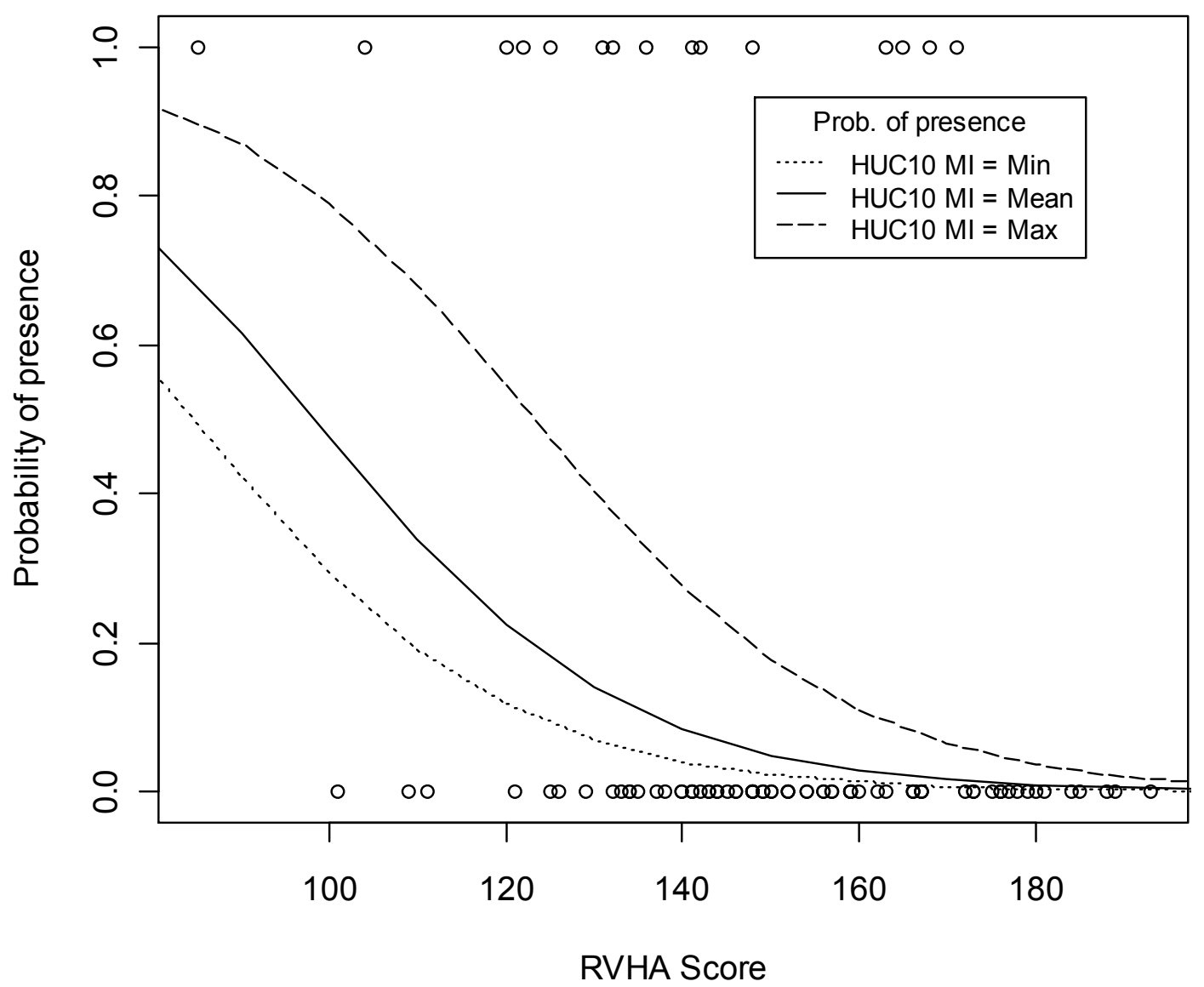




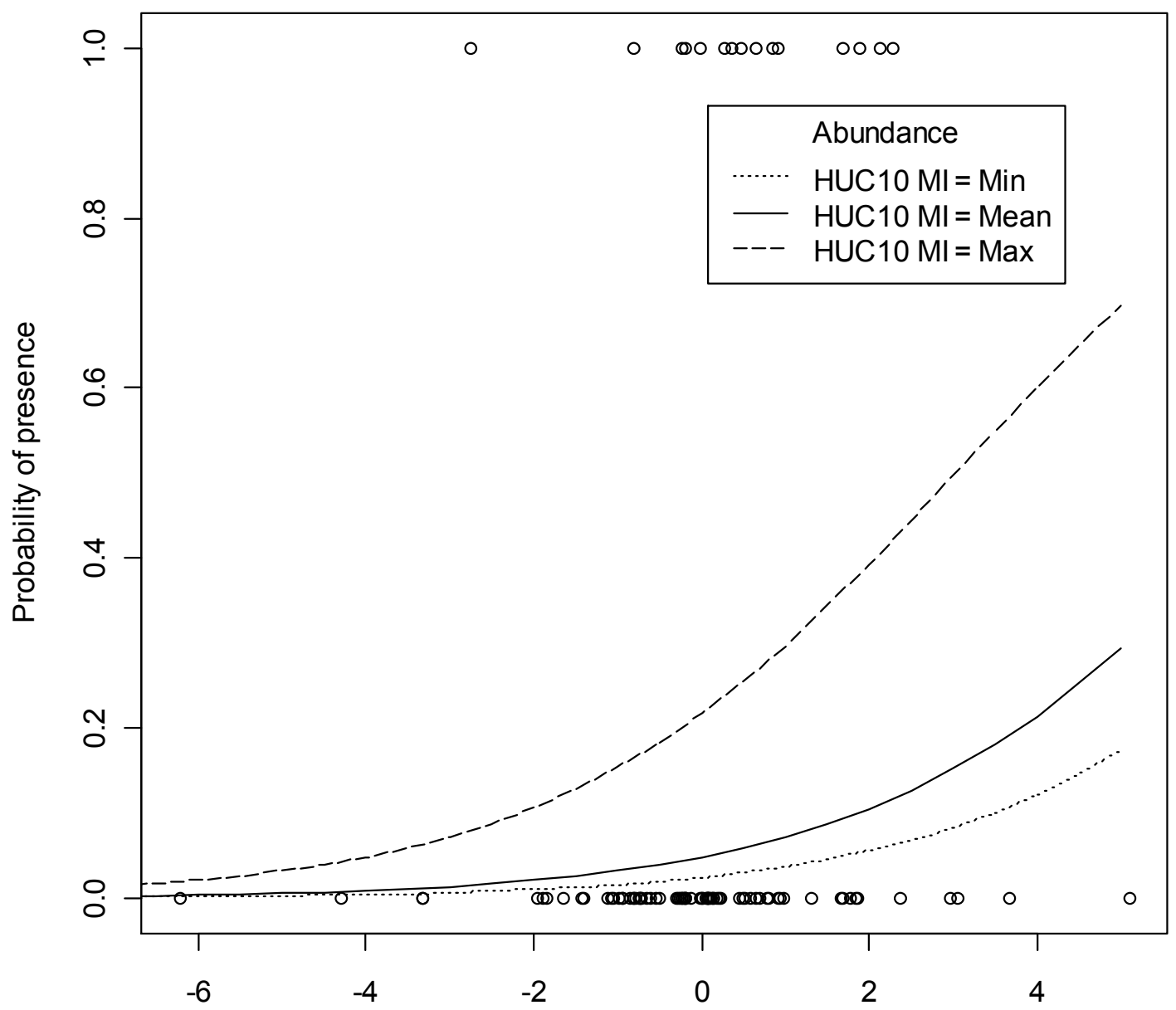

Water chemistry principal component 2 
Figure 7. Plot of local rapid visual habitat assessment (RVHA) score versus abundance for two commonly sampled species - longnose dace (Rhinichthys cataractae, top panel) and rosyside dace (Clinostomus funduloides, bottom panel) - demonstrating a negative relationship between probability of presence and HUC10 mining index based on the negative binomial GLM results presented in Table 3. The trend lines represent the relationship between probability of presence and RVHA at mean conditions for other the variables in the final models (see Table 3), while setting HUC10 mining index (HUC10 $\mathrm{MI}$ ) to the minimum (0), maximum (11.8), and mean (4.2) values for all sites in the dataset.

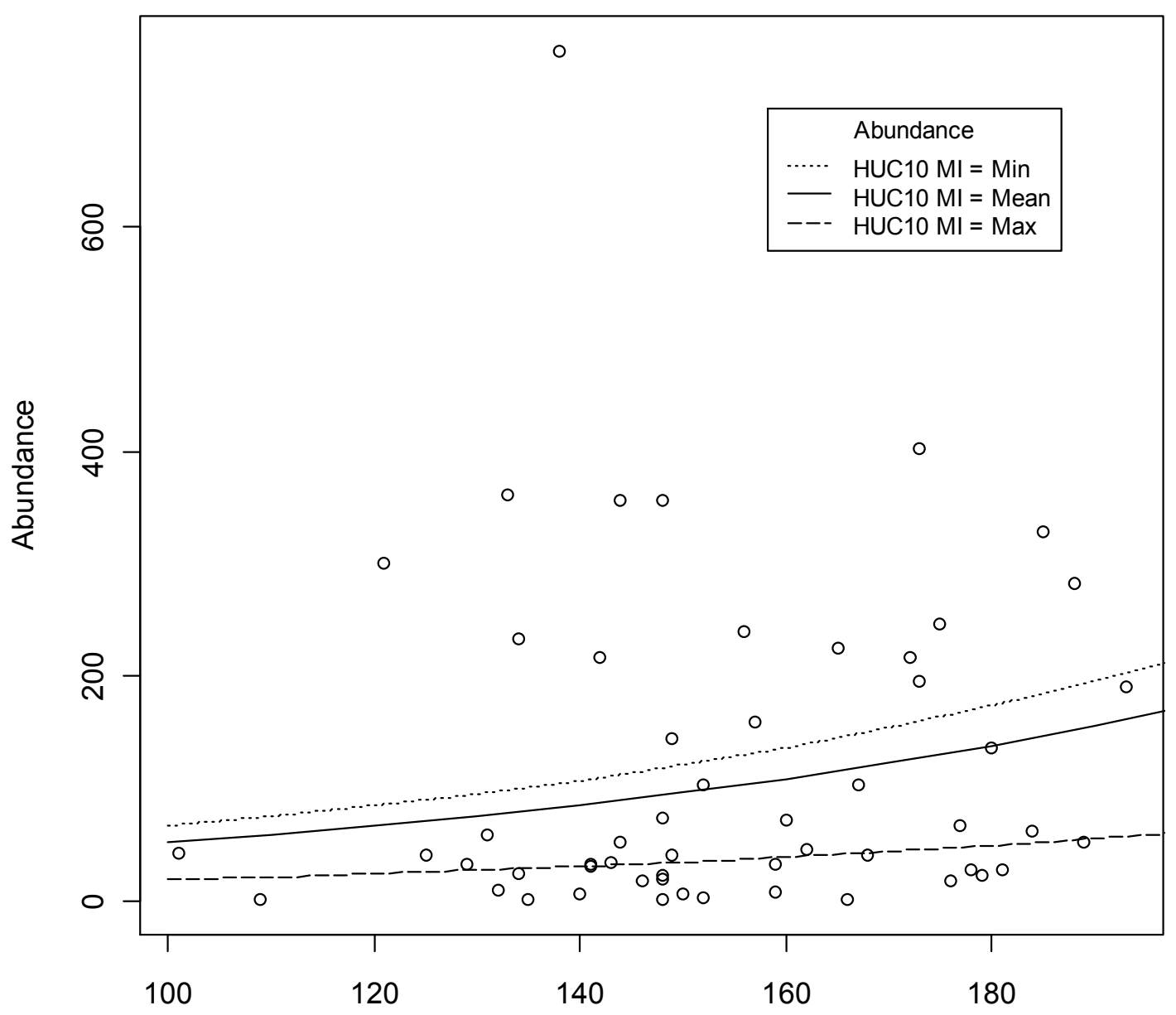

Rapid visual habitat assessment score 


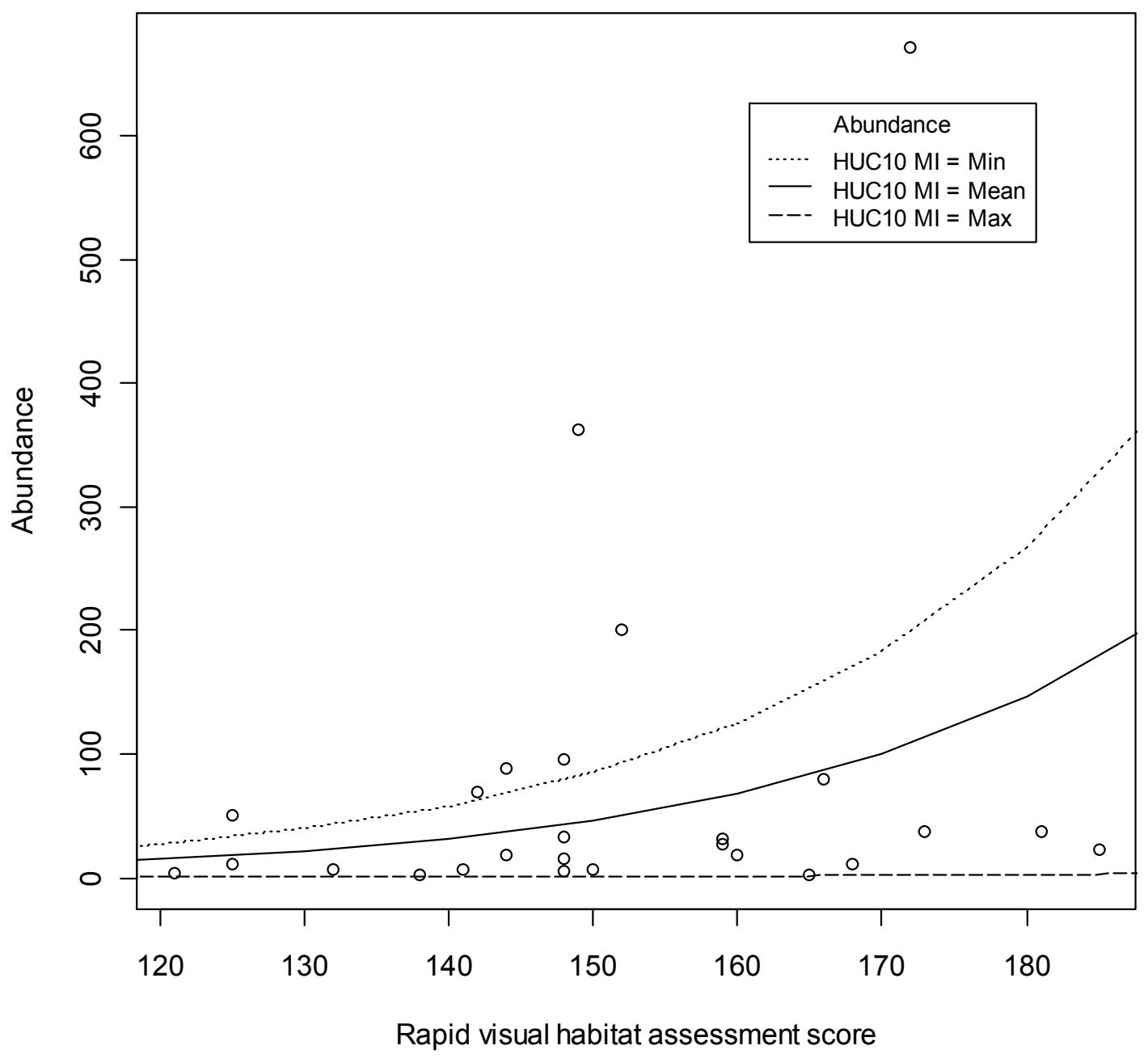


Figure 8. Plot of local water chemistry principal component two (WC2) versus abundance for two commonly sampled species - white sucker (Catostomus commersoni, top panel) and green sunfish (Lepomis cyanellus, bottom panel) - demonstrating a positive relationship between abundance and HUC10 mining index based on the negative binomial GLM results presented in Table 3. The trend lines represent the relationship between probability of presence and $\mathrm{WC} 2$ at mean conditions for other the variables in the final models (see Table 3), while setting HUC10 mining index (HUC10 MI) to the minimum (0), maximum (11.8), and mean (4.2) values for all sites in the dataset.

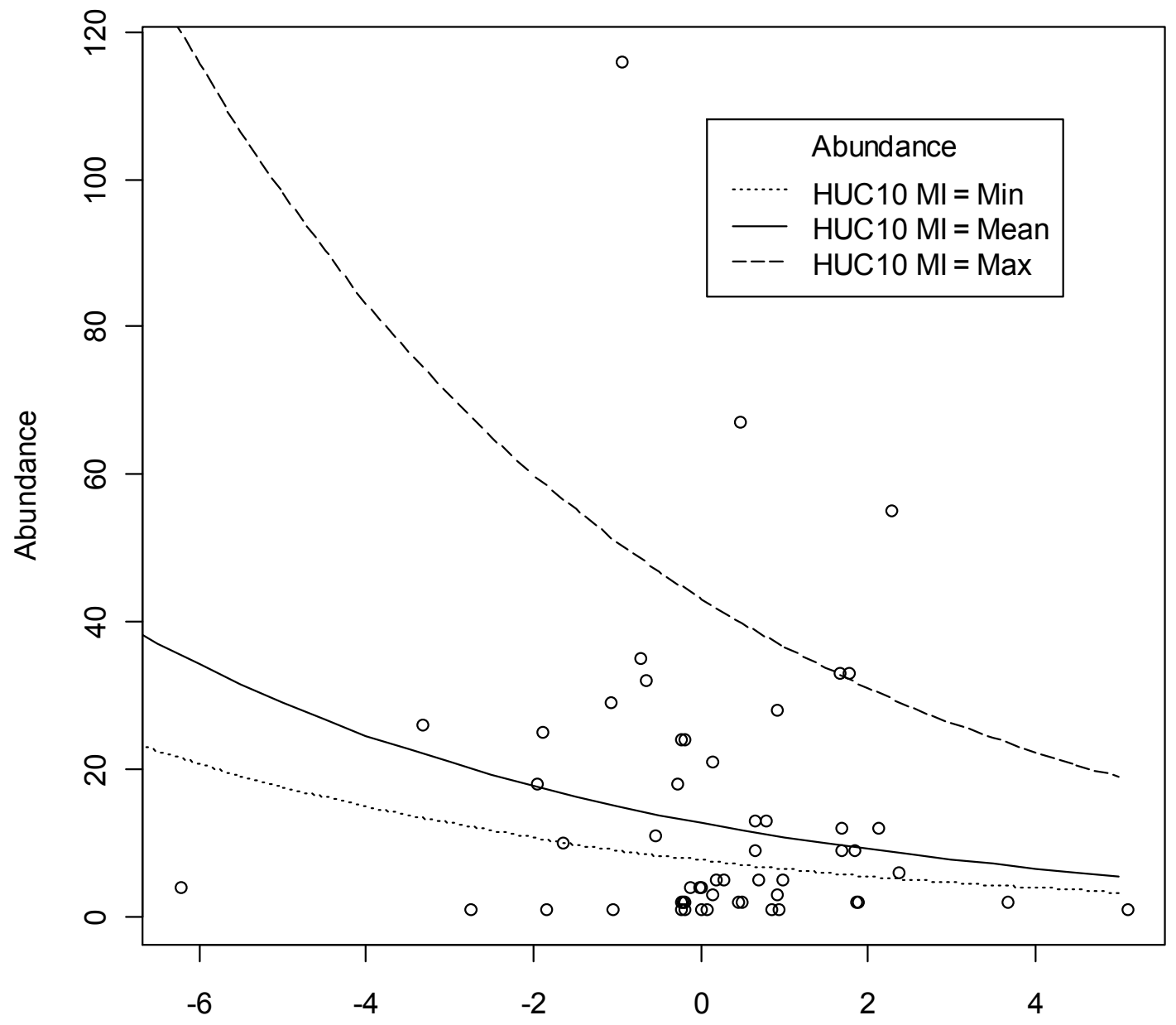

Water chemistry principal component 2 


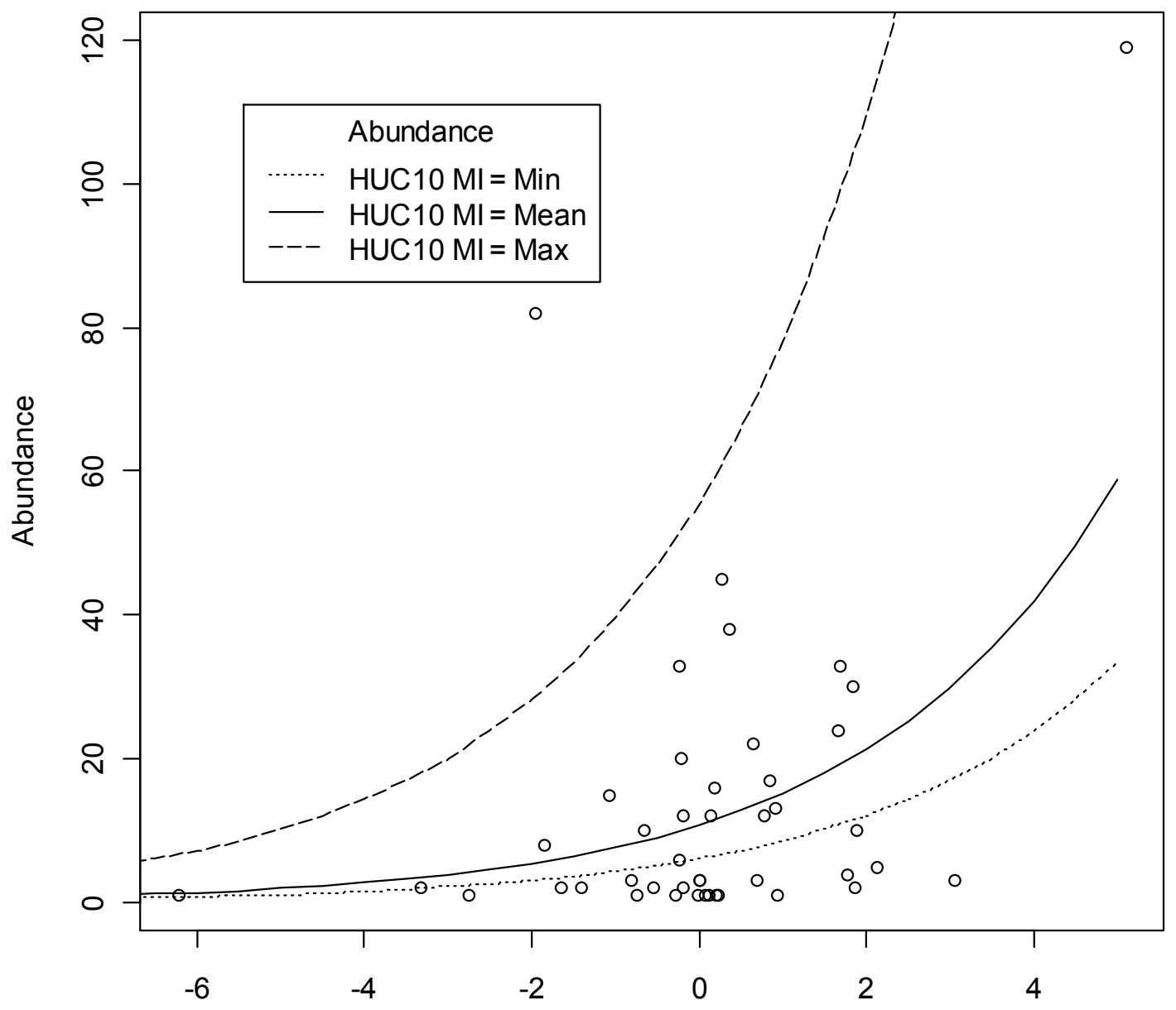

Water chemistry principal component 2 
Figure 9. Stacked bar chart of percent deviance explained by individual predictors for all species in GLM-based presence-absence modeling. AMRU $=$ Ambloplites rupestris, $\mathrm{CAAN}=$ Campostoma anomalum, $\mathrm{COBA}=$ Cottus bairdi, $\mathrm{ETBL}=$ Etheostoma blennioides, $\mathrm{ETFL}=$ Etheostoma flabellare, $\mathrm{HYNI}=$ Hypentelium nigricans, $\mathrm{LECY}=$ Lepomis cyanellus, $\mathrm{MIDO}=$ Micropterus dolomieu, $\mathrm{NOMI}=$ Nocomis micropogon, $\mathrm{PINO}=$ Pimephales notatus, $\mathrm{RHCA}=$ Rhinichthys cataractae, $\mathrm{SAFO}=$ Salvelinus fontinalis, SEAT = Semotilus atromaculatus.

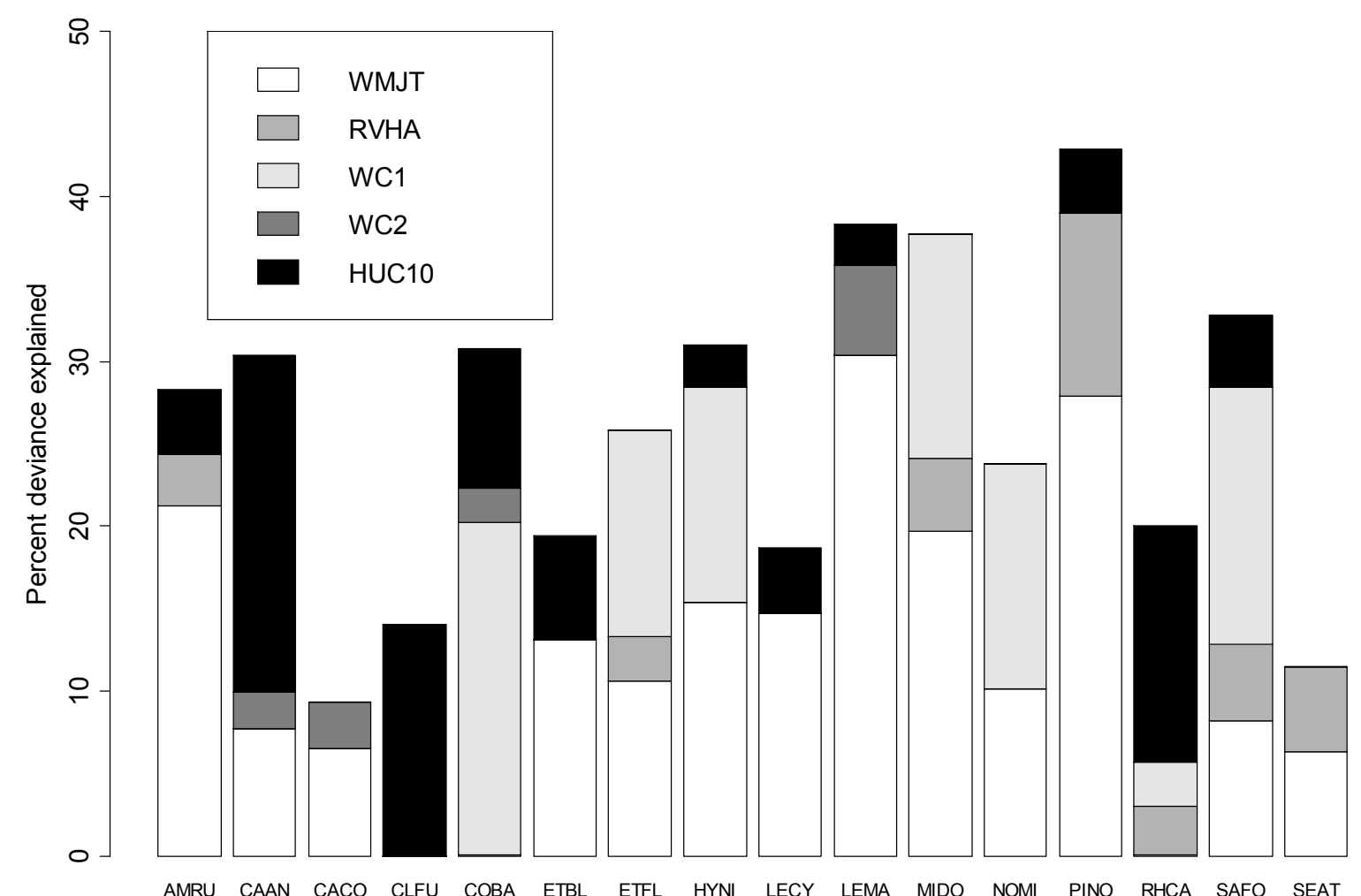


Figure 10. Stacked bar chart of percent deviance explained by individual predictors for all species in GLM-based abundance modeling. AMRU $=$ Ambloplites rupestris, $\mathrm{CAAN}=$ Campostoma anomalum, $\mathrm{COBA}=$ Cottus bairdi, $\mathrm{ETBL}=$ Etheostoma blennioides, $\mathrm{HYNI}=$ Hypentelium nigricans, $\mathrm{LECY}=$ Lepomis cyanellus, $\mathrm{MIDO}=$ Micropterus dolomieu, $\mathrm{NOMI}=$ Nocomis micropogon, $\mathrm{PINO}=$ Pimephales notatus, $\mathrm{RHOB}=$ Rhinichthys obtusus, $\mathrm{RHCA}=$ Rhinichthys cataractae, $\mathrm{SAFO}=$ Salvelinus fontinalis, SEAT = Semotilus atromaculatus.

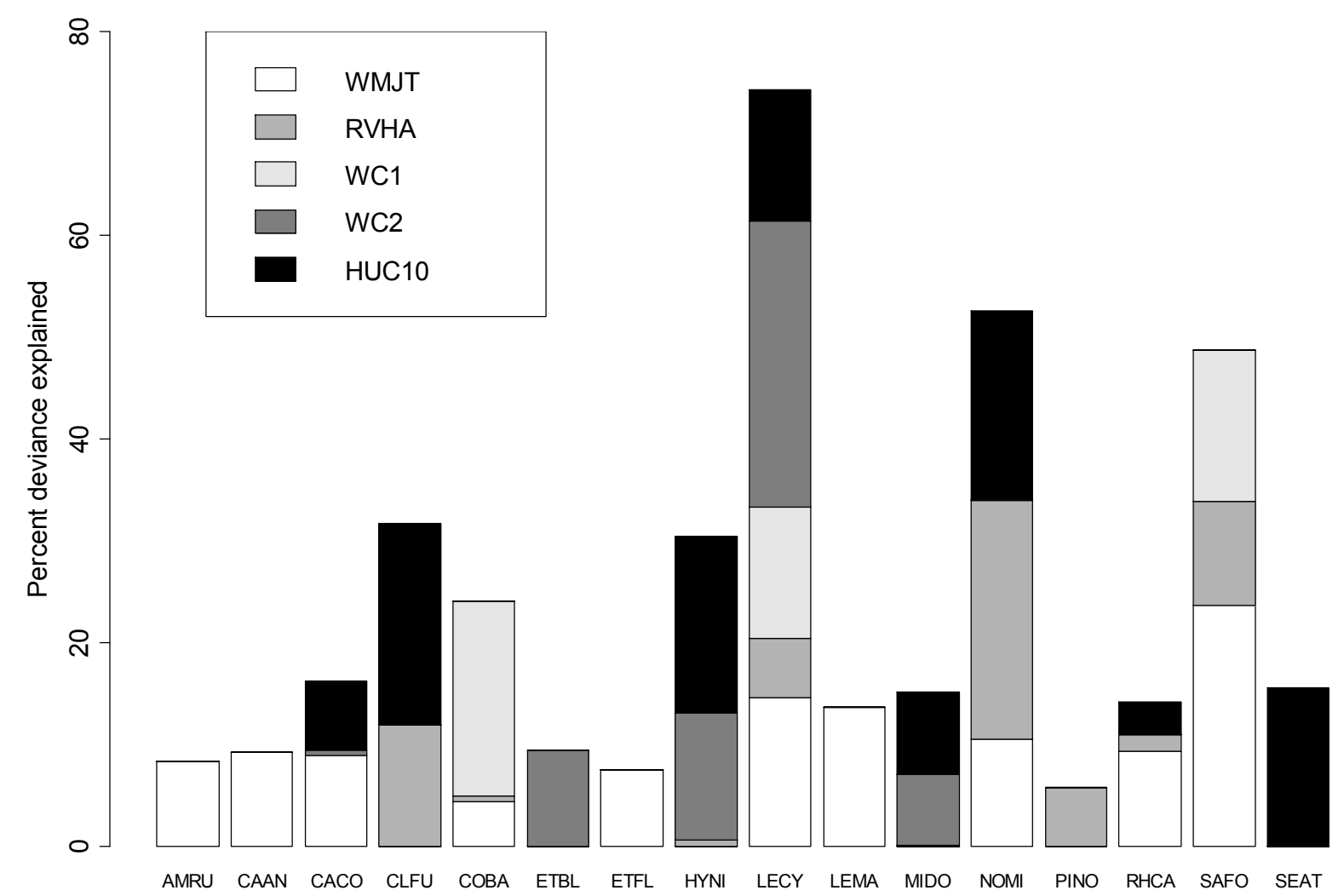


Figure 11. Plot of local water chemistry principal component 1 (WC1, an AMD chemical gradient, refer to table 1) versus index of biotic integrity (IBI) score for all sites sampled. The closed circles indicate sites where the HUC10 mining index was lower than the median value for dataset and open circles indicate sites where the index was higher than the median. The trend lines represent the relationship between IBI score and WC1 at mean conditions for other the variables in the final GLM model (refer to Table 5), while setting HUC10 mining index (HUC10 MI) to the minimum (0), maximum (11.8), and mean (4.2) in the model.

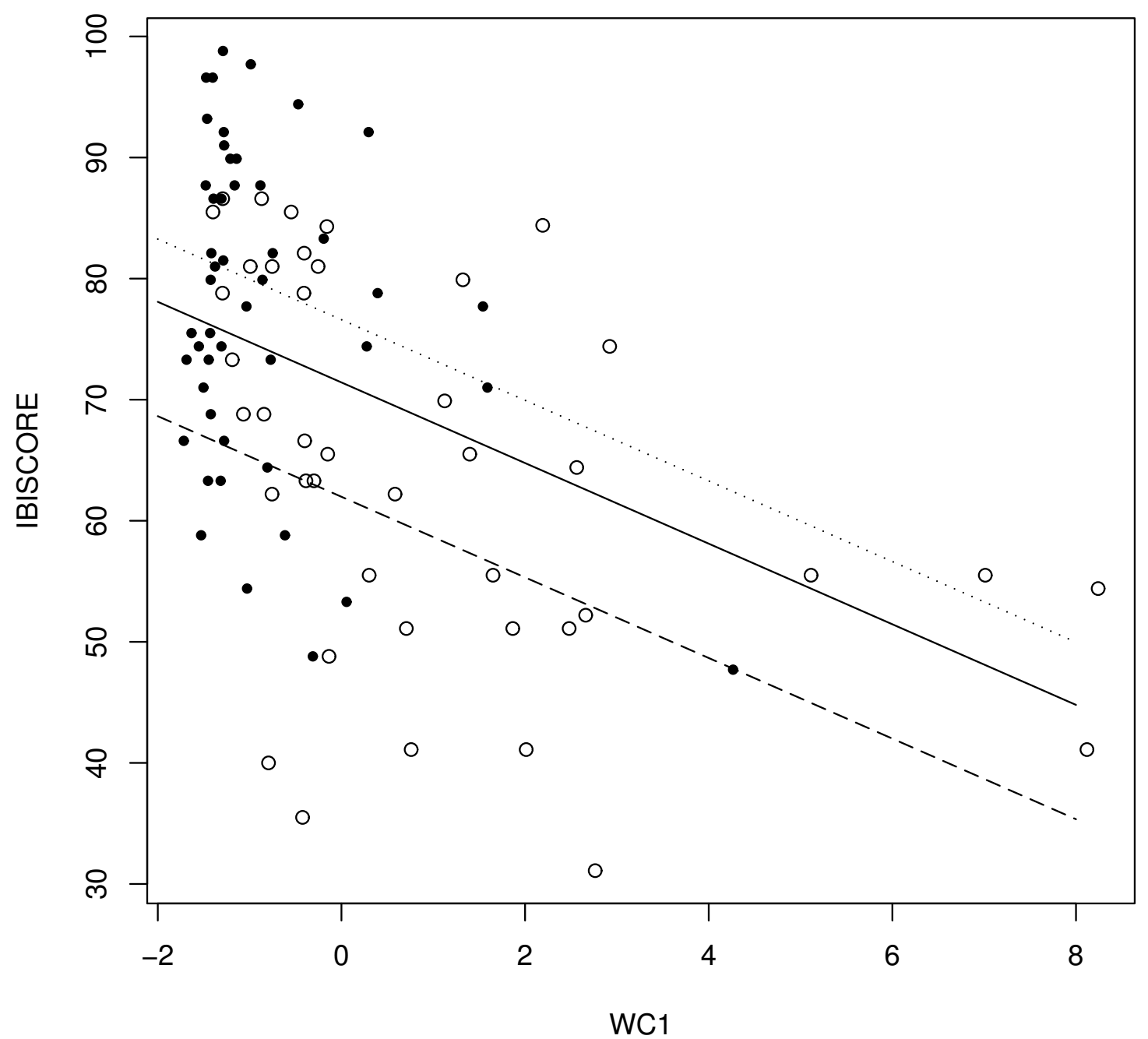


Figure 12. Plot of local water chemistry principal component 1 (WC1) versus site index of biotic integrity (IBI) score. The closed circles indicate sites where the HUC10 mining index was lower than the median value for dataset and open circles indicate sites where the index was higher than the median. The solid trend line is the linear fit of WC1 to the former sites and the dashed is the fit to the latter. Both models were highly significant ( $\mathrm{P}$ $<0.001)$ and the intercepts of the two lines were significantly different $(\mathrm{P}<0.01)$, but the slopes were not $(\mathrm{P}>>0.05)$.

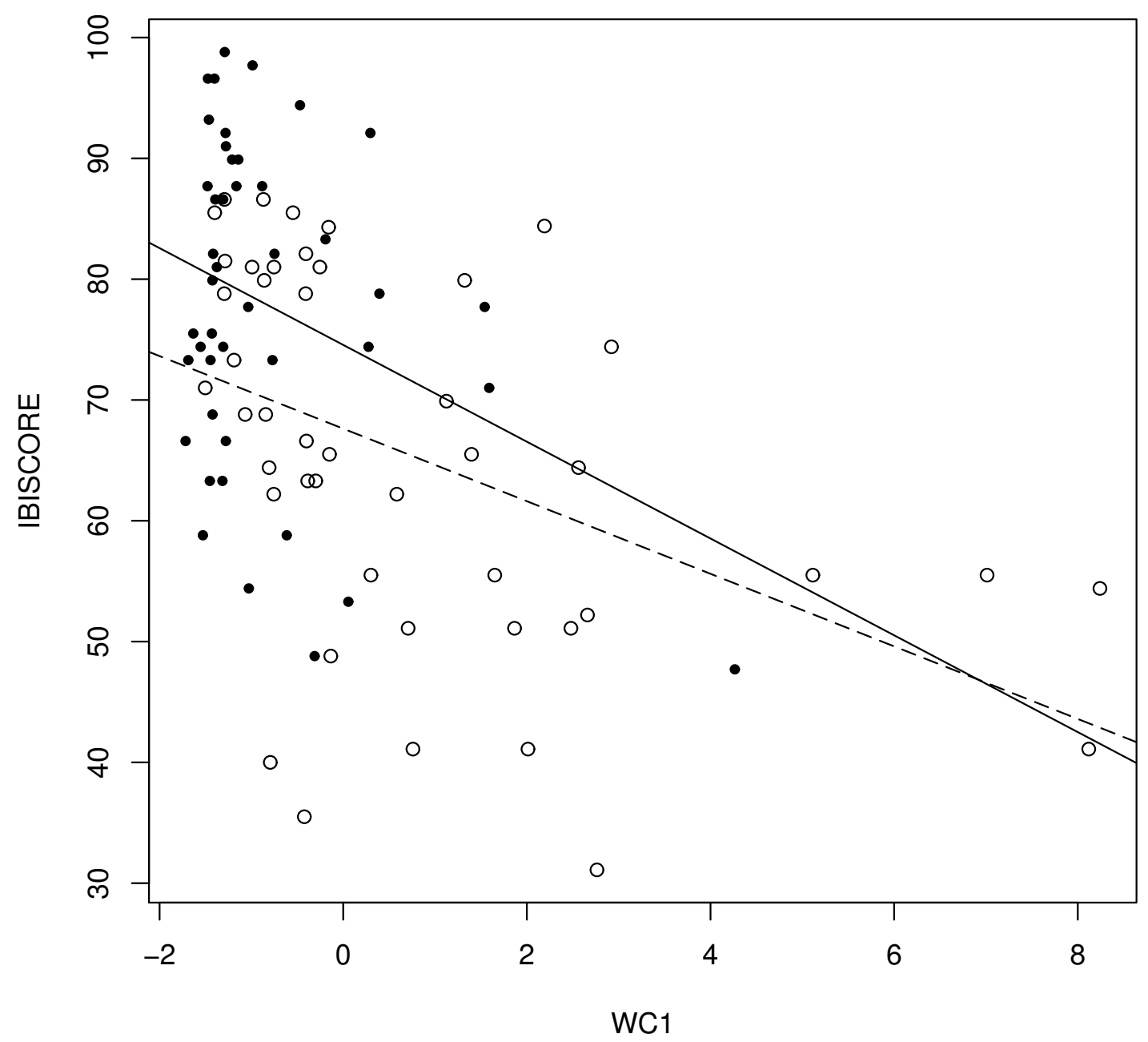


Figure 13. Results of significant zero crossings (SiZer) technique to identify nonlinear trends (i.e., thresholds) in the relationship between HUC10 mining index and site index of biotic integrity (IBI) scores. The dataset was divided into sites with water chemistry principal component 1 scores below (top panel) and above (bottom) the natural break in the data (see Figure 5). The coloring, in the case of the first derivative, indicates whether the function is significantly increasing (blue), decreasing (red), not changing (purple), or whether there is insufficient data (gray). The second derivative panel indicates whether the function is concave up (blue), down (red), not changing (purple), or insufficient data (gray). The $\mathrm{x}$-axis is a logarithmic transformation of the bandwidth parameter $(h)$, which is tuning parameter based on the distance of a particular from the mean value and is, in this case, the standard deviation (i.e., $\log (h)=0$ for normally distributed data). The white lines give a visual representation of the size of the bandwidth, such that the horizontal distance between the lines is $2 h$. This distance visually informs the selection of smoothing parameters in non-parametric regression analyses where high values of $h$ tend toward an over-smoothing of the function and low values tend toward over-fitting.

First derivative

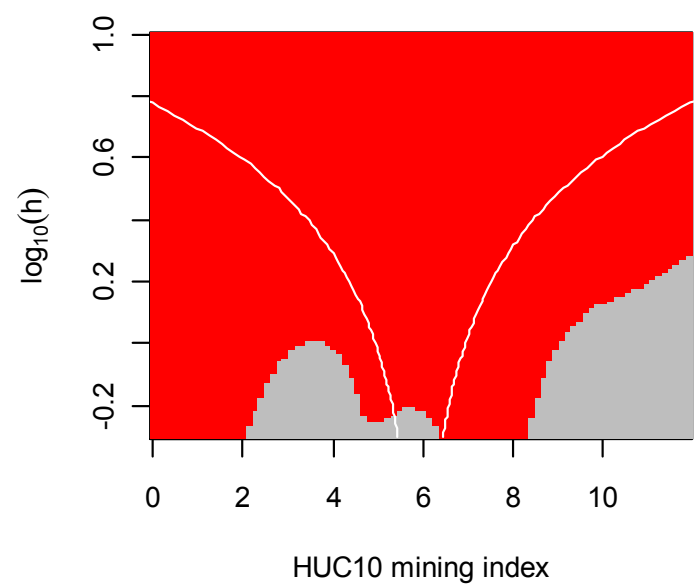

First derivative

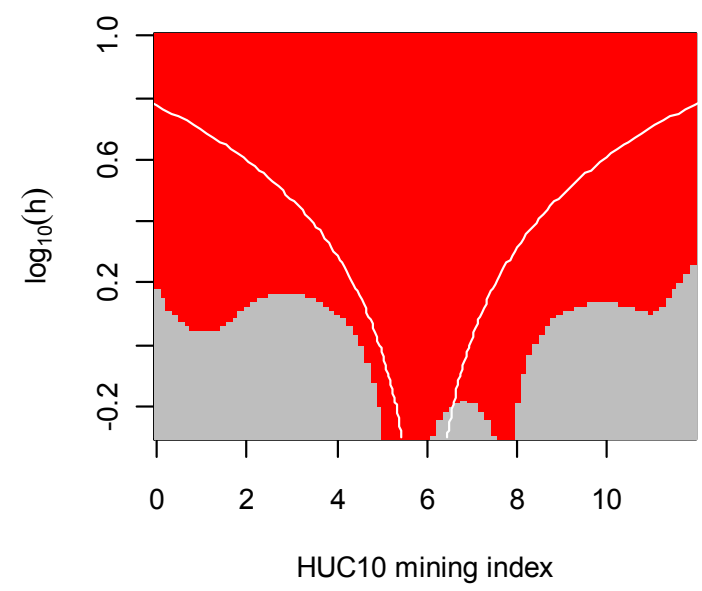

Second derivative

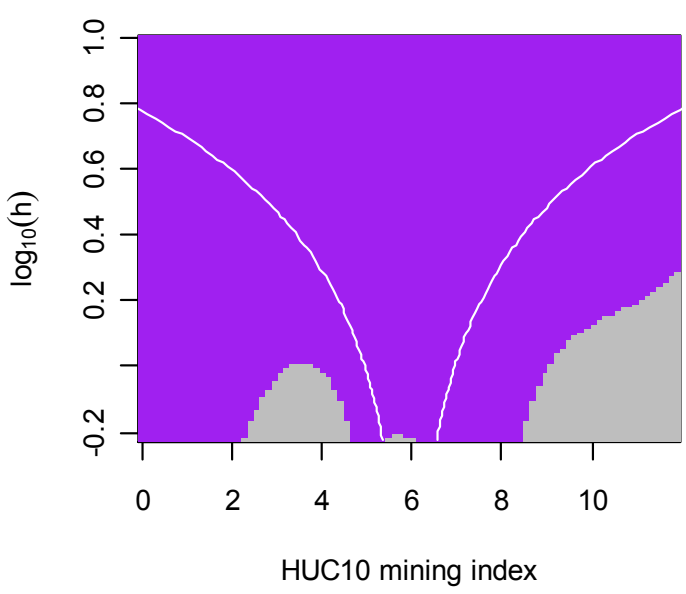

Second derivative

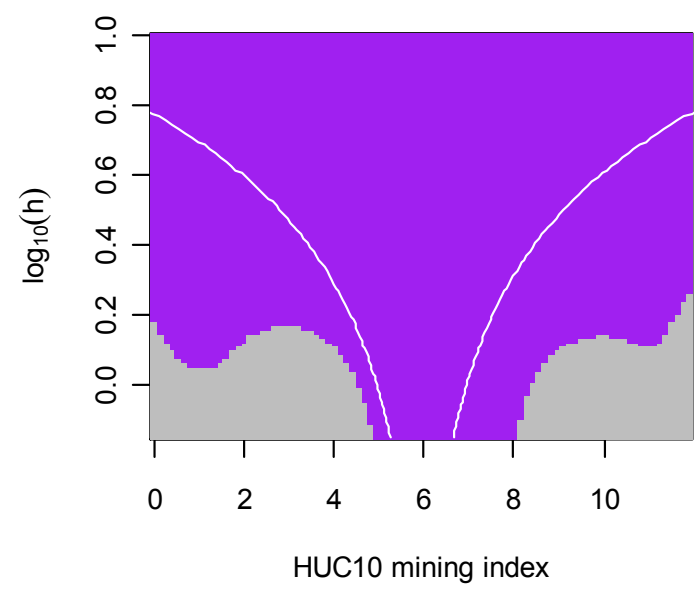




\section{APPENDICES}

\section{Appendix A: Summary of model-averaged capture efficiency model coefficients and estimates}

A1. Summary of logit-scale, model-averaged coefficient estimates, standard errors, and $95 \%$ confidence intervals for predicting capture probability of brook trout. SE $=$ standard error, $\mathrm{LCI}=$ lower $95 \%$ confidence limit, $\mathrm{UCI}=$ upper $95 \%$ confidence limit, $\Sigma \mathrm{wi}=$ sum of Akaike weights for term. The cumulative Akaike weights for each model term are indicated next to the relevant effect. For instance, the cumulative weight for the "age(2)" term (see Table 5 for description) is indicated next to the "Age(YOY)" model effect, while the cumulative weight for the "age" term is indicated next to the "Age" effect. The cumulative Akaike weight for the "date" term is indicated next to the first date effect. The cumulative Akaike weight for the "site" term is indicated next to the first site effect. Astericks (*) indicate where model effect confidence limits did not include 0 (i.e., a "significant" positive or negative slope).

\begin{tabular}{lcccccc}
\multicolumn{1}{c}{ Coefficient } & Estimate & SE & LCl & UCI & & $\mathbf{E w}_{\mathbf{i}}$ \\
\hline Intercept & 0.627 & 0.302 & 0.034 & 1.219 & & \\
Age (YOY) & -0.106 & 0.157 & -0.414 & 0.201 & & 0.447 \\
Age (SAD) & 0.162 & 0.169 & -0.168 & 0.492 & & 0.270 \\
Site (NB) & 0.614 & 0.293 & 0.040 & 1.188 & $*$ & 0.957 \\
Site (OH) & 0.705 & 0.273 & 0.170 & 1.240 & $*$ & \\
Site (BR) & 0.643 & 0.282 & 0.090 & 1.196 & $*$ & \\
Site (SR) & 0.702 & 0.272 & 0.168 & 1.236 & $*$ & \\
Site (LR) & 0.732 & 0.308 & 0.129 & 1.335 & $*$ & \\
Site (LD) & 0.252 & 0.302 & -0.340 & 0.845 & & \\
Site (MR) & 0.847 & 0.398 & 0.066 & 1.628 & $*$ & \\
Site (TR) & 0.503 & 0.410 & -0.300 & 1.305 & & \\
Site (ZH) & 0.432 & 0.341 & -0.235 & 1.100 & & \\
Site (LL) & -0.292 & 0.448 & -1.170 & 0.586 & & \\
Site (GF) & 1.284 & 0.351 & 0.596 & 1.972 & $*$ & \\
Date (Jul-06) & -0.305 & 0.197 & -0.691 & 0.081 & & \multirow{2}{*}{1.000} \\
Date (Aug-06) & 0.210 & 0.214 & -0.210 & 0.630 & & \\
Date (Oct-06) & -0.868 & 0.192 & -1.245 & -0.491 & $*$ & \\
Date (May-07) & -0.955 & 0.178 & -1.304 & -0.605 & & \\
Date (Jul-07) & -0.030 & 0.179 & -0.380 & 0.320 & &
\end{tabular}


A2. Summary of model-averaged capture efficiency parameter estimates, standard errors, and $95 \%$ confidence intervals (i.e., lower $=$ LCI and upper $=$ UCI) for all sites, sampling occasions, and age classes derived from Huggins' closed capture models for brook trout.

\begin{tabular}{|c|c|c|c|c|c|c|}
\hline Site & Date & Age & Estimate & SE & $\mathrm{LCI}$ & $\mathrm{UCl}$ \\
\hline Nan's Branch & Jul-06 & YOY & 0.702 & 0.048 & 0.601 & 0.787 \\
\hline Nan's Branch & Jul-06 & Small Adult & 0.735 & 0.043 & 0.643 & 0.811 \\
\hline Nan's Branch & Jul-06 & Large Adult & 0.726 & 0.044 & 0.633 & 0.803 \\
\hline Nan's Branch & Aug-06 & YOY & 0.797 & 0.040 & 0.707 & 0.865 \\
\hline Nan's Branch & Aug-06 & Small Adult & 0.822 & 0.037 & 0.738 & 0.884 \\
\hline Nan's Branch & Aug-06 & Large Adult & 0.816 & 0.039 & 0.728 & 0.880 \\
\hline Nan's Branch & Oct-06 & YOY & 0.575 & 0.056 & 0.464 & 0.679 \\
\hline Nan's Branch & Oct-06 & Small Adult & 0.614 & 0.055 & 0.502 & 0.715 \\
\hline Nan's Branch & Oct-06 & Large Adult & 0.603 & 0.057 & 0.488 & 0.708 \\
\hline Nan's Branch & May-07 & YOY & 0.553 & 0.055 & 0.444 & 0.656 \\
\hline Nan's Branch & May-07 & Small Adult & 0.592 & 0.055 & 0.481 & 0.695 \\
\hline Nan's Branch & May-07 & Large Adult & 0.581 & 0.056 & 0.469 & 0.686 \\
\hline Nan's Branch & Jul-07 & YOY & 0.755 & 0.040 & 0.668 & 0.825 \\
\hline Nan's Branch & Jul-07 & Small Adult & 0.784 & 0.040 & 0.695 & 0.852 \\
\hline Nan's Branch & Jul-07 & Large Adult & 0.776 & 0.041 & 0.685 & 0.846 \\
\hline Nan's Branch & Oct-07 & YOY & 0.759 & 0.037 & 0.680 & 0.824 \\
\hline Nan's Branch & Oct-07 & Small Adult & 0.788 & 0.038 & 0.703 & 0.853 \\
\hline Nan's Branch & Oct-07 & Large Adult & 0.780 & 0.040 & 0.693 & 0.848 \\
\hline Otis Hollow & Jul-06 & YOY & 0.722 & 0.042 & 0.632 & 0.796 \\
\hline Otis Hollow & Jul-06 & Small Adult & 0.753 & 0.037 & 0.674 & 0.819 \\
\hline Otis Hollow & Jul-06 & Large Adult & 0.745 & 0.038 & 0.662 & 0.813 \\
\hline Otis Hollow & Aug-06 & YOY & 0.812 & 0.036 & 0.731 & 0.873 \\
\hline Otis Hollow & Aug-06 & Small Adult & 0.836 & 0.033 & 0.760 & 0.891 \\
\hline Otis Hollow & Aug-06 & Large Adult & 0.829 & 0.035 & 0.749 & 0.888 \\
\hline Otis Hollow & Oct-06 & YOY & 0.598 & 0.047 & 0.503 & 0.685 \\
\hline Otis Hollow & Oct-06 & Small Adult & 0.636 & 0.046 & 0.542 & 0.721 \\
\hline Otis Hollow & Oct-06 & Large Adult & 0.626 & 0.049 & 0.526 & 0.716 \\
\hline Otis Hollow & May-07 & YOY & 0.576 & 0.044 & 0.488 & 0.659 \\
\hline Otis Hollow & May-07 & Small Adult & 0.615 & 0.045 & 0.525 & 0.698 \\
\hline Otis Hollow & May-07 & Large Adult & 0.604 & 0.047 & 0.509 & 0.692 \\
\hline Otis Hollow & Jul-07 & YOY & 0.772 & 0.032 & 0.704 & 0.828 \\
\hline Otis Hollow & Jul-07 & Small Adult & 0.799 & 0.032 & 0.729 & 0.855 \\
\hline Otis Hollow & Jul-07 & Large Adult & 0.792 & 0.034 & 0.718 & 0.851 \\
\hline Otis Hollow & Oct-07 & YOY & 0.776 & 0.030 & 0.711 & 0.830 \\
\hline Otis Hollow & Oct-07 & Small Adult & 0.803 & 0.032 & 0.732 & 0.859 \\
\hline Otis Hollow & Oct-07 & Large Adult & 0.796 & 0.034 & 0.720 & 0.855 \\
\hline Big Run & Jul-06 & YOY & 0.708 & 0.050 & 0.601 & 0.796 \\
\hline Big Run & Jul-06 & Small Adult & 0.741 & 0.043 & 0.649 & 0.815 \\
\hline Big Run & Jul-06 & Large Adult & 0.732 & 0.043 & 0.641 & 0.807 \\
\hline Big Run & Aug-06 & YOY & 0.802 & 0.038 & 0.716 & 0.866 \\
\hline Big Run & Aug-06 & Small Adult & 0.826 & 0.033 & 0.751 & 0.883 \\
\hline Big Run & Aug-06 & Large Adult & 0.820 & 0.035 & 0.742 & 0.878 \\
\hline Big Run & Oct-06 & YOY & 0.582 & 0.054 & 0.473 & 0.682 \\
\hline
\end{tabular}




\begin{tabular}{|c|c|c|c|c|c|c|}
\hline Big Run & Oct-06 & Small Adult & 0.621 & 0.050 & 0.519 & 0.713 \\
\hline Big Run & Oct-06 & Large Adult & 0.610 & 0.051 & 0.506 & 0.705 \\
\hline Big Run & May-07 & YOY & 0.559 & 0.051 & 0.459 & 0.655 \\
\hline Big Run & May-07 & Small Adult & 0.599 & 0.048 & 0.502 & 0.689 \\
\hline Big Run & May-07 & Large Adult & 0.588 & 0.048 & 0.491 & 0.678 \\
\hline Big Run & Jul-07 & YOY & 0.760 & 0.040 & 0.674 & 0.829 \\
\hline Big Run & Jul-07 & Small Adult & 0.788 & 0.037 & 0.706 & 0.852 \\
\hline Big Run & Jul-07 & Large Adult & 0.781 & 0.038 & 0.698 & 0.846 \\
\hline Big Run & Oct-07 & YOY & 0.764 & 0.035 & 0.689 & 0.826 \\
\hline Big Run & Oct-07 & Small Adult & 0.792 & 0.034 & 0.717 & 0.852 \\
\hline Big Run & Oct-07 & Large Adult & 0.785 & 0.035 & 0.709 & 0.845 \\
\hline Swallow Rock Run & Jul-06 & YOY & 0.719 & 0.043 & 0.627 & 0.796 \\
\hline Swallow Rock Run & Jul-06 & Small Adult & 0.751 & 0.038 & 0.668 & 0.819 \\
\hline Swallow Rock Run & Jul-06 & Large Adult & 0.742 & 0.039 & 0.659 & 0.811 \\
\hline Swallow Rock Run & Aug-06 & YOY & 0.810 & 0.035 & 0.732 & 0.870 \\
\hline Swallow Rock Run & Aug-06 & Small Adult & 0.834 & 0.032 & 0.761 & 0.888 \\
\hline Swallow Rock Run & Aug-06 & Large Adult & 0.828 & 0.034 & 0.751 & 0.884 \\
\hline Swallow Rock Run & Oct-06 & YOY & 0.595 & 0.050 & 0.495 & 0.688 \\
\hline Swallow Rock Run & Oct-06 & Small Adult & 0.633 & 0.049 & 0.534 & 0.723 \\
\hline Swallow Rock Run & Oct-06 & Large Adult & 0.623 & 0.051 & 0.520 & 0.716 \\
\hline Swallow Rock Run & May-07 & YOY & 0.573 & 0.049 & 0.476 & 0.665 \\
\hline Swallow Rock Run & May-07 & Small Adult & 0.612 & 0.049 & 0.513 & 0.703 \\
\hline Swallow Rock Run & May-07 & Large Adult & 0.601 & 0.050 & 0.500 & 0.694 \\
\hline Swallow Rock Run & Jul-07 & YOY & 0.770 & 0.032 & 0.701 & 0.827 \\
\hline Swallow Rock Run & Jul-07 & Small Adult & 0.797 & 0.033 & 0.725 & 0.854 \\
\hline Swallow Rock Run & Jul-07 & Large Adult & 0.790 & 0.034 & 0.716 & 0.848 \\
\hline Swallow Rock Run & Oct-07 & YOY & 0.774 & 0.032 & 0.706 & 0.830 \\
\hline Swallow Rock Run & Oct-07 & Small Adult & 0.801 & 0.034 & 0.727 & 0.859 \\
\hline Swallow Rock Run & Oct-07 & Large Adult & 0.794 & 0.035 & 0.717 & 0.854 \\
\hline Lynn Run & Jul-06 & YOY & 0.725 & 0.050 & 0.618 & 0.811 \\
\hline Lynn Run & Jul-06 & Small Adult & 0.756 & 0.046 & 0.655 & 0.835 \\
\hline Lynn Run & Jul-06 & Large Adult & 0.747 & 0.047 & 0.645 & 0.828 \\
\hline Lynn Run & Aug-06 & YOY & 0.814 & 0.041 & 0.722 & 0.881 \\
\hline Lynn Run & Aug-06 & Small Adult & 0.838 & 0.038 & 0.748 & 0.899 \\
\hline Lynn Run & Aug-06 & Large Adult & 0.831 & 0.040 & 0.738 & 0.896 \\
\hline Lynn Run & Oct-06 & YOY & 0.601 & 0.060 & 0.480 & 0.711 \\
\hline Lynn Run & Oct-06 & Small Adult & 0.640 & 0.060 & 0.515 & 0.748 \\
\hline Lynn Run & Oct-06 & Large Adult & 0.629 & 0.063 & 0.501 & 0.741 \\
\hline Lynn Run & May-07 & YOY & 0.579 & 0.054 & 0.471 & 0.681 \\
\hline Lynn Run & May-07 & Small Adult & 0.618 & 0.057 & 0.503 & 0.722 \\
\hline Lynn Run & May-07 & Large Adult & 0.608 & 0.058 & 0.490 & 0.714 \\
\hline Lynn Run & Jul-07 & YOY & 0.775 & 0.040 & 0.686 & 0.844 \\
\hline Lynn Run & Jul-07 & Small Adult & 0.801 & 0.041 & 0.709 & 0.870 \\
\hline Lynn Run & Jul-07 & Large Adult & 0.794 & 0.042 & 0.699 & 0.865 \\
\hline Lynn Run & Oct-07 & YOY & 0.779 & 0.037 & 0.697 & 0.843 \\
\hline Lynn Run & Oct-07 & Small Adult & 0.805 & 0.039 & 0.716 & 0.871 \\
\hline Lynn Run & Oct-07 & Large Adult & 0.798 & 0.041 & 0.706 & 0.867 \\
\hline Lick Drain & Jul-06 & YOY & 0.623 & 0.059 & 0.503 & 0.730 \\
\hline Lick Drain & Jul-06 & Small Adult & 0.661 & 0.054 & 0.549 & 0.757 \\
\hline Lick Drain & Jul-06 & Large Adult & 0.650 & 0.052 & 0.543 & 0.745 \\
\hline
\end{tabular}




\begin{tabular}{|c|c|c|c|c|c|c|}
\hline Lick Drain & Aug-06 & YOY & 0.734 & 0.052 & 0.620 & 0.823 \\
\hline Lick Drain & Aug-06 & Small Adult & 0.764 & 0.048 & 0.658 & 0.846 \\
\hline Lick Drain & Aug-06 & Large Adult & 0.756 & 0.048 & 0.650 & 0.839 \\
\hline Lick Drain & Oct-06 & YOY & 0.487 & 0.064 & 0.364 & 0.611 \\
\hline Lick Drain & Oct-06 & Small Adult & 0.528 & 0.064 & 0.403 & 0.649 \\
\hline Lick Drain & Oct-06 & Large Adult & 0.516 & 0.063 & 0.394 & 0.637 \\
\hline Lick Drain & May-07 & YOY & 0.464 & 0.063 & 0.346 & 0.587 \\
\hline Lick Drain & May-07 & Small Adult & 0.505 & 0.064 & 0.382 & 0.627 \\
\hline Lick Drain & May-07 & Large Adult & 0.493 & 0.062 & 0.374 & 0.613 \\
\hline Lick Drain & Jul-07 & YOY & 0.683 & 0.051 & 0.576 & 0.774 \\
\hline Lick Drain & Jul-07 & Small Adult & 0.717 & 0.051 & 0.609 & 0.805 \\
\hline Lick Drain & Jul-07 & Large Adult & 0.708 & 0.050 & 0.602 & 0.796 \\
\hline Lick Drain & Oct-07 & YOY & 0.689 & 0.046 & 0.592 & 0.771 \\
\hline Lick Drain & Oct-07 & Small Adult & 0.722 & 0.047 & 0.621 & 0.805 \\
\hline Lick Drain & Oct-07 & Large Adult & 0.713 & 0.047 & 0.614 & 0.796 \\
\hline Mudlick Run & Jul-06 & YOY & 0.746 & 0.067 & 0.596 & 0.854 \\
\hline Mudlick Run & Jul-06 & Small Adult & 0.776 & 0.060 & 0.638 & 0.872 \\
\hline Mudlick Run & Jul-06 & Large Adult & 0.768 & 0.061 & 0.627 & 0.867 \\
\hline Mudlick Run & Aug-06 & YOY & 0.830 & 0.052 & 0.703 & 0.910 \\
\hline Mudlick Run & Aug-06 & Small Adult & 0.852 & 0.047 & 0.735 & 0.923 \\
\hline Mudlick Run & Aug-06 & Large Adult & 0.846 & 0.049 & 0.724 & 0.920 \\
\hline Mudlick Run & Oct-06 & YOY & 0.628 & 0.081 & 0.460 & 0.769 \\
\hline Mudlick Run & Oct-06 & Small Adult & 0.665 & 0.078 & 0.500 & 0.797 \\
\hline Mudlick Run & Oct-06 & Large Adult & 0.655 & 0.080 & 0.487 & 0.791 \\
\hline Mudlick Run & May-07 & YOY & 0.606 & 0.080 & 0.444 & 0.749 \\
\hline Mudlick Run & May-07 & Small Adult & 0.644 & 0.078 & 0.482 & 0.779 \\
\hline Mudlick Run & May-07 & Large Adult & 0.634 & 0.079 & 0.470 & 0.772 \\
\hline Mudlick Run & Jul-07 & YOY & 0.793 & 0.056 & 0.663 & 0.882 \\
\hline Mudlick Run & Jul-07 & Small Adult & 0.819 & 0.052 & 0.694 & 0.900 \\
\hline Mudlick Run & Jul-07 & Large Adult & 0.812 & 0.054 & 0.684 & 0.896 \\
\hline Mudlick Run & Oct-07 & YOY & 0.797 & 0.053 & 0.675 & 0.881 \\
\hline Mudlick Run & Oct-07 & Small Adult & 0.822 & 0.050 & 0.703 & 0.900 \\
\hline Mudlick Run & Oct-07 & Large Adult & 0.815 & 0.052 & 0.693 & 0.896 \\
\hline Tanner Run & Jul-06 & YOY & 0.681 & 0.081 & 0.506 & 0.816 \\
\hline Tanner Run & Jul-06 & Small Adult & 0.715 & 0.074 & 0.551 & 0.837 \\
\hline Tanner Run & Jul-06 & Large Adult & 0.706 & 0.076 & 0.540 & 0.830 \\
\hline Tanner Run & Aug-06 & YOY & 0.780 & 0.064 & 0.631 & 0.881 \\
\hline Tanner Run & Aug-06 & Small Adult & 0.807 & 0.058 & 0.668 & 0.897 \\
\hline Tanner Run & Aug-06 & Large Adult & 0.800 & 0.060 & 0.657 & 0.893 \\
\hline Tanner Run & Oct-06 & YOY & 0.550 & 0.090 & 0.374 & 0.714 \\
\hline Tanner Run & Oct-06 & Small Adult & 0.590 & 0.088 & 0.413 & 0.746 \\
\hline Tanner Run & Oct-06 & Large Adult & 0.579 & 0.090 & 0.401 & 0.739 \\
\hline Tanner Run & May-07 & YOY & 0.527 & 0.091 & 0.353 & 0.695 \\
\hline Tanner Run & May-07 & Small Adult & 0.568 & 0.089 & 0.391 & 0.729 \\
\hline Tanner Run & May-07 & Large Adult & 0.557 & 0.090 & 0.380 & 0.720 \\
\hline Tanner Run & Jul-07 & YOY & 0.736 & 0.070 & 0.578 & 0.850 \\
\hline Tanner Run & Jul-07 & Small Adult & 0.766 & 0.066 & 0.613 & 0.871 \\
\hline Tanner Run & Jul-07 & Large Adult & 0.758 & 0.068 & 0.603 & 0.866 \\
\hline Tanner Run & Oct-07 & YOY & 0.740 & 0.066 & 0.593 & 0.848 \\
\hline Tanner Run & Oct-07 & Small Adult & 0.770 & 0.063 & 0.626 & 0.870 \\
\hline
\end{tabular}




\begin{tabular}{|c|c|c|c|c|c|c|}
\hline Tanner Run & Oct-07 & Large Adult & 0.762 & 0.064 & 0.615 & 0.865 \\
\hline Zinn Hollow & Jul-06 & YOY & 0.663 & 0.068 & 0.521 & 0.781 \\
\hline Zinn Hollow & Jul-06 & Small Adult & 0.698 & 0.063 & 0.564 & 0.806 \\
\hline Zinn Hollow & Jul-06 & Large Adult & 0.689 & 0.062 & 0.556 & 0.797 \\
\hline Zinn Hollow & Aug-06 & YOY & 0.766 & 0.055 & 0.643 & 0.857 \\
\hline Zinn Hollow & Aug-06 & Small Adult & 0.794 & 0.051 & 0.678 & 0.876 \\
\hline Zinn Hollow & Aug-06 & Large Adult & 0.787 & 0.052 & 0.669 & 0.871 \\
\hline Zinn Hollow & Oct-06 & YOY & 0.530 & 0.072 & 0.390 & 0.665 \\
\hline Zinn Hollow & Oct-06 & Small Adult & 0.570 & 0.072 & 0.428 & 0.702 \\
\hline Zinn Hollow & Oct-06 & Large Adult & 0.559 & 0.072 & 0.417 & 0.692 \\
\hline Zinn Hollow & May-07 & YOY & 0.507 & 0.069 & 0.376 & 0.638 \\
\hline Zinn Hollow & May-07 & Small Adult & 0.548 & 0.070 & 0.412 & 0.678 \\
\hline Zinn Hollow & May-07 & Large Adult & 0.537 & 0.069 & 0.402 & 0.666 \\
\hline Zinn Hollow & Jul-07 & YOY & 0.720 & 0.055 & 0.602 & 0.814 \\
\hline Zinn Hollow & Jul-07 & Small Adult & 0.751 & 0.054 & 0.632 & 0.841 \\
\hline Zinn Hollow & Jul-07 & Large Adult & 0.743 & 0.054 & 0.624 & 0.834 \\
\hline Zinn Hollow & Oct-07 & YOY & 0.725 & 0.051 & 0.615 & 0.812 \\
\hline Zinn Hollow & Oct-07 & Small Adult & 0.756 & 0.051 & 0.643 & 0.842 \\
\hline Zinn Hollow & Oct-07 & Large Adult & 0.747 & 0.051 & 0.635 & 0.834 \\
\hline Laurel Lick Run & Jul-06 & YOY & 0.495 & 0.115 & 0.284 & 0.708 \\
\hline Laurel Lick Run & Jul-06 & Small Adult & 0.535 & 0.109 & 0.328 & 0.731 \\
\hline Laurel Lick Run & Jul-06 & Large Adult & 0.524 & 0.107 & 0.323 & 0.718 \\
\hline Laurel Lick Run & Aug-06 & YOY & 0.620 & 0.106 & 0.404 & 0.797 \\
\hline Laurel Lick Run & Aug-06 & Small Adult & 0.657 & 0.097 & 0.452 & 0.817 \\
\hline Laurel Lick Run & Aug-06 & Large Adult & 0.647 & 0.096 & 0.445 & 0.807 \\
\hline Laurel Lick Run & Oct-06 & YOY & 0.361 & 0.105 & 0.188 & 0.579 \\
\hline Laurel Lick Run & Oct-06 & Small Adult & 0.399 & 0.105 & 0.220 & 0.609 \\
\hline Laurel Lick Run & Oct-06 & Large Adult & 0.388 & 0.101 & 0.215 & 0.594 \\
\hline Laurel Lick Run & May-07 & YOY & 0.340 & 0.102 & 0.175 & 0.557 \\
\hline Laurel Lick Run & May-07 & Small Adult & 0.377 & 0.103 & 0.204 & 0.588 \\
\hline Laurel Lick Run & May-07 & Large Adult & 0.367 & 0.099 & 0.200 & 0.572 \\
\hline Laurel Lick Run & Jul-07 & YOY & 0.561 & 0.108 & 0.351 & 0.750 \\
\hline Laurel Lick Run & Jul-07 & Small Adult & 0.600 & 0.102 & 0.394 & 0.776 \\
\hline Laurel Lick Run & Jul-07 & Large Adult & 0.589 & 0.101 & 0.388 & 0.764 \\
\hline Laurel Lick Run & Oct-07 & YOY & 0.567 & 0.099 & 0.372 & 0.743 \\
\hline Laurel Lick Run & Oct-07 & Small Adult & 0.606 & 0.095 & 0.414 & 0.770 \\
\hline Laurel Lick Run & Oct-07 & Large Adult & 0.595 & 0.093 & 0.409 & 0.758 \\
\hline Glady Fork & Jul-06 & YOY & 0.815 & 0.051 & 0.694 & 0.896 \\
\hline Glady Fork & Jul-06 & Small Adult & 0.839 & 0.047 & 0.726 & 0.911 \\
\hline Glady Fork & Jul-06 & Large Adult & 0.832 & 0.049 & 0.714 & 0.908 \\
\hline Glady Fork & Aug-06 & YOY & 0.880 & 0.039 & 0.782 & 0.938 \\
\hline Glady Fork & Aug-06 & Small Adult & 0.896 & 0.035 & 0.804 & 0.948 \\
\hline Glady Fork & Aug-06 & Large Adult & 0.892 & 0.038 & 0.793 & 0.946 \\
\hline Glady Fork & Oct-06 & YOY & 0.717 & 0.067 & 0.571 & 0.829 \\
\hline Glady Fork & Oct-06 & Small Adult & 0.749 & 0.064 & 0.604 & 0.854 \\
\hline Glady Fork & Oct-06 & Large Adult & 0.740 & 0.067 & 0.589 & 0.850 \\
\hline Glady Fork & May-07 & YOY & 0.699 & 0.066 & 0.557 & 0.810 \\
\hline Glady Fork & May-07 & Small Adult & 0.732 & 0.064 & 0.590 & 0.838 \\
\hline Glady Fork & May-07 & Large Adult & 0.723 & 0.067 & 0.576 & 0.833 \\
\hline Glady Fork & Jul-07 & YOY & 0.852 & 0.041 & 0.754 & 0.916 \\
\hline
\end{tabular}




$\begin{array}{lcccccc}\text { Glady Fork } & \text { Jul-07 } & \text { Small Adult } & 0.871 & 0.039 & 0.775 & 0.930 \\ \text { Glady Fork } & \text { Jul-07 } & \text { Large Adult } & 0.866 & 0.041 & 0.764 & 0.928 \\ \text { Glady Fork } & \text { Oct-07 } & \text { YOY } & 0.855 & 0.041 & 0.754 & 0.919 \\ \text { Glady Fork } & \text { Oct-07 } & \text { Small Adult } & 0.874 & 0.040 & 0.774 & 0.933 \\ \text { Glady Fork } & \text { Oct-07 } & \text { Large Adult } & 0.868 & 0.042 & 0.762 & 0.931 \\ \text { Daniels Run } & \text { Jul-06 } & \text { YOY } & 0.563 & 0.077 & 0.412 & 0.704 \\ \text { Daniels Run } & \text { Jul-06 } & \text { Small Adult } & 0.603 & 0.076 & 0.450 & 0.738 \\ \text { Daniels Run } & \text { Jul-06 } & \text { Large Adult } & 0.592 & 0.074 & 0.443 & 0.726 \\ \text { Daniels Run } & \text { Aug-06 } & \text { YOY } & 0.683 & 0.056 & 0.564 & 0.781 \\ \text { Daniels Run } & \text { Aug-06 } & \text { Small Adult } & 0.717 & 0.056 & 0.596 & 0.812 \\ \text { Daniels Run } & \text { Aug-06 } & \text { Large Adult } & 0.708 & 0.056 & 0.588 & 0.804 \\ \text { Daniels Run } & \text { Oct-06 } & \text { YOY } & 0.426 & 0.074 & 0.290 & 0.574 \\ \text { Daniels Run } & \text { Oct-06 } & \text { Small Adult } & 0.466 & 0.079 & 0.319 & 0.619 \\ \text { Daniels Run } & \text { Oct-06 } & \text { Large Adult } & 0.455 & 0.078 & 0.311 & 0.606 \\ \text { Daniels Run } & \text { May-07 } & \text { YOY } & 0.404 & 0.072 & 0.274 & 0.548 \\ \text { Daniels Run } & \text { May-07 } & \text { Small Adult } & 0.444 & 0.077 & 0.302 & 0.595 \\ \text { Daniels Run } & \text { May-07 } & \text { Large Adult } & 0.432 & 0.075 & 0.295 & 0.581 \\ \text { Daniels Run } & \text { Jul-07 } & \text { YOY } & 0.627 & 0.068 & 0.488 & 0.748 \\ \text { Daniels Run } & \text { Jul-07 } & \text { Small Adult } & 0.664 & 0.070 & 0.516 & 0.786 \\ \text { Daniels Run } & \text { Jul-07 } & \text { Large Adult } & 0.654 & 0.070 & 0.509 & 0.776 \\ \text { Daniels Run } & \text { Oct-07 } & \text { YOY } & 0.633 & 0.065 & 0.499 & 0.749 \\ \text { Daniels Run } & \text { Oct-07 } & \text { Small Adult } & 0.670 & 0.069 & 0.525 & 0.788 \\ \text { Daniels Run } & \text { Oct-07 } & \text { Large Adult } & 0.660 & 0.068 & 0.517 & 0.779\end{array}$


A3. Summary of logit-scale, model-averaged coefficient estimates, standard errors, and $95 \%$ confidence intervals for predicting capture probability of mottled sculpin. $\mathrm{SE}=$ standard error, $\mathrm{LCI}=$ lower $95 \%$ confidence limit, $\mathrm{UCI}=$ upper $95 \%$ confidence limit, $\Sigma w i=$ sum of Akaike weights for term. The cumulative Akaike weights for each model term are indicated next to the relevant effect. For instance, the cumulative weight for the "age(2)" term (see Table 5 for description) is indicated next to the "Age(YOY)" model effect, while the cumulative weight for the "age" term is indicated next to the "Age" effect. The cumulative Akaike weight for the "date" term is indicated next to the first date effect. The cumulative Akaike weight for the "site" term is indicated next to the first site effect. Astericks $(*)$ indicate where model effect confidence limits did not include zero (i.e., a "significant" positive or negative slope).

\begin{tabular}{lcccccc}
\multicolumn{1}{c}{ Coefficient } & Estimate & SE & LCI & UCI & & $\mathbf{\Sigma w}_{\mathbf{i}}$ \\
\hline Intercept & -0.319 & 0.149 & -0.611 & -0.027 & $*$ & \\
Age (YOY) & -0.388 & 0.136 & -0.655 & -0.121 & $*$ & 0.673 \\
Age (SAD) & -0.047 & 0.074 & -0.192 & 0.098 & & 0.303 \\
Site (NB) & 0.091 & 0.159 & -0.220 & 0.402 & & 1.000 \\
Site (OH) & 0.385 & 0.239 & -0.083 & 0.852 & & \\
Site (BR) & 0.073 & 0.183 & -0.286 & 0.432 & & \\
Site (SR) & 0.140 & 0.218 & -0.286 & 0.567 & & \\
Site (LR) & 0.855 & 0.154 & 0.553 & 1.157 & $*$ & \\
Site (LD) & 0.598 & 0.152 & 0.300 & 0.896 & $*$ & \\
Site (MR) & 0.835 & 0.153 & 0.535 & 1.136 & $*$ & \\
Site (TR) & 0.579 & 0.152 & 0.281 & 0.878 & $*$ & \\
Site (ZH) & 0.521 & 0.202 & 0.125 & 0.917 & $*$ & \\
Site (LL) & 0.148 & 0.199 & -0.241 & 0.537 & & \\
Site (GF) & 0.507 & 0.196 & 0.122 & 0.892 & $*$ & \\
Date (Jul-06) & 0.449 & 0.125 & 0.204 & 0.694 & $*$ & \multirow{2}{*}{1.000} \\
Date (Aug-06) & 0.483 & 0.129 & 0.230 & 0.737 & $*$ & \\
Date (Oct-06) & -0.321 & 0.120 & -0.556 & -0.087 & $*$ & \\
Date (May-07) & 0.014 & 0.109 & -0.201 & 0.228 & & \\
Date (Jul-07) & 0.395 & 0.114 & 0.173 & 0.618 & &
\end{tabular}


A4. Summary of model-averaged capture efficiency parameter estimates, standard errors, and $95 \%$ confidence intervals (i.e., lower $=$ LCI and upper $=$ UCI) for all sites, sampling occasions, and age classes derived from Huggins' closed capture models for mottled sculpin.

\begin{tabular}{|c|c|c|c|c|c|c|}
\hline Site & Date & Age & Estimate & SE & $\mathrm{LCI}$ & $\mathrm{UCI}$ \\
\hline Nan's Branch & Jul-06 & YOY & 0.461 & 0.050 & 0.366 & 0.559 \\
\hline Nan's Branch & Jul-06 & Small Adult & 0.549 & 0.035 & 0.479 & 0.617 \\
\hline Nan's Branch & Jul-06 & Large Adult & 0.553 & 0.034 & 0.485 & 0.619 \\
\hline Nan's Branch & Aug-06 & YOY & 0.470 & 0.050 & 0.374 & 0.568 \\
\hline Nan's Branch & Aug-06 & Small Adult & 0.558 & 0.036 & 0.487 & 0.627 \\
\hline Nan's Branch & Aug-06 & Large Adult & 0.562 & 0.035 & 0.493 & 0.628 \\
\hline Nan's Branch & Oct-06 & YOY & 0.284 & 0.036 & 0.218 & 0.361 \\
\hline Nan's Branch & Oct-06 & Small Adult & 0.361 & 0.031 & 0.302 & 0.424 \\
\hline Nan's Branch & Oct-06 & Large Adult & 0.364 & 0.030 & 0.307 & 0.426 \\
\hline Nan's Branch & May-07 & YOY & 0.356 & 0.040 & 0.283 & 0.437 \\
\hline Nan's Branch & May-07 & Small Adult & 0.440 & 0.030 & 0.383 & 0.499 \\
\hline Nan's Branch & May-07 & Large Adult & 0.444 & 0.029 & 0.388 & 0.501 \\
\hline Nan's Branch & Jul-07 & YOY & 0.447 & 0.048 & 0.356 & 0.541 \\
\hline Nan's Branch & Jul-07 & Small Adult & 0.535 & 0.033 & 0.470 & 0.598 \\
\hline Nan's Branch & Jul-07 & Large Adult & 0.538 & 0.032 & 0.474 & 0.601 \\
\hline Nan's Branch & Oct-07 & YOY & 0.353 & 0.041 & 0.277 & 0.437 \\
\hline Nan's Branch & Oct-07 & Small Adult & 0.436 & 0.032 & 0.375 & 0.500 \\
\hline Nan's Branch & Oct-07 & Large Adult & 0.440 & 0.032 & 0.379 & 0.502 \\
\hline Otis Hollow & Jul-06 & YOY & 0.535 & 0.065 & 0.407 & 0.657 \\
\hline Otis Hollow & Jul-06 & Small Adult & 0.620 & 0.052 & 0.514 & 0.716 \\
\hline Otis Hollow & Jul-06 & Large Adult & 0.624 & 0.052 & 0.519 & 0.718 \\
\hline Otis Hollow & Aug-06 & YOY & 0.543 & 0.067 & 0.412 & 0.669 \\
\hline Otis Hollow & Aug-06 & Small Adult & 0.629 & 0.054 & 0.518 & 0.728 \\
\hline Otis Hollow & Aug-06 & Large Adult & 0.632 & 0.054 & 0.523 & 0.730 \\
\hline Otis Hollow & Oct-06 & YOY & 0.347 & 0.056 & 0.246 & 0.464 \\
\hline Otis Hollow & Oct-06 & Small Adult & 0.431 & 0.054 & 0.330 & 0.539 \\
\hline Otis Hollow & Oct-06 & Large Adult & 0.434 & 0.054 & 0.334 & 0.541 \\
\hline Otis Hollow & May-07 & YOY & 0.426 & 0.061 & 0.312 & 0.548 \\
\hline Otis Hollow & May-07 & Small Adult & 0.513 & 0.055 & 0.407 & 0.618 \\
\hline Otis Hollow & May-07 & Large Adult & 0.517 & 0.054 & 0.411 & 0.621 \\
\hline Otis Hollow & Jul-07 & YOY & 0.520 & 0.064 & 0.396 & 0.641 \\
\hline Otis Hollow & Jul-07 & Small Adult & 0.606 & 0.051 & 0.503 & 0.701 \\
\hline Otis Hollow & Jul-07 & Large Adult & 0.610 & 0.051 & 0.507 & 0.704 \\
\hline Otis Hollow & Oct-07 & YOY & 0.422 & 0.062 & 0.308 & 0.545 \\
\hline Otis Hollow & Oct-07 & Small Adult & 0.510 & 0.055 & 0.403 & 0.616 \\
\hline Otis Hollow & Oct-07 & Large Adult & 0.513 & 0.055 & 0.407 & 0.619 \\
\hline Big Run & Jul-06 & YOY & 0.457 & 0.055 & 0.354 & 0.564 \\
\hline Big Run & Jul-06 & Small Adult & 0.545 & 0.043 & 0.461 & 0.627 \\
\hline Big Run & Jul-06 & Large Adult & 0.549 & 0.042 & 0.466 & 0.628 \\
\hline Big Run & Aug-06 & YOY & 0.466 & 0.055 & 0.362 & 0.573 \\
\hline Big Run & Aug-06 & Small Adult & 0.554 & 0.043 & 0.469 & 0.636 \\
\hline Big Run & Aug-06 & Large Adult & 0.557 & 0.042 & 0.474 & 0.638 \\
\hline Big Run & Oct-06 & YOY & 0.281 & 0.041 & 0.208 & 0.367 \\
\hline Big Run & Oct-06 & Small Adult & 0.357 & 0.038 & 0.285 & 0.435 \\
\hline
\end{tabular}




\begin{tabular}{|c|c|c|c|c|c|c|}
\hline Big Run & Oct-06 & Large Adult & 0.360 & 0.038 & 0.290 & 0.437 \\
\hline Big Run & May-07 & YOY & 0.352 & 0.044 & 0.272 & 0.442 \\
\hline Big Run & May-07 & Small Adult & 0.436 & 0.037 & 0.366 & 0.509 \\
\hline Big Run & May-07 & Large Adult & 0.440 & 0.036 & 0.371 & 0.511 \\
\hline Big Run & Jul-07 & YOY & 0.443 & 0.052 & 0.345 & 0.545 \\
\hline Big Run & Jul-07 & Small Adult & 0.530 & 0.039 & 0.453 & 0.606 \\
\hline Big Run & Jul-07 & Large Adult & 0.534 & 0.039 & 0.457 & 0.609 \\
\hline Big Run & Oct-07 & YOY & 0.349 & 0.044 & 0.267 & 0.440 \\
\hline Big Run & Oct-07 & Small Adult & 0.432 & 0.038 & 0.360 & 0.507 \\
\hline Big Run & Oct-07 & Large Adult & 0.436 & 0.037 & 0.365 & 0.510 \\
\hline Swallow Rock Run & Jul-06 & YOY & 0.473 & 0.062 & 0.356 & 0.593 \\
\hline Swallow Rock Run & Jul-06 & Small Adult & 0.561 & 0.051 & 0.460 & 0.657 \\
\hline Swallow Rock Run & Jul-06 & Large Adult & 0.565 & 0.050 & 0.464 & 0.660 \\
\hline Swallow Rock Run & Aug-06 & YOY & 0.482 & 0.062 & 0.363 & 0.603 \\
\hline Swallow Rock Run & Aug-06 & Small Adult & 0.570 & 0.052 & 0.467 & 0.667 \\
\hline Swallow Rock Run & Aug-06 & Large Adult & 0.573 & 0.051 & 0.471 & 0.670 \\
\hline Swallow Rock Run & Oct-06 & YOY & 0.294 & 0.048 & 0.209 & 0.396 \\
\hline Swallow Rock Run & Oct-06 & Small Adult & 0.372 & 0.048 & 0.284 & 0.469 \\
\hline Swallow Rock Run & Oct-06 & Large Adult & 0.375 & 0.047 & 0.288 & 0.472 \\
\hline Swallow Rock Run & May-07 & YOY & 0.367 & 0.053 & 0.270 & 0.476 \\
\hline Swallow Rock Run & May-07 & Small Adult & 0.452 & 0.049 & 0.359 & 0.548 \\
\hline Swallow Rock Run & May-07 & Large Adult & 0.456 & 0.049 & 0.363 & 0.551 \\
\hline Swallow Rock Run & Jul-07 & YOY & 0.459 & 0.057 & 0.351 & 0.570 \\
\hline Swallow Rock Run & Jul-07 & Small Adult & 0.546 & 0.046 & 0.456 & 0.634 \\
\hline Swallow Rock Run & Jul-07 & Large Adult & 0.550 & 0.046 & 0.460 & 0.637 \\
\hline Swallow Rock Run & Oct-07 & YOY & 0.364 & 0.054 & 0.266 & 0.474 \\
\hline Swallow Rock Run & Oct-07 & Small Adult & 0.448 & 0.050 & 0.354 & 0.546 \\
\hline Swallow Rock Run & Oct-07 & Large Adult & 0.452 & 0.049 & 0.358 & 0.549 \\
\hline Lynn Run & Jul-06 & YOY & 0.647 & 0.043 & 0.560 & 0.726 \\
\hline Lynn Run & Jul-06 & Small Adult & 0.723 & 0.026 & 0.670 & 0.771 \\
\hline Lynn Run & Jul-06 & Large Adult & 0.726 & 0.025 & 0.674 & 0.772 \\
\hline Lynn Run & Aug-06 & YOY & 0.655 & 0.043 & 0.566 & 0.735 \\
\hline Lynn Run & Aug-06 & Small Adult & 0.730 & 0.027 & 0.674 & 0.780 \\
\hline Lynn Run & Aug-06 & Large Adult & 0.733 & 0.026 & 0.678 & 0.782 \\
\hline Lynn Run & Oct-06 & YOY & 0.460 & 0.043 & 0.378 & 0.544 \\
\hline Lynn Run & Oct-06 & Small Adult & 0.548 & 0.033 & 0.483 & 0.610 \\
\hline Lynn Run & Oct-06 & Large Adult & 0.551 & 0.031 & 0.489 & 0.612 \\
\hline Lynn Run & May-07 & YOY & 0.542 & 0.042 & 0.459 & 0.623 \\
\hline Lynn Run & May-07 & Small Adult & 0.628 & 0.029 & 0.570 & 0.682 \\
\hline Lynn Run & May-07 & Large Adult & 0.631 & 0.028 & 0.574 & 0.684 \\
\hline Lynn Run & Jul-07 & YOY & 0.634 & 0.043 & 0.547 & 0.712 \\
\hline Lynn Run & Jul-07 & Small Adult & 0.711 & 0.025 & 0.659 & 0.758 \\
\hline Lynn Run & Jul-07 & Large Adult & 0.714 & 0.025 & 0.662 & 0.761 \\
\hline Lynn Run & Oct-07 & YOY & 0.538 & 0.044 & 0.453 & 0.622 \\
\hline Lynn Run & Oct-07 & Small Adult & 0.624 & 0.030 & 0.564 & 0.681 \\
\hline Lynn Run & Oct-07 & Large Adult & 0.627 & 0.030 & 0.568 & 0.684 \\
\hline Lick Drain & Jul-06 & YOY & 0.586 & 0.044 & 0.497 & 0.669 \\
\hline Lick Drain & Jul-06 & Small Adult & 0.668 & 0.027 & 0.613 & 0.719 \\
\hline Lick Drain & Jul-06 & Large Adult & 0.671 & 0.026 & 0.618 & 0.721 \\
\hline Lick Drain & Aug-06 & YOY & 0.595 & 0.047 & 0.500 & 0.682 \\
\hline
\end{tabular}




\begin{tabular}{|c|c|c|c|c|c|c|}
\hline Lick Drain & Aug-06 & Small Adult & 0.676 & 0.031 & 0.613 & 0.733 \\
\hline Lick Drain & Aug-06 & Large Adult & 0.679 & 0.030 & 0.618 & 0.735 \\
\hline Lick Drain & Oct-06 & YOY & 0.396 & 0.041 & 0.319 & 0.479 \\
\hline Lick Drain & Oct-06 & Small Adult & 0.483 & 0.033 & 0.420 & 0.546 \\
\hline Lick Drain & Oct-06 & Large Adult & 0.486 & 0.032 & 0.424 & 0.549 \\
\hline Lick Drain & May-07 & YOY & 0.477 & 0.043 & 0.395 & 0.561 \\
\hline Lick Drain & May-07 & Small Adult & 0.565 & 0.030 & 0.505 & 0.623 \\
\hline Lick Drain & May-07 & Large Adult & 0.569 & 0.030 & 0.509 & 0.626 \\
\hline Lick Drain & Jul-07 & YOY & 0.571 & 0.044 & 0.485 & 0.654 \\
\hline Lick Drain & Jul-07 & Small Adult & 0.655 & 0.026 & 0.602 & 0.704 \\
\hline Lick Drain & Jul-07 & Large Adult & 0.658 & 0.026 & 0.605 & 0.707 \\
\hline Lick Drain & Oct-07 & YOY & 0.474 & 0.041 & 0.395 & 0.553 \\
\hline Lick Drain & Oct-07 & Small Adult & 0.561 & 0.027 & 0.508 & 0.613 \\
\hline Lick Drain & Oct-07 & Large Adult & 0.565 & 0.027 & 0.512 & 0.617 \\
\hline Mudlick Run & Jul-06 & YOY & 0.642 & 0.043 & 0.553 & 0.721 \\
\hline Mudlick Run & Jul-06 & Small Adult & 0.718 & 0.026 & 0.664 & 0.767 \\
\hline Mudlick Run & Jul-06 & Large Adult & 0.721 & 0.026 & 0.668 & 0.769 \\
\hline Mudlick Run & Aug-06 & YOY & 0.650 & 0.044 & 0.560 & 0.731 \\
\hline Mudlick Run & Aug-06 & Small Adult & 0.726 & 0.028 & 0.668 & 0.776 \\
\hline Mudlick Run & Aug-06 & Large Adult & 0.728 & 0.027 & 0.672 & 0.778 \\
\hline Mudlick Run & Oct-06 & YOY & 0.454 & 0.042 & 0.374 & 0.536 \\
\hline Mudlick Run & Oct-06 & Small Adult & 0.542 & 0.032 & 0.480 & 0.603 \\
\hline Mudlick Run & Oct-06 & Large Adult & 0.545 & 0.031 & 0.484 & 0.605 \\
\hline Mudlick Run & May-07 & YOY & 0.536 & 0.041 & 0.455 & 0.616 \\
\hline Mudlick Run & May-07 & Small Adult & 0.622 & 0.027 & 0.567 & 0.674 \\
\hline Mudlick Run & May-07 & Large Adult & 0.625 & 0.027 & 0.571 & 0.677 \\
\hline Mudlick Run & Jul-07 & YOY & 0.628 & 0.043 & 0.541 & 0.707 \\
\hline Mudlick Run & Jul-07 & Small Adult & 0.706 & 0.025 & 0.654 & 0.754 \\
\hline Mudlick Run & Jul-07 & Large Adult & 0.709 & 0.026 & 0.657 & 0.757 \\
\hline Mudlick Run & Oct-07 & YOY & 0.533 & 0.042 & 0.449 & 0.614 \\
\hline Mudlick Run & Oct-07 & Small Adult & 0.619 & 0.029 & 0.561 & 0.673 \\
\hline Mudlick Run & Oct-07 & Large Adult & 0.622 & 0.029 & 0.564 & 0.676 \\
\hline Tanner Run & Jul-06 & YOY & 0.582 & 0.047 & 0.487 & 0.670 \\
\hline Tanner Run & Jul-06 & Small Adult & 0.664 & 0.030 & 0.604 & 0.720 \\
\hline Tanner Run & Jul-06 & Large Adult & 0.667 & 0.029 & 0.608 & 0.722 \\
\hline Tanner Run & Aug-06 & YOY & 0.590 & 0.047 & 0.495 & 0.679 \\
\hline Tanner Run & Aug-06 & Small Adult & 0.672 & 0.031 & 0.610 & 0.729 \\
\hline Tanner Run & Aug-06 & Large Adult & 0.675 & 0.030 & 0.614 & 0.731 \\
\hline Tanner Run & Oct-06 & YOY & 0.392 & 0.042 & 0.314 & 0.476 \\
\hline Tanner Run & Oct-06 & Small Adult & 0.478 & 0.032 & 0.416 & 0.541 \\
\hline Tanner Run & Oct-06 & Large Adult & 0.482 & 0.031 & 0.421 & 0.544 \\
\hline Tanner Run & May-07 & YOY & 0.473 & 0.043 & 0.391 & 0.557 \\
\hline Tanner Run & May-07 & Small Adult & 0.561 & 0.029 & 0.503 & 0.617 \\
\hline Tanner Run & May-07 & Large Adult & 0.564 & 0.029 & 0.507 & 0.620 \\
\hline Tanner Run & Jul-07 & YOY & 0.567 & 0.045 & 0.478 & 0.653 \\
\hline Tanner Run & Jul-07 & Small Adult & 0.651 & 0.027 & 0.597 & 0.701 \\
\hline Tanner Run & Jul-07 & Large Adult & 0.654 & 0.027 & 0.600 & 0.705 \\
\hline Tanner Run & Oct-07 & YOY & 0.469 & 0.043 & 0.386 & 0.554 \\
\hline Tanner Run & Oct-07 & Small Adult & 0.557 & 0.029 & 0.499 & 0.614 \\
\hline Tanner Run & Oct-07 & Large Adult & 0.561 & 0.029 & 0.503 & 0.61 \\
\hline
\end{tabular}




\begin{tabular}{|c|c|c|c|c|c|c|}
\hline Zinn Hollow & Jul-06 & YOY & 0.568 & 0.058 & 0.453 & 0.677 \\
\hline Zinn Hollow & Jul-06 & Small Adult & 0.652 & 0.044 & 0.562 & 0.733 \\
\hline Zinn Hollow & Jul-06 & Large Adult & 0.655 & 0.043 & 0.567 & 0.734 \\
\hline Zinn Hollow & Aug-06 & YOY & 0.577 & 0.057 & 0.463 & 0.684 \\
\hline Zinn Hollow & Aug-06 & Small Adult & 0.660 & 0.043 & 0.571 & 0.739 \\
\hline Zinn Hollow & Aug-06 & Large Adult & 0.663 & 0.042 & 0.576 & 0.740 \\
\hline Zinn Hollow & Oct-06 & YOY & 0.379 & 0.051 & 0.286 & 0.482 \\
\hline Zinn Hollow & Oct-06 & Small Adult & 0.465 & 0.046 & 0.377 & 0.555 \\
\hline Zinn Hollow & Oct-06 & Large Adult & 0.468 & 0.045 & 0.382 & 0.556 \\
\hline Zinn Hollow & May-07 & YOY & 0.459 & 0.052 & 0.360 & 0.562 \\
\hline Zinn Hollow & May-07 & Small Adult & 0.547 & 0.043 & 0.463 & 0.629 \\
\hline Zinn Hollow & May-07 & Large Adult & 0.551 & 0.042 & 0.467 & 0.632 \\
\hline Zinn Hollow & Jul-07 & YOY & 0.554 & 0.056 & 0.443 & 0.660 \\
\hline Zinn Hollow & Jul-07 & Small Adult & 0.639 & 0.042 & 0.553 & 0.716 \\
\hline Zinn Hollow & Jul-07 & Large Adult & 0.642 & 0.042 & 0.557 & 0.719 \\
\hline Zinn Hollow & Oct-07 & YOY & 0.456 & 0.054 & 0.354 & 0.561 \\
\hline Zinn Hollow & Oct-07 & Small Adult & 0.544 & 0.044 & 0.456 & 0.629 \\
\hline Zinn Hollow & Oct-07 & Large Adult & 0.547 & 0.044 & 0.460 & 0.631 \\
\hline Laurel Lick Run & Jul-06 & YOY & 0.475 & 0.061 & 0.359 & 0.594 \\
\hline Laurel Lick Run & Jul-06 & Small Adult & 0.563 & 0.049 & 0.466 & 0.655 \\
\hline Laurel Lick Run & Jul-06 & Large Adult & 0.566 & 0.048 & 0.470 & 0.657 \\
\hline Laurel Lick Run & Aug-06 & YOY & 0.484 & 0.056 & 0.376 & 0.593 \\
\hline Laurel Lick Run & Aug-06 & Small Adult & 0.572 & 0.043 & 0.486 & 0.653 \\
\hline Laurel Lick Run & Aug-06 & Large Adult & 0.575 & 0.042 & 0.491 & 0.655 \\
\hline Laurel Lick Run & Oct-06 & YOY & 0.295 & 0.046 & 0.214 & 0.393 \\
\hline Laurel Lick Run & Oct-06 & Small Adult & 0.374 & 0.044 & 0.293 & 0.462 \\
\hline Laurel Lick Run & Oct-06 & Large Adult & 0.377 & 0.043 & 0.297 & 0.464 \\
\hline Laurel Lick Run & May-07 & YOY & 0.369 & 0.052 & 0.273 & 0.476 \\
\hline Laurel Lick Run & May-07 & Small Adult & 0.454 & 0.046 & 0.366 & 0.544 \\
\hline Laurel Lick Run & May-07 & Large Adult & 0.457 & 0.046 & 0.370 & 0.547 \\
\hline Laurel Lick Run & Jul-07 & YOY & 0.460 & 0.059 & 0.349 & 0.575 \\
\hline Laurel Lick Run & Jul-07 & Small Adult & 0.548 & 0.046 & 0.457 & 0.637 \\
\hline Laurel Lick Run & Jul-07 & Large Adult & 0.552 & 0.046 & 0.460 & 0.640 \\
\hline Laurel Lick Run & Oct-07 & YOY & 0.365 & 0.052 & 0.271 & 0.471 \\
\hline Laurel Lick Run & Oct-07 & Small Adult & 0.450 & 0.045 & 0.364 & 0.539 \\
\hline Laurel Lick Run & Oct-07 & Large Adult & 0.454 & 0.045 & 0.368 & 0.542 \\
\hline Glady Fork & Jul-06 & YOY & 0.565 & 0.060 & 0.447 & 0.677 \\
\hline Glady Fork & Jul-06 & Small Adult & 0.649 & 0.045 & 0.557 & 0.732 \\
\hline Glady Fork & Jul-06 & Large Adult & 0.653 & 0.044 & 0.562 & 0.733 \\
\hline Glady Fork & Aug-06 & YOY & 0.574 & 0.054 & 0.466 & 0.676 \\
\hline Glady Fork & Aug-06 & Small Adult & 0.658 & 0.039 & 0.578 & 0.729 \\
\hline Glady Fork & Aug-06 & Large Adult & 0.661 & 0.038 & 0.584 & 0.730 \\
\hline Glady Fork & Oct-06 & YOY & 0.376 & 0.053 & 0.280 & 0.484 \\
\hline Glady Fork & Oct-06 & Small Adult & 0.462 & 0.047 & 0.372 & 0.555 \\
\hline Glady Fork & Oct-06 & Large Adult & 0.465 & 0.046 & 0.377 & 0.556 \\
\hline Glady Fork & May-07 & YOY & 0.457 & 0.055 & 0.353 & 0.564 \\
\hline Glady Fork & May-07 & Small Adult & 0.545 & 0.045 & 0.456 & 0.630 \\
\hline Glady Fork & May-07 & Large Adult & 0.548 & 0.044 & 0.461 & 0.632 \\
\hline Glady Fork & Jul-07 & YOY & 0.551 & 0.058 & 0.437 & 0.660 \\
\hline Glady Fork & Jul-07 & Small Adult & 0.636 & 0.043 & 0.549 & 0.715 \\
\hline
\end{tabular}




$\begin{array}{lccllll}\text { Glady Fork } & \text { Jul-07 } & \text { Large Adult } & 0.639 & 0.042 & 0.553 & 0.717 \\ \text { Glady Fork } & \text { Oct-07 } & \text { YOY } & 0.453 & 0.056 & 0.347 & 0.563 \\ \text { Glady Fork } & \text { Oct-07 } & \text { Small Adult } & 0.541 & 0.046 & 0.450 & 0.629 \\ \text { Glady Fork } & \text { Oct-07 } & \text { Large Adult } & 0.544 & 0.045 & 0.455 & 0.631 \\ \text { Daniels Run } & \text { Jul-06 } & \text { YOY } & 0.437 & 0.053 & 0.338 & 0.542 \\ \text { Daniels Run } & \text { Jul-06 } & \text { Small Adult } & 0.525 & 0.040 & 0.447 & 0.602 \\ \text { Daniels Run } & \text { Jul-06 } & \text { Large Adult } & 0.529 & 0.040 & 0.451 & 0.605 \\ \text { Daniels Run } & \text { Aug-06 } & \text { YOY } & 0.446 & 0.048 & 0.356 & 0.541 \\ \text { Daniels Run } & \text { Aug-06 } & \text { Small Adult } & 0.534 & 0.033 & 0.470 & 0.598 \\ \text { Daniels Run } & \text { Aug-06 } & \text { Large Adult } & 0.538 & 0.032 & 0.474 & 0.600 \\ \text { Daniels Run } & \text { Oct-06 } & \text { YOY } & 0.265 & 0.038 & 0.198 & 0.345 \\ \text { Daniels Run } & \text { Oct-06 } & \text { Small Adult } & 0.339 & 0.034 & 0.276 & 0.409 \\ \text { Daniels Run } & \text { Oct-06 } & \text { Large Adult } & 0.342 & 0.034 & 0.279 & 0.411 \\ \text { Daniels Run } & \text { May-07 } & \text { YOY } & 0.334 & 0.043 & 0.257 & 0.422 \\ \text { Daniels Run } & \text { May-07 } & \text { Small Adult } & 0.417 & 0.035 & 0.350 & 0.486 \\ \text { Daniels Run } & \text { May-07 } & \text { Large Adult } & 0.420 & 0.035 & 0.354 & 0.489 \\ \text { Daniels Run } & \text { Jul-07 } & \text { YOY } & 0.423 & 0.049 & 0.331 & 0.521 \\ \text { Daniels Run } & \text { Jul-07 } & \text { Small Adult } & 0.511 & 0.035 & 0.442 & 0.579 \\ \text { Daniels Run } & \text { Jul-07 } & \text { Large Adult } & 0.514 & 0.035 & 0.445 & 0.583 \\ \text { Daniels Run } & \text { Oct-07 } & \text { YOY } & 0.331 & 0.043 & 0.253 & 0.420 \\ \text { Daniels Run } & \text { Oct-07 } & \text { Small Adult } & 0.413 & 0.035 & 0.346 & 0.483 \\ \text { Daniels Run } & \text { Oct-07 } & \text { Large Adult } & 0.416 & 0.035 & 0.349 & 0.487\end{array}$


Appendix A5. Summary of model-averaged parameter estimates, standard errors, and 95\% confidence intervals (i.e., lower $=$ LCI and upper $=\mathrm{UCI}$ ) of abundance for three size classes of brook trout for all sites and sample dates. Classifications of sites based on treatment and spatial location are also noted.

\begin{tabular}{|c|c|c|c|c|c|c|c|c|}
\hline Site & Date & Age & Removal & Location & Estimate & SE & LCl & UCI \\
\hline Nan's Branch & Jul-06 & Large Adult & Control & Mainstem & 14.376 & 0.636 & 13.129 & 15.622 \\
\hline Nan's Branch & Aug-06 & Large Adult & Control & Mainstem & 6.075 & 0.278 & 5.530 & 6.621 \\
\hline Nan's Branch & Oct-06 & Large Adult & Control & Mainstem & 3.174 & 0.425 & 2.342 & 4.007 \\
\hline Nan's Branch & May-07 & Large Adult & Control & Mainstem & 9.636 & 0.846 & 7.979 & 11.294 \\
\hline Nan's Branch & Jul-07 & Large Adult & Control & Mainstem & 1.015 & 0.121 & 0.777 & 1.252 \\
\hline Nan's Branch & Oct-07 & Large Adult & Control & Mainstem & 5.058 & 0.243 & 4.583 & 5.533 \\
\hline Nan's Branch & Jul-06 & YOY & Control & Mainstem & 22.459 & 0.707 & 21.073 & 23.844 \\
\hline Nan's Branch & Aug-06 & YOY & Control & Mainstem & 13.169 & 0.419 & 12.348 & 13.990 \\
\hline Nan's Branch & Oct-06 & YOY & Control & Mainstem & 29.129 & 1.671 & 25.855 & 32.403 \\
\hline Nan's Branch & May-07 & YOY & Control & Mainstem & 5.416 & 0.684 & 4.076 & 6.756 \\
\hline Nan's Branch & Jul-07 & YOY & Control & Mainstem & 23.348 & 0.602 & 22.169 & 24.528 \\
\hline Nan's Branch & Oct-07 & YOY & Control & Mainstem & 29.391 & 0.639 & 28.139 & 30.643 \\
\hline Nan's Branch & Jul-06 & Small Adult & Control & Mainstem & 11.225 & 0.485 & 10.274 & 12.176 \\
\hline Nan's Branch & Aug-06 & Small Adult & Control & Mainstem & 3.028 & 0.163 & 2.709 & 3.347 \\
\hline Nan's Branch & Oct-06 & Small Adult & Control & Mainstem & 12.871 & 1.012 & 10.888 & 14.855 \\
\hline Nan's Branch & May-07 & Small Adult & Control & Mainstem & 9.832 & 1.000 & 7.872 & 11.792 \\
\hline Nan's Branch & Jul-07 & Small Adult & Control & Mainstem & 9.093 & 0.294 & 8.518 & 9.669 \\
\hline Nan's Branch & Oct-07 & Small Adult & Control & Mainstem & 9.092 & 0.300 & 8.504 & 9.680 \\
\hline Otis Hollow & Jul-06 & Large Adult & Treatment & Mainstem & 14.372 & 0.630 & 13.137 & 15.608 \\
\hline Otis Hollow & Aug-06 & Large Adult & Treatment & Mainstem & 3.037 & 0.193 & 2.658 & 3.416 \\
\hline Otis Hollow & Oct-06 & Large Adult & Treatment & Mainstem & 5.295 & 0.557 & 4.204 & 6.386 \\
\hline Otis Hollow & May-07 & Large Adult & Treatment & Mainstem & 8.561 & 0.786 & 7.020 & 10.102 \\
\hline Otis Hollow & Jul-07 & Large Adult & Treatment & Mainstem & 9.133 & 0.372 & 8.403 & 9.862 \\
\hline Otis Hollow & Oct-07 & Large Adult & Treatment & Mainstem & 2.023 & 0.151 & 1.726 & 2.319 \\
\hline Otis Hollow & Jul-06 & YOY & Treatment & Mainstem & 21.432 & 0.682 & 20.095 & 22.770 \\
\hline Otis Hollow & Aug-06 & YOY & Treatment & Mainstem & 2.026 & 0.160 & 1.712 & 2.339 \\
\hline Otis Hollow & Oct-06 & YOY & Treatment & Mainstem & 43.190 & 2.141 & 38.993 & 47.387 \\
\hline Otis Hollow & May-07 & YOY & Treatment & Mainstem & 47.635 & 2.229 & 43.267 & 52.004 \\
\hline
\end{tabular}




\begin{tabular}{|c|c|c|c|c|c|c|c|c|}
\hline Site & Date & Age & Removal & Location & Estimate & SE & $\mathrm{LCl}$ & $\mathrm{UCI}$ \\
\hline Otis Hollow & Jul-07 & YOY & Treatment & Mainstem & 41.630 & 0.824 & 40.015 & 43.245 \\
\hline Otis Hollow & Oct-07 & YOY & Treatment & Mainstem & 60.793 & 0.921 & 58.988 & 62.598 \\
\hline Otis Hollow & Jul-06 & Small Adult & Treatment & Mainstem & 32.646 & 0.843 & 30.994 & 34.297 \\
\hline Otis Hollow & Aug-06 & Small Adult & Treatment & Mainstem & 5.045 & 0.207 & 4.638 & 5.451 \\
\hline Otis Hollow & Oct-06 & Small Adult & Treatment & Mainstem & 21.470 & 1.357 & 18.811 & 24.130 \\
\hline Otis Hollow & May-07 & Small Adult & Treatment & Mainstem & 33.847 & 2.020 & 29.889 & 37.805 \\
\hline Otis Hollow & Jul-07 & Small Adult & Treatment & Mainstem & 28.296 & 0.531 & 27.255 & 29.338 \\
\hline Otis Hollow & Oct-07 & Small Adult & Treatment & Mainstem & 11.110 & 0.328 & 10.467 & 11.752 \\
\hline Big Run & Jul-06 & Large Adult & Control & Headwater & 13.346 & 0.607 & 12.155 & 14.537 \\
\hline Big Run & Aug-06 & Large Adult & Control & Headwater & 17.210 & 0.473 & 16.283 & 18.137 \\
\hline Big Run & Oct-06 & Large Adult & Control & Headwater & 14.820 & 0.954 & 12.950 & 16.691 \\
\hline Big Run & May-07 & Large Adult & Control & Headwater & 21.408 & 1.292 & 18.877 & 23.939 \\
\hline Big Run & Jul-07 & Large Adult & Control & Headwater & 8.116 & 0.347 & 7.436 & 8.796 \\
\hline Big Run & Oct-07 & Large Adult & Control & Headwater & 10.114 & 0.343 & 9.442 & 10.786 \\
\hline Big Run & Jul-06 & YOY & Control & Headwater & 2.041 & 0.205 & 1.639 & 2.443 \\
\hline Big Run & Aug-06 & YOY & Control & Headwater & 7.090 & 0.302 & 6.498 & 7.682 \\
\hline Big Run & Oct-06 & YOY & Control & Headwater & 14.031 & 1.109 & 11.858 & 16.205 \\
\hline Big Run & May-07 & YOY & Control & Headwater & 22.740 & 1.459 & 19.880 & 25.600 \\
\hline Big Run & Jul-07 & YOY & Control & Headwater & 5.076 & 0.277 & 4.533 & 5.618 \\
\hline Big Run & Oct-07 & YOY & Control & Headwater & 37.493 & 0.719 & 36.083 & 38.903 \\
\hline Big Run & Jul-06 & Small Adult & Control & Headwater & 11.222 & 0.481 & 10.279 & 12.166 \\
\hline Big Run & Aug-06 & Small Adult & Control & Headwater & 22.198 & 0.443 & 21.330 & 23.065 \\
\hline Big Run & Oct-06 & Small Adult & Control & Headwater & 18.243 & 1.234 & 15.825 & 20.661 \\
\hline Big Run & May-07 & Small Adult & Control & Headwater & 22.934 & 1.602 & 19.793 & 26.075 \\
\hline Big Run & Jul-07 & Small Adult & Control & Headwater & 8.083 & 0.277 & 7.540 & 8.626 \\
\hline Big Run & Oct-07 & Small Adult & Control & Headwater & 15.151 & 0.386 & 14.395 & 15.907 \\
\hline Swallow Rock & Jul-06 & Large Adult & Treatment & Headwater & 33.882 & 1.012 & 31.900 & 35.865 \\
\hline Swallow Rock & Aug-06 & Large Adult & Treatment & Headwater & 11.135 & 0.375 & 10.400 & 11.871 \\
\hline Swallow Rock & Oct-06 & Large Adult & Treatment & Headwater & 6.367 & 0.626 & 5.141 & 7.593 \\
\hline Swallow Rock & May-07 & Large Adult & Treatment & Headwater & 10.694 & 0.879 & 8.970 & 12.417 \\
\hline Swallow Rock & Jul-07 & Large Adult & Treatment & Headwater & 7.102 & 0.326 & 6.464 & 7.741 \\
\hline Swallow Rock & Oct-07 & Large Adult & Treatment & Headwater & 6.068 & 0.264 & 5.551 & 6.585 \\
\hline
\end{tabular}




\begin{tabular}{|c|c|c|c|c|c|c|c|c|}
\hline Site & Date & Age & Removal & Location & Estimate & SE & LCI & UCI \\
\hline Swallow Rock & Jul-06 & YOY & Treatment & Headwater & 11.228 & 0.490 & 10.268 & 12.188 \\
\hline Swallow Rock & Aug-06 & YOY & Treatment & Headwater & 17.218 & 0.475 & 16.287 & 18.148 \\
\hline Swallow Rock & Oct-06 & YOY & Treatment & Headwater & 22.720 & 1.501 & 19.777 & 25.663 \\
\hline Swallow Rock & May-07 & YOY & Treatment & Headwater & 14.064 & 1.113 & 11.882 & 16.247 \\
\hline Swallow Rock & Jul-07 & YOY & Treatment & Headwater & 58.884 & 0.988 & 56.948 & 60.821 \\
\hline Swallow Rock & Oct-07 & YOY & Treatment & Headwater & 41.544 & 0.757 & 40.061 & 43.027 \\
\hline Swallow Rock & Jul-06 & Small Adult & Treatment & Headwater & 8.163 & 0.411 & 7.358 & 8.967 \\
\hline Swallow Rock & Aug-06 & Small Adult & Treatment & Headwater & 26.233 & 0.481 & 25.291 & 27.175 \\
\hline Swallow Rock & Oct-06 & Small Adult & Treatment & Headwater & 9.681 & 0.894 & 7.929 & 11.433 \\
\hline Swallow Rock & May-07 & Small Adult & Treatment & Headwater & 17.457 & 1.358 & 14.796 & 20.118 \\
\hline Swallow Rock & Jul-07 & Small Adult & Treatment & Headwater & 21.220 & 0.456 & 20.327 & 22.113 \\
\hline Swallow Rock & Oct-07 & Small Adult & Treatment & Headwater & 9.090 & 0.297 & 8.508 & 9.672 \\
\hline Lynn Run & Jul-06 & Large Adult & Control & Mainstem & 4.109 & 0.335 & 3.452 & 4.766 \\
\hline Lynn Run & Aug-06 & Large Adult & Control & Mainstem & 4.052 & 0.230 & 3.602 & 4.502 \\
\hline Lynn Run & Oct-06 & Large Adult & Control & Mainstem & 2.116 & 0.344 & 1.441 & 2.791 \\
\hline Lynn Run & May-07 & Large Adult & Control & Mainstem & 6.415 & 0.671 & 5.099 & 7.730 \\
\hline Lynn Run & Jul-07 & Large Adult & Control & Mainstem & 3.044 & 0.211 & 2.630 & 3.457 \\
\hline Lynn Run & Oct-07 & Large Adult & Control & Mainstem & 2.023 & 0.153 & 1.724 & 2.322 \\
\hline Lynn Run & Jul-06 & YOY & Control & Mainstem & 5.106 & 0.331 & 4.457 & 5.755 \\
\hline Lynn Run & Aug-06 & YOY & Control & Mainstem & 2.027 & 0.164 & 1.705 & 2.349 \\
\hline Lynn Run & Oct-06 & YOY & Control & Mainstem & 9.707 & 0.901 & 7.942 & 11.473 \\
\hline Lynn Run & May-07 & YOY & Control & Mainstem & 52.998 & 2.352 & 48.388 & 57.607 \\
\hline Lynn Run & Jul-07 & YOY & Control & Mainstem & 20.304 & 0.562 & 19.203 & 21.404 \\
\hline Lynn Run & Oct-07 & YOY & Control & Mainstem & 33.443 & 0.682 & 32.107 & 34.780 \\
\hline Lynn Run & Jul-06 & Small Adult & Control & Mainstem & 11.229 & 0.491 & 10.267 & 12.191 \\
\hline Lynn Run & Aug-06 & Small Adult & Control & Mainstem & 5.048 & 0.215 & 4.627 & 5.469 \\
\hline Lynn Run & Oct-06 & Small Adult & Control & Mainstem & 3.217 & 0.487 & 2.262 & 4.172 \\
\hline Lynn Run & May-07 & Small Adult & Control & Mainstem & 4.363 & 0.640 & 3.109 & 5.617 \\
\hline Lynn Run & Jul-07 & Small Adult & Control & Mainstem & 3.031 & 0.170 & 2.699 & 3.363 \\
\hline Lynn Run & Oct-07 & Small Adult & Control & Mainstem & 10.102 & 0.316 & 9.483 & 10.721 \\
\hline Lick Drain & Jul-06 & Large Adult & Treatment & Mainstem & 12.349 & 0.611 & 11.151 & 13.546 \\
\hline Lick Drain & Aug-06 & Large Adult & Treatment & Mainstem & 4.057 & 0.238 & 3.591 & 4.522 \\
\hline
\end{tabular}




\begin{tabular}{|c|c|c|c|c|c|c|c|c|}
\hline Site & Date & Age & Removal & Location & Estimate & SE & $\mathrm{LCI}$ & $\mathrm{UCI}$ \\
\hline Lick Drain & Oct-06 & Large Adult & Treatment & Mainstem & 5.296 & 0.559 & 4.199 & 6.392 \\
\hline Lick Drain & May-07 & Large Adult & Treatment & Mainstem & 10.690 & 0.875 & 8.974 & 12.406 \\
\hline Lick Drain & Jul-07 & Large Adult & Treatment & Mainstem & 11.167 & 0.419 & 10.347 & 11.987 \\
\hline Lick Drain & Oct-07 & Large Adult & Treatment & Mainstem & 14.159 & 0.405 & 13.364 & 14.953 \\
\hline Lick Drain & Jul-06 & YOY & Treatment & Mainstem & 18.415 & 0.671 & 17.101 & 19.730 \\
\hline Lick Drain & Aug-06 & YOY & Treatment & Mainstem & 5.073 & 0.271 & 4.542 & 5.604 \\
\hline Lick Drain & Oct-06 & YOY & Treatment & Mainstem & 2.160 & 0.418 & 1.340 & 2.980 \\
\hline Lick Drain & May-07 & YOY & Treatment & Mainstem & 6.489 & 0.739 & 5.041 & 7.937 \\
\hline Lick Drain & Jul-07 & YOY & Treatment & Mainstem & 15.237 & 0.495 & 14.267 & 16.208 \\
\hline Lick Drain & Oct-07 & YOY & Treatment & Mainstem & 40.529 & 0.746 & 39.068 & 41.991 \\
\hline Lick Drain & Jul-06 & Small Adult & Treatment & Mainstem & 7.159 & 0.401 & 6.374 & 7.944 \\
\hline Lick Drain & Aug-06 & Small Adult & Treatment & Mainstem & 1.011 & 0.098 & 0.819 & 1.202 \\
\hline Lick Drain & Oct-06 & Small Adult & Treatment & Mainstem & 11.810 & 0.980 & 9.889 & 13.731 \\
\hline Lick Drain & May-07 & Small Adult & Treatment & Mainstem & 6.544 & 0.791 & 4.995 & 8.094 \\
\hline Lick Drain & Jul-07 & Small Adult & Treatment & Mainstem & 5.055 & 0.224 & 4.616 & 5.495 \\
\hline Lick Drain & Oct-07 & Small Adult & Treatment & Mainstem & 15.150 & 0.384 & 14.397 & 15.902 \\
\hline Mudlick Run & Jul-06 & Large Adult & Control & Headwater & 3.083 & 0.292 & 2.511 & 3.654 \\
\hline Mudlick Run & Aug-06 & Large Adult & Control & Headwater & 0.000 & 0.000 & 0.000 & 0.000 \\
\hline Mudlick Run & Oct-06 & Large Adult & Control & Headwater & 10.578 & 0.788 & 9.034 & 12.123 \\
\hline Mudlick Run & May-07 & Large Adult & Control & Headwater & 1.069 & 0.270 & 0.540 & 1.598 \\
\hline Mudlick Run & Jul-07 & Large Adult & Control & Headwater & 0.000 & 0.000 & 0.000 & 0.000 \\
\hline Mudlick Run & Oct-07 & Large Adult & Control & Headwater & 0.000 & 0.000 & 0.000 & 0.000 \\
\hline Mudlick Run & Jul-06 & YOY & Control & Headwater & 0.000 & 0.000 & 0.000 & 0.000 \\
\hline Mudlick Run & Aug-06 & YOY & Control & Headwater & 0.000 & 0.000 & 0.000 & 0.000 \\
\hline Mudlick Run & Oct-06 & YOY & Control & Headwater & 1.079 & 0.291 & 0.509 & 1.648 \\
\hline Mudlick Run & May-07 & YOY & Control & Headwater & 17.305 & 1.242 & 14.870 & 19.740 \\
\hline Mudlick Run & Jul-07 & YOY & Control & Headwater & 4.080 & 0.304 & 3.484 & 4.676 \\
\hline Mudlick Run & Oct-07 & YOY & Control & Headwater & 16.212 & 0.465 & 15.299 & 17.124 \\
\hline Mudlick Run & Jul-06 & Small Adult & Control & Headwater & 5.106 & 0.331 & 4.457 & 5.754 \\
\hline Mudlick Run & Aug-06 & Small Adult & Control & Headwater & 0.000 & 0.000 & 0.000 & 0.000 \\
\hline Mudlick Run & Oct-06 & Small Adult & Control & Headwater & 0.000 & 0.000 & 0.000 & 0.000 \\
\hline Mudlick Run & May-07 & Small Adult & Control & Headwater & 0.000 & 0.000 & 0.000 & 0.000 \\
\hline
\end{tabular}




\begin{tabular}{|c|c|c|c|c|c|c|c|c|}
\hline Site & Date & Age & Removal & Location & Estimate & SE & $\mathrm{LCl}$ & UCI \\
\hline Mudlick Run & Jul-07 & Small Adult & Control & Headwater & 0.000 & 0.000 & 0.000 & 0.000 \\
\hline Mudlick Run & Oct-07 & Small Adult & Control & Headwater & 11.110 & 0.328 & 10.467 & 11.752 \\
\hline Tanner Run & Jul-06 & Large Adult & Treatment & Headwater & 1.027 & 0.165 & 0.704 & 1.350 \\
\hline Tanner Run & Aug-06 & Large Adult & Treatment & Headwater & 2.025 & 0.158 & 1.714 & 2.335 \\
\hline Tanner Run & Oct-06 & Large Adult & Treatment & Headwater & 1.058 & 0.243 & 0.581 & 1.535 \\
\hline Tanner Run & May-07 & Large Adult & Treatment & Headwater & 5.344 & 0.609 & 4.151 & 6.537 \\
\hline Tanner Run & Jul-07 & Large Adult & Treatment & Headwater & 1.015 & 0.122 & 0.776 & 1.253 \\
\hline Tanner Run & Oct-07 & Large Adult & Treatment & Headwater & 2.025 & 0.157 & 1.718 & 2.333 \\
\hline Tanner Run & Jul-06 & YOY & Treatment & Headwater & 2.041 & 0.206 & 1.638 & 2.445 \\
\hline Tanner Run & Aug-06 & YOY & Treatment & Headwater & 3.039 & 0.197 & 2.652 & 3.426 \\
\hline Tanner Run & Oct-06 & YOY & Treatment & Headwater & 5.394 & 0.663 & 4.095 & 6.693 \\
\hline Tanner Run & May-07 & YOY & Treatment & Headwater & 0.000 & 0.000 & 0.000 & 0.000 \\
\hline Tanner Run & Jul-07 & YOY & Treatment & Headwater & 2.030 & 0.176 & 1.686 & 2.375 \\
\hline Tanner Run & Oct-07 & YOY & Treatment & Headwater & 11.161 & 0.406 & 10.366 & 11.956 \\
\hline Tanner Run & Jul-06 & Small Adult & Treatment & Headwater & 4.081 & 0.289 & 3.515 & 4.648 \\
\hline Tanner Run & Aug-06 & Small Adult & Treatment & Headwater & 4.036 & 0.187 & 3.670 & 4.402 \\
\hline Tanner Run & Oct-06 & Small Adult & Treatment & Headwater & 7.507 & 0.758 & 6.022 & 8.993 \\
\hline Tanner Run & May-07 & Small Adult & Treatment & Headwater & 4.362 & 0.638 & 3.112 & 5.612 \\
\hline Tanner Run & Jul-07 & Small Adult & Treatment & Headwater & 1.010 & 0.098 & 0.818 & 1.203 \\
\hline Tanner Run & Oct-07 & Small Adult & Treatment & Headwater & 3.034 & 0.178 & 2.685 & 3.383 \\
\hline Zinn Hollow & Jul-06 & Large Adult & Control & Mainstem & 1.027 & 0.163 & 0.706 & 1.347 \\
\hline Zinn Hollow & Aug-06 & Large Adult & Control & Mainstem & 0.000 & 0.000 & 0.000 & 0.000 \\
\hline Zinn Hollow & Oct-06 & Large Adult & Control & Mainstem & 2.121 & 0.358 & 1.421 & 2.822 \\
\hline Zinn Hollow & Jul-07 & Large Adult & Control & Mainstem & 5.073 & 0.274 & 4.536 & 5.609 \\
\hline Zinn Hollow & Oct-07 & Large Adult & Control & Mainstem & 5.058 & 0.242 & 4.583 & 5.532 \\
\hline Zinn Hollow & Jul-06 & YOY & Control & Mainstem & 1.021 & 0.144 & 0.738 & 1.303 \\
\hline Zinn Hollow & Aug-06 & YOY & Control & Mainstem & 4.051 & 0.226 & 3.608 & 4.495 \\
\hline Zinn Hollow & Oct-06 & YOY & Control & Mainstem & 2.163 & 0.426 & 1.327 & 2.998 \\
\hline Zinn Hollow & Jul-07 & YOY & Control & Mainstem & 17.258 & 0.517 & 16.245 & 18.272 \\
\hline Zinn Hollow & Oct-07 & YOY & Control & Mainstem & 30.403 & 0.649 & 29.131 & 31.676 \\
\hline Zinn Hollow & Jul-06 & Small Adult & Control & Mainstem & 1.020 & 0.142 & 0.741 & 1.299 \\
\hline Zinn Hollow & Aug-06 & Small Adult & Control & Mainstem & 1.009 & 0.092 & 0.828 & 1.190 \\
\hline
\end{tabular}




\begin{tabular}{|c|c|c|c|c|c|c|c|c|}
\hline Site & Date & Age & Removal & Location & Estimate & SE & $\mathrm{LCl}$ & UCI \\
\hline Zinn Hollow & Oct-06 & Small Adult & Control & Mainstem & 2.150 & 0.409 & 1.348 & 2.952 \\
\hline Zinn Hollow & Jul-07 & Small Adult & Control & Mainstem & 1.010 & 0.098 & 0.819 & 1.202 \\
\hline Zinn Hollow & Oct-07 & Small Adult & Control & Mainstem & 4.041 & 0.199 & 3.651 & 4.431 \\
\hline Laurel Lick Run & Jul-06 & Large Adult & Treatment & Mainstem & 1.027 & 0.163 & 0.706 & 1.347 \\
\hline Laurel Lick Run & Aug-06 & Large Adult & Treatment & Mainstem & 4.049 & 0.223 & 3.612 & 4.486 \\
\hline Laurel Lick Run & Oct-06 & Large Adult & Treatment & Mainstem & 3.182 & 0.442 & 2.316 & 4.048 \\
\hline Laurel Lick Run & May-07 & Large Adult & Treatment & Mainstem & 4.278 & 0.548 & 3.204 & 5.351 \\
\hline Laurel Lick Run & Jul-07 & Large Adult & Treatment & Mainstem & 4.059 & 0.247 & 3.576 & 4.542 \\
\hline Laurel Lick Run & Oct-07 & Large Adult & Treatment & Mainstem & 9.104 & 0.327 & 8.463 & 9.746 \\
\hline Laurel Lick Run & Jul-06 & YOY & Treatment & Mainstem & 0.000 & 0.000 & 0.000 & 0.000 \\
\hline Laurel Lick Run & Aug-06 & YOY & Treatment & Mainstem & 0.000 & 0.000 & 0.000 & 0.000 \\
\hline Laurel Lick Run & Oct-06 & YOY & Treatment & Mainstem & 0.000 & 0.000 & 0.000 & 0.000 \\
\hline Laurel Lick Run & May-07 & YOY & Treatment & Mainstem & 2.164 & 0.424 & 1.333 & 2.994 \\
\hline Laurel Lick Run & Jul-07 & YOY & Treatment & Mainstem & 2.031 & 0.177 & 1.685 & 2.377 \\
\hline Laurel Lick Run & Oct-07 & YOY & Treatment & Mainstem & 6.081 & 0.287 & 5.519 & 6.643 \\
\hline Laurel Lick Run & Jul-06 & Small Adult & Treatment & Mainstem & 0.000 & 0.000 & 0.000 & 0.000 \\
\hline Laurel Lick Run & Aug-06 & Small Adult & Treatment & Mainstem & 4.036 & 0.185 & 3.674 & 4.398 \\
\hline Laurel Lick Run & Oct-06 & Small Adult & Treatment & Mainstem & 3.225 & 0.506 & 2.234 & 4.216 \\
\hline Laurel Lick Run & May-07 & Small Adult & Treatment & Mainstem & 3.273 & 0.553 & 2.189 & 4.358 \\
\hline Laurel Lick Run & Jul-07 & Small Adult & Treatment & Mainstem & 0.000 & 0.000 & 0.000 & 0.000 \\
\hline Laurel Lick Run & Oct-07 & Small Adult & Treatment & Mainstem & 5.051 & 0.223 & 4.614 & 5.489 \\
\hline Glady Fork & Jul-06 & Large Adult & Control & Headwater & 2.057 & 0.242 & 1.583 & 2.531 \\
\hline Glady Fork & Aug-06 & Large Adult & Control & Headwater & 0.000 & 0.000 & 0.000 & 0.000 \\
\hline Glady Fork & Oct-06 & Large Adult & Control & Headwater & 6.347 & 0.603 & 5.165 & 7.528 \\
\hline Glady Fork & May-07 & Large Adult & Control & Headwater & 3.207 & 0.471 & 2.285 & 4.130 \\
\hline Glady Fork & Jul-07 & Large Adult & Control & Headwater & 3.044 & 0.210 & 2.631 & 3.456 \\
\hline Glady Fork & Oct-07 & Large Adult & Control & Headwater & 0.000 & 0.000 & 0.000 & 0.000 \\
\hline Glady Fork & Jul-06 & YOY & Control & Headwater & 0.000 & 0.000 & 0.000 & 0.000 \\
\hline Glady Fork & Aug-06 & YOY & Control & Headwater & 15.197 & 0.454 & 14.308 & 16.087 \\
\hline Glady Fork & Oct-06 & YOY & Control & Headwater & 2.157 & 0.412 & 1.349 & 2.965 \\
\hline Glady Fork & May-07 & YOY & Control & Headwater & 19.469 & 1.325 & 16.872 & 22.067 \\
\hline Glady Fork & Jul-07 & YOY & Control & Headwater & 28.424 & 0.667 & 27.117 & 29.730 \\
\hline
\end{tabular}




\begin{tabular}{|c|c|c|c|c|c|c|c|c|}
\hline Site & Date & Age & Removal & Location & Estimate & SE & $\mathrm{LCl}$ & UCI \\
\hline Glady Fork & Oct-07 & YOY & Control & Headwater & 12.158 & 0.401 & 11.372 & 12.944 \\
\hline Glady Fork & Jul-06 & Small Adult & Control & Headwater & 3.066 & 0.262 & 2.553 & 3.579 \\
\hline Glady Fork & Aug-06 & Small Adult & Control & Headwater & 4.037 & 0.189 & 3.667 & 4.408 \\
\hline Glady Fork & Oct-06 & Small Adult & Control & Headwater & 6.434 & 0.696 & 5.070 & 7.798 \\
\hline Glady Fork & May-07 & Small Adult & Control & Headwater & 3.273 & 0.552 & 2.191 & 4.354 \\
\hline Glady Fork & Jul-07 & Small Adult & Control & Headwater & 1.010 & 0.097 & 0.819 & 1.201 \\
\hline Glady Fork & Oct-07 & Small Adult & Control & Headwater & 5.050 & 0.220 & 4.619 & 5.480 \\
\hline Daniel's Run & Jul-06 & Large Adult & Treatment & Headwater & 3.083 & 0.293 & 2.508 & 3.657 \\
\hline Daniel's Run & Aug-06 & Large Adult & Treatment & Headwater & 5.064 & 0.255 & 4.564 & 5.563 \\
\hline Daniel's Run & Oct-06 & Large Adult & Treatment & Headwater & 1.059 & 0.245 & 0.578 & 1.540 \\
\hline Daniel's Run & May-07 & Large Adult & Treatment & Headwater & 4.292 & 0.565 & 3.185 & 5.400 \\
\hline Daniel's Run & Jul-07 & Large Adult & Treatment & Headwater & 2.037 & 0.189 & 1.668 & 2.407 \\
\hline Daniel's Run & Oct-07 & Large Adult & Treatment & Headwater & 4.045 & 0.214 & 3.625 & 4.465 \\
\hline Daniel's Run & Jul-06 & YOY & Treatment & Headwater & 1.022 & 0.148 & 0.731 & 1.312 \\
\hline Daniel's Run & Aug-06 & YOY & Treatment & Headwater & 50.661 & 0.864 & 48.968 & 52.353 \\
\hline Daniel's Run & Oct-06 & YOY & Treatment & Headwater & 9.715 & 0.911 & 7.929 & 11.501 \\
\hline Daniel's Run & May-07 & YOY & Treatment & Headwater & 21.712 & 1.490 & 18.791 & 24.633 \\
\hline Daniel's Run & Jul-07 & YOY & Treatment & Headwater & 8.155 & 0.408 & 7.354 & 8.955 \\
\hline Daniel's Run & Oct-07 & YOY & Treatment & Headwater & 10.132 & 0.367 & 9.413 & 10.851 \\
\hline Daniel's Run & Jul-06 & Small Adult & Treatment & Headwater & 0.000 & 0.000 & 0.000 & 0.000 \\
\hline Daniel's Run & Aug-06 & Small Adult & Treatment & Headwater & 4.037 & 0.189 & 3.667 & 4.408 \\
\hline Daniel's Run & Oct-06 & Small Adult & Treatment & Headwater & 12.879 & 1.021 & 10.877 & 14.880 \\
\hline Daniel's Run & May-07 & Small Adult & Treatment & Headwater & 2.190 & 0.460 & 1.289 & 3.090 \\
\hline Daniel's Run & Jul-07 & Small Adult & Treatment & Headwater & 1.015 & 0.107 & 0.804 & 1.225 \\
\hline Daniel's Run & Oct-07 & Small Adult & Treatment & Headwater & 3.030 & 0.170 & 2.696 & 3.364 \\
\hline
\end{tabular}


Appendix A6. Summary of model-averaged parameter estimates, standard errors, and 95\% confidence intervals (i.e., lower $=$ LCI and upper $=\mathrm{UCI}$ ) of abundance for three size classes of mottled sculpin for all sites and sample dates. Classifications of sites based on treatment and spatial location are also noted.

\begin{tabular}{|c|c|c|c|c|c|c|c|c|}
\hline Site & Date & Age & Removal & Location & Estimate & SE & LCI & $\mathrm{UCI}$ \\
\hline Nan's Branch & Jul-06 & Large Adult & Control & Mainstem & 54.501 & 2.810 & 51.141 & 63.134 \\
\hline Nan's Branch & Aug-06 & Large Adult & Control & Mainstem & 56.458 & 2.512 & 53.593 & 64.476 \\
\hline Nan's Branch & Oct-06 & Large Adult & Control & Mainstem & 236.366 & 13.132 & 216.180 & 268.833 \\
\hline Nan's Branch & May-07 & Large Adult & Control & Mainstem & 200.049 & 9.803 & 185.150 & 224.536 \\
\hline Nan's Branch & Jul-07 & Large Adult & Control & Mainstem & 62.027 & 2.928 & 58.445 & 70.859 \\
\hline Nan's Branch & Oct-07 & Large Adult & Control & Mainstem & 82.686 & 5.186 & 75.501 & 96.756 \\
\hline Nan's Branch & Jul-06 & YOY & Control & Mainstem & 1.193 & 0.483 & 1.012 & 4.051 \\
\hline Nan's Branch & Aug-06 & YOY & Control & Mainstem & 0.000 & 0.000 & 0.000 & 0.000 \\
\hline Nan's Branch & Oct-06 & YOY & Control & Mainstem & 77.528 & 8.261 & 65.902 & 99.633 \\
\hline Nan's Branch & May-07 & YOY & Control & Mainstem & 39.996 & 4.702 & 33.925 & 53.549 \\
\hline Nan's Branch & Jul-07 & YOY & Control & Mainstem & 1.186 & 0.472 & 1.012 & 3.991 \\
\hline Nan's Branch & Oct-07 & YOY & Control & Mainstem & 74.308 & 7.059 & 64.643 & 93.659 \\
\hline Nan's Branch & Jul-06 & Small Adult & Control & Mainstem & 17.796 & 1.479 & 16.438 & 23.358 \\
\hline Nan's Branch & Aug-06 & Small Adult & Control & Mainstem & 8.686 & 0.883 & 8.099 & 12.761 \\
\hline Nan's Branch & Oct-06 & Small Adult & Control & Mainstem & 67.164 & 5.286 & 59.808 & 81.450 \\
\hline Nan's Branch & May-07 & Small Adult & Control & Mainstem & 106.199 & 6.228 & 97.190 & 122.461 \\
\hline Nan's Branch & Jul-07 & Small Adult & Control & Mainstem & 29.906 & 1.913 & 27.896 & 36.421 \\
\hline Nan's Branch & Oct-07 & Small Adult & Control & Mainstem & 43.775 & 3.462 & 39.378 & 53.896 \\
\hline Otis Hollow & Jul-06 & Large Adult & Treatment & Mainstem & 19.198 & 1.237 & 18.225 & 24.365 \\
\hline Otis Hollow & Aug-06 & Large Adult & Treatment & Mainstem & 1.050 & 0.230 & 1.002 & 2.573 \\
\hline Otis Hollow & Oct-06 & Large Adult & Treatment & Mainstem & 55.796 & 4.465 & 50.441 & 69.484 \\
\hline Otis Hollow & May-07 & Large Adult & Treatment & Mainstem & 22.962 & 2.141 & 20.831 & 30.557 \\
\hline Otis Hollow & Jul-07 & Large Adult & Treatment & Mainstem & 22.335 & 1.312 & 21.267 & 27.690 \\
\hline Otis Hollow & Oct-07 & Large Adult & Treatment & Mainstem & 11.352 & 1.341 & 10.267 & 16.846 \\
\hline Otis Hollow & Jul-06 & YOY & Treatment & Mainstem & 2.239 & 0.528 & 2.018 & 5.249 \\
\hline Otis Hollow & Aug-06 & YOY & Treatment & Mainstem & 0.000 & 0.000 & 0.000 & 0.000 \\
\hline Otis Hollow & Oct-06 & YOY & Treatment & Mainstem & 6.539 & 1.523 & 5.305 & 12.774 \\
\hline Otis Hollow & May-07 & YOY & Treatment & Mainstem & 6.242 & 1.333 & 5.223 & 11.907 \\
\hline
\end{tabular}




\begin{tabular}{|c|c|c|c|c|c|c|c|c|}
\hline Site & Date & Age & Removal & Location & Estimate & SE & $\mathrm{LCl}$ & $\mathrm{UCI}$ \\
\hline Otis Hollow & Jul-07 & YOY & Treatment & Mainstem & 1.115 & 0.361 & 1.006 & 3.374 \\
\hline Otis Hollow & Oct-07 & YOY & Treatment & Mainstem & 1.229 & 0.538 & 1.016 & 4.350 \\
\hline Otis Hollow & Jul-06 & Small Adult & Treatment & Mainstem & 12.799 & 0.982 & 12.122 & 17.240 \\
\hline Otis Hollow & Aug-06 & Small Adult & Treatment & Mainstem & 1.050 & 0.230 & 1.002 & 2.573 \\
\hline Otis Hollow & Oct-06 & Small Adult & Treatment & Mainstem & 9.497 & 1.433 & 8.308 & 15.271 \\
\hline Otis Hollow & May-07 & Small Adult & Treatment & Mainstem & 10.333 & 1.330 & 9.261 & 15.801 \\
\hline Otis Hollow & Jul-07 & Small Adult & Treatment & Mainstem & 12.763 & 0.954 & 12.114 & 17.110 \\
\hline Otis Hollow & Oct-07 & Small Adult & Treatment & Mainstem & 7.947 & 1.097 & 7.155 & 12.776 \\
\hline Big Run & Jul-06 & Large Adult & Control & Headwater & 13.327 & 1.276 & 12.272 & 18.481 \\
\hline Big Run & Aug-06 & Large Adult & Control & Headwater & 21.688 & 1.460 & 20.390 & 27.305 \\
\hline Big Run & Oct-06 & Large Adult & Control & Headwater & 77.274 & 6.304 & 68.639 & 94.543 \\
\hline Big Run & May-07 & Large Adult & Control & Headwater & 107.164 & 6.795 & 97.602 & 125.348 \\
\hline Big Run & Jul-07 & Large Adult & Control & Headwater & 56.406 & 2.867 & 53.038 & 65.343 \\
\hline Big Run & Oct-07 & Large Adult & Control & Headwater & 72.785 & 5.003 & 66.101 & 86.792 \\
\hline Big Run & Jul-06 & YOY & Control & Headwater & 0.000 & 0.000 & 0.000 & 0.000 \\
\hline Big Run & Aug-06 & YOY & Control & Headwater & 1.149 & 0.417 & 1.008 & 3.688 \\
\hline Big Run & Oct-06 & YOY & Control & Headwater & 5.829 & 1.705 & 4.388 & 12.615 \\
\hline Big Run & May-07 & YOY & Control & Headwater & 79.725 & 7.988 & 68.810 & 101.659 \\
\hline Big Run & Jul-07 & YOY & Control & Headwater & 9.464 & 1.400 & 8.302 & 15.106 \\
\hline Big Run & Oct-07 & YOY & Control & Headwater & 10.774 & 2.079 & 8.749 & 18.267 \\
\hline Big Run & Jul-06 & Small Adult & Control & Headwater & 2.221 & 0.500 & 2.016 & 5.089 \\
\hline Big Run & Aug-06 & Small Adult & Control & Headwater & 3.253 & 0.530 & 3.020 & 6.222 \\
\hline Big Run & Oct-06 & Small Adult & Control & Headwater & 12.879 & 2.048 & 10.821 & 20.090 \\
\hline Big Run & May-07 & Small Adult & Control & Headwater & 35.721 & 3.263 & 31.728 & 45.560 \\
\hline Big Run & Jul-07 & Small Adult & Control & Headwater & 34.286 & 2.112 & 32.038 & 41.406 \\
\hline Big Run & Oct-07 & Small Adult & Control & Headwater & 64.293 & 4.597 & 58.242 & 77.327 \\
\hline Swallow Rock & Jul-06 & Large Adult & Treatment & Headwater & 13.256 & 1.265 & 12.244 & 18.475 \\
\hline Swallow Rock & Aug-06 & Large Adult & Treatment & Headwater & 11.877 & 1.035 & 11.141 & 16.468 \\
\hline Swallow Rock & Oct-06 & Large Adult & Treatment & Headwater & 22.947 & 2.931 & 19.688 & 32.496 \\
\hline Swallow Rock & May-07 & Large Adult & Treatment & Headwater & 34.183 & 3.352 & 30.286 & 44.723 \\
\hline Swallow Rock & Jul-07 & Large Adult & Treatment & Headwater & 48.414 & 2.648 & 45.489 & 57.085 \\
\hline Swallow Rock & Oct-07 & Large Adult & Treatment & Headwater & 31.275 & 3.041 & 27.846 & 41.072 \\
\hline
\end{tabular}




\begin{tabular}{|c|c|c|c|c|c|c|c|c|}
\hline Site & Date & Age & Removal & Location & Estimate & SE & $\mathrm{LCl}$ & $\mathrm{UCl}$ \\
\hline Swallow Rock & Jul-06 & YOY & Treatment & Headwater & 0.000 & 0.000 & 0.000 & 0.000 \\
\hline Swallow Rock & Aug-06 & YOY & Treatment & Headwater & 0.000 & 0.000 & 0.000 & 0.000 \\
\hline Swallow Rock & Oct-06 & YOY & Treatment & Headwater & 7.191 & 1.906 & 5.503 & 14.542 \\
\hline Swallow Rock & May-07 & YOY & Treatment & Headwater & 12.225 & 2.347 & 9.898 & 20.573 \\
\hline Swallow Rock & Jul-07 & YOY & Treatment & Headwater & 3.522 & 0.806 & 3.060 & 7.544 \\
\hline Swallow Rock & Oct-07 & YOY & Treatment & Headwater & 1.331 & 0.674 & 1.027 & 5.064 \\
\hline Swallow Rock & Jul-06 & Small Adult & Treatment & Headwater & 6.628 & 0.864 & 6.083 & 10.736 \\
\hline Swallow Rock & Aug-06 & Small Adult & Treatment & Headwater & 6.478 & 0.744 & 6.054 & 10.201 \\
\hline Swallow Rock & Oct-06 & Small Adult & Treatment & Headwater & 7.649 & 1.535 & 6.351 & 13.756 \\
\hline Swallow Rock & May-07 & Small Adult & Treatment & Headwater & 10.987 & 1.676 & 9.473 & 17.357 \\
\hline Swallow Rock & Jul-07 & Small Adult & Treatment & Headwater & 56.116 & 2.920 & 52.807 & 65.484 \\
\hline Swallow Rock & Oct-07 & Small Adult & Treatment & Headwater & 7.217 & 1.272 & 6.226 & 12.559 \\
\hline Lynn Run & Jul-06 & Large Adult & Control & Mainstem & 103.545 & 1.753 & 101.750 & 109.634 \\
\hline Lynn Run & Aug-06 & Large Adult & Control & Mainstem & 65.161 & 1.147 & 64.231 & 69.851 \\
\hline Lynn Run & Oct-06 & Large Adult & Control & Mainstem & 75.725 & 2.818 & 72.298 & 84.265 \\
\hline Lynn Run & May-07 & Large Adult & Control & Mainstem & 73.318 & 2.367 & 70.581 & 80.793 \\
\hline Lynn Run & Jul-07 & Large Adult & Control & Mainstem & 66.556 & 1.326 & 65.365 & 71.624 \\
\hline Lynn Run & Oct-07 & Large Adult & Control & Mainstem & 45.426 & 1.713 & 43.697 & 51.440 \\
\hline Lynn Run & Jul-06 & YOY & Control & Mainstem & 0.000 & 0.000 & 0.000 & 0.000 \\
\hline Lynn Run & Aug-06 & YOY & Control & Mainstem & 2.072 & 0.275 & 2.003 & 3.855 \\
\hline Lynn Run & Oct-06 & YOY & Control & Mainstem & 38.910 & 2.730 & 35.776 & 47.578 \\
\hline Lynn Run & May-07 & YOY & Control & Mainstem & 0.000 & 0.000 & 0.000 & 0.000 \\
\hline Lynn Run & Jul-07 & YOY & Control & Mainstem & 0.000 & 0.000 & 0.000 & 0.000 \\
\hline Lynn Run & Oct-07 & YOY & Control & Mainstem & 17.645 & 1.438 & 16.376 & 23.200 \\
\hline Lynn Run & Jul-06 & Small Adult & Control & Mainstem & 43.058 & 1.080 & 42.202 & 47.540 \\
\hline Lynn Run & Aug-06 & Small Adult & Control & Mainstem & 19.345 & 0.602 & 19.034 & 22.502 \\
\hline Lynn Run & Oct-06 & Small Adult & Control & Mainstem & 28.127 & 1.594 & 26.574 & 33.873 \\
\hline Lynn Run & May-07 & Small Adult & Control & Mainstem & 65.880 & 2.223 & 63.366 & 73.025 \\
\hline Lynn Run & Jul-07 & Small Adult & Control & Mainstem & 48.125 & 1.113 & 47.223 & 52.680 \\
\hline Lynn Run & Oct-07 & Small Adult & Control & Mainstem & 10.564 & 0.785 & 10.074 & 14.313 \\
\hline Lick Drain & Jul-06 & Large Adult & Treatment & Mainstem & 93.875 & 2.214 & 91.368 & 100.980 \\
\hline Lick Drain & Aug-06 & Large Adult & Treatment & Mainstem & 23.727 & 0.894 & 23.111 & 27.770 \\
\hline
\end{tabular}




\begin{tabular}{|c|c|c|c|c|c|c|c|c|}
\hline Site & Date & Age & Removal & Location & Estimate & SE & $\mathrm{LCl}$ & $\mathrm{UCl}$ \\
\hline Lick Drain & Oct-06 & Large Adult & Treatment & Mainstem & 88.083 & 4.008 & 82.760 & 99.359 \\
\hline Lick Drain & May-07 & Large Adult & Treatment & Mainstem & 46.232 & 2.350 & 43.532 & 53.690 \\
\hline Lick Drain & Jul-07 & Large Adult & Treatment & Mainstem & 65.584 & 1.745 & 63.777 & 71.592 \\
\hline Lick Drain & Oct-07 & Large Adult & Treatment & Mainstem & 92.775 & 3.295 & 88.506 & 102.245 \\
\hline Lick Drain & Jul-06 & YOY & Treatment & Mainstem & 2.161 & 0.420 & 2.010 & 4.672 \\
\hline Lick Drain & Aug-06 & YOY & Treatment & Mainstem & 2.121 & 0.361 & 2.006 & 4.353 \\
\hline Lick Drain & Oct-06 & YOY & Treatment & Mainstem & 29.266 & 2.874 & 25.936 & 38.326 \\
\hline Lick Drain & May-07 & YOY & Treatment & Mainstem & 4.699 & 0.926 & 4.097 & 9.031 \\
\hline Lick Drain & Jul-07 & YOY & Treatment & Mainstem & 0.000 & 0.000 & 0.000 & 0.000 \\
\hline Lick Drain & Oct-07 & YOY & Treatment & Mainstem & 51.039 & 3.396 & 46.871 & 61.256 \\
\hline Lick Drain & Jul-06 & Small Adult & Treatment & Mainstem & 75.100 & 1.945 & 73.002 & 81.588 \\
\hline Lick Drain & Aug-06 & Small Adult & Treatment & Mainstem & 9.285 & 0.549 & 9.025 & 12.269 \\
\hline Lick Drain & Oct-06 & Small Adult & Treatment & Mainstem & 18.068 & 1.591 & 16.543 & 23.871 \\
\hline Lick Drain & May-07 & Small Adult & Treatment & Mainstem & 72.650 & 3.075 & 68.807 & 81.757 \\
\hline Lick Drain & Jul-07 & Small Adult & Treatment & Mainstem & 122.841 & 2.507 & 119.862 & 130.586 \\
\hline Lick Drain & Oct-07 & Small Adult & Treatment & Mainstem & 133.160 & 4.131 & 127.529 & 144.526 \\
\hline Mudlick Run & Jul-06 & Large Adult & Control & Headwater & 78.001 & 1.533 & 76.528 & 83.579 \\
\hline Mudlick Run & Aug-06 & Large Adult & Control & Headwater & 40.760 & 0.913 & 40.119 & 44.844 \\
\hline Mudlick Run & Oct-06 & Large Adult & Control & Headwater & 75.946 & 2.868 & 72.426 & 84.572 \\
\hline Mudlick Run & May-07 & Large Adult & Control & Headwater & 61.775 & 2.171 & 59.324 & 68.764 \\
\hline Mudlick Run & Jul-07 & Large Adult & Control & Headwater & 26.650 & 0.834 & 26.094 & 30.493 \\
\hline Mudlick Run & Oct-07 & Large Adult & Control & Headwater & 68.816 & 2.206 & 66.332 & 75.929 \\
\hline Mudlick Run & Jul-06 & YOY & Control & Headwater & 3.153 & 0.405 & 3.009 & 5.584 \\
\hline Mudlick Run & Aug-06 & YOY & Control & Headwater & 0.000 & 0.000 & 0.000 & 0.000 \\
\hline Mudlick Run & Oct-06 & YOY & Control & Headwater & 28.737 & 2.305 & 26.228 & 36.375 \\
\hline Mudlick Run & May-07 & YOY & Control & Headwater & 53.626 & 2.973 & 50.127 & 62.882 \\
\hline Mudlick Run & Jul-07 & YOY & Control & Headwater & 9.438 & 0.695 & 9.049 & 12.945 \\
\hline Mudlick Run & Oct-07 & YOY & Control & Headwater & 11.067 & 1.131 & 10.195 & 15.847 \\
\hline Mudlick Run & Jul-06 & Small Adult & Control & Headwater & 49.264 & 1.190 & 48.265 & 54.021 \\
\hline Mudlick Run & Aug-06 & Small Adult & Control & Headwater & 15.285 & 0.546 & 15.025 & 18.251 \\
\hline Mudlick Run & Oct-06 & Small Adult & Control & Headwater & 39.058 & 1.946 & 36.975 & 45.589 \\
\hline Mudlick Run & May-07 & Small Adult & Control & Headwater & 67.101 & 2.277 & 64.484 & 74.329 \\
\hline
\end{tabular}




\begin{tabular}{|c|c|c|c|c|c|c|c|c|}
\hline Site & Date & Age & Removal & Location & Estimate & SE & LCI & $\mathrm{UCl}$ \\
\hline Mudlick Run & Jul-07 & Small Adult & Control & Headwater & 44.076 & 1.087 & 43.208 & 48.568 \\
\hline Mudlick Run & Oct-07 & Small Adult & Control & Headwater & 79.403 & 2.400 & 76.620 & 86.964 \\
\hline Tanner Run & Jul-06 & Large Adult & Treatment & Headwater & 39.707 & 1.407 & 38.416 & 45.000 \\
\hline Tanner Run & Aug-06 & Large Adult & Treatment & Headwater & 35.124 & 1.128 & 34.219 & 39.773 \\
\hline Tanner Run & Oct-06 & Large Adult & Treatment & Headwater & 102.064 & 4.489 & 95.956 & 114.436 \\
\hline Tanner Run & May-07 & Large Adult & Treatment & Headwater & 89.475 & 3.534 & 84.867 & 99.575 \\
\hline Tanner Run & Jul-07 & Large Adult & Treatment & Headwater & 63.612 & 1.760 & 61.787 & 69.666 \\
\hline Tanner Run & Oct-07 & Large Adult & Treatment & Headwater & 96.364 & 3.528 & 91.784 & 106.489 \\
\hline Tanner Run & Jul-06 & YOY & Treatment & Headwater & 1.083 & 0.302 & 1.003 & 3.024 \\
\hline Tanner Run & Aug-06 & YOY & Treatment & Headwater & 0.000 & 0.000 & 0.000 & 0.000 \\
\hline Tanner Run & Oct-06 & YOY & Treatment & Headwater & 15.948 & 2.050 & 13.861 & 23.097 \\
\hline Tanner Run & May-07 & YOY & Treatment & Headwater & 57.862 & 4.040 & 52.781 & 69.770 \\
\hline Tanner Run & Jul-07 & YOY & Treatment & Headwater & 4.320 & 0.596 & 4.029 & 7.526 \\
\hline Tanner Run & Oct-07 & YOY & Treatment & Headwater & 2.331 & 0.628 & 2.029 & 5.728 \\
\hline Tanner Run & Jul-06 & Small Adult & Treatment & Headwater & 39.707 & 1.407 & 38.416 & 45.000 \\
\hline Tanner Run & Aug-06 & Small Adult & Treatment & Headwater & 19.628 & 0.827 & 19.088 & 23.483 \\
\hline Tanner Run & Oct-06 & Small Adult & Treatment & Headwater & 34.021 & 2.298 & 31.419 & 41.398 \\
\hline Tanner Run & May-07 & Small Adult & Treatment & Headwater & 55.232 & 2.640 & 52.057 & 63.306 \\
\hline Tanner Run & Jul-07 & Small Adult & Treatment & Headwater & 91.768 & 2.170 & 89.320 & 98.757 \\
\hline Tanner Run & Oct-07 & Small Adult & Treatment & Headwater & 96.364 & 3.528 & 91.784 & 106.489 \\
\hline Zinn Hollow & Jul-06 & Large Adult & Control & Mainstem & 9.494 & 0.754 & 9.058 & 13.240 \\
\hline Zinn Hollow & Aug-06 & Large Adult & Control & Mainstem & 22.856 & 2.525 & 20.196 & 31.434 \\
\hline Zinn Hollow & Oct-06 & Large Adult & Control & Mainstem & 51.089 & 3.728 & 46.692 & 62.672 \\
\hline Zinn Hollow & May-07 & Large Adult & Control & Mainstem & 48.000 & 0.000 & 0.000 & 0.000 \\
\hline Zinn Hollow & Jul-07 & Large Adult & Control & Mainstem & 14.980 & 1.094 & 14.168 & 19.716 \\
\hline Zinn Hollow & Oct-07 & Large Adult & Control & Mainstem & 24.094 & 2.179 & 21.892 & 31.731 \\
\hline Zinn Hollow & Jul-06 & YOY & Control & Mainstem & 0.000 & 0.000 & 0.000 & 0.000 \\
\hline Zinn Hollow & Aug-06 & YOY & Control & Mainstem & 1.332 & 0.675 & 1.027 & 5.071 \\
\hline Zinn Hollow & Oct-06 & YOY & Control & Mainstem & 0.000 & 0.000 & 0.000 & 0.000 \\
\hline Zinn Hollow & May-07 & YOY & Control & Mainstem & 16.000 & 0.000 & 0.000 & 0.000 \\
\hline Zinn Hollow & Jul-07 & YOY & Control & Mainstem & 0.000 & 0.000 & 0.000 & 0.000 \\
\hline Zinn Hollow & Oct-07 & YOY & Control & Mainstem & 21.203 & 2.770 & 18.296 & 30.638 \\
\hline
\end{tabular}




\begin{tabular}{|c|c|c|c|c|c|c|c|c|}
\hline Site & Date & Age & Removal & Location & Estimate & SE & LCI & $\mathrm{UCI}$ \\
\hline Zinn Hollow & Jul-06 & Small Adult & Control & Mainstem & 1.055 & 0.242 & 1.002 & 2.652 \\
\hline Zinn Hollow & Aug-06 & Small Adult & Control & Mainstem & 10.827 & 1.608 & 9.414 & 17.064 \\
\hline Zinn Hollow & Oct-06 & Small Adult & Control & Mainstem & 6.967 & 1.108 & 6.161 & 11.820 \\
\hline Zinn Hollow & May-07 & Small Adult & Control & Mainstem & 18.000 & 0.000 & 0.000 & 0.000 \\
\hline Zinn Hollow & Jul-07 & Small Adult & Control & Mainstem & 6.420 & 0.690 & 6.045 & 9.955 \\
\hline Zinn Hollow & Oct-07 & Small Adult & Control & Mainstem & 4.589 & 0.848 & 4.074 & 8.700 \\
\hline Laurel Lick Run & Jul-06 & Large Adult & Treatment & Mainstem & 2.087 & 0.303 & 2.004 & 4.022 \\
\hline Laurel Lick Run & Aug-06 & Large Adult & Treatment & Mainstem & 72.402 & 4.575 & 66.562 & 85.718 \\
\hline Laurel Lick Run & Oct-06 & Large Adult & Treatment & Mainstem & 65.666 & 3.669 & 61.147 & 76.669 \\
\hline Laurel Lick Run & May-07 & Large Adult & Treatment & Mainstem & 33.787 & 1.501 & 32.427 & 39.485 \\
\hline Laurel Lick Run & Jul-07 & Large Adult & Treatment & Mainstem & 7.843 & 1.008 & 7.133 & 12.343 \\
\hline Laurel Lick Run & Oct-07 & Large Adult & Treatment & Mainstem & 44.003 & 2.111 & 41.867 & 51.399 \\
\hline Laurel Lick Run & Jul-06 & YOY & Treatment & Mainstem & 0.000 & 0.000 & 0.000 & 0.000 \\
\hline Laurel Lick Run & Aug-06 & YOY & Treatment & Mainstem & 0.000 & 0.000 & 0.000 & 0.000 \\
\hline Laurel Lick Run & Oct-06 & YOY & Treatment & Mainstem & 15.911 & 2.105 & 13.816 & 23.379 \\
\hline Laurel Lick Run & May-07 & YOY & Treatment & Mainstem & 3.306 & 0.592 & 3.026 & 6.533 \\
\hline Laurel Lick Run & Jul-07 & YOY & Treatment & Mainstem & 0.000 & 0.000 & 0.000 & 0.000 \\
\hline Laurel Lick Run & Oct-07 & YOY & Treatment & Mainstem & 7.914 & 1.082 & 7.146 & 12.723 \\
\hline Laurel Lick Run & Jul-06 & Small Adult & Treatment & Mainstem & 0.000 & 0.000 & 0.000 & 0.000 \\
\hline Laurel Lick Run & Aug-06 & Small Adult & Treatment & Mainstem & 50.214 & 3.557 & 45.891 & 60.998 \\
\hline Laurel Lick Run & Oct-06 & Small Adult & Treatment & Mainstem & 23.775 & 1.941 & 21.806 & 30.562 \\
\hline Laurel Lick Run & May-07 & Small Adult & Treatment & Mainstem & 13.726 & 0.910 & 13.108 & 17.874 \\
\hline Laurel Lick Run & Jul-07 & Small Adult & Treatment & Mainstem & 5.602 & 0.843 & 5.078 & 9.641 \\
\hline Laurel Lick Run & Oct-07 & Small Adult & Treatment & Mainstem & 17.172 & 1.202 & 16.222 & 22.176 \\
\hline Glady Fork & Jul-06 & Large Adult & Control & Headwater & 5.335 & 0.613 & 5.031 & 8.603 \\
\hline Glady Fork & Aug-06 & Large Adult & Control & Headwater & 74.552 & 2.322 & 72.103 & 82.437 \\
\hline Glady Fork & Oct-06 & Large Adult & Control & Headwater & 62.968 & 4.751 & 57.106 & 77.198 \\
\hline Glady Fork & May-07 & Large Adult & Control & Headwater & 45.954 & 3.284 & 42.169 & 56.348 \\
\hline Glady Fork & Jul-07 & Large Adult & Control & Headwater & 37.239 & 1.768 & 35.572 & 43.760 \\
\hline Glady Fork & Oct-07 & Large Adult & Control & Headwater & 17.995 & 1.084 & 17.176 & 22.632 \\
\hline Glady Fork & Jul-06 & YOY & Control & Headwater & 0.000 & 0.000 & 0.000 & 0.000 \\
\hline Glady Fork & Aug-06 & YOY & Control & Headwater & 0.000 & 0.000 & 0.000 & 0.000 \\
\hline
\end{tabular}




\begin{tabular}{|c|c|c|c|c|c|c|c|c|}
\hline Site & Date & Age & Removal & Location & Estimate & SE & $\mathrm{LCl}$ & $\mathrm{UCl}$ \\
\hline Glady Fork & Oct-06 & YOY & Control & Headwater & 1.309 & 0.645 & 1.024 & 4.916 \\
\hline Glady Fork & May-07 & YOY & Control & Headwater & 0.000 & 0.000 & 0.000 & 0.000 \\
\hline Glady Fork & Jul-07 & YOY & Control & Headwater & 0.000 & 0.000 & 0.000 & 0.000 \\
\hline Glady Fork & Oct-07 & YOY & Control & Headwater & 9.957 & 1.096 & 9.159 & 14.755 \\
\hline Glady Fork & Jul-06 & Small Adult & Control & Headwater & 0.000 & 0.000 & 0.000 & 0.000 \\
\hline Glady Fork & Aug-06 & Small Adult & Control & Headwater & 7.350 & 0.620 & 7.034 & 10.615 \\
\hline Glady Fork & Oct-06 & Small Adult & Control & Headwater & 10.693 & 1.523 & 9.374 & 16.660 \\
\hline Glady Fork & May-07 & Small Adult & Control & Headwater & 6.893 & 1.056 & 6.143 & 11.583 \\
\hline Glady Fork & Jul-07 & Small Adult & Control & Headwater & 8.512 & 0.764 & 8.061 & 12.270 \\
\hline Glady Fork & Oct-07 & Small Adult & Control & Headwater & 14.819 & 0.974 & 14.130 & 19.160 \\
\hline Daniel's Run & Jul-06 & Large Adult & Treatment & Headwater & 7.975 & 1.086 & 7.168 & 12.672 \\
\hline Daniel's Run & Aug-06 & Large Adult & Treatment & Headwater & 136.206 & 4.924 & 129.511 & 149.786 \\
\hline Daniel's Run & Oct-06 & Large Adult & Treatment & Headwater & 66.168 & 6.099 & 57.735 & 82.742 \\
\hline Daniel's Run & May-07 & Large Adult & Treatment & Headwater & 86.039 & 6.475 & 76.955 & 103.412 \\
\hline Daniel's Run & Jul-07 & Large Adult & Treatment & Headwater & 38.547 & 2.524 & 35.647 & 46.555 \\
\hline Daniel's Run & Oct-07 & Large Adult & Treatment & Headwater & 61.840 & 4.985 & 55.160 & 75.762 \\
\hline Daniel's Run & Jul-06 & YOY & Treatment & Headwater & 0.000 & 0.000 & 0.000 & 0.000 \\
\hline Daniel's Run & Aug-06 & YOY & Treatment & Headwater & 1.185 & 0.472 & 1.011 & 3.989 \\
\hline Daniel's Run & Oct-06 & YOY & Treatment & Headwater & 32.515 & 5.120 & 26.009 & 47.475 \\
\hline Daniel's Run & May-07 & YOY & Treatment & Headwater & 42.105 & 5.557 & 34.906 & 58.077 \\
\hline Daniel's Run & Jul-07 & YOY & Treatment & Headwater & 2.453 & 0.757 & 2.047 & 6.358 \\
\hline Daniel's Run & Oct-07 & YOY & Treatment & Headwater & 28.387 & 4.120 & 23.371 & 40.864 \\
\hline Daniel's Run & Jul-06 & Small Adult & Treatment & Headwater & 3.418 & 0.699 & 3.043 & 7.022 \\
\hline Daniel's Run & Aug-06 & Small Adult & Treatment & Headwater & 130.669 & 4.784 & 124.194 & 143.915 \\
\hline Daniel's Run & Oct-06 & Small Adult & Treatment & Headwater & 35.109 & 4.031 & 29.977 & 46.865 \\
\hline Daniel's Run & May-07 & Small Adult & Treatment & Headwater & 50.083 & 4.489 & 44.162 & 62.791 \\
\hline Daniel's Run & Jul-07 & Small Adult & Treatment & Headwater & 68.024 & 3.588 & 63.474 & 78.529 \\
\hline Daniel's Run & Oct-07 & Small Adult & Treatment & Headwater & 35.337 & 3.476 & 31.037 & 45.724 \\
\hline
\end{tabular}




\section{Appendix B: Length-frequency distributions of brook trout and mottled sculpin}

B1. Diagram of the length-frequency distribution for brook trout among all sites pooled across all sampling occasions with 100 bins. Vertical lines indicate the natural breaks used to distinguish among the young-of-the-year (YOY) age class $(<=75 \mathrm{~mm})$ and the small $(75<\mathrm{x}<=120 \mathrm{~mm})$ and large $(>120 \mathrm{~mm})$ adult segments.

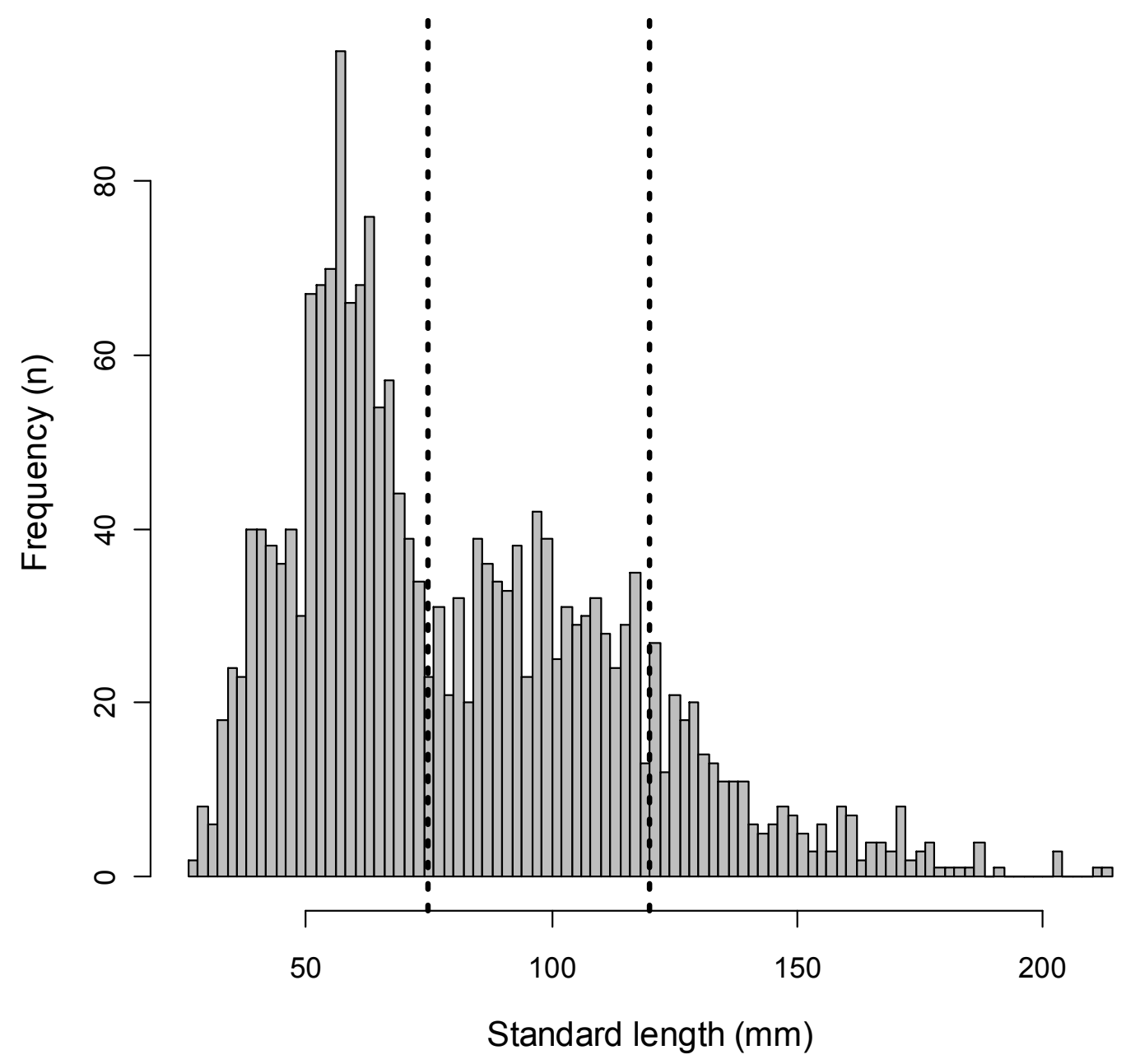


B2. Diagram of the length-frequency distribution for mottled sculpin among all sites pooled across all sampling occasions with 100 bins. Vertical lines indicate the natural breaks used to distinguish among the young-of-the-year (YOY) age class $(<=35 \mathrm{~mm})$ and the small $(35<\mathrm{x}<=50 \mathrm{~mm})$ and large $(>50 \mathrm{~mm})$ adult segments.

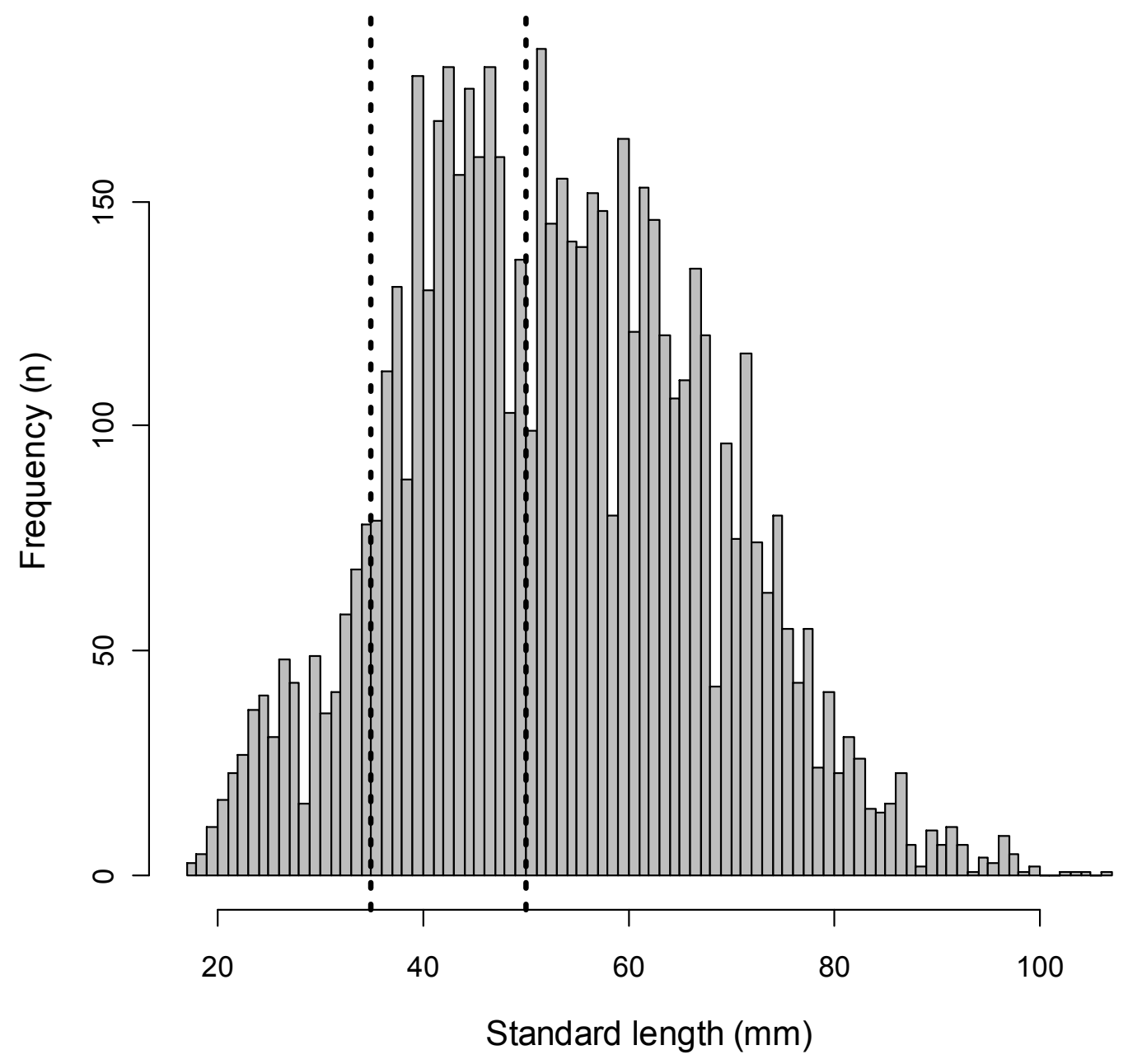




\section{Appendix C: Model-averaged coefficients for estimating log-transformed proportional recovery of abundance}

C1. Model coefficients, standard errors, and confidence limits for the model-averaged coefficients resulting from the $95 \%$ confidence set of linear mixed regression models for estimating the response of log-transformed young-of-the-year (YOY) brook trout proportional recovery (i.e., abundance at time $t$ divided by pre-removal abundance) to fixed effects of fish removal treatment, spatial location, and interactions over the course of the study while accounting for the additional fixed effects of local physical and chemical habitat covariates and sampling date and while modeling site as a random factor. $\Sigma \mathrm{w}_{\mathrm{i}}=$ sum of Akaike weights for term across $95 \%$ confidence model set. The cumulative Akaike weights for each model term are indicated next to the relevant effect. Asterisks (*) indicate where model coefficient confidence limits did not include 0 (i.e., a "significant" positive or negative slope). $\mathrm{g}=$ treatment, $\mathrm{l}=$ spatial location, $\mathrm{t} 3-\mathrm{t} 6=$ sampling date, $1 * \mathrm{t} 3-1 * \mathrm{t} 6=$ location $\mathrm{x}$ sampling date interaction, $\mathrm{g} * 1=$ removal $\mathrm{x}$ location interaction, $\mathrm{W} 1=$ water chemistry principal component $1, \mathrm{H} 1-\mathrm{H} 3=$ physical habitat principal components 1 to 3 , PAREA = proportion of spring wetted channel area in early fall, $\mathrm{CANP}=$ mean percent canopy cover, MWAT $=$ maximum weekly average temperature $\left({ }^{\circ} \mathrm{C}\right)$.

\begin{tabular}{|c|c|c|c|c|c|}
\hline Coefficient & Estimate & SE & $\mathrm{LCl}$ & $\mathrm{UCI}$ & $\Sigma w_{i}$ \\
\hline Intercept & 8.610 & 7.720 & -6.920 & 24.100 & \\
\hline $\mathrm{g}$ (defaunated) & -1.940 & 0.976 & -3.890 & 0.013 & 0.511 \\
\hline I (mainstem) & -0.921 & 0.468 & -1.860 & 0.018 & 0.814 \\
\hline t3 (Oct-2006) & 0.135 & 0.454 & -0.778 & 1.050 & \\
\hline t4 (May-2007) & 0.989 & 0.482 & 0.022 & 1.960 & \\
\hline t5 (Jul-2007) & 0.965 & 0.493 & -0.023 & 1.950 & * \\
\hline t6 (Oct-2007) & 1.630 & 0.489 & 0.654 & 2.610 & \\
\hline$g^{*}$ & -0.113 & 1.160 & -2.440 & 2.210 & 0.252 \\
\hline I*t3 & 0.981 & 0.807 & -0.648 & 2.610 & \\
\hline$I^{*}+4$ & 1.680 & 0.807 & 0.049 & 3.310 & * \\
\hline$I^{*}+5$ & 1.900 & 0.807 & 0.275 & 3.530 & * \\
\hline I*t6 & 1.820 & 0.807 & 0.195 & 3.450 & * \\
\hline W1 & 0.868 & 0.762 & -0.659 & 2.400 & \\
\hline $\mathrm{H} 1$ & -0.033 & 0.184 & -0.399 & 0.334 & \\
\hline $\mathrm{H} 2$ & -0.009 & 0.192 & -0.393 & 0.376 & \\
\hline $\mathrm{H} 3$ & -0.554 & 0.373 & -1.300 & 0.189 & \\
\hline PAREA & -2.480 & 1.230 & -4.950 & -0.013 & * \\
\hline CANP & -0.052 & 0.051 & -0.154 & 0.050 & \\
\hline MWAT & -0.083 & 0.298 & -0.683 & 0.516 & \\
\hline
\end{tabular}


C2. Model coefficients, standard errors, and confidence limits for the model-averaged coefficients resulting from the $95 \%$ confidence set of linear mixed regression models for estimating the response of log-transformed small adult brook trout proportional recovery (i.e., abundance at time $t$ divided by pre-removal abundance) to fixed effects of fish removal treatment, spatial location, and interactions over the course of the study while accounting for the additional fixed effects of local physical and chemical habitat covariates and sampling date and while modeling site as a random factor. $\Sigma \mathrm{w}_{\mathrm{i}}=$ sum of Akaike weights for term across $95 \%$ confidence model set. The cumulative Akaike weights for each model term are indicated next to the relevant effect. Asterisks $(*)$ indicate where model coefficient confidence limits did not include 0 (i.e., a "significant" positive or negative slope). $\mathrm{g}=$ treatment, $\mathrm{l}=$ spatial location, $\mathrm{t} 3-\mathrm{t} 6=$ sampling date, $1 * \mathrm{t} 3-1 * \mathrm{t} 6=$ location $\mathrm{x}$ sampling date interaction, $\mathrm{g} * \mathrm{l}=$ removal $\mathrm{x}$ location interaction, $\mathrm{W} 1=$ water chemistry principal component $1, \mathrm{H} 1-\mathrm{H} 3=$ physical habitat principal components 1 to 3, PAREA = proportion of spring wetted channel area in early fall, $\mathrm{CANP}=$ mean percent canopy cover, MWAT $=$ maximum weekly average temperature $\left({ }^{\circ} \mathrm{C}\right)$.

\begin{tabular}{|c|c|c|c|c|c|}
\hline Coefficient & Estimate & SE & LCI & $\mathrm{UCI}$ & $\Sigma w_{i}$ \\
\hline Intercept & -0.332 & 4.050 & -8.480 & 7.810 & \\
\hline $\mathrm{g}$ (defaunated) & 0.137 & 0.238 & -0.343 & 0.616 & 0.279 \\
\hline I (mainstem) & 0.277 & 0.423 & -0.573 & 1.130 & 0.521 \\
\hline t3 (Oct-2006) & 0.589 & 0.292 & 0.001 & 1.180 & \\
\hline t4 (May-2007) & 0.457 & 0.292 & -0.130 & 1.050 & \\
\hline t5 (Jul-2007) & -0.294 & 0.292 & -0.882 & 0.293 & \\
\hline t6 (Oct-2007) & 0.630 & 0.292 & 0.043 & 1.220 & \\
\hline W1 & 0.318 & 0.316 & -0.317 & 0.953 & \\
\hline H1 & -0.200 & 0.070 & -0.341 & -0.058 & \\
\hline $\mathrm{H} 2$ & -0.265 & 0.088 & -0.443 & -0.087 & \\
\hline H3 & 0.023 & 0.111 & -0.200 & 0.246 & \\
\hline PAREA & -2.100 & 0.521 & -3.150 & -1.050 & \\
\hline CANP & 0.028 & 0.026 & -0.024 & 0.080 & \\
\hline MWAT & -0.059 & 0.150 & -0.360 & 0.241 & \\
\hline
\end{tabular}


C3. Model coefficients, standard errors, and confidence limits for the model-averaged coefficients resulting from the $95 \%$ confidence set of linear mixed regression models for estimating the response of log-transformed large adult brook trout proportional recovery (i.e., abundance at time $t$ divided by pre-removal abundance) to fixed effects of fish removal treatment, spatial location, and interactions over the course of the study while accounting for the additional fixed effects of local physical and chemical habitat covariates and sampling date and while modeling site as a random factor. $\Sigma \mathrm{w}_{\mathrm{i}}=$ sum of Akaike weights for term across $95 \%$ confidence model set. The cumulative Akaike weights for each model term are indicated next to the relevant effect. Asterisks $(*)$ indicate where model coefficient confidence limits did not include 0 (i.e., a "significant" positive or negative slope). $\mathrm{g}=$ treatment, $\mathrm{l}=$ spatial location, $\mathrm{t} 3-\mathrm{t} 6=$ sampling date, $1 * \mathrm{t} 3-1 * \mathrm{t} 6=$ location $\mathrm{x}$ sampling date interaction, $\mathrm{g} * \mathrm{l}=$ removal $\mathrm{x}$ location interaction, $\mathrm{W} 1=$ water chemistry principal component $1, \mathrm{H} 1-\mathrm{H} 3=$ physical habitat principal components 1 to 3, PAREA = proportion of spring wetted channel area in early fall, $\mathrm{CANP}=$ mean percent canopy cover, MWAT $=$ maximum weekly average temperature $\left({ }^{\circ} \mathrm{C}\right)$.

\begin{tabular}{lccccc}
\multicolumn{1}{c}{ Coefficient } & Estimate & SE & LCI & UCI & ${\mathbf{~} \mathbf{w}_{\mathbf{i}}}$ \\
\hline Intercept & -7.180 & 13.700 & -34.700 & 20.400 & \\
g (defaunated) & -1.030 & 0.850 & -2.750 & 0.678 & 0.580 \\
I (mainstem) & 3.180 & 1.920 & -0.656 & 7.020 & 0.798 \\
t3 (Oct-2006) & 0.132 & 0.258 & -0.386 & 0.650 & \\
t4 (May-2007) & 0.613 & 0.258 & 0.095 & 1.130 & \\
t5 (Jul-2007) & 0.123 & 0.258 & -0.395 & 0.641 & \\
t6 (Oct-2007) & 0.328 & 0.258 & -0.191 & 0.846 & \\
g $^{*}$ & -0.931 & 1.840 & -4.630 & 2.770 & 0.500 \\
W1 & 1.870 & 1.460 & -1.040 & 4.780 & \\
H1 & 0.168 & 0.282 & -0.398 & 0.734 & \\
H2 & -0.297 & 0.324 & -0.948 & 0.354 & \\
H3 & 0.723 & 0.707 & -0.684 & 2.130 & \\
PAREA & 1.170 & 2.370 & -3.550 & 5.900 & \\
CANP & -0.043 & 0.083 & -0.211 & 0.124 & \\
MWAT & 0.459 & 0.521 & -0.588 & 1.510 &
\end{tabular}


C4. Model coefficients, standard errors, and confidence limits for the model-averaged coefficients resulting from the $95 \%$ confidence set of regression models for estimating the response of log-transformed young-of-the-year (YOY) mottled sculpin proportional abundance to fish removal, spatial location, and interactions over the course of the study while accounting for the effects of local physical and chemical habitat covariates and sampling date. $\Sigma \mathrm{w}_{\mathrm{i}}=$ sum of Akaike weights for term across $95 \%$ confidence model set. The cumulative Akaike weights for each model term are indicated next to the relevant effect. Asterisks $(*)$ indicate where model coefficient confidence limits did not include 0 (i.e., a "significant" positive or negative slope). $\mathrm{g}=$ treatment, $\mathrm{l}=$ spatial location, $\mathrm{t} 3-\mathrm{t} 6$ $=$ sampling date, $1 * \mathrm{t} 3-1 * \mathrm{t} 6=$ location $\mathrm{x}$ date interaction, $\mathrm{g} * 1=$ removal $\mathrm{x}$ location, $\mathrm{W} 1=$ water chemistry principal component $1, \mathrm{H} 1-\mathrm{H} 3=$ physical habitat principal components 1 to 3 , PAREA = proportion of spring wetted channel area in early fall, CANP = mean percent canopy cover, MWAT $=$ maximum weekly average temperature $\left({ }^{\circ} \mathrm{C}\right)$.

\begin{tabular}{lcccccc}
\multicolumn{1}{c}{ Coefficient } & Estimate & SE & LCI & UCI & $\mathbf{E w}_{\mathbf{i}}$ \\
\hline Intercept & 2.360 & 7.140 & -12.000 & 16.700 & \\
g (defaunated) & -0.544 & 0.441 & -1.430 & 0.342 & 0.310 \\
I (mainstem) & -0.492 & 0.974 & -2.450 & 1.470 & 0.402 \\
t3 (Oct-2006) & 2.430 & 0.435 & 1.550 & 3.300 & $*$ & \\
t4 (May-2007) & 2.420 & 0.445 & 1.530 & 3.320 & $*$ & \\
t5 (Jul-2007) & 0.754 & 0.443 & -0.136 & 1.640 & & \\
t6 (Oct-2007) & 2.130 & 0.436 & 1.250 & 3.000 & $*$ & \\
g*I & 0.499 & 1.900 & -3.340 & 4.330 & & 0.074 \\
I*t3 & 0.335 & 0.815 & -1.310 & 1.980 & & \\
I*t4 & -1.300 & 0.815 & -2.940 & 0.349 & & \\
I*t5 & -1.140 & 0.815 & -2.790 & 0.500 & & \\
I*t6 & 0.489 & 0.815 & -1.150 & 2.130 & & \\
W1 & -0.439 & 0.600 & -1.640 & 0.765 & & \\
H1 & -0.100 & 0.134 & -0.369 & 0.169 & & \\
H2 & -0.421 & 0.161 & -0.744 & -0.098 & $*$ & \\
H3 & 0.192 & 0.225 & -0.260 & 0.643 & & \\
PAREA & 0.081 & 0.968 & -1.860 & 2.030 & & \\
CANP & 0.003 & 0.045 & -0.088 & 0.094 & & \\
MWAT & -0.145 & 0.266 & -0.680 & 0.390 & &
\end{tabular}


C5. Model coefficients, standard errors, and confidence limits for the model-averaged coefficients resulting from the $95 \%$ confidence set of regression models for estimating the response of log-transformed small adult mottled sculpin proportional abundance to fish removal, spatial location, and interactions over the course of the study while accounting for the effects of local physical and chemical habitat covariates and sampling date. $\Sigma \mathrm{w}_{\mathrm{i}}=$ sum of Akaike weights for term across $95 \%$ confidence model set. The cumulative Akaike weights for each model term are indicated next to the relevant effect. Asterisks $(*)$ indicate where model coefficient confidence limits did not include 0 (i.e., a "significant" positive or negative slope). $\mathrm{g}=$ treatment, $1=$ spatial location, $\mathrm{t} 3-\mathrm{t} 6=$ sampling date, $1 * \mathrm{t} 3-1 * \mathrm{t} 6=$ location $\mathrm{x}$ date interaction, $\mathrm{g} * 1=$ removal $\mathrm{x}$ location, $\mathrm{W} 1=$ water chemistry principal component $1, \mathrm{H} 1-\mathrm{H} 3=$ physical habitat principal components 1 to 3 , PAREA = proportion of spring wetted channel area in early fall, CANP = mean percent canopy cover, MWAT $=$ maximum weekly average temperature $\left({ }^{\circ} \mathrm{C}\right)$.

\begin{tabular}{lcccccc}
\multicolumn{1}{c}{ Coefficient } & Estimate & SE & LCI & UCI & & $\mathbf{\Sigma w}_{\mathbf{i}}$ \\
\hline Intercept & 11.900 & 8.770 & -5.710 & 29.500 & & \\
g (defaunated) & -1.510 & 0.364 & -2.250 & -0.781 & $*$ & 0.608 \\
I (mainstem) & 0.755 & 1.040 & -1.320 & 2.830 & & 0.745 \\
t3 (Oct-2006) & 0.571 & 0.306 & -0.044 & 1.190 & & \\
t4 (May-2007) & 1.010 & 0.306 & 0.400 & 1.630 & $*$ & \\
t5 (Jul-2007) & 0.940 & 0.306 & 0.325 & 1.550 & $*$ & \\
t6 (Oct-2007) & 0.954 & 0.306 & 0.339 & 1.570 & $*$ & \\
g*l & 1.930 & 0.787 & 0.344 & 3.510 & $*$ & 0.528 \\
W1 & -0.054 & 0.715 & -1.490 & 1.380 & & \\
H1 & 0.098 & 0.170 & -0.242 & 0.437 & & \\
H2 & -0.424 & 0.265 & -0.950 & 0.103 & & \\
H3 & 0.304 & 0.345 & -0.385 & 0.993 & & \\
PAREA & -4.230 & 1.210 & -6.670 & -1.790 & $*$ & \\
CANP & -0.088 & 0.054 & -0.195 & 0.020 & & \\
MWAT & -0.065 & 0.324 & -0.716 & 0.586 & &
\end{tabular}


C6. Model coefficients, standard errors, and confidence limits for the model-averaged coefficients resulting from the $95 \%$ confidence set of regression models for estimating the response of log-transformed large adult mottled sculpin proportional abundance to fish removal, spatial location, and interactions over the course of the study while accounting for the effects of local physical and chemical habitat covariates and sampling date. $\Sigma \mathrm{w}_{\mathrm{i}}=$ sum of Akaike weights for term across $95 \%$ confidence model set. The cumulative Akaike weights for each model term are indicated next to the relevant effect. Asterisks $(*)$ indicate where model coefficient confidence limits did not include 0 (i.e., a "significant" positive or negative slope). $\mathrm{g}=$ treatment, $1=$ spatial location, $\mathrm{t} 3-\mathrm{t} 6=$ sampling date, $1 * \mathrm{t} 3-1 * \mathrm{t} 6=$ location $\mathrm{x}$ date interaction, $\mathrm{g} * 1=$ removal $\mathrm{x}$ location, $\mathrm{W} 1=$ water chemistry principal component $1, \mathrm{H} 1-\mathrm{H} 3=$ physical habitat principal components 1 to 3, PAREA = proportion of spring wetted channel area in early fall, CANP = mean percent canopy cover, MWAT $=$ maximum weekly average temperature $\left({ }^{\circ} \mathrm{C}\right)$.

\begin{tabular}{lcccccc}
\multicolumn{1}{c}{ Coefficient } & Estimate & SE & LCI & UCI & & $\mathbf{E w}_{\mathbf{i}}$ \\
\hline Intercept & 2.430 & 9.790 & -17.200 & 22.100 & & \\
g (defaunated) & -1.170 & 0.532 & -2.250 & -0.104 & $*$ & 0.396 \\
I (mainstem) & 1.250 & 1.230 & -1.200 & 3.700 & & 0.736 \\
t3 (Oct-2006) & 0.911 & 0.252 & 0.404 & 1.420 & $*$ & \\
t4 (May-2007) & 0.699 & 0.252 & 0.192 & 1.210 & $*$ & \\
t5 (Jul-2007) & 0.189 & 0.252 & -0.318 & 0.696 & & \\
t6 (Oct-2007) & 0.470 & 0.252 & -0.037 & 0.978 & & \\
g*I & 2.170 & 1.150 & -0.140 & 4.490 & & 0.325 \\
W1 & -0.196 & 0.807 & -1.820 & 1.420 & & \\
H1 & 0.207 & 0.206 & -0.205 & 0.619 & & \\
H2 & -0.360 & 0.252 & -0.862 & 0.143 & & \\
H3 & 0.573 & 0.402 & -0.229 & 1.380 & & \\
PAREA & -2.450 & 1.420 & -5.290 & 0.391 & & \\
CANP & -0.050 & 0.061 & -0.173 & 0.072 & & \\
MWAT & 0.213 & 0.369 & -0.527 & 0.953 & &
\end{tabular}




\section{Appendix D: Model-averaged coefficients for estimating population per capita growth rate}

D1. Model coefficients, standard errors, and confidence limits for the model-averaged coefficients resulting from the $95 \%$ confidence set of regression models for estimating the response of young-of-the-year (YOY) brook trout per capita growth rate $(\mathrm{r}=$ $\ln \left(\mathrm{N}_{\mathrm{t}+1} / \mathrm{N}_{\mathrm{t}}\right)$ ) to fish removal, spatial location, and interactions over the course of the study while accounting for the effects of local physical and chemical habitat covariates and sampling date. $\Sigma \mathrm{w}_{\mathrm{i}}=$ sum of Akaike weights for term across $95 \%$ confidence model set. The cumulative Akaike weights for each model term are indicated next to the relevant effect. Asterisks $(*)$ indicate where model coefficient confidence limits did not include 0 (i.e., a "significant" positive or negative slope). $1=$ spatial location, $\mathrm{t} 3-\mathrm{t} 6=$ sampling date, $\mathrm{W} 1=$ water chemistry principal component $1, \mathrm{H} 1-\mathrm{H} 3=$ physical habitat principal components 1 to 3, PAREA = proportion of spring wetted channel area in early fall, $\mathrm{CANP}=$ mean percent canopy cover, $\mathrm{MWAT}=$ maximum weekly average temperature $\left({ }^{\circ} \mathrm{C}\right)$.

\begin{tabular}{cccccc} 
Coefficient & Estimate & SE & LCI & UCI & $\mathbf{\Sigma w}_{\mathbf{i}}$ \\
\hline Intercept & -1.320 & 7.490 & -16.400 & 13.700 & \\
I (mainstem) & -0.259 & 0.785 & -1.840 & 1.320 & 0.176 \\
t2 (Aug - Oct-2006) & -0.966 & 0.540 & -2.050 & 0.120 & \\
t3 (Oct-2006 - May-2007) & -0.423 & 0.540 & -1.510 & 0.664 & \\
t4 (May - Jul-2007) & -1.240 & 0.540 & -2.320 & -0.151 & \\
t5 (Jul - Oct-2007) & -0.488 & 0.540 & -1.570 & 0.599 & \\
W1 & 0.458 & 0.574 & -0.695 & 1.610 & \\
H1 & -0.061 & 0.127 & -0.316 & 0.194 & \\
H2 & 0.038 & 0.162 & -0.288 & 0.364 & \\
H3 & -0.039 & 0.200 & -0.440 & 0.363 & \\
PAREA & 0.289 & 0.955 & -1.630 & 2.210 & \\
CANP & 0.020 & 0.047 & -0.075 & 0.115 & \\
MWAT & 0.018 & 0.276 & -0.537 & 0.572 &
\end{tabular}


D2. Model coefficients, standard errors, and confidence limits for the model-averaged coefficients resulting from the $95 \%$ confidence set of regression models for estimating the response of small adult brook trout per capita growth rate $\left(\mathrm{r}=\ln \left(\mathrm{N}_{\mathrm{t}+1} / \mathrm{N}_{\mathrm{t}}\right)\right)$ to fish removal, spatial location, and interactions over the course of the study while accounting for the effects of local physical and chemical habitat covariates and sampling date. $\Sigma \mathrm{w}_{\mathrm{i}}=$ sum of Akaike weights for term across $95 \%$ confidence model set. The cumulative Akaike weights for each model term are indicated next to the relevant effect. Asterisks $\left.{ }^{*}\right)$ indicate where model coefficient confidence limits did not include 0 (i.e., a "significant" positive or negative slope). $\mathrm{g}=$ treatment, $1=$ spatial location, $\mathrm{t} 3-\mathrm{t} 6=$ sampling date, $\mathrm{g}^{*} \mathrm{t} 3-\mathrm{g} * \mathrm{t} 6=$ removal $\mathrm{x}$ sampling date interaction, $\mathrm{W} 1=$ water chemistry principal component $1, \mathrm{H} 1-\mathrm{H} 3=$ physical habitat principal components 1 to 3, PAREA $=$ proportion of spring wetted channel area in early fall, $\mathrm{CANP}=$ mean percent canopy cover, MWAT $=$ maximum weekly average temperature $\left({ }^{\circ} \mathrm{C}\right)$.

\begin{tabular}{|c|c|c|c|c|c|}
\hline Coefficient & Estimate & SE & $\mathrm{LCl}$ & $\mathrm{UCI}$ & $\Sigma \mathbf{w}_{\mathrm{i}}$ \\
\hline Intercept & -0.579 & 5.690 & -12.000 & 10.900 & \\
\hline$g$ (defaunated) & 1.430 & 0.912 & -0.373 & 3.240 & 0.629 \\
\hline I (mainstem) & -0.129 & 0.619 & -1.380 & 1.120 & 0.155 \\
\hline t2 (Jul - Oct-2006) & 0.313 & 0.580 & -0.843 & 1.470 & \\
\hline t3 (Oct-2006 - May-2007) & -0.289 & 0.689 & -1.660 & 1.080 & \\
\hline t4 (May - Jul-2007) & -0.844 & 0.641 & -2.120 & 0.430 & \\
\hline t5 (Jul - Oct-2007) & 0.686 & 0.724 & -0.748 & 2.120 & \\
\hline$g^{*} \mathrm{t} 2$ & -1.590 & 0.770 & -3.150 & -0.039 & 0.541 \\
\hline$g^{*} \mathrm{t} 3$ & -2.330 & 0.770 & -3.890 & -0.781 & * \\
\hline$g^{*} t 4$ & -2.020 & 0.770 & -3.570 & -0.468 & * \\
\hline$g^{*} \mathrm{t} 5$ & -2.540 & 0.770 & -4.100 & -0.990 & * \\
\hline W1 & -0.065 & 0.097 & -0.261 & 0.130 & \\
\hline $\mathrm{H} 1$ & 0.034 & 0.124 & -0.216 & 0.285 & \\
\hline $\mathrm{H} 2$ & -0.008 & 0.142 & -0.295 & 0.278 & \\
\hline $\mathrm{H} 3$ & 0.255 & 0.465 & -0.680 & 1.190 & \\
\hline PAREA & 0.055 & 0.725 & -1.400 & 1.510 & \\
\hline CANP & 0.012 & 0.036 & -0.060 & 0.084 & \\
\hline MWAT & -0.022 & 0.208 & -0.441 & 0.397 & \\
\hline
\end{tabular}


D3. Model coefficients, standard errors, and confidence limits for the model-averaged coefficients resulting from the $95 \%$ confidence set of regression models for estimating the response of large adult brook trout per capita growth rate $\left(\mathrm{r}=\ln \left(\mathrm{N}_{\mathrm{t}+1} / \mathrm{N}_{\mathrm{t}}\right)\right)$ to fish removal, spatial location, and interactions over the course of the study while accounting for the effects of local physical and chemical habitat covariates and sampling date. $\Sigma \mathrm{w}_{\mathrm{i}}=$ sum of Akaike weights for term across $95 \%$ confidence model set. The cumulative Akaike weights for each model term are indicated next to the relevant effect. Asterisks $\left({ }^{*}\right)$ indicate where model coefficient confidence limits did not include zero (i.e., a "significant" positive or negative slope). $\mathrm{g}=$ treatment, $1=$ spatial location, $\mathrm{t} 3-\mathrm{t} 6=$ sampling date, $\mathrm{g}^{*} \mathrm{t} 3-\mathrm{g} * \mathrm{t} 6=$ removal $\mathrm{x}$ sampling date interaction, $\mathrm{W} 1=$ water chemistry principal component $1, \mathrm{H} 1-\mathrm{H} 3=$ physical habitat principal components 1 to 3, PAREA $=$ proportion of spring wetted channel area in early fall, $\mathrm{CANP}=$ mean percent canopy cover, MWAT $=$ maximum weekly average temperature $\left({ }^{\circ} \mathrm{C}\right)$.

\begin{tabular}{cccccc} 
Coefficient & Estimate & SE & LCI & UCI & $\mathbf{\Sigma w}_{\mathbf{i}}$ \\
\hline Intercept & -1.940 & 5.770 & -13.600 & 9.670 & \\
g (defaunated) & 1.250 & 0.873 & -0.475 & 2.980 & 0.634 \\
I (mainstem) & 0.352 & 0.617 & -0.888 & 1.590 & 0.163 \\
t2 (Aug - Oct-2006) & -0.013 & 0.711 & -1.420 & 1.400 & \\
t3 (Oct-2006 - May-2007) & 0.138 & 0.516 & -0.894 & 1.170 & \\
t4 (May - Jul-2007) & -0.851 & 0.578 & -2.000 & 0.302 & \\
t5 (Jul - Oct-2007) & -0.128 & 0.572 & -1.270 & 1.010 & \\
g $^{*} 2$ & -2.770 & 0.777 & -4.340 & -1.200 & $*$ \\
g*3 & -1.180 & 0.777 & -2.740 & 0.390 & \\
g*t4 & -1.770 & 0.777 & -3.340 & -0.206 & $*$ \\
g*t5 & -1.720 & 0.777 & -3.280 & -0.150 & \\
W1 & 0.250 & 0.476 & -0.707 & 1.210 & \\
H1 & -0.125 & 0.102 & -0.330 & 0.081 & \\
H2 & -0.034 & 0.127 & -0.290 & 0.222 & \\
H3 & -0.018 & 0.154 & -0.328 & 0.292 & \\
PAREA & -0.276 & 0.747 & -1.780 & 1.230 & \\
CANP & 0.022 & 0.037 & -0.051 & 0.095 & \\
MWAT & 0.018 & 0.213 & -0.409 & 0.446 &
\end{tabular}


D4. Model coefficients, standard errors, and confidence limits for the model-averaged coefficients resulting from the $95 \%$ confidence set of regression models for estimating the response of young-of-the-year (YOY) mottled sculpin per capita growth rate $(\mathrm{r}=$ $\ln \left(\mathrm{N}_{\mathrm{t}+1} / \mathrm{N}_{\mathrm{t}}\right)$ ) to fish removal, spatial location, and interactions over the course of the study while accounting for the effects of local physical and chemical habitat covariates and sampling date. $\Sigma \mathrm{w}_{\mathrm{i}}=$ sum of Akaike weights for term across $95 \%$ confidence model set. The cumulative Akaike weights for each model term are indicated next to the relevant effect. Asterisks $(*)$ indicate where model coefficient confidence limits did not include 0 (i.e., a "significant" positive or negative slope). $1=$ spatial location, $\mathrm{t} 3-\mathrm{t} 6=$ sampling date, $\mathrm{W} 1=$ water chemistry principal component $1, \mathrm{H} 1-\mathrm{H} 3=$ physical habitat principal components 1 to 3, PAREA = proportion of spring wetted channel area in early fall, $\mathrm{CANP}=$ mean percent canopy cover, $\mathrm{MWAT}=$ maximum weekly average temperature $\left({ }^{\circ} \mathrm{C}\right)$.

\begin{tabular}{|c|c|c|c|c|c|}
\hline Coefficient & Estimate & SE & LCl & $\mathrm{UCI}$ & $\Sigma \mathbf{w}_{\mathrm{i}}$ \\
\hline Intercept & 1.35 & 8.31 & -15.4 & 18.1 & \\
\hline$g$ (defaunated) & -0.201 & 0.49 & -1.19 & 0.785 & 0.170 \\
\hline I (mainstem) & 0.0248 & 0.872 & -1.73 & 1.78 & 0.221 \\
\hline t2 (Aug - Oct-2006) & 2.44 & 0.6 & 1.23 & 3.65 & * \\
\hline t3 (Oct-2006 - May-2007) & -0.0301 & 0.6 & -1.24 & 1.18 & \\
\hline t4 (May - Jul-2007) & -1.79 & 0.6 & -2.99 & -0.578 & * \\
\hline t5 (Jul - Oct-2007) & 1.54 & 0.6 & 0.331 & 2.75 & * \\
\hline W1 & -0.345 & 0.649 & -1.65 & 0.96 & \\
\hline $\mathrm{H} 1$ & -0.0357 & 0.141 & -0.32 & 0.249 & \\
\hline $\mathrm{H} 2$ & 0.0192 & 0.181 & -0.344 & 0.383 & \\
\hline $\mathrm{H} 3$ & -0.0188 & 0.217 & -0.456 & 0.418 & \\
\hline PAREA & -0.534 & 1.06 & -2.66 & 1.6 & \\
\hline CANP & -0.0145 & 0.0524 & -0.12 & 0.0909 & \\
\hline MWAT & 0.0258 & 0.306 & -0.59 & 0.641 & \\
\hline
\end{tabular}


D5. Model coefficients, standard errors, and confidence limits for the model-averaged coefficients resulting from the $95 \%$ confidence set of regression models for estimating the response of large adult mottled sculpin per capita growth rate $\left(\mathrm{r}=\ln \left(\mathrm{N}_{\mathrm{t}+1} / \mathrm{N}_{\mathrm{t}}\right)\right)$ to fish removal, spatial location, and interactions over the course of the study while accounting for the effects of local physical and chemical habitat covariates and sampling date. $\Sigma \mathrm{w}_{\mathrm{i}}=$ sum of Akaike weights for term across $95 \%$ confidence model set. The cumulative Akaike weights for each model term are indicated next to the relevant effect. Asterisks $\left.{ }^{*}\right)$ indicate where model coefficient confidence limits did not include 0 (i.e., a "significant" positive or negative slope). 1 = spatial location, $\mathrm{t} 3$ - t6 = sampling date, W 1 $=$ water chemistry principal component $1, \mathrm{H} 1-\mathrm{H} 3=$ physical habitat principal components 1 to 3, PAREA = proportion of spring wetted channel area in early fall, $\mathrm{CANP}=$ mean percent canopy cover, $\mathrm{MWAT}=$ maximum weekly average temperature $\left({ }^{\circ} \mathrm{C}\right)$.

\begin{tabular}{|c|c|c|c|c|c|}
\hline Coefficient & Estimate & SE & $\mathrm{LCI}$ & $\mathrm{UCI}$ & $\Sigma \mathbf{w}_{\mathrm{i}}$ \\
\hline Intercept & 2.690 & 6.810 & -11 & 16.400 & \\
\hline g (defaunated) & 1.980 & 0.694 & 0.58 & 3.380 & 0.497 \\
\hline I (mainstem) & 0.340 & 0.731 & -1.13 & 1.810 & 0.172 \\
\hline t2 (Aug - Oct-2006) & -0.318 & 0.796 & -1.9 & 1.260 & \\
\hline t3 (Oct-2006 - May-2007) & -0.479 & 0.762 & -1.99 & 1.030 & \\
\hline t4 (May - Jul-2007) & -1.170 & 0.617 & -2.4 & 0.067 & \\
\hline t5 (Jul - Oct-2007) & -0.855 & 0.815 & -2.47 & 0.762 & \\
\hline$g^{*} \mathrm{t} 2$ & -2.750 & 0.920 & -4.61 & -0.893 & 0.417 \\
\hline$g^{*}+3$ & -2.520 & 0.920 & -4.37 & -0.659 & * \\
\hline$g^{*}+4$ & -1.350 & 0.920 & -3.2 & 0.511 & \\
\hline$g^{*} \mathrm{t} 5$ & -2.880 & 0.920 & -4.73 & -1.020 & * \\
\hline W1 & 0.516 & 0.541 & -0.574 & 1.600 & \\
\hline $\mathrm{H} 1$ & -0.079 & 0.119 & -0.318 & 0.159 & \\
\hline $\mathrm{H} 2$ & -0.046 & 0.149 & -0.346 & 0.254 & \\
\hline H3 & 0.156 & 0.185 & -0.216 & 0.529 & \\
\hline PAREA & -0.005 & 0.877 & -1.77 & 1.760 & \\
\hline CANP & 0.004 & 0.043 & -0.0824 & 0.091 & \\
\hline MWAT & -0.132 & 0.251 & -0.636 & 0.373 & \\
\hline
\end{tabular}


D6. Model coefficients, standard errors, and confidence limits for the model-averaged coefficients resulting from the $95 \%$ confidence set of regression models for estimating the response of large adult mottled sculpin per capita growth rate $\left(\mathrm{r}=\ln \left(\mathrm{N}_{\mathrm{t}+1} / \mathrm{N}_{\mathrm{t}}\right)\right)$ to fish removal, spatial location, and interactions over the course of the study while accounting for the effects of local physical and chemical habitat covariates and sampling date. $\Sigma \mathrm{w}_{\mathrm{i}}=$ sum of Akaike weights for term across $95 \%$ confidence model set. The cumulative Akaike weights for each model term are indicated next to the relevant effect. Asterisks $\left.{ }^{*}\right)$ indicate where model coefficient confidence limits did not include 0 (i.e., a "significant" positive or negative slope). 1 = spatial location, $\mathrm{t} 3$ - t6 = sampling date, W 1 $=$ water chemistry principal component $1, \mathrm{H} 1-\mathrm{H} 3=$ physical habitat principal components 1 to 3, PAREA = proportion of spring wetted channel area in early fall, $\mathrm{CANP}=$ mean percent canopy cover, $\mathrm{MWAT}=$ maximum weekly average temperature $\left({ }^{\circ} \mathrm{C}\right)$.

\begin{tabular}{|c|c|c|c|c|c|}
\hline Coefficient & Estimate & SE & $\mathrm{LCI}$ & $\mathrm{UCI}$ & $\Sigma \mathbf{w}_{\mathrm{i}}$ \\
\hline Intercept & 1.210 & 6.360 & -11.600 & 14.000 & \\
\hline g (defaunated) & 0.924 & 0.829 & -0.714 & 2.560 & 0.465 \\
\hline I (mainstem) & 0.491 & 0.669 & -0.854 & 1.840 & 0.176 \\
\hline t2 (Aug - Oct-2006) & -0.632 & 0.525 & -1.680 & 0.420 & \\
\hline t3 (Oct-2006 - May-2007) & -1.730 & 0.551 & -2.830 & -0.625 & * \\
\hline t4 (May - Jul-2007) & -2.050 & 0.525 & -3.110 & -1.000 & * \\
\hline t5 (Jul - Oct-2007) & -1.250 & 0.537 & -2.320 & -0.174 & * \\
\hline$g^{*} \mathrm{t} 2$ & -1.950 & 0.860 & -3.680 & -0.216 & 0.241 \\
\hline$g^{*}+3$ & -2.540 & 0.860 & -4.270 & -0.804 & * \\
\hline$g^{*}+4$ & -1.950 & 0.860 & -3.680 & -0.215 & * \\
\hline$g^{*} \mathrm{t} 5$ & -2.220 & 0.860 & -3.960 & -0.490 & * \\
\hline W1 & 0.400 & 0.529 & -0.663 & 1.460 & \\
\hline $\mathrm{H} 1$ & -0.069 & 0.115 & -0.300 & 0.162 & \\
\hline $\mathrm{H} 2$ & -0.093 & 0.141 & -0.377 & 0.191 & \\
\hline $\mathrm{H} 3$ & 0.141 & 0.176 & -0.212 & 0.495 & \\
\hline PAREA & 0.109 & 0.831 & -1.560 & 1.780 & \\
\hline CANP & 0.010 & 0.040 & -0.071 & 0.091 & \\
\hline MWAT & -0.053 & 0.235 & -0.525 & 0.420 & \\
\hline
\end{tabular}




\section{Appendix E: Survival and age class transition probability coefficients and estimates}

E1. Model coefficient estimates, standard errors, and confidence limits for the modelaveraged coefficients resulting from the $95 \%$ confidence set of multinomial models for estimating brook trout survival and age-class transition probability in response to fish removal treatment, spatial location, and select interactions over the course of the study while accounting for the effects of local physical and chemical habitat covariates. The multinomial model has two intercepts, one for estimating survival (Intercept 1) and one for estimating age-class transition (Intercept 2). $\Sigma \mathrm{w}_{\mathrm{i}}=$ sum of Akaike weights for term across $95 \%$ confidence model set. The cumulative Akaike weights for each model term are indicated next to the relevant effect. Asterisks $(*)$ indicate where model coefficient confidence limits did not include 0 (i.e., a "significant" positive or negative slope). a1 = YOY age class, $\mathrm{a} 2=$ small adults, $\mathrm{g}=$ fish removal treatment, $\mathrm{l}=$ tributary spatial location, $\mathrm{t} 1-\mathrm{t} 4=$ sampling date, $\mathrm{a} 1 * \mathrm{~g}=$ YOY $\mathrm{x}$ removal interaction effect, $\mathrm{a} 2 * \mathrm{~g}=$ small adult $\mathrm{x}$ removal, $\mathrm{a} 1 * 1=$ YOY $\mathrm{x}$ location, $\mathrm{a} 2 * 1=$ small adult $\mathrm{x}$ location, $\mathrm{g} * 1=$ removal $\mathrm{x}$ location, $\mathrm{g} * \mathrm{t} 1-\mathrm{g} * \mathrm{t} 4=$ removal $\mathrm{x}$ date, $1 * \mathrm{t} 1-1 * \mathrm{t} 4=$ location $\mathrm{x}$ date, $\mathrm{g} * 1 * \mathrm{t} 1-\mathrm{g} * 1 * \mathrm{t} 4=$ removal $\mathrm{x}$ location $\mathrm{x}$ date, $\mathrm{W} 1=$ water chemistry principal component $1, \mathrm{H} 1-\mathrm{H} 3=$ physical habitat principal components 1 to 3 , PAREA = proportion of spring wetted channel area in early fall, CANP = mean percent canopy cover, MWAT $=$ maximum weekly average temperature $\left({ }^{\circ} \mathrm{C}\right)$.

\begin{tabular}{|c|c|c|c|c|c|}
\hline Effect & Estimate & SE & LCl & $\mathrm{UCI}$ & $\Sigma w i$ \\
\hline $\begin{array}{l}\text { Intercept } 1 \\
\text { (Survival) }\end{array}$ & 0.855 & 3.126 & -5.273 & 6.982 & \\
\hline W1 & 0.112 & 0.375 & -0.623 & 0.847 & \\
\hline $\mathrm{H} 1$ & -0.083 & 0.062 & -0.205 & 0.038 & \\
\hline $\mathrm{H} 2$ & 0.119 & 0.075 & -0.029 & 0.266 & \\
\hline H3 & 0.082 & 0.179 & -0.269 & 0.433 & \\
\hline PAREA & 0.488 & 0.673 & -0.831 & 1.806 & \\
\hline CANP & 0.007 & 0.015 & -0.022 & 0.036 & \\
\hline MWAT & -0.043 & 0.114 & -0.266 & 0.179 & \\
\hline a1 & 0.044 & 0.231 & -0.409 & 0.497 & \\
\hline a2 & 0.279 & 0.219 & -0.149 & 0.707 & \\
\hline$g$ & -0.088 & 0.409 & -0.890 & 0.713 & 1.000 \\
\hline I & 0.587 & 0.714 & -0.812 & 1.986 & 1.000 \\
\hline $\mathrm{t} 1$ & -0.915 & 0.490 & -1.876 & 0.046 & \\
\hline t2 & -0.837 & 0.338 & -1.499 & -0.174 & * \\
\hline t3 & 0.380 & 0.318 & -0.243 & 1.004 & \\
\hline $\mathrm{t} 4$ & -0.929 & 0.343 & -1.601 & -0.256 & * \\
\hline$a 1^{*} g$ & 0.426 & 0.276 & -0.115 & 0.968 & \\
\hline$a 2^{*} g$ & 0.297 & 0.291 & -0.274 & 0.867 & \\
\hline a1*I & -0.701 & 0.275 & -1.240 & -0.162 & * \\
\hline$a 2^{*}$ & -0.636 & 0.287 & -1.199 & -0.073 & * \\
\hline$g^{*}$ & -0.521 & 0.699 & -1.890 & 0.849 & 0.999 \\
\hline$g^{*} t 1$ & -26.787 & 1860.043 & -3672.470 & 3618.897 & 1.000 \\
\hline
\end{tabular}




\begin{tabular}{|c|c|c|c|c|c|}
\hline$g^{*}+2$ & -1.198 & 0.455 & -2.090 & -0.305 & \multirow{11}{*}{0.999} \\
\hline$g^{*}+3$ & -0.500 & 0.425 & -1.333 & 0.332 & \\
\hline$g^{*} t 4$ & 0.335 & 0.483 & -0.611 & 1.281 & \\
\hline$I^{*} t 1$ & -0.938 & 0.610 & -2.133 & 0.257 & \\
\hline$I^{*} \mathrm{t} 2$ & 0.574 & 0.515 & -0.436 & 1.585 & \\
\hline$I^{*} \mathrm{t} 3$ & -0.195 & 0.443 & -1.063 & 0.673 & \\
\hline$I^{*}+4$ & -0.340 & 0.470 & -1.261 & 0.581 & \\
\hline$\left.g^{*}\right|^{*} t 1$ & 9.048 & 1639.111 & -3203.610 & 3221.705 & \\
\hline$\left.g^{*}\right|^{*} t 2$ & 1.107 & 0.708 & -0.280 & 2.495 & \\
\hline$\left.g^{*}\right|^{*}$ 3 & 1.508 & 0.586 & 0.360 & 2.656 & \\
\hline$\left.g^{*}\right|^{*} t 4$ & 0.263 & 0.630 & -0.973 & 1.498 & \\
\hline $\begin{array}{l}\text { Intercept } 2 \\
\text { (Transition) }\end{array}$ & 4.663 & 11.750 & -18.366 & 27.692 & \\
\hline W1 & 0.000 & 0.873 & -1.711 & 1.712 & \\
\hline $\mathrm{H} 1$ & -0.179 & 0.261 & -0.690 & 0.333 & \\
\hline $\mathrm{H} 2$ & 0.413 & 0.299 & -0.173 & 0.999 & \\
\hline H3 & -0.252 & 0.672 & -1.569 & 1.064 & \\
\hline PAREA & -3.656 & 1.934 & -7.446 & 0.134 & \\
\hline CANP & -0.055 & 0.055 & -0.164 & 0.054 & \\
\hline MWAT & -0.008 & 0.517 & -1.021 & 1.004 & \\
\hline a & -0.008 & 0.415 & -0.820 & 0.805 & \\
\hline g & 0.489 & 0.515 & -0.520 & 1.498 & 0.380 \\
\hline I & -0.274 & 1.790 & -3.782 & 3.235 & 0.288 \\
\hline $\mathrm{t} 1-2$ & 0.344 & 0.624 & -0.880 & 1.567 & \\
\hline t3 & 4.145 & 0.481 & 3.201 & 5.088 & * \\
\hline
\end{tabular}


E2. Summary of model-averaged parameter estimates, standard errors, and 95\% confidence limits (i.e., lower $=$ LCI and upper $=$ UCI) for apparent survival $(\mathrm{S})$ and age class transition probability $(\psi)$ for brook trout for all sites and sample dates. Experimental classifications of sites based on treatment and spatial location are also noted.

\begin{tabular}{|c|c|c|c|c|c|c|c|c|c|}
\hline Site & Variable & Interval & Age & Removal & Location & Estimate & SE & LCI & $\mathrm{UCl}$ \\
\hline Nan's Branch & Survival (S) & Jul-06 - Aug-06 & YOY & Control & Mainstem & 0.331 & 0.070 & 0.210 & 0.480 \\
\hline Nan's Branch & Survival (S) & Aug-06 - Oct-06 & YOY & Control & Mainstem & 0.709 & 0.070 & 0.556 & 0.825 \\
\hline Nan's Branch & Survival (S) & Oct-06 - May-07 & YOY & Control & Mainstem & 0.792 & 0.037 & 0.709 & 0.856 \\
\hline Nan's Branch & Survival (S) & May-07 - Jul-07 & YOY & Control & Mainstem & 0.471 & 0.070 & 0.340 & 0.606 \\
\hline Nan's Branch & Survival (S) & Jul-07 - Oct-07 & YOY & Control & Mainstem & 0.760 & 0.047 & 0.656 & 0.840 \\
\hline Otis Hollow & Survival (S) & Jul-06 - Aug-06 & YOY & Treatment & Mainstem & 0.000 & 0.000 & 0.000 & 1.000 \\
\hline Otis Hollow & Survival (S) & Aug-06 - Oct-06 & YOY & Treatment & Mainstem & 0.666 & 0.085 & 0.486 & 0.808 \\
\hline Otis Hollow & Survival (S) & Oct-06 - May-07 & YOY & Treatment & Mainstem & 0.903 & 0.020 & 0.857 & 0.936 \\
\hline Otis Hollow & Survival (S) & May-07 - Jul-07 & YOY & Treatment & Mainstem & 0.592 & 0.052 & 0.487 & 0.689 \\
\hline Otis Hollow & Survival (S) & Jul-07 - Oct-07 & YOY & Treatment & Mainstem & 0.739 & 0.036 & 0.664 & 0.803 \\
\hline Big Run & Survival (S) & Jul-06 - Aug-06 & YOY & Control & Headwater & 0.533 & 0.115 & 0.315 & 0.739 \\
\hline Big Run & Survival (S) & Aug-06 - Oct-06 & YOY & Control & Headwater & 0.552 & 0.071 & 0.412 & 0.684 \\
\hline Big Run & Survival (S) & Oct-06 - May-07 & YOY & Control & Headwater & 0.806 & 0.043 & 0.708 & 0.877 \\
\hline Big Run & Survival (S) & May-07 - Jul-07 & YOY & Control & Headwater & 0.529 & 0.064 & 0.405 & 0.650 \\
\hline Big Run & Survival (S) & Jul-07 - Oct-07 & YOY & Control & Headwater & 0.740 & 0.055 & 0.619 & 0.833 \\
\hline Swallow Rock & Survival (S) & Jul-06 - Aug-06 & YOY & Treatment & Headwater & 0.000 & 0.000 & 0.000 & 1.000 \\
\hline Swallow Rock & Survival (S) & Aug-06 - Oct-06 & YOY & Treatment & Headwater & 0.344 & 0.060 & 0.237 & 0.469 \\
\hline Swallow Rock & Survival (S) & Oct-06 - May-07 & YOY & Treatment & Headwater & 0.780 & 0.041 & 0.690 & 0.850 \\
\hline Swallow Rock & Survival (S) & May-07 - Jul-07 & YOY & Treatment & Headwater & 0.689 & 0.063 & 0.555 & 0.797 \\
\hline Swallow Rock & Survival (S) & Jul-07 - Oct-07 & YOY & Treatment & Headwater & 0.800 & 0.035 & 0.723 & 0.860 \\
\hline Lynn Run & Survival (S) & Jul-06 - Aug-06 & YOY & Control & Mainstem & 0.256 & 0.066 & 0.148 & 0.405 \\
\hline Lynn Run & Survival (S) & Aug-06 - Oct-06 & YOY & Control & Mainstem & 0.628 & 0.085 & 0.453 & 0.774 \\
\hline Lynn Run & Survival (S) & Oct-06 - May-07 & YOY & Control & Mainstem & 0.725 & 0.058 & 0.598 & 0.823 \\
\hline Lynn Run & Survival (S) & May-07 - Jul-07 & YOY & Control & Mainstem & 0.381 & 0.061 & 0.271 & 0.505 \\
\hline Lynn Run & Survival (S) & Jul-07 - Oct-07 & YOY & Control & Mainstem & 0.687 & 0.056 & 0.569 & 0.784 \\
\hline Lick Drain & Survival (S) & Jul-06 - Aug-06 & YOY & Treatment & Mainstem & 0.000 & 0.000 & 0.000 & 1.000 \\
\hline Lick Drain & Survival (S) & Aug-06 - Oct-06 & YOY & Treatment & Mainstem & 0.791 & 0.068 & 0.629 & 0.894 \\
\hline Lick Drain & Survival (S) & Oct-06 - May-07 & YOY & Treatment & Mainstem & 0.947 & 0.015 & 0.907 & 0.970 \\
\hline
\end{tabular}




\begin{tabular}{|c|c|c|c|c|c|c|c|c|c|}
\hline Site & Variable & Interval & Age & Removal & Location & Estimate & SE & $\mathrm{LCl}$ & $\mathrm{UCl}$ \\
\hline Mudlick Run & Survival (S) & Jul-06 - Aug-06 & YOY & Control & Headwater & 0.609 & 0.113 & 0.380 & 0.799 \\
\hline Mudlick Run & Survival (S) & Aug-06 - Oct-06 & YOY & Control & Headwater & 0.628 & 0.076 & 0.471 & 0.762 \\
\hline Mudlick Run & Survival (S) & May-07 - Jul-07 & YOY & Control & Headwater & 0.606 & 0.067 & 0.471 & 0.727 \\
\hline Mudlick Run & Survival (S) & Jul-07 - Oct-07 & YOY & Control & Headwater & 0.796 & 0.052 & 0.677 & 0.879 \\
\hline Tanner Run & Survival (S) & Jul-06 - Aug-06 & YOY & Treatment & Headwater & 0.000 & 0.000 & 0.000 & 1.000 \\
\hline Tanner Run & Survival (S) & May-07 - Jul-07 & YOY & Treatment & Headwater & 0.705 & 0.085 & 0.518 & 0.842 \\
\hline Tanner Run & Survival (S) & Jul-07 - Oct-07 & YOY & Treatment & Headwater & 0.812 & 0.057 & 0.676 & 0.900 \\
\hline Zinn Hollow & Survival (S) & Jul-06 - Aug-06 & YOY & Control & Mainstem & 0.261 & 0.077 & 0.139 & 0.435 \\
\hline Zinn Hollow & Survival (S) & Aug-06 - Oct-06 & YOY & Control & Mainstem & 0.634 & 0.094 & 0.439 & 0.793 \\
\hline Zinn Hollow & Survival (S) & Oct-06 - May-07 & YOY & Control & Mainstem & 0.730 & 0.066 & 0.583 & 0.840 \\
\hline Zinn Hollow & Survival (S) & May-07 - Jul-07 & YOY & Control & Mainstem & 0.388 & 0.072 & 0.259 & 0.535 \\
\hline Laurel Lick Run & Survival (S) & May-07 - Jul-07 & YOY & Treatment & Mainstem & 0.536 & 0.090 & 0.362 & 0.701 \\
\hline Laurel Lick Run & Survival (S) & Jul-07 - Oct-07 & YOY & Treatment & Mainstem & 0.693 & 0.073 & 0.536 & 0.815 \\
\hline Glady Fork & Survival (S) & Jul-06 - Aug-06 & YOY & Control & Headwater & 0.543 & 0.118 & 0.320 & 0.751 \\
\hline Glady Fork & Survival (S) & Aug-06 - Oct-06 & YOY & Control & Headwater & 0.563 & 0.073 & 0.418 & 0.697 \\
\hline Glady Fork & Survival (S) & Oct-06 - May-07 & YOY & Control & Headwater & 0.813 & 0.043 & 0.714 & 0.883 \\
\hline Glady Fork & Survival (S) & May-07 - Jul-07 & YOY & Control & Headwater & 0.540 & 0.065 & 0.414 & 0.662 \\
\hline Glady Fork & Survival (S) & Jul-07 - Oct-07 & YOY & Control & Headwater & 0.748 & 0.050 & 0.639 & 0.833 \\
\hline Daniel's Run & Survival (S) & Jul-06 - Aug-06 & YOY & Treatment & Headwater & 0.000 & 0.000 & 0.000 & 1.000 \\
\hline Daniel's Run & Survival (S) & Aug-06 - Oct-06 & YOY & Treatment & Headwater & 0.300 & 0.054 & 0.206 & 0.415 \\
\hline Daniel's Run & Survival (S) & Oct-06 - May-07 & YOY & Treatment & Headwater & 0.744 & 0.048 & 0.640 & 0.826 \\
\hline Daniel's Run & Survival (S) & May-07 - Jul-07 & YOY & Treatment & Headwater & 0.644 & 0.066 & 0.506 & 0.761 \\
\hline Daniel's Run & Survival (S) & Jul-07 - Oct-07 & YOY & Treatment & Headwater & 0.766 & 0.046 & 0.663 & 0.845 \\
\hline
\end{tabular}




\begin{tabular}{|c|c|c|c|c|c|c|c|c|c|}
\hline Site & Variable & Interval & Age & Removal & Location & Estimate & SE & $\mathrm{LCl}$ & $\mathrm{UCl}$ \\
\hline Nan's Branch & Survival (S) & Oct-06 - May-07 & Small Adult & Control & Mainstem & 0.837 & 0.035 & 0.757 & 0.894 \\
\hline Nan's Branch & Survival (S) & Jul-07 - Oct-07 & Small Adult & Control & Mainstem & 0.810 & 0.046 & 0.704 & 0.885 \\
\hline Otis Hollow & Survival (S) & Jul-06 - Aug-06 & Small Adult & Treatment & Mainstem & 0.000 & 0.000 & 0.000 & 1.000 \\
\hline Otis Hollow & Survival (S) & Aug-06 - Oct-06 & Small Adult & Treatment & Mainstem & 0.702 & 0.076 & 0.536 & 0.828 \\
\hline Otis Hollow & Survival (S) & Oct-06 - May-07 & Small Adult & Treatment & Mainstem & 0.917 & 0.018 & 0.874 & 0.947 \\
\hline Big Run & Survival (S) & Jul-06 - Aug-06 & Small Adult & Control & Headwater & 0.590 & 0.104 & 0.383 & 0.770 \\
\hline Big Run & Survival (S) & Aug-06 - Oct-06 & Small Adult & Control & Headwater & 0.609 & 0.061 & 0.485 & 0.721 \\
\hline Big Run & Survival (S) & Oct-06 - May-07 & Small Adult & Control & Headwater & 0.840 & 0.030 & 0.773 & 0.891 \\
\hline Big Run & Survival (S) & May-07 - Jul-07 & Small Adult & Control & Headwater & 0.587 & 0.062 & 0.463 & 0.701 \\
\hline Big Run & Survival (S) & Jul-07 - Oct-07 & Small Adult & Control & Headwater & 0.783 & 0.049 & 0.671 & 0.864 \\
\hline Swallow Rock & Survival (S) & Jul-06 - Aug-06 & Small Adult & Treatment & Headwater & 0.000 & 0.000 & 0.000 & 1.000 \\
\hline Lynn Run & Survival (S) & Jul-06 - Aug-06 & Small Adult & Control & Mainstem & 0.317 & 0.078 & 0.186 & 0.485 \\
\hline Lynn Run & Survival (S) & Aug-06 - Oct-06 & Small Adult & Control & Mainstem & 0.695 & 0.083 & 0.513 & 0.831 \\
\hline Lynn Run & Survival (S) & Oct-06 - May-07 & Small Adult & Control & Mainstem & 0.781 & 0.055 & 0.655 & 0.870 \\
\hline Lynn Run & Survival (S) & May-07 - Jul-07 & Small Adult & Control & Mainstem & 0.454 & 0.071 & 0.322 & 0.593 \\
\hline Lynn Run & Survival (S) & Jul-07 - Oct-07 & Small Adult & Control & Mainstem & 0.747 & 0.058 & 0.619 & 0.843 \\
\hline Lick Drain & Survival (S) & Jul-06 - Aug-06 & Small Adult & Treatment & Mainstem & 0.000 & 0.000 & 0.000 & 1.000 \\
\hline Lick Drain & Survival (S) & Aug-06 - Oct-06 & Small Adult & Treatment & Mainstem & 0.818 & 0.059 & 0.673 & 0.907 \\
\hline Lick Drain & Survival (S) & Oct-06 - May-07 & Small Adult & Treatment & Mainstem & 0.955 & 0.014 & 0.918 & 0.975 \\
\hline Lick Drain & Survival (S) & May-07 - Jul-07 & Small Adult & Treatment & Mainstem & 0.765 & 0.051 & 0.652 & 0.850 \\
\hline Lick Drain & Survival (S) & Jul-07 - Oct-07 & Small Adult & Treatment & Mainstem & 0.864 & 0.032 & 0.788 & 0.916 \\
\hline Mudlick Run & Survival (S) & Jul-06 - Aug-06 & Small Adult & Control & Headwater & 0.664 & 0.104 & 0.443 & 0.831 \\
\hline Mudlick Run & Survival (S) & Aug-06 - Oct-06 & Small Adult & Control & Headwater & 0.681 & 0.072 & 0.528 & 0.803 \\
\hline
\end{tabular}




\begin{tabular}{|c|c|c|c|c|c|c|c|c|c|}
\hline Site & Variable & Interval & Age & Removal & Location & Estimate & SE & $\mathrm{LCI}$ & $\mathrm{UCl}$ \\
\hline Mudlick Run & Survival (S) & Oct-06 - May-07 & Small Adult & Control & Headwater & 0.878 & 0.032 & 0.802 & 0.928 \\
\hline Mudlick Run & Survival (S) & May-07 - Jul-07 & Small Adult & Control & Headwater & 0.661 & 0.070 & 0.514 & 0.782 \\
\hline Mudlick Run & Survival (S) & Jul-07 - Oct-07 & Small Adult & Control & Headwater & 0.831 & 0.049 & 0.713 & 0.907 \\
\hline Tanner Run & Survival (S) & Jul-06 - Aug-06 & Small Adult & Treatment & Headwater & 0.000 & 0.000 & 0.000 & 1.000 \\
\hline Tanner Run & Survival (S) & Aug-06 - Oct-06 & Small Adult & Treatment & Headwater & 0.386 & 0.088 & 0.233 & 0.565 \\
\hline Tanner Run & Survival (S) & Oct-06 - May-07 & Small Adult & Treatment & Headwater & 0.810 & 0.050 & 0.692 & 0.890 \\
\hline Tanner Run & Survival (S) & May-07 - Jul-07 & Small Adult & Treatment & Headwater & 0.726 & 0.077 & 0.555 & 0.850 \\
\hline Tanner Run & Survival (S) & Jul-07 - Oct-07 & Small Adult & Treatment & Headwater & 0.828 & 0.054 & 0.697 & 0.909 \\
\hline Zinn Hollow & Survival (S) & Jul-06 - Aug-06 & Small Adult & Control & Mainstem & 0.323 & 0.089 & 0.176 & 0.515 \\
\hline Zinn Hollow & Survival (S) & Aug-06 - Oct-06 & Small Adult & Control & Mainstem & 0.700 & 0.091 & 0.501 & 0.845 \\
\hline Zinn Hollow & Survival (S) & Oct-06 - May-07 & Small Adult & Control & Mainstem & 0.785 & 0.061 & 0.643 & 0.881 \\
\hline Zinn Hollow & Survival (S) & May-07 - Jul-07 & Small Adult & Control & Mainstem & 0.461 & 0.082 & 0.310 & 0.619 \\
\hline Zinn Hollow & Survival (S) & Jul-07 - Oct-07 & Small Adult & Control & Mainstem & 0.752 & 0.060 & 0.618 & 0.851 \\
\hline Laurel Lick Run & Survival (S) & Jul-06 - Aug-06 & Small Adult & Treatment & Mainstem & 0.000 & 0.000 & 0.000 & 1.000 \\
\hline Laurel Lick Run & Survival (S) & Aug-06 - Oct-06 & Small Adult & Treatment & Mainstem & 0.653 & 0.098 & 0.447 & 0.814 \\
\hline Laurel Lick Run & Survival (S) & Oct-06 - May-07 & Small Adult & Treatment & Mainstem & 0.898 & 0.034 & 0.808 & 0.949 \\
\hline Laurel Lick Run & Survival (S) & May-07 - Jul-07 & Small Adult & Treatment & Mainstem & 0.578 & 0.085 & 0.408 & 0.731 \\
\hline Laurel Lick Run & Survival (S) & Jul-07 - Oct-07 & Small Adult & Treatment & Mainstem & 0.728 & 0.069 & 0.576 & 0.841 \\
\hline Glady Fork & Survival (S) & Jul-06 - Aug-06 & Small Adult & Control & Headwater & 0.601 & 0.114 & 0.372 & 0.792 \\
\hline Glady Fork & Survival (S) & Aug-06 - Oct-06 & Small Adult & Control & Headwater & 0.620 & 0.076 & 0.464 & 0.754 \\
\hline Glady Fork & Survival (S) & Oct-06 - May-07 & Small Adult & Control & Headwater & 0.846 & 0.038 & 0.756 & 0.907 \\
\hline Glady Fork & Survival (S) & May-07 - Jul-07 & Small Adult & Control & Headwater & 0.598 & 0.076 & 0.444 & 0.734 \\
\hline Glady Fork & Survival (S) & Jul-07 - Oct-07 & Small Adult & Control & Headwater & 0.790 & 0.053 & 0.667 & 0.876 \\
\hline Daniel's Run & Survival (S) & Jul-06 - Aug-06 & Small Adult & Treatment & Headwater & 0.000 & 0.000 & 0.000 & 1.000 \\
\hline Daniel's Run & Survival (S) & Aug-06 - Oct-06 & Small Adult & Treatment & Headwater & 0.322 & 0.065 & 0.210 & 0.459 \\
\hline Daniel's Run & Survival (S) & Oct-06 - May-07 & Small Adult & Treatment & Headwater & 0.763 & 0.051 & 0.649 & 0.849 \\
\hline Daniel's Run & Survival (S) & May-07 - Jul-07 & Small Adult & Treatment & Headwater & 0.667 & 0.070 & 0.521 & 0.788 \\
\hline Daniel's Run & Survival (S) & Jul-07 - Oct-07 & Small Adult & Treatment & Headwater & 0.784 & 0.054 & 0.661 & 0.872 \\
\hline Nan's Branch & Survival (S) & Jul-06 - Aug-06 & Large Adult & Control & Mainstem & 0.489 & 0.082 & 0.335 & 0.645 \\
\hline Nan's Branch & Survival (S) & Aug-06 - Oct-06 & Large Adult & Control & Mainstem & 0.824 & 0.054 & 0.693 & 0.907 \\
\hline Nan's Branch & Survival (S) & Oct-06 - May-07 & Large Adult & Control & Mainstem & 0.880 & 0.029 & 0.811 & 0.926 \\
\hline Nan's Branch & Survival (S) & May-07 - Jul-07 & Large Adult & Control & Mainstem & 0.632 & 0.073 & 0.481 & 0.761 \\
\hline
\end{tabular}




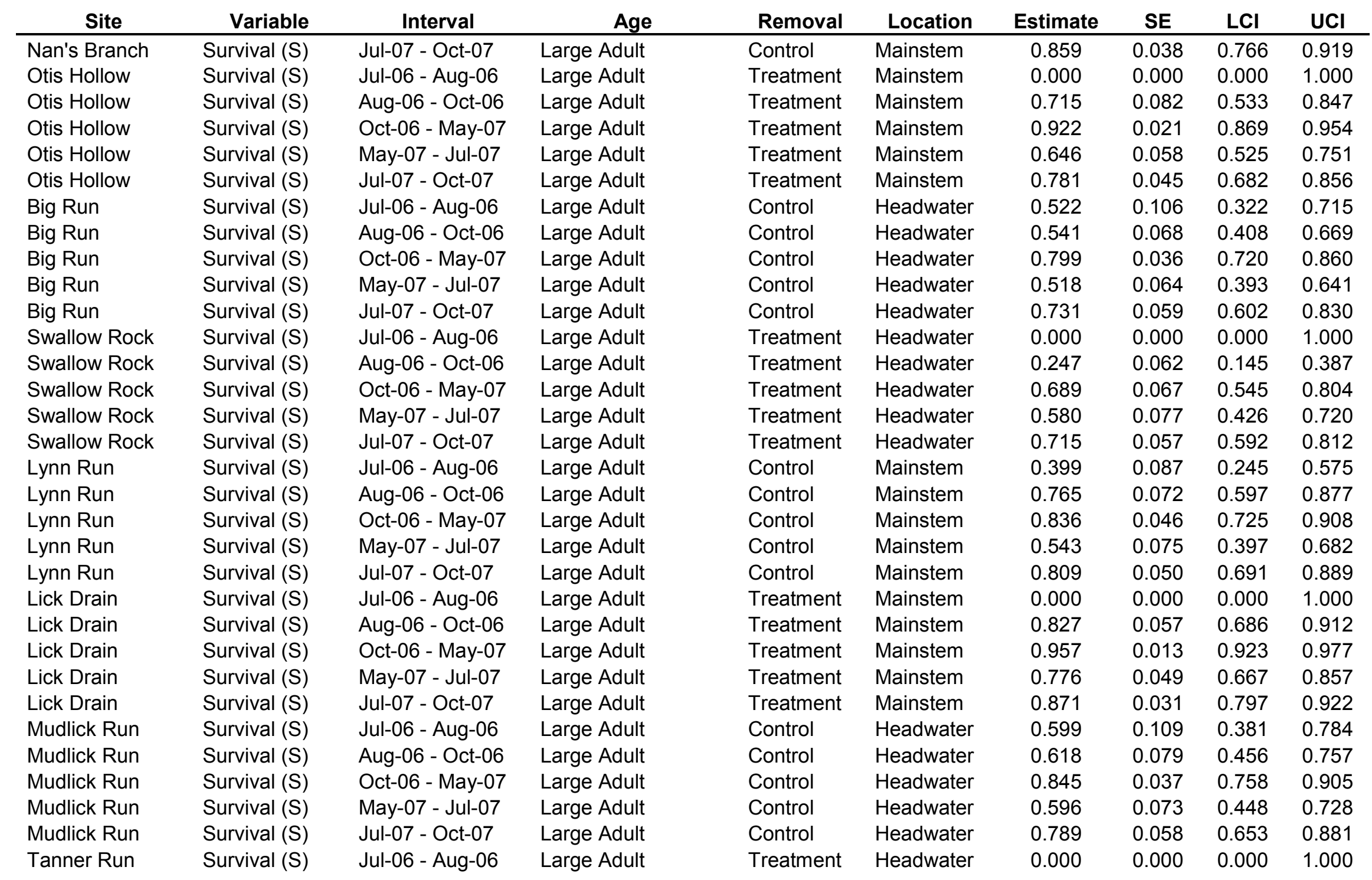




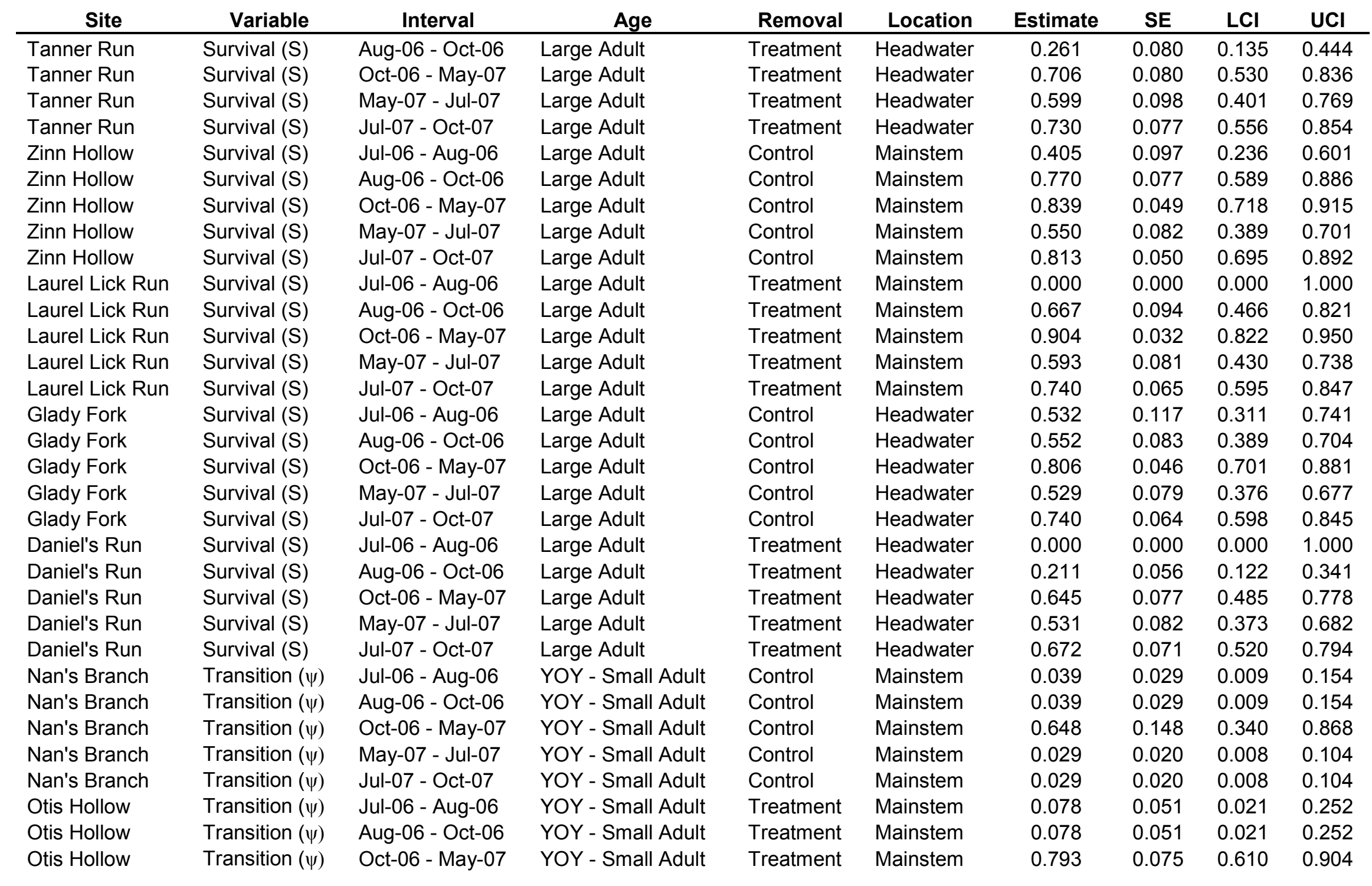




\begin{tabular}{|c|c|c|c|c|c|c|c|c|c|}
\hline Site & Variable & Interval & Age & Removal & Location & Estimate & SE & $\mathrm{LCl}$ & $\mathrm{UCl}$ \\
\hline Big Run & Transition $(\psi)$ & Jul-06 - Aug-06 & YOY - Small Adult & Control & Headwater & 0.032 & 0.028 & 0.005 & 0.162 \\
\hline Big Run & Transition $(\psi)$ & Aug-06 - Oct-06 & YOY - Small Adult & Control & Headwater & 0.032 & 0.028 & 0.005 & 0.162 \\
\hline Big Run & Transition $(\psi)$ & May-07 - Jul-07 & YOY - Small Adult & Control & Headwater & 0.023 & 0.018 & 0.005 & 0.104 \\
\hline Big Run & Transition $(\psi)$ & Jul-07 - Oct-07 & YOY - Small Adult & Control & Headwater & 0.023 & 0.018 & 0.005 & 0.104 \\
\hline Swallow Rock & Transition $(\psi)$ & Jul-06 - Aug-06 & YOY - Small Adult & Treatment & Headwater & 0.110 & 0.075 & 0.027 & 0.359 \\
\hline Swallow Rock & Transition $(\psi)$ & May-07 - Jul-07 & YOY - Small Adult & Treatment & Headwater & 0.083 & 0.040 & 0.031 & 0.200 \\
\hline Swallow Rock & Transition $(\psi)$ & Jul-07 - Oct-07 & YOY - Small Adult & Treatment & Headwater & 0.083 & 0.040 & 0.031 & 0.200 \\
\hline Lynn Run & Transition $(\psi)$ & Jul-06 - Aug-06 & YOY - Small Adult & Control & Mainstem & 0.026 & 0.034 & 0.002 & 0.263 \\
\hline Lynn Run & Transition $(\psi)$ & Aug-06 - Oct-06 & YOY - Small Adult & Control & Mainstem & 0.026 & 0.034 & 0.002 & 0.263 \\
\hline Lynn Run & Transition $(\psi)$ & Oct-06 - May-07 & YOY - Small Adult & Control & Mainstem & 0.547 & 0.316 & 0.090 & 0.936 \\
\hline Lynn Run & Transition $(\psi)$ & May-07 - Jul-07 & YOY - Small Adult & Control & Mainstem & 0.019 & 0.024 & 0.002 & 0.188 \\
\hline Lick Drain & Transition $(\psi)$ & May-07 - Jul-07 & YOY - Small Adult & Treatment & Mainstem & & 0.071 & 0.053 & 0.343 \\
\hline Lick Drain & Transition $(\psi)$ & Jul-07 - Oct-07 & YOY - Small Adult & Treatment & Mainstem & 0.145 & 0.071 & 0.053 & 0.343 \\
\hline Mudlick Run & Transition $(\psi)$ & Jul-06 - Aug-06 & YOY - Small Adult & Control & Headwater & 0.047 & 0.051 & 0.005 & 0.314 \\
\hline Mudlick Run & Transition $(\psi)$ & Aug-06 - Oct-06 & YOY - Small Adult & Control & Headwater & 0.047 & 0.051 & 0.005 & 0.314 \\
\hline Mudlick Run & Transition $(\psi)$ & Oct-06 - May-07 & YOY - Small Adult & Control & Headwater & 0.688 & 0.233 & 0.208 & 0.949 \\
\hline Mudlick Run & Transition $(\psi)$ & May-07 - Jul-07 & YOY - Small Adult & Control & Headwater & 0.034 & 0.036 & 0.004 & 0.235 \\
\hline Mudlick Run & Transition $(\psi)$ & Jul-07 - Oct-07 & YOY - Small Adult & Control & Headwater & 0.034 & 0.036 & 0.004 & 0.235 \\
\hline Tanner Run & Transition $(\psi)$ & Jul-06 - Aug-06 & YOY - Small Adult & Treatment & Headwater & 0.019 & 0.028 & 0.001 & 0.270 \\
\hline Tanner Run & Transition $(\psi)$ & Aug-06 - Oct-06 & YOY - Small Adult & Treatment & Headwater & 0.019 & 0.028 & 0.001 & 0.270 \\
\hline Tanner Run & Transition $(\psi)$ & Oct-06 - May-07 & YOY - Small Adult & Treatment & Headwater & 0.463 & 0.344 & 0.054 & 0.928 \\
\hline Tanner Run & Transition $(\psi)$ & May-07 - Jul-07 & YOY - Small Adult & Treatment & Headwater & 0.014 & 0.020 & 0.001 & 0.191 \\
\hline Tanner Run & Transition $(\psi)$ & Jul-07 - Oct-07 & YOY - Small Adult & Treatment & Headwater & 0.014 & 0.020 & 0.001 & 0.191 \\
\hline
\end{tabular}




\begin{tabular}{|c|c|c|c|c|c|c|c|c|c|}
\hline Site & Variable & Interval & Age & Removal & Location & Estimate & SE & $\mathrm{LCl}$ & $\mathrm{UCl}$ \\
\hline Zinn Hollow & Transition $(\psi)$ & Oct-06 - May-07 & YOY - Small Adult & Control & Mainstem & 0.885 & 0.116 & 0.449 & 0.987 \\
\hline Zinn Hollow & Transition $(\psi)$ & Jul-07 - Oct-07 & YOY - Small Adult & Control & Mainstem & 0.111 & 0.103 & 0.016 & 0.492 \\
\hline Laurel Lick Run & Transition $(\psi)$ & Jul-06 - Aug-06 & YOY - Small Adult & Treatment & Mainstem & 0.179 & 0.246 & 0.008 & 0.853 \\
\hline Laurel Lick Run & Transition $(\psi)$ & Aug-06 - Oct-06 & YOY - Small Adult & Treatment & Mainstem & 0.179 & 0.246 & 0.008 & 0.853 \\
\hline Laurel Lick Run & Transition $(\psi)$ & Oct-06 - May-07 & YOY - Small Adult & Treatment & Mainstem & 0.908 & 0.136 & 0.290 & 0.996 \\
\hline Glady Fork & Transition $(\psi)$ & Jul-06 - Aug-06 & YOY - Small Adult & Control & Headwater & 0.215 & 0.130 & 0.057 & 0.553 \\
\hline Glady Fork & Transition $(\psi)$ & Aug-06 - Oct-06 & YOY - Small Adult & Control & Headwater & 0.215 & 0.130 & 0.057 & 0.553 \\
\hline Glady Fork & Transition $(\psi)$ & Oct-06 - May-07 & YOY - Small Adult & Control & Headwater & 0.925 & 0.047 & 0.765 & 0.979 \\
\hline Glady Fork & Transition $(\psi)$ & May-07 - Jul-07 & YOY - Small Adult & Control & Headwater & 0.166 & 0.073 & 0.067 & 0.358 \\
\hline Glady Fork & Transition $(\psi)$ & Jul-07 - Oct-07 & YOY - Small Adult & Control & Headwater & 0.166 & 0.073 & 0.067 & 0.358 \\
\hline Daniel's Run & Transition $(\psi)$ & Jul-06 - Aug-06 & YOY - Small Adult & Treatment & Headwater & 0.207 & 0.124 & 0.056 & 0.533 \\
\hline Nan's Branch & Transition $(\psi)$ & Jul-06 - Aug-06 & Small - Large Adult & Control & Mainstem & 0.039 & 0.030 & 0.009 & 0.163 \\
\hline Nan's Branch & Transition $(\psi)$ & Aug-06 - Oct-06 & Small - Large Adult & Control & Mainstem & 0.039 & 0.030 & 0.009 & 0.163 \\
\hline Nan's Branch & Transition $(\psi)$ & Oct-06 - May-07 & Small - Large Adult & Control & Mainstem & 0.650 & 0.158 & 0.323 & 0.878 \\
\hline Nan's Branch & Transition $(\psi)$ & May-07 - Jul-07 & Small - Large Adult & Control & Mainstem & 0.029 & 0.021 & 0.007 & 0.114 \\
\hline Nan's Branch & Transition $(\psi)$ & Jul-07 - Oct-07 & Small - Large Adult & Control & Mainstem & 0.029 & 0.021 & 0.007 & 0.114 \\
\hline Otis Hollow & Transition $(\psi)$ & Jul-06 - Aug-06 & Small - Large Adult & Treatment & Mainstem & 0.079 & 0.050 & 0.021 & 0.250 \\
\hline Otis Hollow & Transition $(\psi)$ & Aug-06 - Oct-06 & Small - Large Adult & Treatment & Mainstem & 0.079 & 0.050 & 0.021 & 0.250 \\
\hline Otis Hollow & Transition $(\psi)$ & Oct-06 - May-07 & Small - Large Adult & Treatment & Mainstem & 0.794 & 0.075 & 0.611 & 0.905 \\
\hline Otis Hollow & Transition $(\psi)$ & May-07 - Jul-07 & Small - Large Adult & Treatment & Mainstem & 0.059 & 0.026 & 0.024 & 0.137 \\
\hline Otis Hollow & Transition $(\psi)$ & Jul-07 - Oct-07 & Small - Large Adult & Treatment & Mainstem & 0.059 & 0.026 & 0.024 & 0.137 \\
\hline Big Run & Transition $(\psi)$ & Jul-06 - Aug-06 & Small - Large Adult & Control & Headwater & 0.032 & 0.025 & 0.007 & 0.141 \\
\hline Big Run & Transition $(\psi)$ & Aug-06 - Oct-06 & Small - Large Adult & Control & Headwater & 0.032 & 0.025 & 0.007 & 0.141 \\
\hline
\end{tabular}




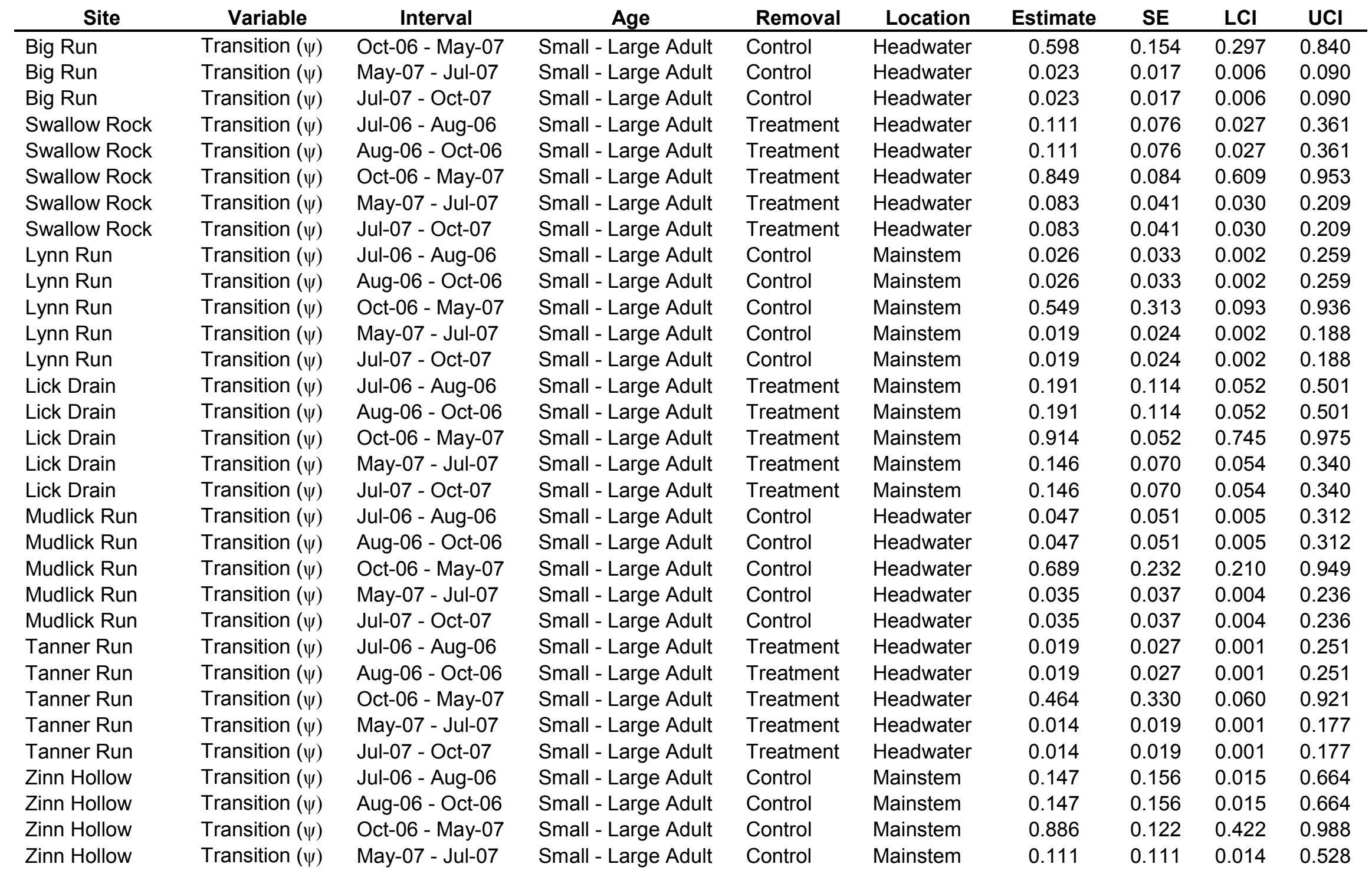




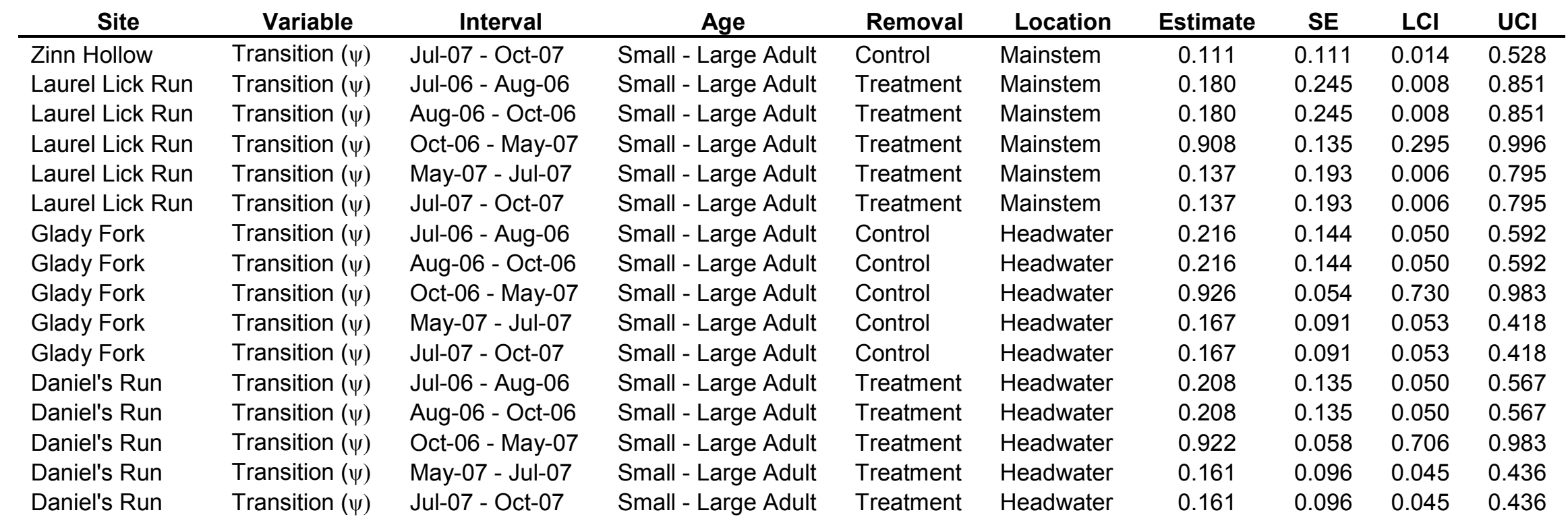


E3. Model coefficient estimates, standard errors, and confidence limits for the modelaveraged coefficients resulting from the $95 \%$ confidence set of multinomial models for estimating mottled sculpin survival and age-class transition probability in response to fish removal treatment, spatial location, and select interactions over the course of the study while accounting for the effects of local physical and chemical habitat covariates. The multinomial model has two intercepts, one for estimating survival (Intercept 1) and one for estimating age-class transition (Intercept 2). $\Sigma \mathrm{w}_{\mathrm{i}}=$ sum of Akaike weights for term across $95 \%$ confidence model set. The cumulative Akaike weights for each model term are indicated next to the relevant effect. Asterisks $\left(^{*}\right)$ indicate where model coefficient confidence limits did not include 0 (i.e., a "significant" positive or negative slope). a1 = YOY age class, $\mathrm{a} 2=$ small adults, $\mathrm{g}=$ fish removal treatment, $\mathrm{l}=$ tributary spatial location, $\mathrm{t} 1-\mathrm{t} 4=$ sampling date, $\mathrm{a} 1 * \mathrm{~g}=$ YOY $\mathrm{x}$ removal interaction effect, $\mathrm{a} 2 * \mathrm{~g}=$ small adult $\mathrm{x}$ removal, $\mathrm{a} 1 * 1=\mathrm{YOY} \mathrm{x}$ location, $\mathrm{a} 2 * 1=$ small adult $\mathrm{x}$ location, $\mathrm{g} * \mathrm{l}=$ removal $\mathrm{x}$ location, $\mathrm{g} * \mathrm{t} 1-\mathrm{g} * \mathrm{t} 4=$ removal $\mathrm{x}$ date, $1 * \mathrm{t} 1-1 * \mathrm{t} 4=$ location $\mathrm{x}$ date, $\mathrm{g} * 1 * \mathrm{t} 1-\mathrm{g} * 1 * \mathrm{t} 4=$ removal $\mathrm{x}$ location $\mathrm{x}$ date, $\mathrm{W} 1=$ water chemistry principal component $1, \mathrm{H} 1-\mathrm{H} 3=$ physical habitat principal components 1 to 3 , PAREA = proportion of spring wetted channel area in early fall, CANP $=$ mean percent canopy cover, MWAT $=$ maximum weekly average temperature $\left({ }^{\circ} \mathrm{C}\right)$.

\begin{tabular}{|c|c|c|c|c|c|}
\hline Effect & Estimate & SE & $\mathrm{LCI}$ & $\mathrm{UCI}$ & Ewi \\
\hline Intercept & -1.416 & 1.778 & -4.901 & 2.068 & \\
\hline W1 & 0.204 & 0.221 & -0.229 & 0.636 & \\
\hline $\mathrm{H} 1$ & 0.095 & 0.038 & 0.021 & 0.170 & * \\
\hline $\mathrm{H} 2$ & 0.006 & 0.052 & -0.095 & 0.107 & \\
\hline H3 & 0.201 & 0.094 & 0.016 & 0.386 & * \\
\hline PAREA & 0.384 & 0.279 & -0.163 & 0.930 & \\
\hline CANP & -0.045 & 0.011 & -0.066 & -0.023 & * \\
\hline MWAT & 0.234 & 0.072 & 0.092 & 0.375 & * \\
\hline a1 & -1.421 & 0.280 & -1.970 & -0.872 & * \\
\hline a2 & -0.251 & 0.139 & -0.523 & 0.021 & \\
\hline g & 0.161 & 0.225 & -0.281 & 0.602 & 1.000 \\
\hline I & 0.307 & 0.380 & -0.437 & 1.051 & 1.000 \\
\hline $\mathrm{t} 1$ & -0.238 & 0.252 & -0.733 & 0.257 & \\
\hline t2 & 0.932 & 0.239 & 0.463 & 1.400 & * \\
\hline $\mathrm{t} 3$ & 0.536 & 0.213 & 0.118 & 0.954 & * \\
\hline $\mathrm{t} 4$ & 0.236 & 0.218 & -0.191 & 0.663 & \\
\hline$a 1^{*} g$ & -0.375 & 0.377 & -1.113 & 0.364 & \\
\hline$a 2^{*} g$ & -0.127 & 0.161 & -0.442 & 0.187 & \\
\hline$a 1^{*} \mid$ & -0.355 & 0.408 & -1.154 & 0.444 & \\
\hline$a 2 * 1$ & -0.420 & 0.162 & -0.738 & -0.101 & * \\
\hline$g^{*}$ & -0.069 & 0.419 & -0.890 & 0.752 & 0.989 \\
\hline$g^{*}+1$ & -2.648 & 0.654 & -3.930 & -1.366 & 1.000 \\
\hline$g^{*}+2$ & -1.129 & 0.302 & -1.721 & -0.537 & * \\
\hline$g^{*}+3$ & 0.075 & 0.291 & -0.495 & 0.645 & \\
\hline$g^{*} t 4$ & -0.027 & 0.295 & -0.605 & 0.550 & \\
\hline$I^{*}+1$ & 0.948 & 0.341 & 0.280 & 1.616 & 0.995 \\
\hline
\end{tabular}




\begin{tabular}{|c|c|c|c|c|c|}
\hline I*t2 & 0.214 & 0.333 & -0.439 & 0.866 & \multirow{7}{*}{0.974} \\
\hline I*t3 & 0.125 & 0.298 & -0.459 & 0.710 & \\
\hline$\left.\right|^{*} t 4$ & 0.054 & 0.311 & -0.556 & 0.664 & \\
\hline$\left.g^{*}\right|^{*} t 1$ & -13.374 & 253.406 & -510.049 & 483.302 & \\
\hline$\left.g^{*}\right|^{*} t 2$ & -0.383 & 0.476 & -1.316 & 0.550 & \\
\hline$\left.g^{*}\right|^{*} \mathrm{t} 3$ & -1.061 & 0.431 & -1.905 & -0.217 & \\
\hline$\left.\mathrm{g}^{*}\right|^{*} \mathrm{t} 4$ & -0.050 & 0.451 & -0.935 & 0.834 & \\
\hline Intercept 2 & -23.718 & 12.218 & -47.666 & 0.230 & \\
\hline W1 & -0.244 & 1.410 & -3.008 & 2.521 & \\
\hline $\mathrm{H} 1$ & -0.029 & 0.241 & -0.501 & 0.444 & \\
\hline $\mathrm{H} 2$ & 0.677 & 0.335 & 0.020 & 1.333 & \\
\hline $\mathrm{H} 3$ & 0.166 & 0.736 & -1.276 & 1.608 & \\
\hline PAREA & -1.666 & 1.509 & -4.624 & 1.293 & \\
\hline CANP & 0.082 & 0.067 & -0.050 & 0.214 & \\
\hline MWAT & 0.872 & 0.597 & -0.299 & 2.042 & \\
\hline a & 3.821 & 0.700 & 2.450 & 5.193 & * \\
\hline g & 0.892 & 0.967 & -1.003 & 2.788 & 0.540 \\
\hline I & 1.644 & 2.144 & -2.559 & 5.846 & 0.420 \\
\hline $\mathrm{t} 1$ & 1.861 & 0.877 & 0.143 & 3.579 & * \\
\hline t2 & 1.578 & 0.576 & 0.449 & 2.707 & * \\
\hline $\mathrm{t} 3$ & 5.338 & 0.657 & 4.050 & 6.627 & * \\
\hline t4 & 0.839 & 0.518 & -0.177 & 1.855 & \\
\hline$g^{*} \mid$ & -2.571 & 2.130 & -6.746 & 1.604 & 0.182 \\
\hline
\end{tabular}


E4. Summary of model-averaged parameter estimates, standard errors, and $95 \%$ confidence limits (i.e., lower $=$ LCI and upper $=\mathrm{UCI}$ ) for apparent survival (S) and age class transition probability $(\psi)$ for mottled sculpin for all sites and sample dates. Experimental classifications of sites based on treatment and spatial location are also noted.

\begin{tabular}{|c|c|c|c|c|c|c|c|c|c|}
\hline Site & Variable & Interval & Age & Removal & Location & Estimate & SE & LCI & $\mathrm{UCl}$ \\
\hline Nan's Branch & Survival (S) & Jul-06 - Aug-06 & YOY & Control & Mainstem & 0.096 & 0.034 & 0.047 & 0.184 \\
\hline Nan's Branch & Survival (S) & Aug-06 - Oct-06 & YOY & Control & Mainstem & 0.140 & 0.047 & 0.071 & 0.259 \\
\hline Nan's Branch & Survival (S) & Oct-06 - May-07 & YOY & Control & Mainstem & 0.092 & 0.028 & 0.049 & 0.164 \\
\hline Nan's Branch & Survival (S) & May-07 - Jul-07 & YOY & Control & Mainstem & 0.065 & 0.022 & 0.033 & 0.124 \\
\hline Nan's Branch & Survival (S) & Jul-07 - Oct-07 & YOY & Control & Mainstem & 0.049 & 0.018 & 0.024 & 0.100 \\
\hline Otis Hollow & Survival (S) & Jul-06 - Aug-06 & YOY & Treatment & Mainstem & 0.000 & 0.000 & 0.000 & 0.000 \\
\hline Otis Hollow & Survival (S) & Aug-06 - Oct-06 & YOY & Treatment & Mainstem & 0.028 & 0.015 & 0.010 & 0.081 \\
\hline Otis Hollow & Survival (S) & Oct-06 - May-07 & YOY & Treatment & Mainstem & 0.030 & 0.015 & 0.011 & 0.076 \\
\hline Otis Hollow & Survival (S) & May-07 - Jul-07 & YOY & Treatment & Mainstem & 0.050 & 0.025 & 0.018 & 0.127 \\
\hline Otis Hollow & Survival (S) & Jul-07 - Oct-07 & YOY & Treatment & Mainstem & 0.041 & 0.021 & 0.015 & 0.106 \\
\hline Big Run & Survival (S) & Jul-06 - Aug-06 & YOY & Control & Headwater & 0.072 & 0.024 & 0.037 & 0.136 \\
\hline Big Run & Survival (S) & Aug-06 - Oct-06 & YOY & Control & Headwater & 0.200 & 0.056 & 0.112 & 0.330 \\
\hline Big Run & Survival (S) & Oct-06 - May-07 & YOY & Control & Headwater & 0.144 & 0.039 & 0.083 & 0.238 \\
\hline Big Run & Survival (S) & May-07 - Jul-07 & YOY & Control & Headwater & 0.111 & 0.028 & 0.066 & 0.179 \\
\hline Big Run & Survival (S) & Jul-07 - Oct-07 & YOY & Control & Headwater & 0.089 & 0.026 & 0.050 & 0.155 \\
\hline Swallow Rock & Survival (S) & Jul-06 - Aug-06 & YOY & Treatment & Headwater & 0.004 & 0.003 & 0.001 & 0.016 \\
\hline Swallow Rock & Survival (S) & Aug-06 - Oct-06 & YOY & Treatment & Headwater & 0.059 & 0.020 & 0.030 & 0.113 \\
\hline Swallow Rock & Survival (S) & Oct-06 - May-07 & YOY & Treatment & Headwater & 0.124 & 0.037 & 0.068 & 0.217 \\
\hline Swallow Rock & Survival (S) & May-07 - Jul-07 & YOY & Treatment & Headwater & 0.086 & 0.026 & 0.047 & 0.152 \\
\hline Swallow Rock & Survival (S) & Jul-07 - Oct-07 & YOY & Treatment & Headwater & 0.071 & 0.023 & 0.038 & 0.130 \\
\hline Lynn Run & Survival (S) & Jul-06 - Aug-06 & YOY & Control & Mainstem & 0.146 & 0.047 & 0.076 & 0.265 \\
\hline Lynn Run & Survival (S) & Aug-06 - Oct-06 & YOY & Control & Mainstem & 0.210 & 0.063 & 0.111 & 0.360 \\
\hline Lynn Run & Survival (S) & Oct-06 - May-07 & YOY & Control & Mainstem & 0.141 & 0.042 & 0.077 & 0.243 \\
\hline Lynn Run & Survival (S) & May-07 - Jul-07 & YOY & Control & Mainstem & 0.101 & 0.033 & 0.052 & 0.187 \\
\hline Lynn Run & Survival (S) & Jul-07 - Oct-07 & YOY & Control & Mainstem & 0.078 & 0.028 & 0.038 & 0.152 \\
\hline Lick Drain & Survival (S) & Jul-06 - Aug-06 & YOY & Treatment & Mainstem & 0.000 & 0.000 & 0.000 & 1.000 \\
\hline Lick Drain & Survival (S) & Aug-06 - Oct-06 & YOY & Treatment & Mainstem & 0.048 & 0.024 & 0.018 & 0.123 \\
\hline Lick Drain & Survival (S) & Oct-06 - May-07 & YOY & Treatment & Mainstem & 0.050 & 0.023 & 0.021 & 0.118 \\
\hline
\end{tabular}




\begin{tabular}{|c|c|c|c|c|c|c|c|c|c|}
\hline Site & Variable & Interval & Age & Removal & Location & Estimate & SE & $\mathrm{LCl}$ & $\mathrm{UCl}$ \\
\hline Mudlick Run & Survival (S) & Jul-06 - Aug-06 & YOY & Control & Headwater & 0.109 & 0.033 & 0.059 & 0.192 \\
\hline Mudlick Run & Survival (S) & Aug-06 - Oct-06 & YOY & Control & Headwater & 0.282 & 0.068 & 0.170 & 0.431 \\
\hline Mudlick Run & Survival (S) & May-07 - Jul-07 & YOY & Control & Headwater & 0.164 & 0.038 & 0.102 & 0.253 \\
\hline Mudlick Run & Survival (S) & Jul-07 - Oct-07 & YOY & Control & Headwater & 0.134 & 0.037 & 0.077 & 0.223 \\
\hline Tanner Run & Survival (S) & Jul-06 - Aug-06 & YOY & Treatment & Headwater & 0.007 & 0.005 & 0.002 & 0.026 \\
\hline Tanner Run & Survival (S) & May-07 - Jul-07 & YOY & Treatment & Headwater & 0.140 & 0.035 & 0.084 & 0.225 \\
\hline Tanner Run & Survival (S) & Jul-07 - Oct-07 & YOY & Treatment & Headwater & 0.117 & 0.033 & 0.066 & 0.198 \\
\hline Zinn Hollow & Survival (S) & Jul-06 - Aug-06 & YOY & Control & Mainstem & 0.086 & 0.033 & 0.040 & 0.176 \\
\hline Zinn Hollow & Survival (S) & Aug-06 - Oct-06 & YOY & Control & Mainstem & 0.127 & 0.046 & 0.061 & 0.246 \\
\hline Zinn Hollow & Survival (S) & Oct-06 - May-07 & YOY & Control & Mainstem & 0.083 & 0.028 & 0.042 & 0.158 \\
\hline Zinn Hollow & Survival (S) & May-07 - Jul-07 & YOY & Control & Mainstem & 0.058 & 0.021 & 0.028 & 0.117 \\
\hline Laurel Lick Run & Survival (S) & May-07 - Jul-07 & YOY & Treatment & Mainstem & 0.091 & 0.041 & 0.037 & 0.208 \\
\hline Laurel Lick Run & Survival (S) & Jul-07 - Oct-07 & YOY & Treatment & Mainstem & 0.075 & 0.035 & 0.030 & 0.177 \\
\hline Glady Fork & Survival (S) & Jul-06 - Aug-06 & YOY & Control & Headwater & 0.107 & 0.037 & 0.054 & 0.204 \\
\hline Glady Fork & Survival (S) & Aug-06 - Oct-06 & YOY & Control & Headwater & 0.279 & 0.071 & 0.163 & 0.435 \\
\hline Glady Fork & Survival (S) & Oct-06 - May-07 & YOY & Control & Headwater & 0.207 & 0.054 & 0.120 & 0.333 \\
\hline Glady Fork & Survival (S) & May-07 - Jul-07 & YOY & Control & Headwater & 0.162 & 0.043 & 0.094 & 0.264 \\
\hline Glady Fork & Survival (S) & Jul-07 - Oct-07 & YOY & Control & Headwater & 0.132 & 0.040 & 0.072 & 0.231 \\
\hline Daniel's Run & Survival (S) & Jul-06 - Aug-06 & YOY & Treatment & Headwater & 0.008 & 0.005 & 0.002 & 0.029 \\
\hline Daniel's Run & Survival (S) & Aug-06 - Oct-06 & YOY & Treatment & Headwater & 0.107 & 0.030 & 0.060 & 0.183 \\
\hline Daniel's Run & Survival (S) & Oct-06 - May-07 & YOY & Treatment & Headwater & 0.212 & 0.052 & 0.127 & 0.331 \\
\hline Daniel's Run & Survival (S) & May-07 - Jul-07 & YOY & Treatment & Headwater & 0.152 & 0.038 & 0.091 & 0.243 \\
\hline Daniel's Run & Survival (S) & Jul-07 - Oct-07 & YOY & Treatment & Headwater & 0.127 & 0.036 & 0.072 & 0.215 \\
\hline
\end{tabular}




\begin{tabular}{|c|c|c|c|c|c|c|c|c|c|}
\hline Site & Variable & Interval & Age & Removal & Location & Estimate & SE & $\mathrm{LCl}$ & $\mathrm{UCl}$ \\
\hline Nan's Branch & Survival (S) & Jul-06 - Aug-06 & Small Adult & Control & Mainstem & 0.242 & 0.039 & 0.174 & 0.326 \\
\hline Nan's Branch & Survival (S) & Aug-06 - Oct-06 & Small Adult & Control & Mainstem & 0.330 & 0.048 & 0.245 & 0.429 \\
\hline Nan's Branch & Survival (S) & Oct-06 - May-07 & Small Adult & Control & Mainstem & 0.233 & 0.032 & 0.176 & 0.302 \\
\hline Nan's Branch & Survival (S) & May-07 - Jul-07 & Small Adult & Control & Mainstem & 0.173 & 0.025 & 0.130 & 0.227 \\
\hline Nan's Branch & Survival (S) & Jul-07 - Oct-07 & Small Adult & Control & Mainstem & 0.136 & 0.024 & 0.094 & 0.191 \\
\hline Otis Hollow & Survival (S) & Jul-06 - Aug-06 & Small Adult & Treatment & Mainstem & 0.000 & 0.000 & 0.000 & 1.000 \\
\hline Otis Hollow & Survival (S) & Aug-06 - Oct-06 & Small Adult & Treatment & Mainstem & 0.102 & 0.033 & 0.053 & 0.186 \\
\hline Otis Hollow & Survival (S) & Oct-06 - May-07 & Small Adult & Treatment & Mainstem & 0.106 & 0.030 & 0.060 & 0.180 \\
\hline Otis Hollow & Survival (S) & May-07 - Jul-07 & Small Adult & Treatment & Mainstem & 0.168 & 0.043 & 0.099 & 0.271 \\
\hline Otis Hollow & Survival (S) & Jul-07 - Oct-07 & Small Adult & Treatment & Mainstem & 0.141 & 0.036 & 0.084 & 0.227 \\
\hline Big Run & Survival (S) & Jul-06 - Aug-06 & Small Adult & Control & Headwater & 0.200 & 0.040 & 0.132 & 0.291 \\
\hline Big Run & Survival (S) & Aug-06 - Oct-06 & Small Adult & Control & Headwater & 0.446 & 0.060 & 0.332 & 0.565 \\
\hline Big Run & Survival (S) & Oct-06 - May-07 & Small Adult & Control & Headwater & 0.351 & 0.049 & 0.261 & 0.453 \\
\hline Big Run & Survival (S) & May-07 - Jul-07 & Small Adult & Control & Headwater & 0.286 & 0.039 & 0.215 & 0.369 \\
\hline Big Run & Survival (S) & Jul-07 - Oct-07 & Small Adult & Control & Headwater & 0.240 & 0.035 & 0.178 & 0.316 \\
\hline Swallow Rock & Survival (S) & Jul-06 - Aug-06 & Small Adult & Treatment & Headwater & 0.017 & 0.011 & 0.005 & 0.056 \\
\hline Swallow Rock & Survival (S) & Aug-06 - Oct-06 & Small Adult & Treatment & Headwater & 0.206 & 0.036 & 0.144 & 0.286 \\
\hline Swallow Rock & Survival (S) & Oct-06 - May-07 & Small Adult & Treatment & Headwater & 0.369 & 0.055 & 0.269 & 0.481 \\
\hline Swallow Rock & Survival (S) & May-07 - Jul-07 & Small Adult & Treatment & Headwater & 0.281 & 0.046 & 0.200 & 0.379 \\
\hline Swallow Rock & Survival (S) & Jul-07 - Oct-07 & Small Adult & Treatment & Headwater & 0.241 & 0.035 & 0.179 & 0.315 \\
\hline Lynn Run & Survival (S) & Jul-06 - Aug-06 & Small Adult & Control & Mainstem & 0.341 & 0.043 & 0.262 & 0.430 \\
\hline Lynn Run & Survival (S) & Aug-06 - Oct-06 & Small Adult & Control & Mainstem & 0.445 & 0.050 & 0.350 & 0.544 \\
\hline Lynn Run & Survival (S) & Oct-06 - May-07 & Small Adult & Control & Mainstem & 0.331 & 0.041 & 0.255 & 0.415 \\
\hline Lynn Run & Survival (S) & May-07 - Jul-07 & Small Adult & Control & Mainstem & 0.254 & 0.033 & 0.194 & 0.325 \\
\hline Lynn Run & Survival (S) & Jul-07 - Oct-07 & Small Adult & Control & Mainstem & 0.203 & 0.032 & 0.147 & 0.274 \\
\hline Lick Drain & Survival (S) & Jul-06 - Aug-06 & Small Adult & Treatment & Mainstem & 0.000 & 0.000 & 0.000 & 1.000 \\
\hline Lick Drain & Survival (S) & Aug-06 - Oct-06 & Small Adult & Treatment & Mainstem & 0.164 & 0.038 & 0.102 & 0.253 \\
\hline Lick Drain & Survival (S) & Oct-06 - May-07 & Small Adult & Treatment & Mainstem & 0.170 & 0.034 & 0.113 & 0.247 \\
\hline Lick Drain & Survival (S) & May-07 - Jul-07 & Small Adult & Treatment & Mainstem & 0.259 & 0.043 & 0.184 & 0.353 \\
\hline Lick Drain & Survival (S) & Jul-07 - Oct-07 & Small Adult & Treatment & Mainstem & 0.221 & 0.031 & 0.165 & 0.288 \\
\hline Mudlick Run & Survival (S) & Jul-06 - Aug-06 & Small Adult & Control & Headwater & 0.282 & 0.044 & 0.205 & 0.375 \\
\hline Mudlick Run & Survival (S) & Aug-06 - Oct-06 & Small Adult & Control & Headwater & 0.559 & 0.054 & 0.452 & 0.660 \\
\hline
\end{tabular}




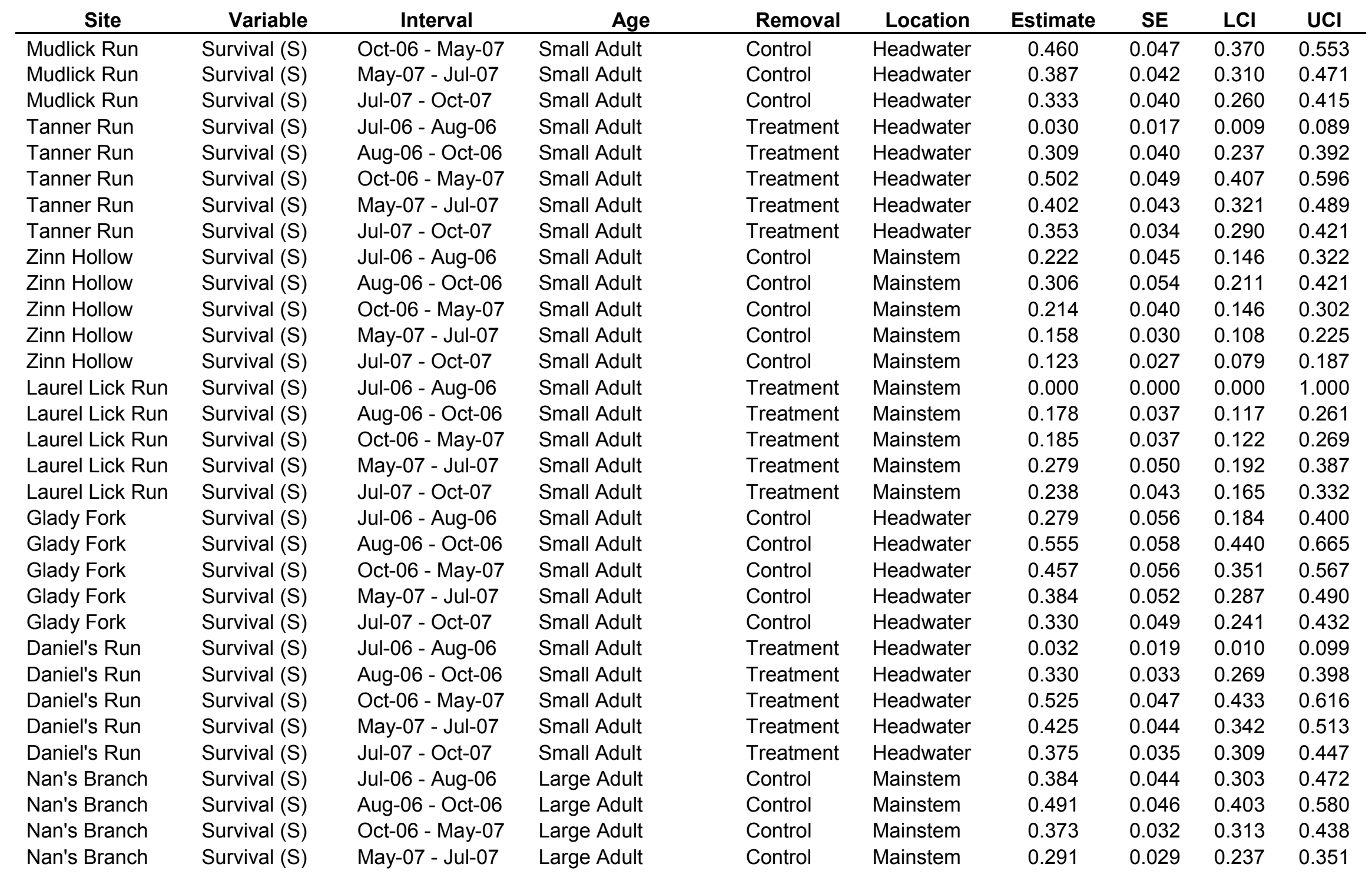




\begin{tabular}{|c|c|c|c|c|c|c|c|c|c|}
\hline Site & Variable & Interval & Age & Removal & Location & Estimate & SE & $\mathrm{LCl}$ & $\mathrm{UCl}$ \\
\hline Otis Hollow & Survival (S) & Aug-06 - Oct-06 & Large Adult & Treatment & Mainstem & 0.201 & 0.053 & 0.116 & 0.326 \\
\hline Otis Hollow & Survival (S) & May-07 - Jul-07 & Large Adult & Treatment & Mainstem & 0.310 & 0.060 & 0.206 & 0.437 \\
\hline Otis Hollow & Survival (S) & Jul-07 - Oct-07 & Large Adult & Treatment & Mainstem & 0.267 & 0.055 & 0.173 & 0.387 \\
\hline Big Run & Survival (S) & Jul-06 - Aug-06 & Large Adult & Control & Headwater & 0.243 & 0.043 & 0.168 & 0.337 \\
\hline Big Run & Survival (S) & Aug-06 - Oct-06 & Large Adult & Control & Headwater & 0.508 & 0.055 & 0.402 & 0.613 \\
\hline Big Run & Survival (S) & Jul-07 - Oct-07 & Large Adult & Control & Headwater & 0.289 & 0.036 & 0.224 & 0.364 \\
\hline Swallow Rock & Survival (S) & Jul-06 - Aug-06 & Large Adult & Treatment & Headwater & 0.025 & 0.015 & 0.008 & 0.079 \\
\hline Swallow Rock & Survival (S) & Aug-06 - Oct-06 & Large Adult & Treatment & Headwater & 0.275 & 0.042 & 0.201 & 0.364 \\
\hline Swallow Rock & Survival (S) & Oct-06 - May-07 & Large Adult & Treatment & Headwater & 0.460 & 0.053 & 0.359 & 0.564 \\
\hline Swallow Rock & Survival (S) & May-07 - Jul-07 & Large Adult & Treatment & Headwater & 0.363 & 0.047 & 0.276 & 0.459 \\
\hline Swallow Rock & Survival (S) & Jul-07 - Oct-07 & Large Adult & Treatment & Headwater & 0.316 & 0.040 & 0.243 & 0.400 \\
\hline Lynn Run & Survival (S) & Jul-07 - Oct-07 & Large Adult & Control & Mainstem & 0.332 & 0.040 & 0.260 & 0.414 \\
\hline Lick Drain & Survival (S) & Jul-06 - Aug-06 & Large Adult & Treatment & Mainstem & 0.000 & 0.000 & 0.000 & 1.000 \\
\hline Lick Drain & Survival (S) & Aug-06 - Oct-06 & Large Adult & Treatment & Mainstem & 0.304 & 0.056 & 0.205 & 0.424 \\
\hline Lick Drain & Survival (S) & Oct-06 - May-07 & Large Adult & Treatment & Mainstem & 0.313 & 0.044 & 0.233 & 0.405 \\
\hline Lick Drain & Survival (S) & May-07 - Jul-07 & Large Adult & Treatment & Mainstem & 0.438 & 0.051 & 0.341 & 0.539 \\
\hline Lick Drain & Survival (S) & Jul-07 - Oct-07 & Large Adult & Treatment & Mainstem & 0.386 & 0.044 & 0.304 & 0.475 \\
\hline Mudlick Run & Survival (S) & Jul-06 - Aug-06 & Large Adult & Control & Headwater & 0.336 & 0.045 & 0.253 & 0.429 \\
\hline Mudlick Run & Survival (S) & Aug-06 - Oct-06 & Large Adult & Control & Headwater & 0.620 & 0.047 & 0.524 & 0.707 \\
\hline Mudlick Run & Survival (S) & Oct-06 - May-07 & Large Adult & Control & Headwater & 0.523 & 0.041 & 0.443 & 0.602 \\
\hline Mudlick Run & Survival (S) & May-07 - Jul-07 & Large Adult & Control & Headwater & 0.448 & 0.039 & 0.373 & 0.526 \\
\hline Mudlick Run & Survival (S) & Jul-07 - Oct-07 & Large Adult & Control & Headwater & 0.391 & 0.041 & 0.314 & 0.474 \\
\hline Tanner Run & Survival (S) & Jul-06 - Aug-06 & Large Adult & Treatment & Headwater & 0.043 & 0.024 & 0.014 & 0.124 \\
\hline
\end{tabular}




\begin{tabular}{|c|c|c|c|c|c|c|c|c|c|}
\hline Site & Variable & Interval & Age & Removal & Location & Estimate & SE & $\mathrm{LCl}$ & $\mathrm{UCl}$ \\
\hline Tanner Run & Survival (S) & May-07 - Jul-07 & Large Adult & Treatment & Headwater & 0.495 & 0.038 & 0.421 & 0.570 \\
\hline Tanner Run & Survival (S) & Jul-07 - Oct-07 & Large Adult & Treatment & Headwater & 0.443 & 0.037 & 0.373 & 0.516 \\
\hline Zinn Hollow & Survival (S) & Aug-06 - Oct-06 & Large Adult & Control & Mainstem & 0.463 & 0.056 & 0.357 & 0.572 \\
\hline Zinn Hollow & Survival (S) & Oct-06 - May-07 & Large Adult & Control & Mainstem & 0.347 & 0.045 & 0.264 & 0.440 \\
\hline Zinn Hollow & Survival (S) & May-07 - Jul-07 & Large Adult & Control & Mainstem & 0.268 & 0.038 & 0.200 & 0.350 \\
\hline Laurel Lick Run & Survival (S) & Aug-06 - Oct-06 & Large Adult & Treatment & Mainstem & 0.325 & 0.050 & 0.236 & 0.429 \\
\hline Laurel Lick Run & Survival (S) & Oct-06 - May-07 & Large Adult & Treatment & Mainstem & 0.335 & 0.045 & 0.253 & 0.428 \\
\hline Laurel Lick Run & Survival (S) & May-07 - Jul-07 & Large Adult & Treatment & Mainstem & 0.462 & 0.056 & 0.357 & 0.572 \\
\hline Laurel Lick Run & Survival (S) & Jul-07 - Oct-07 & Large Adult & Treatment & Mainstem & 0.410 & 0.056 & 0.307 & 0.522 \\
\hline Glady Fork & Survival (S) & Jul-06 - Aug-06 & Large Adult & Control & Headwater & 0.332 & 0.056 & 0.234 & 0.449 \\
\hline Glady Fork & Survival (S) & Aug-06 - Oct-06 & Large Adult & Control & Headwater & 0.616 & 0.047 & 0.521 & 0.703 \\
\hline Daniel's Run & Survival (S) & Aug-06 - Oct-06 & Large Adult & Treatment & Headwater & 0.418 & 0.035 & 0.352 & 0.488 \\
\hline Daniel's Run & Survival (S) & Oct-06 - May-07 & Large Adult & Treatment & Headwater & 0.618 & 0.040 & 0.536 & 0.693 \\
\hline Daniel's Run & Survival (S) & May-07 - Jul-07 & Large Adult & Treatment & Headwater & 0.519 & 0.040 & 0.441 & 0.596 \\
\hline Daniel's Run & Survival (S) & Jul-07 - Oct-07 & Large Adult & Treatment & Headwater & 0.467 & 0.039 & 0.391 & 0.544 \\
\hline Nan's Branch & Transition $(\psi)$ & Jul-06 - Aug-06 & YOY - Small Adult & Control & Mainstem & 0.961 & 0.040 & 0.752 & 0.995 \\
\hline Nan's Branch & Transition $(\psi)$ & Aug-06 - Oct-06 & YOY - Small Adult & Control & Mainstem & 0.949 & 0.046 & 0.739 & 0.992 \\
\hline Nan's Branch & Transition $(\psi)$ & Oct-06 - May-07 & YOY - Small Adult & Control & Mainstem & 0.999 & 0.001 & 0.990 & 1.000 \\
\hline Nan's Branch & Transition $(\psi)$ & May-07 - Jul-07 & YOY - Small Adult & Control & Mainstem & 0.900 & 0.074 & 0.643 & 0.978 \\
\hline Nan's Branch & Transition $(\psi)$ & Jul-07 - Oct-07 & YOY - Small Adult & Control & Mainstem & 0.795 & 0.150 & 0.391 & 0.959 \\
\hline Otis Hollow & Transition $(\psi)$ & Jul-06 - Aug-06 & YOY - Small Adult & Treatment & Mainstem & 0.954 & 0.119 & 0.093 & 1.000 \\
\hline Otis Hollow & Transition $(\psi)$ & Aug-06 - Oct-06 & YOY - Small Adult & Treatment & Mainstem & 0.940 & 0.150 & 0.079 & 1.000 \\
\hline Otis Hollow & Transition $(\psi)$ & Oct-06 - May-07 & YOY - Small Adult & Treatment & Mainstem & 0.999 & 0.004 & 0.810 & 1.000 \\
\hline
\end{tabular}




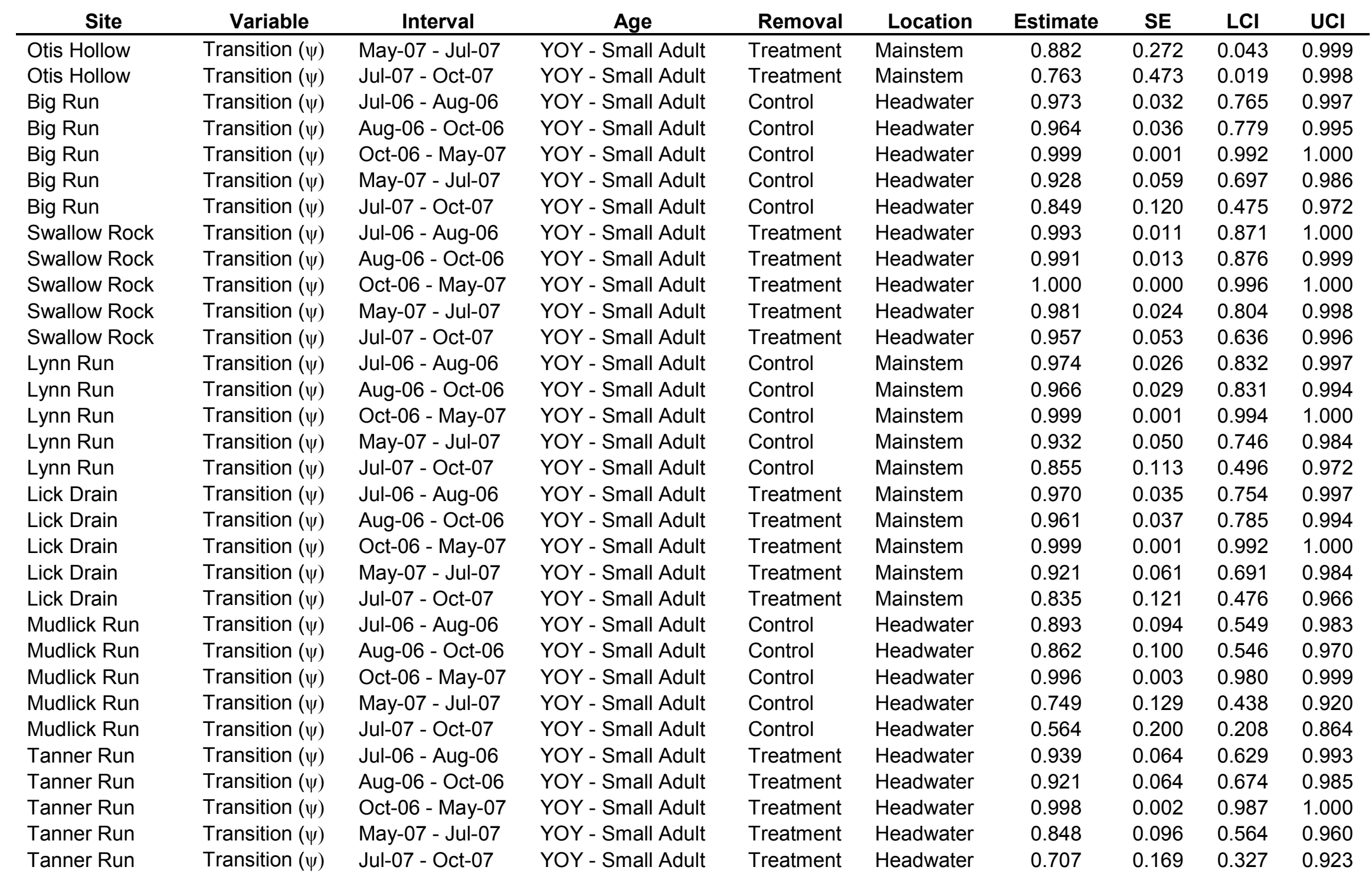




\begin{tabular}{|c|c|c|c|c|c|c|c|c|c|}
\hline Site & Variable & Interval & Age & Removal & Location & Estimate & SE & $\mathrm{LCl}$ & $\mathrm{UCl}$ \\
\hline Zinn Hollow & Transition $(\psi)$ & Oct-06 - May-07 & YOY - Small Adult & Control & Mainstem & 1.000 & 0.000 & 0.996 & 1.000 \\
\hline Zinn Hollow & Transition $(\psi)$ & Jul-07 - Oct-07 & YOY - Small Adult & Control & Mainstem & 0.955 & 0.057 & 0.613 & 0.997 \\
\hline Laurel Lick Run & Transition $(\psi)$ & Jul-06 - Aug-06 & YOY - Small Adult & Treatment & Mainstem & 0.972 & 0.039 & 0.676 & 0.998 \\
\hline Laurel Lick Run & Transition $(\psi)$ & Aug-06 - Oct-06 & YOY - Small Adult & Treatment & Mainstem & 0.963 & 0.042 & 0.719 & 0.996 \\
\hline Laurel Lick Run & Transition $(\psi)$ & Oct-06 - May-07 & YOY - Small Adult & Treatment & Mainstem & 0.999 & 0.001 & 0.989 & 1.000 \\
\hline Glady Fork & Transition $(\psi)$ & Jul-06 - Aug-06 & YOY - Small Adult & Control & Headwater & 0.997 & 0.004 & 0.946 & 1.000 \\
\hline Glady Fork & Transition $(\psi)$ & Aug-06 - Oct-06 & YOY - Small Adult & Control & Headwater & 0.996 & 0.005 & 0.953 & 1.000 \\
\hline Glady Fork & Transition $(\psi)$ & Oct-06 - May-07 & YOY - Small Adult & Control & Headwater & 1.000 & 0.000 & 0.999 & 1.000 \\
\hline Glady Fork & Transition $(\psi)$ & May-07 - Jul-07 & YOY - Small Adult & Control & Headwater & 0.992 & 0.010 & 0.913 & 0.999 \\
\hline Glady Fork & Transition $(\psi)$ & Jul-07 - Oct-07 & YOY - Small Adult & Control & Headwater & 0.983 & 0.023 & 0.810 & 0.999 \\
\hline Daniel's Run & Transition $(\psi)$ & Jul-06 - Aug-06 & YOY - Small Adult & Treatment & Headwater & 0.949 & 0.055 & 0.669 & 0.994 \\
\hline Nan's Branch & Transition $(\psi)$ & Jul-06 - Aug-06 & Small - Large Adult & Control & Mainstem & 0.353 & 0.182 & 0.103 & 0.723 \\
\hline Nan's Branch & Transition $(\psi)$ & Aug-06 - Oct-06 & Small - Large Adult & Control & Mainstem & 0.292 & 0.139 & 0.100 & 0.605 \\
\hline Nan's Branch & Transition $(\psi)$ & Oct-06 - May-07 & Small - Large Adult & Control & Mainstem & 0.946 & 0.037 & 0.806 & 0.987 \\
\hline Nan's Branch & Transition $(\psi)$ & May-07 - Jul-07 & Small - Large Adult & Control & Mainstem & 0.164 & 0.079 & 0.060 & 0.378 \\
\hline Nan's Branch & Transition $(\psi)$ & Jul-07 - Oct-07 & Small - Large Adult & Control & Mainstem & 0.078 & 0.046 & 0.023 & 0.231 \\
\hline Otis Hollow & Transition $(\psi)$ & Jul-06 - Aug-06 & Small - Large Adult & Treatment & Mainstem & 0.313 & 0.566 & 0.003 & 0.988 \\
\hline Otis Hollow & Transition $(\psi)$ & Aug-06 - Oct-06 & Small - Large Adult & Treatment & Mainstem & 0.255 & 0.492 & 0.002 & 0.982 \\
\hline Otis Hollow & Transition $(\psi)$ & Oct-06 - May-07 & Small - Large Adult & Treatment & Mainstem & 0.936 & 0.149 & 0.099 & 0.999 \\
\hline Otis Hollow & Transition $(\psi)$ & May-07 - Jul-07 & Small - Large Adult & Treatment & Mainstem & 0.141 & 0.310 & 0.001 & 0.962 \\
\hline Otis Hollow & Transition $(\psi)$ & Jul-07 - Oct-07 & Small - Large Adult & Treatment & Mainstem & 0.066 & 0.158 & 0.000 & 0.915 \\
\hline Big Run & Transition $(\psi)$ & Jul-06 - Aug-06 & Small - Large Adult & Control & Headwater & 0.441 & 0.241 & 0.104 & 0.843 \\
\hline Big Run & Transition $(\psi)$ & Aug-06 - Oct-06 & Small - Large Adult & Control & Headwater & 0.373 & 0.177 & 0.118 & 0.725 \\
\hline
\end{tabular}




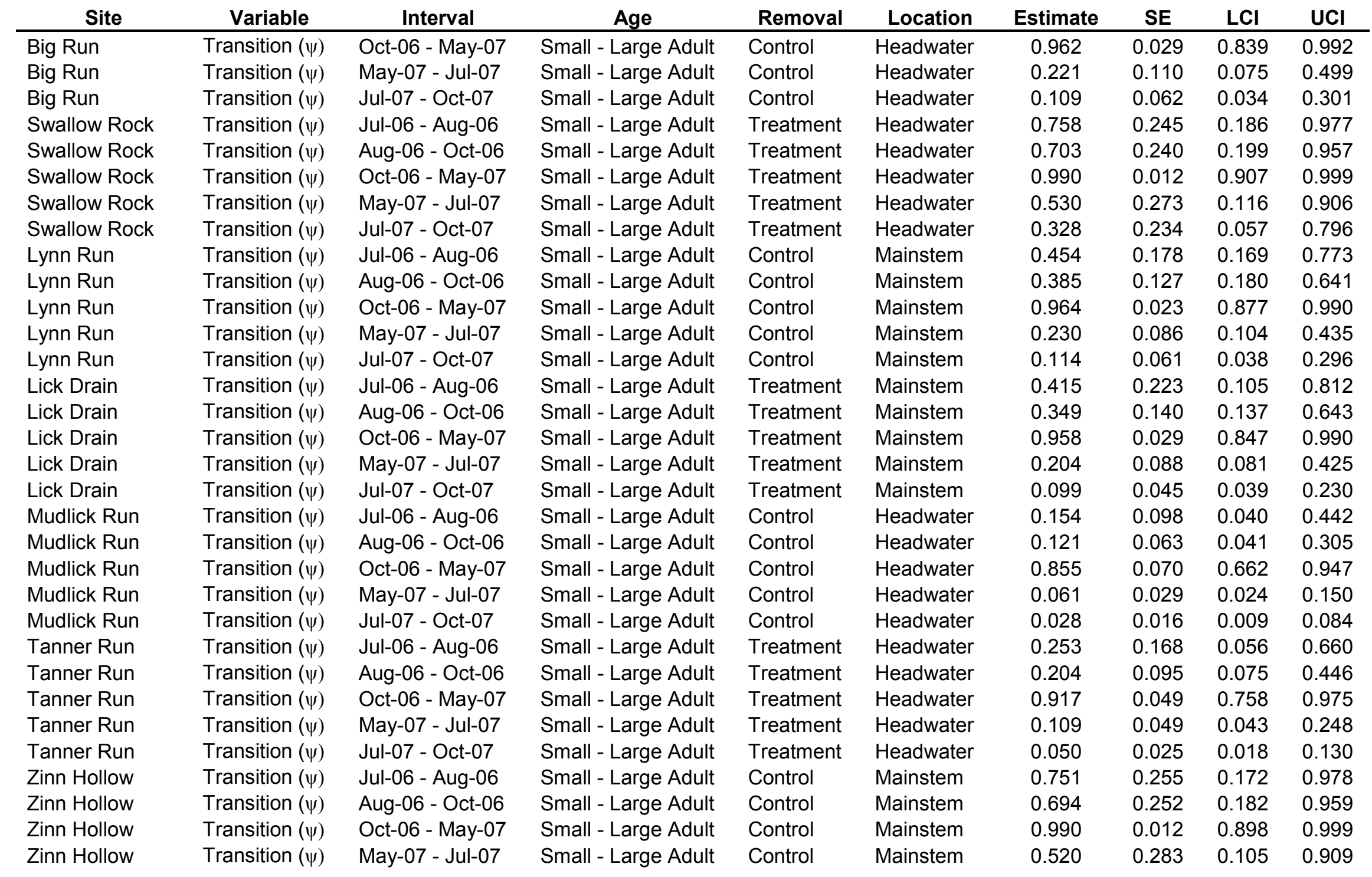




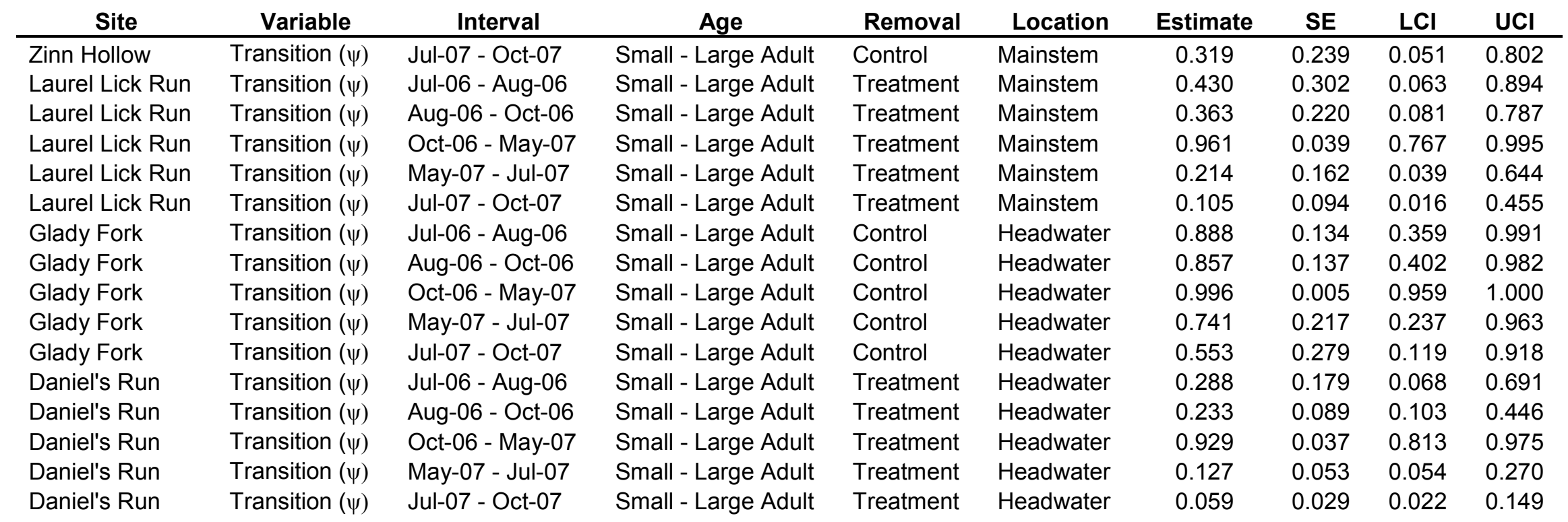




\section{Appendix F: Model coefficients and parameter estimates for estimating components of instantaneous population growth rate}

F1. Model coefficient estimates, standard errors, and confidence limits for the modelaveraged coefficients resulting from the $95 \%$ confidence set of multinomial models for estimating the proportion of the instantaneous population growth rate $(\lambda)$ due to survival $\left(\gamma_{\mathrm{ii}}\right)$ and local recruitment $\left(\gamma_{\mathrm{ji}}\right)$ for brook trout in response to fish removal treatment, spatial location, and interactions of interest over the course of the study while accounting for the effects of age, sampling interval and local physical and chemical habitat covariates. The multinomial model has two intercepts, one for estimating survival (Intercept $\gamma_{\mathrm{ii}}$ ) and one for estimating age-class transition (Intercept $\gamma_{\mathrm{ji}}$ ). $\Sigma \mathrm{w}_{\mathrm{i}}=$ sum of Akaike weights for experimental effect terms across $95 \%$ confidence model set. The cumulative Akaike weights for each model term are indicated next to the relevant effect. Asterisks (*) indicate where model coefficient confidence limits did not include 0 (i.e., a "significant" positive or negative slope). $a 1=$ YOY age class, $a 2=$ small adults, $g=$ fish removal treatment site, $1=$ mainstem tributary site, $\mathrm{t} 1-\mathrm{t} 4=$ sampling interval, $\mathrm{a} 1 * \mathrm{~g}=$ YOY $\mathrm{x}$ removal interaction effect, $\mathrm{a} 2 * \mathrm{~g}=$ small adult $\mathrm{x}$ removal, $\mathrm{a} 1 * \mathrm{l}=\mathrm{YOY} \times \mathrm{x}$ location, a2*1 $=$ small adult $x$ location, $g * l=$ removal $x$ location, $g * t 1-g * t 4=$ removal $x$ date, $1 * \mathrm{t} 1-1 * \mathrm{t} 4=$ location $\mathrm{x}$ date, $\mathrm{g} * \mathrm{l}^{*} \mathrm{t} 1-\mathrm{g} * 1 * \mathrm{t} 4=$ removal $\mathrm{x}$ location $\mathrm{x}$ date, $\mathrm{W} 1=$ water chemistry principal component $1, \mathrm{H} 1-\mathrm{H} 3=$ physical habitat principal components 1 to 3 , PAREA $=$ proportion of spring wetted channel area in early fall, CANP $=$ mean percent canopy cover, MWAT $=$ maximum weekly average temperature $\left({ }^{\circ} \mathrm{C}\right)$.

\begin{tabular}{|c|c|c|c|c|c|c|}
\hline Effect & Estimate & SE & $\mathrm{LCl}$ & $\mathrm{UCI}$ & & $\Sigma w_{i}$ \\
\hline Intercept $\left(\gamma_{\mathrm{ii}}\right)$ & 3.711 & 4.685 & -5.471 & 12.893 & & \\
\hline W1 & -0.326 & 0.525 & -1.354 & 0.703 & & \\
\hline $\mathrm{H} 1$ & -0.026 & 0.086 & -0.195 & 0.143 & & \\
\hline $\mathrm{H} 2$ & 0.072 & 0.112 & -0.149 & 0.292 & & \\
\hline H3 & -0.247 & 0.264 & -0.764 & 0.270 & & \\
\hline PAREA & -0.079 & 1.009 & -2.057 & 1.899 & & \\
\hline CANP & -0.007 & 0.023 & -0.052 & 0.037 & & \\
\hline MWAT & -0.161 & 0.166 & -0.486 & 0.164 & & \\
\hline a1 & -0.701 & 0.310 & -1.309 & -0.093 & * & \\
\hline a2 & 0.227 & 0.325 & -0.409 & 0.864 & & \\
\hline$g$ & 1.023 & 0.498 & 0.046 & 2.000 & * & 1.000 \\
\hline 1 & 0.051 & 0.912 & -1.736 & 1.837 & & 0.171 \\
\hline $\mathrm{t} 1$ & 0.079 & 0.331 & -0.570 & 0.728 & & \\
\hline t2 & -0.404 & 0.314 & -1.019 & 0.210 & & \\
\hline t3 & -1.824 & 0.363 & -2.536 & -1.112 & * & \\
\hline $\mathrm{t} 4$ & 0.071 & 0.307 & -0.531 & 0.673 & & \\
\hline$a 1^{*} g$ & -0.216 & 0.382 & -0.964 & 0.532 & & \\
\hline$a 2^{*} g$ & 0.693 & 0.424 & -0.138 & 1.524 & & \\
\hline $\mathrm{a} 1^{*} \mathrm{I}$ & -1.229 & 0.369 & -1.953 & -0.505 & * & \\
\hline a2*I & -0.723 & 0.413 & -1.533 & 0.087 & & \\
\hline$g^{*} \mid$ & -0.157 & 0.868 & -1.859 & 1.544 & & \\
\hline$g^{*}+1$ & -6.367 & 1.401 & -9.114 & -3.620 & * & 1.000 \\
\hline$g^{*} \mathrm{t} 2$ & -1.912 & 0.462 & -2.818 & -1.006 & * & \\
\hline
\end{tabular}




\begin{tabular}{cccccc}
$\mathrm{g}^{*} \mathrm{t} 3$ & -1.357 & 0.519 & -2.374 & -0.340 & $*$ \\
$\mathrm{~g}^{*} \mathrm{t} 4$ & -0.989 & 0.406 & -1.785 & -0.192 & $*$ \\
\hline Intercept $\left(\mathrm{Y}_{\mathrm{ji}}\right)$ & 6.093 & 8.151 & -9.884 & 22.070 & \\
$\mathrm{~W} 1$ & 0.131 & 0.567 & -0.981 & 1.243 & \\
$\mathrm{H} 1$ & 0.304 & 0.136 & 0.038 & 0.570 & $*$ \\
$\mathrm{H} 2$ & 0.620 & 0.165 & 0.297 & 0.944 & $*$ \\
$\mathrm{H} 3$ & 0.452 & 0.251 & -0.040 & 0.944 & \\
PAREA & 0.400 & 1.207 & -1.965 & 2.766 & \\
CANP & -0.122 & 0.051 & -0.222 & -0.021 & $*$ \\
MWAT & 0.112 & 0.318 & -0.512 & 0.736 & \\
a & 0.085 & 0.322 & -0.547 & 0.717 & \\
t12 & -2.118 & 0.580 & -3.254 & -0.982 & $*$ \\
t3 & 1.638 & 0.335 & 0.980 & 2.295 & $*$
\end{tabular}


F2. Summary of model-averaged parameter estimates, standard errors, and 95\% confidence limits (i.e., lower $=$ LCI and upper $=$ UCI) for proportion of the brook trout instantaneous population growth rate $(\lambda)$ attributable to local survival $\left(\gamma_{\mathrm{ii}}\right)$ and in-situ recruitment $\left(\gamma_{\mathrm{ji}}\right)$ for all sites and sample dates. Experimental classifications of sites based on treatment and spatial location are also noted.

\begin{tabular}{|c|c|c|c|c|c|c|c|c|c|}
\hline Site & Variable & Interval & Age & Removal & Location & EST & SE & $\mathrm{LCL}$ & UCL \\
\hline Nan's Branch & Survival $\left(\gamma_{\text {ii }}\right)$ & Jul-06 - Aug-06 & YOY & Control & Mainstem & 0.250 & 0.066 & 0.120 & 0.380 \\
\hline Nan's Branch & Survival $\left(\gamma_{\mathrm{ii}}\right)$ & Oct-06 - May-07 & YOY & Control & Mainstem & 0.047 & 0.018 & 0.013 & 0.082 \\
\hline Nan's Branch & Survival $\left(\gamma_{\mathrm{ii}}\right)$ & Jul-07 - Oct-07 & YOY & Control & Mainstem & 0.236 & 0.049 & 0.139 & 0.332 \\
\hline Otis Hollow & Survival $\left(\gamma_{\mathrm{ii}}\right)$ & Jul-06 - Aug-06 & YOY & Treatment & Mainstem & 0.001 & 0.001 & -0.002 & 0.003 \\
\hline Otis Hollow & Survival $\left(\gamma_{\mathrm{ii}}\right)$ & Aug-06 - Oct-06 & YOY & Treatment & Mainstem & 0.046 & 0.016 & 0.015 & 0.076 \\
\hline Otis Hollow & Survival $\left(\gamma_{\mathrm{ii}}\right)$ & Jul-07 - Oct-07 & YOY & Treatment & Mainstem & 0.326 & 0.048 & 0.232 & 0.420 \\
\hline Big Run & Survival $\left(\gamma_{\mathrm{ii}}\right)$ & Jul-06 - Aug-06 & YOY & Control & Headwater & 0.271 & 0.068 & 0.137 & 0.405 \\
\hline Big Run & Survival $\left(\gamma_{i i}\right)$ & Aug-06 - Oct-06 & YOY & Control & Headwater & 0.187 & 0.053 & 0.083 & 0.290 \\
\hline Big Run & Survival $\left(\gamma_{\mathrm{ii}}\right)$ & Oct-06 - May-07 & YOY & Control & Headwater & 0.053 & 0.019 & 0.015 & 0.090 \\
\hline Big Run & Survival $\left(\gamma_{\mathrm{ii}}\right)$ & May-07 - Jul-07 & YOY & Control & Headwater & 0.270 & 0.064 & 0.143 & 0.396 \\
\hline Swallow Rock & Survival $\left(\gamma_{\mathrm{ii}}\right)$ & May-07 - Jul-07 & YOY & Treatment & Headwater & 0.244 & 0.046 & 0.153 & 0.335 \\
\hline Swallow Rock & Survival $\left(\gamma_{\mathrm{ii}}\right)$ & Jul-07 - Oct-07 & YOY & Treatment & Headwater & 0.447 & 0.062 & 0.326 & 0.568 \\
\hline Lynn Run & Survival $\left(\gamma_{\mathrm{ii}}\right)$ & Jul-06 - Aug-06 & YOY & Control & Mainstem & 0.251 & 0.071 & 0.113 & 0.390 \\
\hline Lynn Run & Survival $\left(\gamma_{\mathrm{ii}}\right)$ & Aug-06 - Oct-06 & YOY & Control & Mainstem & 0.172 & 0.051 & 0.071 & 0.272 \\
\hline Lynn Run & Survival $\left(\gamma_{\mathrm{ii}}\right)$ & Oct-06 - May-07 & YOY & Control & Mainstem & 0.048 & 0.018 & 0.013 & 0.082 \\
\hline Lynn Run & Survival $\left(\gamma_{\mathrm{ii}}\right)$ & May-07 - Jul-07 & YOY & Control & Mainstem & 0.250 & 0.061 & 0.130 & 0.370 \\
\hline Lynn Run & Survival $\left(\gamma_{\mathrm{ii}}\right)$ & Jul-07 - Oct-07 & YOY & Control & Mainstem & 0.237 & 0.054 & 0.132 & 0.342 \\
\hline
\end{tabular}




\begin{tabular}{|c|c|c|c|c|c|c|c|c|c|}
\hline Lick Drain & Survival $\left(\gamma_{\mathrm{ii}}\right)$ & Jul-06 - Aug-06 & YOY & Treatment & Mainstem & 0.001 & 0.001 & -0.001 & 0.003 \\
\hline Lick Drain & Survival $\left(\gamma_{\mathrm{ii}}\right)$ & Aug-06 - Oct-06 & YOY & Treatment & Mainstem & 0.039 & 0.015 & 0.008 & 0.069 \\
\hline Lick Drain & Survival $\left(\gamma_{\mathrm{ii}}\right)$ & Oct-06 - May-07 & YOY & Treatment & Mainstem & 0.017 & 0.007 & 0.002 & 0.031 \\
\hline Lick Drain & Survival $\left(\gamma_{\mathrm{ii}}\right)$ & May-07 - Jul-07 & YOY & Treatment & Mainstem & 0.140 & 0.040 & 0.061 & 0.218 \\
\hline Lick Drain & Survival $\left(\gamma_{\mathrm{ii}}\right)$ & Jul-07 - Oct-07 & YOY & Treatment & Mainstem & 0.289 & 0.057 & 0.176 & 0.402 \\
\hline Mudlick Run & Survival $\left(\gamma_{\mathrm{ii}}\right)$ & Jul-06 - Aug-06 & YOY & Control & Headwater & 0.390 & 0.097 & 0.201 & 0.579 \\
\hline Mudlick Run & Survival $\left(\gamma_{\mathrm{ii}}\right)$ & Aug-06 - Oct-06 & YOY & Control & Headwater & 0.283 & 0.077 & 0.132 & 0.433 \\
\hline Mudlick Run & Survival $\left(\gamma_{\mathrm{ii}}\right)$ & Oct-06 - May-07 & YOY & Control & Headwater & 0.087 & 0.034 & 0.021 & 0.153 \\
\hline Mudlick Run & Survival $\left(\gamma_{\mathrm{ii}}\right)$ & May-07 - Jul-07 & YOY & Control & Headwater & 0.388 & 0.090 & 0.212 & 0.564 \\
\hline Mudlick Run & Survival $\left(\gamma_{\mathrm{ii}}\right)$ & Jul-07 - Oct-07 & YOY & Control & Headwater & 0.371 & 0.077 & 0.221 & 0.521 \\
\hline Tanner Run & Survival $\left(\gamma_{\mathrm{ii}}\right)$ & Jul-06 - Aug-06 & YOY & Treatment & Headwater & 0.001 & 0.002 & -0.003 & 0.005 \\
\hline Tanner Run & Survival $\left(\gamma_{\mathrm{ii}}\right)$ & Aug-06 - Oct-06 & YOY & Treatment & Headwater & 0.071 & 0.035 & 0.003 & 0.139 \\
\hline Tanner Run & Survival $\left(\gamma_{\mathrm{ii}}\right)$ & Oct-06 - May-07 & YOY & Treatment & Headwater & 0.031 & 0.017 & -0.003 & 0.065 \\
\hline Tanner Run & Survival $\left(\gamma_{\mathrm{ii}}\right)$ & May-07 - Jul-07 & YOY & Treatment & Headwater & 0.236 & 0.089 & 0.062 & 0.410 \\
\hline Tanner Run & Survival $\left(\gamma_{\mathrm{ii}}\right)$ & Jul-07 - Oct-07 & YOY & Treatment & Headwater & 0.436 & 0.113 & 0.214 & 0.658 \\
\hline Zinn Hollow & Survival $\left(\gamma_{\mathrm{ii}}\right)$ & Jul-06 - Aug-06 & YOY & Control & Mainstem & 0.174 & 0.060 & 0.056 & 0.293 \\
\hline Zinn Hollow & Survival $\left(\gamma_{\mathrm{ii}}\right)$ & Aug-06 - Oct-06 & YOY & Control & Mainstem & 0.115 & 0.042 & 0.032 & 0.198 \\
\hline Zinn Hollow & Survival $\left(\gamma_{\mathrm{ii}}\right)$ & Oct-06 - May-07 & YOY & Control & Mainstem & 0.030 & 0.013 & 0.004 & 0.057 \\
\hline Zinn Hollow & Survival $\left(\gamma_{\mathrm{ii}}\right)$ & May-07 - Jul-07 & YOY & Control & Mainstem & 0.173 & 0.053 & 0.070 & 0.276 \\
\hline Zinn Hollow & Survival $\left(\gamma_{\mathrm{ii}}\right)$ & Jul-07 - Oct-07 & YOY & Control & Mainstem & 0.163 & 0.047 & 0.072 & 0.254 \\
\hline Laurel Lick Run & Survival $\left(\gamma_{\mathrm{ii}}\right)$ & Jul-06 - Aug-06 & YOY & Treatment & Mainstem & 0.000 & 0.001 & -0.001 & 0.002 \\
\hline Laurel Lick Run & Survival $\left(\gamma_{\mathrm{ii}}\right)$ & Aug-06 - Oct-06 & YOY & Treatment & Mainstem & 0.023 & 0.013 & -0.001 & 0.048 \\
\hline Laurel Lick Run & Survival $\left(\gamma_{\mathrm{ii}}\right)$ & Oct-06 - May-07 & YOY & Treatment & Mainstem & 0.010 & 0.006 & -0.002 & 0.021 \\
\hline Laurel Lick Run & Survival $\left(\gamma_{\mathrm{ii}}\right)$ & May-07 - Jul-07 & YOY & Treatment & Mainstem & 0.088 & 0.041 & 0.008 & 0.168 \\
\hline Laurel Lick Run & Survival $\left(\gamma_{\mathrm{ii}}\right)$ & Jul-07 - Oct-07 & YOY & Treatment & Mainstem & 0.194 & 0.073 & 0.051 & 0.337 \\
\hline Glady Fork & Survival $\left(\gamma_{\mathrm{ii}}\right)$ & Jul-06 - Aug-06 & YOY & Control & Headwater & 0.255 & 0.072 & 0.114 & 0.395 \\
\hline Glady Fork & Survival $\left(\gamma_{\mathrm{ii}}\right)$ & Aug-06 - Oct-06 & YOY & Control & Headwater & 0.174 & 0.054 & 0.068 & 0.280 \\
\hline Glady Fork & Survival $\left(\gamma_{\mathrm{ii}}\right)$ & Oct-06 - May-07 & YOY & Control & Headwater & 0.048 & 0.020 & 0.010 & 0.087 \\
\hline Glady Fork & Survival $\left(\gamma_{\mathrm{ii}}\right)$ & May-07 - Jul-07 & YOY & Control & Headwater & 0.253 & 0.062 & 0.133 & 0.374 \\
\hline Glady Fork & Survival $\left(\gamma_{\mathrm{ii}}\right)$ & Jul-07 - Oct-07 & YOY & Control & Headwater & 0.240 & 0.060 & 0.122 & 0.358 \\
\hline
\end{tabular}




\begin{tabular}{|c|c|c|c|c|c|c|c|c|c|}
\hline Daniel's Run & Survival $\left(\gamma_{\mathrm{ii}}\right)$ & Jul-06 - Aug-06 & YOY & Treatment & Headwater & 0.001 & 0.002 & -0.003 & 0.005 \\
\hline Daniel's Run & Survival $\left(\gamma_{\mathrm{ii}}\right)$ & Aug-06 - Oct-06 & YOY & Treatment & Headwater & 0.072 & 0.030 & 0.014 & 0.130 \\
\hline Daniel's Run & Survival $\left(\gamma_{\mathrm{ii}}\right)$ & Oct-06 - May-07 & YOY & Treatment & Headwater & 0.032 & 0.015 & 0.002 & 0.061 \\
\hline Daniel's Run & Survival $\left(\gamma_{\mathrm{ii}}\right)$ & May-07 - Jul-07 & YOY & Treatment & Headwater & 0.239 & 0.072 & 0.098 & 0.380 \\
\hline Daniel's Run & Survival $\left(\gamma_{\mathrm{ii}}\right)$ & Jul-07 - Oct-07 & YOY & Treatment & Headwater & 0.440 & 0.092 & 0.260 & 0.620 \\
\hline Nan's Branch & Survival $\left(\gamma_{\mathrm{ii}}\right)$ & Jul-06 - Aug-06 & Small Adult & Control & Mainstem & 0.583 & 0.090 & 0.408 & 0.759 \\
\hline Nan's Branch & Survival $\left(\gamma_{i i}\right)$ & Aug-06 - Oct-06 & Small Adult & Control & Mainstem & 0.463 & 0.083 & 0.300 & 0.626 \\
\hline Nan's Branch & Survival $\left(\gamma_{\mathrm{ii}}\right)$ & Oct-06 - May-07 & Small Adult & Control & Mainstem & 0.173 & 0.057 & 0.061 & 0.285 \\
\hline Nan's Branch & Survival $\left(\gamma_{\mathrm{ii}}\right)$ & May-07 - Jul-07 & Small Adult & Control & Mainstem & 0.581 & 0.086 & 0.413 & 0.750 \\
\hline Nan's Branch & Survival $\left(\gamma_{\mathrm{ii}}\right)$ & Jul-07 - Oct-07 & Small Adult & Control & Mainstem & 0.564 & 0.083 & 0.402 & 0.726 \\
\hline Otis Hollow & Survival $\left(\gamma_{\mathrm{ii}}\right)$ & Jul-06 - Aug-06 & Small Adult & Treatment & Mainstem & 0.009 & 0.012 & -0.015 & 0.034 \\
\hline Otis Hollow & Survival $\left(\gamma_{\mathrm{ii}}\right)$ & Aug-06 - Oct-06 & Small Adult & Treatment & Mainstem & 0.332 & 0.067 & 0.201 & 0.464 \\
\hline Otis Hollow & Survival $\left(\gamma_{\mathrm{ii}}\right)$ & Oct-06 - May-07 & Small Adult & Treatment & Mainstem & 0.173 & 0.046 & 0.083 & 0.263 \\
\hline Otis Hollow & Survival $\left(\gamma_{\mathrm{ii}}\right)$ & May-07 - Jul-07 & Small Adult & Treatment & Mainstem & 0.668 & 0.067 & 0.537 & 0.799 \\
\hline Otis Hollow & Survival $\left(\gamma_{\mathrm{ii}}\right)$ & Jul-07 - Oct-07 & Small Adult & Treatment & Mainstem & 0.834 & 0.042 & 0.752 & 0.917 \\
\hline Big Run & Survival $\left(\gamma_{\mathrm{ii}}\right)$ & Jul-06 - Aug-06 & Small Adult & Control & Headwater & 0.485 & 0.080 & 0.328 & 0.642 \\
\hline Big Run & Survival $\left(\gamma_{\mathrm{ii}}\right)$ & Aug-06 - Oct-06 & Small Adult & Control & Headwater & 0.367 & 0.074 & 0.222 & 0.512 \\
\hline Big Run & Survival $\left(\gamma_{\mathrm{ii}}\right)$ & Oct-06 - May-07 & Small Adult & Control & Headwater & 0.123 & 0.041 & 0.043 & 0.203 \\
\hline Big Run & Survival $\left(\gamma_{\mathrm{ii}}\right)$ & May-07 - Jul-07 & Small Adult & Control & Headwater & 0.483 & 0.087 & 0.312 & 0.654 \\
\hline Big Run & Survival $\left(\gamma_{\mathrm{ii}}\right)$ & Jul-07 - Oct-07 & Small Adult & Control & Headwater & 0.465 & 0.077 & 0.315 & 0.616 \\
\hline Swallow Rock & Survival $\left(\gamma_{\mathrm{ii}}\right)$ & Jul-06 - Aug-06 & Small Adult & Treatment & Headwater & 0.009 & 0.013 & -0.015 & 0.034 \\
\hline Swallow Rock & Survival $\left(\gamma_{\mathrm{ii}}\right)$ & Aug-06 - Oct-06 & Small Adult & Treatment & Headwater & 0.334 & 0.080 & 0.177 & 0.491 \\
\hline Swallow Rock & Survival $\left(\gamma_{\mathrm{ii}}\right)$ & Oct-06 - May-07 & Small Adult & Treatment & Headwater & 0.174 & 0.056 & 0.064 & 0.285 \\
\hline Swallow Rock & Survival $\left(\gamma_{\mathrm{ii}}\right)$ & May-07 - Jul-07 & Small Adult & Treatment & Headwater & 0.670 & 0.079 & 0.515 & 0.825 \\
\hline Swallow Rock & Survival $\left(\gamma_{\mathrm{ii}}\right)$ & Jul-07 - Oct-07 & Small Adult & Treatment & Headwater & 0.835 & 0.052 & 0.733 & 0.937 \\
\hline Lynn Run & Survival $\left(\gamma_{\mathrm{ii}}\right)$ & Jul-06 - Aug-06 & Small Adult & Control & Mainstem & 0.585 & 0.104 & 0.382 & 0.788 \\
\hline Lynn Run & Survival $\left(\gamma_{\mathrm{ii}}\right)$ & Aug-06 - Oct-06 & Small Adult & Control & Mainstem & 0.465 & 0.102 & 0.266 & 0.665 \\
\hline Lynn Run & Survival $\left(\gamma_{\mathrm{ii}}\right)$ & Oct-06 - May-07 & Small Adult & Control & Mainstem & 0.174 & 0.062 & 0.052 & 0.295 \\
\hline Lynn Run & Survival $\left(\gamma_{\mathrm{ii}}\right)$ & May-07 - Jul-07 & Small Adult & Control & Mainstem & 0.583 & 0.100 & 0.388 & 0.779 \\
\hline Lynn Run & Survival $\left(\gamma_{\mathrm{ii}}\right)$ & Jul-07 - Oct-07 & Small Adult & Control & Mainstem & 0.566 & 0.097 & 0.375 & 0.757 \\
\hline
\end{tabular}




\begin{tabular}{|c|c|c|c|c|c|c|c|c|c|}
\hline Lick Drain & Survival $\left(\gamma_{\mathrm{ii}}\right)$ & Jul-06 - Aug-06 & Small Adult & Treatment & Mainstem & 0.008 & 0.011 & -0.013 & 0.029 \\
\hline Lick Drain & Survival $\left(\gamma_{\mathrm{ii}}\right)$ & Aug-06 - Oct-06 & Small Adult & Treatment & Mainstem & 0.295 & 0.077 & 0.144 & 0.446 \\
\hline Lick Drain & Survival $\left(\gamma_{\mathrm{ii}}\right)$ & Oct-06 - May-07 & Small Adult & Treatment & Mainstem & 0.150 & 0.051 & 0.049 & 0.250 \\
\hline Lick Drain & Survival $\left(\gamma_{\mathrm{ii}}\right)$ & May-07 - Jul-07 & Small Adult & Treatment & Mainstem & 0.628 & 0.086 & 0.459 & 0.798 \\
\hline Lick Drain & Survival $\left(\gamma_{\mathrm{ii}}\right)$ & Jul-07 - Oct-07 & Small Adult & Treatment & Mainstem & 0.809 & 0.055 & 0.700 & 0.918 \\
\hline Mudlick Run & Survival $\left(\gamma_{\mathrm{ii}}\right)$ & Jul-06 - Aug-06 & Small Adult & Control & Headwater & 0.618 & 0.102 & 0.417 & 0.819 \\
\hline Mudlick Run & Survival $\left(\gamma_{\mathrm{ii}}\right)$ & Aug-06 - Oct-06 & Small Adult & Control & Headwater & 0.499 & 0.101 & 0.301 & 0.698 \\
\hline Mudlick Run & Survival $\left(\gamma_{\mathrm{ii}}\right)$ & Oct-06 - May-07 & Small Adult & Control & Headwater & 0.194 & 0.072 & 0.052 & 0.336 \\
\hline Mudlick Run & Survival $\left(\gamma_{\mathrm{ii}}\right)$ & May-07 - Jul-07 & Small Adult & Control & Headwater & 0.616 & 0.106 & 0.409 & 0.823 \\
\hline Mudlick Run & Survival $\left(\gamma_{\mathrm{ii}}\right)$ & Jul-07 - Oct-07 & Small Adult & Control & Headwater & 0.599 & 0.100 & 0.402 & 0.796 \\
\hline Tanner Run & Survival $\left(\gamma_{\mathrm{ii}}\right)$ & Jul-06 - Aug-06 & Small Adult & Treatment & Headwater & 0.009 & 0.012 & -0.015 & 0.033 \\
\hline Tanner Run & Survival $\left(\gamma_{\mathrm{ii}}\right)$ & Aug-06 - Oct-06 & Small Adult & Treatment & Headwater & 0.324 & 0.112 & 0.103 & 0.544 \\
\hline Tanner Run & Survival $\left(\gamma_{\mathrm{ii}}\right)$ & Oct-06 - May-07 & Small Adult & Treatment & Headwater & 0.168 & 0.078 & 0.015 & 0.321 \\
\hline Tanner Run & Survival $\left(\gamma_{\mathrm{ii}}\right)$ & May-07 - Jul-07 & Small Adult & Treatment & Headwater & 0.660 & 0.123 & 0.419 & 0.901 \\
\hline Tanner Run & Survival $\left(\gamma_{\mathrm{ii}}\right)$ & Jul-07 - Oct-07 & Small Adult & Treatment & Headwater & 0.829 & 0.076 & 0.681 & 0.977 \\
\hline Zinn Hollow & Survival $\left(\gamma_{\mathrm{ii}}\right)$ & Jul-06 - Aug-06 & Small Adult & Control & Mainstem & 0.470 & 0.115 & 0.245 & 0.694 \\
\hline Zinn Hollow & Survival $\left(\gamma_{\mathrm{ii}}\right)$ & Aug-06 - Oct-06 & Small Adult & Control & Mainstem & 0.353 & 0.103 & 0.151 & 0.555 \\
\hline Zinn Hollow & Survival $\left(\gamma_{\mathrm{ii}}\right)$ & Oct-06 - May-07 & Small Adult & Control & Mainstem & 0.117 & 0.050 & 0.018 & 0.215 \\
\hline Zinn Hollow & Survival $\left(\gamma_{\mathrm{ii}}\right)$ & May-07 - Jul-07 & Small Adult & Control & Mainstem & 0.468 & 0.108 & 0.255 & 0.680 \\
\hline Zinn Hollow & Survival $\left(\gamma_{\mathrm{ii}}\right)$ & Jul-07 - Oct-07 & Small Adult & Control & Mainstem & 0.450 & 0.105 & 0.244 & 0.655 \\
\hline Laurel Lick Run & Survival $\left(\gamma_{\mathrm{ii}}\right)$ & Jul-06 - Aug-06 & Small Adult & Treatment & Mainstem & 0.005 & 0.007 & -0.008 & 0.017 \\
\hline Laurel Lick Run & Survival $\left(\gamma_{\mathrm{ii}}\right)$ & Aug-06 - Oct-06 & Small Adult & Treatment & Mainstem & 0.198 & 0.083 & 0.035 & 0.361 \\
\hline Laurel Lick Run & Survival $\left(\gamma_{\mathrm{ii}}\right)$ & Oct-06 - May-07 & Small Adult & Treatment & Mainstem & 0.094 & 0.048 & 0.001 & 0.188 \\
\hline Laurel Lick Run & Survival $\left(\gamma_{\mathrm{ii}}\right)$ & May-07 - Jul-07 & Small Adult & Treatment & Mainstem & 0.500 & 0.134 & 0.238 & 0.762 \\
\hline Laurel Lick Run & Survival $\left(\gamma_{\mathrm{ii}}\right)$ & Jul-07 - Oct-07 & Small Adult & Treatment & Mainstem & 0.715 & 0.105 & 0.508 & 0.922 \\
\hline Glady Fork & Survival $\left(\gamma_{\mathrm{ii}}\right)$ & Jul-06 - Aug-06 & Small Adult & Control & Headwater & 0.464 & 0.101 & 0.267 & 0.661 \\
\hline Glady Fork & Survival $\left(\gamma_{\mathrm{ii}}\right)$ & Aug-06 - Oct-06 & Small Adult & Control & Headwater & 0.348 & 0.091 & 0.170 & 0.526 \\
\hline Glady Fork & Survival $\left(\gamma_{\mathrm{ii}}\right)$ & Oct-06 - May-07 & Small Adult & Control & Headwater & 0.114 & 0.047 & 0.023 & 0.206 \\
\hline Glady Fork & Survival $\left(\gamma_{\mathrm{ii}}\right)$ & May-07 - Jul-07 & Small Adult & Control & Headwater & 0.462 & 0.099 & 0.267 & 0.656 \\
\hline Glady Fork & Survival $\left(\gamma_{\mathrm{ii}}\right)$ & Jul-07 - Oct-07 & Small Adult & Control & Headwater & 0.444 & 0.103 & 0.243 & 0.645 \\
\hline
\end{tabular}




\begin{tabular}{|c|c|c|c|c|c|c|c|c|c|}
\hline Daniel's Run & Survival $\left(\gamma_{\mathrm{ii}}\right)$ & Jul-06 - Aug-06 & Small Adult & Treatment & Headwater & 0.009 & 0.012 & -0.015 & 0.033 \\
\hline Daniel's Run & Survival $\left(\gamma_{\mathrm{ii}}\right)$ & Aug-06 - Oct-06 & Small Adult & Treatment & Headwater & 0.328 & 0.098 & 0.136 & 0.519 \\
\hline Daniel's Run & Survival $\left(\gamma_{\mathrm{ii}}\right)$ & Oct-06 - May-07 & Small Adult & Treatment & Headwater & 0.170 & 0.070 & 0.033 & 0.308 \\
\hline Daniel's Run & Survival $\left(\gamma_{\mathrm{ii}}\right)$ & May-07 - Jul-07 & Small Adult & Treatment & Headwater & 0.664 & 0.107 & 0.455 & 0.872 \\
\hline Daniel's Run & Survival $\left(\gamma_{\mathrm{ii}}\right)$ & Jul-07 - Oct-07 & Small Adult & Treatment & Headwater & 0.832 & 0.067 & 0.701 & 0.962 \\
\hline Nan's Branch & Survival $\left(\gamma_{\mathrm{ii}}\right)$ & Jul-06 - Aug-06 & Large Adult & Control & Mainstem & 0.697 & 0.087 & 0.526 & 0.868 \\
\hline Nan's Branch & Survival $\left(\gamma_{\mathrm{ii}}\right)$ & Aug-06 - Oct-06 & Large Adult & Control & Mainstem & 0.586 & 0.091 & 0.408 & 0.764 \\
\hline Nan's Branch & Survival $\left(\gamma_{\mathrm{ii}}\right)$ & Oct-06 - May-07 & Large Adult & Control & Mainstem & 0.255 & 0.076 & 0.105 & 0.405 \\
\hline Nan's Branch & Survival $\left(\gamma_{\mathrm{ii}}\right)$ & May-07 - Jul-07 & Large Adult & Control & Mainstem & 0.695 & 0.082 & 0.534 & 0.856 \\
\hline Nan's Branch & Survival $\left(\gamma_{\mathrm{ii}}\right)$ & Jul-07 - Oct-07 & Large Adult & Control & Mainstem & 0.680 & 0.080 & 0.522 & 0.837 \\
\hline Otis Hollow & Survival $\left(\gamma_{\mathrm{ii}}\right)$ & Jul-06 - Aug-06 & Large Adult & Treatment & Mainstem & 0.008 & 0.010 & -0.013 & 0.028 \\
\hline Otis Hollow & Survival $\left(\gamma_{\mathrm{ii}}\right)$ & Aug-06 - Oct-06 & Large Adult & Treatment & Mainstem & 0.290 & 0.084 & 0.125 & 0.454 \\
\hline Otis Hollow & Survival $\left(\gamma_{\mathrm{ii}}\right)$ & Oct-06 - May-07 & Large Adult & Treatment & Mainstem & 0.147 & 0.051 & 0.047 & 0.246 \\
\hline Otis Hollow & Survival $\left(\gamma_{\mathrm{ii}}\right)$ & May-07 - Jul-07 & Large Adult & Treatment & Mainstem & 0.623 & 0.079 & 0.469 & 0.778 \\
\hline Otis Hollow & Survival $\left(\gamma_{\mathrm{ii}}\right)$ & Jul-07 - Oct-07 & Large Adult & Treatment & Mainstem & 0.805 & 0.055 & 0.697 & 0.913 \\
\hline Big Run & Survival $\left(\gamma_{\mathrm{ii}}\right)$ & Jul-06 - Aug-06 & Large Adult & Control & Headwater & 0.429 & 0.086 & 0.260 & 0.597 \\
\hline Big Run & Survival $\left(\gamma_{\mathrm{ii}}\right)$ & Aug-06 - Oct-06 & Large Adult & Control & Headwater & 0.316 & 0.069 & 0.181 & 0.451 \\
\hline Big Run & Survival $\left(\gamma_{\mathrm{ii}}\right)$ & Oct-06 - May-07 & Large Adult & Control & Headwater & 0.101 & 0.035 & 0.032 & 0.169 \\
\hline Big Run & Survival $\left(\gamma_{\mathrm{ii}}\right)$ & May-07 - Jul-07 & Large Adult & Control & Headwater & 0.427 & 0.085 & 0.259 & 0.594 \\
\hline Big Run & Survival $\left(\gamma_{\mathrm{ii}}\right)$ & Jul-07 - Oct-07 & Large Adult & Control & Headwater & 0.409 & 0.077 & 0.257 & 0.561 \\
\hline Swallow Rock & Survival $\left(\gamma_{\mathrm{ii}}\right)$ & Jul-06 - Aug-06 & Large Adult & Treatment & Headwater & 0.004 & 0.005 & -0.006 & 0.014 \\
\hline Swallow Rock & Survival $\left(\gamma_{\mathrm{ii}}\right)$ & Aug-06 - Oct-06 & Large Adult & Treatment & Headwater & 0.166 & 0.062 & 0.045 & 0.287 \\
\hline Swallow Rock & Survival $\left(\gamma_{\mathrm{ii}}\right)$ & Oct-06 - May-07 & Large Adult & Treatment & Headwater & 0.077 & 0.032 & 0.015 & 0.140 \\
\hline Swallow Rock & Survival $\left(\gamma_{\mathrm{ii}}\right)$ & May-07 - Jul-07 & Large Adult & Treatment & Headwater & 0.447 & 0.091 & 0.269 & 0.625 \\
\hline Swallow Rock & Survival $\left(\gamma_{\mathrm{ii}}\right)$ & Jul-07 - Oct-07 & Large Adult & Treatment & Headwater & 0.669 & 0.086 & 0.500 & 0.838 \\
\hline Lynn Run & Survival $\left(\gamma_{\mathrm{ii}}\right)$ & Jul-06 - Aug-06 & Large Adult & Control & Mainstem & 0.698 & 0.093 & 0.515 & 0.881 \\
\hline Lynn Run & Survival $\left(\gamma_{\mathrm{ii}}\right)$ & Aug-06 - Oct-06 & Large Adult & Control & Mainstem & 0.588 & 0.101 & 0.389 & 0.787 \\
\hline Lynn Run & Survival $\left(\gamma_{\mathrm{ii}}\right)$ & Oct-06 - May-07 & Large Adult & Control & Mainstem & 0.257 & 0.078 & 0.103 & 0.410 \\
\hline Lynn Run & Survival $\left(\gamma_{\text {ii }}\right)$ & May-07 - Jul-07 & Large Adult & Control & Mainstem & 0.697 & 0.088 & 0.524 & 0.870 \\
\hline Lynn Run & Survival $\left(\gamma_{\mathrm{ii}}\right)$ & Jul-07 - Oct-07 & Large Adult & Control & Mainstem & 0.681 & 0.087 & 0.511 & 0.852 \\
\hline
\end{tabular}




\begin{tabular}{|c|c|c|c|c|c|c|c|c|c|}
\hline Lick Drain & Survival $\left(\gamma_{\mathrm{ii}}\right)$ & Jul-06 - Aug-06 & Large Adult & Treatment & Mainstem & 0.006 & 0.009 & -0.011 & 0.024 \\
\hline Lick Drain & Survival $\left(\gamma_{\mathrm{ii}}\right)$ & Aug-06 - Oct-06 & Large Adult & Treatment & Mainstem & 0.255 & 0.077 & 0.105 & 0.406 \\
\hline Lick Drain & Survival $\left(\gamma_{\mathrm{ii}}\right)$ & Oct-06 - May-07 & Large Adult & Treatment & Mainstem & 0.126 & 0.046 & 0.036 & 0.216 \\
\hline Lick Drain & Survival $\left(\gamma_{\mathrm{ii}}\right)$ & May-07 - Jul-07 & Large Adult & Treatment & Mainstem & 0.581 & 0.081 & 0.423 & 0.739 \\
\hline Lick Drain & Survival $\left(\gamma_{\mathrm{ii}}\right)$ & Jul-07 - Oct-07 & Large Adult & Treatment & Mainstem & 0.776 & 0.057 & 0.664 & 0.889 \\
\hline Mudlick Run & Survival $\left(\gamma_{\mathrm{ii}}\right)$ & Jul-06 - Aug-06 & Large Adult & Control & Headwater & 0.563 & 0.105 & 0.356 & 0.770 \\
\hline Mudlick Run & Survival $\left(\gamma_{\mathrm{ii}}\right)$ & Aug-06 - Oct-06 & Large Adult & Control & Headwater & 0.443 & 0.092 & 0.263 & 0.622 \\
\hline Mudlick Run & Survival $\left(\gamma_{\mathrm{ii}}\right)$ & Oct-06 - May-07 & Large Adult & Control & Headwater & 0.161 & 0.059 & 0.045 & 0.277 \\
\hline Mudlick Run & Survival $\left(\gamma_{\text {ii }}\right)$ & May-07 - Jul-07 & Large Adult & Control & Headwater & 0.561 & 0.102 & 0.360 & 0.762 \\
\hline Mudlick Run & Survival $\left(\gamma_{\text {ii }}\right)$ & Jul-07 - Oct-07 & Large Adult & Control & Headwater & 0.543 & 0.098 & 0.351 & 0.735 \\
\hline Tanner Run & Survival $\left(\gamma_{\mathrm{ii}}\right)$ & Jul-06 - Aug-06 & Large Adult & Treatment & Headwater & 0.004 & 0.005 & -0.006 & 0.014 \\
\hline Tanner Run & Survival $\left(\gamma_{\mathrm{ii}}\right)$ & Aug-06 - Oct-06 & Large Adult & Treatment & Headwater & 0.160 & 0.076 & 0.010 & 0.310 \\
\hline Tanner Run & Survival $\left(\gamma_{\mathrm{ii}}\right)$ & Oct-06 - May-07 & Large Adult & Treatment & Headwater & 0.074 & 0.041 & -0.005 & 0.154 \\
\hline Tanner Run & Survival $\left(\gamma_{\mathrm{ii}}\right)$ & May-07 - Jul-07 & Large Adult & Treatment & Headwater & 0.436 & 0.134 & 0.173 & 0.699 \\
\hline Tanner Run & Survival $\left(\gamma_{\mathrm{ii}}\right)$ & Jul-07 - Oct-07 & Large Adult & Treatment & Headwater & 0.659 & 0.120 & 0.425 & 0.893 \\
\hline Zinn Hollow & Survival $\left(\gamma_{\mathrm{ii}}\right)$ & Jul-06 - Aug-06 & Large Adult & Control & Mainstem & 0.592 & 0.109 & 0.378 & 0.807 \\
\hline Zinn Hollow & Survival $\left(\gamma_{\mathrm{ii}}\right)$ & Aug-06 - Oct-06 & Large Adult & Control & Mainstem & 0.473 & 0.109 & 0.260 & 0.685 \\
\hline Zinn Hollow & Survival $\left(\gamma_{\mathrm{ii}}\right)$ & Oct-06 - May-07 & Large Adult & Control & Mainstem & 0.178 & 0.065 & 0.050 & 0.306 \\
\hline Zinn Hollow & Survival $\left(\gamma_{\mathrm{ii}}\right)$ & May-07 - Jul-07 & Large Adult & Control & Mainstem & 0.590 & 0.101 & 0.392 & 0.789 \\
\hline Zinn Hollow & Survival $\left(\gamma_{\mathrm{ii}}\right)$ & Jul-07 - Oct-07 & Large Adult & Control & Mainstem & 0.573 & 0.098 & 0.380 & 0.766 \\
\hline Laurel Lick Run & Survival $\left(\gamma_{\mathrm{ii}}\right)$ & Jul-06 - Aug-06 & Large Adult & Treatment & Mainstem & 0.004 & 0.005 & -0.007 & 0.014 \\
\hline Laurel Lick Run & Survival $\left(\gamma_{\mathrm{ii}}\right)$ & Aug-06 - Oct-06 & Large Adult & Treatment & Mainstem & 0.169 & 0.070 & 0.031 & 0.307 \\
\hline Laurel Lick Run & Survival $\left(\gamma_{\mathrm{ii}}\right)$ & Oct-06 - May-07 & Large Adult & Treatment & Mainstem & 0.079 & 0.038 & 0.004 & 0.153 \\
\hline Laurel Lick Run & Survival $\left(\gamma_{\mathrm{ii}}\right)$ & May-07 - Jul-07 & Large Adult & Treatment & Mainstem & 0.451 & 0.114 & 0.229 & 0.674 \\
\hline Laurel Lick Run & Survival $\left(\gamma_{\mathrm{ii}}\right)$ & Jul-07 - Oct-07 & Large Adult & Treatment & Mainstem & 0.673 & 0.099 & 0.480 & 0.866 \\
\hline Glady Fork & Survival $\left(\gamma_{\mathrm{ii}}\right)$ & Jul-06 - Aug-06 & Large Adult & Control & Headwater & 0.408 & 0.104 & 0.203 & 0.613 \\
\hline Glady Fork & Survival $\left(\gamma_{\mathrm{ii}}\right)$ & Aug-06 - Oct-06 & Large Adult & Control & Headwater & 0.298 & 0.084 & 0.133 & 0.464 \\
\hline Glady Fork & Survival $\left(\gamma_{\mathrm{ii}}\right)$ & Oct-06 - May-07 & Large Adult & Control & Headwater & 0.093 & 0.040 & 0.016 & 0.171 \\
\hline Glady Fork & Survival $\left(\gamma_{\mathrm{ii}}\right)$ & May-07 - Jul-07 & Large Adult & Control & Headwater & 0.406 & 0.097 & 0.216 & 0.596 \\
\hline Glady Fork & Survival $\left(\gamma_{\mathrm{ii}}\right)$ & Jul-07 - Oct-07 & Large Adult & Control & Headwater & 0.389 & 0.102 & 0.190 & 0.588 \\
\hline
\end{tabular}




\begin{tabular}{|c|c|c|c|c|c|c|c|c|c|}
\hline Daniel's Run & Survival $\left(\gamma_{\mathrm{ii}}\right)$ & Jul-06 - Aug-06 & Large Adult & Treatment & Headwater & 0.004 & 0.005 & -0.006 & 0.014 \\
\hline Daniel's Run & Survival $\left(\gamma_{\mathrm{ii}}\right)$ & Aug-06 - Oct-06 & Large Adult & Treatment & Headwater & 0.162 & 0.066 & 0.033 & 0.292 \\
\hline Daniel's Run & Survival $\left(\gamma_{\mathrm{ii}}\right)$ & Oct-06 - May-07 & Large Adult & Treatment & Headwater & 0.076 & 0.036 & 0.005 & 0.146 \\
\hline Daniel's Run & Survival $\left(\gamma_{\mathrm{ii}}\right)$ & May-07 - Jul-07 & Large Adult & Treatment & Headwater & 0.440 & 0.112 & 0.221 & 0.659 \\
\hline Daniel's Run & Survival $\left(\gamma_{\mathrm{ii}}\right)$ & Jul-07 - Oct-07 & Large Adult & Treatment & Headwater & 0.663 & 0.101 & 0.464 & 0.862 \\
\hline Nan's Branch & Recruitment $\left(\gamma_{\mathrm{ji}}\right)$ & Jul-06 - Aug-06 & YOY - Small Adult & Control & Mainstem & 0.008 & 0.006 & -0.004 & 0.020 \\
\hline Nan's Branch & Recruitment $\left(\gamma_{\mathrm{ji}}\right)$ & Aug-06 - Oct-06 & YOY - Small Adult & Control & Mainstem & 0.008 & 0.006 & -0.004 & 0.020 \\
\hline Nan's Branch & Recruitment $\left(\gamma_{\mathrm{ji}}\right)$ & Oct-06 - May-07 & YOY - Small Adult & Control & Mainstem & 0.262 & 0.105 & 0.057 & 0.467 \\
\hline Nan's Branch & Recruitment $\left(\gamma_{\mathrm{ji}}\right)$ & May-07 - Jul-07 & YOY - Small Adult & Control & Mainstem & 0.065 & 0.034 & -0.003 & 0.132 \\
\hline Nan's Branch & Recruitment $\left(\gamma_{\mathrm{ji}}\right)$ & Jul-07 - Oct-07 & YOY - Small Adult & Control & Mainstem & 0.065 & 0.034 & -0.003 & 0.132 \\
\hline Otis Hollow & Recruitment $\left(\gamma_{\mathrm{ji}}\right)$ & Jul-06 - Aug-06 & YOY - Small Adult & Treatment & Mainstem & 0.021 & 0.012 & -0.003 & 0.044 \\
\hline Otis Hollow & Recruitment $\left(\gamma_{\mathrm{ji}}\right)$ & Aug-06 - Oct-06 & YOY - Small Adult & Treatment & Mainstem & 0.021 & 0.012 & -0.003 & 0.044 \\
\hline Otis Hollow & Recruitment $\left(\gamma_{\mathrm{ji}}\right)$ & Oct-06 - May-07 & YOY - Small Adult & Treatment & Mainstem & 0.475 & 0.080 & 0.318 & 0.633 \\
\hline Otis Hollow & Recruitment $\left(\gamma_{\mathrm{ji}}\right)$ & May-07 - Jul-07 & YOY - Small Adult & Treatment & Mainstem & 0.150 & 0.042 & 0.068 & 0.232 \\
\hline Otis Hollow & Recruitment $\left(\gamma_{\mathrm{ji}}\right)$ & Jul-07 - Oct-07 & YOY - Small Adult & Treatment & Mainstem & 0.150 & 0.042 & 0.068 & 0.232 \\
\hline Big Run & Recruitment $\left(\gamma_{\mathrm{ji}}\right)$ & Jul-06 - Aug-06 & YOY - Small Adult & Control & Headwater & 0.007 & 0.004 & -0.002 & 0.016 \\
\hline Big Run & Recruitment $\left(\gamma_{\mathrm{ji}}\right)$ & Aug-06 - Oct-06 & YOY - Small Adult & Control & Headwater & 0.007 & 0.004 & -0.002 & 0.016 \\
\hline Big Run & Recruitment $\left(\gamma_{\mathrm{ji}}\right)$ & Oct-06 - May-07 & YOY - Small Adult & Control & Headwater & 0.229 & 0.068 & 0.096 & 0.362 \\
\hline Big Run & Recruitment $\left(\gamma_{\mathrm{ji}}\right)$ & May-07 - Jul-07 & YOY - Small Adult & Control & Headwater & 0.055 & 0.023 & 0.009 & 0.100 \\
\hline Big Run & Recruitment $\left(\gamma_{\mathrm{ji}}\right)$ & Jul-07 - Oct-07 & YOY - Small Adult & Control & Headwater & 0.055 & 0.023 & 0.009 & 0.100 \\
\hline Swallow Rock & Recruitment $\left(\gamma_{\mathrm{ji}}\right)$ & Jul-06 - Aug-06 & YOY - Small Adult & Treatment & Headwater & 0.009 & 0.005 & -0.002 & 0.019 \\
\hline Swallow Rock & Recruitment $\left(\gamma_{\mathrm{ji}}\right)$ & Aug-06 - Oct-06 & YOY - Small Adult & Treatment & Headwater & 0.009 & 0.005 & -0.002 & 0.019 \\
\hline Swallow Rock & Recruitment $\left(\gamma_{\mathrm{ji}}\right)$ & Oct-06 - May-07 & YOY - Small Adult & Treatment & Headwater & 0.269 & 0.073 & 0.125 & 0.413 \\
\hline Swallow Rock & Recruitment $\left(\gamma_{\mathrm{ji}}\right)$ & May-07 - Jul-07 & YOY - Small Adult & Treatment & Headwater & 0.067 & 0.026 & 0.016 & 0.117 \\
\hline Swallow Rock & Recruitment $\left(\gamma_{\mathrm{ji}}\right)$ & Jul-07 - Oct-07 & YOY - Small Adult & Treatment & Headwater & 0.067 & 0.026 & 0.016 & 0.117 \\
\hline Lynn Run & Recruitment $\left(\gamma_{\mathrm{ji}}\right)$ & Jul-06 - Aug-06 & YOY - Small Adult & Control & Mainstem & 0.005 & 0.005 & -0.005 & 0.014 \\
\hline Lynn Run & Recruitment $\left(\gamma_{\mathrm{ji}}\right)$ & Aug-06 - Oct-06 & YOY - Small Adult & Control & Mainstem & 0.005 & 0.005 & -0.005 & 0.014 \\
\hline Lynn Run & Recruitment $\left(\gamma_{\mathrm{ji}}\right)$ & Oct-06 - May-07 & YOY - Small Adult & Control & Mainstem & 0.171 & 0.127 & -0.078 & 0.420 \\
\hline Lynn Run & Recruitment $\left(\gamma_{\mathrm{ji}}\right)$ & May-07 - Jul-07 & YOY - Small Adult & Control & Mainstem & 0.039 & 0.035 & -0.029 & 0.106 \\
\hline Lynn Run & Recruitment $\left(\gamma_{\mathrm{ji}}\right)$ & Jul-07 - Oct-07 & YOY - Small Adult & Control & Mainstem & 0.039 & 0.035 & -0.029 & 0.106 \\
\hline
\end{tabular}




\begin{tabular}{|c|c|c|c|c|c|c|c|c|c|}
\hline Lick Drain & Recruitment $\left(\gamma_{\mathrm{ji}}\right)$ & Jul-06 - Aug-06 & YOY - Small Adult & Treatment & Mainstem & 0.022 & 0.014 & -0.006 & 0.050 \\
\hline Lick Drain & Recruitment $\left(\gamma_{\mathrm{ji}}\right)$ & Aug-06 - Oct-06 & YOY - Small Adult & Treatment & Mainstem & 0.022 & 0.014 & -0.006 & 0.050 \\
\hline Lick Drain & Recruitment $\left(\gamma_{\mathrm{ji}}\right)$ & Oct-06 - May-07 & YOY - Small Adult & Treatment & Mainstem & 0.492 & 0.120 & 0.258 & 0.727 \\
\hline Lick Drain & Recruitment $\left(\gamma_{\mathrm{ji}}\right)$ & May-07 - Jul-07 & YOY - Small Adult & Treatment & Mainstem & 0.159 & 0.059 & 0.043 & 0.274 \\
\hline Lick Drain & Recruitment $\left(\gamma_{\mathrm{ji}}\right)$ & Jul-07 - Oct-07 & YOY - Small Adult & Treatment & Mainstem & 0.159 & 0.059 & 0.043 & 0.274 \\
\hline Mudlick Run & Recruitment $\left(\gamma_{\mathrm{ji}}\right)$ & Jul-06 - Aug-06 & YOY - Small Adult & Control & Headwater & 0.041 & 0.028 & -0.013 & 0.096 \\
\hline Mudlick Run & Recruitment $\left(\gamma_{\mathrm{ji}}\right)$ & Aug-06 - Oct-06 & YOY - Small Adult & Control & Headwater & 0.041 & 0.028 & -0.013 & 0.096 \\
\hline Mudlick Run & Recruitment $\left(\gamma_{\mathrm{ji}}\right)$ & Oct-06 - May-07 & YOY - Small Adult & Control & Headwater & 0.648 & 0.146 & 0.363 & 0.934 \\
\hline Mudlick Run & Recruitment $\left(\gamma_{\mathrm{ji}}\right)$ & May-07 - Jul-07 & YOY - Small Adult & Control & Headwater & 0.264 & 0.124 & 0.022 & 0.506 \\
\hline Mudlick Run & Recruitment $\left(\gamma_{\mathrm{ji}}\right)$ & Jul-07 - Oct-07 & YOY - Small Adult & Control & Headwater & 0.264 & 0.124 & 0.022 & 0.506 \\
\hline Tanner Run & Recruitment $\left(\gamma_{\mathrm{ji}}\right)$ & Jul-06 - Aug-06 & YOY - Small Adult & Treatment & Headwater & 0.017 & 0.013 & -0.008 & 0.043 \\
\hline Tanner Run & Recruitment $\left(\gamma_{\mathrm{ji}}\right)$ & Aug-06 - Oct-06 & YOY - Small Adult & Treatment & Headwater & 0.017 & 0.013 & -0.008 & 0.043 \\
\hline Tanner Run & Recruitment $\left(\gamma_{\mathrm{ji}}\right)$ & Oct-06 - May-07 & YOY - Small Adult & Treatment & Headwater & 0.428 & 0.161 & 0.113 & 0.744 \\
\hline Tanner Run & Recruitment $\left(\gamma_{\mathrm{ji}}\right)$ & May-07 - Jul-07 & YOY - Small Adult & Treatment & Headwater & 0.127 & 0.077 & -0.023 & 0.277 \\
\hline Tanner Run & Recruitment $\left(\gamma_{\mathrm{ji}}\right)$ & Jul-07 - Oct-07 & YOY - Small Adult & Treatment & Headwater & 0.127 & 0.077 & -0.023 & 0.277 \\
\hline Zinn Hollow & Recruitment $\left(\gamma_{\mathrm{ji}}\right)$ & Jul-06 - Aug-06 & YOY - Small Adult & Control & Mainstem & 0.004 & 0.004 & -0.004 & 0.012 \\
\hline Zinn Hollow & Recruitment $\left(\gamma_{\mathrm{ji}}\right)$ & Aug-06 - Oct-06 & YOY - Small Adult & Control & Mainstem & 0.004 & 0.004 & -0.004 & 0.012 \\
\hline Zinn Hollow & Recruitment $\left(\gamma_{\mathrm{ji}}\right)$ & Oct-06 - May-07 & YOY - Small Adult & & Mainstem & 0.148 & 0.102 & -0.053 & 0.348 \\
\hline Zinn Hollow & Recruitment $\left(\gamma_{\mathrm{ji}}\right)$ & May-07 - Jul-07 & YOY - Small Adult & Control & Mainstem & 0.033 & 0.026 & -0.018 & 0.083 \\
\hline Zinn Hollow & Recruitment $\left(\gamma_{\mathrm{ji}}\right)$ & Jul-07 - Oct-07 & YOY - Small Adult & Control & Mainstem & 0.033 & 0.026 & -0.018 & 0.083 \\
\hline Laurel Lick Run & Recruitment $\left(\gamma_{\mathrm{ji}}\right)$ & Jul-06 - Aug-06 & YOY - Small Adult & Treatment & Mainstem & 0.009 & 0.005 & -0.002 & 0.019 \\
\hline Laurel Lick Run & Recruitment $\left(\gamma_{\mathrm{ji}}\right)$ & Aug-06 - Oct-06 & YOY - Small Adult & Treatment & Mainstem & 0.009 & 0.005 & -0.002 & 0.019 \\
\hline Laurel Lick Run & Recruitment $\left(\gamma_{\mathrm{ji}}\right)$ & Oct-06 - May-07 & YOY - Small Adult & Treatment & Mainstem & 0.273 & 0.082 & 0.112 & 0.435 \\
\hline Laurel Lick Run & Recruitment $\left(\gamma_{\mathrm{ji}}\right)$ & May-07 - Jul-07 & YOY - Small Adult & Treatment & Mainstem & 0.068 & 0.028 & 0.014 & 0.122 \\
\hline Laurel Lick Run & Recruitment $\left(\gamma_{\mathrm{ji}}\right)$ & Jul-07 - Oct-07 & YOY - Small Adult & Treatment & Mainstem & 0.068 & 0.028 & 0.014 & 0.122 \\
\hline Glady Fork & Recruitment $\left(\gamma_{\mathrm{ji}}\right)$ & Jul-06 - Aug-06 & YOY - Small Adult & Control & Headwater & 0.110 & 0.063 & -0.013 & 0.233 \\
\hline Glady Fork & Recruitment $\left(\gamma_{\mathrm{ji}}\right)$ & Aug-06 - Oct-06 & YOY - Small Adult & Control & Headwater & 0.110 & 0.063 & -0.013 & 0.233 \\
\hline Glady Fork & Recruitment $\left(\gamma_{\mathrm{ji}}\right)$ & Oct-06 - May-07 & YOY - Small Adult & Control & Headwater & 0.841 & 0.086 & 0.673 & 1.009 \\
\hline Glady Fork & Recruitment $\left(\gamma_{\mathrm{ji}}\right)$ & May-07 - Jul-07 & YOY - Small Adult & Control & Headwater & 0.507 & 0.149 & 0.215 & 0.799 \\
\hline Glady Fork & Recruitment $\left(\gamma_{\mathrm{ji}}\right)$ & Jul-07 - Oct-07 & YOY - Small Adult & Control & Headwater & 0.507 & 0.149 & 0.215 & 0.799 \\
\hline
\end{tabular}




\begin{tabular}{|c|c|c|c|c|c|c|c|c|c|}
\hline Daniel's Run & Recruitment $\left(\gamma_{\mathrm{ji}}\right)$ & Jul-06 - Aug-06 & YOY - Small Adult & Treatment & Headwater & 0.011 & 0.010 & -0.009 & 0.031 \\
\hline Daniel's Run & Recruitment $\left(\gamma_{\mathrm{ji}}\right)$ & Aug-06 - Oct-06 & YOY - Small Adult & Treatment & Headwater & 0.011 & 0.010 & -0.009 & 0.031 \\
\hline Daniel's Run & Recruitment $\left(\gamma_{\mathrm{ji}}\right)$ & Oct-06 - May-07 & YOY - Small Adult & Treatment & Headwater & 0.323 & 0.184 & -0.038 & 0.683 \\
\hline Daniel's Run & Recruitment $\left(\gamma_{\mathrm{ji}}\right)$ & May-07 - Jul-07 & YOY - Small Adult & Treatment & Headwater & 0.085 & 0.066 & -0.044 & 0.214 \\
\hline Daniel's Run & Recruitment $\left(\gamma_{\mathrm{ji}}\right)$ & Jul-07 - Oct-07 & YOY - Small Adult & Treatment & Headwater & 0.085 & 0.066 & -0.044 & 0.214 \\
\hline Nan's Branch & Recruitment $\left(\gamma_{\mathrm{ji}}\right)$ & Jul-06 - Aug-06 & Small - Large Adult & Control & Mainstem & 0.008 & 0.006 & -0.004 & 0.019 \\
\hline Nan's Branch & Recruitment $\left(\gamma_{\mathrm{ji}}\right)$ & Aug-06 - Oct-06 & Small - Large Adult & Control & Mainstem & 0.008 & 0.006 & -0.004 & 0.019 \\
\hline Nan's Branch & Recruitment $\left(\gamma_{\mathrm{ji}}\right)$ & Oct-06 - May-07 & Small - Large Adult & Control & Mainstem & 0.246 & 0.103 & 0.045 & 0.447 \\
\hline Nan's Branch & Recruitment $\left(\gamma_{\mathrm{ji}}\right)$ & May-07 - Jul-07 & Small - Large Adult & Control & Mainstem & 0.060 & 0.033 & -0.004 & 0.123 \\
\hline Nan's Branch & Recruitment $\left(\gamma_{\mathrm{ji}}\right)$ & Jul-07 - Oct-07 & Small - Large Adult & Control & Mainstem & 0.060 & 0.033 & -0.004 & 0.123 \\
\hline Otis Hollow & Recruitment $\left(\gamma_{\mathrm{ji}}\right)$ & Jul-06 - Aug-06 & Small - Large Adult & Treatment & Mainstem & 0.019 & 0.012 & -0.005 & 0.043 \\
\hline Otis Hollow & Recruitment $\left(\gamma_{\mathrm{ji}}\right)$ & Aug-06 - Oct-06 & Small - Large Adult & Treatment & Mainstem & 0.019 & 0.012 & -0.005 & 0.043 \\
\hline Otis Hollow & Recruitment $\left(\gamma_{\mathrm{ji}}\right)$ & Oct-06 - May-07 & Small - Large Adult & Treatment & Mainstem & 0.454 & 0.097 & 0.265 & 0.643 \\
\hline Otis Hollow & Recruitment $\left(\gamma_{\mathrm{ji}}\right)$ & May-07 - Jul-07 & Small - Large Adult & Treatment & Mainstem & 0.139 & 0.047 & 0.047 & 0.232 \\
\hline Otis Hollow & Recruitment $\left(\gamma_{\mathrm{ji}}\right)$ & Jul-07 - Oct-07 & Small - Large Adult & Treatment & Mainstem & 0.139 & 0.047 & 0.047 & 0.232 \\
\hline Big Run & Recruitment $\left(\gamma_{\mathrm{ji}}\right)$ & Jul-06 - Aug-06 & Small - Large Adult & Control & Headwater & 0.006 & 0.004 & -0.002 & 0.015 \\
\hline Big Run & Recruitment $\left(\gamma_{\mathrm{ji}}\right)$ & Aug-06 - Oct-06 & Small - Large Adult & Control & Headwater & 0.006 & 0.004 & -0.002 & 0.015 \\
\hline Big Run & Recruitment $\left(\gamma_{\mathrm{ji}}\right)$ & Oct-06 - May-07 & Small - Large Adult & & Headwater & 0.214 & 0.065 & 0.087 & 0.342 \\
\hline Big Run & Recruitment $\left(\gamma_{\mathrm{ji}}\right)$ & May-07 - Jul-07 & Small - Large Adult & Control & Headwater & 0.050 & 0.022 & 0.008 & 0.093 \\
\hline Big Run & Recruitment $\left(\gamma_{\mathrm{ji}}\right)$ & Jul-07 - Oct-07 & Small - Large Adult & Control & Headwater & 0.050 & 0.022 & 0.008 & 0.093 \\
\hline Swallow Rock & Recruitment $\left(\gamma_{\mathrm{ji}}\right)$ & Jul-06 - Aug-06 & Small - Large Adult & Treatment & Headwater & 0.008 & 0.005 & -0.002 & 0.018 \\
\hline Swallow Rock & Recruitment $\left(\gamma_{\mathrm{ji}}\right)$ & Aug-06 - Oct-06 & Small - Large Adult & Treatment & Headwater & 0.008 & 0.005 & -0.002 & 0.018 \\
\hline Swallow Rock & Recruitment $\left(\gamma_{\mathrm{ji}}\right)$ & Oct-06 - May-07 & Small - Large Adult & Treatment & Headwater & 0.252 & 0.067 & 0.120 & 0.385 \\
\hline Swallow Rock & Recruitment $\left(\gamma_{\mathrm{ji}}\right)$ & May-07 - Jul-07 & Small - Large Adult & Treatment & Headwater & 0.062 & 0.023 & 0.017 & 0.106 \\
\hline Swallow Rock & Recruitment $\left(\gamma_{\mathrm{ji}}\right)$ & Jul-07 - Oct-07 & Small - Large Adult & Treatment & Headwater & 0.062 & 0.023 & 0.017 & 0.106 \\
\hline Lynn Run & Recruitment $\left(\gamma_{\mathrm{ji}}\right)$ & Jul-06 - Aug-06 & Small - Large Adult & Control & Mainstem & 0.004 & 0.005 & -0.004 & 0.013 \\
\hline Lynn Run & Recruitment $\left(\gamma_{\mathrm{ji}}\right)$ & Aug-06 - Oct-06 & Small - Large Adult & Control & Mainstem & 0.004 & 0.005 & -0.004 & 0.013 \\
\hline Lynn Run & Recruitment $\left(\gamma_{\mathrm{ji}}\right)$ & Oct-06 - May-07 & Small - Large Adult & Control & Mainstem & 0.160 & 0.117 & -0.070 & 0.389 \\
\hline Lynn Run & Recruitment $\left(\gamma_{\mathrm{ji}}\right)$ & May-07 - Jul-07 & Small - Large Adult & Control & Mainstem & 0.036 & 0.031 & -0.026 & 0.097 \\
\hline Lynn Run & Recruitment $\left(\gamma_{\mathrm{ji}}\right)$ & Jul-07 - Oct-07 & Small - Large Adult & Control & Mainstem & 0.036 & 0.031 & -0.026 & 0.097 \\
\hline
\end{tabular}




\begin{tabular}{|c|c|c|c|c|c|c|c|c|c|}
\hline Lick Drain & Recruitment $\left(\gamma_{\mathrm{ji}}\right)$ & Jul-06 - Aug-06 & Small - Large Adult & Treatment & Mainstem & 0.020 & 0.013 & -0.005 & 0.046 \\
\hline Lick Drain & Recruitment $\left(\gamma_{\mathrm{ji}}\right)$ & Aug-06 - Oct-06 & Small - Large Adult & Treatment & Mainstem & 0.020 & 0.013 & -0.005 & 0.046 \\
\hline Lick Drain & Recruitment $\left(\gamma_{\mathrm{ji}}\right)$ & Oct-06 - May-07 & Small - Large Adult & Treatment & Mainstem & 0.471 & 0.108 & 0.259 & 0.683 \\
\hline Lick Drain & Recruitment $\left(\gamma_{\mathrm{ji}}\right)$ & May-07 - Jul-07 & Small - Large Adult & Treatment & Mainstem & 0.148 & 0.049 & 0.052 & 0.244 \\
\hline Lick Drain & Recruitment $\left(\gamma_{\mathrm{ji}}\right)$ & Jul-07 - Oct-07 & Small - Large Adult & Treatment & Mainstem & 0.148 & 0.049 & 0.052 & 0.244 \\
\hline Mudlick Run & Recruitment $\left(\gamma_{\mathrm{ji}}\right)$ & Jul-06 - Aug-06 & Small - Large Adult & Control & Headwater & 0.038 & 0.026 & -0.012 & 0.088 \\
\hline Mudlick Run & Recruitment $\left(\gamma_{\mathrm{ji}}\right)$ & Aug-06 - Oct-06 & Small - Large Adult & Control & Headwater & 0.038 & 0.026 & -0.012 & 0.088 \\
\hline Mudlick Run & Recruitment $\left(\gamma_{\mathrm{ji}}\right)$ & Oct-06 - May-07 & Small - Large Adult & Control & Headwater & 0.629 & 0.142 & 0.350 & 0.907 \\
\hline Mudlick Run & Recruitment $\left(\gamma_{\mathrm{ji}}\right)$ & May-07 - Jul-07 & Small - Large Adult & Control & Headwater & 0.248 & 0.113 & 0.027 & 0.469 \\
\hline Mudlick Run & Recruitment $\left(\gamma_{\mathrm{ji}}\right)$ & Jul-07 - Oct-07 & Small - Large Adult & Control & Headwater & 0.248 & 0.113 & 0.027 & 0.469 \\
\hline Tanner Run & tment $\left(\gamma_{\mathrm{ji}}\right)$ & Jul-06 - Aug-06 & Small - Large Adult & Treatment & Headwater & 0.016 & 0.012 & -0.008 & 0.039 \\
\hline Tanner Run & Recruitment $\left(\gamma_{\mathrm{ji}}\right)$ & Aug-06 - Oct-06 & Small - Large Adult & Treatment & Headwater & 0.016 & 0.012 & -0.008 & 0.039 \\
\hline Tanner Run & Recruitment $\left(\gamma_{\mathrm{ji}}\right)$ & Oct-06 - May-07 & Small - Large Adult & Treatment & Headwater & 0.408 & 0.152 & 0.109 & 0.706 \\
\hline Tanner Run & Recruitment $\left(\gamma_{\mathrm{ji}}\right)$ & May-07 - Jul-07 & Small - Large Adult & Treatment & Headwater & 0.118 & 0.069 & -0.017 & 0.253 \\
\hline Tanner Run & Recruitment $\left(\gamma_{\mathrm{ji}}\right)$ & Jul-07 - Oct-07 & Small - Large Adult & Treatment & Headwater & 0.118 & 0.069 & -0.017 & 0.253 \\
\hline Zinn Hollow & Recruitment $\left(\gamma_{\mathrm{ji}}\right)$ & Jul-06 - Aug-06 & Small - Large Adult & Control & Mainstem & 0.004 & 0.004 & -0.004 & 0.011 \\
\hline Zinn Hollow & Recruitment $\left(\gamma_{\mathrm{ji}}\right)$ & Aug-06 - Oct-06 & Small - Large Adult & Control & Mainstem & 0.004 & 0.004 & -0.004 & 0.011 \\
\hline Zinn Hollow & Recruitment $\left(\gamma_{\mathrm{ji}}\right)$ & Oct-06 - May-07 & Small - Large Adult & & Mainstem & 0.137 & 0.096 & -0.051 & 0.325 \\
\hline Zinn Hollow & Recruitment $\left(\gamma_{\mathrm{ji}}\right)$ & May-07 - Jul-07 & Small - Large Adult & Control & Mainstem & 0.030 & 0.024 & -0.017 & 0.077 \\
\hline Zinn Hollow & Recruitment $\left(\gamma_{\mathrm{ji}}\right)$ & Jul-07 - Oct-07 & Small - Large Adult & Control & Mainstem & 0.030 & 0.024 & -0.017 & 0.077 \\
\hline Laurel Lick Run & Recruitment $\left(\gamma_{\mathrm{ji}}\right)$ & Jul-06 - Aug-06 & Small - Large Adult & Treatment & Mainstem & 0.008 & 0.005 & -0.002 & 0.018 \\
\hline Laurel Lick Run & Recruitment $\left(\gamma_{\mathrm{ji}}\right)$ & Aug-06 - Oct-06 & Small - Large Adult & Treatment & Mainstem & 0.008 & 0.005 & -0.002 & 0.018 \\
\hline Laurel Lick Run & Recruitment $\left(\gamma_{\mathrm{ji}}\right)$ & Oct-06 - May-07 & Small - Large Adult & Treatment & Mainstem & 0.257 & 0.067 & 0.125 & 0.388 \\
\hline Laurel Lick Run & Recruitment $\left(\gamma_{\mathrm{ji}}\right)$ & May-07 - Jul-07 & Small - Large Adult & Treatment & Mainstem & 0.063 & 0.022 & 0.020 & 0.106 \\
\hline Laurel Lick Run & Recruitment $\left(\gamma_{\mathrm{ji}}\right)$ & Jul-07 - Oct-07 & Small - Large Adult & Treatment & Mainstem & 0.063 & 0.022 & 0.020 & 0.106 \\
\hline Glady Fork & Recruitment $\left(\gamma_{\mathrm{ji}}\right)$ & Jul-06 - Aug-06 & Small - Large Adult & Control & Headwater & 0.102 & 0.061 & -0.017 & 0.221 \\
\hline Glady Fork & Recruitment $\left(\gamma_{\mathrm{ji}}\right)$ & Aug-06 - Oct-06 & Small - Large Adult & Control & Headwater & 0.102 & 0.061 & -0.017 & 0.221 \\
\hline Glady Fork & Recruitment $\left(\gamma_{\mathrm{ji}}\right)$ & Oct-06 - May-07 & Small - Large Adult & Control & Headwater & 0.829 & 0.090 & 0.652 & 1.006 \\
\hline Glady Fork & Recruitment $\left(\gamma_{\mathrm{ji}}\right)$ & May-07 - Jul-07 & Small - Large Adult & Control & Headwater & 0.486 & 0.147 & 0.197 & 0.775 \\
\hline Glady Fork & Recruitment $\left(\gamma_{\mathrm{ji}}\right)$ & Jul-07 - Oct-07 & Small - Large Adult & Control & Headwater & 0.486 & 0.147 & 0.197 & 0.775 \\
\hline
\end{tabular}


Daniel's Run

Daniel's Run

Daniel's Run

Daniel's Run

Daniel's Run
Recruitment $\left(\gamma_{\mathrm{ji}}\right)$

Recruitment $\left(\gamma_{\mathrm{ji}}\right)$

Recruitment $\left(\gamma_{\mathrm{ji}}\right)$

Recruitment $\left(\gamma_{\mathrm{ji}}\right)$

Recruitment $\left(\gamma_{\mathrm{ji}}\right)$
Jul-06 - Aug-06

Aug-06 - Oct-06

Oct-06 - May-07

May-07 - Jul-07

Jul-07 - Oct-07
Small - Large Adult

Small - Large Adult

Small - Large Adult

Small - Large Adult

Small - Large Adult
Treatment

Treatment

Treatment

Treatment

Treatment
Headwater

Headwater

Headwater

Headwater

Headwater
0.010

0.010

0.304

0.078

0.078
0.009

0.009

0.164

0.056

0.056
$-0.007$

$-0.007$

$-0.017$

$-0.032$

$-0.032$
0.027

0.027

0.626

0.189

0.189 
F3. Model coefficient estimates, standard errors, and confidence limits for the modelaveraged coefficients resulting from the $95 \%$ confidence set of multinomial models for estimating the proportion of the instantaneous population growth rate $(\lambda)$ due to survival $\left(\gamma_{\mathrm{ii}}\right)$ and local recruitment $\left(\gamma_{\mathrm{ji}}\right)$ for mottled sculpin in response to fish removal treatment, spatial location, and interactions of interest over the course of the study while accounting for the effects of age, sampling interval and local physical and chemical habitat covariates. The multinomial model has two intercepts, one for estimating survival (Intercept $\gamma_{\mathrm{ii}}$ ) and one for estimating age-class transition (Intercept $\gamma_{\mathrm{ji}}$ ). $\Sigma \mathrm{w}_{\mathrm{i}}=$ sum of Akaike weights for experimental effect terms across $95 \%$ confidence model set. The cumulative Akaike weights for each model term are indicated next to the relevant effect. Asterisks (*) indicate where model coefficient confidence limits did not include 0 (i.e., a "significant" positive or negative slope). $a 1=$ YOY age class, $a 2=$ small adults, $g=$ fish removal treatment site, $1=$ mainstem tributary site, $\mathrm{t} 1-\mathrm{t} 4=$ sampling interval, $\mathrm{a} 1 * \mathrm{~g}=$ YOY $\mathrm{x}$ removal interaction effect, $\mathrm{a} 2 * \mathrm{~g}=$ small adult $\mathrm{x}$ removal, $\mathrm{a} 1 * 1=$ YOY $\mathrm{x}$ location, a2*1 $=$ small adult $x$ location, $g * 1=$ removal $x$ location, $g * t 1-g * t 4=$ removal $x$ date, $1 * \mathrm{t} 1-1 * \mathrm{t} 4=$ location $\mathrm{x}$ date, $\mathrm{g} * 1 * \mathrm{t} 1-\mathrm{g} * 1 * \mathrm{t} 4=$ removal $\mathrm{x}$ location $\mathrm{x}$ date, $\mathrm{W} 1=$ water chemistry principal component $1, \mathrm{H} 1-\mathrm{H} 3=$ physical habitat principal components 1 to 3 , PAREA $=$ proportion of spring wetted channel area in early fall, CANP $=$ mean percent canopy cover, MWAT $=$ maximum weekly average temperature $\left({ }^{\circ} \mathrm{C}\right)$.

\begin{tabular}{|c|c|c|c|c|c|}
\hline Effect & Estimate & SE & LCI & $\mathrm{UCl}$ & $\Sigma w_{i}$ \\
\hline Intercept $\left(\gamma_{i i}\right)$ & 5.214 & 1.736 & 1.812 & 8.616 & * \\
\hline W1 & -0.710 & 0.199 & -1.100 & -0.320 & * \\
\hline $\mathrm{H} 1$ & 0.203 & 0.043 & 0.118 & 0.287 & * \\
\hline $\mathrm{H} 2$ & 0.055 & 0.053 & -0.048 & 0.159 & \\
\hline H3 & -0.047 & 0.099 & -0.241 & 0.147 & \\
\hline PAREA & -1.334 & 0.287 & -1.896 & -0.772 & * \\
\hline CANP & -0.094 & 0.013 & -0.119 & -0.070 & * \\
\hline MWAT & 0.262 & 0.077 & 0.111 & 0.412 & * \\
\hline a1 & -3.615 & 0.688 & -4.963 & -2.268 & * \\
\hline a2 & -1.142 & 0.149 & -1.434 & -0.850 & * \\
\hline$g$ & 0.255 & 0.244 & -0.223 & 0.733 & 1.000 \\
\hline I & -0.131 & 0.269 & -0.657 & 0.396 & 1.000 \\
\hline $\mathrm{t} 1$ & -0.715 & 0.203 & -1.113 & -0.317 & * \\
\hline $\mathrm{t} 2$ & -0.983 & 0.177 & -1.330 & -0.636 & * \\
\hline $\mathrm{t} 3$ & -0.694 & 0.169 & -1.024 & -0.363 & * \\
\hline $\mathrm{t} 4$ & -0.123 & 0.195 & -0.506 & 0.259 & \\
\hline$a 1^{*} g$ & -0.329 & 0.885 & -2.063 & 1.405 & \\
\hline$a 2^{*} g$ & -0.309 & 0.175 & -0.652 & 0.035 & \\
\hline$a 1 * \mid$ & -0.688 & 1.072 & -2.789 & 1.414 & \\
\hline$a 2 * \mid$ & 0.108 & 0.171 & -0.228 & 0.443 & \\
\hline$g^{*}+1$ & -4.116 & 0.533 & -5.161 & -3.072 & 1.000 \\
\hline$g^{*} \mathrm{t} 2$ & 0.275 & 0.275 & -0.264 & 0.813 & \\
\hline$g^{*}+3$ & 0.224 & 0.264 & -0.292 & 0.741 & \\
\hline$g^{*}+4$ & -0.468 & 0.279 & -1.014 & 0.078 & \\
\hline Intercept $\left(\gamma_{\mathrm{ji}}\right)$ & 1.975 & 4.332 & -6.515 & 10.465 & \\
\hline W1 & -0.099 & 0.407 & -0.896 & 0.699 & \\
\hline $\mathrm{H} 1$ & 0.221 & 0.085 & 0.055 & 0.387 & * \\
\hline
\end{tabular}




$\begin{array}{cccccccc}\text { H3 } & 0.016 & 0.108 & -0.195 & 0.227 & & \\ \text { H3 } & 0.124 & 0.133 & -0.137 & 0.384 & & \\ \text { PAREA } & -0.750 & 0.454 & -1.640 & 0.141 & & \\ \text { CANP } & -0.119 & 0.025 & -0.167 & -0.070 & * & \\ \text { MWAT } & 0.296 & 0.193 & -0.082 & 0.674 & & \\ \text { a } & -0.656 & 0.203 & -1.053 & -0.259 & * & \\ \text { g } & -0.111 & 0.340 & -0.778 & 0.555 & & 0.076 \\ \text { t1 } & -1.493 & 0.599 & -2.667 & -0.319 & * & \\ \text { t2 } & 1.054 & 0.362 & 0.344 & 1.763 & * & \\ \text { t3 } & 1.709 & 0.339 & 1.044 & 2.374 & * & \\ \text { t4 } & 0.984 & 0.368 & 0.263 & 1.705 & * & \end{array}$


F4. Summary of model-averaged parameter estimates, standard errors, and 95\% confidence limits (i.e., lower $=$ LCI and upper $=$ UCI) for proportion of the mottled sculpin instantaneous population growth rate $(\lambda)$ attributable to local survival $\left(\gamma_{\mathrm{ii}}\right)$ and in-situ recruitment $\left(\gamma_{\mathrm{ji}}\right)$ for all sites and sample dates. Experimental classifications of sites based on treatment and spatial location are also noted.

\begin{tabular}{|c|c|c|c|c|c|c|c|c|c|}
\hline Site & Variable & Interval & Age & Removal & Location & Est & SE & LCL & UCL \\
\hline Nan's Branch & Survival $\left(\gamma_{i i}\right)$ & Jul-06 - Aug-06 & YOY & Control & Mainstem & 0.012 & 0.007 & -0.002 & 0.025 \\
\hline Nan's Branch & Survival $\left(\gamma_{i i}\right)$ & Aug-06 - Oct-06 & YOY & Control & Mainstem & 0.009 & 0.005 & -0.001 & 0.019 \\
\hline Nan's Branch & Survival $\left(\gamma_{\mathrm{ii}}\right)$ & Oct-06 - May-07 & YOY & Control & Mainstem & 0.012 & 0.007 & -0.002 & 0.026 \\
\hline Nan's Branch & Survival $\left(\gamma_{\mathrm{ii}}\right)$ & May-07 - Jul-07 & YOY & Control & Mainstem & 0.022 & 0.013 & -0.004 & 0.047 \\
\hline Nan's Branch & Survival $\left(\gamma_{\mathrm{ii}}\right)$ & Jul-07 - Oct-07 & YOY & Control & Mainstem & 0.023 & 0.013 & -0.003 & 0.050 \\
\hline Otis Hollow & Survival $\left(\gamma_{i i}\right)$ & Jul-06 - Aug-06 & YOY & Treatment & Mainstem & 0.000 & 0.000 & 0.000 & 0.000 \\
\hline Otis Hollow & Survival $\left(\gamma_{i i}\right)$ & Aug-06 - Oct-06 & YOY & Treatment & Mainstem & 0.004 & 0.003 & -0.002 & 0.010 \\
\hline Otis Hollow & Survival $\left(\gamma_{\mathrm{ii}}\right)$ & Oct-06 - May-07 & YOY & Treatment & Mainstem & 0.005 & 0.004 & -0.003 & 0.013 \\
\hline Otis Hollow & Survival $\left(\gamma_{i i}\right)$ & May-07 - Jul-07 & YOY & Treatment & Mainstem & 0.005 & 0.004 & -0.003 & 0.012 \\
\hline Otis Hollow & Survival $\left(\gamma_{\mathrm{ii}}\right)$ & Jul-07 - Oct-07 & YOY & Treatment & Mainstem & 0.008 & 0.007 & -0.004 & 0.021 \\
\hline Big Run & Survival $\left(\gamma_{\mathrm{ii}}\right)$ & Jul-06 - Aug-06 & YOY & Control & Headwater & 0.008 & 0.005 & -0.002 & 0.017 \\
\hline Big Run & Survival $\left(\gamma_{\mathrm{ii}}\right)$ & Aug-06 - Oct-06 & YOY & Control & Headwater & 0.006 & 0.004 & -0.001 & 0.013 \\
\hline Big Run & Survival $\left(\gamma_{\mathrm{ii}}\right)$ & Oct-06 - May-07 & YOY & Control & Headwater & 0.008 & 0.005 & -0.001 & 0.017 \\
\hline Big Run & Survival $\left(\gamma_{\mathrm{ii}}\right)$ & May-07 - Jul-07 & YOY & Control & Headwater & 0.014 & 0.009 & -0.003 & 0.032 \\
\hline Big Run & Survival $\left(\gamma_{\mathrm{ii}}\right)$ & Jul-07 - Oct-07 & YOY & Control & Headwater & 0.015 & 0.009 & -0.003 & 0.033 \\
\hline Swallow Rock & Survival $\left(\gamma_{\mathrm{ii}}\right)$ & Jul-06 - Aug-06 & YOY & Treatment & Headwater & 0.000 & 0.000 & 0.000 & 0.000 \\
\hline Swallow Rock & Survival $\left(\gamma_{i i}\right)$ & Aug-06 - Oct-06 & YOY & Treatment & Headwater & 0.008 & 0.006 & -0.004 & 0.021 \\
\hline Swallow Rock & Survival $\left(\gamma_{\mathrm{ii}}\right)$ & Oct-06 - May-07 & YOY & Treatment & Headwater & 0.011 & 0.008 & -0.005 & 0.027 \\
\hline Swallow Rock & Survival $\left(\gamma_{\mathrm{ii}}\right)$ & May-07 - Jul-07 & YOY & Treatment & Headwater & 0.010 & 0.007 & -0.005 & 0.024 \\
\hline Swallow Rock & Survival $\left(\gamma_{\mathrm{ii}}\right)$ & Jul-07 - Oct-07 & YOY & Treatment & Headwater & 0.018 & 0.013 & -0.008 & 0.044 \\
\hline Lynn Run & Survival $\left(\gamma_{\mathrm{ii}}\right)$ & Jul-06 - Aug-06 & YOY & Control & Mainstem & 0.023 & 0.014 & -0.004 & 0.050 \\
\hline Lynn Run & Survival $\left(\gamma_{\mathrm{ii}}\right)$ & Aug-06 - Oct-06 & YOY & Control & Mainstem & 0.017 & 0.010 & -0.003 & 0.037 \\
\hline Lynn Run & Survival $\left(\gamma_{\mathrm{ii}}\right)$ & Oct-06 - May-07 & YOY & Control & Mainstem & 0.024 & 0.014 & -0.004 & 0.051 \\
\hline Lynn Run & Survival $\left(\gamma_{i i}\right)$ & May-07 - Jul-07 & YOY & Control & Mainstem & 0.042 & 0.025 & -0.006 & 0.090 \\
\hline Lynn Run & Survival $\left(\gamma_{\mathrm{ii}}\right)$ & Jul-07 - Oct-07 & YOY & Control & Mainstem & 0.045 & 0.026 & -0.006 & 0.095 \\
\hline
\end{tabular}




\begin{tabular}{|c|c|c|c|c|c|c|c|c|c|}
\hline Site & Variable & Interval & Age & Removal & Location & Est & SE & LCL & UCL \\
\hline Lick Drain & Survival $\left(\gamma_{i i}\right)$ & Jul-06 - Aug-06 & YOY & Treatment & Mainstem & 0.000 & 0.000 & 0.000 & 0.000 \\
\hline Lick Drain & Survival $\left(\gamma_{i i}\right)$ & Aug-06 - Oct-06 & YOY & Treatment & Mainstem & 0.005 & 0.004 & -0.002 & 0.012 \\
\hline Lick Drain & Survival $\left(\gamma_{\mathrm{ii}}\right)$ & Oct-06 - May-07 & YOY & Treatment & Mainstem & 0.006 & 0.005 & -0.003 & 0.016 \\
\hline Lick Drain & Survival $\left(\gamma_{\mathrm{ii}}\right)$ & May-07 - Jul-07 & YOY & Treatment & Mainstem & 0.006 & 0.004 & -0.003 & 0.014 \\
\hline Lick Drain & Survival $\left(\gamma_{\mathrm{ii}}\right)$ & Jul-07 - Oct-07 & YOY & Treatment & Mainstem & 0.010 & 0.008 & -0.005 & 0.025 \\
\hline Mudlick Run & Survival $\left(\gamma_{\mathrm{ii}}\right)$ & Jul-06 - Aug-06 & YOY & Control & Headwater & 0.019 & 0.011 & -0.003 & 0.041 \\
\hline Mudlick Run & Survival $\left(\gamma_{i i}\right)$ & Aug-06 - Oct-06 & YOY & Control & Headwater & 0.014 & 0.008 & -0.002 & 0.031 \\
\hline Mudlick Run & Survival $\left(\gamma_{\mathrm{ii}}\right)$ & Oct-06 - May-07 & YOY & Control & Headwater & 0.019 & 0.011 & -0.003 & 0.042 \\
\hline Mudlick Run & Survival $\left(\gamma_{i i}\right)$ & May-07 - Jul-07 & YOY & Control & Headwater & 0.035 & 0.020 & -0.005 & 0.075 \\
\hline Mudlick Run & Survival $\left(\gamma_{\mathrm{ii}}\right)$ & Jul-07 - Oct-07 & YOY & Control & Headwater & 0.037 & 0.021 & -0.005 & 0.079 \\
\hline Tanner Run & Survival $\left(\gamma_{\mathrm{ii}}\right)$ & Jul-06 - Aug-06 & YOY & Treatment & Headwater & 0.000 & 0.000 & 0.000 & 0.000 \\
\hline Tanner Run & Survival $\left(\gamma_{i i}\right)$ & Aug-06 - Oct-06 & YOY & Treatment & Headwater & 0.009 & 0.007 & -0.004 & 0.022 \\
\hline Tanner Run & Survival $\left(\gamma_{i i}\right)$ & Oct-06 - May-07 & YOY & Treatment & Headwater & 0.012 & 0.009 & -0.005 & 0.029 \\
\hline Tanner Run & Survival $\left(\gamma_{\mathrm{ii}}\right)$ & May-07 - Jul-07 & YOY & Treatment & Headwater & 0.011 & 0.008 & -0.005 & 0.026 \\
\hline Tanner Run & Survival $\left(\gamma_{\mathrm{ii}}\right)$ & Jul-07 - Oct-07 & YOY & Treatment & Headwater & 0.019 & 0.014 & -0.008 & 0.047 \\
\hline Zinn Hollow & Survival $\left(\gamma_{\mathrm{ii}}\right)$ & Jul-06 - Aug-06 & YOY & Control & Mainstem & 0.008 & 0.005 & -0.002 & 0.019 \\
\hline Zinn Hollow & Survival $\left(\gamma_{\mathrm{ii}}\right)$ & Aug-06 - Oct-06 & YOY & Control & Mainstem & 0.006 & 0.004 & -0.001 & 0.014 \\
\hline Zinn Hollow & Survival $\left(\gamma_{i i}\right)$ & Oct-06 - May-07 & YOY & Control & Mainstem & 0.009 & 0.005 & -0.002 & 0.019 \\
\hline Zinn Hollow & Survival $\left(\gamma_{\mathrm{ii}}\right)$ & May-07 - Jul-07 & YOY & Control & Mainstem & 0.016 & 0.010 & -0.003 & 0.035 \\
\hline Zinn Hollow & Survival $\left(\gamma_{\mathrm{ii}}\right)$ & Jul-07 - Oct-07 & YOY & Control & Mainstem & 0.017 & 0.010 & -0.003 & 0.036 \\
\hline Laurel Lick Run & Survival $\left(\gamma_{\mathrm{ii}}\right)$ & Jul-06 - Aug-06 & YOY & Treatment & Mainstem & 0.000 & 0.000 & 0.000 & 0.000 \\
\hline Laurel Lick Run & Survival $\left(\gamma_{\mathrm{ii}}\right)$ & Aug-06 - Oct-06 & YOY & Treatment & Mainstem & 0.010 & 0.007 & -0.004 & 0.023 \\
\hline Laurel Lick Run & Survival $\left(\gamma_{\mathrm{ii}}\right)$ & Oct-06 - May-07 & YOY & Treatment & Mainstem & 0.013 & 0.009 & -0.006 & 0.031 \\
\hline Laurel Lick Run & Survival $\left(\gamma_{i i}\right)$ & May-07 - Jul-07 & YOY & Treatment & Mainstem & 0.011 & 0.008 & -0.005 & 0.028 \\
\hline Laurel Lick Run & Survival $\left(\gamma_{i i}\right)$ & Jul-07 - Oct-07 & YOY & Treatment & Mainstem & 0.020 & 0.015 & -0.009 & 0.050 \\
\hline Glady Fork & Survival $\left(\gamma_{\mathrm{ii}}\right)$ & Jul-06 - Aug-06 & YOY & Control & Headwater & 0.026 & 0.016 & -0.005 & 0.057 \\
\hline Glady Fork & Survival $\left(\gamma_{\mathrm{ii}}\right)$ & Aug-06 - Oct-06 & YOY & Control & Headwater & 0.020 & 0.012 & -0.004 & 0.044 \\
\hline Glady Fork & Survival $\left(\gamma_{\mathrm{ii}}\right)$ & Oct-06 - May-07 & YOY & Control & Headwater & 0.027 & 0.016 & -0.005 & 0.059 \\
\hline Glady Fork & Survival $\left(\gamma_{i i}\right)$ & May-07 - Jul-07 & YOY & Control & Headwater & 0.049 & 0.029 & -0.008 & 0.105 \\
\hline
\end{tabular}




\begin{tabular}{|c|c|c|c|c|c|c|c|c|c|}
\hline Site & Variable & Interval & Age & Removal & Location & Est & SE & LCL & UCL \\
\hline Glady Fork & Survival $\left(\gamma_{i i}\right)$ & Jul-07 - Oct-07 & YOY & Control & Headwater & 0.052 & 0.030 & -0.007 & 0.110 \\
\hline Daniel's Run & Survival $\left(\gamma_{i i}\right)$ & Jul-06 - Aug-06 & YOY & Treatment & Headwater & 0.000 & 0.000 & 0.000 & 0.001 \\
\hline Daniel's Run & Survival $\left(\gamma_{\mathrm{ii}}\right)$ & Aug-06 - Oct-06 & YOY & Treatment & Headwater & 0.022 & 0.016 & -0.009 & 0.053 \\
\hline Daniel's Run & Survival $\left(\gamma_{\mathrm{ii}}\right)$ & Oct-06 - May-07 & YOY & Treatment & Headwater & 0.029 & 0.021 & -0.011 & 0.069 \\
\hline Daniel's Run & Survival $\left(\gamma_{\mathrm{ii}}\right)$ & May-07 - Jul-07 & YOY & Treatment & Headwater & 0.026 & 0.018 & -0.011 & 0.062 \\
\hline Daniel's Run & Survival $\left(\gamma_{\mathrm{ii}}\right)$ & Jul-07 - Oct-07 & YOY & Treatment & Headwater & 0.046 & 0.032 & -0.017 & 0.109 \\
\hline Nan's Branch & Survival $\left(\gamma_{i i}\right)$ & Jul-06 - Aug-06 & Small Adult & Control & Mainstem & 0.152 & 0.025 & 0.102 & 0.202 \\
\hline Nan's Branch & Survival $\left(\gamma_{\mathrm{ii}}\right)$ & Aug-06 - Oct-06 & Small Adult & Control & Mainstem & 0.119 & 0.018 & 0.085 & 0.154 \\
\hline Nan's Branch & Survival $\left(\gamma_{i i}\right)$ & Oct-06 - May-07 & Small Adult & Control & Mainstem & 0.157 & 0.020 & 0.117 & 0.197 \\
\hline Nan's Branch & Survival $\left(\gamma_{\mathrm{ii}}\right)$ & May-07 - Jul-07 & Small Adult & Control & Mainstem & 0.254 & 0.031 & 0.193 & 0.314 \\
\hline Nan's Branch & Survival $\left(\gamma_{i i}\right)$ & Jul-07 - Oct-07 & Small Adult & Control & Mainstem & 0.266 & 0.032 & 0.203 & 0.328 \\
\hline Otis Hollow & Survival $\left(\gamma_{i i}\right)$ & Jul-06 - Aug-06 & Small Adult & Treatment & Mainstem & 0.001 & 0.001 & 0.000 & 0.003 \\
\hline Otis Hollow & Survival $\left(\gamma_{i i}\right)$ & Aug-06 - Oct-06 & Small Adult & Treatment & Mainstem & 0.073 & 0.022 & 0.031 & 0.116 \\
\hline Otis Hollow & Survival $\left(\gamma_{\mathrm{ii}}\right)$ & Oct-06 - May-07 & Small Adult & Treatment & Mainstem & 0.096 & 0.028 & 0.041 & 0.151 \\
\hline Otis Hollow & Survival $\left(\gamma_{\mathrm{ii}}\right)$ & May-07 - Jul-07 & Small Adult & Treatment & Mainstem & 0.085 & 0.024 & 0.038 & 0.133 \\
\hline Otis Hollow & Survival $\left(\gamma_{\mathrm{ii}}\right)$ & Jul-07 - Oct-07 & Small Adult & Treatment & Mainstem & 0.147 & 0.039 & 0.070 & 0.223 \\
\hline Big Run & Survival $\left(\gamma_{\mathrm{ii}}\right)$ & Jul-06 - Aug-06 & Small Adult & Control & Headwater & 0.106 & 0.021 & 0.064 & 0.147 \\
\hline Big Run & Survival $\left(\gamma_{i i}\right)$ & Aug-06 - Oct-06 & Small Adult & Control & Headwater & 0.082 & 0.015 & 0.053 & 0.112 \\
\hline Big Run & Survival $\left(\gamma_{\mathrm{ii}}\right)$ & Oct-06 - May-07 & Small Adult & Control & Headwater & 0.109 & 0.018 & 0.075 & 0.144 \\
\hline Big Run & Survival $\left(\gamma_{\mathrm{ii}}\right)$ & May-07 - Jul-07 & Small Adult & Control & Headwater & 0.183 & 0.027 & 0.131 & 0.236 \\
\hline Big Run & Survival $\left(\gamma_{\mathrm{ii}}\right)$ & Jul-07 - Oct-07 & Small Adult & Control & Headwater & 0.193 & 0.028 & 0.137 & 0.248 \\
\hline Swallow Rock & Survival $\left(\gamma_{\mathrm{ii}}\right)$ & Jul-06 - Aug-06 & Small Adult & Treatment & Headwater & 0.003 & 0.001 & 0.000 & 0.005 \\
\hline Swallow Rock & Survival $\left(\gamma_{\mathrm{ii}}\right)$ & Aug-06 - Oct-06 & Small Adult & Treatment & Headwater & 0.145 & 0.033 & 0.080 & 0.209 \\
\hline Swallow Rock & Survival $\left(\gamma_{i i}\right)$ & Oct-06 - May-07 & Small Adult & Treatment & Headwater & 0.185 & 0.036 & 0.115 & 0.256 \\
\hline Swallow Rock & Survival $\left(\gamma_{i i}\right)$ & May-07 - Jul-07 & Small Adult & Treatment & Headwater & 0.166 & 0.029 & 0.110 & 0.223 \\
\hline Swallow Rock & Survival $\left(\gamma_{\mathrm{ii}}\right)$ & Jul-07 - Oct-07 & Small Adult & Treatment & Headwater & 0.269 & 0.049 & 0.173 & 0.364 \\
\hline Lynn Run & Survival $\left(\gamma_{\mathrm{ii}}\right)$ & Jul-06 - Aug-06 & Small Adult & Control & Mainstem & 0.262 & 0.038 & 0.188 & 0.337 \\
\hline Lynn Run & Survival $\left(\gamma_{\mathrm{ii}}\right)$ & Aug-06 - Oct-06 & Small Adult & Control & Mainstem & 0.212 & 0.030 & 0.154 & 0.270 \\
\hline Lynn Run & Survival $\left(\gamma_{i i}\right)$ & Oct-06 - May-07 & Small Adult & Control & Mainstem & 0.269 & 0.032 & 0.207 & 0.331 \\
\hline
\end{tabular}




\begin{tabular}{|c|c|c|c|c|c|c|c|c|c|}
\hline Site & Variable & Interval & Age & Removal & Location & Est & SE & LCL & UCL \\
\hline Lynn Run & Survival $\left(\gamma_{i i}\right)$ & May-07 - Jul-07 & Small Adult & Control & Mainstem & 0.403 & 0.039 & 0.326 & 0.479 \\
\hline Lynn Run & Survival $\left(\gamma_{\mathrm{ii}}\right)$ & Jul-07 - Oct-07 & Small Adult & Control & Mainstem & 0.418 & 0.043 & 0.332 & 0.503 \\
\hline Lick Drain & Survival $\left(\gamma_{\mathrm{ii}}\right)$ & Jul-06 - Aug-06 & Small Adult & Treatment & Mainstem & 0.002 & 0.001 & 0.000 & 0.003 \\
\hline Lick Drain & Survival $\left(\gamma_{i i}\right)$ & Aug-06 - Oct-06 & Small Adult & Treatment & Mainstem & 0.089 & 0.018 & 0.053 & 0.124 \\
\hline Lick Drain & Survival $\left(\gamma_{\mathrm{ii}}\right)$ & Oct-06 - May-07 & Small Adult & Treatment & Mainstem & 0.116 & 0.021 & 0.074 & 0.158 \\
\hline Lick Drain & Survival $\left(\gamma_{i i}\right)$ & May-07 - Jul-07 & Small Adult & Treatment & Mainstem & 0.103 & 0.017 & 0.071 & 0.136 \\
\hline Lick Drain & Survival $\left(\gamma_{\mathrm{ii}}\right)$ & Jul-07 - Oct-07 & Small Adult & Treatment & Mainstem & 0.175 & 0.026 & 0.123 & 0.227 \\
\hline Mudlick Run & Survival $\left(\gamma_{\mathrm{ii}}\right)$ & Jul-06 - Aug-06 & Small Adult & Control & Headwater & 0.225 & 0.033 & 0.160 & 0.291 \\
\hline Mudlick Run & Survival $\left(\gamma_{\mathrm{ii}}\right)$ & Aug-06 - Oct-06 & Small Adult & Control & Headwater & 0.180 & 0.025 & 0.131 & 0.230 \\
\hline Mudlick Run & Survival $\left(\gamma_{\mathrm{ii}}\right)$ & Oct-06 - May-07 & Small Adult & Control & Headwater & 0.232 & 0.028 & 0.178 & 0.286 \\
\hline Mudlick Run & Survival $\left(\gamma_{i i}\right)$ & May-07 - Jul-07 & Small Adult & Control & Headwater & 0.356 & 0.037 & 0.283 & 0.428 \\
\hline Mudlick Run & Survival $\left(\gamma_{\mathrm{ii}}\right)$ & Jul-07 - Oct-07 & Small Adult & Control & Headwater & 0.370 & 0.037 & 0.298 & 0.442 \\
\hline Tanner Run & Survival $\left(\gamma_{\mathrm{ii}}\right)$ & Jul-06 - Aug-06 & Small Adult & Treatment & Headwater & 0.003 & 0.001 & 0.000 & 0.006 \\
\hline Tanner Run & Survival $\left(\gamma_{i i}\right)$ & Aug-06 - Oct-06 & Small Adult & Treatment & Headwater & 0.154 & 0.027 & 0.101 & 0.207 \\
\hline Tanner Run & Survival $\left(\gamma_{i i}\right)$ & Oct-06 - May-07 & Small Adult & Treatment & Headwater & 0.197 & 0.030 & 0.137 & 0.256 \\
\hline Tanner Run & Survival $\left(\gamma_{\mathrm{ii}}\right)$ & May-07 - Jul-07 & Small Adult & Treatment & Headwater & 0.177 & 0.025 & 0.129 & 0.225 \\
\hline Tanner Run & Survival $\left(\gamma_{i i}\right)$ & Jul-07 - Oct-07 & Small Adult & Treatment & Headwater & 0.283 & 0.036 & 0.213 & 0.354 \\
\hline Zinn Hollow & Survival $\left(\gamma_{\mathrm{ii}}\right)$ & Jul-06 - Aug-06 & Small Adult & Control & Mainstem & 0.114 & 0.025 & 0.065 & 0.163 \\
\hline Zinn Hollow & Survival $\left(\gamma_{\mathrm{ii}}\right)$ & Aug-06 - Oct-06 & Small Adult & Control & Mainstem & 0.089 & 0.018 & 0.053 & 0.124 \\
\hline Zinn Hollow & Survival $\left(\gamma_{\mathrm{ii}}\right)$ & Oct-06 - May-07 & Small Adult & Control & Mainstem & 0.117 & 0.022 & 0.075 & 0.160 \\
\hline Zinn Hollow & Survival $\left(\gamma_{\mathrm{ii}}\right)$ & May-07 - Jul-07 & Small Adult & Control & Mainstem & 0.196 & 0.034 & 0.130 & 0.262 \\
\hline Zinn Hollow & Survival $\left(\gamma_{\mathrm{ii}}\right)$ & Jul-07 - Oct-07 & Small Adult & Control & Mainstem & 0.206 & 0.034 & 0.139 & 0.273 \\
\hline Laurel Lick Run & Survival $\left(\gamma_{\mathrm{ii}}\right)$ & Jul-06 - Aug-06 & Small Adult & Treatment & Mainstem & 0.003 & 0.002 & 0.000 & 0.006 \\
\hline Laurel Lick Run & Survival $\left(\gamma_{\mathrm{ii}}\right)$ & Aug-06 - Oct-06 & Small Adult & Treatment & Mainstem & 0.162 & 0.032 & 0.100 & 0.224 \\
\hline Laurel Lick Run & Survival $\left(\gamma_{\mathrm{ii}}\right)$ & Oct-06 - May-07 & Small Adult & Treatment & Mainstem & 0.206 & 0.037 & 0.133 & 0.279 \\
\hline Laurel Lick Run & Survival $\left(\gamma_{\mathrm{ii}}\right)$ & May-07 - Jul-07 & Small Adult & Treatment & Mainstem & 0.186 & 0.031 & 0.125 & 0.247 \\
\hline Laurel Lick Run & Survival $\left(\gamma_{\mathrm{ii}}\right)$ & Jul-07 - Oct-07 & Small Adult & Treatment & Mainstem & 0.296 & 0.042 & 0.213 & 0.379 \\
\hline Glady Fork & Survival $\left(\gamma_{i i}\right)$ & Jul-06 - Aug-06 & Small Adult & Control & Headwater & 0.292 & 0.047 & 0.200 & 0.384 \\
\hline Glady Fork & Survival $\left(\gamma_{\mathrm{ii}}\right)$ & Aug-06 - Oct-06 & Small Adult & Control & Headwater & 0.238 & 0.040 & 0.159 & 0.316 \\
\hline
\end{tabular}




\begin{tabular}{|c|c|c|c|c|c|c|c|c|c|}
\hline Site & Variable & Interval & Age & Removal & Location & Est & SE & LCL & UCL \\
\hline Glady Fork & Survival $\left(\gamma_{i i}\right)$ & Oct-06 - May-07 & Small Adult & Control & Headwater & 0.299 & 0.044 & 0.212 & 0.386 \\
\hline Glady Fork & Survival $\left(\gamma_{i i}\right)$ & May-07 - Jul-07 & Small Adult & Control & Headwater & 0.439 & 0.053 & 0.335 & 0.542 \\
\hline Glady Fork & Survival $\left(\gamma_{\mathrm{ii}}\right)$ & Jul-07 - Oct-07 & Small Adult & Control & Headwater & 0.454 & 0.055 & 0.347 & 0.561 \\
\hline Daniel's Run & Survival $\left(\gamma_{\mathrm{ii}}\right)$ & Jul-06 - Aug-06 & Small Adult & Treatment & Headwater & 0.007 & 0.003 & 0.000 & 0.014 \\
\hline Daniel's Run & Survival $\left(\gamma_{\mathrm{ii}}\right)$ & Aug-06 - Oct-06 & Small Adult & Treatment & Headwater & 0.309 & 0.048 & 0.215 & 0.403 \\
\hline Daniel's Run & Survival $\left(\gamma_{i i}\right)$ & Oct-06 - May-07 & Small Adult & Treatment & Headwater & 0.375 & 0.046 & 0.285 & 0.465 \\
\hline Daniel's Run & Survival $\left(\gamma_{i i}\right)$ & May-07 - Jul-07 & Small Adult & Treatment & Headwater & 0.345 & 0.038 & 0.270 & 0.420 \\
\hline Daniel's Run & Survival $\left(\gamma_{\mathrm{ii}}\right)$ & Jul-07 - Oct-07 & Small Adult & Treatment & Headwater & 0.492 & 0.050 & 0.395 & 0.590 \\
\hline Nan's Branch & Survival $\left(\gamma_{i i}\right)$ & Jul-06 - Aug-06 & Large Adult & Control & Mainstem & 0.355 & 0.040 & 0.277 & 0.433 \\
\hline Nan's Branch & Survival $\left(\gamma_{\mathrm{ii}}\right)$ & Aug-06 - Oct-06 & Large Adult & Control & Mainstem & 0.294 & 0.028 & 0.238 & 0.349 \\
\hline Nan's Branch & Survival $\left(\gamma_{\mathrm{ii}}\right)$ & Oct-06 - May-07 & Large Adult & Control & Mainstem & 0.363 & 0.031 & 0.302 & 0.424 \\
\hline Nan's Branch & Survival $\left(\gamma_{i i}\right)$ & May-07 - Jul-07 & Large Adult & Control & Mainstem & 0.510 & 0.038 & 0.435 & 0.586 \\
\hline Nan's Branch & Survival $\left(\gamma_{i i}\right)$ & Jul-07 - Oct-07 & Large Adult & Control & Mainstem & 0.526 & 0.038 & 0.451 & 0.600 \\
\hline Otis Hollow & Survival $\left(\gamma_{\mathrm{ii}}\right)$ & Jul-06 - Aug-06 & Large Adult & Treatment & Mainstem & 0.005 & 0.003 & -0.001 & 0.011 \\
\hline Otis Hollow & Survival $\left(\gamma_{\mathrm{ii}}\right)$ & Aug-06 - Oct-06 & Large Adult & Treatment & Mainstem & 0.248 & 0.055 & 0.140 & 0.356 \\
\hline Otis Hollow & Survival $\left(\gamma_{\mathrm{ii}}\right)$ & Oct-06 - May-07 & Large Adult & Treatment & Mainstem & 0.307 & 0.065 & 0.179 & 0.436 \\
\hline Otis Hollow & Survival $\left(\gamma_{\mathrm{ii}}\right)$ & May-07 - Jul-07 & Large Adult & Treatment & Mainstem & 0.280 & 0.060 & 0.163 & 0.397 \\
\hline Otis Hollow & Survival $\left(\gamma_{i i}\right)$ & Jul-07 - Oct-07 & Large Adult & Treatment & Mainstem & 0.417 & 0.073 & 0.275 & 0.560 \\
\hline Big Run & Survival $\left(\gamma_{\mathrm{ii}}\right)$ & Jul-06 - Aug-06 & Large Adult & Control & Headwater & 0.266 & 0.039 & 0.190 & 0.342 \\
\hline Big Run & Survival $\left(\gamma_{\mathrm{ii}}\right)$ & Aug-06 - Oct-06 & Large Adult & Control & Headwater & 0.216 & 0.028 & 0.160 & 0.271 \\
\hline Big Run & Survival $\left(\gamma_{\mathrm{ii}}\right)$ & Oct-06 - May-07 & Large Adult & Control & Headwater & 0.273 & 0.031 & 0.212 & 0.335 \\
\hline Big Run & Survival $\left(\gamma_{\mathrm{ii}}\right)$ & May-07 - Jul-07 & Large Adult & Control & Headwater & 0.408 & 0.040 & 0.329 & 0.487 \\
\hline Big Run & Survival $\left(\gamma_{\mathrm{ii}}\right)$ & Jul-07 - Oct-07 & Large Adult & Control & Headwater & 0.423 & 0.040 & 0.343 & 0.502 \\
\hline Swallow Rock & Survival $\left(\gamma_{i i}\right)$ & Jul-06 - Aug-06 & Large Adult & Treatment & Headwater & 0.011 & 0.006 & 0.000 & 0.022 \\
\hline Swallow Rock & Survival $\left(\gamma_{i i}\right)$ & Aug-06 - Oct-06 & Large Adult & Treatment & Headwater & 0.413 & 0.059 & 0.298 & 0.529 \\
\hline Swallow Rock & Survival $\left(\gamma_{\mathrm{ii}}\right)$ & Oct-06 - May-07 & Large Adult & Treatment & Headwater & 0.487 & 0.056 & 0.377 & 0.596 \\
\hline Swallow Rock & Survival $\left(\gamma_{\mathrm{ii}}\right)$ & May-07 - Jul-07 & Large Adult & Treatment & Headwater & 0.454 & 0.049 & 0.358 & 0.551 \\
\hline Swallow Rock & Survival $\left(\gamma_{\mathrm{ii}}\right)$ & Jul-07 - Oct-07 & Large Adult & Treatment & Headwater & 0.605 & 0.057 & 0.493 & 0.717 \\
\hline Lynn Run & Survival $\left(\gamma_{i i}\right)$ & Jul-06 - Aug-06 & Large Adult & Control & Mainstem & 0.521 & 0.044 & 0.436 & 0.607 \\
\hline
\end{tabular}




\begin{tabular}{|c|c|c|c|c|c|c|c|c|c|}
\hline Site & Variable & Interval & Age & Removal & Location & Est & SE & LCL & UCL \\
\hline Lynn Run & Survival $\left(\gamma_{i i}\right)$ & Aug-06 - Oct-06 & Large Adult & Control & Mainstem & 0.452 & 0.038 & 0.378 & 0.526 \\
\hline Lynn Run & Survival $\left(\gamma_{\mathrm{ii}}\right)$ & Oct-06 - May-07 & Large Adult & Control & Mainstem & 0.530 & 0.036 & 0.459 & 0.601 \\
\hline Lynn Run & Survival $\left(\gamma_{\mathrm{ii}}\right)$ & May-07 - Jul-07 & Large Adult & Control & Mainstem & 0.674 & 0.035 & 0.606 & 0.742 \\
\hline Lynn Run & Survival $\left(\gamma_{i i}\right)$ & Jul-07 - Oct-07 & Large Adult & Control & Mainstem & 0.687 & 0.037 & 0.615 & 0.759 \\
\hline Lick Drain & Survival $\left(\gamma_{i i}\right)$ & Jul-06 - Aug-06 & Large Adult & Treatment & Mainstem & 0.006 & 0.003 & 0.000 & 0.013 \\
\hline Lick Drain & Survival $\left(\gamma_{i i}\right)$ & Aug-06 - Oct-06 & Large Adult & Treatment & Mainstem & 0.289 & 0.040 & 0.211 & 0.367 \\
\hline Lick Drain & Survival $\left(\gamma_{\mathrm{ii}}\right)$ & Oct-06 - May-07 & Large Adult & Treatment & Mainstem & 0.353 & 0.043 & 0.268 & 0.439 \\
\hline Lick Drain & Survival $\left(\gamma_{\mathrm{ii}}\right)$ & May-07 - Jul-07 & Large Adult & Treatment & Mainstem & 0.324 & 0.037 & 0.253 & 0.396 \\
\hline Lick Drain & Survival $\left(\gamma_{\mathrm{ii}}\right)$ & Jul-07 - Oct-07 & Large Adult & Treatment & Mainstem & 0.469 & 0.042 & 0.387 & 0.551 \\
\hline Mudlick Run & Survival $\left(\gamma_{\mathrm{ii}}\right)$ & Jul-06 - Aug-06 & Large Adult & Control & Headwater & 0.471 & 0.043 & 0.386 & 0.557 \\
\hline Mudlick Run & Survival $\left(\gamma_{i i}\right)$ & Aug-06 - Oct-06 & Large Adult & Control & Headwater & 0.403 & 0.035 & 0.334 & 0.472 \\
\hline Mudlick Run & Survival $\left(\gamma_{\mathrm{ii}}\right)$ & Oct-06 - May-07 & Large Adult & Control & Headwater & 0.480 & 0.036 & 0.410 & 0.551 \\
\hline Mudlick Run & Survival $\left(\gamma_{\mathrm{ii}}\right)$ & May-07 - Jul-07 & Large Adult & Control & Headwater & 0.628 & 0.038 & 0.555 & 0.702 \\
\hline Mudlick Run & Survival $\left(\gamma_{i i}\right)$ & Jul-07 - Oct-07 & Large Adult & Control & Headwater & 0.643 & 0.035 & 0.573 & 0.712 \\
\hline Tanner Run & Survival $\left(\gamma_{i i}\right)$ & Jul-06 - Aug-06 & Large Adult & Treatment & Headwater & 0.012 & 0.006 & 0.000 & 0.023 \\
\hline Tanner Run & Survival $\left(\gamma_{\mathrm{ii}}\right)$ & Aug-06 - Oct-06 & Large Adult & Treatment & Headwater & 0.431 & 0.043 & 0.346 & 0.517 \\
\hline Tanner Run & Survival $\left(\gamma_{i i}\right)$ & Oct-06 - May-07 & Large Adult & Treatment & Headwater & 0.505 & 0.044 & 0.420 & 0.591 \\
\hline Tanner Run & Survival $\left(\gamma_{\mathrm{ii}}\right)$ & May-07 - Jul-07 & Large Adult & Treatment & Headwater & 0.473 & 0.039 & 0.396 & 0.550 \\
\hline Tanner Run & Survival $\left(\gamma_{\mathrm{ii}}\right)$ & Jul-07 - Oct-07 & Large Adult & Treatment & Headwater & 0.623 & 0.039 & 0.547 & 0.698 \\
\hline Zinn Hollow & Survival $\left(\gamma_{i i}\right)$ & Jul-06 - Aug-06 & Large Adult & Control & Mainstem & 0.282 & 0.045 & 0.194 & 0.371 \\
\hline Zinn Hollow & Survival $\left(\gamma_{\mathrm{ii}}\right)$ & Aug-06 - Oct-06 & Large Adult & Control & Mainstem & 0.230 & 0.034 & 0.162 & 0.297 \\
\hline Zinn Hollow & Survival $\left(\gamma_{i i}\right)$ & Oct-06 - May-07 & Large Adult & Control & Mainstem & 0.290 & 0.039 & 0.214 & 0.365 \\
\hline Zinn Hollow & Survival $\left(\gamma_{\mathrm{ii}}\right)$ & May-07 - Jul-07 & Large Adult & Control & Mainstem & 0.427 & 0.049 & 0.331 & 0.524 \\
\hline Zinn Hollow & Survival $\left(\gamma_{\mathrm{ii}}\right)$ & Jul-07 - Oct-07 & Large Adult & Control & Mainstem & 0.443 & 0.048 & 0.349 & 0.536 \\
\hline Laurel Lick Run & Survival $\left(\gamma_{i i}\right)$ & Jul-06 - Aug-06 & Large Adult & Treatment & Mainstem & 0.013 & 0.006 & 0.000 & 0.025 \\
\hline Laurel Lick Run & Survival $\left(\gamma_{\mathrm{ii}}\right)$ & Aug-06 - Oct-06 & Large Adult & Treatment & Mainstem & 0.446 & 0.050 & 0.349 & 0.544 \\
\hline Laurel Lick Run & Survival $\left(\gamma_{i i}\right)$ & Oct-06 - May-07 & Large Adult & Treatment & Mainstem & 0.520 & 0.052 & 0.419 & 0.622 \\
\hline Laurel Lick Run & Survival $\left(\gamma_{i i}\right)$ & May-07 - Jul-07 & Large Adult & Treatment & Mainstem & 0.488 & 0.048 & 0.393 & 0.583 \\
\hline Laurel Lick Run & Survival $\left(\gamma_{\mathrm{ii}}\right)$ & Jul-07 - Oct-07 & Large Adult & Treatment & Mainstem & 0.637 & 0.043 & 0.552 & 0.721 \\
\hline
\end{tabular}




\begin{tabular}{|c|c|c|c|c|c|c|c|c|c|}
\hline Site & Variable & Interval & Age & Removal & Location & Est & SE & LCL & UCL \\
\hline Glady Fork & Survival $\left(\gamma_{\mathrm{ii}}\right)$ & Jul-06 - Aug-06 & Large Adult & Control & Headwater & 0.558 & 0.050 & 0.460 & 0.656 \\
\hline Glady Fork & Survival $\left(\gamma_{\mathrm{ii}}\right)$ & Aug-06 - Oct-06 & Large Adult & Control & Headwater & 0.489 & 0.049 & 0.393 & 0.584 \\
\hline Glady Fork & Survival $\left(\gamma_{\mathrm{ii}}\right)$ & Oct-06 - May-07 & Large Adult & Control & Headwater & 0.567 & 0.048 & 0.473 & 0.661 \\
\hline Glady Fork & Survival $\left(\gamma_{\mathrm{ii}}\right)$ & May-07 - Jul-07 & Large Adult & Control & Headwater & 0.705 & 0.043 & 0.622 & 0.789 \\
\hline Glady Fork & Survival $\left(\gamma_{\mathrm{ii}}\right)$ & Jul-07 - Oct-07 & Large Adult & Control & Headwater & 0.718 & 0.042 & 0.635 & 0.801 \\
\hline Daniel's Run & Survival $\left(\gamma_{\mathrm{ii}}\right)$ & Jul-06 - Aug-06 & Large Adult & Treatment & Headwater & 0.029 & 0.013 & 0.002 & 0.055 \\
\hline Daniel's Run & Survival $\left(\gamma_{\mathrm{ii}}\right)$ & Aug-06 - Oct-06 & Large Adult & Treatment & Headwater & 0.651 & 0.047 & 0.558 & 0.743 \\
\hline Daniel's Run & Survival $\left(\gamma_{\mathrm{ii}}\right)$ & Oct-06 - May-07 & Large Adult & Treatment & Headwater & 0.715 & 0.038 & 0.639 & 0.790 \\
\hline Daniel's Run & Survival $\left(\gamma_{\mathrm{ii}}\right)$ & May-07 - Jul-07 & Large Adult & Treatment & Headwater & 0.688 & 0.036 & 0.616 & 0.759 \\
\hline Daniel's Run & Survival $\left(\gamma_{\mathrm{ii}}\right)$ & Jul-07 - Oct-07 & Large Adult & Treatment & Headwater & 0.802 & 0.031 & 0.740 & 0.863 \\
\hline Nan's Branch & Recruitment $\left(\gamma_{\mathrm{ji}}\right)$ & Jul-06 - Aug-06 & YOY - Small Adult & Control & Mainstem & 0.001 & 0.001 & 0.000 & 0.002 \\
\hline Nan's Branch & Recruitment $\left(\gamma_{\mathrm{ji}}\right)$ & Aug-06 - Oct-06 & YOY - Small Adult & Control & Mainstem & 0.018 & 0.006 & 0.006 & 0.030 \\
\hline Nan's Branch & Recruitment $\left(\gamma_{\mathrm{ji}}\right)$ & Oct-06 - May-07 & YOY - Small Adult & Control & Mainstem & 0.031 & 0.010 & 0.011 & 0.050 \\
\hline Nan's Branch & Recruitment $\left(\gamma_{\mathrm{ji}}\right)$ & May-07 - Jul-07 & YOY - Small Adult & Control & Mainstem & 0.014 & 0.005 & 0.004 & 0.023 \\
\hline Nan's Branch & Recruitment $\left(\gamma_{\mathrm{ji}}\right)$ & Jul-07 - Oct-07 & YOY - Small Adult & Control & Mainstem & 0.006 & 0.003 & 0.001 & 0.011 \\
\hline Otis Hollow & Recruitment $\left(\gamma_{\mathrm{ji}}\right)$ & Jul-06 - Aug-06 & YOY - Small Adult & Treatment & Mainstem & 0.001 & 0.001 & 0.000 & 0.002 \\
\hline Otis Hollow & Recruitment $\left(\gamma_{\mathrm{ji}}\right)$ & Aug-06 - Oct-06 & YOY - Small Adult & Treatment & Mainstem & 0.012 & 0.007 & -0.002 & 0.027 \\
\hline Otis Hollow & Recruitment $\left(\gamma_{\mathrm{ji}}\right)$ & Oct-06 - May-07 & YOY - Small Adult & Treatment & Mainstem & 0.021 & 0.012 & -0.003 & 0.046 \\
\hline Otis Hollow & Recruitment $\left(\gamma_{\mathrm{ji}}\right)$ & May-07 - Jul-07 & YOY - Small Adult & Treatment & Mainstem & 0.009 & 0.006 & -0.002 & 0.021 \\
\hline Otis Hollow & Recruitment $\left(\gamma_{\mathrm{ji}}\right)$ & Jul-07 - Oct-07 & YOY - Small Adult & Treatment & Mainstem & 0.004 & 0.003 & -0.001 & 0.009 \\
\hline Big Run & Recruitment $\left(\gamma_{\mathrm{ji}}\right)$ & Jul-06 - Aug-06 & YOY - Small Adult & Control & Headwater & 0.001 & 0.001 & 0.000 & 0.003 \\
\hline Big Run & Recruitment $\left(\gamma_{\mathrm{ji}}\right)$ & Aug-06 - Oct-06 & YOY - Small Adult & Control & Headwater & 0.020 & 0.007 & 0.006 & 0.033 \\
\hline Big Run & Recruitment $\left(\gamma_{\mathrm{ji}}\right)$ & Oct-06 - May-07 & YOY - Small Adult & Control & Headwater & 0.034 & 0.011 & 0.012 & 0.055 \\
\hline Big Run & Recruitment $\left(\gamma_{\mathrm{ji}}\right)$ & May-07 - Jul-07 & YOY - Small Adult & Control & Headwater & 0.015 & 0.005 & 0.005 & 0.025 \\
\hline Big Run & Recruitment $\left(\gamma_{\mathrm{ji}}\right)$ & Jul-07 - Oct-07 & YOY - Small Adult & Control & Headwater & 0.006 & 0.003 & 0.001 & 0.012 \\
\hline Swallow Rock & Recruitment $\left(\gamma_{\mathrm{ji}}\right)$ & Jul-06 - Aug-06 & YOY - Small Adult & Treatment & Headwater & 0.001 & 0.001 & 0.000 & 0.003 \\
\hline Swallow Rock & Recruitment $\left(\gamma_{\mathrm{ji}}\right)$ & Aug-06 - Oct-06 & YOY - Small Adult & Treatment & Headwater & 0.025 & 0.009 & 0.009 & 0.042 \\
\hline Swallow Rock & Recruitment $\left(\gamma_{\mathrm{ji}}\right)$ & Oct-06 - May-07 & YOY - Small Adult & Treatment & Headwater & 0.043 & 0.013 & 0.018 & 0.069 \\
\hline Swallow Rock & Recruitment $\left(\gamma_{\mathrm{ji}}\right)$ & May-07 - Jul-07 & YOY - Small Adult & Treatment & Headwater & 0.019 & 0.006 & 0.007 & 0.031 \\
\hline
\end{tabular}




\begin{tabular}{|c|c|c|c|c|c|c|c|c|c|}
\hline Site & Variable & Interval & Age & Removal & Location & Est & SE & LCL & UCL \\
\hline Swallow Rock & Recruitment $\left(\gamma_{\mathrm{ji}}\right)$ & Jul-07 - Oct-07 & YOY - Small Adult & Treatment & Headwater & 0.008 & 0.004 & 0.001 & 0.015 \\
\hline Lynn Run & Recruitment $\left(\gamma_{\mathrm{ji}}\right)$ & Jul-06 - Aug-06 & YOY - Small Adult & Control & Mainstem & 0.003 & 0.002 & -0.001 & 0.006 \\
\hline Lynn Run & Recruitment $\left(\gamma_{\mathrm{ji}}\right)$ & Aug-06 - Oct-06 & YOY - Small Adult & Control & Mainstem & 0.046 & 0.015 & 0.018 & 0.075 \\
\hline Lynn Run & Recruitment $\left(\gamma_{\mathrm{ji}}\right)$ & Oct-06 - May-07 & YOY - Small Adult & Control & Mainstem & 0.078 & 0.021 & 0.036 & 0.119 \\
\hline Lynn Run & Recruitment $\left(\gamma_{\mathrm{ji}}\right)$ & May-07 - Jul-07 & YOY - Small Adult & Control & Mainstem & 0.035 & 0.011 & 0.014 & 0.056 \\
\hline Lynn Run & Recruitment $\left(\gamma_{\mathrm{ji}}\right)$ & Jul-07 - Oct-07 & YOY - Small Adult & Control & Mainstem & 0.015 & 0.006 & 0.003 & 0.027 \\
\hline Lick Drain & Recruitment $\left(\gamma_{\mathrm{ji}}\right)$ & Jul-06 - Aug-06 & YOY - Small Adult & Treatment & Mainstem & 0.001 & 0.001 & 0.000 & 0.003 \\
\hline Lick Drain & Recruitment $\left(\gamma_{\mathrm{ji}}\right)$ & Aug-06 - Oct-06 & YOY - Small Adult & Treatment & Mainstem & 0.020 & 0.008 & 0.004 & 0.035 \\
\hline Lick Drain & Recruitment $\left(\gamma_{\mathrm{ji}}\right)$ & Oct-06 - May-07 & YOY - Small Adult & Treatment & Mainstem & 0.034 & 0.013 & 0.009 & 0.058 \\
\hline Lick Drain & Recruitment $\left(\gamma_{\mathrm{ji}}\right)$ & May-07 - Jul-07 & YOY - Small Adult & Treatment & Mainstem & 0.015 & 0.006 & 0.004 & 0.026 \\
\hline Lick Drain & Recruitment $\left(\gamma_{\mathrm{ji}}\right)$ & Jul-07 - Oct-07 & YOY - Small Adult & Treatment & Mainstem & 0.006 & 0.003 & 0.001 & 0.012 \\
\hline Mudlick Run & Recruitment $\left(\gamma_{\mathrm{ji}}\right)$ & Jul-06 - Aug-06 & YOY - Small Adult & Control & Headwater & 0.004 & 0.002 & -0.001 & 0.008 \\
\hline Mudlick Run & Recruitment $\left(\gamma_{\mathrm{ji}}\right)$ & Aug-06 - Oct-06 & YOY - Small Adult & Control & Headwater & 0.061 & 0.016 & 0.029 & 0.093 \\
\hline Mudlick Run & Recruitment $\left(\gamma_{\mathrm{ji}}\right)$ & Oct-06 - May-07 & YOY - Small Adult & Control & Headwater & 0.102 & 0.023 & 0.056 & 0.148 \\
\hline Mudlick Run & Recruitment $\left(\gamma_{\mathrm{ji}}\right)$ & May-07 - Jul-07 & YOY - Small Adult & Control & Headwater & 0.047 & 0.013 & 0.022 & 0.072 \\
\hline Mudlick Run & Recruitment $\left(\gamma_{\mathrm{ji}}\right)$ & Jul-07 - Oct-07 & YOY - Small Adult & Control & Headwater & 0.020 & 0.007 & 0.005 & 0.035 \\
\hline Tanner Run & Recruitment $\left(\gamma_{\mathrm{ji}}\right)$ & Jul-06 - Aug-06 & YOY - Small Adult & Treatment & Headwater & 0.003 & 0.002 & -0.001 & 0.006 \\
\hline Tanner Run & Recruitment $\left(\gamma_{\mathrm{ji}}\right)$ & Aug-06 - Oct-06 & YOY - Small Adult & Treatment & Headwater & 0.049 & 0.014 & 0.022 & 0.076 \\
\hline Tanner Run & Recruitment $\left(\gamma_{\mathrm{ji}}\right)$ & Oct-06 - May-07 & YOY - Small Adult & Treatment & Headwater & 0.082 & 0.020 & 0.043 & 0.121 \\
\hline Tanner Run & Recruitment $\left(\gamma_{\mathrm{ji}}\right)$ & May-07 - Jul-07 & YOY - Small Adult & Treatment & Headwater & 0.037 & 0.010 & 0.017 & 0.057 \\
\hline Tanner Run & Recruitment $\left(\gamma_{\mathrm{ji}}\right)$ & Jul-07 - Oct-07 & YOY - Small Adult & Treatment & Headwater & 0.016 & 0.006 & 0.004 & 0.028 \\
\hline Zinn Hollow & Recruitment $\left(\gamma_{\mathrm{ji}}\right)$ & Jul-06 - Aug-06 & YOY - Small Adult & Control & Mainstem & 0.001 & 0.001 & 0.000 & 0.002 \\
\hline Zinn Hollow & Recruitment $\left(\gamma_{\mathrm{ji}}\right)$ & Aug-06 - Oct-06 & YOY - Small Adult & Control & Mainstem & 0.015 & 0.008 & -0.001 & 0.031 \\
\hline Zinn Hollow & Recruitment $\left(\gamma_{\mathrm{ji}}\right)$ & Oct-06 - May-07 & YOY - Small Adult & Control & Mainstem & 0.026 & 0.013 & 0.000 & 0.052 \\
\hline Zinn Hollow & Recruitment $\left(\gamma_{\mathrm{ji}}\right)$ & May-07 - Jul-07 & YOY - Small Adult & Control & Mainstem & 0.012 & 0.006 & 0.000 & 0.024 \\
\hline Zinn Hollow & Recruitment $\left(\gamma_{\mathrm{ji}}\right)$ & Jul-07 - Oct-07 & YOY - Small Adult & Control & Mainstem & 0.005 & 0.003 & -0.001 & 0.011 \\
\hline Laurel Lick Run & Recruitment $\left(\gamma_{\mathrm{ji}}\right)$ & Jul-06 - Aug-06 & YOY - Small Adult & Treatment & Mainstem & 0.002 & 0.001 & -0.001 & 0.005 \\
\hline Laurel Lick Run & Recruitment $\left(\gamma_{\mathrm{ji}}\right)$ & Aug-06 - Oct-06 & YOY - Small Adult & Treatment & Mainstem & 0.039 & 0.012 & 0.016 & 0.062 \\
\hline Laurel Lick Run & Recruitment $\left(\gamma_{\mathrm{ji}}\right)$ & Oct-06 - May-07 & YOY - Small Adult & Treatment & Mainstem & 0.065 & 0.017 & 0.032 & 0.099 \\
\hline
\end{tabular}




\begin{tabular}{|c|c|c|c|c|c|c|c|c|c|}
\hline Site & Variable & Interval & Age & Removal & Location & Est & SE & LCL & UCL \\
\hline Laurel Lick Run & Recruitment $\left(\gamma_{\mathrm{ji}}\right)$ & May-07 - Jul-07 & YOY - Small Adult & Treatment & Mainstem & 0.029 & 0.008 & 0.013 & 0.046 \\
\hline Laurel Lick Run & Recruitment $\left(\gamma_{\mathrm{ji}}\right)$ & Jul-07 - Oct-07 & YOY - Small Adult & Treatment & Mainstem & 0.013 & 0.005 & 0.003 & 0.022 \\
\hline Glady Fork & Recruitment $\left(\gamma_{\mathrm{ji}}\right)$ & Jul-06 - Aug-06 & YOY - Small Adult & Control & Headwater & 0.004 & 0.003 & -0.001 & 0.010 \\
\hline Glady Fork & Recruitment $\left(\gamma_{\mathrm{ji}}\right)$ & Aug-06 - Oct-06 & YOY - Small Adult & Control & Headwater & 0.071 & 0.026 & 0.020 & 0.122 \\
\hline Glady Fork & Recruitment $\left(\gamma_{\mathrm{ji}}\right)$ & Oct-06 - May-07 & YOY - Small Adult & Control & Headwater & 0.117 & 0.039 & 0.040 & 0.194 \\
\hline Glady Fork & Recruitment $\left(\gamma_{\mathrm{ji}}\right)$ & May-07 - Jul-07 & YOY - Small Adult & Control & Headwater & 0.054 & 0.020 & 0.015 & 0.094 \\
\hline Glady Fork & Recruitment $\left(\gamma_{\mathrm{ji}}\right)$ & Jul-07 - Oct-07 & YOY - Small Adult & Control & Headwater & 0.023 & 0.011 & 0.002 & 0.045 \\
\hline Daniel's Run & Recruitment $\left(\gamma_{\mathrm{ji}}\right)$ & Jul-06 - Aug-06 & YOY - Small Adult & Treatment & Headwater & 0.007 & 0.004 & -0.001 & 0.016 \\
\hline Daniel's Run & Recruitment $\left(\gamma_{\mathrm{ji}}\right)$ & Aug-06 - Oct-06 & YOY - Small Adult & Treatment & Headwater & 0.115 & 0.030 & 0.055 & 0.174 \\
\hline Daniel's Run & Recruitment $\left(\gamma_{\mathrm{ji}}\right)$ & Oct-06 - May-07 & YOY - Small Adult & Treatment & Headwater & 0.184 & 0.039 & 0.108 & 0.260 \\
\hline Daniel's Run & Recruitment $\left(\gamma_{\mathrm{ji}}\right)$ & May-07 - Jul-07 & YOY - Small Adult & Treatment & Headwater & 0.089 & 0.022 & 0.046 & 0.132 \\
\hline Daniel's Run & Recruitment $\left(\gamma_{\mathrm{ji}}\right)$ & Jul-07 - Oct-07 & YOY - Small Adult & Treatment & Headwater & 0.039 & 0.015 & 0.011 & 0.068 \\
\hline Nan's Branch & Recruitment $\left(\gamma_{\mathrm{ji}}\right)$ & Jul-06 - Aug-06 & Small - Large Adult & Control & Mainstem & 0.002 & 0.001 & 0.000 & 0.004 \\
\hline Nan's Branch & Recruitment $\left(\gamma_{\mathrm{ji}}\right)$ & Aug-06 - Oct-06 & Small - Large Adult & Control & Mainstem & 0.031 & 0.010 & 0.013 & 0.050 \\
\hline Nan's Branch & Recruitment $\left(\gamma_{\mathrm{ji}}\right)$ & Oct-06 - May-07 & Small - Large Adult & Control & Mainstem & 0.053 & 0.015 & 0.024 & 0.083 \\
\hline Nan's Branch & Recruitment $\left(\gamma_{\mathrm{ji}}\right)$ & May-07 - Jul-07 & Small - Large Adult & Control & Mainstem & 0.024 & 0.008 & 0.008 & 0.039 \\
\hline Nan's Branch & Recruitment $\left(\gamma_{\mathrm{ji}}\right)$ & Jul-07 - Oct-07 & Small - Large Adult & Control & Mainstem & 0.010 & 0.004 & 0.002 & 0.018 \\
\hline Otis Hollow & Recruitment $\left(\gamma_{\mathrm{ji}}\right)$ & Jul-06 - Aug-06 & Small - Large Adult & Treatment & Mainstem & 0.001 & 0.001 & -0.001 & 0.003 \\
\hline Otis Hollow & Recruitment $\left(\gamma_{\mathrm{ji}}\right)$ & Aug-06 - Oct-06 & Small - Large Adult & Treatment & Mainstem & 0.022 & 0.012 & -0.002 & 0.046 \\
\hline Otis Hollow & Recruitment $\left(\gamma_{\mathrm{ji}}\right)$ & Oct-06 - May-07 & Small - Large Adult & Treatment & Mainstem & 0.037 & 0.021 & -0.003 & 0.078 \\
\hline Otis Hollow & Recruitment $\left(\gamma_{\mathrm{ji}}\right)$ & May-07 - Jul-07 & Small - Large Adult & Treatment & Mainstem & 0.017 & 0.010 & -0.003 & 0.036 \\
\hline Otis Hollow & Recruitment $\left(\gamma_{\mathrm{ji}}\right)$ & Jul-07 - Oct-07 & Small - Large Adult & Treatment & Mainstem & 0.007 & 0.004 & -0.002 & 0.016 \\
\hline Big Run & Recruitment $\left(\gamma_{\mathrm{ji}}\right)$ & Jul-06 - Aug-06 & Small - Large Adult & Control & Headwater & 0.002 & 0.001 & -0.001 & 0.005 \\
\hline Big Run & Recruitment $\left(\gamma_{\mathrm{ji}}\right)$ & Aug-06 - Oct-06 & Small - Large Adult & Control & Headwater & 0.034 & 0.010 & 0.014 & 0.054 \\
\hline Big Run & Recruitment $\left(\gamma_{\mathrm{ji}}\right)$ & Oct-06 - May-07 & Small - Large Adult & Control & Headwater & 0.058 & 0.016 & 0.027 & 0.089 \\
\hline Big Run & Recruitment $\left(\gamma_{\mathrm{ji}}\right)$ & May-07 - Jul-07 & Small - Large Adult & Control & Headwater & 0.026 & 0.008 & 0.010 & 0.042 \\
\hline Big Run & Recruitment $\left(\gamma_{\mathrm{ji}}\right)$ & Jul-07 - Oct-07 & Small - Large Adult & Control & Headwater & 0.011 & 0.004 & 0.002 & 0.020 \\
\hline Swallow Rock & Recruitment $\left(\gamma_{\mathrm{ji}}\right)$ & Jul-06 - Aug-06 & Small - Large Adult & Treatment & Headwater & 0.003 & 0.002 & -0.001 & 0.006 \\
\hline Swallow Rock & Recruitment $\left(\gamma_{\mathrm{ji}}\right)$ & Aug-06 - Oct-06 & Small - Large Adult & Treatment & Headwater & 0.044 & 0.012 & 0.020 & 0.068 \\
\hline
\end{tabular}




\begin{tabular}{|c|c|c|c|c|c|c|c|c|c|}
\hline Site & Variable & Interval & Age & Removal & Location & Est & SE & LCL & UCL \\
\hline Swallow Rock & Recruitment $\left(\gamma_{\mathrm{ji}}\right)$ & Oct-06 - May-07 & Small - Large Adult & Treatment & Headwater & 0.075 & 0.018 & 0.039 & 0.111 \\
\hline Swallow Rock & Recruitment $\left(\gamma_{\mathrm{ji}}\right)$ & May-07 - Jul-07 & Small - Large Adult & Treatment & Headwater & 0.034 & 0.009 & 0.015 & 0.052 \\
\hline Swallow Rock & Recruitment $\left(\gamma_{\mathrm{ji}}\right)$ & Jul-07 - Oct-07 & Small - Large Adult & Treatment & Headwater & 0.014 & 0.006 & 0.003 & 0.025 \\
\hline Lynn Run & Recruitment $\left(\gamma_{\mathrm{ji}}\right)$ & Jul-06 - Aug-06 & Small - Large Adult & Control & Mainstem & 0.005 & 0.003 & -0.001 & 0.011 \\
\hline Lynn Run & Recruitment $\left(\gamma_{\mathrm{ji}}\right)$ & Aug-06 - Oct-06 & Small - Large Adult & Control & Mainstem & 0.079 & 0.020 & 0.039 & 0.119 \\
\hline Lynn Run & Recruitment $\left(\gamma_{\mathrm{ji}}\right)$ & Oct-06 - May-07 & Small - Large Adult & Control & Mainstem & 0.130 & 0.029 & 0.073 & 0.187 \\
\hline Lynn Run & Recruitment $\left(\gamma_{\mathrm{ji}}\right)$ & May-07 - Jul-07 & Small - Large Adult & Control & Mainstem & 0.061 & 0.017 & 0.028 & 0.094 \\
\hline Lynn Run & Recruitment $\left(\gamma_{\mathrm{ji}}\right)$ & Jul-07 - Oct-07 & Small - Large Adult & Control & Mainstem & 0.026 & 0.010 & 0.007 & 0.046 \\
\hline Lick Drain & Recruitment $\left(\gamma_{\mathrm{ji}}\right)$ & Jul-06 - Aug-06 & Small - Large Adult & Treatment & Mainstem & 0.002 & 0.001 & -0.001 & 0.005 \\
\hline Lick Drain & Recruitment $\left(\gamma_{\mathrm{ji}}\right)$ & Aug-06 - Oct-06 & Small - Large Adult & Treatment & Mainstem & 0.034 & 0.013 & 0.010 & 0.059 \\
\hline Lick Drain & Recruitment $\left(\gamma_{\mathrm{ji}}\right)$ & Oct-06 - May-07 & Small - Large Adult & Treatment & Mainstem & 0.058 & 0.020 & 0.019 & 0.098 \\
\hline Lick Drain & Recruitment $\left(\gamma_{\mathrm{ji}}\right)$ & May-07 - Jul-07 & Small - Large Adult & Treatment & Mainstem & 0.026 & 0.010 & 0.007 & 0.045 \\
\hline Lick Drain & Recruitment $\left(\gamma_{\mathrm{ji}}\right)$ & Jul-07 - Oct-07 & Small - Large Adult & Treatment & Mainstem & 0.011 & 0.005 & 0.001 & 0.021 \\
\hline Mudlick Run & Recruitment $\left(\gamma_{\mathrm{ji}}\right)$ & Jul-06 - Aug-06 & Small - Large Adult & Control & Headwater & 0.007 & 0.004 & -0.001 & 0.014 \\
\hline Mudlick Run & Recruitment $\left(\gamma_{\mathrm{ji}}\right)$ & Aug-06 - Oct-06 & Small - Large Adult & Control & Headwater & 0.104 & 0.023 & 0.059 & 0.148 \\
\hline Mudlick Run & Recruitment $\left(\gamma_{\mathrm{ji}}\right)$ & Oct-06 - May-07 & Small - Large Adult & Control & Headwater & 0.167 & 0.032 & 0.105 & 0.230 \\
\hline Mudlick Run & Recruitment $\left(\gamma_{\mathrm{ji}}\right)$ & May-07 - Jul-07 & Small - Large Adult & Control & Headwater & 0.080 & 0.020 & 0.041 & 0.120 \\
\hline Mudlick Run & Recruitment $\left(\gamma_{\mathrm{ji}}\right)$ & Jul-07 - Oct-07 & Small - Large Adult & Control & Headwater & 0.035 & 0.012 & 0.011 & 0.059 \\
\hline Tanner Run & Recruitment $\left(\gamma_{\mathrm{ji}}\right)$ & Jul-06 - Aug-06 & Small - Large Adult & Treatment & Headwater & 0.005 & 0.003 & -0.001 & 0.011 \\
\hline Tanner Run & Recruitment $\left(\gamma_{\mathrm{ji}}\right)$ & Aug-06 - Oct-06 & Small - Large Adult & Treatment & Headwater & 0.083 & 0.019 & 0.045 & 0.122 \\
\hline Tanner Run & Recruitment $\left(\gamma_{\mathrm{ji}}\right)$ & Oct-06 - May-07 & Small - Large Adult & Treatment & Headwater & 0.137 & 0.027 & 0.084 & 0.189 \\
\hline Tanner Run & Recruitment $\left(\gamma_{\mathrm{ji}}\right)$ & May-07 - Jul-07 & Small - Large Adult & Treatment & Headwater & 0.064 & 0.016 & 0.033 & 0.096 \\
\hline Tanner Run & Recruitment $\left(\gamma_{\mathrm{ji}}\right)$ & Jul-07 - Oct-07 & Small - Large Adult & Treatment & Headwater & 0.028 & 0.010 & 0.009 & 0.047 \\
\hline Zinn Hollow & Recruitment $\left(\gamma_{\mathrm{ji}}\right)$ & Jul-06 - Aug-06 & Small - Large Adult & Control & Mainstem & 0.002 & 0.001 & -0.001 & 0.004 \\
\hline Zinn Hollow & Recruitment $\left(\gamma_{\mathrm{ji}}\right)$ & Aug-06 - Oct-06 & Small - Large Adult & Control & Mainstem & 0.027 & 0.013 & 0.001 & 0.053 \\
\hline Zinn Hollow & Recruitment $\left(\gamma_{\mathrm{ji}}\right)$ & Oct-06 - May-07 & Small - Large Adult & Control & Mainstem & 0.046 & 0.021 & 0.004 & 0.087 \\
\hline Zinn Hollow & Recruitment $\left(\gamma_{\mathrm{ji}}\right)$ & May-07 - Jul-07 & Small - Large Adult & Control & Mainstem & 0.020 & 0.010 & 0.000 & 0.041 \\
\hline Zinn Hollow & Recruitment $\left(\gamma_{\mathrm{ji}}\right)$ & Jul-07 - Oct-07 & Small - Large Adult & Control & Mainstem & 0.009 & 0.005 & -0.001 & 0.018 \\
\hline Laurel Lick Run & Recruitment $\left(\gamma_{\mathrm{ji}}\right)$ & Jul-06 - Aug-06 & Small - Large Adult & Treatment & Mainstem & 0.004 & 0.002 & -0.001 & 0.009 \\
\hline
\end{tabular}




\begin{tabular}{|c|c|c|c|c|c|c|c|c|c|}
\hline Site & Variable & Interval & Age & Removal & Location & Est & SE & LCL & UCL \\
\hline Laurel Lick Run & Recruitment $\left(\gamma_{\mathrm{ji}}\right)$ & Aug-06 - Oct-06 & Small - Large Adult & Treatment & Mainstem & 0.067 & 0.016 & 0.035 & 0.099 \\
\hline Laurel Lick Run & Recruitment $\left(\gamma_{\mathrm{ji}}\right)$ & Oct-06 - May-07 & Small - Large Adult & Treatment & Mainstem & 0.110 & 0.023 & 0.065 & 0.156 \\
\hline Laurel Lick Run & Recruitment $\left(\gamma_{\mathrm{ji}}\right)$ & May-07 - Jul-07 & Small - Large Adult & Treatment & Mainstem & 0.051 & 0.013 & 0.027 & 0.076 \\
\hline Laurel Lick Run & Recruitment $\left(\gamma_{\mathrm{ji}}\right)$ & Jul-07 - Oct-07 & Small - Large Adult & Treatment & Mainstem & 0.022 & 0.008 & 0.006 & 0.038 \\
\hline Glady Fork & Recruitment $\left(\gamma_{\mathrm{ji}}\right)$ & Jul-06 - Aug-06 & Small - Large Adult & Control & Headwater & 0.008 & 0.005 & -0.002 & 0.017 \\
\hline Glady Fork & Recruitment $\left(\gamma_{\mathrm{ji}}\right)$ & Aug-06 - Oct-06 & Small - Large Adult & Control & Headwater & 0.119 & 0.036 & 0.048 & 0.190 \\
\hline Glady Fork & Recruitment $\left(\gamma_{\mathrm{ji}}\right)$ & Oct-06 - May-07 & Small - Large Adult & Control & Headwater & 0.190 & 0.052 & 0.087 & 0.292 \\
\hline Glady Fork & Recruitment $\left(\gamma_{\mathrm{ji}}\right)$ & May-07 - Jul-07 & Small - Large Adult & Control & Headwater & 0.092 & 0.031 & 0.032 & 0.152 \\
\hline Glady Fork & Recruitment $\left(\gamma_{\mathrm{ji}}\right)$ & Jul-07 - Oct-07 & Small - Large Adult & Control & Headwater & 0.041 & 0.017 & 0.007 & 0.075 \\
\hline Daniel's Run & Recruitment $\left(\gamma_{\mathrm{ji}}\right)$ & Jul-06 - Aug-06 & Small - Large Adult & Treatment & Headwater & 0.013 & 0.007 & -0.002 & 0.028 \\
\hline Daniel's Run & Recruitment $\left(\gamma_{\mathrm{ji}}\right)$ & Aug-06 - Oct-06 & Small - Large Adult & Treatment & Headwater & 0.187 & 0.039 & 0.110 & 0.264 \\
\hline Daniel's Run & Recruitment $\left(\gamma_{\mathrm{ji}}\right)$ & Oct-06 - May-07 & Small - Large Adult & Treatment & Headwater & 0.285 & 0.046 & 0.195 & 0.376 \\
\hline Daniel's Run & Recruitment $\left(\gamma_{\mathrm{ji}}\right)$ & May-07 - Jul-07 & Small - Large Adult & Treatment & Headwater & 0.148 & 0.032 & 0.085 & 0.211 \\
\hline Daniel's Run & Recruitment $\left(\gamma_{\mathrm{ji}}\right)$ & Jul-07 - Oct-07 & Small - Large Adult & Treatment & Headwater & 0.068 & 0.023 & 0.023 & 0.112 \\
\hline
\end{tabular}

